Günter Ott

Internationale

Verteilungswirkungen im Finanzausgleich

Gemeinschaften

Europäischen

Gemeinschaften 


\author{
Günter Ott
}

\title{
Internationale Verteilungswirkungen im Finanzausgleich Gemeinschaften Europäischen Gemeinschaften
}

Ein seit langem ungelöstes Problem der EG ist eine allgemein akzeptierte Verteilung der Finanzierungslasten und Ausgaben auf die Mitgliedsstaaten. Dabei sind die Fragen nach den Größenordnungen der Distributionswirkungen sowie nach einem normativen Rahmen immer wieder von aktuellem politischen Interesse. Die vorliegende Studie versucht nun, zum einen genauere und umfassendere empirische Ergebnisse als bisher zu liefern. Zum anderen werden diese Verteilungswirkungen anhand der für die Finanzwirtschaft der Gemeinschaft ableitbaren Zielsetzungen und postulierbaren normativen Maßstäben beurteilt und wird die Richtung möglicher Korrekturen aufgezeigt.

Günter Ott wurde 1946 in Neumünster geboren. Nach Volksschulabschluß Banklehre; anschließend neben der Berufstätigkeit Abitur am Abendgymnasium. Danach Studium der Volkswirtschaft an der Christian-Albrechts-Universität Kiel mit abschließender Diplom-Prüfung. 1977 Wissenschaftlicher Angestellter und nach Promotion 1981 Hochschulassistent am Institut für Finanzwissenschaft der Universität Kiel. Seit 1985 Direktor der Hanseatischen Sparkassenschule in Hamburg. 
Internationale Verteilungswirkungen im Finanzausgleich der Europäischen Gemeinschaften 


\section{FINANZWISSENSCHAFTLICHE SCHRIFTEN}

Herausgegeben von den Professoren

Albers, Krause-Junk, Littmann, Oberhauser, Pohmer, Schmidt

Band 34

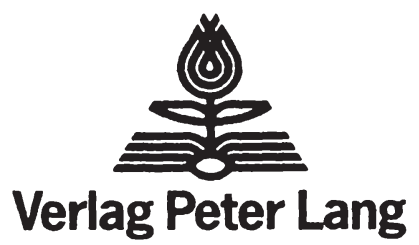

Frankfurt am Main · Bern · New York · Paris 


\section{Günter Ott}

\section{Internationale Verteilungswirkungen im Finanzausgleich der Europäischen Gemeinschaften}

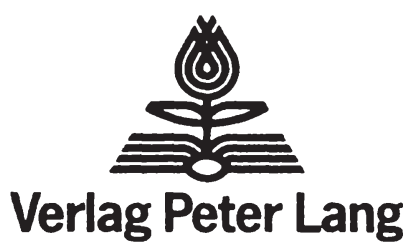

Frankfurt am Main - Bern - New York - Paris 
CIP-Kurztitelaufnahme der Deutschen Bibliothek

\title{
Ott, Günter:
}

Internationale Verteilungswirkungen im Finanzausgleich

der Europäischen Gemeinschaften / Günter Ott. -

Frankfurt am Main ; Bern ; New York ; Paris :

Lang, 1987.

(Finanzwissenschaftliche Schriften ; Bd. 34)

ISBN 3-8204-0196-2

NE: GT

Open Access: The online version of this publication is published on www.peterlang.com and www.econstor.eu under the international Creative Commons License CC-BY 4.0. Learn more on how you can use and share this work: http://creativecommons. org/licenses/by/4.0.

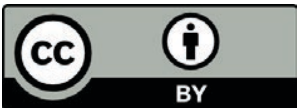

This book is available Open Access thanks to the kind support of ZBW - Leibniz-Informationszentrum Wirtschaft.

\author{
ISSN 0170-8252 \\ ISBN 3-8204-0196-2 \\ ISBN 978-3-631-75256-2 (eBook) \\ (c) Verlag Peter Lang GmbH, Frankfurt am Main 1987 \\ Alle Rechte vorbehalten.
}

Das Werk einschließlich aller seiner Teile ist urheberrechtlich geschützt. Jede Verwertung außerhalb der engen Grenzen des Urheberrechtsgesetzes ist ohne Zustimmung des Verlages unzulässig und strafbar. Das gilt insbesondere für Vervielfältigungen, Übersetzungen, Mikroverfilmungen und die Einspeicherung und Verarbeitung in elektronischen Systemen.

Druck und Bindung: Weihert-Druck GmbH, Darmstadt 


\section{Vorwort}

Die vorliegende Arbeit entstand größtenteils während meiner Tätigkeit als Hochschulassistent am Institut für Finanzwissenschaft der Christian-Albrechts-Universität Kiel. Sie stellt die erweiterte und aktualisierte Fassung einer Studie über 'Die Nettopositionen als Indikator der nationalen Vor- und Nachteile der europäischen Integration' dar, die durch ein Forschungsstipendium der Kommission der Europäischen Gemeinschaften gefördert wurde.

Für diese Unterstützung sowie die zahlreichen Gespräche mit Mitarbeitern der Kommission - hier möchte ich insbesondere Herrn Gerhard Hipp von der Generaldirektion Haushalt hervorheben - bin ich sehr zu Dank verpflichtet. Ein besonderer Dank gilt auch meinen akademischen Lehrern, Herrn Prof. Dr. W. Albers, Herrn Prof. Dr. W. Kitterer und Herrn Prof. Dr. R. Peffekoven für wertvolle Verbesserungsvorschläge bei der kritischen Durchsicht des Manuskripts. 
Günter Ott - 978-3-631-75256-2

Downloaded from PubFactory at 01/11/2019 06:38:19AM

via free access 


\section{Inhalt}

Inhaltsverzeichnis

Verzeichnis der Tabellen 5

Verzeichnis der Abbildungen 9

Verzeichnis der Abkürzungen $\quad 10$

1. Einleitung

2. Theoretisch-methodische Grundfragen der Inzidenzanalyse

2.1. Abgrenzung des Untersuchungsgegenstandes

2.2. Ansätze zur verteilungspolitischen Ausgestaltung von Finanzausgleichssystemen

2.3. Verteilungspolitische Zielsetzungen der EG

2.4. Inzidenzanalyse unter dem Meßaspekt

2.4.1. Inzidenzkonzepte

2.4.2. Inzidenzmaßstab

2.4.3. Zeitliche Dimension und rechnungsmäß1ge Abgrenzung der Analyse

3. Das Konzept der Nettopositionen zur Erfassung der innergemeinschaftlichen $\mathrm{Z}$ ahlungsströme

4. Verteilungswirkungen der finanzwirtschaftlichen Gemeinschaftstätigkeit

4.1. Gesamthaushalt

4.1.1. Finanzierungssystem

4.1.1.1. Mehrwertsteuer-Eigenmittel

4.1.1.1.1. Determinanten der mitgliedsstaatlichen Verteilung

4.1.1.1.2. Probleme der verwaltungstechnischen Erfassung und Abwicklung

4.1.1.2. Zölle 
4.1.1.2.1. Handels- und währungspolitische Bestimmungsgründe der mitgliedsstaatlichen Verteilung

4.1.1.2.2. Verteilungseffekte der Erfassung und Verwaltung

4.1.1.2.3. Probleme des formalen Verteilungsansatzes

4.1.1.3. Agrarabschöpfungen

102
4.1.1.3.1. Agrar- und wirtschafts- politische Bestimmungs- grinde der mitglieds- staatlichen Verteilung

106

4.1.1.3.2. Probleme des formalen Verteilungsansatzes

4.1.1.4. Zuckerabgaben

4.1.1.5. Sonstige Einnahmen

4.1.1.6. Gesamte Eigenmittel

4.1.2. Korrekturmechanismen und Entlastungsregelungen

4.1.2.1. Der Dubliner Korrekturmechanismus

4.1.2.2. Der Brlisseler KompromiB

4.1.2.3. We1tere Ubergangsregelungen

4.1.3.1. Agrarfonds - Abteilung Garantie

4.1.3.1.1. Orqanisation und Instrumentarium der Gemeinsamen Agrarpolitik

4.1.3.1.2. Mitgliedsstaatliche Ausgabenverteilung

4.1.3.1.3. Problemat1k des formalen Vertellungsansatzes 
4.1.3.1.3.1. Exporterstattungen 165

4.1.3.1.3.2. Lagerhaltungskosten

4.1.3.1.3.3. Währungsausgleichsbeträge

4.1.3.1.3.4. Zurechnung nach Maßqabe der landwirtschaftlichen Produktion

4.1.3.1.4. Exkurs: Außerbudgetäre Verte1lungswirkungen des innergemeinschaftlichen Agrarhandels

4.1.3.2. Interventionen im Bereich der Strukturpolitik

4.1.3.2.1. Agrarfonds - Abteilung Ausrichtung

4.1.3.2.2. Europä1scher Fonds für regionale Entwicklung

$4 \cdot 1 \cdot 3 \cdot 2 \cdot 3$. Europä1scher Sozialfonds

4.1.3.2.4. Spezifische Maßnahmen

4.1.3.3. Ausgaben fur Forschung, Energie, Wirtschaft und Verkehr

4.1.3.4. Entwicklungshilfe

4.1.3.5. Erstattungen an die Mitgliedsstaaten

4.1.3.6. Verwaltungsausgaben

4.2. Außerbudgetäre Aktivitäten

4.2.1. Europäischer Entwicklungsfonds

4.2.2. Funktionshaushalt der Europäischen Gemeinschaft für Kohle und Stahl

4.2.3. Anleihe- und Darlehenstransaktionen 
4.2.3.1. Mitgliedsstaatliche Verteilung der Kapitaltransaktionen

4.2.3.2. Mitgliedsstaatliche Verteilungsund Belastungswirkungen

5. Erweiterter Maßstab der finanzwirtschaftlichen Vertellungswirkungen

6. Normative Ansätze zur Beurteilung der Verteilungswirkungen

6.1. Vergleich der Nettopositionen mit der nationalen Wirtschaftskraft

6.2. Verminderung der Differenzen zwischen den mitgliedsstaatlichen Pro-Kopf Sozialprodukten als Maßstab normativer Umverteilungskraft

7. Schlußbemerkungen 


\section{Verzeichnis der Tabelien}

Tabelle 1: Bruttoinlandsprodukte der Mitgliedsstaaten $1980-1984$

Tabelle 2: Nettopositionen der Mitgliedsstaaten 1976 - 1984

Tabelle 3: Finanztätigkeit der EG 1975 - 1984

Tabelle 4: Einnahmen des EG-Gesamthaushalts 1975 - 1984

Tabelle 5: Mehrwertsteuer-Elgenmittel nach Mitgliedsstaaten 1980 - 1984

Tabelle 6: Mehrwertsteuer-Eigenmittel in Relation zu den Bruttoinlandsprodukten der Mitgliedsstaaten 1980 - 1984

Tabelle 7: Regression der Mehrwertsteuerbelastung pro Kopf in bezug auf das Pro-Kopf-BIP der Mitgliedsstaaten 1980 - 1984

Tabelle 8: Mehrwertsteuerbelastung und gesamtwirtschaftliche Größen der Mitgliedsstaaten 1982

Tabelle 9: Inflationsraten und Wechselkurse der Mitgliedsstaaten 1980 - 1984

Tabelle 10: Zolleinnahmen nach Mitgliedsstaaten $1980-1984$

Tabelle 11: Importstruktur und Zollaufkommen der Mitglledsstaaten 1983

Tabelle 12: Zollabführungen in Relation zu den Bruttoinlandsprodukten der Mitgliedsstaaten $1980-1984$

Tabelle 13: Ausgefallene Zollerträge für die Bundesrepublik Deutschland 1980 - 1984

Tabelle 14: Agrarabschöpfungen nach M1tgliedsstaaten $1980-1984$

Tabelle 15: Agrarabschöpfungen und Drittlandsimporte von Getreide nach Mitgliedsstaaten 1982

Tabelle 16: Agrarabschöpfungen in Relation zu den Bruttoinlandsprodukten der Mitgliedsstaaten $1980-1984$ 
Tabelle 17: Ausgefallene Agrarabschöpfungen für die Bundesrepublik Deutschland 1980 - 1984

Tabelle 18: Zucker- und Isoglucoseabgaben nach Mitgliedsstaaten 1980 - 1984

Tabelle 19: Eigenmittel nach Mitgliedsstaaten $1980-1984$

Tabelle 20: Eigenmittel in Relation zu den Bruttoinlandsprodukten der Mitgliedsstaaten $1980-1984$

Tabelle 21: Regression der Finanzierungsanteile in bezug auf die BIP-Anteile der Mitgliedsstaaten $1980-1984$

Tabelle 22: Erfüllung bzw. Nichterfüllung der Voraussetzungen des Korrektur- und Finanzmechanismus durch das Vereinigte Königreich

Tabelle 23: Vorausgeschätzter und tatsächlicher Ausgleich zugunsten des Verelnigten Könlqreichs 1980 und 1981

Tabelle 24: Ausgaben (Zahlungen) des EG-Gesamthaushalts $1975-1984$

Tabelle 25: Ausgaben des EAGFL-Garantie nach wirtschaftlichen Arten und Erzeugnissen 1980 - 1984

Tabelle 26: Ausgaben des EAGFL-Garantle nach M1tgliedsstaaten 1980 - 1984

Tabelle 27: Landwirtschaftliche Enderzeugung nach Mitgliedsstaaten und im Verhältnis zu den Marktordnungsausgaben 1980 - 1984

Tabelle 28: Exporterstattungen, Produktion und Selbstversorgungsgrad ausgewählter Agrarerzeugnisse nach Mitgliedsstaaten 1980 - 1983

Tabelle 29: Lagerhaltungskosten, Produktion und Selbstversorgungsgrad ausgewählter Agrarerzeugnisse nach Mitglledsstaaten 1980 - 1983

Tabelle 30: Im Handelsverkehr angewendete WAB-Sätze $1980-1984$

Tabelle 31: Währungsausgleichsbeträge der M1tgliedsstaaten 1983 
Tabelle 32: Verteilung der EAGFL-Garantleausgaben nach der landwirtschaftlichen Enderzeuqung und Differenzen zur Verteilung der Zahlungsströme $1980-1984$

Tabelle 33: Ausgaben des EAGFL-Ausrichtung nach Mitgliedsstaaten $1980-1984$

Tabelle 34: Länderquoten im Regionalfonds

Tabelle 35: Ausgaben des Regionalfonds nach Mitgliedsstaaten 1980 - 1984

Tabelle 36: Ausgaben des Sozialfonds nach Mitgliedsstaaten $1980-1984$

Tabelle 37: Zinszuschüsse des Gesamthaushalts $1980-1984$

Tabelle 38: Ausgaben im Zusammenhang mit den britischen Entlastungsmaßnahmen - Ausführung und Auswirkungen auf die Nettopositionen $1980-1984$

Tabelle 39: Ausgaben für speziflsche Maßnahmen im Sozialbereich 1980 - 1984

Tabelle 40: Ausgaben für Forschung, Energie, Wirtschaft und Verkehr nach Mitgliedsstaaten 1980 - 1984

Tabelle 41: Leistungen der EG für die Zusammenarbeit mit Entwicklungsländern und Drittländern $1980-1984$

Tabelle 42: Erstattungen an die Mitqliedsstaten $1980-1984$

Tabelle 43: Verwaltungsalisgaben 1980 - 1984

Tabelle 44: Auswirkungen elnes veränderten Zurechnungsverfahrens der Verwaltungsausqaben auf die Höhe der Nettopositionen 1980 - 1984

Tabelle 45: Finanzierung des 5. FEF nach Beltragsschlüssel und nach Maßgabe der Mehrwertsteuer-Antelle

Tabelle 46: Aufschlüsselung der vom 5. EEF finanzierten Aufträge nach Nationalitäten. Stand: 31. Dez. 1982

Tabelle 47: Ausführung der Funktionshaushaltspläne der EGKS 1981 - 1984

Tabel le 48: Aufkommen aus der F.GKS-Umlage nach Mitgliedsstaaten 1981 - 1984 
Tabelle 49: Anpassungsbeihilfen der EGKS nach Mitgliedsstaaten 1981 - 1984

Tabelle 50: Sozialmaßnahmen zur Umstrukturierung der Eisen- und Stahlindustrie nach Mitqliedsstaaten 1981 - 1984

Tabelle 51: EGKS-Zinszuschüsse nach Mitgliedsstaaten $1981-1984$

256

Tabelle 52: Umverteilungswirkungen ausgewählter Einnahmen und Ausgaben des EGKS-Funktionshaushalts 1983

Tabelle 53: Anleihe- und Darlehenstätiqkeit der EG 1980 - 1984

Tabelle 54: Gemeinschaftsanlelhen nach Anleihewährungen $1980-1984$

Tabelle 55: Gemeinschaftsdarlehen nach Empfängerländern $1980-1984$

268

Tabelle 56: Zusammensetzung des EIB-Kapitals

270

Tabelle 57: Ausstehende EG-Darlehen nach Mitgliedsstaaten 277 $1980-1983$

Tabelle 58: Korrekturen der traditionellen Nettopositionen 1983

Tabelle 59: Traditionelle und korrigierte Nettopositionen 1983

Tabelle 60: Mitgliedsstaatliche Bruttoinlandsprodukte und Nettopositionen pro Kopf 1983

Tabelle 61: Normative und reale Nettopositionen 1983

Tabelle 62: Normative Nettopositionen der erweiterten Gemeinschaft auf der Basis des Jahres 1983 


\section{Verzelchnis der Abbildungen}

Abbildung 1: Mehrwertsteuerbelastung der Pro-Kopf Bruttoinlandsprodukte der Mitaliedsstaaten

Abbildung 2: Transfers im innergemeinschaftlichen Handel mit ausgewählten Agrarprodukten 1976/77 $1979 / 80$ 


\section{Verzeichnis der Abkürzungen}

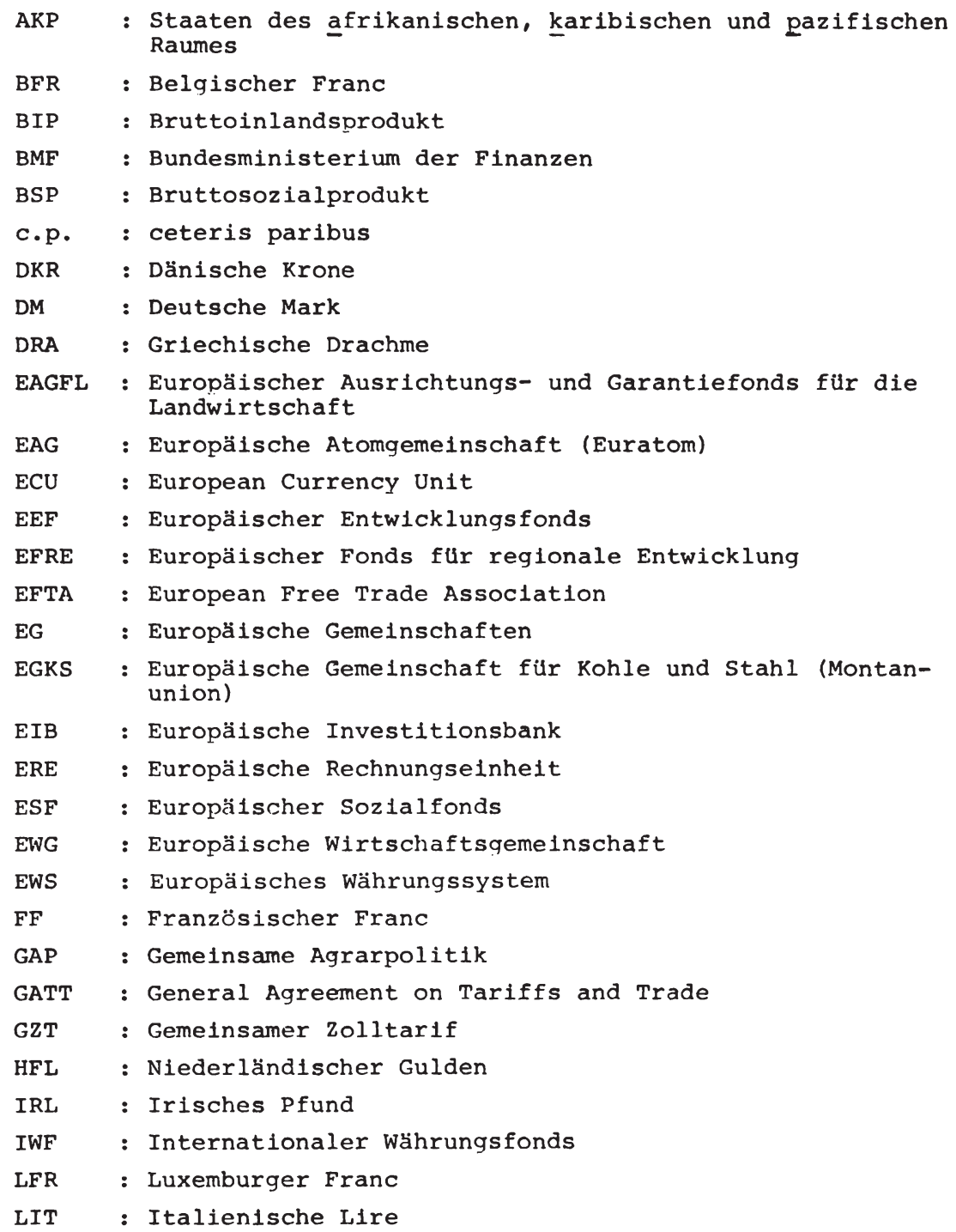




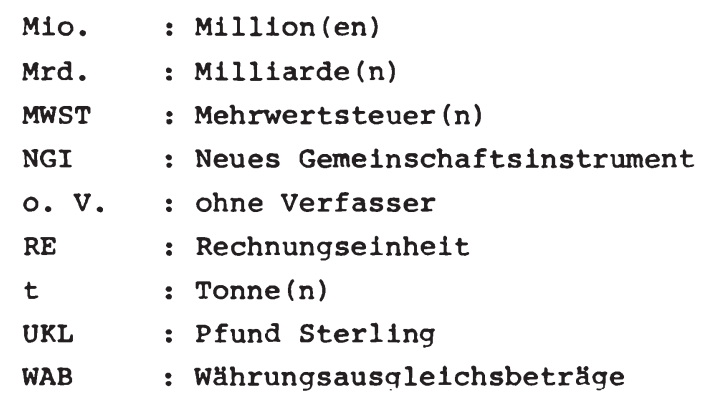




\section{Einleitung}

Die Diskussion uber die budgetären Ungleichgewichte im Finanzierungs- und Ausqabensystem der Europäischen Gemeinschaften (EG) und Versuche ihrer Korrektur haben bereits eine lange Tradition. Die zugrunde liegende Problematik entstand schon zu Beginn der siebziger Jahre mit dem britischen Finanzierungsproblem; mehr und mehr trat aber auch die Stellung der Bundesrepublik Deutschland als vielzitierter Zahlmeister der EG hinzu. Angesichts der erneuten Finanzierungsschwierigkeiten sowie der dritten Erweitung der Gemeinschaft um Spanien und Portugal werden diese Fragen eine noch weiterreichende Dimension gewinnen und erscheinen grundlegende Lösungen immer dringlicher.

In diesem Zusammenhang sind vor allem zwei Problemfelder von besonderem Interesse:

- welche Größenordnungen weisen die Verteilungswirkungen der finanzwirksamen Gemeinschaftstätigkeit tatsächlich auf und

- welcher normative Rahmen läßt sich aufstellen, an dem die aktuellen Verteilungswirkungen beurteilt werden können und nach dem sie gegebenenfalls zu korrigieren sind?

Diese Fragen werden stets von dem Tatbestand überlagert, daß die im Gemeinschaftshaushalt erfaßten Finanzierungs- und Ausgabenströme nur elnen Tellaspekt der europäischen Integration darstellen. Daneben werden auf dem handels- und wirtschaftspolitischen Gebiet sowie im allgemeinpolitischen Bereich bedeutsame Ergebnisse erzielt, die die unmittelbar finanziellen Wirkungen möglicherweise in den Hintergrund drängen, sie aber zumindest relativieren.

Eine solche Sichtweise ist allerdings im gewissen Sinne auch umkehrbar, denn die mitgliedsstaatliche verteilung der Finanzierungsund Ausgabenströme dürfte auch Rückwirkungen auf die Zusammenarbeit 
in den übrigen Bereichen der Gemeinschaftspolitik haben, und scheinen dauerhafte Integrationsfortschritte nur möglich, wenn auch die Struktur der finanziellen Vorteile und Belastungen allgemein gültigen und akzeptierten Prinziplen genügt. In jedem Fall hat sich gezeigt, daß in der politischen Auseinandersetzung haushalts- und finanzpolitische Probleme einen zentralen Stellenwert für die weitere Entwicklung der Gemeinschaft besitzen, so daß es allein von daher ebenso geboten wie gerechtfertigt erscheint, diesen Fragen auch isoliert, d.h. unabhängig von den übrigen Feldern der Gemeinschaftspolitik nachzugehen.

Als Maßstab für die Verteilungswirkungen der finanzwirksamen Gemeinschaftspolitik werden stets die sogenannten Nettopositionen der Mitgliedsstaaten herangezogen. Dies sind die Differenzen aus den Finanzierungsleistungen der Gemeinschaftsländer an den Gesamthaushalt der EG und den daraus empfangenen Rückflüssen. Dabei handelt es sich allerdings um rein formale, an Zahlungsvorgänge anknüpfende Zurechnungen der Haushaltseinnahmen und -ausgaben, die nicht immer den tatsăchlichen auf die Mitgliedsstaaten entfallenden Belastungen und Begünstigungen entsprechen, und die sich zudem auf den Haushalt der EG beschränken. Diese Größen sind jedoch gegenwärtig der einzig quantitativ erfaßbare Indikator der finanzwirksamen Vor- und Nachteile der Gemeinschaft.

Die vorliegende Arbeit verfolgt nun zum einen die Zielsetzung, die Aussagefähigkeit des bisherigen Konzepts der Nettopositionen zu verbessern bzw. zu erweitern, um damit sowohl zu einem genaueren als auch umfassenderen Indikator zu gelangen. Dazu sind die im Rahmen der finanzwirtschaftlichen Ebene liegenden Schwächen in der zurechnung der Einnahmen und Ausgaben auf die Mitgliedsstaaten abzubauen und daneben die außerbudgetären Aktivitäten in die Betrachtung einzubeziehen. 
Auf dieser Grundlage soll dann versucht werden, die aktuelle Verteilungsstruktur anhand der für die Finanzwirtschaft der EG geltenden $\mathrm{zielsetzungen} \mathrm{und} \mathrm{postulierbaren} \mathrm{normativen} \mathrm{Maßstäbe} z u$ beurteilen und die Richtung möglicher Korrekturen aufuzeigen. Damit werden grundlegende Fragen des internationalen Finanzausgleichs und der ihm zugrundeliegenden Prinzipien angesprochen, zu deren methodischer Vertiefung und anwendungsbezogener Umsetzung diese Arbeit beitragen soll.

Ein solches Vorhaben erfordert zunächst die Behandlung der für die empirische Analyse relevanten theoretisch-methodischen Grundfragen. Diese betreffen die Abgrenzung des Untersuchungsgegenstandes, allgemeine Ansătze zur verteilungspolitischen Ausgestaltung von Finanzlerungs- und Ausgabensystemen sowie speziell die für die EG ableitbaren Verteilungszielsetzungen; daneben Fragen des Meßaspekts der Inzidenzanalyse. Als Ausgangspunkt für die quantitative Untersuchung ist dann das bisher verwendete Konzept zur Ermittlung der Nettopositionen darzustellen. Danach erfolgt eine umfassende Verteilungsanalyse der einzelnen Einnahmen- und Ausgabenpositionen des Gesamthaushalts mit der doppelten Zielrichtung, die jeweiligen Verteilungswirkungen aufzuzeigen sowie generell die im Konzept der Nettopositionen durchgeführten Berechnungen auf ihre zuverlässigkeit hin zu uberprüfen. Im AnschluB daran werden auch die im Rahmen der Nettopositionen bisher nicht berücksichtigten Verteilungswirkungen der außerbudgetären Aktivitäten zu ermitteln versucht. Als Ergebnis erhält man damit einen im Vergleich zu den derzeit vorliegenden Informationen verbesserten und umfassenderen Indikator für die Verteilungswirkungen der finanzwirksamen Aktivitäten der EG und damit gleichzeitig einen erweiterten Ansatz zur Beurteilung der nationalen Vor- und Nachteile der europäischen Integration. 
Dle Analyse dieser Zusammenhänge kann vor allem an die jüngsten Arbeiten von May ${ }^{1}$, Nottelmann ${ }^{2}$, M $l \operatorname{ler}^{3}$ und Petersen ${ }^{4}$ anknüpfen, die teilweise einen umfassenden Kosten-Nutzen-Begriff zugrunde legen, dafür aber den finanzwirtschaftlichen Aspekt zwangsläufig weniger intensiv behandeln (May), teils eine sehr geraffte Darstellung bieten (Nottelmann), vornehmlich auf die Agrarpolitik ausqerichtet sind (Petersen), aber auch die finanzwirksamen Aktivitäten der Gemeinschaft ausführlich analysieren (Mфller). Die vorliegende Arbelt unterscheidet sich von diesen Untersuchungen zunächst durch eine aktualisierte Darstellung sowie durch eine unterschiedliche Intensität bei der Behandlung der verteilungsrelevanten Fragestellungen und liefert damit eine Reihe zusätzlicher Ergebnisse. Vor allem aber geht sie tuber die vorhandenen Arbeiten hinaus, indem versucht wird, zumindest ansatzweise die eingangs angesprochenen, bisher ungeklärten Fragen der Zurechnung bestimmter Einnahmen- und Ausgabenarten des Gemeinschaftshaushalts auf die Mitgliedsstaaten zu lösen, und indem auch die außerbudgetären Aktivitäten der Gemeinschaft im Hinblick auf ihre Verteilungswirkungen analysiert und in den Gesamtzusammenhang einbezogen werden.

Die abschließende Uberprüfung der so ermittelten Verteilungswirkungen an geeigneten Referenzsystemen stellt zunächst auf die in den nationalen Sozialprodukten zum Ausdruck kommende Wirtschaftskraft der Mitgliedsländer ab. Daran anschließend wird ein Ansatz der Sachverständigengruppe zur Untersuchung der Rolle der öffentlichen

${ }^{1}$ B. May, Kosten und Nutzen der deutschen EG-Mitgliedschaft, Bonn 1982.

2A. Nottelmann, Der Haushalt der Europäischen Gemeinschaften Probleme und Reformvorstellungen -, Hamburg 1982.

${ }^{3} \mathrm{~J}$. M $\not 1 l e r$, Member States and the Community Budget, Kopenhagen 1982 .

${ }^{4}$ v. Petersen, Agrarpolitik und EG-Haushalt, Gemeinsame Agrarpolitik und der Haushalt der Europäischen Gemeinschaft, Eine Untersuchung des Finanztransfers innerhalb der EG sowie Analyse alternativer Finanzmodelle, Schriftenreihe des Bundesministers für Ernähruna, Landwirtschaft und Forsten, Reihe A: Angewandte Wissenschaft Heft 278 , Münster-Hiltrup 1983. 
Finanzen bei der Europäischen Integration (MacDougal1-Kommission) ${ }^{1}$ zur Messung der nationalen Umverteilungskraft, der von Reichenbach ${ }^{2}$ zu einem konzeptionellen Rahmen normativer Redistributionseffekte weiterentwickelt wurde, aufgegriffen und auf die aktuellen Größenordnungen der finanziellen Verteilungswirkungen angewerdet.

${ }^{1}$ Bericht der Sachverständigengruppe zur Untersuchung der Rolle der offentlichen Finanzen bei der Europäischen Integration, hrsg. von der Kommission der Europäischen Gemeinschaften, Brüssel 1977, Bd. II, Einzelbeitrăge und Arbeitsunterlagen, Brüssel 1977, S. $140 \mathrm{ff}$

${ }^{2}$ H. Relchenbach, Les déséquilibres des flux budgétaires, in: Revue francalse de Finances publiques, 1983, S. $35 \mathrm{ff.;}$ ders., EC Budgetary Imbalances: A Conceptual Framework, in: Finanzarchiv, N.F. Bd. 43, 1984, S. 452 ff. 


\section{Theoret1sch-methodische Grundfragen der Inzidenzanalyse}

Vor einer empirischen Untersuchung ist der Rahmen $z u$ beschreiben, innerhalb dessen die Analyse erfolgen soll. Dazu gehören zum einen die Prinzipien und zielsetzungen, an denen die Verteilungswirkungen gemessen und anhand derer sie beurteilt werden. Daneben ist aufzuzeigen, was die Analvse zu leisten vermaq und wo ihre Grenzen lieaen.

\subsection{Abgrenzung des Untersuchungsgegenstandes}

Das Spektrum der Vor- und Nachteile einer EG-Mitgliedschaft umfaBt die finanzwirtschaftliche, die handels- und wirtschaftspolitische und die allgemeinpolitische Ebene. Diese Determinanten sind im folgenden uberblickartig zu beschreiben, um den Untersuchungsgegenstand der vorliegenden Arbeit abzugrenzen, exinzuordnen und damit von vornherein $\mathrm{zu}$ relativieren.

Betrachtet man zunächst die finanzwirtschaftliche Ebene, so fallen hierunter vor allem die über den Gemeinschaftshaushalt abgewickelten Transaktionen, deren Salden die Nettopositionen in ihrer gegenwärtig ermittelten Form darstellen. Dabei handelt es sich allerdings um rein formale, an Zahlungsvorgänge anknüpfende Zurechnungen der Haushaltseinnahmen und -ausgaben, die nicht immer den tatsächlichen auf die Mitgliedsstaaten entfallenden Belastungen und Begünstigungen entsprechen. Solche Abweichungen resultieren etwa daraus, daß die mit dem Drittlandshandel verbundenen Einnahmen und Ausgaben in der Weise abgerechnet werden, daß die Mittelabführungen an den Gemeinschaftshaushalt nicht immer gleichzeitig auch Belastungen der Mitgliedsstaaten darstellen und die Rückflüsse nicht stets mit entsprechenden Vortellen gleichzusetzen sind. Weiterhin bedeutet die Ausgabenzurechnung nach dem Zahlungsprinzip, daß Mitgliedsländerndort Vorteile zugewiesen werden, wo die Ausgaben auch von gemeinschaftlichem Nutzen sind, 
also auch die übrigen Gemeinschaftsstaten daran partizipieren.

Eine Verteilungsanalyse der finanzwirtschaftlichen Ebene hat nicht nur die über den Gemeinschaftshaushalt ausgeführten Transaktionen, sondern auch die auBerbudgetären Aktivitäten zu berücksichtigen. Dazu gehören vor allem die in den letzten Jahren zunehmend stärker ausgeweiteten Anleihe- und Darlehenstransaktionen, aber auch die uber einen besonderen Haushalt bzw. Fonds abgewickelten Finanztätigkeiten der Europäischen Gemeinschaft für Kohle und Stahl (Montanunion) und des Europäischen Entwicklungsfonds.

Unter der wirtschaftspolitischen Ebene könnendie nicht unmittelbar finanz- bzw. ausgabenwirksamen Aktivitäten im Rahmen der gemeinschaftlichen Bestrebungen nach einer Wirtschafts- und währungsunion zusammengefaßt werden. Darunter fallen zunächst die Regelungen für einen freien Waren-, Personen- und Kapitalverkehr innerhalb der Gemeinschaft sowle die ökonomischen Auswirkungen des Europäischen wăhrungssystems (EWS). Daneben versucht die Gemeinschaft eine Koordination der elnzelstaatlichen Wirtschaftspolitiken und besitzt Kompetenzen in zahlreichen Politikbereichen. Neben den vorwiegend ausgabenwirksamen Feldern der Agrar-, Regional- und Sozialpolitik erfolgen etwa auf den Gebieten der Wettbewerbs-, Verkehrs-, Industrie- und Umweltpolitik Bemühungen um gemeinschaftliche Aktionen, ohne daß damit unmittelbar finanzielle Auswirkungen verbunden sind. Auch wenn diese Bestrebungen zumeist noch in Ansätzen stecken und praktisch nicht monetär quantifizierbar sind, können die daraus resultierenden Effekte in einer umfassenden Kosten-Nutzen-Analyse nicht vernachlässigt werden.

Als Sonderfall der ökonomischen Wirkungen lassen sich auf der handelspolit1schen Ebene die mit dem Zusammenschluß der Mitgliedsstaaten $z u$ elner Zollunion verbundenen Effekte einordnen. 1

${ }^{1}$ Die folgenden Ausführungen sind entnommen aus G. Ot.t, Zur Diskussion um "Zahlmeister" und "Nutznießer" der Europäischen Gemeinschaften, in: W.A.S. Koch, H.-G. Petersen (Hrsg.), Staat, Steuern und Finanzausgleich, Probleme nationaler und internationaler Finanzwirtschaften im zeitlichen Wandel, Festschrift für Heinz Kolms zum 70. Geburtstag, Berlin 1984, S. $333 \mathrm{ff.,} \mathrm{hier:} \mathrm{S.} 349 \mathrm{ff}$. 
Nach der traditionellen Theorie ${ }^{1}$ sind mit der Bildung einer 2ollunion grundsätzlich handelsschaffende (trade creation) und handelsumlenkende (trade diversion) Effekte verbunden. Dabel wird unter Handelsschaffung jener Aufschließungseffekt verstanden, durch den die Produktion zum kostengünstigeren Land innerhalb einer Zollunion wandert und über erhöhte Arbeitsteilung und Produktivitätssteigerungen positive Wohlfahrtseffekte entstehen. Mit Handel,sumlenkung ist der Abschließungseffekt aufgrund des gemeinsamen AuBenzolls gemeint, bei dem die Produktion eines kostenungünstigeren Landes innerhalb der Zollunion an die Stelle eines vergleichsweise kostengünstigeren Drittlandes tritt. Die Differenz zwischen den handelsschaffenden und -umlenkenden Wirkungen wird als wohlfahrtseffekt der Zollunion interpretiert. Uber diese sog. statischen Wirkungen hinaus können sich auch weitere, dynamische Effekte ergeben. Als solche werden vor allem die aus der Ausdehnuna der Betriebsgrößen resultierende Kostendegression (economies of scale) und die mit einer höheren Wettbewerbsintensität auf einem nun größeren gemeinsamen Markt slch einstellende effizientere produktion angesehen, ${ }^{2}$ die insgesamt auch das wirtschaftliche wachstum positiv beeinflussen können. 3

Zur empirischen Uberprüfung dieser Sachverhalte wird häufig ein Vergleich der Entwicklung des Intrahandels mit der des Extrahandels herangezogen. Danach scheint die EG offenbar ein Musterbeispiel für

${ }^{1}$ Zuruckgehend vor allem auf J. Viner, The Customs Union Issue, New York 1950 sowie J. E. Meade, The Theory of Customs Unions, Amsterdam 1955 und R. G. Lipsey, The Theory of Customs Unions: Trade Diversion and Welfare, in: Economica, 5/1957, S. $40 \mathrm{ff}$.

2 Dabei ist allerdings nicht auszuschließen, daB sich innerhalb des erweiterten zollfreien Gebiets neue und auch größere monopolistische Marktsituationen bilden.

${ }^{3}$ Vgl. hierzu etwa H. Siebert, Zölle IV: Zollunion und Präferenzzonen, in: Handwörterbuch der Wirtschaftswissenschaft (HdWW), Bd. 9, Stuttgart, New York u.a. 1982, S. $666 \mathrm{ff}$. 
die Außenhandelswirkungen einer Zollunion zu sein. ${ }^{1}$ Denn der Intrahandel expandierte wesentlich stärker als der Handel mit Drittländern und der Anteil der innergemeinschaftlichen Exporte bzw. Importe an der Gesamtausfuhr (-einfuhr) stieg von 1958 bis Anfang der achtziger Jahre von ungefähr $35 \%$ auf jeweils etwa $50 \%$ an. ${ }^{2}$ Auch eine nach Agrar- und Industrieerzeugnissen differenzierende Betrachtung der gehandelten Güter scheint den Uberlegungen der Zolltheorie $z u$ entsprechen: Die im Vergleich zum Industriesektor wesentlich geringeren Zuwachsraten des Agrarhandels mit Drittländern sind danach teilweise als handelsumlenkende Wirkunoen anzusehen, die auf den protektionistischen Charakter des F.G-Acrarsystems zuriickzuführen sind.

Zu dem Ergebnis, daß seit der Gründung der Zollunion überwiegend handelsschaffende Effekte entstanden sind, kommt auch eine Reihe anderer, auf unterschiedlichen Ansätzen beruhender Untersuchungen. ${ }^{3}$ Allerdings sind solche Resultate mit Vorsicht zu interpretieren; denn neben der Zollunionsbildung werden stets auch andere Faktoren, wie etwa die Handelsliberalisierung ${ }^{4}$ im Rahmen der weltweiten Bestrebungen zum Abbau der Zollschranken in den Verhandlungen des Allgemeinen Zoll- und Handelsabkommens (GATT), die den Güterhandel substituierenden ausländischen Direktinvestitionen in der $\mathrm{EG}^{5}$ oder

${ }^{1}$ Vgl. R. Böhnke: Der Intrahandel in der EWG, in: Wirtschaftsdienst, 55. Jg., 1975, S. $208 \mathrm{ff.,} \mathrm{hier:} \mathrm{S.} 208$.

${ }^{2}$ Vgl. EG-Kommission, Europäische Wirtschaft, Nr. 18, November 1983, S. 234 .

${ }^{3} \mathrm{Vgl}$. hierzu die vergleichende tbersicht bei B. Balassa, Trade creation and diversion in the European Common Market: an appraisal of the evidence, in: B. Balassa (Hrsg.), European Economic Integration, Amsterdam, Oxford u.a. 1975, S. $79 \mathrm{ff} .$, hier: S. $103 \mathrm{ff}$. sowie W. Sellekaerts, How Meaningful Are Empirical Studies on Trade Creation and Diversion?, in: Weltwirtschaftliches Archiv, Bd. 109, 1973, S. 519 ff.

${ }^{4} \mathrm{Vgl}$. W. Sellekaerts, S. 548

${ }^{5}$ Vgl. G. Heiduk, Integraionseffekte - Theorie und Praxis, in: Wirtschaftsdienst, 55. Jg., 1975, S. 303 ff., hier: S. 306. 
die räumlichen Wettbewerbsbedingungen eine Rolle gespielt haben. Eine isolierte Aussage über den EinfluB der europäischen Integration ist praktisch nicht möglich. ${ }^{1}$ Die Problematik, aus globalen Entwicklungen auf die integrationsinduzierten Wohlfahrtseffekte zu schließen, zeigt sich auch, wenn man die Wachstumsraten der EG-Länder mit jenen anderer Industriestaaten vergleicht. Dabei ergeben sich nämlich positive zuwächse in ähnlichem Ausmaß sowohl für die Gemeinschaft als auch für andere vergleichbare Staaten (USA, Japan u.a.). ${ }^{2}$ zur Erklärung integrationsbedingter Wirkungen ist man deshalb weiterhin auch auf Plausibilitätsüberlegungen angewiesen.

Die hier skizzenhaft dargestellten Ergebnisse empirischer Untersuchungen über Handels- und Wohlfahrtswirkungen der EG-Zollunion führen zwar zu keinen eindeutigen Aussagen. Sie widerlegen allerdings auch nicht die These, daß Handels- und Wohlfahrtseffekte durch die europäische Integration zumindest mitverursacht worden sind, ohne daß sich allerdings ihr quantitativer Einfluß sowie eine Aufteilung dieser Vorteile auf die einzelnen Mitgliedsländer angeben 1 äßt.

Schließlich ist auch die allgemeinpolitische Dimension zu berücksichtigen. Es wird zu Recht argumentiert, daB die EG nicht allein mit der materiellen Elle $z u$ messen ist und an den Geist und die Konzeption der Gründungsverträge erinnert, die neben dem Wohlstand auch dem Frieden und der Freiheit Europas dienen sollen. ${ }^{3}$ zudem

${ }^{1}$ Vgl. C. Watrin, Integrationstheorie. Was ist die EG wert?, in: Der Volkswirt, Nr. 31/1970, S. 35 ff.

2vgl. G. Heiduk, S. 304 .

${ }^{3}$ So in der Präambel zum Vertrag zur Gründung der Europäischen Wirtschaftsgemeirischaft. 
werden die aus der gemeinschaftlich vertretenen Politik nach außen resultierenden wirkungen als unbestreitbare - wenngleich von den einzelnen Mitgliedsstaaten unterschiedlich bewertete - Vorteile angesehen, die für sich bereits als hinreichende Legitimation für eine Mitgliedschaft betrachtet werden können. ${ }^{1}$ In diesem Rahmen wären solche Bereiche $\mathrm{zu}$ analysieren wie die gemeinsame Entwicklungspolitik sowie die gemeinschaftliche Politik der wirtschaftlichen Sicherheit bis hin zur Verflechtung zwischen der einzelstaatlichen und den Ansätzen einer europäischen AuBenpolitik. Dabei handelt es sich zwar überwiegend um immaterielle und einer quantitativen Analyse zumeist nicht zugängliche Faktoren, deren Auswirkungen jedoch im Rahmen einer weit gefaßten Kosten-Nutzen-Betrachtung der europäischen Integration ebenfalls nicht vernachlässigt werden dürfen.

Damit steht außer Frage, daß die Nettopositionen kein vollständiger Indikator für die Vor- und Nachteile einer EG-Mitgliedschaft oder gar der europäischen Integration sein können, denn eine umfassende Kosten-Nutzen-Analyse hätte sämtliche der zuvor skizzierten Bereiche einzubeziehen. Die Aussagefähigkeit des partiellen finanzwirtschaftlichen Ansatzes wird zudem dadurch eingeschränkt, daß zwischen dem finanziellen Bereich und den übrigen Feldern gemeinschaftlicher Tätigkeit teilweise bewuBt gestaltete Beziehungen, die sogenannten Kopplungsgeschäfte (package deals), bestehen.

Danach kann es etwa für einige Mitgliedsstaaten vorteilhaft sein, Nettozahlerpositionen hinzunehmen, wenn diese zugeständnisse im Rahmen der Integrationsfortschritte auf anderen Gebieten abgegolten werden. ${ }^{2}$ Solche Verbindungen haben vor allem bei der Gründung der

\footnotetext{
${ }^{1}$ Vgl. hierzu etwa K. H. Narjes, Die EG ist mehr als ein Haushalt, in: Wirtschaftsdienst, $62 \mathrm{Jg} ., 1982, \mathrm{~s} .111 \mathrm{ff} . ; \mathrm{H}$. Stadlmann, Was man in Bonn vor lauter Sparen an Europa nicht vergessen sollte, in: EG-Kommission (Hrsg.), EG-Magazin 10/1981, S. 3 ff.; J. Wahl, Die "weitsichtigen" Enkel der Gründungsväter, in: EG-Magazin 7-8/ 1982 , S. 3 f.

2Vgl. I. E. Druker, Financing the European Community, Leyden 1975 , S. 397.
} 
EWG eine wichtige Rolle gespielt und ihre Gültigkeit bis heute behalten. So wird ein Interessenausgleich zwischen den seinerzeit entscheidenden Partnern Frankreich und Deutschland als die gemeinsame Geschäftsgrundlage des EWG-Vertrages angesehen, indem die deutsche zustimmung zur gemeinsamen Agrarpolitik Voraussetzung für die Beteiligung Frankreichs an der EWG überhaupt war. Dabei erwartete man von der Agrarpolitik finanzielle Vorteile für die AgrarüberschuBländer und hier insbesondere für Frankreich, das bereits in den fünfiger Jahren den Selbstversorgungsgrad erheblich überschritten und die Produktionskapazitäten der Agrarerzeugung noch nicht ausgeschöpft hatte. Für die Bundesrepublik, die bereits einen größeren industriellen Sektor als Frankreich aufwies, vermutete man vornehmlich Integrationsvorteile im industriellen Bereich. 1 Diese Zusammenhänge haben sich im Prinzip bis in die Gegenwart erhalten, und es wird vor allem in der Bundesrepublik häufig auf die Vorteile aus der Handelsliberalisierung in der Gemeinschaft hingewiesen, wenn die - obwohl als zu hoch empfundene - Nettozahlerposition im finanzwirtschaftlichen Bereich zu rechtfertigen ist. Solche Vereinbarungen im Sinne des "do ut des" werden nicht nur als legitim, sondern sogar als notwendig zur Erzielung qualitativer Integrationsfortschritte angesehen. ${ }^{2}$

Gleichwohl macht man es sich aus angesichts der vielfältigen Bestimmungsgründe der europäischen Integration und der wechselseitigen Verflechtungen zu einfach, wenn man die Berechnungen der Nettopositionen und die damit verbundenen Aussagen, Beurteilungen und

${ }^{1}$ Vgl. hierzu U. Koester, EG-Agrarpolitik in der Sackgasse, BadenBaden 1977, S. 33; F. Siebels, H. Lenke, Kapitalistische Vergesellschaftung in der Landwirtschaft und "Gemeinsamer Agrarmarkt" in der EWG, in: F. Deppe (Hrsg.), Europäische Wirtschaftsgemeinschaft (EWG). Zur politischen ökonomie der westeuropäischen Integration, Reinbek 1975, S. 388 ff., hier: S. 362 ff. 2 Vgl. F. Franzmeyer, Regionalpolitische Erfordernisse für die weitere Entwicklung der Europäischen Gemeinschaft, in: Konjunkturpolitik, 18. Jg. 1972, S. 28 ff., hier: S. 39. 
Forderungen als "Krämergeist" und "Buchhaltermentalität" abqualifiziert. Denn selbst wenn man davon ausgeht, daß mit dem europäischen Zusammenschluß allgemein wohlfahrtssteigernde Effekte ausgelöst werden, an denen mehr oder weniger sämtliche Mitgliedsstaaten teilhaben, und die allgemeinpolitischen Vorteile durchaus würdigt, besitzen die Verteilungswirkungen der finanzwirksamen Gemeinschaftsaktivitäten ihren eigenen stellenwert.

So ist zum einen das legitime Interesse der Mitgliedsstaaten zu berücksichtigen, sich zumindest im quantitativ erfaßbaren Bereich der Gemeinschaftspolitik Klarheit über die finanziellen Auswirkungen $\mathrm{zu}$ verschaffen. Die daraus resultierenden nationalen Verteilungspositionen wären dann gegebenenfalls als "Preis" zu interpretieren, zu dem andere Vorteile "erkauft" werden, bzw. würden den "Gewinn" ausdrücken, zu dem möglicherweise Nachteile auf anderen Gebieten hinzunehmen sind. Eine solche Sichtweise kann in dem Zusammenhang bedeutsam sein, daß mit der Zugehörigkeit zur EG insbesondere auch wirtschaftliche Vorteile erwartet werden, und dauerhafte Integrationsfortschritte nur zu erzielen sein dürften, wenn die Länder von einer Vorteilhaftigkeit ihrer Mitgliedschaft überzeugt sind. 1

Die Verteilungswirkungen des gemeinschaftlichen Finanzierungs- und Ausgabensystems spielen für sich genommen aber vor allem dann eine Rolle, wenn man die EG an ihrer - zugegeben langfristigen - zielsetzung der Errichtung einer Wirtschafts- und Währungsunion miBt, und ihrer Finanzwirtschaft - bezogen auf föderal organisierte Mitgliedsstaaten - gewissermaßen die Position einer vierten Ebene des öffentlichen Sektors zuweist. ${ }^{2}$ In diesem Rahmen fallen, wie in einzelstaatlichen Finanzausgleichssystemen, auch der Gemeinschaft ver-

${ }^{1}$ Vgl. A. Nottelmann, 1982, s. 22 .

2 Vgl. G. Denton, Finanzföderalismus und der Haushalt der Europäischen Gemeinschaft, in: Integration, 2. Jg., 1979, S. 1 ff., hier: S. 13 . 
teilungspolitische Aufgaben $\mathrm{zu}^{1}$ deren $\mathrm{ziele} \mathrm{zu}$ definieren und deren Wirkungen zu analysieren und $\mathrm{zu}$ beurteilen sind.

Schließlich haben die budgetären Ungleichgewichte auch einen erheblichen politischen stellenwert erlangt, und fortdauernde Auseinandersetzungen um diese problematik dürften auf die Integrationsfortschritte in den übrigen Bereichen gemeinschaftlicher Politik nicht ohne negative Auswirkungen bleiben. Deshalb kann allein auch dieser Tatbestand Legitimation dafür sein, wenigstens in den einer quantitativen Analyse zugänglichen Teilbereich der Integrationsdiskussion mehr Licht hineinzutragen.

\subsection{Ansätze zur verteilungspolitischen Ausgestaltung von Finanz- ausgleichssystemen}

Akzeptiert man die EG als supranationale Finanzwirtschaft, die mit ihren Mitteln auch verteilungspolitische $\mathrm{zielsetzungen} \mathrm{zu}$ realisieren hat, dann ist zunächst nach den grundsätzlichen Möglichkeiten der Ausgestaltung solcher systeme zu fragen.

Die ordnung der finanzwirtschaftlichen Beziehungen zwischen verschiedenen Gebletskörperschaften umfaßt die Verteilung der öffentlichen Aufgaben und der daraus resultierenden Ausgaben ("passiver" Finanzausgleich) sowie die Verteilung der Finanzierungsmittel ("aktiver" Finanzausgleich). ${ }^{2}$ Dabei geht es zunächst um die Frage, welche diesbezüglichen Wirkungen bereits mit der ursprünglichen Ausgabenstruktur und der Aufbringung der Finanzierungsmittel verbunden sind. Davon zu unterscheiden sind Maßnahmen, die gewissermaßen in einem zweiten Schritt versuchen, die aus der ursprünglichen Aus-

${ }^{1}$ Vgl. H. Reichenbach (1984), S. 457.

2Vgl. H. Kolms, Finanzwissenschaft IV, 2. Aufl., Berlin, New York 1976 , S. 156 . 
gaben- und Mitteiverteilung resultierenden Wirkungen in einem supranationalen Finanzausgleich mit veränderter Mittelverteilung in Richtung auf eine gewünschte Verteilungsstruktur zu korrigieren.

Im Kontext der vorliegenden Arbeit und Im folgenden geht es nicht speziell um nachträgliche Korrekturen bereits realisierter Verteilungswirkungen, sondern wird der grundsätzlichen Frage nachgegangen, welche Vertellungsprinzipien zur ursprünglichen Ausgestaltung der Ausgabenverteilung und insbesondere der Finanzierungsbeteiligung im Finanzwirtschaftssystem der EG herangezogen werden können. Dabei spielen folgende Grundsätze eine Rolle:

Nach dem Äquivalenzprinzip müssen die an den Gemeinschaftshaushalt abgeführten Finanzierungsbeiträge der elnzelnen Mitgliedsstaaten den daraus empfangenen Leistungen entsprechen, d.h. es gilt der Grundsatz von Leistung und Gegenlelstung. Bel einer strikten Anwendung dieses "juste retour" kann ex definitione die Distributionsfunktion des EG-Haushalts nicht wirksam werden. Damit ist dieses Prinzip ungeelgnet, insoweit Integrationsfortschritte im Sinne einer konvergenten Entwicklung der nationalen Volkswirtschaften liber Umvertellungen der Haushaltsmittel verwirklicht werden sollen. Das Aquivalenzprinzip ist zudem kaum im Rahmen eines Eigenmittelsystems vollständig zu realisieren, da die mitgliedsstaatliche Verteilung der Ausgaben ständig mit der Entwicklung der Finanzierungsleistungen abgestimmt werden mußte. ${ }^{1}$

Bei der Verteilung der Finanzierungslasten auf die einzelnen Mitglieder der Gemeinschaft spielt das Leistungsfähigkeitsprinzip eine zentrale Rolle. Bezogen auf die Steuerlastverteilung im nationalen

${ }^{1}$ Vgl. U Nittka, Das Finanzierungssystem der Europäischen Gemeinschaften, Bochum 1979, S. 229. 
Bereich fordert dieser Grundsatz, "die Summe der den einzelnen zufallenden Steuertraglasten so zu bemessen, daß diese unter Berücksichtigung aller steuerlich relevanten persönlichen Momente das Verhältnis der individuellen Leistungsfähigkeiten widerspiegeln, so daß die steuerbedingten Einbußen an ökonomisch-finanzieller Dispositionsfähigkeit flir die Individuen relativ gleich sind." 1

Wird dieses Konzept der individuellen steuerlichen Leistungsfähigkeit auf die Ebene der zwischenstaatlichen Finanzbeziehungen übertragen, dann unterstellt man, daß die persönlichen Leistungsfähigkeitspositionen sich ohne weiteres zu makroökonomischen Größen aggregieren lassen. Denn die Wirtschaftseinheiten sind nunmehr die jewellige Gesamtheit der Individuen in den einzelnen Mitgliedsstaaten der Gemeinschaft. Ebenso wie im nationalen Rahmen können verschiedene Indikatoren der Leistungsfahigkeit als Bemessungsgrundlagen herangezogen werden: das Sozlalprodukt, das Volkseinkommen, der private Konsum oder das Volksvermögen. Dabei lassen sich sowohl eindimensionale als auch mehrdimensionale Indikatoren verwenden. 2

Aus dem Prinzip der Leistungsfahigkelt folgt jedoch keine konkrete Anwelsung für die Ausgestaltung des Steuertarlfs. Ublicherweise geht man zwar davon aus, daß die Leistungsfähigkelt mit steigendem Einkommen oder Vermögen überproportional steigt, doch läßt sich ein häufig daraus abgeleiteter progressiver Tarif nicht wissenschaftlich rechtfertigen und ermöglicht dieses Theorem damit keine eindeutige steuerbemessung. ${ }^{3}$ Der Leistungsfähigkeitsgrundsatz läßt sich

\footnotetext{
${ }^{1}$ F. Neumark, Steuern I: Grundlaqen, in: Handwörterbuch der Wirtschaftswissenschaft, Bd. 7, S. 295 f.f., hier: S. 304.

${ }^{2}$ Vgl. hierzu etwa R. Peffekoven, Probleme der internationalen Finanzordnung, in: F. Neumark (Hrsg.), Handbuch der Finanzwissenschaft, 3. Aufl., Bd. IV, Tübingen 1983, S. 219 ff., hier: S. 254 ff.

${ }^{3}$ Vgl. S. F. Franke, Entwicklung und Begründung der Einkommensbesteuerung, Darmstadt 1981, s. $32 \mathrm{ff}$.
} 
in der Praxis denn auch nicht eindeutig trennen von dem Redistributionsprinzip. Dieser Grundsatz geht insofern weiter als das Leistungsfähigkeitspostulat, als er über die so gestaltete Verteilung der Steuerlasten hinaus die Verteilung der Einkommen oder Vermögen gleichmäßiger im sinne von "gerechter" gestalten will. 1

Mit welchem Tarif man dem Leistungsfähigkeitspostulat auch Rechnung tragen will und welchen Grad der Redistribution man daruber hinaus auch wăhlt, die Einflußnahme auf die Vertellungspositionen der Wirtschaftssubjekte - hier der EG-Mitgliedsstaaten - kann nur partieller Natur sein. ${ }^{2}$ Denn wăhrend das Aquivalenzprinzip eine Abstimmung der nationalen Finanzierungsbeteiligungen mit den Mittelrückflüssen erfordert, stellen der Leistungsfähigkeitsgrundsatz und das darüber hinausgehende Redlstributionsprinzip allein auf die Finanzierungsselte $\mathrm{ab}$ und lassen die aus den Ausgaben resultierende Nutzenvertellung unberlucksichtigt.

Für elne Einflußnahme auf die Vertellungspositionen der betroffenen Länder im Rahmen der gesamten finanzwirtschaftlichen Aktivitäten ist es erforderlich, die Finanzlerungsleistungen zu den Ausgabenzuflüs* sen in Bezlehung zu setzen und diese beiden Komponenten zur sog. fiskalischen Restgröße (fiscal residuum) zu saldieren. ${ }^{3}$ Diese Größen entsprechen im Rahmen der Finanzbeziehungen der EG-Mitgliedsstaaten - abgesehen von methodischen und institutionellen Problemen ihrer Ermittlung - den nationalen Nettopositionen. Ein Spezialfall des Kon-

${ }^{1}$ Vgl. F. Neumark, 198, S. 304 .

2Vgl. R. A. Musgrave, P. B. Musgrave, L. Kullmer, Die öffentlichen Finanzen in Theorie und Praxis, Bd. 3, Tübingen 1977, S. 9.

${ }^{3}$ Vgl. hierzu etwa J. M. Buchanan, Fiscal Theory and Political Economy, 1960 , S. 17 und $176 \mathrm{ff}$; K. Littmann, Ein Valet dem Leistungsfähigkeitsprinzip, in: H. Haller u.a. (Hrsg.), Theorie und Praxis des finanzpolitischen Interventionismus. Fritz Neumark zum $70 . \mathrm{Ge}-$ burtstag, Tubingen 1970, S. 113 ff.; hier: S. 123. 
zepts des fiscal residuums stellt das Äquivalenzprinzip mit dem Saldo Null dar. Werden dagegen Umverteilungszielsetzungen in diesem umfassenden Sinne nach Maßgabe der wirtschaftlichen Leistungskraft der Mitgliedsstaaten angestrebt, dann müssen die wirtschaftlich schwächeren Länder einen tuberschuß der Ausgabenzuflüsse über thre Finanzierungsleistungen, den man als positiven fiskalischen Restwert bezeichnen kann, aufweisen, während die leistungsstärkeren Länder negative Restwerte in Kauf $\mathrm{zu}$ nehmen haben. Eine derart motivierte Ausgestaltung des Finanzierungs- und Ausgabensystems läßt sich als Prinzip des Ressourcen-Transfers charakteristeren.

\subsection{Verte1lungspolit1sche Zielsetzungen der EG}

Nach den allgemeinen Betrachtungen ist nun zu fragen, welche verteilungspolitischen ziele im Rahmen der zwischenstaatlichen Finanzbeziehungen der EG-Mitglledsstaaten angestrebt werden, und welche Vertellungsgrundsatze ihnen entsprechen. Hinweise darauf finden sich zunachst im EWG-Vertrag. So wird in der Präambel das Bestreben ausgedrückt, "den Abstand zwlschen einzelnen Gebieten und den Rückstand weniger begunstigter Gebiete (zu) verringern", und weist Art. 2 des Vertrages der Gemeinschaft u.a. die Aufgabe zu, "die schrittweise Annäherung der Wirtschaftspolitik der Mitgliedsstaaten" und eine "ausgewogene Wirtschaftsausweitung" zu fördern. Daneben hat die Kommission stets die Ausrichtung der Gemeinschaftspolitiken am Grundsatz der Solidarität der wohlhabenden Länder gegenüber den weniger wohlhabenderen Mitgliedsstaaten betont. ${ }^{1}$

Pruft man zunächst das Äquivalenzprinzip im Hinblick auf seine Vereinbarkeit mit den gemeinschaftlichen Finanzierungs- und verteilungspolitischen zlelsetzungen, so ist festzustellen, daß es den Inten-

\footnotetext{
${ }^{1} \mathrm{Vgl}$. EG-Kommission, Berlcht der Kommission der Europäischen Gemeinschaften an den Rat in Durchfuhrung des Mandats vom 30. Ma1 1980, Bulletin der EG, Beilage 1/81, Tz. 43 .
} 
tionen und dem Wortlaut des EWG-Vertrages nicht entspricht. Es wird allgemein als integrationsfeindlich abgelehnt und stand als Finanzierungsmaxime der EG auch nie zur Diskussion, obwohl sich dieser Grundsatz mehr oder weniger offen hinter den Kontroversen der Mitgliedsstaaten um ihre Finanzierungsbeteiligung verbirgt. ${ }^{1}$ Dabei hat sich zwischen diesem Prinzlp und den Nettopositionen insofern eine unglickliche Beziehung entwickelt, als allein der Tatbestand, daß Finanzierungsleistungen und Ausgabenrückflüsse einander gegenübergestellt werden, zumeist als ein Versuch angesehen wird, um äquivalenzmäßige Vorstellungen zu stützen. Daraus haben sich starke Vorbehalte - insbesondere von Seiten der Kommission - gegenüber der Ermittlung der Nettopositionen ergeben und trat ihre Funktion als - wenn auch unvollkommener - Beurteilungsmaßstab der Verteilungswirkungen in den Hintergrund.

Dagegen tragen der Grundsatz der Leistungsfähigkeit und das Redistributionsprinzip dem Wortlaut der Präambel und dem Art. 2 des Gründungsvertrages ebenso Rechnung ${ }^{2}$ wie sie dem Grundsatz der gemeinschaftlichen Solidaritat entsprechen. Allerdings bietet eine so bewirkte Vertellung der Finanzierungslasten keine hinreichende Gewähr dafür, daß die gesamten finanzwirksamen Transaktionen zu einer Umverteilung zugunsten der wirtschaftlich schwächeren Länder führen, da dies auch von der Ausgabeninzidenz abhängt. Die gemeinschaftlichen Verteilungszielsetzungen müßten sich deshalb am Grundsatz des Ressourcen-Transfers orientieren. Damit sind die Nettopositionen in ihrer Bezlehung $z u$ einem Indikator der wirtschaftlichen Leistungskraft der Mitgliedsstaaten entscheidendes Beurteilungskriterium dafür, in welchem Umfang dieser Zielsetzung entsprochen wird.

${ }^{1}$ Siehe hierzu Abschnitt 4.1.2.

2Vgl. N. Andel, Zur Harmonisierung öffentlicher Ausgaben in einem gemeinsamen Markt, in: Finanzarchiv, N.F. Bd. 24, 1965, S. 1 ff., hier: S. 24 f.; R. Peffekoven, Verteilung internationaler Finanhierungslasten, in: List-Forum, Bd. 11, 1981/82, S. $14 \mathrm{ff}$. , hier: S. 19 . 


\subsection{Inzidenzanalyse unter dem Meßaspekt}

Nach den Ausführungen über allgemeine verteilungspolitische Grundsätze und deren Relevanz für das Finanzierungs- und Ausgabensystem der EG ist nunmehr auf die Durchführung der Inzidenzanalyse einzugehen. Dabei geht es um die Wahl des zugrundezulegenden Konzepts zur Messung der Verteilungswirkungen sowie um spezifische Fragen der zeitlichen und sachlichen Abgrenzung des Untersuchungsgegenstandes.

\subsubsection{Inzidenzkonzepte}

Gegenstand von Inzidenzanalysen sind Verteilungswirkungen, die sich aus Änderungen finanzwirtschaftlicher Staataktivitäten ergeben. Sie fordern die Festlegung eines Referenzsystems, dessen Verteilungsrelationen mit den aktuellen Werten zu vergleichen sind. Dabei lassen sich grundsätzlich die Konzepte der effektiven (materiellen) und der formalen Inzidenz unterscheiden.

Bei der effektiven Inzidenz handelt es sich um die Messung der Verteilungswirkungen, die sich nach dem staatlichen Eingriff einschlieBlich aller daraus resultierender Anpassungswirkungen ergeben, die also auf die endgültige Verteilung der Lasten und Vorteile abstellt. ${ }^{1}$ Eine solche Analyse würde erfordern, sämtliche mit den staatsaktivitäten verbundenen Anpassungsvorgänge wie Produktions-, Sozialprodukts-, Einkommens- und Beschäftigungswirkungen zu berücksichtigen und die daraus resultierenden Verteilungsrelationen zu quantifizieren. In diesem umfassenden Sinne wird eine Inzidenz wahrscheinlich nie empirisch erfaßbar sein.

${ }^{1}$ Vgl. etwa H. Zimmermann, K.-D. Henke, Einführung in die Finanzwissenschaft, 3. Aufl., München 1982, S. 378. 
Das Konzept der formalen Inzidenz verfolgt zwar insofern eine bescheidenere Zielsetzung, als es auf die Verteilunsänderungen nach Staatseingriff, aber vor weiteren Reaktionen der Betroffenen abstellt. Sie ist aber wegen der Möglichkeit vorweggenommener oder gleichzeitiger Anpassungsvorgänge in der Regel ebenfalls nicht meBbar. Empirisch zu identifizieren sind lediglich die gezahlten und empfangenen Finanzströme. ${ }^{1}$ Ihr Nettoeffekt soll hier als (formale) Zahlungsinzidenz interpretiert werden.

Dieses an reine $\mathrm{Z}$ ahlungsvorgänge anknüpfende Konzept ist allerdings dann ohne größeren Aussagewert, wenn die Finanzströme - etwa aus erhebungs- und abrechnungstechnischen Gründen - nicht gleichzeitig den finanziellen Vorteilen und Belastungen der beteiligten wirtschaftseinheiten entsprechen. Ein Ansatz, der solche Zusammenhänge berücksichtigt, erfordert ausgehend von der zahlungsinzidenz eine Korrektur dieser Verteilungswirkungen, indem danach $z u$ fragen ist, ob die Mittelabführungen an den Haushalt auch mit unmittelbaren Belastungen und die Rückflüsse aus dem Budget mit unmittelbaren finanziellen Vortellen für die jeweils leistenden bzw. empfangenden Mitgliedsländer bzw. Ihrer Bürger verbunden sind. Das Konzept der so spezifizierten Inzldenz liegt der folgenden Verteilungsanalyse zugrunde.

Während mit dieser inzidenztheoretischen Einordnung noch einmal die generelle Aussagefähigkeit ihrer Ergebnisse verdeutlicht wurde, ist abschließend kurz auf die Frage einzugehen, ob ein solcher vergleich der finanziellen Vor- und Nachteile und die damit möglicherweise verbundenen Forderungen nach Anderungen des Finanzierungs- und Ausgabensystems im Rahmen eigener Einnahmen der Gemeinschaft überhaupt

${ }^{1}$ Vgl. M. Rose, Finanzwissenschaftliche Verteilungslehre, München 1977, S. $23 \mathrm{f}$. 
zulässig ist. In diesem Zusammenhang werden solche Verteilungsrechnungen deshalb als bedenklich angesehen, weil die Zahlungen an den EG-Haushalt nicht den Mitgliedsstaaten, sondern unwiderruflich der EG zustehen. Dabei wird teilweise differenziert in die sog. natürlichen Einnahmen, die Resultate der gemeinschaftlich verwirklichten Politikbereichesind, nämlich die allein der Gemeinschaft zustehenden zölle und Agrarabschöpfungen, sowie in die Beteiligungen der EG an den nationalen Mehrwertsteuereinnahmen. ${ }^{1}$ Mit derartigen Einwänden kann allerdings nicht die verteilungsökonomische motivierte Fragestellung abgewiesen werden, in deren Mittelpunkt nicht $\mathrm{Zah}-$ lungsverpflichtungen und Zuständigkeiten, sondern Belastungs- und Vorteilswirkungen stehen. 2

\subsubsection{Inzidenzmaßstab}

Als geeignete Maßstäbe zur Messung der Verteilungsänderungen kommen grundsätzlich die Konzepte der absoluten und der relativen Inzidenz in Betracht. Dabei versteht man unter absoluter Inzidenz die niveaumäßige Anderung der auf die einzelnen Verteilungsträger entfallenden Verteilungsobjekte, während bei der relativen Inzidenz diese Verteilungsänderungen auf Indikatoren wirtschaftlicher Leistungskraft der Verteilungsträger bezogen werden können. ${ }^{3}$

Im Kontext der vorliegenden Untersuchung erfaßt das Konzept der absoluten Inzidenz die nominellen Änderungen einzelner Einnahmen- bzw. Ausgabenarten (Einnahmen- bzw. Ausgabeninzidenz) und mit der Saldie-

1Vgl. zur Diskussion dieser Fragen u.a. C. D. Ehlermann, The Financing of the Community: The Districtions between Financial Contributions and own Resources, in: Common Market Law Review, Vol. 19, 1982 , S. 571 ff.

${ }^{2} \mathrm{Vgl}$. K. $-\mathrm{D}$. Henke, S. $37 \mathrm{f}$.

${ }^{3} \mathrm{Vgl}$. hierzu K. Rose, s. 21 . 
rung sämtlicher Einnahmen und Ausgaben die absoluten Budgetwirkungen (Budgetinzidenz). Diese Größen sind zur Beurteilung der Verteilungswirkungen insofern von untergeordneter Bedeutung, als die Verteilungsträger - die EG-Mitgliedsländer - in der Gemeinschaft ein unterschiedliches ökonomisches Gewicht besitzen. Gleichwohl können auch die größenmäßigen Änderungen eine Rolle spielen, wenn über das Niveau der Verteilungswirkungen und deren politische Durchsetzbarkeit entschieden werden soll.

Stellt man darauf $a b$, wie die Verteilungsänderungen im Hinblick auf die Wirtschaftskraft der Mitgliedsländer und den daran ausgerichteten verteilungspolitischen $\mathrm{zielsetzungen} \mathrm{zu}$ beurteilen sind, dann ist das Konzept der relativen Inzidenz heranzuziehen. Die Verteilungswirkungen einzelner Einnahmen- und Ausgabenarten bzw. die daraus resultierenden Budgetsalden werden auf einen Indikator für die Wirtschaftskraft der Mitgliedsstaaten bezogen. Als ein solcher Maßstab ist das Sozialprodukt seit langem anerkannt:

- Die Sozialproduktrelationen spielten bereits bei der Bemessung der Beitragsquoten nach der Wirtschaftskraft der Mitgliedsstaaten im ursprünglichen system der Finanzbeiträge eine Rolle. ${ }^{1}$

- Sie bestimmten den Finanzbeitragsschlüssel für die sog. Restfinanzierung im Rahmen der tbergangsregelungen bis zur vollständigen Verwirklichung des Eigenmittelsystems. 2

- Das Bruttosozialprodukt (BSP) und später das Bruttoinlandsprodukt (BIP) dienten als Beurteilungsmaßstab für die wirtschaftliche Situation eines Mitgliedslandes bei dem in das Finanzierungssystem integrierten Korrekturmechanismus. ${ }^{3}$

- Schließlich wird in jüngsten Ubberlegungen, die auf eine stärkere Orientierung der Finanzierungsleistungen an der Leistungsfähigkeit abzielen, stets auch das Sozialprodukt als geeigneter Indikator hierfür herangezogen. ${ }^{4}$

1,2 Siehe hierzu Abschnitt 4.1.1.
${ }^{3,4}$ Siehe hierzu Abschnitt 4.1.2. 
Im Hinblick auf die zwischenstaatliche Vergleichbarkeit der Sozialproduktgrößen dürften insofern kaum Verzerrungen auftreten, als die vom Statistischen Amt der EG veröffentlichten Angaben einheitlich definiert, bewertet und erfaßt werden. ${ }^{1}$ Dies gilt allerdings nicht für die gewählte Technik ihrer Ermittlung. So werden etwa im Vereinigten Königreich die Sozialproduktgrößen aus der. Steuerstatistiken abgeleitet. Dies führt $z u$ relativ geringeren Ansätzen und stört die Vergleichbarkeit mit den Größen der anderen Mitgliedsländer.

Als konkrete Bezugsgrößen für das Sozialprodukt werden zumeist das BSP oder das BIP gewählt. Da die statistischen Veröffentlichungen neuerdings fast ausschlieBlich auf das BIP abstellen, relevante volkswirtschaftliche Größen hierauf beziehen und BSP-Größen nur mit zeitlicher Verzögerung zur Verfügung stehen, wird im folgenden das BIP als grundlegende Sozialproduktgröße verwendet.

In der Tabelle 1 sind die im Zusammenhang mit den BIP der Mitgliedsstaaten relevanten Größen für die Jahre 1980 bis 1984 zusammengestellt: die nominellen Beträge, die nationalen Anteile am Gemeinschaftssozialprodukt sowie die Pro-Kopf-Werte. ${ }^{2}$ zur Erläuterung scheinen vor allem die folgenden Bemerkungen angebracht.

Die Verwendung des BIP als Bezugsgröße anstelle des BSP führt dazu, $\mathrm{da} B$ die aus den Auslandsbeziehungen resultierenden Nettofaktoreinkommen aus unselbständiger Arbeit, Unternehmertätigkeit und Ver-

${ }^{1}$ Vgl. K.-D. Henke, Die Finanzierung der Europäischen Gemeinschaften. zur integrationsfördernden Fortentwicklung des europäischen Einnahmensystems, in: D. Pohmer (Hrsg.), Probleme des Finanzausgleichs III. Finanzausgleich im Rahmen der Europäischen Gemeinschaften. Schriften des Vereins für Socialpolitik, N.F., Bd. 36/III, Berlin 1981, S. $11 \mathrm{ff}$. , hier: S. 63.

${ }^{2}$ Hier und im folgenden werden die über mehrere zurückliegende Jahre sich erstreckenden Angaben sämtlich mit ECU (European Currency Unit) bezeichnet, auch wenn die Gemeinschaftswährung von 1978 bis 1980 die ERE (Europäische Rechnungseinheit) und zuvor die RE (rechnungseinheit) war. Einer ECU entsprechen im Jahresdurchschnitt 1984 DM 2,24 . 
Tabelle 1

Bruttoinlandsprodukte der Mitgliedsstaaten 1980 - 1984

\begin{tabular}{|c|c|c|c|c|c|}
\hline Mitgliedsland & 1980 & 1981 & 1982 & 1983 & 1984 \\
\hline & \multicolumn{5}{|c|}{ BIP in Mrd. ECU } \\
\hline Belgien & 84,4 & 86,2 & 86,1 & 90,0 & 96,2 \\
\hline Dänemark & 47,8 & 51,8 & 57,6 & 63,7 & 69,6 \\
\hline Deutschland & 586,9 & 613,6 & 673,0 & 733,9 & 777,6 \\
\hline Frankreich & 471,2 & 514,3 & 552,0 & 581,0 & 624,3 \\
\hline Griechenland & 28,8 & 33,0 & 38,5 & 38,7 & 41,7 \\
\hline Irland & 13,1 & 15,3 & 18,0 & 19,3 & 21,0 \\
\hline Italien & 284,8 & 317,7 & 354,9 & 395,6 & 438,6 \\
\hline Luxemburg & 3,3 & 3,5 & 3,4 & 3,6 & 3,9 \\
\hline Niederlande & 122,0 & 127,3 & 140,6 & 148,4 & 156,1 \\
\hline Verein. Königreich & 378,0 & 450,6 & 487,6 & 505,8 & 532,7 \\
\hline \multirow[t]{2}{*}{ EG } & 2020,3 & 2213,3 & 2411,7 & 2580,1 & 2761,7 \\
\hline & \multicolumn{5}{|c|}{ BIP-Anteile in 8} \\
\hline Belgien & 4,18 & 3,89 & 3,57 & 3,49 & 3,48 \\
\hline Dänemark & 2,36 & 2,34 & 2,39 & 2,47 & 2,52 \\
\hline Deutschland & 29,05 & 27,73 & 27,90 & 28,44 & 28,16 \\
\hline Frankreich & 23,32 & 23,24 & 22,89 & 22,52 & 22,61 \\
\hline Griechenland & 1,43 & 1,49 & 1,60 & 1,50 & 1,51 \\
\hline Irland & 0,65 & 0,69 & 0,75 & 0,75 & 0,76 \\
\hline Italien & 14,10 & 14,35 & 14,71 & 15,33 & 15,88 \\
\hline Luxemburg & 0,16 & 0,16 & 0,14 & 0,14 & 0,14 \\
\hline Niederlande & 6,04 & 5,75 & 5,83 & 5,75 & 5,65 \\
\hline Verein. Königreich & 18,71 & 20,36 & 20,22 & 19,60 & 19,29 \\
\hline EG & 100,00 & 100,00 & 100,00 & 100,00 & 100,00 \\
\hline
\end{tabular}


Tabelle 1 (Fortsetzung)

\begin{tabular}{|c|c|c|c|c|c|}
\hline Mitgliedsland & 1980 & 1981 & 1982 & 1983 & 1984 \\
\hline & & BIP pro & Kopf in E & & \\
\hline Belgien & 8571 & 8749 & 8739 & 9141 & 9776 \\
\hline Dänemark & 9321 & 10118 & 11254 & 12458 & 13620 \\
\hline Deutschland & 9532 & 9948 & 10919 & 11947 & 12684 \\
\hline Frankreich & 8772 & 9529 & 10180 & 10672 & 11422 \\
\hline Griechenland & 2990 & 3392 & 3935 & 3924 & 4190 \\
\hline Irland & 3856 & 4445 & 5171 & 5503 & 5943 \\
\hline Italien & 4991 & 5554 & 6266 & 6969 & 7712 \\
\hline Luxemburg & 9139 & 9501 & 9337 & 9754 & 10610 \\
\hline Niederlande & 8623 & 8935 & 9821 & 10327 & 10828 \\
\hline Verein. Königreich & 6749 & 8044 & 8696 & 9013 & 9479 \\
\hline EG & 7458 & 8148 & 8883 & 9491 & 10144 \\
\hline
\end{tabular}

Quelle: EG-Kommission, Europäische Wirtschaft, Nr. 22, Nov. 1984, S. $223 \mathrm{f} . ;$ eigene Berechnungen.

mögen nicht mit erfaßt werden, denn um diesen Betrag unterscheiden sich die beiden Sozialproduktgrößen: BSP = BIP + (Faktoreinkommen aus dem Ausland - Faktoreinkommen an das Ausland). 1 Während dieser Unterschiedsbetrag für die übrigen Mitgliedsstaaten vernachlässigbar gering ist, besitzt er für Luxemburg erhebliche quantitative Relevanz, denn hier liegt das BSP aufgrund des relativ hohen Volumens der vom Ausland gezahlten Faktoreinkommen um etwa $25 \&$ über dem BIP. Dies hat zur Folge, daß in bezug auf das BSP Luxemburg

${ }^{1}$ Vgl. A. Stobbe, Volkswirtschaftslehre I. Volkswirtschaftliches Rechnungswesen, 5. Aufl., Berlin, Heidelberg u.a. 1980, S. 144. 
an der Spitze der Pro-Kopf-Einkommensskala der Mitgliedsstaaten liegt. Obwohl diese Sozialproduktgröße der geeignetere Indikator zur Beurteilung der wirtschaftlichen Leistungsfähigkeit eines Landes sein dürfte, da hier auch die über das Ausland erzielten Einkommen seiner Bürger einbezogen werden, wird aus den oben genannten Gründen das BIP herangezogen. Bei der Interpretation der Verteilungswirkungen werden diese Umstände allerdings stets $\mathrm{zu}$ berücksichtigen sein.

Weiterhin ist auf mögliche Verzerrungen der realwirtschaftlichen Aussagen der Ergebnisse hinzuweisen. Denn die in der Gemeinschaftswährung angegebenen Sozialproduktgrößen hängen neben realen Veränderungen auch von den nominellen Entwicklungen der Landeswährungen sowie den Wechselkursentwicklungen in bezug auf die ECU ab. Ein an die BIP anknüpfender Vergleich der zwischenstaatlichen Wirtschaftsund Leistungskraft kann damit von Preis- und Währungsentwicklungen beeinträchtigt werden.

Bezüglich der mitgliedsstaatlichen Pro-Kopf-Sozialprodukte ist an dieser Stelle zu konstatieren, daß ihre Struktur bzw. Rangfolge im Rahmen einer relativ kurzfristigen Betrachtung weitgehend konstant ist. Daneben ist bemerkenswert, daß die Abstände zwischen den einzelnen Ländern teilweise erheblich sind: So macht das Pro-Kopf-BIP des Vereinigten Königreichs etwa drei Viertel, das Italiens weniger als zwei Drittel, das Irlands weniger als die Hälfte und das Griechenlands nur etwa ein Drittel des Pro-Kopf-BIP der wohlhabendsten Länder Dänemark ${ }^{1}$ und Deutschland aus.

${ }^{1}$ Die Spitzenposition Dänemarks in der Pro-Kopf-Einkommensskala ist maßgeblich auf seine vergleichsweise hohe Beschäftigtenquote der Frauen zurückzuführen. Diese Erwerbsquote liegt in Dänemark um 50 \& und mehr über denen der übrigen Gemeinschaftsländer. Vgl. Amt für amtliche Veröffentlichungen der EG, Eurostat Revue 1974-1983, Luxemburg 1985, S. 117. 


\subsubsection{Zeitliche Dimension und rechnungsmäßige Abgrenzung der Analyse}

Als Untersuchungszeitraum für die empirische Verteilungsanalyse werden die Jahre 1980 bis 1984 zugrundegelegt. Damit wird eine Periode betrachtet, in der das Eigenmittelsystem der Gemeinschaft vollständig angewendet worden ist, und mit der geringfügigen Ausnahme einiger Sonderregelungen für das 1981 der Gemeinschaft neu beigetretene Mitglied Griechenlands keine Ubbergangsbestimmungen zu berücksichtigen sind.

Mit dieser zeitlichen Abgrenzung sind auch Probleme gegenstandslos, die sich in den Jahren bis 1979 aus der Verwendung der ursprünglichen IWF-Rechnungseinheit (RE) und aus den Sonderregelungen für den Ubergang zur Europäischen Rechnungseinheit (ERE) ergaben. ${ }^{1}$ Die ERE, die von 1978 bis einschließlich 1980 für den EG-Haushalt galt, ist eine Korbwährungseinheit, die sich aus der Summe unveränderlicher währungsbeträge der Mitgliedsstaaten zusammensetzt. Die Anteile der einzelnen Währungen am Korb wurden insbesondere auf der Grundlage der mitgliedsstaatlichen Anteile am Gemeinschaftssozialprodukt und am innergemeinschaftlichen Handel festgelegt. Die seit 1981 für alle haushaltsrelevanten Vorgänge geltenden ECU ist hinsichtlich ihrer Definition und Berechnungsweise identisch mit der ERE; sie weist aber im Unterschied dazu eine Revisionsklausel auf, die Änderungen in ihrer Zusammensetzung ermöglicht. ${ }^{2}$ Eine solche Revision erfolgte im Juni 1984, indem auch die griechische Drachme

${ }^{1}$ Vgl. hierzu etwa E. Mohr, Die Finanzprobleme der Gemeinschaft Spiegelbild des Integrationsstandes und -willens der EG -, in: Ifo-Schnelldienst, 33. Jg., 1980, Heft 8, S. 5 ff.; A. Nottelmann, S. $31 \mathrm{ff.;}$ W. Heck, Die neue Europäische Rechnungseinheit, in: Wirtschaftsdienst, 58. Jg. 1978, S. $87 \mathrm{ff}$.

${ }^{2}$ Vgl. hierzu etwa EG-Kommission, Die ECU, Europäische Dokumentation 6/1984. 
in den Währungskorb einbezogen und die Gewichtsanteile der einzelnen Währungen neu festgelegt wurden. 1

Die Untersuchungsperiode von fünf Jahren erscheint dennoch lang genug, um außergewöhnliche Veränderungen der Verteilungswirkungen entsprechend $z u$ bewerten und in die mittelfristige Entwicklung der Verteilungsstruktur einordnen zu können. Zudem werden die Jahre des Untersuchungszeitraumes stets im Anschluß an die bisherige Entwicklung betrachtet und wird auf sich abzeichnende künftige Veränderungen hingewiesen.

Im Hinblick auf die rechnungsmäßige Erfassung der Finanzierungsströme stellt die Analyse grundsätzlich auf die Ist-Werte ab. Eine solche Vorgehensweise besitzt den Vorteil, die tatsächliche Entwicklung der finanzwirksamen Aktivitäten zu erfassen und vermeidet Interpretationsprobleme, die mit den teilweise starken Abweichungen zwischen Soll- und Ist-Werten verbunden sind. Andererseits ist damit der Untersuchungsrahmen insofern eng abgesteckt, als vor allem die Größenordnungen der Verpflichtungsermächtigungen ${ }^{2}$ nicht explizit berücksichtigt werden. In den Fällen starker Abweichungen von den tatsächlich realisierten Werten ist deshalb auch auf die Größenordnungen der Verpflichtungen einzugehen, um damit sowohl die Ansprüche aus den bisherigen Haushaltsentscheidungen einzubeziehen als auch den Trend der Zahlungen in künftigen Perioden berücksichtigen zu können.

${ }^{1}$ Veroranung (EWG) Nr. 2626/84 des Rates vom 15. September 1984 zur Anderung von Artikel 1 der Verordnung (EWG) Nr. 3180/78 zur Änderung des Wertes der vom Europäischen Fonds für währungspolitische Zusammenarbeit verwendeten Rechnungseinheit, in: Amtsblatt der EG, Nr. L 247 vom 16.9.1984, S. 1 f.

2 Verpflichtungsermächtigungen bestimmen den Rahmen, in dem während des laufenden Haushaltsjahres Verpflichtungen eingegangen werden dürfen. Nicht ausgenutzte Mittelbindungen sind ohne weiteres ein zweites Jahr gültig und können in einem vereinfachten Verfahren auch auf ein drittes Haushaltsjahr übertragen werden. Zahlungsermächtigungen stellen die Höchtsgrenze für die Ausgaben dar, die während des Haushaltsjahres geleistet werden können. Davon zu unterscheiden sind dann die tatsächlich ausgeführten Zahlungen, die hier als Ist-Werte bezeichnet werden. 


\section{Das Konzept der Nettopositionen zur Erfassung der innergemeinschaftlichen Zahlungsströme}

Unter Nettopositionen, Netto(ressourcen)transfers oder Nettoüberweisungen werden derzeit die Differenzen aus den Mittelübertragungen der Mitgliedsstaaten an den Gesamthaushalt der EG und den daraus empfangenen Rückflüssen verstanden. Ein Mitgliedsland, dessen Zahlungen die Rückflüsse übersteigen, wird als Nettozahler bezeichnet; ein Mitgliedsland, das höhere Mittelzuweisungen erhält als es Zahlungen leistet, Nettoempfänger genannt.

Die Methode, aus den Gegenüberstellungen von Mittelabführungen und empfangenen Leistungen Aussagen über die Verteilungswirkungen gemeinschaftlicher Aktivitäten zu machen, ist nicht neu. Solche Rechnungen wurden bereits für die ersten Entwicklungsphasen der Gemeinschaft vorgenommen. Sie konnten nach verschiedenen Ausgabenbereichen gesondert durchgeführt werden, da die finanziellen Aktivitäten der Gemeinschaft noch über einzelne Fonds abgewickelt wurden, die in Ausgaben und Finanzierungsbetelligungen jeweils abgeschlossene Systeme darstellten.

Die Umverteilungswirkungen vollzogen sich vor allem über den seit jeher dominierenden Agrarfonds, der von 1962 bis 1970 bei einem Gesamtvolumen von 7,8 Mrd. RE eine Umverteilungsmasse zwischen den Mitgliedsländern von 1,3 Mrd. RE und damit eine Umverteilungsquote von $17 \&$ aufwies. Der Nettotransfer begünstigte Frankreich $\left(\begin{array}{ll}69 & 8\end{array}\right)$ und die Niederlande (31 8 ) und wurde vor allem von Deutschland $(61 \%)$, aber auch Italien $(27 \%)$ und Belgien (11 \%) getragen. Die beiden größten Nettozahlerländer wurden zwar über den Sozialfonds begünstigt, der aber angesichts seines Gesamtvolumens von 0,4 Mrd. RE (1960 - 1872) nur eine sehr begrenzte Kompensation bieten konnte. 1

${ }^{1}$ Vgl. E. Reister, S. 98 ff. 
Obwohl es über die Art und den Umfang der Ausgaben des Agrarfonds sowie über die - für die einzelnen Fondswirtschaftsjahre gesondert geregelten - Finanzierungsbeteiligungen durchaus Auseinandersetzungen zwischen den Mitgliedsstaaten gab ${ }^{1}$, wurde die grundsätzliche Rollenverteilung als Nettozahler bzw. Nettoempfänger akzeptiert. Die Situation änderte sich mit der Institutionalisierung des Eigenmi.ttelsystems im Jahre 1971 und der Erweiterung der Gemeinschaft 1973. Hinsichtlich der Durchführbarkeit von Verteilungsanalysen konnten die Finanztransfers nach 1971 nicht mehr unmittelbar durch eine Gegenüberstellung der Ausgaben und Einnahmen der einzelnen Fonds ermittelt werden. Denn im System der Eigenmittel werden diese nicht mehr einzeln ausgeglichen, sondern über den Haushalt insgesamt abgerechnet, und die Einnahmen dienen unterschiedslos zur Finanzierung sämtlicher Ausgaben (Nonaffektationsprinzip).

Vor allem aber erwies sich die Situation des Vereinigten Königreichs als Problemfall hinsichtlich der Akzeptanz der aus dem Eigenmittelsystem und den ausgabenwirksamen Gemeinschaftspolitiken resultierenden Verteilungswirkungen. ${ }^{2}$ Denn es war vorauszusehen, daß dieses $z u$ den wirtschaftsschwächeren Gemeinschaftsstaaten gehörende Land nach dem Auslaufen der Beitrittsübergangsbestimmungen im Jahre 1980 aufgrund seiner Agrar- und Außenhandelsstruktur überproportional viel Eigenmittel an den Gemeinschaftshaushalt abzuführen hatte und nur unterproportional an den Rückflüssen beteiligt sein würde. zudem wurden Wettbewerbsnachteile aus der Liberalisierung des innergemeinschaftlichen Handels erwartet. Mit der Gründung des Regionalfonds im Jahre 1975 wurde zwar ein Instrument geschaffen, um diese "Vorleistungen" durch eine bevorzugte Mittelvergabe zur Sanierung

\footnotetext{
${ }^{1}$ Vgl. hierzu J. H. Weber, The Financing of the Common Agricultural Policy, in: Common Market Law Review, Vol. 4, 1966, S. $263 \mathrm{ff}$. ${ }^{2}$ siehe hierzu ausfihrlleher Kapitel 4.1.2.
} 
der britischen Entwicklungsregionen zu kompensieren. 1 Es zeigte sich aber bald, daß angesichts des relativ geringen Fondsvolumens die britische Nettozahlerposition nur geringfügig vermindert werden konnte. Da sich auch der im Rahmen der Bej.trittsneuverhandlungen installierte Korrekturmechanismus zum Ausgleich einer wirtschaftlich unhaltbaren situation als unwirksam erwies, und mit dem Auslaufen der Ubergangsbestimmungen das Vereinigte Königreich größter Nettozahler der Gemeinschaft zu werden drohte, erhielten die Nettopositionen zunehmend stärkeres Gewicht bei der Beurteilung der Kosten und Nutzen der Gemeinschaft und spielten diese Zahlen eine immer größere Rolle in der Integrationsdiskussion.

Zur quantitativen Ermittlung der Nettopositionen stand bis zum Jahre 1978 kein Zahlenmaterial der Kommission zur Verfügung. Es lagen zwar diesbezügliche Anfragen von Abgeordneten des Europäischen Parlaments vor. ${ }^{2}$ In ihren Antworten verneinte die Kommission jedoch stets das Vorhandensein solcher zusammenfassender Darstellungen und verwies auf die Haushaltsrechnungen, in denen die von den Mitgliedsstaaten abgeführten Beiträge und die ihnen aus den einzelnen Fonds zugeflossenen Mittel aufgeführt waren. ${ }^{3}$ Ausschlaggebend für die negative Beantwortung dieser Fragen war offensichtlich weniger die technische Durchführbarkeit solcher Berechnungen. Vielmehr ist dies auf die Auffassung der Kommission zurückzuführen, daß solche Saldenberechnungen einen falschen Eindruck von den wirtschaftlichen Beiträgen und Vorteilen der Mitgliedsstaaten vermittelten und "daß es nicht dem Geist europäischer Zusammenarbeit för-

'Auch dieser Fall wird als ein sog. Kopplungsgeschäft angesehen. Vgl. K. Reding, Zur Problematik eines Finanzausgleichs in der Europäischen Gemeinschaft in: H. von der Groeben, H. Möller, Möglichkeiten und Grenzen einer Europäischen Union, Bd. 2, Verteilung der wirtschaftlichen Kräfte im Raum, Baden-Baden 1976, S. 199 ff., hier: S. 203 .

${ }^{2} \mathrm{Vgl}$. Schriftliche Anfrage Nr. $888 / 77$ von Herrn L'Estrange, vom 16. Dezember 1977, in: Amtsblatt der EG, Nr. C 56 vom 6.3.1978, S. 28 .

${ }^{3}$ Vgl. EG-Kommission, Antwort auf die schriftliche Anfrage von Herrn L'Estrange vom 31. Januar 1978, in: Amtsblatt der EG, C 56 vom 6. März 1978, S. $28 \mathrm{f}$. 
derlich (sei), wenn Berechnungen über die Adäquanz der Gegenleistungen angestellt werden." 1

Zur Veröffentlichung von Informationen über die Finanzströme zwischen den Mitgliedsstaaten sah sich die Kommission allerdings veranlaßt, als sowohl von der Presse als auch von Abgeordneten des Parlaments teilweise unrichtige Berechnungen über die finanziellen Auswirkungen des EG-Haushalts auf die einzelnen Mitgliedsstaaten publik gemacht wurden. ${ }^{2}$ Die Berechnung der Nettopositionen war zudem erforderlich geworden, nachdem diese Größen in der Verordnung des Rates zur Schaffung eines Finanzmechanismus, mit dessen Hilfe unzumutbar hohe Finanzierungsleistungen eines Mitgliedslandes korrigiert werden sollten, als ein Kriterium für die Rückzahlungsbegrenzung verankert worden waren. ${ }^{3}$ In diesem zusammenhang wies die Kommission darauf hin, daB die ihren Berechnungen zugrunde liegende Definition der Nettopositionen auf finanztechnischen Uberweisungen im Zuge der Ausführung des Haushaltsplans basiert und nicht auf die effektiven wirtschaftlichen Auswirkungen der Gemeinschaftsaktivitäten abstellt. Sie wiederholte zudem ihre Bedenken, daß die in Richtung eines "juste retour" gehenden Berechnungen "dem Entstehen eines europäischen zusammengehörigkeitsgefühls nicht förderlich (seien)." 4

Daneben wies die Kommission auf die Problematik einer die verteilungspolitischen Auswirkungen zutreffend erfassenden Aufteilung bestimmter Haushaltseinnahmen und -ausgaben hin, die die Aussage-

${ }^{1}$ Vgl. EG-Kommission, Antwort auf die schriftliche Anfrage von Herrn L'Estrange vom 31. Januar 1978, in: Amtsblatt der EG, C 56 vom 6. März 1978, S. 29.

${ }^{2} \mathrm{Vgl}$. EG-Kommission, Gemeinsame Antwort der auf die schriftlichen Anfragen Nr. 1020/77 von Lord Bessborough, Nr. 604/78 von Herrn Cointat und $\mathrm{Nr} .607 / 78$ von Lord Bessborough vom 22. Dezember 1978, in: Amtsblatt der EG, Nr. C 28 vom 31.1.1979, S. 2 f.

${ }^{3}$ Siehe hierzu Abschnitt 4.1.2.1.

${ }^{4}$ Vgl. EG-Kommission, Gemeinsame Antwort auf die schriftlichen Anfragen Nrn. 1020/77,604/78 und 607/78, S. 2 . 
fähigkeit der ermittelten Nettopositionen erheblich beeinträchtigen: ${ }^{1}$

- die Zölle und Agrarabschöpfungen werden jeweils den Mitgliedsländern als Zahlungen an den Gesamthaushalt zugewiesen, in denen diese Einnahmen aus Drittlandsimporten anfallen. Insoweit die Importländer nicht gleichzeitig Bestimmungsländer der Einfuhren sind, werden ihnen Mittel anerkannt, die sie nicht aufzubringen haben. Für die Bestimmungsländer gilt die umgekehrte Argumentation.

- Das generell praktizierte Verfahren, die Ausgaben denjenigen Mitgliedsstaaten zuzurechnen, in denen die Zahlungen geleistet werden, entspricht nicht immer den diesen Ländern zukommenden Vorteilen. Als Beispiele nennt die Kommission die in Brüssel und Luxemburg anfallenden und Belgien bzw. Luxemburg "gutgeschriebenen" Verwaltungsaufwendungen der Gemeinschaft sowie die Nahrungsmittelhilfeausgaben für Entwicklungsländer, die den Mitgliedsländern zugerechnet werden, in denen der Ankauf der versandten Nahrungsmittel erfolgt. ${ }^{2}$

- Die von der Kommission vorgenommene zurechnung der im innergemeinschaftlichen Agrarhandel erhobenen und gezahlten währungsausgleichsbeträge auf die Mitgliedsländer ist problematisch und entspricht nicht unbedingt den damit verbundenen Aufwendungen und Erträgen.

Die von der Kommission veröffentlichten Nettopositionen bezogen sich zunächst auf die Haushaltsjahre 1976 und 1977 und wurden im Rahmen einer Antwort auf eine erneute schriftliche Anfrage ${ }^{3}$ um die Berechnungen zum Jahre 1978 ergänzt. Eine eingehende Analyse der verschiedenen Aspekte, die neben den auf reinen Finanzströmen ba-

\footnotetext{
${ }^{1}$ Vgl. EG-Kommission, S. 2 f.; dies., Antwort auf die schriftliche Anfrage $\mathrm{Nr}$. 50/79 von Lord Bessborough vom 8. Juni 1979, in: Amtsblatt der EG Nr. C 104 vom 2.6.1979, S. $9 \mathrm{ff}$.

2 Inzwischen werden die Entwicklungshilfeausgaben aus der Berechnung der Nettopositionen ausgeklammert.

${ }^{3}$ EG-Kommission, Antwort auf die schriftliche Anfrage Nr. 50/79, S. $9 \mathrm{ff}$.
} 
sierenden Nettopositionen berücksichtigt werden müssen, um die Auswirkungen der Gemeinschaftsaktivitäten auf die Mitgliedsstaaten vollständig $\mathrm{zu}$ erfassen, legte die Kommission in einem Arbeitsdokument 1979 vor. ${ }^{1}$ In diesem Zusammenhang wurde auch eine detaillierte Aufgliederung der Einnahmearten und der einzelnen Ausgabengruppen auf die Mitgliedsländer vorgenommen. Dabei handelte es sich zwar um Schätzgrößen für die Jahre 1979 und 1980, die insofern nicht mit den für die Vorjahre veröffentlichten, auf tatsächlich transferierte Mittel aufbauenden Ergebnissen vergleichbar sind. ${ }^{2}$ Die grundsätzliche Berechnungsweise der Nettopositionen war damit aber erstmals nachvollziehbar geworden.

Diese Ergebnisse stellen allerdings die letzten von der Kommission offiziell vorgelegten Daten über die Nettotransfers zwischen den Mitgliedsstaaten dar. Auf verschiedene Anfragen von Abgeordneten des Europäischen Parlaments in den letzten Jahren ${ }^{3}$ hat die Kommission stets erneut darauf verwiesen, daß die Erfassung allein der uber den Haushalt abgewickelten Zahlungsströme eine unvollständige Vorstellung von den Kosten und Nutzen der EG-Mitgliedschaft vermitteln würde, und daß die Schwierigkeit, die darüber hinausgehenden wirtschaftlichen, sozialen und politischen Vorteile aus der zugehörigkeit zur Gemeinschaft zu messen, es nicht rechtfertige, diese außer acht zu lassen. 4 Die Nettopositionen werden von der Kommission zwar weiterhin ermittelt; sind aber offenbar nur für den

1 EG-Kommission, Arbeitsdokument über Haushaltsfragen, KOM (79) 462 endg., Brüssel, 12.9.1979.

${ }^{2}$ Vgl. Ebenda, S. 14.

${ }^{3}$ Schriftliche Anfrage Nr. 1260/81 der Herren Seeler und Rogalla vom 5. Nov. 1981, in: Amtsblatt der EG, Nr. C 47 vom 22.2.1982, S. 15; Schriftliche Anfrage $\mathrm{Nr} .2258 / 82$ von Herrn E. Kellett-Bowman, EGKommission, Mitteilungen der Sprechergruppe vom 6.5.1983. Schriftliche Anfrage Nr. 289/83 von Herrn E. Kellett-Bowman, EG-Kommission, Mitteilungen der Sprechergruppe vom 9.8.1983. Schriftliche Anfrage Nr. 1682/83 von Herrn I. Paisley, EG-Kommission, Mitteilungen der Sprechergruppe vom 30.5.1984.

${ }^{4} \mathrm{Vgl}$. EG-Kommission, Antwort auf die schriftliche Anfrage Nr. 1260/ 81 der Herren Seeler und Rogalla, in: Amtsblatt der EG Nr. C 47 vom 22.2.1982. 
internen Gebrauch innerhalb der organe der Gemeinschaft vorgesehen. 1

Für eine - zumindest annähernde - Bestimmung der Nettoressourcentransfers über den EG-Haushalt ist man allerdings auf die Berechnungen der Kommission nicht unbedingt angewiesen. Es besteht nämlich die Möglichkeit - und auf diese Informationsquelle weist die Kommission ausdrücklich hin ${ }^{2}$ - aus den Haushaltsrechnungen des EGRechnungshofes eine Gegenüberstellung der von den Mitgliedsstaaten geleisteten Finanzierungsbeiträge und den ihnen zugeflossenen Gemeinschaftsausgaben vorzunehmen. Hierin werden die Eigenmittel und damit fast sämtliche Einnahmen - vollständig nach den abführenden Mitgliedsstaaten ausgewiesen; die Ausgaben allerdings nur zu 80 bis 85 \& des Haushaltsvolumens ${ }^{3}$, wobei im Unterschied zu den Kommissionsrechnungen vor allem die Verwaltungsausgaben sowie die Ausgaben in den Bereichen Forschung, Industrie, Energie und Verkehr unberücksichtigt bleiben. ${ }^{4}$ Berechnungen der Nettopositionen werden auch $^{5}$ vom Bundesministerium der Finanzen (BMF) vorgenommen, das sich dabei auf interne Rommissionsangaben stützt. 6

${ }^{1}$ Vgl. EG-Kommission, Antwort auf die schriftliche Anfrage Nr. 289/ 83 von Herrn Kellett-Bowman, Mitteilungen der Sprechergruppe vom 9.8.1983

${ }^{2}$ Vgl. EG-Kommission, Antwort von Herrn Tugendhat im Namen der Kommission auf die schriftlichen Anfragen Nrn. 1680/83, 1681/83 und $1682 / 83$ von Herrn Paisley, Mitteilungen der Sprechergruppe vom 30.5.1984.

${ }^{3} \mathrm{Vgl}$. Rechnungshof der EG, Jahresbericht zu dem Haushaltsjahr ... zusammen mit den Antworten der Organe, in: Amtsblatt der EG, versch. Jg. Die Entwicklungshilfeausgaben werden sowohl in den Berechnungen der Kommission als auch in den Haushaltsrechnungen nicht berücksichtigt. Siehe dazu auch weiter unten.

${ }^{5}$ Daneben werden in der deutschen und internationalen Presse ständig Angaben über die Nettopositionen publiziert, die vermuten lassen, das die für den internen Gebrauch bestimmten Kommissionsdaten auch Außenstehenden zugänglich sind.

${ }^{6} \mathrm{Vgl}$. etwa Bundesministerium der Finanzen, Finanznachrichten $30 /$ 1983, vom 10.11.1983, S. 6 . 
Die in Tabelle 2 dargestellten Nettopositionen beziehen sich bis zum Jahre $1978^{1}$ auf die von der Kommission veröffentlichten Angaben und danach auf die Berechnungen des $\mathrm{BMF}^{2}$. Sie sind unterteilt in die Größen vor und nach der Durchführung der Entlastungsmaßnahmen zugunsten des Vereinigten Königreichs, die von den übrigen Mitgliedsstaaten finanziert werden und die damit ihre Verteilungsposition verschlechtern. ${ }^{3}$ Die Ergebnisse zeigen, daß über den gesamten Zeitraum allein die Bundesrepublik und das Vereinigte Königreich Nettozahler slnd, während die übrigen Mitgliedsländer lange Zeit (Frankreich) bzw. ausschließlich zu den Nettoempfängern gehören. Die beiden Nettozahler weisen ab 1980, also nach dem Auslaufen der Beitrittsübergangsbestimmungen für die 1973 neu aufgenommenen Mitgliedsstaaten, einen etwa gleichhohen Finanzierungssaldo auf, der sich allerdings für das Vereinigte Königreich infolge der Ausgleichsmaßnahmen erheblich reduziert.

Das von der Kommission angewandte Verfahren zur Berechnung der Nettopositionen, auf dem die hier dargestellten Ergebnisse im Grundsatz beruhen, läßt sich auf folgende Weise darstellen: ${ }^{4}$

- Die Nettopositionen der einzelnen Mitgliedsstaaten werden zwar grundsätzlich aus der Differenz zwischen ihren Zahlungen an den Gemeinschaftshaushalt und den daraus empfangenen Rückflüssen be-

IEs sei hier nochmals darauf verwiesen, das die Werte der bis 1977 geltenden, auf festen Paritäten basierenden IWF-Rechnungseinheiten (RE) stark von der danach verwendeten, an Marktkursen orientierten Europäischen Rechnungseinheit (ERE und ab 1981 ECU) abweichen, so daß sich die Ausgaben bis 1977 nicht ohne weiteres mit denen der folgenden Jahre vergleichen lassen.

${ }^{2}$ Ein Vergleich mit den bei Reichenbach (1984), S. 454 angegebenen Daten, bei denen es sich offenbar um die internen Berechnungen der Kommission handelt, zeigt nur relativ geringfügige Abweichungen.

${ }^{3}$ Dabei ist $z u$ beachten, daß die Ausgleichsregelungen in dem dafür vorgesehenen Jahr in den Werten der Tabelle berücksichtigt sind, sie aber erst liber den Haushalt des jeweils folgenden Jahres tatsächlich abgewickelt werden.

${ }^{4} \mathrm{Vgl}$. Britisches Schatzamt, The European Community budget: net contributions and recelpts, in: Economic Progress Report, Supplement Oktober 1982, S. 3; A. Edwards, Der Haushalt der Europäischen Gemeinschaft: das Konzept der Nettopositionen, in: Europa-Archiv, 37. Jg., 1982, S. $747 \mathrm{ff}$. 
Tabelle 2

Nettopositionen der Mitgliedsstaaten 1976 - 1984 (in Mio. ECU) ${ }^{a}$

\begin{tabular}{|c|c|c|c|c|c|c|c|c|}
\hline \multirow[t]{2}{*}{ Mitgliedsland } & \multirow[t]{2}{*}{1976} & \multirow[t]{2}{*}{1977} & \multirow[t]{2}{*}{1978} & \multirow[t]{2}{*}{1979} & \multicolumn{2}{|c|}{1980} & \multicolumn{2}{|c|}{1981} \\
\hline & & & & & vor & $\begin{array}{l}\text { nach } \\
\text { britisch }\end{array}$ & $\begin{array}{c}\text { vor } \\
\text { Entla }\end{array}$ & $\begin{array}{l}\text { nach } \\
\text { tung }\end{array}$ \\
\hline Belgien & 346,2 & 328,8 & 350,3 & 610 & $+\quad 236$ & +174 & $+\quad 317$ & 247 \\
\hline Luxemburg & & & & & +206 & +203 & +270 & +266 \\
\hline Dänemark & 294,0 & 293,0 & 381,3 & 380 & 326 & +295 & +281 & 241 \\
\hline Deutschland & $-1053,6$ & $-1466,9$ & 519,8 & -1430 & -1531 & -1982 & -1770 & -2285 \\
\hline Griechenland & -- & -- & -- & -- & -- & -- & +164 & +156 \\
\hline Frankreich & 58,1 & 309,9 & 321,0 & 78 & 422 & 83 & +536 & 119 \\
\hline Irland & 155,1 & 212,0 & 320,1 & 545 & 650 & 639 & +588 & 575 \\
\hline Italien & 247,8 & 293,6 & 304,9 & 534 & 739 & 544 & $+\quad 775$ & 525 \\
\hline Niederlande & 220,8 & 87,5 & 57,0 & 288 & +459 & 376 & $+\quad 215$ & 122 \\
\hline Verein. Königreich & 89,8 & 125,8 & 407,0 & 849 & -1507 & 332 & -1376 & 34 \\
\hline
\end{tabular}

a Nettoempfänger: +; Nettozahler: - 
Tabelle 2 (Fortsetzung)

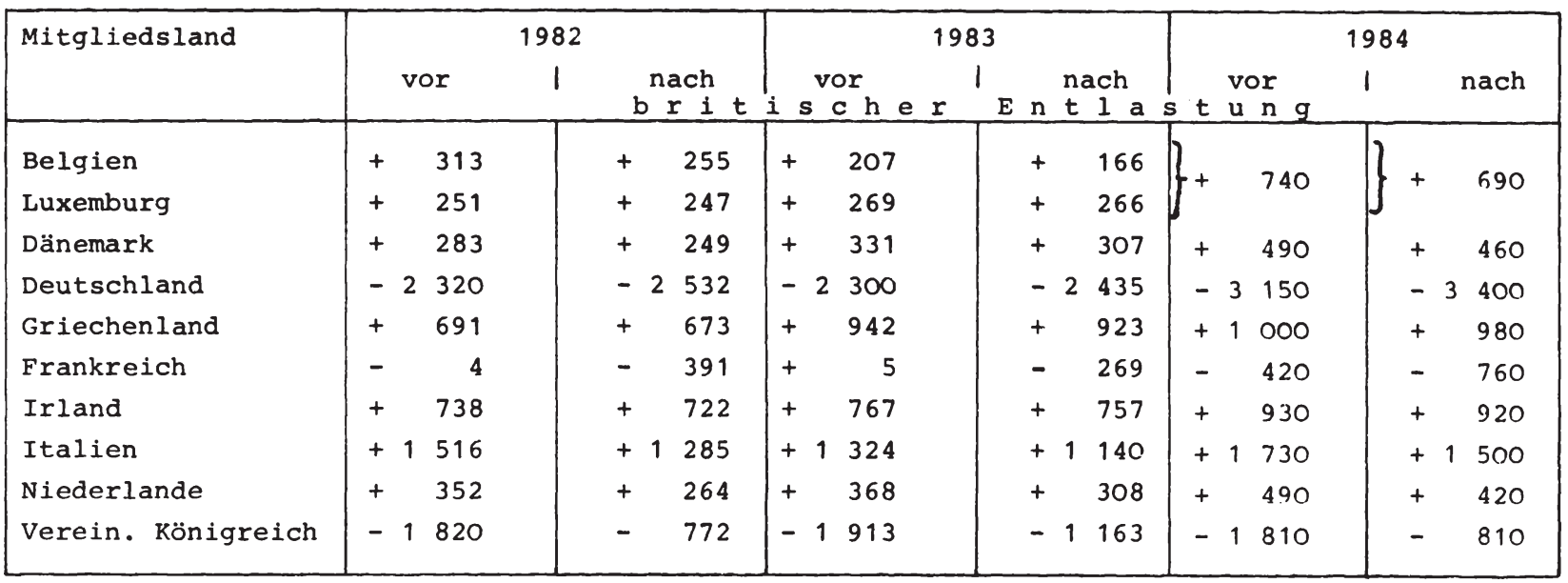

Quelle: bis 1978: EG-Kommission, Antwort auf die schriftliche Anfrage Nr. 50/79, S. 11; ab 1979: Bundesministerium der Finanzen, Der Haushalt der Europäischen Gemeinschaften, Dokumentation, Bonn, versch. Jahre; 1984: Berechnungen des BMF, die für die Zwecke dieser Arbeit zur Verfiiquna aestellt wurden. 
rechnet. Dabei sind allerdings nicht sämtliche Einnahmen und Ausgaben des Gesamthaushalts, sondern nur diejenigen im Rahmen des sog. zugewiesenen Haushalts zu berücksichtigen.

- Das Volumen des zugewiesenen Haushalts wird determiniert durch diejenigen Haushaltsausgaben, die die Kommission den Mitgliedsstaaten zuweist, d.h. auch im Sinne von Mittelübertragungen zurechnen kann. In diesem Rahmen werden etwa 90 bis 95 \& des Gesamthaushaltsvolumens erfaßt. ${ }^{1}$ Die übrigen Ausgaben, die hauptsächlich zum Bereich der Zusammenarbeit mit Entwicklungsländern und Drittländern gehören, werden als sog. nicht zugewiesener Haushalt bezeichnet und aus den Berechnungen ausgeklammert.

- Die Leistungen der Mitgliedsstaaten zum zugewiesenen Haushalt werden nach Maßgabe ihrer Finanzierungsanteile am Gesamthaushalt berechnet. Man geht hier also vom Proportionalansatz aus, d.h. unterstellt, daß für den zugewiesenen wie für den nicht zugewiesenen Haushalt die gleiche Finanzierungsstruktur gilt.

- Da sämtlichen Finanzierungsleistungen an den zugewiesenen Haushalt auch Rückflüsse an die Mitgliedsstaaten gegenüberstehen, ist die Summe aus Nettozahlungen und Nettoempfängen gleich Null.

- Die Ausgaben im Rahmen der EntlastungsmaBnahmen zugunsten GroBbritanniens sowie die Finanzierungsbeteiligungen der ubrigen Mitgliedsstaaten an diesen Rückerstattungen werden außerhalb des $\mathbf{z u}-$ gewiesenen Haushalts behandelt und die um diese Korrekturen veränderten Verteilungspositionen gesondert ausgewiesen.

Nachdem das bislang praktizierte Verfahren zur Berechnung der Nettopositionen dargestelit und einige unmittelbar sichtbare Unzulänglichkeiten aufgezeigt worden sind, erfolgt nun eine systematische Analyse der Finanztätigkeit der Gemeinschaft. Die daraus resultierenden Ergebnisse sollen einem zweifachen Zweck dienen: der Beurteilung des

${ }^{1}$ Vgl. hierzu die detallliert dargestellten Berechnungen der Kommission für die Jahre 1979 und 1980 in: EG-Kommission, KOM (79) 462 endg., Tabelien 3 und 4 des Anhangs. 
Finanzierungssystems und der ausgabenwirksamen Gemeinschaftspolitiken im Hinblick auf die im Abschnitt 2. behandelten verteilungspolitischen Prinzipien und zielsetzungen sowie der tberprüfung der Aussagefähigkeit des gegenwärtig verwendeten Konzepts der Nettopositionen und darauf aufbauend der Ermittlung eines erweiterten Indikators. 


\section{Verteilungswirkungen der finanzwirtschaftlichen Gemeinschafts-} tätigkeit

Die quantitative Bedeutung der einzelnen finanziellen Aktivitäten der Gemeinschaft zeigt Tabelle 3. Der überwiegende Teil der Transaktionen wird über den Gesamthaushalt abgewickelt, während der EGKS-Funktionshaushalt und das Finanzvolumen des Entwicklungsfonds mit gegenwärtig nur etwa 1,5 \& bzw. 3 \& des Gesamthaushaltsvolumens eine gegenwärtig untergeordnete Rolle spielen. Quantitativ bedeutsam sind dagegen die auBerhalb des Budgets durchgeführten Anleihe- und Darlehensaktivitäten der Gemeinschaft, die in den letzten Jahren immerhin mehr als ein Viertel und 1983 sogar fast die Hälfte des Gesamthaushaltsumfangs ausmachten.

Die größenmäßige Relevanz der Gemeinschaftstransaktionen kann weiterhin relativiert werden, wenn die Finanzvolumina zum Umfang des BIP bzw. der nationalen Haushalte der Mitgliedsstaaten in Beziehung gesetzt werden. So beträgt das Gesamthaushaltsvolumen gegenwärtig lediglich 1 \& des gemeinschaftlichen BIP, während die nationalen Budgets der Mitgliedsstaaten zwischen 30 und 60 \& ihres Sozialprodukts ausmachen. Eine in diesem Sinne doppelt so große Bedeutung wie der Gesamthaushalt besitzen die gemeinschaftlichen Anleihe- und Darlehensaktivitäten. Sie betragen in den letzten Jahren etwa 0,2 \& (1983: 0,4 \%) des Gemeinschaftssozialprodukts gegenüber einem gut 5 sigen Anteil der mitgliedsstaatlichen Haushaltsdefizite. ${ }^{1}$

\subsection{Gesamthaushalt}

Der Gesamthaushalt der EG umfaßt den Haushalt der EWG, den Verwaltungshaushalt und den Forschungs- urd Investitionshaushalt der EAG sowie den Verwaltungshaushalt der EGKS. Er besteht in dieser Form

${ }^{1} \mathrm{Vgl}$. EG-Kommission, Europäische Wirtschaft, Nr. 22, s. $222 \mathrm{ff}$. 240; Bundesministerium der Finanzen (Hrsg.), Finanzbericht 1986, Bonn 1985, S. 249. 
Tabelle 3

Finanztatigkeit der EG $1975-1984$

(In Mio ECU)

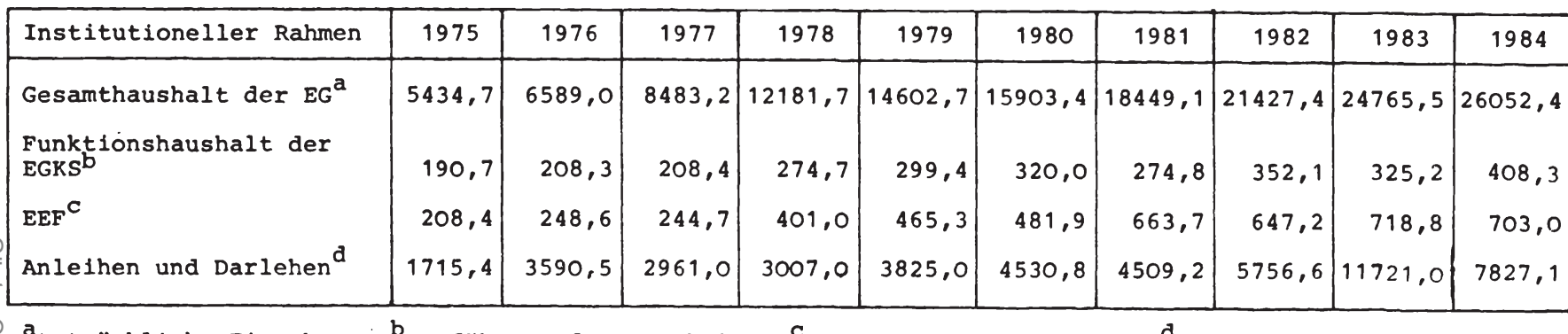

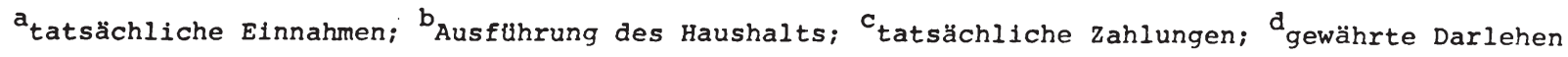

Quelle: EG-Rechnungshof, Jahresberlchte zu den Haushaltsjahren; EG-Komission, Finanzberichte der EGKS; dies., Gesamtberlchte uber die Tätigkeit der EG, jeweils versch. Jg.; D. Strasser, S. $402 \mathrm{f}$. 
seit dem Jahre 1971. Zuvor waren durch den Brüsseler Fusionsvertrag 1968 bereits der Haushalt der EWG und die beiden Verwaltungshaushalte der EAG und EGKS zu einem Gesamthaushalt zusammengefaßt worden, in den durch den Vertrag von Luxemburg 1971 auch der Forschungsund Investitionshaushalt der EAG integriert wurde. Für die drei rechtlich selbständigen Gemeinschaften gilt demnach ein Haushaltsplan; als Ausnahme besteht neben diesem Budget noch der separate Funktionshaushalt der EGKS.

\subsubsection{Finanzierungssystem}

Da die vorgesehene Analyse der Verteilungswirkungen primär auf den Zeitraum nach der vollständigen Anwendung des Eigenmittelsystems abstellen soll, ist das Hauptaugenmerk auf die Ausgestaltung des Finanzierungssystems ab 1980 zu richten. Zum besseren Verständnis und zur Einordnung dieser Zusammenhänge scheint es allerdings zweckmäßig zu sein, auch kurz auf die früheren Finanzierungsverfahren einzugehen. Die bisherige Entwicklung des Finanzierungssystems der EG läßt sich in drei Phasen einteilen:

- in das ursprüngliche System der Finanzbeiträge seit der Gründung der Gemeinschaft bis zum Jahre 1970;

- in die anschließende zeit der Ubergangsregelungen bis zur vollständigen Verwirklichung des Eigenmittelbeschlusses, in die auch die erste Erweiterung der Gemeinschaft um Dänemark, Irland und das Vereinigte königreich mit den zugehörigen Beitrittsübergangsregelungen fällt;

- und schließlich in das seit 1980 praktizierte system der eigenen Mittel, das lediglich durch Ubergangsregelungen für das 1981 neu aufgenommene Mitglied Griechenlands lind neuerdings auch für die 1986 beigetretenen Mitgliedsländer Spanien und Portugal modifiziert wird. 
Von ihrer Grüdung bis zum Jahre 1970 finanzierte sich die EWG und ähnlich auch die EAG - nahezu ausschlieBlich über Beiträge der Mitgliedsstaaten. Dabei hing die Höhe des Beitragsaufkommens von dem Umfang der bewilligten Ausgaben ab. Die Finanzierung erfolgte nicht nach dem Nonaffektationsprinzip, sondern getrennt nach unterschiedlichen Ausgabenzwecken über dafür vorgesehene Fonds. Die Verteilung der Finanzierungslasten auf die Mitgliedsstaaten ergab sich aus den Beitragsschlüsseln, die für die einzelnen Fonds festgelegt wurden. Diese Beitragsquoten entsprachen insbesondere beim Sozialfonds und beim Investitions- und Forschungshaushalt der EAG den BSP-Relationen der Mitgliedsländer und orientierten sich damit an ihrer wirtschaftlichen Leistungsfähigkeit. ${ }^{1}$ Bei der - häufig revidierten - Beitragsregelung für den quantitativ dominierenden Agrarfonds spielten in zunehmendem Maße die Höhe der Nettoimporte aus Drittländern bzw. die daraus resultierenden Abgaben eine Rolle, so daß der Bezug zum Leistungsfähigkeitsprinzip mehr und mehr in den Hintergrund trat. ${ }^{2}$ Der Beitragsschlüssel des Verwaltungshaushalts schließlich wurde auch von politischen Faktoren: der in der Bevölkerungsgröß $\mathrm{e}^{3}$ liegenden politischen Bedeutung der Mitgliedsländer sowie ihren stimmverhältnissen in der alten Versammlung und im Rat ${ }^{4}$ beeinfluBt.

In der Systematik des Finanzausgleichs stellen diese Finanzierungsregelungen ein Zuweisungssystem dar. Ein solches Verfahren besitzt Vorteile, da es technisch relativ einfach durchzuführen ist, den Mitgliedsstaaten die Art der Mittelaufbringung freigestellt bleibt und bei der Festlegung der Beitragsschlüssel die verschiedenen zielsetzungen in geeigneter Weise berücksichtigt werden können. 5

${ }^{1}$ Vgl. N. Andel, Finanzwissenschaftliche Aspekte regionaler wirtschaftlicher Zusammenschlüsse, a Europäische Gemeinschaften, in: F. Neumark (Hrsg.), Handbuch der Finanzwissenschaft, 3. Aufl., Bd. 4, Tübingen 1983 , S. 329 f.

${ }^{2}$ Vgl. U. Nittka, S. $94 \mathrm{ff}$. und $283 \mathrm{ff}$.

${ }^{3}$ Vgl. E. Reister, S. 28.

${ }^{4}$ Vgl. N. Andel (1983), S. 329.

${ }^{5} \mathrm{Vgl}$. R. Peffekoven, Das Finanzierungssystem der Europäischen Gemeinschaften, in: Wirtschaftswissenschaftliches studium (WIST), 11. Jg., 1982, S. $413 \mathrm{ff}$, hier: S. 424. 
Insofern ist Reister zuzustimmen, daß das System der Finanzbeiträge "der ersten Entwicklungsetappe dieser Gemeinschaften durchaus angemessen (war), da Volumen und Natur der künftigen Ausgaben Funktion des erst eingeleiteten Integrationsprozesses waren und ein einfaches und flexibles Finanzierungssystem bedingten". 1

Die Finanzierung der Gemeinschaftsausgaben über Finanzbeiträge war allerdings von vornherein nicht als endgültige Lösung gedacht und die Möglichkeit ihrer Substituierung durch eigene Einnahmen in den Gemeinschaftsverträgen bereits vorgesehen. Nach Art. 201 des EWGVertrages hatte die Kommission zu prüfen, "unter welchen Bedingungen die Finanzbeiträge der Mitgliedsstaaten durch eigene Mittel, insbesondere durch Einnahmen aus dem gemeinsamen Zolltarif nach dessen endgültiger Einführung ersetzt werden können." Zugunsten einer solchen Weiterentwicklung $\mathrm{zu}$ einem Eigenmittelsystem konnten zum einen gemeinschaftspolitische Argumente angefihrt werden: die Sicherung und Stärkung des eigenständigen Charakters der Gemeinschaft, eine größere Autonomie in bezug auf das Haushalts- und Finanzgebaren sowie auch die Hoffnung des Parlaments, im Rahmen dieser Finanzierungsreform effektive Budgetbefugnisse zu erhalten. 2

Daneben gebot auch die in den 60er Jahren weitgehend verwirklichte Zollunion mit der innergemeinschaftlichen zollfreiheit und dem gemeinsamen Außenzoll sowie die im Rahmen der gemeinsamen Agrarpolitik erhobenen Abschöpfungen auf Drittlandsimporte eine tbertragung dieser Mittel an die Gemeinschaft. Denn zum einen sind diese Abgaben das Ergebnis von Gemeinschaftsaktivitäten und damit ihrem Wesen nach dazu bestimmt, der Gemeinschaft zuzufließen. ${ }^{3}$ zum anderen führte die nationale Ertragshoheit für diese Mittel zu Diskrepanzen

${ }^{1}$ E. Reister, S. 27.

${ }^{2}$ Vgl. N. Andel. (1983), S. 331; U. Nittka, S. $199 \mathrm{ff}$.

${ }^{3} \mathrm{Vgl}$. EG-Parlament, Bericht im Namen des Finanz- und Haushaltsausschusses über die Vorschläge der Kommission der Europäischen Gemeinschaften an den Rat (Dok. 99/69 und 147/69) für die Schaffung von Eigenmitteln der Gemeinschaften und die Erweiterung der Haushaltsbefugnisse des Europäischen Parlaments, Berichterstatter: Herr Spénale, Europäisches Parlament, Sitzungsdokumente 1969 1970, Dok. 174 vom 8.12.1969, S. 41. 
zwischen dem nationalen Aufkommen und der nationalen Belastung. Denn der Weg von Drittlandsimporten in die Gemeinschaft wird häufig von verkehrs- und transporttechnischen Gegebenheiten bestimmt, so daß das abgabenerhebende Mitgliedsland oft nicht mit dem Land identisch ist, in dem die Einfuhren verbraucht und die Einfuhrabgaben letztlich aufgebracht werden. Daher stellte auch unter finanzierungstechnischen Gesichtspunkten eine zufriedenstellende Lösung nur die Zuweisung der Zölle und Agrarabschöpfungen an den Gemeinschaftshaushalt dar. 1

Der entscheidende Schritt zum Eigenmittelsystem erfolgte mit dem BeschluB des Rates vom 21. April 1970 über die Ersetzung der Finanzbeiträge der Mitgliedsstaaten durch eigene Mittel der Gemeinschaften ${ }^{2}$, der nach der Ratifizierung durch die Mitgliedsstaten zum 1. Januar 1971 wirksam wurde. ${ }^{3}$ Eigene Mittel sind die Agrarabschöpfungen einschließlich der Zuckerabgaben, die ab 1971 in vollem Umfang in den Haushaltsplan eingesetzt wurden, sowie die zölle des gemeinsamen Agrarzolltarifs, die zunächst stufenweise und ab 1975 vollständig der Gemeinschaft zufließen. Die zum Ausgleich des Haushalts noch erforderlichen Einnahmen, die sog. Restfinanzierung, solite im tbergangszeitraum bis 1975 weiterhin aus Finanzbeiträgen der Mitgliedsstaaten bestehen. Hierfür galt ein einheitlicher Beitragsschlüssel, der aus den Sozialproduktrelationen und den übrigen bisher geltenden Schlüsseln gebildet wurde. Die hieraus resultierenden Finanzbeiträge wurden allerdings korrigiert, um Schwankungen in den gesamten Finanzierungsanteilen der einzelnen Mitgliedsstaaten $\mathrm{zu}$ begrenzen.

Der Eigenmittelbeschluß sah ab 1.1.1975 den vollständigen Ersatz der Finanzbeiträge durch die dritte Eigenmittelkategorie, eine Beteiligung an dem Mehrwertsteueraufkommen der Mitgliedsstaaten, vor,

\footnotetext{
${ }^{1}$ Vgl. Eg-Parlament, Sitzungsdokumente 1969 - 1970, Dok. 174, S. 41. ${ }^{2}$ Amtsblatt der EG Nr. L 94 vom 28.4.1970, S. $19 \mathrm{ff}$.

${ }^{3}$ Das Eigenmittelsystem erstreckte sich nun auch auf die in den Haushaltsplan einzustellenden Ausgaben der EAG.
} 
wobei der Ertragsanspruch der Gemeinschaft auf maximal 1 \& der steuerpflichtigen Bemessungsgrundlage beschränkt war. Die dazu erforderliche Harmonisierung der nationalen Bemessungsgrundlagen ${ }^{1}$ konnte allerdings nur mit großen Verzögerungen erreicht werden, so daß die Mehrwertsteuereinnahmen erstmals 1979 und von sämtlichen Mitgliedsstaaten mit Beginn des Jahres 1980 an die Gemeinschaft abgeführt werden. Während dieser (zweiten) Ubergangszeit von 1975 bis 1979 erfolgte die Restfinanzierung weiterhin über Finanzbeiträge der Mitgliedsstaaten, die nun allerdings ausschließlich nach dem Sozialproduktschlüssel bemessen wurden. Zu den Ubergangsregelungen für die Gründungsmitglieder der Gemeinschaft traten für die ab 1973 neu beigetretenen Mitgliedsstaaten Dänemark, Großbritannien und Irland Sonderbestimmungen gemäß der Beitrittsakte hinzu, die bis Ende 1979 die Finanzierungsanteile dieser Länder gesondert begrenzten. $^{2}$

Die Entwicklung der Finanzierungsmittel während des Ubergangszeitraums und nach vollständiger Anwendung des Eigenmittelsystems zeigt Tabelle 4. Dabei wird zunächst deutlich, daß die quantitative Bedeutung der Zölle und Agrarabschöpfungen als Finanzierungsquelle des Gesamthaushalts zurückgegangen ist. Während bei den zöllen in der Regel absolut steigende Einnahmen zu verzeichnen waren, sanken die Agrarabschöpfungen nicht nur relativ, sondern teilweise auch in absoluten Beträgen und schwankte das Aufkommen zudem relativ stark. Diese Einnahmen können von der Gemeinschaft auch nicht nach finanzpolitischen Erfordernissen ausgestaltet werden, sondern sind das

\footnotetext{
${ }^{1}$ Der an die EG abzuführende Mehrwertsteueranteil kann wegen der unterschiedlichen Höhe und Anzahl der nationalen Mehrwertsteuersätze (vgl. O.V., EC: The Evolution of VAT Rates Applicable in the Member states of the Community, in: Intertax, $1984, \mathrm{~S} .252 \mathrm{ff}$.$) nicht$ an die einzelstaatlichen Mehrwertsteueraufkommen anknüpfen. Hierzu wäre eine Harmonisierung auch der Mehrwertsteuersätze erforderlich gewesen, die insbesondere wegen der Auswirkungen auf die Höhe der nationalen steueraufkommen wesentlich schwieriger herbeizuführen ist, als es die Vereinheitlichung der Bemessungsgrundlage war. ${ }^{2} \mathrm{zu}$ den Einzelheiten dieser Regelungen vgl. U. Nittka, S. $106 \mathrm{ff}$.
} 
Tabelle 4

Einnahmen des EG-Gesamthaushalts ${ }^{a} 1975$ - 1984

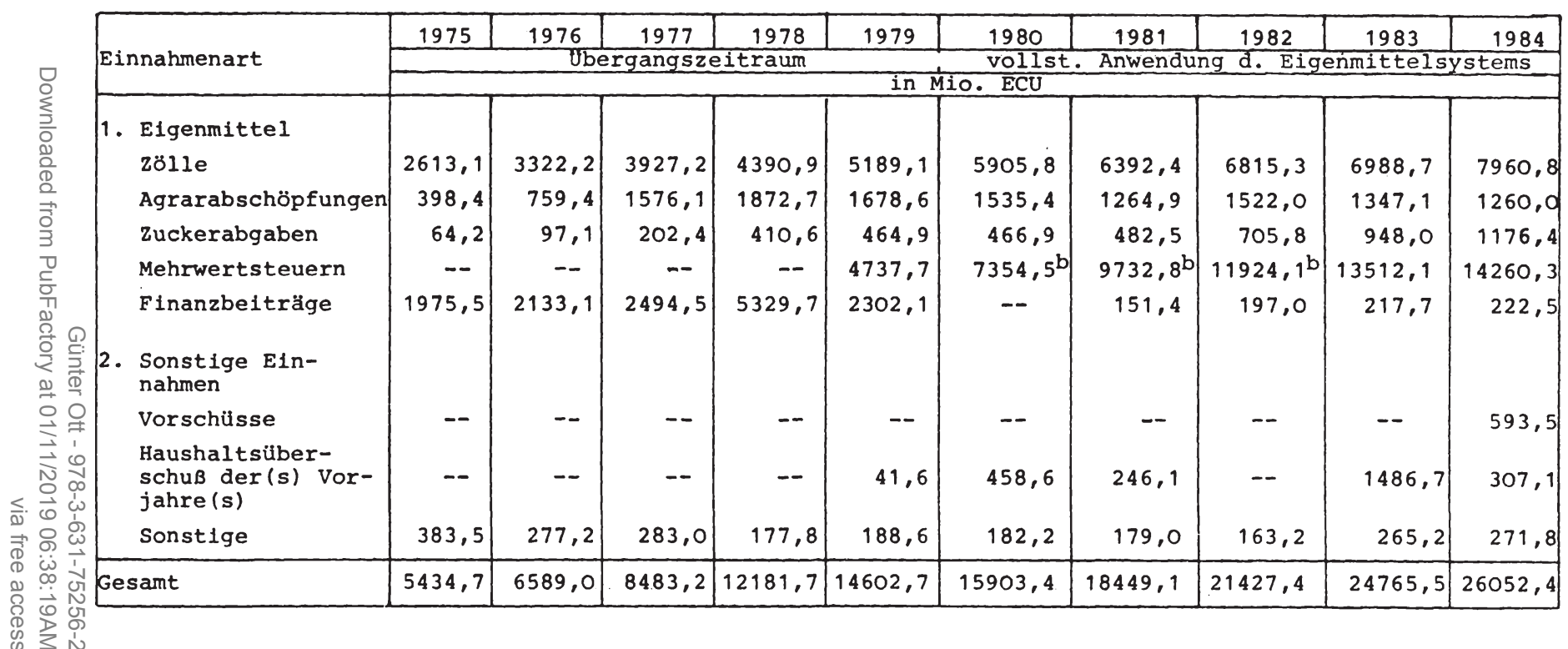


Tabelle 4 (Fortsetzung)

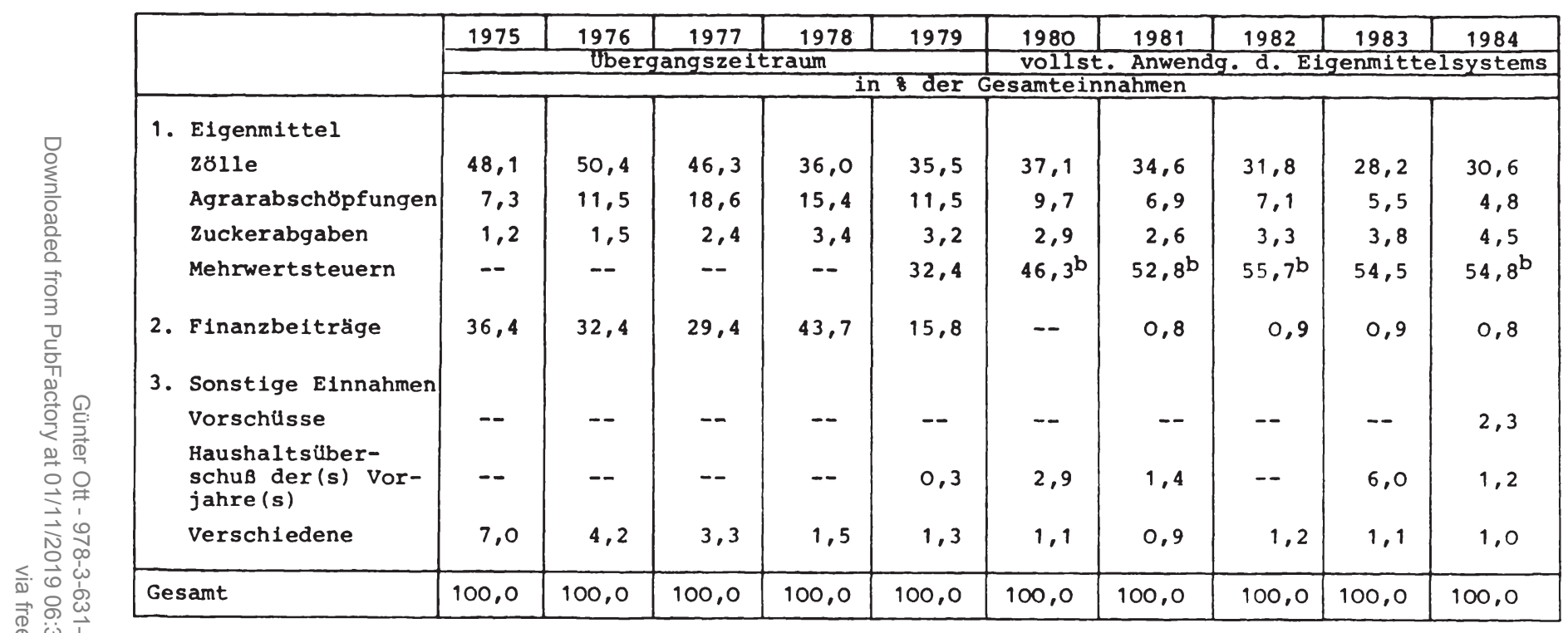

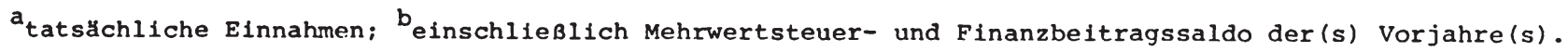
Quelle: EG-Rechnungshof, Jahresberichte zu den Haushaltsjahren 1975 bis 1984 . 
Ergebnis handels- und agrarpolitischer Gegebenheiten und Entwicklungen. 1

Mit der Entwicklung der Zölle, Agrarabschöpfungen und Zuckerabgaben korrespondieren die Finanzbeiträge bzw. Mehrwertsteuereinnahmen in der Weise, daß sie - unter Einschluß der sonstigen Einnahmen - als Restfinanzierung den zum Ausgleich der Haushaltsausgaben noch ausstehenden Betrag bereitstellen. Damit ergibt sich ihre erforderliche Höhe sowohl aus der Entwicklung aller übrigen Einnahmen als auch aus dem Volumen der zu finanzierenden Gesamtausgaben. Der Finanzierungsspielraum ist nach dem EigenmittelbeschluB von 1970 allerdings durch die 18 -Grenze limitiert. Während im Haushaltsjahr 1980 der Ausschöpfungssatz erst 0,73 \& betrug, ${ }^{2}$ wurde diese Einnahmequelle bereits 1983 mit $0,9988^{3}$ fast vollständig ausgeschöpft. Um den insgesamt weiter stark wachsenden Finanzbedarf der Gemeinschaft zu decken, ist der Mehrwertsteuer-Eigenmittel-Plafonds mit Beginn des Jahres 1986 auf 1,4 \& der Bemessungsgrundlage heraufgesetzt worden. 4 Die über den erschöpften Eigenmittelrahmen hinausgehenden Ausgabenverpflichtungen der Haushaltsjahre 1984 und 1985 wurden zwischenzeitlich durch Vorschüsse auf die erhöhten Eigenmittel finanziert.

\footnotetext{
${ }^{1}$ Vgl. EG-Parlament, Bericht im Namen des Haushaltsausschusses über die Eigenmittel der Gemeinschaft, Berichterstatter: Herr Altiero Spinelli, Europäisches Parlament, Sitzungsdokumente 1980 - 1981, Dok. 1-772/80, 5.1.1981, S. 31.

${ }^{2}$ Vgl. EG-Rechnungshof, Jahresbericht zum Haushaltsjahr 1980 zusammen mit den Antworten der Organe, in: Amtsblatt der EG Nr. C 344 , 31.12.1981, S. 181 .

${ }^{3}$ Vgl. EG-Kommission, Siebzehnter Gesamtbericht über die Tätigkeit der Europäischen Gemeinschaften 1983, Brüssel, Luxemburg 1984, S. $44 \mathrm{f}$.

${ }^{4}$ EG-Rat, BeschluB des Rates vom 7. Mai 1985 über das System der eigenen Mittel der Gemeinschaften, in: Amtsblatt der EG Nr. L 128, 14.5 .1985 , S. 15 ff.
} 


\subsubsection{Mehrwertsteuer-Eigenmittel}

Bei einer Analyse der zwischenstaatlichen Belastungswirkungen der Mehrwertsteuer-Eigenmittel ist zunächst auf die relative Bedeutung dieser Einnahmenkategorie hinzuweisen. Wie aus der Tabelle 4 hervorgeht, hat der Anteil der Mehrwertsteuern an den gesamten Finanzierungsaufwendungen in der Vergangenheit ständig zugenommen und macht gegenwärtig mehr als die Hälfte der gesamten Einnahmen aus. Insofern werden die Vertellungswirkungen des gesamten Finanzierungssystems auch maßgeblich von den Mehrwertsteuer-Eigenmitteln beeinfluBt.

Der Haushalt des Jahres 1980 ist der erste, bei dem das Eigenmittelsystem vollständig zur Anwendung gelangte und mithin jeder Mitgliedsstaat die im Eigenmittelbeschluß festgelegten Mehrwertsteuereinnahmen abzuführen hatte. Dieses System wird seit dem Jahre 1981 lediglich durch eine Ubergangsregelung für das neu aufgenommene Mitglied Griechenland modifiziert, ${ }^{1}$ das bis zum Ende des Jahres 1985 anstelle der Mehrwertsteuer Finanzbeiträge nach dem BSP leistet. Diese Ausnahmebestimmung schränkt - auch angesichts der wirtschaftlichen Größe Griechenlands - die Wirkungsweise des Eigenmittelsystems und speziell der Mehrwertsteuerregelung allerdings nur unwesentlich ein. Die von den Mitgliedsländern seit 1980 abgeführten Mehrwertsteuern sind in der Abgrenzung der sog. Zahlungs-Definition in Tabelle 5 ausgewiesen. Dabei handelt es sich um die von der Gemeinschaft tatsächlich eingenommenen Beträge, d.h. die laufenden Zahlungen der Mitgliedsstaaten im Haushaltsjahr einschließlich der Salden und Berichtigungen der vorhergehenden Haushaltsjahre.

${ }^{1}$ Die zu Beginn des Jahres 1986 der Gemeinschaft beigetretenen Mitglieder Spanien und Portugal führen bereits im ersten Jahr ihrer Mitgliedschaft Elgenmittel zu denselben Bedingungen wie die übrigen Mitgliedsstaaten ab. Sie erhalten allerdings einen finanziellen Ausgleich, der mit dem Grad ihrer Integration geringer wird. Vgl. EGKommission, Bulletin der EG Nr. 6/1985, S. 31. 
Tabelle 5

Mehrwertsteuer-Eigenmittel nach Mitgliedsstaaten $1980-1984^{\mathrm{a}}$

\begin{tabular}{|c|c|c|c|c|c|}
\hline Mitgliedsland & 1980 & 1981 & 1982 & 1983 & 1984 \\
\hline & \multicolumn{5}{|c|}{ in MiO. ECU } \\
\hline Belgien & 337,7 & 375,9 & 461,6 & 432,4 & 473,4 \\
\hline Dänemark & 189,4 & 189,8 & 226,8 & 274,3 & 289,6 \\
\hline Deutschland & 2456,7 & 2806,5 & 3339,9 & 4038,8 & 4233,9 \\
\hline Frankreich & 1842,4 & 2256,2 & 2872,8 & 3082,1 & 3201,8 \\
\hline Griechenland ${ }^{b}$ & - & 151,5 & 196,3 & 215,6 & 220,3 \\
\hline Irland & 63,7 & 68,1 & 107,7 & 150,6 & 135,9 \\
\hline Italien & 858,7 & 1582,9 & 1457,8 & 1923,4 & 2319,9 \\
\hline Luxemburg & 15,2 & 22,6 & 25,7 & 39,2 & 45,3 \\
\hline Niederlande & 461,6 & 499,8 & 649,8 & 713,7 & 738,3 \\
\hline Verein. Königreich & 1294,1 & 1930,8 & 2782,7 & 2859,7 & 2824,4 \\
\hline \multirow[t]{2}{*}{ EG } & 7519,5 & 9884,1 & 12121,1 & 13729,8 & 14482,8 \\
\hline & \multicolumn{5}{|c|}{ in 8} \\
\hline Belgien & 4,49 & 3,80 & 3,81 & 3,14 & 3,27 \\
\hline Dänemark & 2,52 & 1,92 & 1,87 & 2,00 & 2,00 \\
\hline Deutsch land & 32,67 & 28,39 & 27,56 & 29,42 & 29,23 \\
\hline Frankreich & 24,50 & 22,83 & 23,70 & 22,45 & 22,12 \\
\hline Griechen land $^{b}$ & - & 1,53 & 1,62 & 1,57 & 1,52 \\
\hline Irland & 0,85 & 0,69 & 0,88 & 1,10 & 0,94 \\
\hline Italien & 11,42 & 16,02 & 12,03 & 14,00 & 16,02 \\
\hline Luxemburg & 0,20 & 0,23 & 0,21 & 0,29 & 0,31 \\
\hline Niederlande & 6,14 & 5,06 & 5,36 & 5,20 & 5,10 \\
\hline Verein. Königreich & 17,21 & 19,53 & 22,96 & 20,83 & 19,49 \\
\hline EG & 100,00 & 100,00 & 100,00 & 100,00 & 100,00 \\
\hline
\end{tabular}

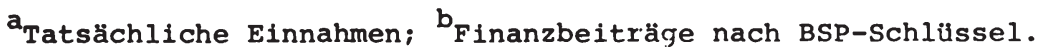

Quelle: EG-Rechnungshof, Jahresberichte, versch. Jg.; eigene Berechnungen. 
Ausgangspunkt für die Höhe der von den Mitgliedsstaaten abzuführenden Mehrwertsteuern ist die Aufstellung des Haushaltsplans. Hier wird zunächst aus der Differenz zwischen den geplanten Ausgaben und geschätzten übrigen Einnahmen der globale Restfinanzierungsantell festgelegt und nach Maßgabe der nationalen Bemessungsgrundlagen zu einem einheitlichen Prozentsatz auf die Mitgliedsstaaten verteilt. Dieses Verfahren sei am Beispiel des Haushaltsplans für 1982 veranschaulicht. 1

Gesamtbetrag der geplanten Ausgaben

Verschiedene Einnahmen einschließlich

Uberschlisse aus den vorhergehenden

Haushaltsjahren

Gemäв Eigenmittelbeschluß zu

finanzierende Ausgaben

Zölle, Agrarabschöpfungen, Zuckerund Isoglucoseabgaben

Noch zu finanzierender Betrag

Finanzbeiträge Griechenlands gemä $\beta$ BSP-Schlüssel

Aus Mehrwertsteuer-Eigenmitteln zu finanzierender Betrag
21.984 Mio. ECU

164 Mio. ECU

21.820 Mio. ECU

9.624 Mio. ECU

12.196 Mio. ECU

198 Mio. ECU

11.998 MiO. ECU

\begin{tabular}{lcc}
\hline Mitgliedsstaaten & $\begin{array}{c}1 \text { \% der MWSt-Bemessungs- } \\
\text { grundlage }\end{array}$ & $\begin{array}{c}\text { Abzuführende MWSt- } \\
\text { Eigenmittel }\end{array}$ \\
\hline Belgien & 510 & 472 \\
Dänemark & 265 & 245 \\
Deutschland & 3610 & 3339 \\
Frankreich & 3150 & 2913 \\
Irland & 100 & 92 \\
Italien & 1725 & 1595 \\
Luxemburg & 24 & 22 \\
Niederlande & 690 & 638 \\
Verein. Königreich & 2900 & 2682 \\
Gesamt & 12974 & 11998 \\
\hline
\end{tabular}

Ausschöpfungssatz: $\frac{11 \cdot 998}{12 \cdot 974}=0,925$

1Vgl. EG-Parlament, Endgültige Feststellung des Gesamthaushaltsplans der Europäischen Gemeinschaften für das Haushaltsjahr 1982, in: Amtsblatt der EG, Nr. L 31, 8.2.1982, S. 57 f. 
Die hier verwendeten nationalen Bemessungsgrundlagen basieren auf den Schätzungen der einzelnen Mitgliedsstaaten für das Haushaltsvorjahr. Diese werden von der Kommission zum Wechselkurs des

1. Februar des Haushaltsvorjahres in ECU umgerechnet und so im Haushaltsplan ausgewiesen. Die von den Mitgliedsstaaten abzuführenden Beträge bleiben allerdings in ihren nationalen währungen fixiert. Die Abführung an den Gemeinschaftshaushalt erfolgt in der Welse, daß die Mitgliedsstaaten monatlich ein Zwölftel des festgesetzten Betrages auf die Konten der Gemeinschaft zu zahlen haben. 1 $\mathrm{Zu}$ diesen Terminen werden die in nationaler Währung geleisteten Beträge in ECU umgerechnet. ${ }^{2}$ Die im Haushaltsplan veranschlagten Beträge können damit je nach Entwicklung der nationalen Währungen zur ECU über- oder unterschritten werden.

Eine Korrektur der Steuerschuld wird vorgenommen, nachdem die Mitgliedsstaaten die endgültigen steuerpflichtigen Transaktionen ermittelt haben. $\mathrm{Zu}$ diesem Zweck sind der Kommission bis zum 1 . Juli des auf den Haushaltsplan folgenden Jahres entsprechende Ubersichten vorzulegen. ${ }^{3}$ Die aus den ursprünglichen Haushaltsansätzen und den endgultig festgelegten Steuerschuldbeträgen resultierenden salden werden den Mitgliedsstaaten dann gutgeschrieben bzw. angelastet. ${ }^{4}$ Allerdings hat slch gezeigt, daß auch zu diesem Zeitpunkt die Veranlagung der Mehrwertsteuerzahlungen noch nicht endgültig abgeschlossen werden kann, sondern $d a \beta$ vor allem infolge der bestehenden ubergangsregelungen ${ }^{5}$ auch in den folgenden Jahren noch Ausgleichszahlun-

\footnotetext{
${ }^{1}$ Art. 10 Abs. 3 der Verordnung (EWG, Euratom, EGKS) Nr. 2891/77 des Rates vom 19. Dezember 1977 zur Durchführung des Beschlusses vom 21. April 1970 über die Ersetzung der Finanzbeiträge der Mitgliedsstaaten durch eigene Mittel der Gemeinschaften, in: Amtsblatt der EG, Nr. L 336 vom 27.12.1977, S. 1 ff.

2 Art. 9 Abs. 3 der Verordnung $2891 / 77$.

${ }^{3}$ Art. 10 Abs. 1 der Verordnung (EWG, Euratom, EGKS) Nr. 2892/77 des Rates vom 19. Dezember 1977 liber die Anwendung des Beschlusses vom 21. April 1970 liber die Ersetzung der Finanzbeiträge der Mitgliedsstaaten durch elgene Mittel der Gemeinschaften auf die Mehrwertsteuer-Elgenmittel, in: Amtsblatt der EG, Nr. L 336 vom 27.12.1977, S. $8 \mathrm{ff}$.

${ }^{4}$ Art. 10 Abs. 4 der Verordnung $2891 / 77$.

${ }^{5}$ siehe hierzu Abschnitt 4.1.1.1.2.
} 
gen erforderlich sind. So mußten etwa zu den laufenden zahlungen des Jahres 1980 in Höhe von 7.258,5 Mio. ECU ${ }^{1}$ nicht nur im Jahre 1981 auf der Grundlage der am 1. Juli 1981 von den Mitgliedsstaaten übermittelten Ubersichten Nachzahlungen von 558,2 Mio. ECU geleistet werden, sondern wurden auch noch in den drei folgenden Jahren Nettobertchtigungen in Höhe von 60,9 Mio. ECU bzw. 35,6 Mio. ECU bzw. 12,7 Mio. ECU erforderlich. ${ }^{2}$ Die so korrigierten Werte der ursprünglichen Mehrwertsteuerabführungen bezeichnet man auch als Veranlagungs-Definition der Mehrwertsteuer.

\subsection{Determinanten der mitgliedsstaatlichen Verteilung}

Einen ersten Eindruck von den Distributionswirkungen der Mehrwertsteuer-Eigenmittel erhält man, wenn die Anteile der Mitgliedsländer am gesamten Mehrwertsteueraufkommen ihren BIP-Anteilen am Gemeinschaftssozialprodukt gegenübergestellt werden (Tabelle 6). Die Mehrwertsteuer-Antelle sind dabei nach der Veranlagungs-Definition abgegrenzt, um abrechnungs- und zahlungstechnische Verzerrungen $z \mathrm{u}$ vermelden. Ein Vergleich der in Tabelle 6 dargestellten Mehrwertsteuer-Anteile mit den in Tabelle 5 ausgewiesenen tatsächlich eingenommenen Mitteln zeigt, daß sich bei Verwendung der Zahlungs-Definition nicht unerhebliche Verzerrungen ergeben würden. Während die Anteilswerte der Mitgliedsstaaten bei der Veranlagungsdefinition einen im Zeitablauf kontinuierlichen Verlauf aufweisen, ergeben sich bei der Zahlungs-Definition häufig starke Schwankungen, wie es besonders im Falle Italiens deutlich wird. Insgesamt ist $z u$ beachten, daB die ausgewiesenen Werte der Veranlagungsdefinition umso genauer sind, je mehr Korrekturen in den Folgejahren berïcksichtigt werden konnten.

${ }^{1}$ Dle Differenz zu dem in Tabelle 5 ausgewiesenen Betrag in Höhe von 261,0 Mio. ECU resultiert aus Anpassungen des Jahres 1979.

${ }^{2}$ Vgl. EG, Compte de gestion et bilan financier. Afferents aux operations du budget de I'exercise 1980 - 1984, Vol. I, Analyse de la gestion financière. 
Tabelle 6

Mehrwertsteuer-Eigenmittel in Relation zu den Bruttoinlandsprodukten der Mitgliedsstaaten 1980 - 1984

\begin{tabular}{|l|r|r|r|r|r|r|r|r|r|r|}
\hline Mitgliedsland & \multicolumn{4}{|c|}{ Mehrwertsteuer-Anteilea } & \multicolumn{4}{|c|}{ Relation Mehrwertsteuer/BIP } \\
& 1980 & 1981 & 1982 & 1983 & 1984 & 1980 & 1981 & 1982 & 1983 & 1984 \\
\hline Belgien & 4,17 & 3,94 & 3,54 & 3,27 & 3,46 & 1,00 & 1,01 & 0,99 & 0,94 & 0,99 \\
Dänemark & 2,14 & 2,06 & 2,01 & 2,02 & 1,99 & 0,91 & 0,88 & 0,84 & 0,82 & 0,79 \\
Deutschland & 30,96 & 29,61 & 28,26 & 29,26 & 28,80 & 1,07 & 1,07 & 1,01 & 1,03 & 1,02 \\
Frankreich & 23,13 & 23,64 & 23,38 & 22,30 & 22,82 & 0,99 & 1,02 & 1,02 & 0,99 & 1,01 \\
Griechenland & - & 1,63 & 1,63 & 1,57 & 1,53 & - & 1,09 & 1,02 & 1,05 & 1,01 \\
Irland & 0,79 & 0,93 & 0,92 & 1,02 & 0,86 & 1,22 & 1,35 & 1,23 & 1,36 & 1,13 \\
Italien & 13,30 & 13,69 & 13,31 & 13,87 & 15,31 & 0,94 & 0,95 & 0,90 & 0,90 & 0,96 \\
Luxemburg & 0,22 & 0,25 & 0,25 & 0,29 & 0,25 & 1,38 & 1,56 & 1,79 & 2,07 & 1,79 \\
Niederlande & 5,85 & 5,43 & 5,23 & 5,39 & 4,99 & 0,97 & 0,94 & 0,90 & 0,94 & 0,88 \\
Verein. König- & 19,44 & 18,82 & 21,47 & 20,99 & 19,99 & 1,04 & 0,92 & 1,06 & 1,07 & 1,04 \\
reich & & & & & & & & & & 1,00 \\
EG & 100,00 & 100,00 & 100,00 & 100,00 & 100,00 & 1,00 & 1,00 & 1,00 & 1,00 \\
\hline
\end{tabular}

aeranlagungs-Definition.

Quelle: EG-Kommission, Europäische Wirtschaft, Nr. 22; EG, Compte de gestion et bilan financier; eigene Berechnungen. 
Als Ergebnis der Relationen in Tabelle 6 läßt sich festhalten, daB in vielen Fällen die Mehrwertsteuer-Anteile den Sozialproduktanteilen etwa entsprechen, daneben allerdings einige signifikante Ausnahmen bestehen. Sieht man einmal vom Falle Luxemburgs ab, dessen weit überproportionale Steuerleistungen auf die Verwendung des Bruttoinlands- anstelle des Bruttosozialprodukts als BezugsgröBe zurückzuführen sind, ${ }^{1}$ dann fallen vor allem die relativ hohen $A b-$ führungen Irlands und die vergleichsweise geringen Mehrwertsteuerzahlungen Dänemarks, aber auch Italiens und der Niederlande ins Auge.

Zur weiteren Analyse der zwischenstaatlichen Verteilungswirkungen der Mehrwertsteuer-Eigenmittel bietet es sich an, eine Regressionsrechnung zwischen den Pro Kopf-Sozialprodukten der Mitgliedsländer

\section{Tabelle 7}

Regression der Mehrwertsteuerbelastung pro Kopf in bezug auf das Pro Kopf-BIP der Mitgliedsstaaten 1980 - 1984

\begin{tabular}{|c|c|c|}
\hline Jahr & $\begin{array}{l}\text { Korrelations- } \\
\text { koeffizient }\end{array}$ & Steigung \\
\hline 1980 & $-0,64$ & $-0,08$ \\
1981 & $-0,59$ & $-0,17$ \\
1982 & $-0,58$ & $-0,16$ \\
1983 & $-0,64$ & $-0,23$ \\
1984 & $-0,68$ & $-0,14$ \\
\hline
\end{tabular}

$x_{i}$ : Pro Kopf-BIP in 1000 ECU

$Y_{i}$ : Mehrwertsteuerbelastung des Pro Kopf-BIP in 80

$i=1, \ldots, 8$ : Mitgliedsstaaten ohne Griechenland und Luxemburg

Quelle: Eigene Berechnungen nach EG-Kommission, Europäische Wirtschaft Nr. 22; EG, Compte de gestion et bilan financier.

${ }^{1}$ Siehe hierzu Abschnitt 2.4.2. 
als erklärende Variable $\left(x_{i}\right)$ und den dazugehörigen relativen steuerbelastungen als unabhängige Variable $\left(y_{i}\right)$ vorzunehmen. Dabei ist es zweckmäßig, diese Berechnungen unter Ausschluß Griechenlands und Luxemburgs durchzuführen, um die nicht in der Mehrwertsteuerbemessungsgrundlage liegenden Einflüsse zu eliminieren.

Die Ergebnisse dieser Querschnittsanalyse in Tabelle 7 zeigen für jedes Jahr des Untersuchungszeitraums, daß Mitgliedsländer mit einem relativ höheren Pro Kopf-BIP eine geringere prozentuale Mehrwertsteuerbelastung $\mathrm{zu}$ trager haben als Länder mit einem vergleichsweise geringeren BIP, also insgesamt eine regressive Wirkung vorliegt. Diese Zusammenhänge sind über die Jahre unterschiedlich stark ausgeprägt. 1 sie bestehen aber vor allem - wie die relativ geringen (absoluten) Werte der Korrelationskoeffizienten bereits andeuten - nicht durchgehend. Dies veranschaulicht die Abbildung 1, in der die zur Regressionsrechnung gehörigen Mehrwertsteuerbelastungen der mitgliedsstaatlichen Pro Kopf-Bruttoinlandsprodukte noch einmal dargestellt sind. Dabei ergibt sich ein recht uneinheitliches Blld und wird erkennbar, daß der berechnete regressive Verlauf maßgeblich durch die Positionen Irlands und Dänemarks bestimmt wird. Insgesamt deutet diese Darstellung darauf hin, daß die Verteilungswirkungen der Mehrwertsteuer nicht monokausal zu erklären sind, sondern sie vielmehr durch eine Reihe von besonderen Einflußfaktoren bestimnt werden.

Auf der Suche nach solchen. Bestimmungsgründen ist gleichwohl zunächst danach zu fragen, ob und in welchem Umfang sich für die global beobachtete Regressivwirkung Erklärungsgründe finden lassen.

\footnotetext{
${ }^{1}$ Dabel ist noch elnmal darauf hinzuweisen, daß die hier zugrundeliegenden Mehrwertsteuerabfuhrungen nach der Veranlagungs-Definition insbesondere fur die letzten Jahre des Untersuchungszeitraums durch künftige Korrekturen noch leicht verändert werden durften, also vergleichsweise weniger genau sind.
} 


\section{Abbildung 1}

Mehrwertsteuerbelastuna der Pro-Kopf. Bruttninlandsprodukte der Mitaliedsstaaten

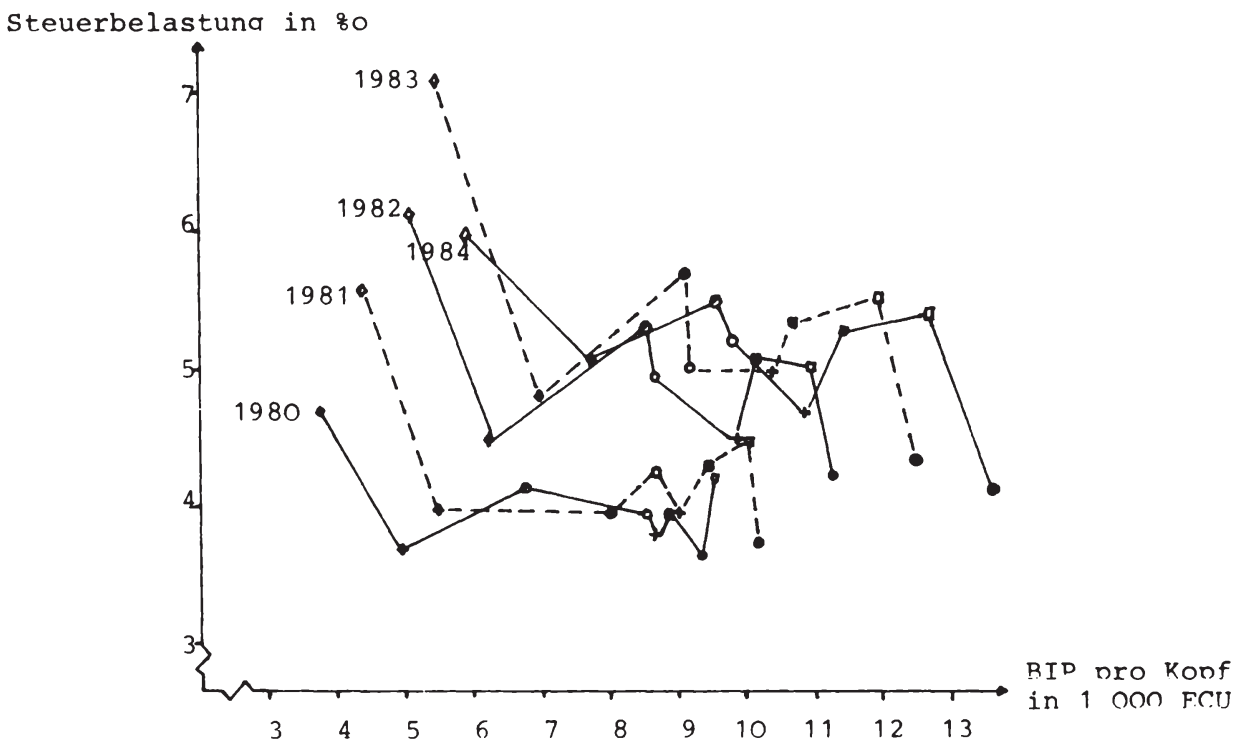

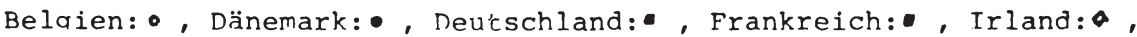
Italien: , Niederlande: $\bullet$, Verein. Könirreich: • .

Ouelle: Eiqene Berechnunaen nach FG-Kommission, Furonäische Wirtschaft, Nr. 22; EG, Combte de aestion et bilan financier. 
Von der Regressivwirkung einer steuer spricht man allgemein, wenn mit steigendem Einkommen die prozentuale steuerbelastung abnimmt. Da regressive steuertarife in der Regel keine Rolle spielen, kommt die Regressivwirkung auf indirekte Weise zustande. ${ }^{1}$ Bei den Verbrauchsteuern und den allgemeinen Umsatzsteuern, die den Konsum der Wirtschaftssubjekte belasten, kann dieser Mechanismus durch eine mit wachsendem Einkommen sinkende marginale Konsumquote ausgelöst werden: trotz eines proportionalen steuertarifs sinkt mit steigendem Einkommen der Anteil der Konsumausgaben und damit die auf das Einkommen bezogene prozentuale Belastung durch diese Steuern.

So wird etwa der deutschen Mehrwertsteuer eine regressive Wirkung zumindest im Bereich höherer Einkommen zugesprochen: Während in den unteren und mittleren Einkommensklassen vor allem der ermäßigte Steuersatz für die meisten Nahrungsmittel sowie auch die vollständigen steuerbefreiungen einer Reihe von Gütern eine Regressivwirkung verhindern, kann dieser Effekt mit zunehmend sinkender Konsumquote höherer Einkommensklassen nicht mehr kompensiert werden. 2

Im Rahmen der personellen Verteilungswirkungen einer nationalen Steuer bezieht man die steuerbelastung auf die Einkommen der einzelnen Wirtschaftssubjekte. Stellt eine solche Inzidenzanalyse auf den zwischenstaatlichen Vergleich der EG-Mitgliedsländer ab, dann sind für diese Wirtschaftseinheiten die Volkseinkommen bzw.

\footnotetext{
'Vgl. R. Peffekoven, Einführung in die Grunabegriffe der Finanzwissenschaft, 2. Aufl., Darmstadt 1985, S. 52.

${ }^{2}$ Vgl. hierzu etwa D. Pohmer, Allgemeine Umsatzsteuern, in: Handbuch der Finanzwissenschaft, 3. Aufl., Bd. II, Tübingen 1980, S. $647 \mathrm{ff}$, hier: S. 706; W. Kitterer, Belastungswirkungen der Umsatzsteuer; Gutachten im Auftrage des Bundesministers für Wirtschaft. Unter Mitarbeit von J. Fronia, Tübingen: Institut für Angewandte Wirtschaftsforschung, 1981, S. $152 \mathrm{ff}$.
} 
die Sozialprodukte und im Hinblick auf die empirische Handhabung speziell die Bruttoinlandsprodukte heranzuziehen. Die Begründung für eine vermutete Regressionswirkung der an die EG abgeführten Mehrwertsteuer hätte sich in diesem Fall auf eine allgemein mit steigendem Volkseinkommen bzw. Sozialprodukt sinkende nationale Konsumquote zu stützen. ${ }^{1}$ Da sich die Mehrwertsteuerabführungen allein an der Bemessungsgrundlage ausrichten, können ermäßigte Steuersätze und Steuerbefreiungen verschiedener Güter keinen Einfluß auf die Verteilungswirkungen ausüben.

Eine solche Hypothese ist allerdings kaum haltbar. Der fiir individuelle Einkommen empirisch erwiesene Tatbestand sinkender marginaler Konsumquoten mit wachsenden Einkommen ${ }^{2}$ läßt sich nicht auf den zwischenstaatlichen Vergleich in der EG übertragen. Wie Tabelle 8 ausweist, varieren die privaten Konsumquoten der Mitgliedsstaaten keineswegs durchgehend invers mit der Höhe ihrer Pro Kopf-Sozialprodukte. Trotz der teilweise großen Unterschiede in den nationalen Pro Kopf-BIP liegen die entsprechenden Konsumquoten auf einem relativ einheitlichen Niveau; sie lassen auch in ihrer geringen Differenzierung kaum einen Einfluß der Sozialprodukte erkennen.

Nun ist der private Konsum zwar der quantitativ bedeutsamste, nicht aber der einzige Bestimmungsgrund für die Mehrwertsteuerbemessungsgrundlage, nach der sich die Höhe der abzuführenden Mittel bemißt. Die gemeinschaft].iche Mehrwertsteuer soll den jeweiligen heimischen

\footnotetext{
${ }^{1}$ So etwa G. Denton, Der Beitrag Großbritanniens zum EG-Haushalt eine Untersuchung aus britischer Sicht, in: Integration, 2. Jg., 1979 , S. 149 ff., hier: S. 152.

2Vgl. F. X. Bea, Umsatzsteuern I: Ziele und Ausgestaltung, in: Handwörterbuch der Wirtschaftswissenschaft, Bd. 8, stuttgart u.a.o. 1980 , S. 27 ff., hier: S. 38.
} 
Verbrauch belasten ${ }^{1}$ und man spricht in diesen Fällen von einer Mehrwertsteuer vom Konsumtyp. ${ }^{2}$ Neben der großen Anzahl von steuerbefreiungen, $z u$ denen vor allem bestimmte dem Gemeinwohl dienende Leistungen gesundheitlicher, bildungs- und sozialpolitischer Art gehören, wird diese Konzeption einer den privaten Konsum belastenden allgemeinen Verbrauchsteuer vor allem deshalb nicht konsequent verwirklicht, weil auch die Leistungen und Lieferungen der Unternehmen an den staat mehrwertsteuerpflichtig sind. ${ }^{3}$ Damit umfassen die nationalen Bemessungsgrundlagen nicht nur den privaten Konsum, sondern enthalten vor allem auch die vom staat getätiaten Käufe von Unternehmen.

Die in der Tabelle 8 dargestellten Ergebnisse ${ }^{4}$ zeigen allerdings, daB sich auch unter Berücksichtigung der in den Staatsausgaben enthaltenen mehrwertsteuerpflichtigen Umsätze ${ }^{5}$ keine eindeutige Beziehung zwischen den Bemessungsgrundlagen und der relativen Höhe der Mehrwertsteuerbelastungen ableiten läßt. Zwar sind - wie auch bei Betrachtung allein der privaten Konsumquoten - gewisse zusammenhänge etwa zwischen den relativ geringen Quoten Dänemarks und

1Vgl. EG-Rat, Erste Richtlinien vom 11. April 1967 zur Harmonisierung der Rechtsvorschriften der Mitgliedsstaaten über die Umsatzsteuern, in: Amtsblatt der EG, 1967, s. 1301 ff., Art. 2.

${ }^{2} \mathrm{Zu}$ dieser und anderen Formen allgemeiner Umsatzsteuern vgl. etwa F. X. Bea (1980), S. $30 \mathrm{ff}$, D. Pohmer (1980), S. $695 \mathrm{ff}$.

${ }^{3}$ Vgl. hiexzu D. Pohmer, Zur systematik und Praxis der Mehrwertsteuern unter besonderer Berücksichtigung der Entwicklung in Europa, in: L. Fischer (Hrsg.), Unternehmung und Steuer, Festschrift zur Vollendung des 80 . Lebensjahres von Peter Scherpf, Wiesbaden 1983, S. 375 ff.

${ }^{4}$ Dabei ist auf unterschiedliche Abgrenzungsmöglichkeiten der gesamtwirtschaftlichen Aggregate hinzuweisen. So beträgt etwa nach den Angaben des Statistischen Bundesamtes der private Konsum für die Bundesrepublik 56,2 8 statt $63,3 \%$ und der staatliche Konsum $20,7 \%$ statt 13,9 \%. Da allerdings die mitgliedsstaatlichen Größen in den EG-Statistiken nach einheitlichen Maßstäben erfaßt werden, dürften die Ergebnisse der Tabelle 8 für den zwischenstaatlichen Vergleich durchaus aussagefähig sein.

${ }^{5}$ Als Schätzgrößen für die durch Käufe des Staates bewirkte Erhöhung der Bemessungsgrundlage wurden die Bruttoanlageinvestitionen sowie die im Staatsverbrauch enthaltenen Käufe von anderen Unternehmen (Vorleistungen) herangezogen. 
der Niederlande bzw. der vergleichsweise hohen Quote Irlands und den dazugehörigen prozentualen Mehrwertsteuerbelastungen erkennbar. Doch ist die Differenzierung der Quoten hier wie dort zu gering, als daß sie einen signifikanten Teil der belastungsmäßiqen Unterschiede erklären könnte.

Im Rahmen der verteilungsorientierten Analyse der MehrwertsteuerEigenmittel wird häufig auch mit den Investitions-, Export- und Importquoten argumentiert. Dabei wird etwa behauptet, daß "die Mehrwertsteuerregelung (...) tendenziell Mitgliedsstaaten (entlastet), die im Vergleich zum Durchschnitt der Gemeinschaft eine relativ hohe Export- und/oder Investitionsquote erzielen", da die privaten Investitionen sowie die Exporte ${ }^{1}$ nicht in die Bemessungsgrundlage eingehen, ${ }^{2}$ oder werden zur möglichen Erklärung relativ hoher Mehrwertsteuer-Anteile eine hohe Import- und eine vergleichsweise geringere Investitionsquote herangezogen. ${ }^{3}$ Dabei wird ähnlich wie im Zusammenhang mit der Konsumquote darauf abgestellt, daß wirtschaftsschwächere Länder eine geringe Investitions- und Exportquote, aber eine relativ hohe Importquote aufweisen und vice versa. Insoweit solche Beziehungen insgesamt zutreffen, könnte daraus eine regressive Wirkung der Mehrwertsteuer-Eigenmittel resultieren.

Bei der empirischen Überprüfung dieser Zusammenhänge stellt man allerdings sofort fest, daß für die Gemeinschaftsländer die beschriebenen Konstellationen nicht vorliegen. Wie Tabelle 8 zeigt,

\footnotetext{
${ }^{1}$ Nach dem fur international gehandelte Güter geltenden Bestimmungslandprinzip zur Vermeidung der Doppelbesteuerung werden Exporte von der heimlschen Mehrwertsteuer freigestellt und Importe mit dem heimischen Steuersatz belastet. Vgl. hierzu R. Peffekoven, Das Bestimungs- und Ursprungslandprinzip be1 Steuern im grenzüberschreitenden Verkehr, in: W. Albers (Hrsg.), Besteuerung und Zahlungsbilanz, Schriften des Vereins für Socialpolitik, N.F. Bd. 68, Berlin 1982 , S. 33 ff., hier: S. 35 f.

2Vgl. U. Nittka, S. 276 .

${ }^{3}$ Vgl. V. Petersen (1983), S. 29.
} 


\section{Tabelle 8}

Mehrwertsteuerbelastung und gesamtwirtschaftliche Größen der Mitgliedsstaaten 1982

\begin{tabular}{|c|c|c|c|c|c|c|c|c|c|c|c|c|}
\hline Mitgliedsland & $\begin{array}{l}\text { BIP pro } \\
\text { Kopf in } \\
\text { ECU }\end{array}$ & $\begin{array}{l}\text { MWSt-Be- } \\
\text { lastung } \\
\text { des BIP } \\
\text { in } 80\end{array}$ & $\mathrm{C}_{\mathrm{pr}}$ & $c_{\text {st }}$ & $\begin{array}{l}\text { darunter: } \\
\text { Vorleist. }\end{array}$ & $\mathrm{I}_{\mathrm{pr}}^{\mathrm{br}}$ & $\mathrm{I}_{\mathrm{St}}^{\mathrm{bra}}$ & Ex & Im & $E x-I m$ & Gesamt & $\mid \begin{array}{l}\text { darunter: } \\
C_{p r}+\text { Vor- } \\
\text { leist.+ I } I_{\text {st }}\end{array}$ \\
\hline & & & \multicolumn{10}{|c|}{ in 8 BIP } \\
\hline Dänemark & 11.254 & 4,2 & 55,1 & 28,0 & $9,6^{a)}$ & 13,4 & 3,0 & 36,2 & 35,7 & $+0,5$ & 100 & 67,7 \\
\hline Deutschland & 10.919 & 5,0 & 63,3 & 13,9 & 5,2 & 17,4 & 3,0 & 30,9 & 28,4 & $+2,5$ & 100 & 71,5 \\
\hline Frankreich & 10.180 & 5,1 & 64,6 & 16,2 & 5,6 & 18,9 & 2,7 & 23,1 & 25,5 & $-2,4$ & 100 & 72,9 \\
\hline Niederlande & 9.821 & 4,5 & 60,3 & 17,7 & 5,3 & 14,8 & 3,0 & 57,5 & 53,3 & $+4,2$ & 100 & 68,6 \\
\hline Belgien & 8.739 & 4,9 & 65,4 & 18,4 & 4,0 & 13,5 & 3,6 & 73,7 & 74,7 & $-1,0$ & 100 & 73,0 \\
\hline $\begin{array}{l}\text { Verein. König- } \\
\text { reich }\end{array}$ & 8.696 & 5,3 & 59,8 & 21,8 & 9,6 & 14,3 & 1,8 & 26,7 & 24,4 & $+2,3$ & 100 & 71,2 \\
\hline Italien & 6.266 & 4,5 & 62,3 & 18,6 & 5,1 & 16,5 & 3,6 & 26,5 & 27,6 & $-1,1$ & 100 & 71,0 \\
\hline Irland & 5.171 & 6,1 & 62,1 & 20,3 & $6,0^{b)}$ & 20,8 & 4,4 & 49,5 & 57,1 & $-7,6$ & 100 & 72,5 \\
\hline
\end{tabular}

a) 1981 ; b) geschätzt, da nicht gesondert ausgewiesen.

Symbolik: $\mathrm{C}_{\mathrm{pr}}$ : Privater Verbrauch im Wirtschaftsgebiet; $\mathrm{C}_{\mathrm{St}}$ : Staatlicher Verbrauch; $I_{\mathrm{pr}}$ : Private Bruttoanlageninvestitionen und Vorratsänderungen; $I_{S t}$ : Bruttoanlageninvestitionen des Staates; Ex, Im: Ausfuhr, Einfuhr von Waren und Dienstleistungen.

Quelle: Amt für amtliche Veröffentlichungen der EG; eurostat revue 1974 - 1983, Luxemburg 1985, S. $47 \mathrm{ff.;}$ Statistisches Amt der EG, Konten und Statistiken des Staates 1970 - 1982, Luxemburg 1984; elgene Berechnungen. 
weisen die Investitionsquoten größtenteils ein ähnliches Niveau auf und korrespondieren im Kreislaufzusammenhang mit hohen bzw. geringen Exportquoten stets auch entsprechende Importquoten. Fin empirischer Nachweis zwischen der Höhe der einzelstaatlichen Mehrwertsteuerbelastungen und den Investitions-, Export- und Importquoten läßt sich damit ebensowenig führen wie im Hinblick auf die nationalen Konsumquoten.

Als welterer möglicher Bestimmungsfaktor der mitgliedsstaatlichen Vertellungswirkungen der Mehrwertsteuer-Eigenmittel sind die unterschiedlichen nationalen Preissteigerungen in Betracht zu ziehen. Denn da die Mehrwertsteuer eine an nominelle Größen anknüpfende Abgabe ist, hängt die Höhe ihrer Bemessungsgrundlage auch von der Inflationsrate ab. Damit beeinflussen ceteris paribus (c.p.) - insbesondere bel Konstanz der Wechselkurse in bezug auf die ECU - unterschiedliche nationale Preissteigerungen die zugunsten der $\mathrm{Ge}-$ meinschaft abzufuhrenden Mehrwertsteuerbeträge und so die Länderantelle am gesamten Finanzierungsaufkommen. Auf diesen Zusammenhang bezieht sich auch die Deutsche Bundesbank, wenn sie den zu Beqinn der achtziger Jahre gesunkenen Finanzierungsanteil der Bundesrenublik u.a. mit der geringeren Inflationsrate im vergleich $z u$ den Partnerstaaten erklärt. ${ }^{1}$ Aus einer inflationsinduzierten Veränderunq der Mittelaufbringung bzw. der Finanzierungsanteile läßt sich allerdings nicht ohne weiteres auf tatsächliche Belastunasverschiebungen im Sinne von nationalem Ressourcenverzicht bzw. -qewinn schließen. Zum einen läßt nämlich elne allein inflatorisch bedinqte Erhöhunq der Mehrwertsteuerbemessungsgrundlage und die damit verbundene hohere Mtttelabfuhrung an die Gemeinschaft rie reale innere Auf-

${ }^{1}$ Val. Deutsche Bundesbank, Monatsberichte, 34. Ja., 1982, Nr. 1, S. 22, sowie 37 . Ja., 1985, S. 40 . 
bringung der Mittel unverändert. ${ }^{1}$ zum anderen werden die aus der Betrachtung allein der Mehrwertsteuer-Finanzierungsanteile resultierenden Verschiebungen relativiert, wenn sie auf die jeweiligen Sozialprodukte, die ja im gleichen Maße von der Erhöhung des Preisniveaus betroffen sind, bezogen werden.

Diese tberlegungen relativieren auch die Hypothese einer durch unterschiedliche nationale Preissteigerungsraten induzierten Regressivwirkung der Mehrwertsteuer-Eigenmittel. Dabei wird davon ausgegangen, daß die Inflationsraten in wirtschaftsschwächeren Ländern relativ hoch sind und diese über nominell überproportionale steuerabführungen regressiv belastet werden. ${ }^{2}$ zwar lassen die Preissteigerungsraten der Mitgliedsstaaten in Tabelle 9 im Vergleich mit den zugehörigen Pro Kopf-BIP in Tabelle 1 - wenn auch nicht durchgehend - einen Zusammenhang zwischen Inflationsrate und der Wirtschaftskraft durchaus erkennen: die drei ärmsten Gemeinschaftsländer Griechenland, Irland und Italien weisen die mit Abstand höchste Preissteigerung auf. Unter Berücksichtigung der realen Wirkungen inflationsinduzierter Mehrwertsteuerabführungen und ihrer Relativierung am Sozialprodukt läßt sich diese Hypothese jedoch nicht halten. Ein solcher Zusammenhang ist denn auch nicht aus den - wenngleich durch vielfältige Einflußfaktoren überlagerten - Mehrwertsteuer/BIP-Relationen der Tabelle 6 erkennbar.

Schließlich ist danach zu fragen, ob und inwieweit wechselkursänderungen der nationalen währungen in bezug auf die ECU die Verteilungswirkungen der Mehrwertsteuer-Eigenmittel beeinflussen. Solche

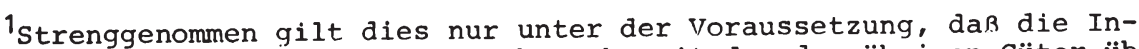
flationsrate des nrivaten Verbrauchs mit der der iibrigen Güter übereinstimmt. Die Unterschiede zwischen den Inflationsraten des BIP und des privaten Verbrauchs sind allerdings vernachlässigbar gering, จgl. Kommission der EG, Europäische Wirtschaft, Nr. 22, S. 229. ${ }^{2}$ Vgl. V. Petersen (1983), s. $29 \mathrm{f}$.
} 
Tabelle 9

Inflationsraten und Wechselkurse der Mitgliedsstaaten 1980 - 1984

\begin{tabular}{|c|c|c|}
\hline \multirow[t]{2}{*}{ Mitgliedsland } & $\begin{array}{l}\text { Inflationsraten des } \\
\text { privaten Verbrauchs }\end{array}$ & $\begin{array}{c}\text { Wechselkurse gegenüber } \\
\text { der ECU b,c }\end{array}$ \\
\hline & \multicolumn{2}{|c|}{ Veränderungen 1984 gegenüber 1980 in 8} \\
\hline Belgien & $+42,1$ & $-12,2$ \\
\hline Dänemark & $+53,0$ & $-4,1$ \\
\hline Deutschland & $+23,3$ & $+11,4$ \\
\hline Frankreich & $+66,5$ & $-17,0$ \\
\hline Griechenland & $+155,2$ & $-47,8$ \\
\hline Irland & $+99,1$ & $-7,8$ \\
\hline Italien & $+112,5$ & $-16,1$ \\
\hline Luxemburg & $+47,6$ & $-12,2$ \\
\hline Niederlande & $+27,0$ & $+8,6$ \\
\hline Verein. Königreich & $+55,2$ & $+1,1$ \\
\hline EG & $+48,5$ & \\
\hline
\end{tabular}

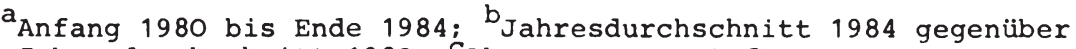
Jahresdurchschnitt 1980; CAbwertung; +: Aufwertung.

Quelle: Kommission der EG, Europäische Wirtschaft, Nr. 22, S. 229 und 241; eigene Berechnungen.

Änderungen können nach der Kaufkraftparitätentheorie ${ }^{1}$ im Gefolge einer unterschiedlichen Entwicklung der nationalen Inflationsraten erwartet werden, indem Länder mit relativ stabilem Preisniveau zur Aufwertung ihrer Währungen tendieren und vice versa. Ein solcher

${ }^{1}$ Vgl. etwa K. Rose, Theorie der Außenwirtschaft, 3. Aufl., Berlin 1970 , S. 94 ff. 
Zusammenhang scheint nach den Ergebnissen der Tabelle 9 zwar für die Bundesrepublik und die Niederlande als Aufwertungsländer mit den geringsten Preissteigerungsraten vorzuliegen; für die übrigen Gemeinschaftsländer ergibt sich allerdings kein einheitliches Bild. Dies deutet darauf hin, daB die Wechselkurse auch von einer vielzahl anderer Faktoren beeinflußt werden.

Auf die Höhe der an den Gemeinschaftshaushalt abzuführenden Mehrwertsteuer-Eigenmittel haben Wechselkursänderungen c. p. folgenden EinfluB: Bei einer Höherbewertung der nationalen Währung nehmen die in ECU umgerechneten steuerabführungen $z u$, während sie sich bei abgewerteten währungen verringern. Insoweit nun der nach dem Kaufkraftparitätenargument $\mathrm{zu}$ erwartende Zusammenhang zwischen den Inflationsraten und Wechselkursänderungen besteht, verlaufen diese belden Elnflüsse entgegengesetzt, so daß es vom daraus resultierenden Nettoeffekt abhängt, wie sich die nationalen Anteile am gesamten Finanzierungsvolumen entwickeln.

Im Hinblick auf eine verteilungspolitisch motivierte Beurteilung der Mehrwertsteuerabführungen gelten die bereits oben angebrachten Vorbehalte gegen elne alleinige Betrachtung der Finanzierungsante1le. Auch hier ist eine Relativierung an den entsprechenden Sozialproduktgrößen erforderlich. Da Inflationsraten wie Währungsänderungen prinzipiell in gleicher Weise auf die abzuführenden Finanzierungsmittel wie auf die Sozialprodukte wirken, besitzt der Einfluß dieser Größen im Hinblick auf eine am Sozialprodukt orientierte Beurtellung der Mehrwertsteuerverteilungswirkungen kaum Relevanz. 
4.1.1.1.2. Probleme der verwaltungstechnischen Erfassung und Abwicklung

Die bisherigen Uberlegungen - sollen sie allein die Belastungswirkungen der Mehrwertsteuer erklären - setzen eine einheitliche und vollständige Erfassung der Bemessungsgrundlage sowie eine korrekte Abrechnung und Verwaltung der Mittel voraus. Das ist allerdings in der Gemeinschaft nicht der Fall, so daß sich aus diesen Unzulänglichkeiten ebenfalls Auswirkungen auf die Verteilung der Finanzierungslasten ergeben. Sie resultieren aus der noch nicht vollständig abgeschlossenen Harmonisierung der Mehrwertsteuer mit den bestehenden Ubergangsregelungen sowie aus der offensichtlich unterschiedlich ausgeprägten Steuermoral und Effizienz der einzelnen nationalen Finanzverwaltungen.

Trotz des vereinheitlichten Grundtyps der Mehrwertsteuer ist die Harmonisierung damit noch nicht abgeschlossen. Die 6. Mehrwertsteuerrichtlinte und die ergänzend erlassenen Verordnungen $\mathrm{zu}$ ihrer Durchführung gewähren den Mitgliedsstaaten während einer Ubergangszeit noch erhebliche Spielräume für die Ausgestaltung einzelner Vorschriften. Unter den hier interessierenden Verteilungsgesichtspunkten ist vor allem relevant, daß den Mitgliedsstaaten die Wahlmöglichkelt bei der Ermittlung der Erhebungsgrundlage zugestanden wird. Dabei kann zwischen zwei Methoden gewählt werden:

- Die direkte oder Erklärungsmethode erfaßt den Mehrwert, indem sie die Besteuerungsgrundlage anhand der Steuererklärungen als Differenz zwischen den steuerpflichtigen Umsätzen und den entsprechenden Vorumsätzen ermittelt.

- Bei der indirekten, statistischen oder Einnahmenmethode wird von dem (aus der volkswirtschaftlichen Gesamtrechnung ermittelten) Mehrwertsteueraufkommen mittels Division durch den Mehrwertsteuersatz (bzw. bei mehreren Steuersätzen durch ihr gewogenes Mittel) auf die Bemessungsgrundlage geschlossen. 
Mit dem Inkrafttreten der 6. Mehrwertsteuer-Richtlinie war vorgesehen, bis zum Ende des Jahres 1982 die direkte Methode als endgültiges und für alle Mitgliedsstaaten verbindliches Erhebungssystem einzuführen. Deshalb war die Geltungsdauer der Utbergangsregelungen ursprünglich bis zu diesem zeitpunkt befristet. Infolge der Verzögerungen bei der Einführung der Mehrwertsteuer in den Mitgliedsstaaten mußten diese Regelungen jedoch bis zum Ende 1985 verlängert werden. Da auch gegenwärtig noch vielschichtige probleme einer endgültigen einheitlichen Methode entgegenstehen, soll die Ubergangszeit erneut bis zum Ende 1988 ausgedehnt werden. ${ }^{1}$

Von den Mitgliedsstaaten haben sich von Beginn an nur Dänemark und Irland für das direkte Verfahren entschieden. Dänemark machte allerdings 1983 von seinem Recht Gebrauch, auf die indirekte Methode überzuwechseln. "Die 'Ubergangszeit' für die Anwendung der Verordnung Nr. 2892/77 hat somit in der Praxis ein Ergebnis gebracht, welches das genaue Gegenteil des von der Kommission und den Europäischen Parlament angestrebten Zieles ist." 2

\footnotetext{
${ }^{1}$ Vgl. EG-Kommission, Bericht der Kommission über die Anwendung der Verordnungen (EWG, Euratom, EGKS) Nr. 2891/77 und Nr. 2892/77 des Rates vom 19. Dezember 1977 zur Durchführung des Beschlusses vom 21. April 1970 uber die Ersetzung der Finanzbeiträge der Mitgliedsstaaten durch eigene Mittel der Gemeinschaften sowie Vorschlag für eine Verordnung (EGKS, EWG, Euratom) des Rates zur Verlängerung der Verordnung (EWG, Euratom, EGKS) Nr. 2892/77 über die Anwendung des Beschlusses vom 21. April 1970 über die Ersetzung der Finanzbeiträge der Mitgliedsstaaten durch eigene Mittel der Gemeinschaften auf die Mehrwertsteuereigenmittel, KOM (85) 170 endg., Brüssel, 19.4.1985; EG-Parlament, Bericht im Namen des Haushaltsausschusses über den Vorschlag der Kommission der Europäischen Gemeinschaften an den Rat (Dok. C2-33/85-KOM (85) 170 endg.) für eine Verordnung zur Verlängerung der Verordnung (EWG, Euratom, EGKS) Nr. 2892/77 über die Anwendung des Beschlusses vom 21. April 1970 über die Ersetzung der Finanzbeiträge der Mitgliedsstaaten durch eigene Mittel der Gemeinschaften auf die MehrwertsteuerEigenmittel und luber den Bericht der Kommission über die Anwendung der Verordnungen (EWG, Euratom, EGKS) Nr. 2891/77 und 2892/77 des Rates vom 19. Dezember 1977 zur Durchführung des Beschlusses vom 21. April 1970 über die Ersetzung der Finanzbeiträge der Mitgliedsstaaten durch Eigenmittel der Gemeinschaften, Berichterstatter: Herr Petrus A. M. Cornelissen, Europäisches Parlament, Sitzungsdokumente, Dok. A 2-126/85 vom 21.10.1985.

${ }^{2}$ EG-Kommission, КОМ (85) 170 endg., S. 17.
} 
Für die Wahl zugunsten des statistischen Verfahrens können die möglicherweise geringeren Verwaltungskosten sprechen, da hier im Vergleich zur Erklärungsmethode grundsätzlich auf Daten abgestellt wird, die bei den mitgliedsstaatlichen Verwaltungen bereits weitgehend bekannt sind. ${ }^{1}$ Aus welchen Gründen sich etwa Dänemark für diese Methode neu entschieden hat, ist allerdings nicht erklärt worden. 2

$O b$ und in welchem AusmaB die Höhe der abzuführenden MehrwertsteuerEigenmittel von der Wahl des Berechnungsverfahrens abhängt, ist umstritten. Zwar ging man bei der Konzeption und Einführung der Ubergangsregelungen davon aus, daß beide Methoden ergebnisneutral sein würden, und hat die Kommission in ihrem jüngsten Bericht keine groBen Unterschiede hinsichtlich des jeweiligen Endergebnisses der Berechnungen festgestellt. ${ }^{3}$ Gestützt auf Untersuchungen spezieller Aspekte ist der Rechnungshof allerdings zu der Auffassung gelangt, da $\beta$ die beiden Berechnungsmethoden keineswegs ergebnisneutral sind, sondern das von fast sämtlichen Mitgliedsstaaten angewandte indirekte Verfahren zu nicht unerheblich geringeren Mehrwertsteuerabführungen im Vergleich zur direkten Methode führt. ${ }^{4}$

Im diesem Zusammenhang ist vor allem eine vom Statistischen Amt der Gemeinschaft durchgeführte Untersuchung aufschluBreich, in der die anhand der volkswirtschaftlichen Gesamtrechnungen (VGR) geschätzten Mehrwertsteuergrundlagen in Beziehung zu den von den Mit-

${ }^{1}$ Vgl. EG-Kommission, KOM (85) 170 endg., S. 19.

2 Vgl. EG-Parlament, Dok. A $2126 / 85$, S. 23.

${ }^{3} \mathrm{Vgl}$. EG-Kommission, KOM (85) 170 endg., S. 18 f.; EG-Parlament, Dok. A $2126 / 85$, S. 14.

${ }^{4} \mathrm{Vgl}$. E'G-Rechnungshof, Jahresbericht 1984, S. 31 . 
gliedsstaaten in den Jahresübersichten mitgeteilten Bemessungsgrundlagen gesetzt werden. ${ }^{1}$ Dabei zeigt sich, daß die VGR-Grundlagen fast ausnahmslos höher sind als die der Jahresübersichten, nach denen die tatsächlich abzuführenden Mehrwertsteuern berechnet werden. Bemerkenswert ist weiterhin, daß die Unterschiede für Irland am geringsten und für Dänemark und vor allem Italien am stärksten ausgeprägt sind.

Diese Ergebnisse lassen in ihrer globalen Aussage "auf den ersten Blick gewisse Zweifel an der Vollständigkeit der Bemessungsgrundlage der Mitgliedsstaaten für die Mehrwertsteuer-Eigenmittel aufkommen" 2 und unterstützen damit die vom Rechnungshof vorgebrachten Bedenken. Sie decken sich im Hinblick auf die zwischenstaatlichen Unterschiede aber auch weitgehend mit den oben dargestellten $\mathrm{Be}-$ lastungswirkungen der Mehrwertsteuer-Eigenmittel in bezug auf die jeweiligen mitgliedsstaatlichen Sozialprodukte.

Weitgehend ungeklärt sind die konkreten Ursachen dieser beobachteten Diskrepanzen. Es lassen sich lediglich einige Anhaltspunkte geben. So hat der Rechnungshof feststellen können, daß die Angaben aus der VGR, auf deren Grundlage der gewogene Durchschnitt der nationalen Mehrwertsteuersätze ermittelt werden, in den einzelnen Mit-

\begin{tabular}{lr|r|r}
\hline 1Für die Jahre 1980 bis 1982 ergaben sich folgende Werte: \\
& 1980 & 1981 & 1982 \\
\cline { 2 - 4 } Belgien & 113,3 & 109,6 & 110,9 \\
Dänemark & 115,4 & 118,6 & 120,7 \\
Deutschland & 106,6 & 105,8 & 103,9 \\
Frankreich & 112,3 & 113,2 & 110,9 \\
Irland & 109,7 & 101,3 & 96,3 \\
Italien & 133,6 & 140,5 & 145,4 \\
Niederlande & 110,0 & 110,1 & 113,7 \\
Verein. Königreich & 114,3 & 125,7 & 107,6 \\
Quelle: EG-Kommission, KOM (85) 170 endg., S. 39. \\
2EG-Rechnungshof, Jahresbericht 1984, S. 32.
\end{tabular}


gliedsländern unterschiedlich aufgegliedert und nicht im gleichen Maße aktuell sind. Dies würde bei einigen Mitgliedsstaaten höhere Mehrwertsteuerabführungen als auf der Grundlage der Jahresübersichten erwarten lassen. 1 Weiterhin ist festgestellt worden, daB die statistische Methode in den Mitgliedsstaaten, die eine Pauschalbesteuerung der Landwirte anwenden, dazu tendiert, die Mehrwertsteuer-Bemessungsgrundlage zu unterschätzen. ${ }^{2}$ Daneben sind auch die zahlreichen Ausnahmegenehmigungen von der harmonisierten Bemessungsgrundlage $z u$ berücksichtigen, die einzelnen Ländern während der Ubergangszeit zugestanden werden, und die ebenfalls gewisse Einflüsse auf die mitgliedsstaatliche Verteilung der Mehrwertsteuer-Eigenmittel ausüben dürften. ${ }^{3}$

Schließlich ist $z$ vermuten, daß die Verteilung der Finanzierungslasten auch von der unterschiedlich ausgeprägten Steuermoral in den Gemeinschaftsländern sowie von der unterschiedlichen Effizienz der nationalen Finanzverwaltungen beeinflußt wird. Die hiermit verbundenen Einnahmeausfälle betreffen allerdings nicht nur den Gemeinschaftshaushalt, sondern in einem absolut stärkeren Maße die Mitgliedsstaaten selbst, denen der Hauptteil des Erlöses dieser Steuer zufließt. In einem Verbundsystem ${ }^{4}$ bleibt also das Interesse der nationalen Regierungen, den Steueranspruch voll durchzusetzen, durchaus bestehen. Konflikte zwischen den beteiligten Ebenen im Rahmen der Beseitigung solcher Unausgewogenheiten sind damit kaum zu befürchten.

1Vgl. EG-Rechnungshof, Jahresbericht 1981, S. 21; EG-Parlamert, Dok. A $2126 / 85$, S. 13.

${ }^{2}$ Vgl. G. Spanakakis, Mehrwertsteuer-Eigenmittel für die EG-Verteilung der Lasten auf die Mitgliedsstaaten nicht unproblematisch -, in: Ifo-Schnelldienst, 32. Jg., 1979, Heft 11/12, S. 9 ff.

3 Vgl. A. Nottelmann (1982), s. 47.

${ }^{4} \mathrm{Vgl}$. zu diesen Begriffsbestimmungen etwa R. Peffekoven, Finanzausgleich I: Wirtschaftstheoretische Grundlagen, in: Handwörterbuch der Wirtschaftswissenschaft (HdWW), Bd. 2, stuttgart, Tübingen u.a. 1980, S. 608 ff., hier: S. 620 . 
Die Steuerhinterziehung weist vielfältige Formen auf, die von Land zu Land variieren können. ${ }^{1}$ Hinsichtlich des dadurch bedingten steuerausfalls lassen sich nur grobe Schätzungen angeben. So geht der Spinelli-Bericht des Europäischen Parlaments davon aus, daß die Hinterziehung in bestimmten Mitgliedsstaaten bis zu 20 bis $30 \%$ des möglichen Erlöses der Mehrwertsteuer ausmachen kann. ${ }^{2}$ Andere Untersuchungen halten für möglich, daß in Italien 40 \& und mehr des theoretischen Erlöses dieser Steuer hinterzogen werden ${ }^{3}$, während solche Steuerausfälle für andere Gemeinschaftsländer erheblich geringer veranschlagt werden: für Belgien etwa $8 \%^{4}$, die Niederlande ca. $68^{5}$ und für das Vereinigte Königreich nur $1-58^{6}$ des Aufkommens dieser Steuer. Insoweit sich diese Ergebnisse - zumindest der Tendenz nach - bestätigen, würden die aus dem Rahmen fallenden italienischen Verhältnisse einen Erklärungsgrund für die unterdurchschnittliche Belastung durch die Mehrwertsteuer abgeben.

1Vgl. Controller General's Report to the Congress of the United States, The Value-Added Tax in the European Economic Community, Washington, 5. Dez. 1980, S. $14 \mathrm{ff}$.

${ }^{2}$ Vgl. EG-Parlament, Sitzungsdokumente 1980 - 1981, Dok. 1-772/80, S. 35 .

3Vgl. A. Pedone, Italy, in: H. J. Aaron (Hrsg.), The Value-Added Tax, Experiences of some European Countries, Antwerpen, Boston u.a. 1982 , S. 203 ff., hier: S. 225. Von beträchtlichen Steuerhinterziehungen spricht auch D. Strasser, S. 125.

${ }^{4}$ Vgl. Prof. M. Frank over belastingontduiking en fiscale onderschatting, in: Maandblad Belastingbeschouwingen, 49/78, 1980, S. $154 \mathrm{f.}$, zitiert nach S. Cnossen, The Netherlands, in: H. J. Aaron (Hrsg.), 1982, S. 78 .

${ }^{5}$ Vgl. S. Cnossen, S. 67.

${ }^{6} \mathrm{Vgl}$. R. Hemming and J. A. Kay, United Kingdom, in: H. J. Aaron (Hrsg.), 1982, S. 103. 
Zusammenfassend läßt sich feststellen, daß im Rahmen der verwaltungstechnischen Abwicklung und Erfassung der Mehrwertsteuer-Eigenmittel offenbar maßgebliche Gründe vorliegen, die die mitgliedsstaatliche Verteilung beeinflussen. Eine Isolierung oder gar Quantifizierung dieser Effekte ist derzeit jedoch kaum möglich. Dieses Ergebnis weist gleichzeitig aber auch darauf hin, daß die Mehrwertsteuer-Erhebung gegenwärtig nicht uneingeschränkt geeignet ist, die am pro Kopf-Sozialprodukt gemessene Wirtschaftskraft der Mitgliedsstaaten zu erfassen.

\subsubsection{Zölle}

Die Zolleinnahmen sind nach der Mehrwertsteuer die zweitgrößte Einnahmequelle der Gemeinschaft - wenn auch mit abnehmender relativer Bedeutung (Tabelle 4). Angesichts ihres Volumens kommt ihrer Verteilung auf die einzelnen Mitgliedsstaaten ebenfalls ein erhebliches Gewicht bei der Bestimmung der nationalen Finanzierungspositionen zu.

Die Zolleinnahmen erwachsen aus der Anwendung des Gemeinsamen Zolltarifs (GZT) auf die aus Drittländern eingeführten Waren. Der GZT wird ergänzt durch die Agrarabschöpfungen, die bei den meisten Produkten, die der gemeinsamen Agrarmarktordnung unterliegen, an die Stelle der Zollsätze treten. ${ }^{1}$ Diese Abgaben werden im Abschnitt 4.1.1.3. gesondert behandelt.

Die Einführung des GZT war am 1. Juli 1968 abgeschlossen - eineinhalb Jahre eher, als die Bestimmungen des EWG-Vertrages es vorsahen. Die Binnenzölle zwischen den sechs Gründungssaaten waren ab-

${ }^{1}$ Ausnahmen von diesem Grundsatz bestehen insofern, als einige Waren sowohl zoll- als auch abschöpfungspflichtig sind. Vgl. U. Nittka, S. 69. 
geschafft und die Zuständigkeit, verbindliche tarifrechtliche Regelungen $z u$ erlassen, war vom nationalen Recht auf das Gemeinschaftsrecht übergegangen. 1 Seit dem 1. Juli 1977 ist auch für die 1973 beigetretenen Mitgliedsstaaten die Ubergangszeit für den innergemeinschaftlichen Zollabbau und die Ubernahme des gemeinsamen Außenzolltarifs abgeschlossen. Für Griechenland wurde eine solche Anpassungsfrist bis zum Jahre 1986 gewährt.

Das Schema des GZT umfaßt gegenwärtig etwa 3.000 Warenpositionen, für die die jeweiligen zölle festgelegt sind. ${ }^{2}$ Dabei handelt es sich fast ausnahmslos um Wertzölle $e^{3}$, also um Zollbelastungen, die in einem Vomhundertsatz des zollwertes der eingeführten Waren ausgedrückt werden. Die zölle werden von den Zollverwaltungen der Mitgliedsstaaten gemä $\beta$ ihren Rechts- und Verwaltungsvorschriften erhoben und dann als Eigenmittel an die Konten der Gemeinschaft abgeführt. Die Umrechnung der nationalen Währungen in ECU erfolgt auf der Grundlage der Notierungen des Devisenmarktes. 4

\subsection{Handels- und währungspolitische Bestimmungsgründe der} mitgliedsstaatlichen Verteilung

Das gemeinschaftliche Zollaufkommen und speziell die Höhe der von einem Mitgliedsland abzuführenden zolleinnahmen wird durch das zusammenwirken einer Reihe von Einflußfaktoren bestimmt. Dazu gehören allgemein das Volumen der Drittlandsimporte sowie das hierauf lastende Zollniveau und in diesem Rahmen die Zusammensetzung der Drittlandsimporte nach Einfuhrländern und warengruppen.

${ }^{1}$ Verordnung (EWG) Nr. 950/68 des Rates vom 28. Juni 1968 über den Gemeinsamen Zolltarif, in: Amtsblatt der EG, Nr. L 172 vom 22.7 .1968 .

${ }^{2}$ Vgl. EG-Amt für amtliche Veröffentlichungen, Die Zollunion, Europäische Dokumentation 6/1983, Luxemburg 1983, S. 19.

${ }^{3}$ Vgl. M. Lux, Allgemeines Zolltarifrecht, in: R. Regul (Hrsg.), Gemeinschaftszollrecht, Baden-Baden 1982, S. 59 ff., hier: S. 93.

${ }^{4}$ Vgl. Art. 1 ff. der Verordnung Nr. $2891 / 77$. 
Tabelle 10

Zolleinnahmen nach Mitgliedsstaaten 1980 - 1984

\begin{tabular}{|c|c|c|c|c|c|}
\hline \multirow[t]{2}{*}{ Mitgliedsland } & 1980 & 1981 & 1982 & 1983 & 1984 \\
\hline & \multicolumn{5}{|c|}{ in Mio. ECU } \\
\hline Belgien & 392,6 & 408,4 & 403,7 & 423,8 & 470,0 \\
\hline Dänemark & 130,2 & 135,2 & 142,6 & 159,8 & 197,3 \\
\hline Deutschland & 1799,1 & 1943,8 & 1966,5 & 2019,8 & 2309,7 \\
\hline Frankreich & 900,0 & 977,3 & 1071,2 & 1051,8 & 1100,0 \\
\hline Griechenland & - & 84,6 & 108,7 & 107,4 & 99,4 \\
\hline Irland & 67,1 & 81,9 & 87,8 & 100,4 & 126,7 \\
\hline Italien & 636,9 & 633,0 & 660,8 & 657,1 & 750,9 \\
\hline Luxemburg & 4,3 & 4,5 & 4,3 & 4,6 & 5,9 \\
\hline Niederlande & 535,5 & 572,8 & 585,2 & 633,2 & 740,8 \\
\hline Verein. Königreich & 1440,1 & 1550,9 & 1784,5 & 1830,8 & 2160,1 \\
\hline \multirow[t]{2}{*}{ EG } & 5905,8 & 6392,4 & 6815,3 & 6988,7 & 7960,8 \\
\hline & \multicolumn{5}{|c|}{ in 8} \\
\hline Belgien & 6,6 & 6,4 & 5,9 & 6,1 & 5,9 \\
\hline Dänemark & 2,2 & 2,1 & 2,1 & 2,3 & 2,5 \\
\hline Deutschland & 30,5 & 30,4 & 28,8 & 28,9 & 29,0 \\
\hline Frankreich & 15,2 & 15,3 & 15,7 & 15,0 & 13,8 \\
\hline Griechenland & - & 1,3 & 1,6 & 1,5 & 1,3 \\
\hline Irland & 1,1 & 1,3 & 1,3 & 1,4 & 1,6 \\
\hline Italien & 10,8 & 9,9 & 9,7 & 9,4 & 9,4 \\
\hline Luxemburg & 0,1 & 0,1 & 0,1 & 0,1 & 0,1 \\
\hline Niederlande & 9,1 & 9,0 & 8,6 & 9,1 & 9,3 \\
\hline Verein. Königreich & 24,4 & 24,3 & 26,2 & 26,2 & 27,1 \\
\hline EG & 100,0 & 100,0 & 100,0 & 100,0 & 100,0 \\
\hline
\end{tabular}

Quelle: EG-Rechnungshof, Jahresberichte, versch. Jg.; eigene Berechnungen. 
Das gegenwärtige Zollniveau ist maßgeblich durch die Verhandlungen im Rahmen des Allgemeinen Zoll- und Handelsabkommens (GATT) mitbestimmt worden, das weltweit eine Liberalisierung des Handels durch den Abbau tarifärer Hemmnisse zum $\mathrm{Z}$ iel hat. Bereits vor dem Inkrafttreten des GZT hatte die Gemeinschaft im Rahmen der sogenannten Dillon-Runde (1960 bis 1962) und Kennedy-Runde (1964 bis 1967) Zollsenkungen zugestimmt. Diese Bestrebungen wurden in der TokioRunde (1973 bis 1979) fortgesetzt. Betrug das durchschnittliche Zollniveau der zollpflichtigen Güter bei Einführung des gemeinsamen Außenzolltarifs noch 11 \%, so ist es bis 1979 auf 9,8 \& reduziert worden und soll 1987, dem Abschlußjahr der Tokio-Runde, auf 7,5 \% vermindert werden. 1

Von einer allgemeinen, d.h. unterschiedslos auf sämtliche eingeführten Waren und unabhängig von ihrer Herkunft bezogenen Anwendung des GZT wurde im Laufe der zeit in einem erheblichen Umfang abgewichen. Diese Entwicklung lag in den Zielsetzungen der Gemeinschaft begründet, einen regionalen Freihandelsraum in Europa zu schaffen und durch die Gewährung von zollpräferenzen entwicklungspolitische Aufgaben zu erfüllen.

Den Bemühungen um einen Zollabbau mit den übrigen europäischen Ländern waren zunächst insofern Grenzen gesetzt, als sich mit der 1960 geschaffenen Europäischen Freihandelszone (EFTA) ein zweiter Handelsblock in Europa etablierte, und dieser Dualismus eine Annäherung durch Präferenzbeziehungen erschwerte. In diese phase fielen denn auch lediglich Assozilerungsabkommen mit Griechenland (1961) und der Türkei (1963), die als Vorstufe zu einem späteren Beitritt zur Gemeinschaft die schrittweise Herstellung einer Zollunion zum ziel hatten. Zu Beginn der siebziger Jahre wurden darüber hinaus Freihandelsabkommen mit den meisten Anrainerstaaten des Mittelmeeres geschlossen, dile sich allerdings auf die Ursprungswaren beschränkten. Eine Verbindung mit der EFTA erfolgte schließlich 1973 im Zuge

${ }^{1}$ Vgl. EG-Amt für amtliche Veröffentlichungen, Die Zollunion, s. 13. 
der Erweiterung der Gemeinschaft, deren neue Mitglieder Vereinigtes Königreich und Dänemark zuvor der EFTA angehört hatten. Es wurden nun Abkommen mit den verbleibenden sieben EFTA-Mitgliedsländern geschlossen, die die Bildung einer Freihandelszone für gewerbliche (Ursprungs-)Waren bewirkten. Der schrittweise beiderseitige Zollabbau konnte mit den in der EG-Beitrittsakte vorgesehenen Fristen koordiniert werden, so daß das allgemeine Zollabbauverfahren 1977 weitgehend abgeschlossen war. ${ }^{1}$

Hatten bereits die Mittelmeerabkommen entwicklungspolitischen Charakter, indem hier das Prinzip der Gegenseitigkeit der zollzugeständnisse gelockert war, so wurde diese zielsetzung vor allem durch die Präferenzabkommen mit den Entwicklungsländern in Ubersee verfolgt. Die Entwicklung dieser Politik begann 1963 mit dem ersten Assoziierungsabkommen mit 18 staaten und basiert bis 1985 auf dem auf fünf Jahre befristeten Abkommen Lomé II, das inzwischen 63 Staaten Afrikas, der Karibik und des Pazifiks (AKP-Staaten) umfaßt. 2 Dieses Präferenzabkommen gewährt den begünstigten Staaten einen zollfreien zugang zum Gemeinschaftsmarkt für fast alle gewerblichen Exportgüter, die dort ihren Ursprung haben. Gleichzeitig verzichtet die Gemeinschaft auf den Grundsatz der Gegenseitigkeit. Daneben besteht seit 1971 das im Rahmen der Handels- und Entwicklungskonferenz der Vereinten Nationen (UNCTAD) entwickelte Allgemeine Zollpräferenzsystem, das auch den nicht zur AKP-Gruppe gehörenden Staaten weitgehende Zollfreiheit für alle gewerblichen Fertig- und Halbfertigwaren mit Ursprung in den Entwicklungsländern einräumt. ${ }^{3}$

Die den Entwicklungsländern eingeräumten Präferenzen gelten allerdings nicht uneingeschränkt, sondern werden durch mengenmäßige Beschränkungen teilweise limitiert. Solche Mengenregulierungen erfol-

\footnotetext{
${ }^{1}$ Vgl. N. Vaulant, Die Zollunion der Europäischen Wirtschaftsgemeinschaft, Luxemburg 1981, S. $35 \mathrm{ff}$.

2 Als Anschlußregelung wurde im Dezember 1984 in Lomé das III. AKPEWG-Abkommen geschlossen, das ebenfalls eine fünfjähriqe Gültigkeitsdauer besitzt und $z u$ dem nunmehr 65 AKP-Staaten gehören. 3 Vgl. N. Vaulant, S. $40 \mathrm{ff}$.
} 
gen durch zollkontingente, bei denen die Aussetzung der zölle automatisch erlischt, sobald die eingeführten Waren mengen- oder wertmäßig eine bestimmte Höchstgrenze erreicht haben, oder durch Zollplafonds, bei denen die Wiedereinsetzung der zölle aufgrund eines Rechtsaktes möalich ist. Diese Maßnahmen werden ergänzt durch Export-Selbstbeschränkungsabkommen, mit denen die Herstellerländer sich verpflichten, nur eine bestimmte warenmenge auf den Gemeinschaftsmarkt zu bringen, und die vor allem im Textilsektor Anwendung gefunden haben. 1 Die Ausgestaltung des allgemeinen Präferenzsystems läßt also nur einen kontrollierten Präferenzhandel zu. ${ }^{2}$ Damit wird allgemein das ziel verfolgt, die heimische Wirtschaft vor einem ungehindert großen Angebot preisgünstiger Güter zu schützen. Trotz dieser Einschränkungen ist der entwicklungspolitisch motivierte Verzicht auf zolleinnahmen nicht unbeträchtlich: nach Schätzungen der Kommission betrug er etwa im Jahre 1982590 bis 673 Mio. ECU. ${ }^{3}$ Allgemein stellen die zahlreichen zollabkommen und ihre Ausgestaltung unter Beiweis, daß die Handelspolitik der Gemeinschaft nicht darauf abzielt, möglichst hohe zolleinnahmen zu erzielen, sondern daß es sich in erster Linie um schutzzölle handelt. 4

Insgesamt bewirken die Zollabkommen und die Ausgestaltung der Präferenzregelungen zum einen, daß der GZT für große geographische Bereiche nicht oder nur in vermindertem Ausmaß angewandt wird. Voll gilt der Tarif praktisch nur noch für den Warenverkehr mit Nordamerika, Japan, den Staatshandelsländern des Ostens, Australien und

${ }^{1}$ Vgl. EG-Amt für amtliche Veröffentlichungen, Die Zollunion, S. 18.

${ }^{2}$ Vgl. A. Bormann, C. Bormann, M. Stegger, Das Allgemeine Zollpräferenzsystem der EG und seine Auswirkungen auf die Einfuhren aus Entwicklungsländern - Zusammenfassung - HWWA - Institut für Wirtschaftsforschung Hamburg, Sept. 1979, S. 3.

${ }^{3}$ Vgl. EG-Rechnungshof, Jahresbericht zum Haushaltsjahr 1982 , S. $31 \mathrm{f}$.

${ }^{4} \mathrm{Vgl}$. U. Nittka, S. 266 f. 
Südafrika. ${ }^{1}$ zum anderen unterliegen von den importierten Gütern zahlreiche Agrarprodukte nicht dem Zolltarifrecht, sondern werden im Rahmen der gemeinsamen Agrarpolitik mit Agrarabschöpfungen belegt. Daneben werden die meisten Rohstoffe einschließlich der quantitativ stark zu Buche schlagenden Rohöle zollfrei eingeführt. Im wesentlichen sind daher nur noch die chemischen Erzeugnisse, Maschinen und Transporteinrichtungen sowie eine Reihe verarbeiteter gewerblicher Waren von der zollerhebung betroffen. ${ }^{2}$

Zieht man diese geographischen und güterspezifischen Abgrenzungen der zollbelasteten Drittlandsimporte als Näherungsgröße zur Erklärung der Höhe der Zolleinnahmen heran, dann erhält man als Ergebnis einer einfachen Regressionsrechnung für das Jahr 1983 die Gleichung $^{3}$

$$
y=-0,04+0,117 x
$$

mit $y$ : Zolleinnahmen der Gemeinschaftsländer in Mrd. ECU

x: Importe chemischer Erzeugnisse, Maschinen und Fahrzeuge, verschiedene verarbeitete Produkte aus Nordamerika, Japan, Osteuropa in Mrd. ECU 5

und für den Korrelationskoeffizienten den Wert 0,99. Der im Regressionskoeffizienten dieses Modells zum Ausdruck kommende durchschnittliche Zollsatz von 11,7 \& liegt zwar über dem tatsächlichen zollsatz. Dies erklärt sich allerdings daraus, daß das modellmäßig erfaßte Importvolumen nicht die gesamten zollpflichtigen Einfuhren repräsentlert.

\footnotetext{
${ }^{1}$ Vgl. R. Christiansen, Die Europäische Zollunion, Erreichtes und Erstrebtes, in: Zeltschrift für Zölle und Verbrauchsteuern, 54 . Jg., 1978, S. 354 ff., hier: S. 356; EG-Amt für amtliche Veröffentlichungen, Die Zollunion, S. 16.

${ }^{2}$ Vgl. D. Strasser, S. 391.

${ }^{3}$ Errechnet aus den der Tabelle 11 zugrundeliegenden Daten.

${ }^{4}$ Belgien und Luxemburg als ein Wirtschaftsraum.

${ }^{5}$ Die zuvor genannten Länder Australien und Südafrika wurden wegen der geringen quantitativen Bedeutung vernachlässigt.
} 
Tabelle 11

Importstruktur und Zollaufkommen der Mitgliedsstaaten 1983

\begin{tabular}{|c|c|c|c|c|c|}
\hline \multirow[t]{2}{*}{ Mitgliedsland } & \multicolumn{2}{|c|}{$\begin{array}{l}\text { Drittlandsimpor- } \\
\text { te von Nicht- } \\
\text { Agrarprodukten }\end{array}$} & \multicolumn{2}{|c|}{$\begin{array}{l}\text { darunter: } \\
\text { chem. Erzeugnisse, } \\
\text { Maschinen u. Fahr- } \\
\text { zeuge, versch.ver- } \\
\text { arbeitete Produkte } \\
\text { aus Nordamerika, } \\
\text { Japan, Osteuropa }\end{array}$} & \multirow[t]{2}{*}{$\begin{array}{l}\text { Zollaufkommen } \\
\text { in \& der } \\
\text { Drittlandsim- } \\
\text { porte von } \\
\text { Nicht-Agrar- } \\
\text { produkten }\end{array}$} \\
\hline & $\begin{array}{l}\text { in } 8 \\
\text { des BIP }\end{array}$ & $\begin{array}{l}\text { in Mrd. } \\
\text { ECU }\end{array}$ & in Mio. ECU & in 8 & \\
\hline $\begin{array}{l}\text { Belgien/ } \\
\text { Luxemburg }\end{array}$ & 20,4 & 19,1 & 4.330 & 22,5 & 2,2 \\
\hline Dänemark & 12,1 & 7,7 & 1.421 & 18,2 & 2,1 \\
\hline peutschland & 10,0 & 73,3 & 16.266 & 22,1 & 2,8 \\
\hline Frankreich & 8,2 & 47,4 & 10.253 & 21,5 & 2,2 \\
\hline Griechenland & 12,9 & 5,0 & 1.194 & 24,0 & 2,1 \\
\hline Irland & 11,9 & 2,3 & 1.503 & 65,2 & 4,3 \\
\hline Italien & 11,2 & 44,3 & 5.063 & 11,3 & 1,5 \\
\hline Niederlande & 17,3 & 25,7 & 5.758 & 22,2 & 2,5 \\
\hline $\begin{array}{l}\text { Verein. König- } \\
\text { reich }\end{array}$ & 10,9 & 55,2 & 16.800 & 30,4 & 3,3 \\
\hline EG & 10,8 & 279,9 & 62.627 & 22,4 & 2,5 \\
\hline
\end{tabular}

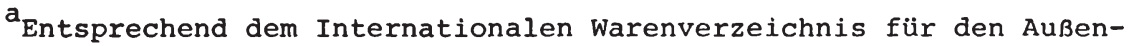
handel (SITC), Gruppen 5, 6, 7, 8 .

Quelle: Eurostat, Monatsbulletin der Außenhandelsstatistik, 6 und 8/1984; 2/1985; EG-Kommission, Europäische Wirt.schaft, Nr. 22, S. 222; eigene Berechnungen.

Die Abhängigkeit des Zollaufkommens von den Handelsverbindungen und der Warenstruktur der eingeführten Güter belegt auch die Tabelle 11: Die zollbelastung in bezug auf den Gesamtwert der importierten waren ist in den Gemeinschaftsländern besonders hoch, bei denen der Anteil zolltariflich erfaßter Güter überdurchschnittlich groß ist 
(Irland, Vereinigtes Königreich). Umgekehrt ist die Zollbelastung im allgemeinen in Ländern mit einem unterdurchschnittlichen Anteil zollbelasteter Güter relativ gering (Italien). In Verbindung mit den unterschiedlich hohen Importquoten der Mitgliedsstaaten ergeben sich hieraus ihre zollabführungen an den Gemeinschaftshaushalt, die in Relation zum Sozialprodukt in Tabelle 12 dargestellt sind.

Tabelle 12

Zollabführungen in Relation zu den Bruttoinlandsprodukten der Mitgliedsstaaten 1980 - 1984

\begin{tabular}{|l|c|c|c|c|c|}
\hline Mitgliedsland & \multicolumn{5}{|c|}{ Anteil der Zollabeürungen an den Gesamtzoll- } \\
& \multicolumn{4}{|c|}{ Anteil des BIP am Gemeinschafts-BIP } \\
& 1980 & 1981 & 1982 & 1983 & 1984 \\
\hline Belgien & 1,58 & 1,64 & 1,64 & 1,74 & 1,69 \\
Dänemark & 0,92 & 0,91 & 0,88 & 0,92 & 1,00 \\
Deutschland & 1,03 & 1,10 & 1,03 & 1,02 & 1,03 \\
Frankreich & 0,64 & 0,66 & 0,69 & 0,67 & 0,61 \\
Griechenland & - & 0,87 & 1,00 & 1,00 & 0,87 \\
Irland & 1,78 & 1,86 & 1,62 & 1,75 & 2,00 \\
Italien & 0,75 & 0,69 & 0,66 & 0,61 & 0,59 \\
Luxemburg & 0,41 & 0,44 & 0,45 & 0,46 & 0,53 \\
Niederlande & 1,49 & 1,55 & 1,48 & 1,57 & 1,63 \\
Verein. Königreich & 1,29 & 1,19 & 1,30 & 1,34 & 1,40 \\
EG & 1,00 & 1,00 & 1,00 & 1,00 & 1,00 \\
\hline
\end{tabular}

Quelle: EG-Rechnungshof, Jahresberichte, versch. Jg.; Kommission der EG, Europäische Wirtschaft, Nr. 22, S. 223; eigene Berechnungen.

So sind etwa die in bezug auf das BIP hohen Zollabführungen Belgiens und der Niederlande aus ihren weit überdurchschnittlichen Importquoten - auf deren Ursachen im Abschnitt 4.1.1.2.3. gesondert 
einzugehen sein wird - zu erklären, während die hohen Werte für Irland und das Vereinigte Königrelch offenbar in erster Linie aus der Handels- und Warenstruktur und der damit verbundenen zollbelastung resultieren. Auf der anderen Seite werden etwa die relativ geringen zollaufkommen Italiens zu einem großen Teil ais der relativ geringen Belastung der importierten Güter zurückzuführen sein.

Die Entwicklung der Zolleinnahmen im Zeitablauf sowie die damit verbundenen Wirkungen auf thre Verteilung innerhalb der Mitgliedsstaaten hängt von einer Reihe von Einflubfaktoren $a b$. So ist im großen und ganzen ein positiver Zusammenhang mit der Höhe des Sozialprodukts festzustellen. Dies drückt sich allgemein darin aus, daß im Zeitraum von 1971 bis 1980 das jahresdurchschnittliche reale Wachstum des Gemeinschaftssozialprodukts 2,9 \& und das der zolleinnahmen uber 30 \& betrug, wahrend ab 1981 bei Wachstumsraten von zumeist unter 1 \& auch das zollaufkommen durchschnittlich um weniger als $10 \%$ anstleg. 1 Ahnlich wie diese globale Entwicklung stellen sich auch die zusammenhänge in den einzelnen Mitgliedsstaaten dar, ${ }^{2}$ ohne daß sich daraus allerdings nachhaltige Veränderungen auf die zwischenstaatliche Verteilung der zolleinnahmen ergeben haben.

Weiterhın können von der Senkung der Zollsätze im Rahmen bilateraler und internationaler Vereinbarungen Einflüsse auf das zollaufkommen ausgehen. Denn nach zoll- und preistheoretischen überlegungen ist allgemein mit sinkender zollbelastung ein steigendes Importvolumen zu erwarten, und es hängt von der Summe aus Preis- und Mengeneffekten $a b$, ob das Zollaufkommen sinkt, steigt oder unverändert bleibt. Vergleicht man die Entwicklung der Drittlandsimporte mit der Entwicklung des Zollaufkommens, so zeigt sich, daß das wachstum der zolleinnahmen nicht mit dem der Drittlandsimporte

${ }^{1}$ Vgl. EG-Kommission, Europäische Wirtschaft, Nr. 22, S. 224 und 246; eigene Berechnungen.

${ }^{2}$ Vgl. J. $\varnothing$. Mфller, s. $36 \mathrm{ff}$. 
Schritt halten konnte. ${ }^{1}$ Dieses Ergebnis dürfte zum wesentlichen Teil auf die Verminderung des allgemeinen zollniveaus während dieser zeit zurückzufuhren sein. ${ }^{2}$ Inwieweit die zolleinnahmen der einzelnen Mitgliedsstaaten von dieser Entwicklung unterschiedlich betroffen werden, hängt von ihrer Importstruktur und der auf den einzelnen Güterkategorien lastenden Zollhöhe $a b$ und ist praktisch nicht zu quantifizieren.

Eine besondere Entwicklung der Importstruktur vollzog sich im Falle des Vereinigten Königreichs, das seit Mitte der siebziger Jahre seinen Anteil an Importen aus den Mitgliedsstaaten zu Lasten des ExtraEG-Handels ausgedehnt hat. Dies fuhrt tendenziell $z$ u verminderten Zolleinnahmen, wenn von der Reduktion des gesamten Extra-EG-Handels auch die zollpflichtigen Guter betroffen sind. ${ }^{3}$ Allerdings hat sich dieser Sachverhalt nicht in der Entwicklung der Zolleinnahmen GroBbritanniens niedergeschlagen. Sie weisen während des Untersuchungszeitraums vielmehr mit den vergleichswelse stärksten Anstieg auf (Tabelle 10), der demzufolge auf andere Ursachen zurückzuführen sein mus.

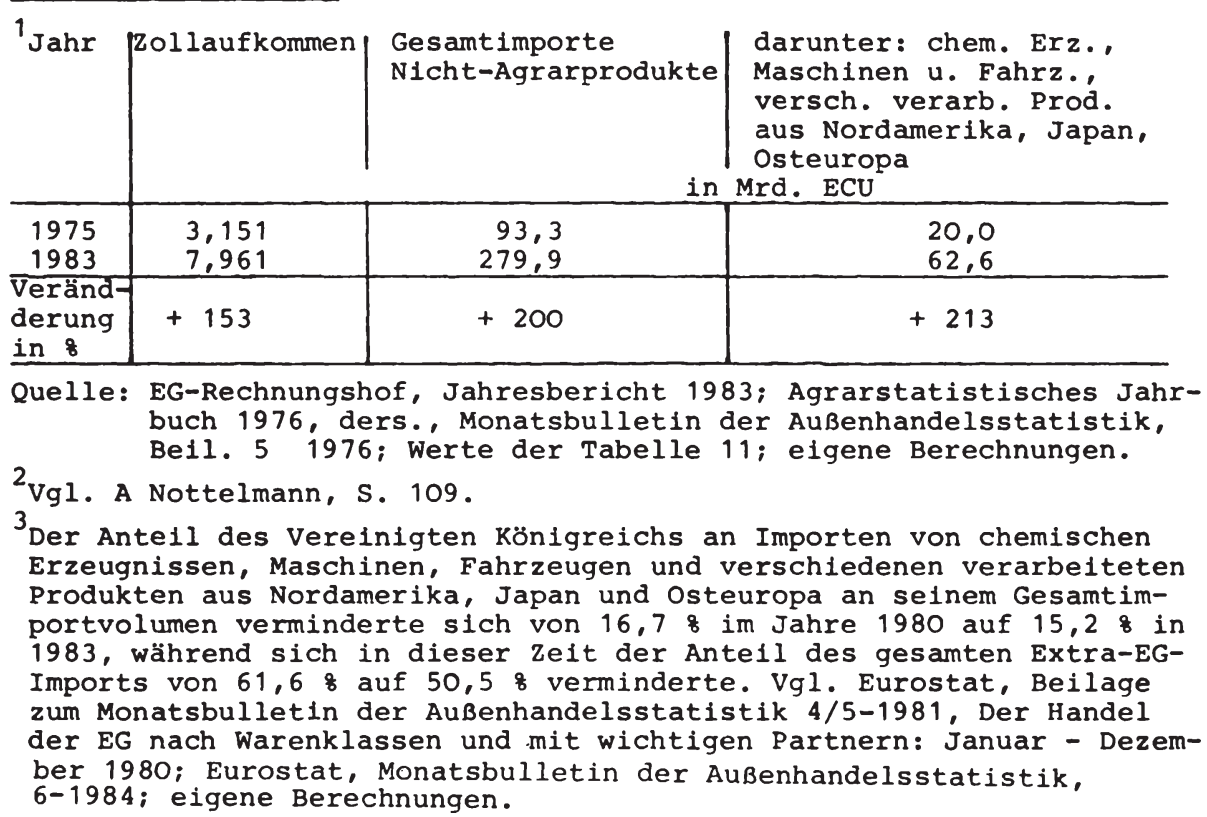


Zu diesen Bestimmungsgründen gehört die Währungsentwicklung, denn das Zollaufkommen und seine Verteilung auf die Mitgliedsstaaten wird auch von Veränderungen der Wechselkurse beeinflußt. Solche Effekte resultieren zum einen aus mitgliedsstaatlichen Wechselkursänderungen in bezug auf die Währungen der Exportländer. Abwertungen der Drittlandswährungen vermindern die Zollbemessungsgrundlage und Aufwertungen erhöhen sie. Das endgültige Ergebnis wird allerdings auch davon abhängig sein, inwieweit solche Währungsänderungen von Mengeneffekten begleitet werden und/oder in welchem Maße die Exporteure mit Variationen der Angebotspreise reagieren. ${ }^{1}$ Daneben ist - ebenso wie bei den Mehrwertsteuer-Eigenmitteln - das Verhältnis der Wăhrung des Mitgliedslandes in bezug auf die ECU von Bedeutung, da die in nationaler währung erhobenen zolleinnahmen $z u$ den laufenden Devisenmarktkursen in ECU umgerechnet und auf die Konten der Gemelnschaft transferiert werden. Dabei führt eine Höherbewertung der Mitgliedsstaatswährung zu steigenden Zollabführungen in ECU und vice versa. Je nach der konkreten Situation können sich die einzelnen Effekte gegenseitig verstärken oder kompensieren. In welchem Maße sich solche Entwicklungen auf das zollaufkommen auswirken, und speziell wie die elnzelnen Gemeinschaftsländer in ihrer Finanzierungsposition davon betroffen werden, läßt sich nur schwer abschätzen. Im Falle des Vereinigten Königreichs dürfte die Aufwertung des Pfund Sterling zu Beginn der achtziger Jahre dazu beigetragen haben, das aus dem verminderten Extra-EG Importvolumen prinzipiell resultierende geringere zollaufkommen $\mathrm{zu}$ kompensieren.

\subsection{Vertellungseffekte der Erfassung und Verwaltung}

Auf der Suche nach weiteren Einflußfaktoren, die die Verteilungswirkungen der zölle bestimmen, ist analog zu den Uberlegungen bei den Mehrwertsteuer-Eigenmitteln auch hier zu fragen, ob und in welchem Maße eine unzureichende und/oder unterschiedlich effiziente Er-

1Vgl. J. Ф. Mфller, S. 45. 
fassung dieser Einnahmen durch die nationalen Verwaltungsstellen das Zollaufkommen und seine Verteilung auf die Mitgliedsstaaten beeinflussen. Dabei ist anders als bei der Mehrwertsteuer, deren Erlöse ja zur Hauptsache den Mitgliedsstaaten zugute kommen, das Risiko einer Demobilisierung der nationalen Administrationen bei den zöllen insofern gegeben, als diese Mittel vollständig der Gemeinschaft zufließen. Auf diese Zusammenhänge weist der SpinelliBericht hin und forderte eine wirksamere kontrolle. ${ }^{1}$ In diesem $\mathrm{Zu}-$ sammenhang prüfte der EG-Rechnungshof 1982 das in Italien bestehende system der zentralen nachträglichen Kontrolle der Zollbelege. Dabei stellte sich im Rahmen einer Stichprobenerhebung heraus, daß bei der Bearbeitung der Zollbelege so große Rückstände auftraten, die elne Nacherhebung der Zollschulden wegen der Uberschreitung zeitlich vorgeschriebener Fristen gefährdeten. ${ }^{2}$ Dies mag als Indiz für administrative Probleme bei der Zollerhebung gelten. Uber mögliche Größenordnungen oder gar längerspezifische Unterschiede lassen sich gegenwärtig allerdings kelne Aussagen machen.

Die hier angesprochenen Risiken einer nicht vollständigen Erfassung der warenimporte gelten grundsätzlich auch für die abschöpfungspflichtigen Agrarimporte, so daB im Rahmen der Agrarabschöpfungen hierauf nicht mehr gesondert einzugehen ist.

\subsection{Probleme des formalen Verteilungsansatzes}

Der Aussagewert der vorliegenden Ergebnisse als Maßstab für die finanziellen Belastungen der Mitgliedsstaaten wird durch die an reine Zahlungsströme anknüpfende Erfassung eingeschränkt. Die Problematik besteht darin, daß die zölle - und gleichermaßen die Agrarabschöpfungen $^{3}$ - zumeist denjenigen Mitgliedsländern als zahlungen an den

\footnotetext{
${ }^{1}$ Vgl. EG-Parlament, Sitzungsdokumente 1980 - 1981, Dok. 1-772/80, S. $35 \mathrm{f}$.

2Vgl. EG-Rechnungshof, Jahresbericht 1982, S. 32.

${ }^{3}$ siehe hierzu Abschnitt 4.1.1.3.3.
} 
Gemeinschaftshaushalt zugerechnet werden, in denen diese Einnahmen anfallen. Insoweit nun die Importländer nicht gleichzeitig die Bestimmungsländer der Waren sind, werden Ihnen Abführungen anerkannt, für die sie keinen Verzicht leisten bzw. die ihre Bürger nicht belasten; mit anderen Worten: das Aufkommen der Abgaben und ihre Traglast fallen auseinander.

Auf diese zusammenhänge hat bereits die Kommission bei ihrer ersten Veröffentlichung der Nettopositionen als Einschränkung ihres Aussagewertes hingewiesen. ${ }^{1}$ Sie sind seither als kritischer Einwand gegen die Tragfähigkeit der Ergebnisse oft wiederholt worden.

Die Gründe für ein Auseinanderfallen von Importland und Bestimmungsland liegen hauptsächlich in geografischen Gegebenheiten und transporttechnischen Bedingungen, die es oft günstiger erscheinen lassen, die Importe aus tbersee in einen verkehrsgünstig gelegenen und kapazitätsmäßig geeigneten Hafen eines anderen Gemeinschaftslandes entladen zu lassen. Daneben dürften aber auch traditionelle Handelsverbindungen und die damit einhergehenden gewohnten Formen der $\mathrm{Ab}-$ wicklung für die Wahl der Best1mmungshäfen eine Rolle spielen.

Von diesen Gegebenheiten werden naturgemäß weniger diejenigen Mitgliedsländer betroffen, die an der Peripherie der Gemeinschaft liegen, sondern hauptsächlich die verkehrsgünstig und zentral gelegenen Staaten im Zentrum der Gemeinschaft. In diesem Rahmen kommt den großen Häfen Rotterdam und Antwerpen erhebliche Bedeutung für den Warenverkehr zwischen Belgien und den Niederlanden einerseits und der Bundesrepublik und auch Luxemburg, das als einziges Gemeinschaftsland über keinen Zugang zum Meer verfügt, andererseits, zu. ${ }^{2}$ zur Charakterisierung der Problematik des Auseinanderfallens von

\footnotetext{
${ }^{1}$ Siehe hierzu Abschnitt 3. 2Vgl. A. Edwards, s. 751.
} 
Zollerhebungs- und Bestimmungsland hat sich dann auch der Begriff "Rotterdam-Antwerpen-Effekt" herausgebildet. Für die übrigen Mitgliedsstaaten wie etwa das Vereinigte Königreich und Italien werden solche Wirkungen aufgrund der anderen geografischen und verkehrstechnischen Gegebenheiten nur eine untergeordnete Rolle spielen. ${ }^{1}$

Einen Eindruck von den Größenordnungen des "Rotterdam-AntwerpenEffekts" vermitteln Berechnungen des Statistischen Bundesamtes über die sogenannten ausgefallenen Zollerträge der Bundesrepublik. Diese Beträge, die durch die bereits erfolgte Verzollung in einem anderen Gemeinschaftsstaat bei den deutschen zollerträgen ausfallen, werden auf der Basis der glelchzeitigen Erfassung des Ursprungs- und Versendungslandes der Importe ermittelt. Sie sind in der Tabelle 13 angegeben.

Tabelle 13

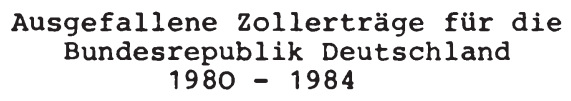

\begin{tabular}{|l|c|}
\hline Jahr & Mio. ECU \\
\hline 1980 & 75 \\
1981 & 79 \\
1982 & 90 \\
1983 & 131 \\
1984 & 168 \\
\hline
\end{tabular}

$a_{\text {Umgerechnet von DM in ECU auf der }}$ Basis der jahresdurchschnittlichen Wechselkurse

Quelle: Für die Zwecke dieser Arbeit zur Verfügung gestellt vom Statistischen Bundesamt, Wiesbaden.

\footnotetext{
${ }^{1}$ Vgl. G. Denton, S. 158; EG-Kommission, KOM (79) 462 endg., S. 10 f.
} 
Die ausgefallenen Zollerträge betreffen die Reexporte aus sämtlichen Mitgliedsländern und nicht nur die über die Niederlande und Belgien abgewickelten Importe. Folgt man allerdings den eingangs angestellten tberlegungen bezüglich der verkehrstechnischen Handelsverbindungen, dann dürfte der weit überwiegende Teil dieser Erträge in diesen beiden Ländern erhoben worden sein.

Für den umgekehrten Fall, daß die für andere Mitgliedsländer bestimmten Drittlandsimporte in der Bundesrepublik bereits zum freien Verkehr in der EG abgefertigt und hier Zolleinnahmen erhoben worden sind, liegen bisher keine zuverlässigen Schätzungen vor. Sie werden aber in jedem Fall den bisher dargestellten Effekten entgegenwirken.

Zusammenfassend läßt sich feststellen, daß die Existenz des bisher nur qualitativ formulierbaren "Rotterdam-Antwerpen-Effekts" zwar durchaus gegeben ist. Bezieht man die ausgefallenen zollerträge der Tabelle 13 auf das Gesamtzollaufkommen der Bundesrepublik bzw. Belgiens und der Niederlande, dann betragen diese Anteile allerdings nur 48 bis $7 \%$ bzw. $8 \%$ bis $14 \%$, wobei sich der Anstieg dieser Prozentzahlen kontinuierlich über die Jahre des Untersuchungszeitraums vollzogen hat. ${ }^{1}$ Die dadurch ausgelösten Verzerrungen in der zurechnung der Einnahmen auf die betroffenen Mitgliedsstaaten sind damit relativ gering.

\subsubsection{Agrarabschöpfungen}

Die Agrarabschöpfungen stellen die drittgrößte Einnahmequelle der Gemeinschaft dar. Ihr Aufkommen ist allerdings im Vergleich zu den Mehrwertsteuern und zöllen mit gegenwärtig etwa 5 \& der gesamten Eigenmittel relativ gering (vgl. Tabelle 4). Bemerkenswert sind wei-

${ }^{1}$ Nach Angaben des Statistischen Bundesamtes ist von einem im Zeitablauf zunehmenden Grad der Genauigkeit der Ergebnisse auszugehen. 
terhin die relativ starken Schwankungen im Zeitablauf, die temporär auch zu absolut sinkenden Abschöpfungseinnahmen geführt haben.

Die Agrarabschöpfungen gehören zum Instrumentarium des gemeinschaftlichen Marktordnungssystems für landwirtschaftliche Erzeugnisse, dessen Kern die Festlegung eines einheitlichen Preisniveaus auf dem Binnenmarkt und die Sicherung dieses Niveaus durch Binnenmarktinterventionen und Außenschutzmaßnahmen ist. In diesem Rahmen haben sie die Funktion, bei Agrarimporten aus Drittländern die Differenz zwischen den niedrigeren Weltmarktpreisen und den höheren garantierten Inlandspreisen auszugleichen. Damit nimmt dieser Ausgleichsmechanismus eine Schutzfunktion wahr, indem er gewährleistet, daß die in der Gemeinschaft angestrebten Preise nicht von Drittländern unterboten werden können. Gleichzeitig erfüllen die Agrarabschöpfungen eine Stabilisierungsfunktion, indem sie das Binnenmarktpreisniveau vor Schwankungen der Weltmarktpreise absichern. 1

Neben den Einfuhrabschöpfungen können grundsätzlich auch Ausfuhrabschöpfungen erhoben werden. Diese Möglichkeit besteht, wenn die Weltmarktpreise über den Preisen der Gemeinschaft liegen. Sie hat den Zweck, innergemeinschaftliche Preisniveausteigerungen und Versorgungsschwierigkeiten $z u$ vermeiden. Da das EG-Preisniveau bei Marktordnungsproduktion im allgemeinen deutlich über dem Weltmarktniveau liegt, sind diese Situationen allerdings nur relativ selten für eine begrenzte zeit bei einigen Erzeugnissen eingetreten. ${ }^{2}$

\footnotetext{
'Vgl. hierzu etwa H. E. Buchholz, Agrarmarkt: EWG-Marktordnungen, in: Handwörterbuch der Wirtschaftswissenschaft (HDWW), Bd. 1, Stuttgart, New York, Tübingen u.a. 1977, S. $87 \mathrm{ff}$., hier: S. $90 \mathrm{f.;}$ o. Gottsmann, Der gemeinsame Agrarmarkt, Texte mit Kommentar, Baden-Baden, Loseblattsammlung, IC 51 (30. Lieferung - 1983), S. 2 . ${ }^{2} \mathrm{Vgl}$. hierzu etwa P. Hrubesch, Regelungen des Außen- und Binnenhandels der Europäischen Gemeinschaft mit Agrarprodukten, in: Deutsches Institut für Wirtschaftsforschung, Vierteljahresheft 1/1975, S. $33 \mathrm{ff}$. hier: S. 35 f.; U. Nittka, S. 82 f.; EG-Kommission (Hrsg.), Die Landwirtschafts- und Agrarhandelspolitik der Gemeinschaft, Grünes Europa, Nr. 187, Brüssel 1981, S. 4.
} 
Tabelle 14

Agrarabschöpfungen nach Mitgliedsstaaten

$1980-1984$

\begin{tabular}{|c|c|c|c|c|c|}
\hline Mitgliedsland & 1980 & 1981 & 1982 & 1983 & 1984 \\
\hline & \multicolumn{5}{|c|}{ in Mio. ECU } \\
\hline Belgien & 193,1 & 178,4 & 238,6 & 293,9 & 211,0 \\
\hline Dänemark & 7,5 & 10,1 & 7,5 & 6,9 & 8,0 \\
\hline Deutschland & 223,7 & 179,9 & 201,9 & 143,0 & 158,6 \\
\hline Frankreich & 91,6 & 103,1 & 70,9 & 78,9 & 94,2 \\
\hline Griechenland & -- & 17,4 & 63,5 & 38,0 & 19,5 \\
\hline Irland & 3,9 & 4,3 & 6,0 & 7,0 & 6,9 \\
\hline Italien & 379,3 & 247,6 & 285,1 & 323,2 & 288,5 \\
\hline Luxemburg & 0,1 & 0,1 & 0,1 & 0,1 & 0,1 \\
\hline Niederlande & 234,7 & 175,4 & 172,5 & 135,8 & 131,8 \\
\hline Verein. Königreich & 401,5 & 384,6 & 475,9 & 320,3 & 341,4 \\
\hline \multirow[t]{2}{*}{ EG } & 1535,4 & 1264,9 & 1522,0 & 1347,1 & 1260,0 \\
\hline & \multicolumn{5}{|c|}{ in 8} \\
\hline Belgien & 12,6 & 14,1 & 15,7 & 21,8 & 16,7 \\
\hline Dänemark & 0,5 & 0,8 & 0,5 & 0,5 & 0,6 \\
\hline Deutschland & 14,6 & 14,2 & 13,3 & 10,6 & 12,6 \\
\hline Frankreich & 6,0 & 8,1 & 4,6 & 5,9 & 7,5 \\
\hline Griechen land & -- & 1,4 & 4,2 & 2,8 & 1,6 \\
\hline Irland & 0,2 & 0,3 & 0,4 & 0,5 & 0,5 \\
\hline Italien & 24,7 & 19,6 & 18,7 & 24,0 & 22,9 \\
\hline Luxemburg & 0,0 & 0,0 & 0,0 & 0,0 & 0,0 \\
\hline Niederlande & 15,3 & 13,9 & 11,3 & 10,1 & 10,5 \\
\hline Verein. Königreich & 26,1 & 27,6 & 31,3 & 23,8 & 27,1 \\
\hline EG & 100,0 & 100,0 & 100,0 & 100,0 & 100,0 \\
\hline
\end{tabular}

Quelle: EG-Rechnungshof, Jahresberichte, versch. Jg.; eigene Berechnungen. 
Agrarabschöpfungen haben prinzipiell die gleiche Wirkung wie zölle und sind deshalb nur im Einklang mit den zollrechtlichen Bestimmungen des GATT zu erheben. 1 Ihre Funktion kann allerdings weder durch Wertzölle noch durch spezifische zölle wahrgenommen werden, da diese nicht an die auszugleichende Differenz zwischen dem Weltmarkt- und Binnenmarktpreis anknüpfen. Diese Forderung erfüllt zwar der Gleitzol1 ${ }^{2}$, und er entspricht in seiner schutzwirkung damit den Abschöpfungen. Gleitzölle haben sich allerdings in der Praxis wegen administrativ bedingter Verzögerungen bei der Fixierung wechselnder Zollsätze gegenüber den Weltmarktpreisänderungen nicht bewährt. ${ }^{3}$ Die Festsetzung der Abschöpfungsbeträge erfolgt verwaltungstechnisch wie beim spezifischen 2011 auf der Grundlage von Gewicht, MaB oder Stückzahl der Importe; sie wird allerdings ständig den Unterschieden zwischen den Gemeinschafts- und Weltmarktpreisen angepaßt.

Die Agrarabschöpfungen sind eng mit den Mechanismen des Zollrechts verbunden. Sie werden in den melsten Mitgliedsstaten von den Zollverwaltungen erhoben, und die Anwendungsvorschriften sind Bestandteile des Zollrechts. ${ }^{4}$ Die Abschöpfungen werden in den für den gemeinsamen Agrarmarkt geltenden (fiktiven) Rechnungseinheiten fest-

${ }^{1}$ Vgl. H. -J. Rohr, G. Cordts, EWG Abschöpfungen und Ausfuhrerstattungen, Kommentar und Texte, Loseblattausgabe, Frankfurt a. M. ○.J., Anmerkung $7 \mathrm{zu} \S 1$ Abschöpfungserhebungsgesetz, 8. Ergänzungslieferung (1.10.1969).

${ }^{2}$ Beim Gleitzoll ist die Zollbelastung vom Wert der Ware abhängig, jedoch findet hier eine Umkehrung des Wertzollprinzips statt: Mit steigenden (sinkenden) Weltmarktpreisen sinkt (steigt) die Zollbelastung absolut und relativ, vgl. hierzu etwa $\mathrm{H}$. Conrad, Der Gleitzoll, Hamburg, Berlin, Bonn 1955, S. $41 \mathrm{f}$.

${ }^{3} \mathrm{Vgl}$. O. Wagner, Die preisdifferenzierenden Wirkungen von AuBenhandelsinstrumenten, Abschöpfungen, Zölle und Kontingente im AuBenhandel der EWG, Hamburg 1968, S. $30 \mathrm{f}$.

${ }^{4}$ Vgl. D. Ehle, G. Meier, EWG-Warenverkehr. Außenhandel-Zölle-Subventionen, Köln 1971, S. 540. 
gesetzt, in der Währung der importierenden Mitgliedsländer bezahlt ${ }^{1}$ und von diesen unter Umrechnung der Beträge in ECU an die Konten der Gemeinschaft abgeführt.

\subsection{Agrar- und wirtschaftspolitische Bestimmungsgründe} der mitgliedsstaatlichen Verteilung

Der Arwendungsbereich der Agrarabschöpfungen erstreckt sich nicht auf sämtliche Importe landwirtschaftlicher Produkte, sondern nur auf Erzeugnisse, die den gemeinsamen Agrarmarktordnungen unterliegen und auch hier nur auf einen Teil davon. ${ }^{2}$ Dazu gehören vor allem Getreide, Reis, Schweine- und Rindfleisch, Milcherzeugnisse, zukker, Eier und Geflügel. Die Abschöpfungsbeträge werden bei den meisten Produkten im Prinzip aus der Differenz zwischen innergemeinschaftlichem Prels und dem Angebotspreis zu Weltmarktbedingungen ${ }^{3}$ unter Berücksichtigung der Transport-, Versicherungs- und Umschlagskosten bestimmt. ${ }^{4}$ Sie werden je nach der Art der Erzeugnisse täglich, wöchentlich, vierzehntägig, monatlich oder vierteljährlich festgelegt. 5 Neben den Agrarabschöpfungen werden teilweise Zusatzbeträge und Ausgleichsabgaben erhoben, die ebenfalls zu den Agrarabschöpfungen zählen, ${ }^{6}$ und die den Zweck haben, die Einfuhrabschöp-

${ }^{1}$ Vgl. A. Ries, Das ABC der Europäischen Agrarpolitik, Baden-Baden 1979 , S. 60 .

${ }^{2}$ Vgl. O. Gottsmann, IB 27, S. 7 (29. Lieferung - 1983) und IC 51, S. 1 (30. Lieferung - 1983).

${ }^{3}$ Den Weltmarktpreis berechnet die Kommission auf der Grundlage der günstigsten Einkaufsmöglichkeiten auf dem Weltmarkt. Vgl. B. Börner, Das Interventionssystem der landwirtschaftlichen Marktordnungen der EWG, in: Agrarrecht der EWG, Kölner Schriften zum Europarecht, Köln, Berlin, Bonn u.a. 1969, s. 39.

${ }^{4} \mathrm{Zu}$ Einzelheiten vgl. O. Gottsmann, IC $51, \mathrm{~S} .2$ f. (30. Lieferung 1983) und U. Nittka, S. $78 \mathrm{ff}$.

${ }^{5}$ Vgl. O. Gottsmann, IB 36, s. 1 ff. (24. Lieferung - 1982).

${ }^{6} \mathrm{Vgl}$. Art. 2 des Eigenmittelbeschlusses. Von den dort weiterhin genannten Abgaben werden gegenwärtig lediglich noch Terminprämien im Rahmen der Getreide- und der Reismarktordnung erhoben. Vgl. o. Gottsmann (24. Lieferung - 1982), IB 36, S. 1. 
fungen in ihren preisstabilisierenden Wirkungen zu ergänzen. 1

Analog zu den Zöllen bestehen auch im System der Agrarabschöpfungen handels- und entwicklungshilfepolitisch motivierte Ausnahmeregelungen. So werden im Abkommen von Lomé und in einigen bilateralen präferenzabkommen für bestimmte abschöpfungspflichtige Marktordnungserzeugnisse - bei gleichzeitiger Erhebung einer Ausfuhrabgabe des Exportlandes - weitgehende ErmäBigungen gewährt. ${ }^{2}$ Daneben hat sich die Gemeinschaft in den GATT-Verhandlungen etwa zu besonderen Abschöpfungssenkungen für Rindfleisch im Rahmen festgelegter Einfuhrkontingente verpflichtet, ${ }^{3}$ die teilweise speziell zugunsten einiger EFTA-Länder ausgerichtet sind. ${ }^{4}$ Insgesamt dürften die mit diesen Ausnahmeregelungen verbundenen quantitativen Effekte auf die Abschöpfungseinnahmen allerdings wesentlich geringer sein als die vergleichbaren Wirkungen im Bereich der $20 ̈ l l e$.

Die Höhe der Agrarabschöpfungen und ihre Verteilung auf die Mitgliedsstaaten wird von den abschöpfungspflichtigen Agrarimporten bestimmt, die nur etwa 20 \& der gesamten Drittlandseinfuhren von Agrarerzeugnissen ausmachen. ${ }^{5}$ Dabei sind die Abschöpfungsbeträge zum einen abhängig von den jewelligen Importmengen, die ihrerseits maßgeblich von den innergemeinschaftlichen Ernteerzeugnissen beeinfluBt werden, sowie von der Differenz zwischen den Weltmarktpreisen, die starken Schwankungen unterliegen können, und dem Gemein-

${ }^{1}$ Vgl. D. Ehle, G. Meier, S. 538.

${ }^{2}$ Vgl. EG-Kommission (Hrsg.), Gemeinsame Agrarpolitik und Agrarhandel mit den Entwicklungsländern, Grünes Europa, Nr. 192, Brüssel, Nov. 1982, S. 10 und 13.

${ }^{3} \mathrm{Vgl}$. H. R. Krämer, Instrumente und Gestaltung der Gemeinsamen Agrarpolitik, Publikation des Instituts für Weltwirtschaft an der Universität Kiel, Kiel 1981, S. 37.

${ }^{4} \mathrm{Vgl}$. EG-Kommission (Hrsg.), Die Landwirtschafts- und Agrarpolitik der Gemeinschaft, Grünes Europa, Nr. 187, S. 11.

${ }^{5}$ Vgl. ebenda, s. 9 . 
schaftspreisniveau, das in der Vergangenheit durch die kontinuierliche Erhöhung der innergemeinschaftlichen Agrarpreise stetig angestiegen ist. 1

Eine Aufgliederung der Agrarabschöpfungen nach einzelnen Produkten ist nicht verfügbar; es ist lediglich bekannt, daB in den letzten Jahren etwa drei Viertel der Abschöpungseinnahmen auf die Marktordnung für Getreide entfallen. ${ }^{2}$ Damit spielt die diesbezügliche Importstruktur der Mitgliedsländer die ausschlaggebende Rolle für die innergemeinschaftliche Verteilung dieser Einnahmen, und bestimmen vor allem Weltmarktpreisschwankungen auf diesem Markt die Höhe der Agrarabschöpfungen.

Diese Zusammenhänge verdeutlicht Tabelle 15 und auch die zugehörige Regressionsbezlehung zwischen den Abschöpfungseinnahmen $\left(y_{i}\right)$ und dem wertmäßigen Volumen der Getreideimporte der Mitgliedsländer ( $\left.x_{1}\right)$, die $z u$ der Gleichung

$$
y=34,3+0,485 x
$$

fuhrt und mit einem Korrelationskoeffizienten von 0,83 einen relativ guten statistischen Erklärungswert aufweist. Der Regressionskoeffizient von 0,485 bestätigt, daß die Abschöpfungsbeträge einen relativ großen Tell der Gemeinschaftspreise ausmachen.

Die Einflüsse der produktspezifischen Importstruktur der Mitgliedsländer auf thre Abschöpfungseinnahmen spiegeln sich auch in den entsprechenden Relationen zum Sozialprodukt wieder (Tabelle 16), so daß im allgemeinen diejenigen Mitgliedsstaaten mit den relativ höchsten Getreideimporten auch die höchsten Relationen der Abschöpfungen

\footnotetext{
${ }^{1}$ Vgl. H. R. Krämer, Tabellen S. 7, 13, 21, 30, 40, 50 für einzelne Marktordnungsprodukte.

2vgl. D. Strasser, S. 388 .
} 
Tabelle 15

Agrarabschöpfungen und Drittlandsimporte von Getreide nach Mitgliedsstaaten 1982

\begin{tabular}{|c|c|c|c|c|}
\hline Mitgliedsland & \multicolumn{2}{|c|}{ Agrarabschŏpfungen } & \multicolumn{2}{|c|}{$\begin{array}{l}\text { Drittlandsimporte Getrei- } \\
\text { de und Getreideerzeugnis- }\end{array}$} \\
\hline Belgien/Luxemburg & 238,7 & 15,7 & 454,7 & 18,1 \\
\hline Dănemark & 7,5 & 0,5 & 17,2 & 0,7 \\
\hline Deutsch land & 201,9 & 13,3 & 228,4 & 9,1 \\
\hline Frankreich & 70,9 & 4,6 & 202,2 & 8,1 \\
\hline Griechenland & 63,5 & 4,2 & 59,9 & 2,4 \\
\hline Irland & 6,0 & 0,4 & 4,2 & 0,2 \\
\hline Italien & 285,1 & 18,7 & 773,0 & 30,8 \\
\hline Niederlande & 172.5 & 11,3 & 221,3 & 8,8 \\
\hline Verein. Königreich & 475,9 & 31,3 & 546,2 & 21,8 \\
\hline EG & 1522,0 & 100,0 & 2507,2 & 100,0 \\
\hline
\end{tabular}

Quelle: Eurostat, Agrarstatistisches Jahrbuch 1984, S. 15; EG-Rechnungshof, Jahresbericht 1982, S. 183; eigene Berechnungen.

im Verhältnis zum BIP aufweisen (Belgien, Griechenland, Italien, Niederlande) und vice versa (Dänemark, Deutschland, Frankreich, Irland).

Währungsverschiebungen können insofern die Höhe der Abschöpfungseinnahmen beeinflussen, als Auf-bzw. Abwertungen der Wăhrungen, in denen die Drittländer die Agrarimporte anbieten, zu einem veränderten in ECU ausgedrückten Weltmarktprels führen. Diese Verschiebungen werden bei der flexiblen Festsetzung der Einfuhrabschöpfungen berücksichtigt: Abwertungen der Drittlandswährungen haben grundsätzlich einen höheren und Aufwertungen einen geringeren Abschöpfungsbetrag zur Folge. 
Tabelle 16

Agrarabschöpfungen in Relation zu den Bruttoinlandsprodukten der Mitgliedsstaaten 1980 - 1984

\begin{tabular}{|l|c|c|c|c|c|}
\hline Mitgliedsland & \multicolumn{5}{|c|}{ Anteil der Agrarabschöpfungen an den Gesamt- } \\
& \multicolumn{5}{|c|}{ abschöpfungseinnahmen } \\
& 1980 & 1981 & 1982 & 1983 & 1984 \\
\hline Belgien & 2,97 & 3,62 & 4,36 & 6,23 & 4,77 \\
Dänemark & 0,20 & 0,35 & 0,21 & 0,20 & 0,23 \\
Deutschland & 0,49 & 0,51 & 0,48 & 0,37 & 0,45 \\
Frankreich & 0,25 & 0,35 & 0,20 & 0,26 & 0,33 \\
Griechenland & -- & 0,93 & 2,63 & 1,87 & 1,07 \\
Irland & 0,39 & 0,43 & 0,50 & 0,63 & 0,63 \\
Italien & 1,72 & 1,36 & 1,27 & 1,57 & 1,44 \\
Luxemburg & 0,04 & 0,00 & 0,00 & 0,00 & 0,00 \\
Niederlande & 2,50 & 2,40 & 1,95 & 1,74 & 1,84 \\
Verein. Königreich & 1,38 & 1,35 & 1,55 & 1,21 & 1,40 \\
Eg & 1,00 & 1,00 & 1,00 & 1,00 & 1,00 \\
\hline
\end{tabular}

Quelle: EG-Rechnungshof, Jahresbericht, versch. Jg.; EG-Kommission, Europäische Wirtschaft, Nr. 22, S. 223; eigene Berechnungen.

Im Ergebnis wird die Verteilung der Abschöpfungseinnahmen auf die Mitgliedsstaaten maßgeblich von ihren landwirtschaftlichen Erzeugungs-, Verarbeitungs- und Verbrauchsstrukturen, insbesondere im Getreidesektor, bestimmt. In diesem Rahmen spielen vor allem erntebedingte Mengen- und Preisentwicklungen eine Rolle. Damit scheiden bei der Bemessung der Agrarabschöpfungen - ebenso wie bei den Zöllen - fiskalische Gesichtspunkte aus und können auch keine verteilungspolitischen $\mathrm{zielsetzungen} \mathrm{berücksichtigt} \mathrm{werden.}$ 


\subsection{Probleme des formalen Verteilungsansatzes}

Die hier vorliegende Problemstellung ist analoq der des Rotterdam-Antwerpen-Effekts im Zusammenhang mit den Zolleinnahmen, ${ }^{1}$ so daß darauf nicht erneut einzugehen ist.

Zur Schätzung der damit verbundenen Verzerrungen in der mitqliedsstaatlichen Zurechnung werden Berechnungen der Deutschen Bundesbank über die ausgefallenen Agrarabschöpfungen herangezogen, die zusammen mit den vom Statistischen Bundesamt ermittelten ausgefallenen zollerträgen in der Ubertragungsbilanz unter der Position 'Erstattungen an andere FG-Länder' ausgewiesen werden. ${ }^{2}$ Diese Schätzungen erfolgen in der Weise, daß auf die Agrarimporte, die aus einem EG-Land als Versendungsland und einem Nicht-EG-Land als Herstellungsland stammen, die jeweiligen Abgabensätze des deutschen Gebrauchszolltarifs angewendet werden. Die Eraebnisse sind in der Tabelle 17 darqestellt.

Diese Angaben beziehen sich ebenso wie die ausgefallenen zollerträge auf die Reexporte aus sämtlichen Mitgliedsstaaten, wobei der weit uberwiegende Tell allerdings wiederum den Niederlanden und Belgien als an den Gemeinschaftshaushalt abqefühte, jedoch nicht ihre Bürger belastende Agrarabschöpfungen zuzurechnen sein dürfte. Für den umgekehrten Fall der in der Bundesrepublik erhobenen Abschn̈pfungen auf die in andere Mitgliedsländer reexportierten Aqrarimporte liegen ebenfalls keine Schätzungen vor. Sie würden die Wirkungen der in Tabelle 17 ausgewiesenen Agrarabschöpfunqen abschwächen.

\footnotetext{
${ }^{1}$ Siehe Abschnitt 4.1.1.2.3.

${ }^{2} \mathrm{Vgl}$. Deutsche Bundesbank, Statistische Beihefte zu den Monatsberichten der Deutschen Bundesbank, Rethe 3 Zahlungsbilanzstatistik, Tabelle $4 a$, Spalte 23 .
} 
Tabelle 17

Ausgefallene Agrarabschöpfungen ${ }^{a}$ für die

Bundesrepublik Deutschland 1980 - 1984

\begin{tabular}{|c|c|}
\hline Jahr & Mio. ECU \\
\hline 1980 & 82 \\
1981 & 85 \\
1982 & 106 \\
1983 & 97 \\
1984 & 34 \\
\hline
\end{tabular}

a einsch1. Währungsausgle1chsbetrăge

bumgerechnet von DM in ECU auf der Basis der jahresdurchschnittlichen Wechselkurse.

Quelle: Deutsche Bundesbank, Statistische Beihefte zu den Monatsberichten, Rethe 3 Zahlungsbilanzstatistik, Tabelle $4 a$, Spalte 23 (abzüglich der ausgefallenen zollerträge aus Tabelle 13). 


\subsubsection{Zuckerabgaben}

Ein in den letzten Jahren zunehmender Anteil an den Gemeinschaftseinnahmen mit zuletzt etwa $4,5 \%$ (Tabelle 4) resultiert aus den Zucker- und Isoglucoseabgaben, ${ }^{1}$ die in den Haushaltsplänen ebenfalls als eigene Mittel ausgewiesen werden. Dabei handelt es sich um zweckgebundene Abgaben der Zuckerproduzenten und Isoglucosehersteller zur Finanzierung der Interventionsausgaben im Rahmen der gemeinsamen Marktorganisation für zucker. ${ }^{2}$

Die Zuckerabgaben bestehen in Form einer Produktions- und einer Lagerkostenabgabe. Produktionsabgaben werden erhoben, wenn eine Gesamtrechnung der innergemeinschaftlichen Produktion einerseits und der Absatzmöglichkeiten innerhalb der Gemeinschaft und auf den Exportmärkten andererseits voraussichtlich elnen finanziellen Verlust ergeben. Die Bemessung richtet sich nach den von den Zuckerherstellern produzierten Mengen unter Berücksichtigung der ihnen (jährlich erneut) zugewiesenen Produktionsquoten. Dabel kann auf eine Grundproduktionsmenge (sog. A- und B-Produktion) eine Abgabe bis zur Höhe von 2 ies Interventionspreises erhoben werden, die um eine bis zu 30 \&ige Abgabe auf die die untere A-Produktion überschreitende B-Erzeugermenge ergänzt werden kann. Für eine über diese quotengebundenen Produktionsmengen hinausgehende Zuckererzeugung besteht ein innergemeinschaftliches Vermarktungsverbot bzw. ein Exportgebot; hierauf kann eine zusätzliche Abgabe erhoben werden.

Das Aufkommen der Lagerkostenabgabe ist zum Ausgleich der mit der Lagerung der zuckerproduktion verbundenen Kosten zu verwenden. Wahrend diese Kosten den einlagernden Produzenten pauschal vergütet werden, wird die Abgabe von jedem Hersteller, Importeur oder Raffinierer nach Maßgabe seiner Gesamtproduktionsmenge erhoben, gleichgultig ob er lagertoder nicht.

\footnotetext{
${ }^{1}$ Von dem Gesamtaufkommen dieser Abgaben entfielen in den Jahren seit 1980 etwa 99 \& auf die Zuckerabgaben.

2 Diese Vorschriften sind seit 1981 in der Verordnung (EWG) $\mathrm{Nr}$. $1785 / 81$ des Rates vom 30. Juni 1981 ubber die gemeinsame Marktorganisation für Zucker, in: Amtsblatt der EG, Nr. L 177 vom 1.7.1981, s. $4 \mathrm{ff}$. elnheitlich für die Sektoren zucker und Isoglucose gereqelt.
} 
Tabelle 18

Zucker- und Isoglucoseabgaben nach Mitgliedsstaaten 1980 - 1984

\begin{tabular}{|c|c|c|c|c|c|}
\hline Mitgliedsland & 1980 & 1981 & 1982 & 1983 & 1984 \\
\hline & \multicolumn{5}{|c|}{ in MiO. ECU } \\
\hline Belgien & 27,2 & 27,8 & 44,4 & 65,8 & 83,9 \\
\hline Dänemark & 19,1 & 19,3 & 25,3 & 38,9 & 38,0 \\
\hline Deutschland & 130,7 & 126,9 & 190,2 & 270,5 & 350,2 \\
\hline Frankreich & 158,0 & 154,5 & 211,0 & 293,7 & 406,3 \\
\hline Griechenland & -- & 1,0 & 13,1 & 16,7 & 16,6 \\
\hline Irland & 4,6 & 4,6 & 7,0 & 11,6 & 16,8 \\
\hline Italien & 54,5 & 62,6 & 83,5 & 95,0 & 84,1 \\
\hline Luxemburg & -- & -- & -- & -- & -- \\
\hline Niederlande & 40,8 & 39,1 & 58,9 & 82,2 & 76,9 \\
\hline Verein. Königreich & 32,1 & 46,9 & 72,4 & 73,6 & 103,6 \\
\hline \multirow[t]{2}{*}{ EG } & 466,9 & 482,6 & 705,8 & 948,0 & 1176,4 \\
\hline & \multicolumn{5}{|c|}{ in 8} \\
\hline Belgien & 5,8 & 5,8 & 6,3 & 6,9 & 7,2 \\
\hline Dänemark & 4,1 & 4,0 & 3,6 & 4,1 & 3,2 \\
\hline Deutschland & 28,0 & 26.3 & 26,9 & 28,5 & 29,8 \\
\hline Frankreich & 33,8 & 32,0 & 29,9 & 31,0 & 34,5 \\
\hline Griechen land & -- & 0,2 & 1,9 & 1,8 & 1,4 \\
\hline Irland & 1,0 & 1,0 & 1,0 & 1,2 & 1,4 \\
\hline Italien & 11,7 & 12,9 & 11,8 & 10,0 & 7,2 \\
\hline Luxemburg & -- & -- & -- & -- & -- \\
\hline Niederlande & 8,7 & 8,1 & 8,3 & 8,7 & 6,5 \\
\hline Verein. Königreich & 6,9 & 9,7 & 10,3 & 7,8 & 8,8 \\
\hline EG & 100,0 & 100,0 & 100,0 & 100,0 & 100,0 \\
\hline
\end{tabular}

Quelle: EG-Rechnungshof, Jahresberichte, versch. Jg.; eigene Berechnungen. 
Mit der Isoglucoseabgabe soll ein zumeist aus Stärke gewonnenes Konkurrenzprodukt von zucker verteuert werden. ${ }^{1}$ Für ihre Erhebung gilt analog dem zucker ein an den Produktionsquoten orientiertes Abgabensystem. Ein Lagerkostenausgleich erfolgt jedoch nicht.

Die Höhe der Zucker- und Isoglucoseabgaben hänat demnach von dem Volumen der Interventionsausgaben auf den zuckermarkt ab, die im Durchschnitt der Jahre ab 1980 zu etwa zwei Drittel durch diese Abgaben finanziert wurden. ${ }^{2}$ Die Interventionskosten wiederum werden determiniert durch das AusmaB der gemeinschaftlichen Zuckerproduktion und ihr Verhältnis zum heimischen Verbrauch sowie von den Weltmarktproduktions- und -preisverhältnissen, die u.a. die Höhe der Ausfuhrerstattungen mitbestimmen. So waren in der Zeit von 1972 bis 1975 aufgrund einer allgemeinen Zuckerknappheit die Einnahmen aus dieser Abgabe gering und konnte die Produktionsabgabe zeitweilig sogar aufgehoben werden. Ab 1976 führte dann eine zunehmend höhere Gemeinschafts- und Weltmarktproduktion zu allgemein wachsenden tberschüssen und sinkenden Weltmarktpreisen, so daß sich die Abgaben wieder erhöhten. ${ }^{3}$

Die Verteilung der Zucker- und Isoglucoseabgaben auf die einzelnen Mitgliedsländer hängt von dem Umfang der Zucker- und Isoglucoseherstellung ihrer Produzenten ab. Die nationalen Finanzierungsanteile an den Gesamtabgaben variieren mit den entsprechenden Produktionsgrößen.

Im Gegensatz zu den übrigen Eigenmittelarten, die nach dem Nonaffektationsprinzip zur Finanzierung sämtlicher Ausgaben dienen, handelt es sich bei den diesen Abgaben um zweckgebundene Einnahmen, die ausschließlich zur Finanzierung der Interventionsausgaben innerhalb der gemeinsamen Marktorganisation für zucker herangezogen werden.

\footnotetext{
${ }^{1}$ Vgl. N. Andel (1983), S. 332 f.; U. Nittka, S. 86.

${ }^{2}$ Vgl. EG-Kommission, Die Lage der Landwirtschaft in der Gemeinschaft. Bericht 1983, Brússel, Luxemburg 1984, S. 262 und 266. ${ }^{3}$ Vgl. D. Strasser, S. 389 .
} 
Sie führen hier zu einer Schmälerung der durch die Preis- und $\mathrm{Ab}-$ satzgarantie gewährten Zuflüsse. Die Abgaben weisen damit insoweit Merkmale einer kostenäquivalenten Finanzierung auf. Dieser Zusammenhang wird lediglich dadurch gelockert, daß im Rahmen der Lagerkostenabgabe auch die nicht eingelagerte produktion erfaßt wird, und bei der Produktionsabgabe unterschiedliche Sätze erhoben werden.

Im Hinblick auf eine korrekte effektive Zurechnung dieser Abgaben auf die einzelnen Mitgliedsländer sind keine Vorgänge erkennbar, die die Ergebnisse verzerren könnten. Die Abgaben werden durch die Mitgliedsstaaten von den produzenten ihres Gebietes erhoben und dann an die Konten der Gemeinschaft weitergeleitet.

\subsubsection{Sonstige Einnahmen}

Die neben den Eigenmitteln der Gemeinschaft zur Finanzierung des Gesamthaushalts zur Verfügung stehenden Einnahmen machten in den Jahren seit $1980 \quad 2,5$ bis 7 \% der Gesamteinnahmen aus (Tabelle 4). Sie lassen sich im Hinblick auf ihre quantitative Bedeutung unterteilen in die stark schwankenden Haushaltsüberschüsse der(s) Vorjahre(s) sowie in eine Reihe verschiedener Einnahmen, die mit einem Anteil von etwa 1 \& stets in einer annähernd konstanten Größenordnung angefallen sind.

$\mathrm{zu}$ den hier genannten verschiedenen Einnahmen gehören

- Beiträge der EGKS zur pauschalen Deckung der gemeinsamen Verwaltungsausgaben; dieser Betrag ist seit 1978 auf 5 Mio. ECU festgesetzt; $^{1}$

\footnotetext{
${ }^{1}$ Er war im Art. 20 des Fusionsvertrages ursprünglich auf 18 Mio. Rechnungseinheiten festgelegt, wurde aber zur Entlastung der EGKS 1978 reduziert. Vgl. D. Strasser, S. $118 \mathrm{f}$. Dieser Betrag deckt damit nur einen geringen Teil der zugunsten der EGKS erbrachten Verwaltungsleistungen $\mathrm{ab}$.
} 
- Finanzbeiträge der Mitgliedsstaaten zur Beteiligung an zusätzlichen Gemeinschaftsprogrammen sowie Beiträge im Rahmen von Kooperationsabkommen mit Nichtmitgliedsstaaten;

- Einnahmen aus der Besteuerung der Bezüge der Gemeinschaftsbediensteten und aus ihren Beiträgen zur Altersversorgung;

- weitere kleinere Einnahmen wie Vergütungen für entgeltliche Leistungen, aus dem Verkauf von Publikationen und sonstige Verwaltungseinnahmen.

Unter verteilungspolitischen Gesichtspunkten besitzen diese - teilweise zweckgebundenen - Einnahmen allein wegen ihrer Größenordnung nur eine geringe Relevanz. Ihre zurechnung auf die einzelnen Mitgliedsstaaten ist zudem in vielen Fällen praktisch nicht möglich.

Die Haushaltsüberschüsse der Vorjahre stellten 1980, 1981, 1983 und 1984 mit $2,98,1,48,68$ und 1,28 eine nicht unerhebliche Haushaltseinnahme dar. Die Salden - ob Uberschuß oder Defizit jedes Haushaltsjahres sind in der darauffolgenden Budgetperiode in einem Berichtigungshaushaltsplan den übrigen Finanzmitteln hinzuzufügen. ${ }^{1}$ Da dies bei dem Saldo des Haushaltsjahres 1981 aus zeitlichen Gründen nicht mehr möglich war, wurde er mit in den Haushaltsplan 1983 eingestellt. ${ }^{2}$ Die Uberschüsse resultieren allgemein aus der Differenz zwischen den geschätzten bzw. geplanten und den tatsächlichen Einnahmen und Ausgaben.

Die Verteilungswirkungen der Haushaltsüberschüsse stehen in einem engen Zusammenhang mit der Restfinanzierung durch die Mehrwertsteuer-Eigenmittel. Die im Jahr des entstehenden Uberschusses faktisch zuviel abgeführten Mehrwertsteuern reduzieren sich im darauffolgenden Jahr, in dem der Uberschuß als Einnahmequelle angesetzt wird,

1Art. 27 der Haushaltsordnung vom 21. Dez. 1977, Amtsblatt der EG Nr. L 356 vom 31.12.1977, S. 1

${ }^{2}$ Vgl. Endgültige Feststellung des Haushaltsplans der Europäischen Gemeinschaften für das Haushaltsjahr 1983, in: Amtsblatt der EG, Nr. L 60 vom 7.3.1983. 
sowohl hinsichtlich des Volumens als auch in ihrer Verteilung auf die Mitgliedsstaaten im gleichen Maße. ${ }^{1}$ Bei einer Betrachtung über mehrere Perioden beeinflussen die Haushaltsüberschüsse die einzelstaatlichen Finanzierungspositionen damit nicht.

Eine zeitlich befristete besondere Finanzierungsquelle stellen die rückzahlbaren Vorschüsse dar. ${ }^{2}$ Erhebungskriterium sind die Relationen der mitgliedsstaatlichen Mehrwertsteuer-Bemessungsgrundlagen, so daß diese Einnahmen unter verteilungspolitischen Gesichtspunkten den Mehrwertsteuer-Eigenmitteln zuzuordnen sind.

\section{4:1.1.6. Gesamte Eigenmittel}

Abschließend sind die Verteilungswirkungen der einzelnen Einnahmenarten zusammenzufassen, um damit die gesamten Finanzierungspositionen der Mitgliedsstaaten betrachten zu können. Eine Beschränkung auf die Eigenmittel tut der Aussagefähigkeit faktisch keinen $\mathrm{Ab}-$ bruch, da diese Mittel im allgemeinen über 95 \& der gesamten Haushaltseinnahmen ausmachen.

Die Finanzierungspositionen sind in der Tabelle 19 dargestellt. Darin ist teilweise der dominierende Einfluß der Mehrwertsteuer-Eigenmittel erkennbar; in anderen Fällen bestimmen die mit dem Außenhandel verbundenen Einnahmen maßgeblich die Höhe des Finanzierungsanteils. Letzteres gilt vor allem für die Niederlande und Belgien, deren relativ hohe Finanzierungsanteile vor allem aus ihren überproportional hohen Zöllen und Agrarabschöpfungen resultieren, die auch bei einer belastungsmäBig korrekten Zurechnung unter Ausschaltung des Rotterdam-Antwerpen-Effekts nicht entscheidend geringer sein würden. Daneben wird der Einfluß der Außenhandelseinnahmen

\footnotetext{
${ }^{1}$ Abgesehen davon, daß die Relationen der Mehrwertsteuerbemessungsgrundlagen sich verändern können.

${ }^{2}$ Siehe hierzu ausführlicher Abschnitt 4.1.2.3.
} 
Tabelle 19

Eigenmittel nach Mitgliedsstaaten 1980 - 1984

\begin{tabular}{|c|c|c|c|c|c|}
\hline Mitgliedsland & 1980 & 1981 & 1982 & 1983 & 1984 \\
\hline & \multicolumn{5}{|c|}{ in Mio. ECU } \\
\hline Belgien & 950,6 & 990,5 & 1148,3 & 1215,9 & 1283,3 \\
\hline Dänemark & 346,2 & 354,4 & 402,2 & 479,9 & 532,9 \\
\hline Deutschland & 4610,2 & 5057,1 & 5698,5 & 6472,1 & 7052,4 \\
\hline Frankreich & 2992,0 & 3491,1 & 4225,9 & 4506,6 & 4802,3 \\
\hline Griechenland & -- & 254,5 & 381,6 & 377,7 & 355,8 \\
\hline Irland & 139,3 & 158,9 & 208,5 & 269,6 & 286,3 \\
\hline Italien & 1929,3 & 2526,0 & 2487,2 & 2998,7 & 3443,4 \\
\hline Luxemburg & 19,6 & 27,2 & 30,1 & 43,9 & 51,3 \\
\hline Niederlande & 1276,6 & 1287,1 & 1466,4 & 1564,9 & 1687,8 \\
\hline Verein. Königreich & 3167,8 & 3877,2 & 5115,5 & 5084,4 & 5429,5 \\
\hline \multirow[t]{2}{*}{ EG } & 15427,6 & 18024,0 & 21164,2 & 23013,6 & 24880,0 \\
\hline & \multicolumn{5}{|c|}{ in 8} \\
\hline Belgien & 6,2 & 5,5 & 5,4 & 5,3 & 5,0 \\
\hline Dänemark & 2,2 & 2,0 & 1,9 & 2,1 & 2,1 \\
\hline Deutschland & 29,9 & 28,1 & 26,9 & 28,1 & 28,4 \\
\hline Frankreich & 19,4 & 19,4 & 20,0 & 19,6 & 19,3 \\
\hline Griechenland & -- & 1,4 & 1,8 & 1,6 & 1,4 \\
\hline Irland & 0,9 & 0,9 & 1,0 & 1,2 & 1,2 \\
\hline Italien & 12,5 & 14,0 & 11,8 & 13,0 & 13,8 \\
\hline Luxemburg & 0,1 & 0,1 & 0,1 & 0,2 & 0,2 \\
\hline Niederlande & 8,3 & 7,1 & 6,9 & 6,8 & 6,8 \\
\hline Verein. Königreich & 20,5 & 21,5 & 24,2 & 22,1 & 21,8 \\
\hline EG & 100,0 & 100,0 & 100,0 & 100,0 & 100,0 \\
\hline
\end{tabular}

Quelle: EG-Rechnungshof, Jahresbericht, versch. Jg. 
auch in der Finanzierungsposition des Vereinigten Königreichs aufgrund der relativ hohen außergemeinschaftlichen Importe ebenfalls in einer Erhöhung des Finanzierungsanteils - deutlich, während die relativ geringen 2011 - und Abschöpfungseinnahmen im Falle Frankreichs $z u$ einem vergleichsweise niedrigen Finanzierungsanteil führen.

Diese Zusammenhänge wirken auch auf die Ergebnisse der Tabelle 20, in der die an den Gemeinschaftshaushalt abgeführten Eigenmittel in Relation zu den Bruttoinlandsprodukten der einzelnen Mitgliedsstaaten angegeben sind. Sie zeigen noch einmal insgesamt', daß das Einnahmensystem keineswegs den Forderungen einer an der wirtschaftlichen Leistungskraft der Mitgliedsstaaten orientierten Finanzierung entspricht.

Tabelle 20

Eigenmittel in Relation zu den Bruttoinlandsprodukten der Mitgliedsstaaten 1980 - 1984

\begin{tabular}{|l|c|c|c|c|c|}
\hline \multirow{2}{*}{ Mitgliedsland } & \multicolumn{5}{|c|}{$\begin{array}{l}\text { Anteil der Eigenmittel an den gesamten } \\
\text { mitteln }\end{array}$} \\
& $\begin{array}{l}\text { in Relation } \\
\text { meinschafts-BIP } \\
\text { 1980 }\end{array}$ & 1981 & 1982 & 1983 & 1984 \\
\hline Belgien & 1,47 & 1,41 & 1,50 & 1,51 & 1,43 \\
Dänemark & 0,92 & 0,87 & 0,79 & 0,84 & 0,84 \\
Deutschland & 1,01 & 1,01 & 0,96 & 0,99 & 1,01 \\
Frankreich & 0,82 & 0,83 & 0,87 & 0,87 & 0,85 \\
Griechenland & -- & 0,93 & 1,13 & 1,07 & 0,93 \\
Irland & 1,38 & 1,29 & 1,25 & 1,50 & 1,50 \\
Italien & 0,87 & 0,97 & 0,80 & 0,82 & 0,87 \\
Luxemburg & 0,75 & 0,94 & 1,02 & 1,36 & 1,00 \\
Niederlande & 1,36 & 1,22 & 1,19 & 1,17 & 1,19 \\
Verein. Königreich & 1,08 & 1,05 & 1,20 & 1,13 & 1,13 \\
Eg & 1,00 & 1,00 & 1,00 & 1,00 & 1,00 \\
\hline
\end{tabular}

Quelle: EG-Rechnungshof, Jahresberichte, versch. Jg.; EG-Kommission, Europäische Wirtschaft, Nr. 22, S. 223; eigene Berechnungen.

${ }^{1}$ Siehe hierzu die mitgliedsstaatlichen Pro-Kopf BIP in Tabelle 1. 
Dem Erfordernis, zumindest den Sozialprodukt-Relationen zu entsprechen, kommen - trotz der vorhandenen Unzulänglichkeiten ${ }^{1}$ die Mehrwertsteuerabführungen noch am nächsten. Es ist deshalb zu erwarten, daß mit steigendem Anteil der Mehrwertsteuern an den Gesamteinnahmen auch die Anpassung der gesamten mitgliedsstatichen Finanzierungsanteile an die Relationen der Sozialprodukte besser wird. Diese Beziehungen sind in der Tabelle 21 in Form von Regressionsrechnungen für den Untersuchungszeitraum angegeben, in dem sich der Mehrwertsteuer-Anteil von 46,3 \& im Jahre 1980 auf 55,3 \& bzw. 54,8 \& in den Jahren 1983 bzw. 1984 erhöhte ${ }^{2}$.

Tabelle 21

Regression der Finanzierungsanteile ${ }^{a}$ in bezug auf die BIP-Anteile der Mitgliedsstaaten 1980 - 1984

\begin{tabular}{|l|c|c|}
\hline Jahr & $\begin{array}{l}\text { Korrelations } \\
\text { koeffizient }\end{array}$ & steigung \\
\hline 1980 & 0,977 & 1,045 \\
1981 & 0,987 & 1,037 \\
1982 & 0,981 & 1,018 \\
1983 & 0,981 & 1,029 \\
1984 & 0,982 & 1,021 \\
\hline
\end{tabular}

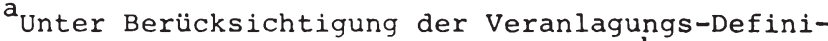
tion der Mehrwertsteuer-Eigenmittel;bohne Griechenland und Luxemburg

Quelle: Eigene Berechnungen nach EG-Kommission, Europäische Wirtschaft, Nr. 22; EG-Rechnungshof, Jahresberichte, versch. Jg.; EG, Compte de gestion et bilan financier.

\footnotetext{
'Siehe hierzu die Abschnitte 4.1.1.1.1. und 4.1.1.1.2. ${ }^{2}$ Siehe Tabelle 4 .
} 
Dabei deutet die im Zeitablauf näher an den Wert 1 heranrückende Steigung zumindest der Tendenz nach darauf hin, daB der zusammenhang zwischen dem Finanzierungsbeitrag und der Sozialprodukthöhe enger geworden ist. Betrachtet man die Entwicklung über diesen Untersuchungszeitraum hinaus, die nach den gegenwärtig gültigen Ergebnissen der dreijährigen finanziellen Vorausschau der Kommission bereits im Jahre 1988 zu einem Mehrwertsteuer-Anteil an den Gesamteinnahmen von 65 \& führen wird, ${ }^{1}$ dann ist eine Fortsetzung des beobachteten Trends zu erwarten. Er wird verstärkt, wenn es gelingt, die bei den Mehrwertsteuern liegenden Verzerrungen durch die nicht einheitliche Erfassung der Bemessungsgrundlage abzubauen.

${ }^{1} \mathrm{Vgl}$. EG-Kommission, Bulletin der EG, 7/8-1985, s. 112. 


\subsubsection{Korrekturmechanismen und Entlastungsregelungen}

Die Verteilung der Finanzierungslasten auf die einzelnen Mitgliedsstaaten resultiert nicht nur aus dem Einnahmensystem, sondern wird durch spezielle Korrekturmechanismen und Entlastungsregelungen ergänzt. Die Notwendigkeit solcher Verfahren ergab sich daraus, daß das Finanzierungs- und Ausgabensystem sowohl im Hinblick auf eine angemessene Finanzierungsbeteiligung als auch in bezug auf die Begünstigung durch die ausgabenwirksame Politik nicht in der Lage ist, von allen Mitgliedsstaaten akzeptierte Verteilungswirkungen zu gewährleisten. Dies betrifft vor allem das Vereinigte Königreich, das als eines der weniger wohlhabenden Gemeinschaftsländer nicht nur einen überproportionalen Finanzierungsanteil zu leisten hat, sondern auch an den Rückflüssen aus dem EG-Budget nur unterdurchschnittlich partizipiert. Angesichts dieser Situation sind bereits seit Mitte der siebziger Jahre Korrekturmechanismen institutionalisiert und Entlastungsregelungen getroffen worden. Die Entwicklung dieser zusammenhänge ist im folgenden nachzuzeichnen und $z u$ analysieren. 1

\subsubsection{Der Dubliner Korrekturmechanismus}

Bereits bei den Verhandlungen, die der Erweiterung der Gemeinschaft im Jahre 1973 vorausgingen, hatte die britische Regierung darauf hingewiesen, daß sie mit Beginn der vollständigen Anwendung des Eigenmittelbeschlusses unverhältnismäßig starke Belastungen zu tragen haben würde. Die daraus abgeleitete Forderung nach einer Korrektur des Finanzierungssystems ${ }^{2}$ wurde von der 1974 neugewählten britischen

${ }^{1}$ Dieser Abschnitt stellt teilweise eine enge Anlehnung an G. Ott, Das britische Haushaltsproblem im Finanzausgleich der Europäischen Gemeinschaften, Diskussionsbeitrag aus dem Institut für Finanzwissenschaft der Universität Kiel, Nr. 10, Sept. 1983, dar.

${ }^{2} \mathrm{Vgl}$. J. R. Dodsworth, European Community Financing: An Analysis of the Dublin Amendement, in: Journal of Common Market Studies, Vol. 14,1976, S. 129 ff., hier: S. 129. 
Regierung in die sog. Neuverhandlungen der Beitrittsbedingungen eingebracht, über deren Ergebnisse und damit uber einen Verbleib des Vereinigten Königreichs in der Gemeinschaft in einem Referendum abgestimmt werden sollte. Die EG-Kommission wurde mit der uberprüfung der künftigen finanziellen Lage der Gemeinschaft unter besonderer Berücksichtigung der Situation Großbritanniens beauftragt ${ }^{1}$ und entwickelte einen Vorschlag für einen Korrekturmechanismus ${ }^{2}$, dem der Europäische Rat schließlich auf seinem Gipfeltreffen am 11. März 1975 in Dublin - mit einigen Modifikationen - zustimmte ${ }^{3}$ und der seither als der "Dubliner" Korrekturmechanismus bekannt ist. Er ist am 1. Januar 1976 zunächst für eine Probezeit von sieben Jahren in Kraft getreten. Der Mechanismus war zwar grundsätzlich auf sämtliche Gemeinschaftsländer anwendbar. Die konkrete Situation, die Anlaß zu seiner Einführung war, sowie die Ausgestaltung der einzelnen Voraussetzungen zeigen jedoch, daß es sich um die Lösung eines spezifisch britischen Problems handelte. ${ }^{4}$

Der Korrekturmechanismus sollte in Kraft treten, wenn ein Mitgliedsstaat sich in einer besonderen Wirtschaftslage befand und eine unangemessene Belastung bei der Finanzierung des Gemeinschaftshaushalts zu tragen hatte. Eine solche Wirtschaftssituation erforderte nach den Vorschriften der Verordnung, daß das Pro-Kopf-BSP des Mitgliedsstaates weniger als 85 \% des gemeinschaftsdurchschnittlichen Pro-kopf-BSP betrug und die reale Wachstumsrate seines BSP pro Kopf geringer als $120 \%$ der Durchschnittsrate innerhalb der Gemeinschaft war. Eine unangemessene Finanzierungsbelastung war dann gegeben, wenn der Haushaltsfinanzierungsbetrag des Mitgliedsstaates den Be-

${ }^{1}$ Vgl. EG-Kommission: Bestandsaufnahme der Wirtschafts- und Finanzlage in der Gemeinschaft seit der Erweiterung und Uberblick über die künftige Entwicklung, KOM (74) 1800 endg., Brüssel, 25.10.1974, s. $17 \mathrm{ff}$.

2 Kommission der EG, Unannehmbare Situation und Korrekturmechanismus, KOM (75) 40, Brüssel, 30.01.1975.

${ }^{3}$ Niedergelegt in der Verordnung (EWG) Nr. 1172/76 des Rates vom 17. Mai 1976 zur Schaffung eines Finanzmechanismus, in: Amtsblatt der EG Nr. L 131 vom 20.05.1976, S. 7 ff.

${ }^{4} \mathrm{Vgl}$. Kommission der EG, Mandat vom 30. Mai 1980. Bericht über die Anwendung des Finanzmechanismus, KOM (81) 704 endg., Brüssel, 13.11 .1981 , S. 1 
trag, den er nach Maßgabe seines Anteils am BSP der Gemeinschaft zu zahlen hätte, um mehr als 10 \& überstieg. In diesen Fällen bildete der gesamte Mehrbetrag die Grundlage für die Rückerstattung. Voraussetzung hierfür war allerdings ein negativer Leistungsbilanzsaldo; anderenfalls bezog sich die Berechnung des Mehrbetrages nur auf die abzuführende Mehrwertsteuer (bzw. in der Ubergangszeit ersatzweise auf die Finanzbeiträge).

Die finanzielle Tragweite des Korrekturmechanismus wurde durch verschiedene Rückzahlungsbegrenzungen eingeschränkt. Zum einen dadurch, daß der Mehrbetrag nicht voll, sondern nach einem Tranchensystem mit gestaffelten Zuwendungssätzen nur teilweise erstattet werden konnte. Zum anderen durften die so errechneten Zuwendungen bestimmte Höchstgrenzen nicht überschreiten: den niedrigeren Betrag aus den Nettoüberweisungen oder den Mehrwertsteuerzahlungen (bzw. Finanzbeiträgen) bzw. den höheren Betrag aus 250 Mio. ERE oder 3 \& der Gesamtausgaben. Abgesehen von den Nettoüberweisungen, auf deren Bedeutung unten noch einzugehen sein wird, kam angesichts des schnell ansteigenden EG-Haushaltsvolumens und des wachsenden Finanzierungsanteils der Mehrwertsteuereinnahmen nur der 3 \&-Grenze Bedeutung zu. 1

Ungeachtet ihrer praktischen Relevanz kamen in den Rückzahlungsbegrenzungen aber auch grundsätzliche Positionen des Finanzausgleichssystems der Gemeinschaft zum Ausdruck. ${ }^{2}$ Indem nämlich der Betrag der Rückzahlung auf die Mehrwertsteuereinnahmen limitiert wurde, war sichergestellt, daß die abgeführten Zölle und Agrarabschöpfungen unter keinen Umständen an die Mitgliedsstaaten zurücktransferiert werden konnten. Diese Trennung der Eigenmittel in zwei Klassen entsprach der Sichtweise, zölle und Abschöpfungen als den

1Vgl. U. Nittka, S. $290 \mathrm{ff}$.

${ }^{2} \mathrm{Vgl}$. M. R. Emmerson und T. W. Scott, The Financial Mechanism in the Budget of the European Community: The Hard core of the British "Renegotiations" of 1974 - 1975, in: Common Market Law Review, Vol 14, 1977, S. 209 ff., hier: S. $220 \mathrm{f}$. 
unantastbaren Bestand des Eigenmittelsystems bzw. als die "natürlichen", d.h. aus den beiden bisher vollständig realisierten Bereichen gemeinschaftlicher Politik (Zollunion, Agrarmarkt) resultierenden Einnahmen anzusehen.

Eine solche Klassifizierung der Eigenmittelarten entstand ebenfalls durch die Berücksichtigung der Zahlungsbilanzsituation bei der Berechnung des überschüssigen Finanzierungsbetrages, indem diese bei einem positiven Leistungsbilanzsaldo nur auf der Grundlage der Mehrwertsteuereinnahmen (bzw. Finanzbeiträge) durchzuführen war. Die in dieser Regelung zum Ausdruck kommende Trennung der Eigenmittel entsprang allerdings weniger gemeinschaftlichen Finanzierungsprinzipien als vielmehr einem politischen Kompromiß: Denn die Kommission hatte in ihrem Vorschlag zum Korrekturmechanismus vorgesehen, ein Leistungsbilanzdefizit und die damit verbundene Devisenbelastung bei der Aufbringung der Gemeinschaftsfinanzierungsmittel - neben den am BSP orientierten Indikatoren - als zusätzliche Voraussetzung für die Erstattungsberechtigung eines Mitgliedsstaates aufzunehmen. 1 Die dann letztlich vom Europäischen Rat beschlossene Vorschrift ${ }^{2}$ stellte einen Kompromiß zwischen dem britischen standpunkt, der eine Nichtberücksichtigung des Zahlungsbilanzkriteriums forderte, und der französischen Auffassung, die den Korrekturmechanismus ausschließlich auf die Mehrwertsteuermittel beschränken wollte, $\operatorname{dar} .^{3}$

Schließlich wurde durch die Vorschriften des Korrekturmechanismus ausgeschlossen, daß durch die Ausgleichszahlungen ein Land vom Nettozahler zum Nettoempfänger werden konnte. Damit sollte vermieden werden, daß relativ wirtschaftsschwache Mitgliedsstaaten, wie z.B. Irland, die bereits aus der Umverteilung über den Gesamthaushalt begünstigt wurden, zusätzliche Mittel aus dem Korrekturmechanismus erhielten. Gleichwohl war diese Regelung nicht unproblematisch;

\footnotetext{
${ }^{1}$ Vgl. EG-Kommission, KOM (75) 40 endg., S. $4 \mathrm{ff}$.

2 Dies ist im übrigen der einzige wesentliche Fall, in dem die endgültigen Regelungen von dem Kommissionsvorschlag abweichen.

${ }^{3} \mathrm{Vgl}$. M. R. Emmerson und T. W. K. Scott, S. 221.
} 
denn die Kommission hatte stets die Auffassung vertreten, daß einem Mitgliedsstaat weder Rechte noch Verpflichtungen aus seiner Nettoposition entstehen dürften, da diese das Ergebnis der Gemeinschaftspolitiken sei. Mit der Berücksichtigung der Nettoposition als Rückzahlungsbegrenzung wurde das Finanzierungsprinzip der Leistungsfähigkeit, das allein auf die Einnahmenseite des Haushalts abstellt, verlassen und auch die Ausgabensituation einbezogen.

Der Dubliner Korrekturmechanismus gelangte während der zeit der Beitrittsübergangsbestimmungen (bis 1979) in keinem Falle - weder für das Vereinigte Königreich noch zugunsten eines anderen Mitgliedsstaates - zur Anwendung, da die Anspruchsvoraussetzungen nicht erfüllt waren. Großbritannien befand sich stets in der situation, daß sein Finanzierungsanteil am Gemeinschaftshaushalt geringer als sein Sozialproduktanteil war bzw. (1979) diesen nicht um die erforderlichen 10 \& überstieg (Tabelle 22). Ursache hierfür waren im wesentlichen die die Finanzierungsbeiträge begrenzenden Beitrittsgarantien.

Solange diese Ubergangsbestimmungen wirksam waren, hätte es demnach eines solchen Mechanismus nicht bedurft. Seine Bedeutung liegt denn auch hauptsächlich darin, daß im Falle einer wirtschaftlichen und belastungsmäßig ungünstigen Situation eines Mitgliedsstaates Ausgleichszahlungen grundsätzlich anerkannt und damit die Finanzierungsprinzipien des Eigenmittelsystems erstmals druchbrochen wurden. Zudem trug die Einführung des Korrekturmechanismus dazu bei, die Bedenken der britischen Regierung bezüglich der Beitrittsbedingungen auszuräumen. Das Referendum der britischen Bevölkerung im Juni 1975 endete jedenfalls mit einem deutlichen Votum für einen Verbleib des Landes in der Gemeinschaft. 1

${ }^{1}$ Vgl. Bulletin der EG, Nr. $5-1975$, S. 5 . 


\subsubsection{Der Brüsseler KompromiB}

Nach dem Auslaufen der Beitrittsübergangsregelungen wäre das Vereinigte Königreich 1980 zum ersten Mal im vollen Umfang zur Finanzierung des Gemeinschaftshaushalts herangezogen worden. Dies hätte nach ersten Schätzungen der Kommission ${ }^{1}$ dazu geführt, daß es zum größten - und neben der Bundesrepublik einzigen - Nettozahler der Gemeinschaft geworden wäre. Im Hinblick auf die Entlastungswirkungen des Dubliner Korrekturmechanismus ergaben Berechnungen, die von einem um über 25 \& höheren britischen Haushaltsfinanzierungsanteil im Vergleich zum BSP-Anteil ausgingen, einen entsprechenden Mehrbetrag von 520 Mio. ERE. Durch die teilwelse Erstattung dieses Betrages nach dem Tranchensystem resultierte hieraus allerdings nur eine effektive Zuwendung von 250 Mio. ERE. Von den übrigen im Korrekturmechanismus vorgesehenen Begrenzungen hätte diejenige auf $3 \&$ des Haushaltsvolumens den Zuwendungsbetrag auf 400 Mio. ERE limitiert, während die Begrenzung der Rückzahlungen auf die Höhe der abgeführten Mehrwertsteuereinnahmen angesichts der ständig gestiegenen quantitativen. Bedeutung dieser Einnahmeart praktisch keine Rolle mehr spielte. ${ }^{2}$ Die stärkste Begrenzung erfuhr der Mechanismus durch die an die Zahlungsbilanzsituation anknüpfende Voraussetzung: Während bei den genannten Entlastungsbeträgen von einem Leistungsbilanzdefizit ausgegangen wurde, wären diese im Falle eines Leistungsbilanzüberschusses gleich Null gewesen.

Angesichts dieser GröBenverhältnisse war offensichtlich, daß über den Korrekturmechanismus keine wirksame Entlastung des Vereinigten Könlgreichs erfolgen konnte. Ebenso wurde deutlich, daß die Gründe hierfur nicht nur in den restriktiven Voraussetzungen des Mechanis-

\footnotetext{
${ }^{1} \mathrm{Zu}$ den folgenden Angaben vgl. EG-Kommission, KOM (79) 462 endg., S. $18 \mathrm{ff}$.

${ }^{2}$ So betrug das auf GroBbritannien entfallende Mehrwertsteueraufkommen im Haushaltsplan 1980 bereits 1,2 Mrd. ERE. Vgl. Endgültige Feststellung des Gesamthaushaltsplans der Europäischen Gemeinschaften für das Haushaltsjahr 1980, in: Amtsblatt der EG, Nr. I $242,15.09 .1980$, S. 45.
} 
mus und damit auf der Finanzierungsseite lagen. Denn der Nettobeitrag des Vereinigten Königreichs war nicht nur darauf zurückzuführen, daß sein Haushaltsfinanzierungsanteil über den BSP-Anteil hinausging, sondern $z u$ einem ebenso großen Tell auch darauf, daß sein Ausgabenanteil unter dem Gemeinschaftsdurchschnitt lag, wofür vor allem die Agrarlastigkeit des Haushalts verantwortlich war. ${ }^{1}$

Die britischen Forderungen nach einer Korrektur ihrer finanziellen situation richteten slch auf eine langfristige Lösung, die ein umfassendes Gleichgewicht zwischen ihren $\mathrm{Z}$ ahlungen an den Gemeinschaftshaushalt und den Rückflüssen, zumindest aber eine "gerechte Bürde" gewährleisten sollte. ${ }^{2}$ Dazu wurde vorgeschlagen, die ProKopf-Ausgaben zugunsten des Vereinigten Königreichs auf den Gemeinschaftsdurchschnitt anzuheben oder alternativ den globalen Nettobeitrag nach oben $z u$ begrenzen. Dieser vom Äquivalenzgedanken geprägten Position des "broad balance" bzw. "juste retour" begegnete die Kommission mit dem bekannten Einwand, daß sich kein Mitgliedsland den finanziellen Auswirkungen eines einmal gewählten Finanzierungssystems und der gemeinsam beschlossenen Politik entziehen dürfe, ${ }^{3}$ und empfahl stattdessen eine Änderung des Dubliner Korrekturmechanismus durch die Abschaffung der die Rückzahlungsbeträge limitierenden Obergrenzen. ${ }^{4}$ Zur Ergänzung des neuen Finanzierungsmechanismus entwickelte die Kommission erstmals Vorschläge, die eine Verbesserung der britischen Situation über die Ausgabenseite herbeiführen sollte. Dabei war an zeitlich befristete Sondermaßnahmen gedacht, die sich aus einer Intensivierung der Gemeinschaftspolitiken ergeben und zur konvergenten Entwicklung der Gemeinschaftsstaaten beitragen.

${ }^{1}$ Vgl. EG-Kommission, KOM (79) 462 endg., S. 18.

${ }^{2}$ Vgl. D. Strasser, s. 130.

${ }^{3} \mathrm{Vgl}$. Kommission der EG, Konvergenz- und Haushaltsfragen, KOM (79) 620 endg. , Brüssel, 31.10.1979, S. $8 \mathrm{f}$.

${ }^{4} \mathrm{Vgl}$. dies., Konvergenz- und Haushaltsfragen (Mitteilung der Kommission an den Europäischen Rat - Dublin, 29. und 30. November 1979), KOM (79) 680 endg., Brüssel, 21.11.1979, S. 4 ff. 
Nach langwierigen und schwierigen Verhandlungen - drei Tagungen des Europäischen Rates verliefen ergebnislos, und man stand zunehmend unter dem Druck neu zu fixierender Agrarpreise und eines noch nicht verabschiedeten Haushalts ${ }^{1}$ - kam es schließlich auf der Ministerratstagung am 30. Mai 1980 in Brüssel zu einer Einigung über die britischen Entlastungsbeiträge im sog. Brüsseler Kompromiß. ${ }^{2}$ Diese Regelungen sahen Ausgleichszahlungen für die Jahre 1980 und 1981 von insgesamt 2585 Mio. ERE vor. Ihre Höhe wurde auf der Basis der seinerzeit vorliegenden Schätzungen der Kommission über die britischen Nettobeiträge festgelegt, die für die Jahre 1980 und 1981 auf 1784 Mio. ERE bzw. 2140 Mio. ERE beziffert wurden. Davon sollten fast genau zwei Drittel (1175 Mio. ERE und $1410 \mathrm{Mio}$. ERE) zurücktransferiert werden, so daß die effektiven Nettobeiträge mit 609 Mio. ERE für 1980 und 730 Mio. ERE für 1981 festgelegt wurden. Weiterhin waren zusätzliche Entlastungsbeträge für den Fall vorgesehen, daß die tatsächlichen Nettozahlungen des Vereinigten Königreichs die vorausgeschätzten übersteigen. Die Ausgleichszahlungen sollten zunächst im Rahmen eines modifizierten Korrekturmechanismus geleistet werden; der Restbetrag war durch zusätzliche Maßnahmen abzudecken.

Der sog. angepaßte Finanzmechanismus ${ }^{3}$ sah folgende inhaltliche Anderungen vor: die Abschaffung der Rückzahlungsbeschränkungen bei einem positiven Leistungsbilanzsaldo, den Ersatz des Tranchensystems durch die Erstattung des gesamten Mehrbetrages sowie die Aufhebung der Rückzahlungsbegrenzung von maximal 250 Mio. ERE oder 3 \% der Gesamtausgaben. Damit wurden - abgesehen von der grundlegenden Voraussetzung, daß durch die Ausgleichszahlungen keine Nettoempfän-

\footnotetext{
${ }^{1}$ Vgl. hierzu M. Jenkins, Britain and the Community Budget: The End of a Chapter, in: Common Market Law Review, Vol. 17, 1980, S. $493 \mathrm{ff}$.

${ }^{2}$ Schlußfolgerungen des Rates von der Tagung vom 30. Mai 1980 betreffend den britischen Beitrag zur Finanzierung des Haushaltsplans der Gemeinschaft, in: Amtsblatt der EG, Nr. C 158 vom 27.06 .1980 , S. 1 .

${ }^{3}$ Verordnung (EWG) Nr. 2743/80 des Rates vom 27. Oktober 1980 zur Anderung der Verordnung (EWG) Nr. 1172/76 zur Schaffunq eines Finanzmechanismus, in: Amtsblatt der EG, Nr. L 284 vom 29.10.1980, S. $1 \mathrm{ff}$.
} 
gerposition entstehen dürfe - die bisher geltenden Obergrenzen, die die Rückzahlung der überschüssigen Finanzierungsbeiträge limitieren, abgeschafft. ${ }^{1}$ Das Wirksamwerden der Entlastungsmaßnahmen hing nun nur noch von der Höhe und der wachstumsrate des britischen Pro-Kopf-BIP ${ }^{2}$ im Vergleich zum Gemeinschaftsdurchschnitt sowie vom Verhältnis des britischen Finanzierungs- zu seinem BIPAnteil ab. Bezüglich dieser Indikatoren vertrat die Kommission die Auffassung, daB sie unter den seinerzeitigen Gegebenheiten Ausgleichszahlungen gewährleisten würden. ${ }^{3}$ Trotz der britischen Anregungen, diese Voraussetzungen zu lockern, wurde eine solche weitergehende Regelung nicht getroffen.

Der angepaßte Finanzmechanismus galt allein für das Vereinigte Königreich und war zunächst auf die Jahre 1980 und 1981 begrenzt. Eine Verlängerungsmöglichkeit war $z$ war vorgesehen. Diese erübrigte sich jedoch, als im Laufe der zeit sichtbar wurde, daß auch die verbliebenen Voraussetzungen von dem Vereinigten Königreich nicht erfültt wurden und der Mechanismus damit nicht wirksam werden konnte. Wie Tabelle 2. 2 zeigt, befand sich das Vereinigte Königreich zwar in einer besonderen wirtschaftlichen Lage, doch war sein Finanzierungsbeitrag noch nicht als unangemessen einzustufen, da der Haushaltsfinanzierungs- den BIP-Anteil nicht um die hierfür erforderlichen $10 \&$ überstieg. Diese Entwicklung lag hauptsächlich in der zwischen 1979 bis 1981 erfolgten Höherbewertung des Pfund Sterling gegenüber der ERE begründet, die 1980 und 1981 den BIP-Anteil stärker als den Haushaltsfinanzierungsanteil erhöhte und 1982 dafür sorgte, daB der Anstieg des (als Durchschnitt der vergangenen Jahre zu ermittelnden) Pro-Kopf-BIP den Qualifikationsstandard von 85 \& überschreiten würde. 4

${ }^{1}$ Die Begrenzung der Rückzahlungen auf die Höhe der abgeführten Mehrwertsteuereinnahmen besa $\beta$ ohnehin keine praktische Relevanz mehr.

2 Anstelle der Bruttosozialprodukte wurden nun die Bruttoinlandsprodukte als BezugsgröBen gewählt.

${ }^{3}$ Vgl. EG-Kommission, KOM (79) 620 endg., S. 5.

${ }^{4} \mathrm{Vgl}$. EG-Kommission, KOM (81) 704 endg., s. $10 \mathrm{ff}$. 
Tabelle 22

Erfüllung bzw. Nichterfüllung der Voraussetzungen des Korrekturund Finanzmechanismus durch das Vereiniqte Königreich

\begin{tabular}{|c|c|c|c|c|c|c|}
\hline \multirow[t]{2}{*}{ Jahr } & \multirow[t]{2}{*}{$\begin{array}{l}\text { Nettobei- } \\
\text { tragszahler }\end{array}$} & \multirow{2}{*}{$\begin{array}{l}\text { Pro-Kopf-BSP/BIP } \\
\text { in \& des Gemein- } \\
\text { schaftsdurch- } \\
\text { schnitts }\end{array}$} & \multirow{2}{*}{$\begin{array}{l}\text { Zuwachsrate d. Pro- } \\
\text { Kopf-BSD/BIP in } 8 \\
\text { d. Gemeinschafts- } \\
\text { durchschnitts }\end{array}$} & \multicolumn{3}{|c|}{$\begin{array}{c}\text { Haushaltsanteil im Verọleich } \\
\text { zum BSP/BIP-Anteil }\end{array}$} \\
\hline & & & & $\begin{array}{c}\text { Haushalts- } \\
\text { anteil in } \\
8\end{array}$ & $\begin{array}{c}\text { BSP/BIP- } \\
\text { Anteil in } \\
8\end{array}$ & $\begin{array}{l}\text { Unter- } \\
\text { schied } \\
\text { in } 8\end{array}$ \\
\hline 1976 & $\mathrm{Ja}$ & 77 & 100 & 10,7 & 15,7 & $-31,6^{+}$ \\
\hline 1977 & $\mathrm{Nein}^{+}$ & 75 & 27 & 12,2 & 15,4 & $-20,9^{+}$ \\
\hline 1978 & $\mathrm{Ja}$ & 74 & 74 & 15,4 & 15,7 & $-2,5^{+}$ \\
\hline 1979 & $\mathrm{Ja}$ & 72 & 88 & 17,5 & 16,7 & $+5,0^{+}$ \\
\hline 1980 & $\mathrm{Ja}$ & 74 & 60 & 20,5 & 18,9 & $-8,5^{+}$ \\
\hline 1981 & $\mathrm{Ja}$ & 82 & 34 & 21,2 & 20,2 & $+5,2^{+}$ \\
\hline 1982 & $\mathrm{Ja}$ & $89^{+}$ & neqativ & 22,3 & 19,3 & $+15,5$ \\
\hline
\end{tabular}

+Voraussetzung nicht erfüllt.

Quelle: EG-Kommission, KOM (81) 704 endg., Anlage B. 
Damit gelangte auch dieser Mechanismus nicht zur Anwendung. Offenbar war es selbst für relativ kurze Zeiträume nicht möglich, verläßliche Prognosen über die künftige Wirtschafts- und Finanzentwicklung abzugeben und die Voraussetzungen für ein Inkrafttreten des Mechanismus hiermit abzustimmen. Mit dem Scheitern der auf die Korrektur des Finanzierungssystems abstellenden Mechanismen erschienen auch die Finanzierungsprinzipien der Gemeinschaft in einem veränderten Licht. Während bis dahin die Konzeption der finanziellen Solidarität der Mitgliedsstaaten noch weitgehend aufrechterhalten werden konnte, ist diese Finanzierungsmaxime mit den nun allein über die Ausgabenseite erfolgenden Ausgleichszahlungen durch den Äquivalenzgedanken in den Hintergrund gedrängt worden. 1

Die im Rahmen des Brüsseler Kompromisses vereinbarten Haushaltsentlastungen zugunsten des Vereinigten Königreichs waren nunmehr in voller Höhe über die sog. zusätzlichen Maßnahmen ${ }^{2}$ abzuwickeln. Im Gegensatz zu den ohne Verwendungsauflagen zu erstattenden Beträgen des Finanzmechanismus handelt es sich bei diesen Maßnahmen um finanzielle Beteiligungen der Gemeinschaft an mehrjährigen britischen Sonderprogrammen, die der Kommission zur Genehmigung vorzulegen waren. Diese Programme betreffen den Ausbau der wirtschaftlichen und sozialen Infrastruktur sowie Investitionen im Energiebereich und sollten damit zu einer ausgewogenen wirtschaftsentwicklung der Gemeinschaftsstaaten beitragen. Die hierfür vorgesehenen Mittel wurden in die Haushalte 1980 bis 1982 eingestellt und auch in voller Höhe verausgabt. ${ }^{3}$

Im Laufe der Haushaltsentwicklung dieser Jahre stellte sich allerdings heraus, daß die Nettobeiträge des Vereinigten Königreichs erheblich überschätzt worden waren (Tabelle 23 ). Für das Jahr

1Vgl. N. Andel, S. 335; R. Peffekoven (1982), S. 416.

2 Verordnung (EWG) Nr. 2744/80 des Rates vom 27. Oktober 1980 betreffend zusätzliche Maßnahmen zugunsten des Vereinigten Königreichs, in: Amtsblatt der EG, Nr. L 284 vom 29.10.1980, S. 4 ff. ${ }^{3} \mathrm{Vgl}$. EG-Kommission, Vierter Bericht der Kommission an Rat und Parlament zur Anwendung der Verordnung (EWG) des Rates Nr. 2744/ 80 vom 27. Oktober 1980 betreffend zusätzliche MaBnahmen zugunsten des Vereinigten Königreichs, KOM (83) 100 endg., Brüssel, 1.3 .1983 , S. 5 . 
1981 verwandelte sich dadurch die ursprüngliche britische Nettozahlerposition sogar in einen - wenn auch geringen - Nettoempfängersaldo. Da in den Entlastungs- und Finanzierungsregelungen zwar Vorsorge für den Fall einer Unterschätzung der Nettobeiträge getroffen worden war, nicht jedoch Bestimmungen über Kürzungen der Zuwendungen im umgekehrten Fall, bestand Unklarheit über die tatsächliche Höhe der britischen Ausgleichsbeträge: Sollten die in der Verordnung festgelegten Rückerstattungsbeträge oder die hier gleichfalls fixierten Nettobeiträge maßgeblich sein? Nach den Vorstellungen der Kommission und des Rates sollen die gegenüber den ursprünglich fixierten Nettobeiträgen bestehenden Abweichungen bei Entlastungsmaßnahmen für die Jahre 1983 bis 1985 berücksichtigt werden, das Parlament forderte dagegen einen sofortigen Ausgleich. 1

Tabelle 23

Vorausgeschätzter und tatsächlicher Ausgleich zugunsten des Vereinigten Königreichs 1980 und 1981 (in MiO. ECU)

\begin{tabular}{|c|c|c|c|c|c|c|}
\hline \multirow[t]{2}{*}{ Jahr } & \multirow{2}{*}{\multicolumn{2}{|c|}{$\begin{array}{c}\text { Nettobeiträge vor } \\
\text { Ausgleich }\end{array}$}} & \multicolumn{2}{|c|}{ Empfangener Aus- } & \multirow{2}{*}{\multicolumn{2}{|c|}{$\begin{array}{l}\text { Nettobeiträge nach } \\
\text { Ausgleich } \\
\text { geschätzt|tatsächlich }\end{array}$}} \\
\hline & & & netto & brutto & & \\
\hline 1980 & -1784 & -1507 & 1175 & 1438 & -609 & -332 \\
\hline 1981 & -2140 & -1376 & 1410 & 1804 & -730 & 34 \\
\hline
\end{tabular}

Quelle: EG-Parlament, Sitzungsdokumente 1982 - 1983, Dok. 1-1004/82, S. 23 .

1EG-Parlament, Bericht im Namen des Haushaltsausschusses über den Vorschlag der Europäischen Gemeinschaften an den Rat (Dok. 1-936) 82 - KOM (82) 728 endg.) für eine Verordnung zur Änderung der Verordnung (EWG) Nr. 2744/80 betreffend zusätzliche Maßnahmen zugunsten des Vereinigten Königreichs, Berichterstatterin: Frau Carla Barbarella, Europäisches Parlament, Sitzungsdokumente 1982 - 1983 , Dok. 1-1004/82, 13.12.1982, S. 27. 
Die Finanzierung der britischen Entlastung erfolgte über die Einnahmen des Gemeinschaftshaushalts, so daß sämtliche Mitgliedsstaaten nach Maßgabe ihres zusätzlichen Haushaltsfinanzierungsanteils hierzu herangezogen wurden. Damit trug die Bundesrepublik nicht nur den größten Teil der Ausgleichsmaßnahmen, sondern wurde wieder zum mit Abstand größten und 1981 sogar alleinigen Nettozahler der Gemeinschaft in Höhe von über $2 \mathrm{Mrd}$. ECU jährlich. Eine solche Position hielt die Bundesregierung nicht mit dem Gemeinschaftsgedanken vereinbar, insbesondere auch deshalb nicht, weil gleichzeitig wirtschaftlich gleichgestelite Staaten erhebliche Nettobezüge aus der Gemeinschaft erhielten, und forderte eine korrektur der Ungleichgewichte. ${ }^{1}$ spätestens damit trat neben das britische auch ein deutsches Haushaltsproblem und wurde eine globale Lösung der Lastenverteilung immer dringlicher.

\subsubsection{Weitere Übergangsregelungen}

Mit dem Brüsseler Beschluß hatte die Gemeinschaft erklärt, die Probleme ab 1982 durch strukturelle Änderungen lösen zu wollen. Dazu wurde die Kommission in einem Mandat ${ }^{2}$ formell beauftragt, bis Juni 1981 Vorschläge zu einer Reform der Gemeinschaftspolitiken zu erarbeiten, die künftig das Eintreten einer unannehmbaren Situation für einen Mitgliedsstaat verhindern sollten. In ihrem Bericht in Ausführung des Mandats ${ }^{3}$ wandte sich die Kommission erneut gegen Versuche, das Finanzierungsproblem auf der Basis von Gegenüberstellungen von Zahlungen und Rückflüssen lösen $\mathrm{zu}$ wollen. Stattdessen entwikkelte sie Vorstellungen, wie die Ungleichgewichte durch eine Reform der Agrarpolitik und die Intensivierung der Struktur-, Energie- und wirtschaftspolitik verhindert werden können. Allerdings würden sol-

\footnotetext{
${ }^{1}$ Vgl. etwa Presse- und Informationsamt der Bundesregierung, Bulleti.n Nr. 68 vom 10.06.1980, S. 574; Bundesministerium der Finanzen, Finanzbericht 1982, S. 144 .

${ }^{2}$ EG-Rat, SchluBfolgerungen von der Tagung vom 30. Mai 1980.

${ }^{3}$ EG-Kommission, Bericht zu dem Mandat vom 30. Mai 1980, KOM (81) 300 endg., Brüssel, 24.06.1981.
} 
che Veränderungen nicht kurzfristig zu realisieren sein, so daß zur Lösung des britischen Haushaltsproblems erneut tbergangsregelungen getroffen werden müßten. Hierzu empfahl sie einen Ausgleichsmechanismus, der sich an der Differenz zwischen dem britischen Anteil am Gemeinschaftssozialprodukt und dem britischen Rückflußanteil aus den Agrarausgaben orientiert, ohne allerdings zu konkretisieren, wieviel von dieser Differenz ausgeglichen werden soll. Konkrete Vorschläge, die als Grundlage neuer Beschlüsse hätten dienen können, enthielt der Kommissionsbericht damit nicht, so daß er eher als ein "erster Schritt in der Reformdiskussion verstanden werden" 1 kann.

Diese Auseinandersetzungen waren nach wie vor durch die unterschiedlichen nationalen Interessen gekennzeichnet: Die Bundesrepublik akzeptierte nicht, quasi unbegrenzt zur Finanzierung der Umverteilungen herangezogen zu werden; das Vereinigte Königreich erkannte zwar die Bedeutung langfristig angelegter Reformen an, verwies aber auf die bis dahin noch bestehenden Ungleichgewichte und forderte stattdessen an der Wirtschaftskraft der Mitgliedsstaaten orientierte dauerhafte Ausgleichsregelungen. Diese Position wurde jedoch von den übrigen Mitgliedsstaaten nicht akzeptiert. ${ }^{2}$ Damit wir man von einer endgültigen Lösung des Finanzierungsproblems weit entfernt, so daß man auf die vorsorglich in die Brüsseler Beschlüsse eingebaute Option zurückgreifen mußte, nach der ohne Vorhandensein einer neuen Konzeption auch 1982 nach dem Lösungsmuster der Jahre 1980/81 verfahren werden sollte.

Auf der Basis der Schätzungen der Kommission über die Nettobeiträge des Vereinigten Königreichs in Höhe von 1530 Mio. ECU wurde der Ausgleich vom Rat auf 850 Mio. ECU netto (bzw. 1092 Mio. ECU brutto) festgesetzt. Gleichzeitig wurde ein Verfahren zur Anpassung der

${ }^{1}$ G. Stahl, Die Lösung läßt noch auf sich warten, in: Wirtschaftsdienst, 61. Jg., 1981, S. $326 \mathrm{ff.,} \mathrm{hier:} \mathrm{S.} 329$.

${ }^{2} \mathrm{Vgl}$. hierzu M. Vanden Abeele, The Mandate of 30 May 1980, Budget Financing and the Revitalization of the Community: An Unfinished Journey, in: Common Market Law Review, Vol. 19, 1982, S. 501 ff., hier: S. $518 \mathrm{ff}$. 
Ausgleichszahlungen festgelegt, das bei Abweichungen der tatsächlich sich ergebenden Nettobeiträge von den geschätzten werten also sowohl bei Unter- als auch bei Uberschätzungen - angewendet werden sollte. ' Diese Beschlüsse wurden zusammen mit einem Sonderprogramm auf dem Gebiet der Energie in Höhe von 210 Mio. ECU zugunsten der Bundesrepublik - das eine gewisse Kompensation ihres hohen Anteils an der Finanzierung der Entlastungsmaßnahmen darstellen sollte - in den Entwurf des ersten Berichtigungs- und Nachtragshaushalts 1982 gebracht.

Dieser Haushalt ist allerdings im Laufe des Haushaltsverfahrens vom Parlament abgelehnt worden und konnte damit nicht inkrafttreten. Als Begründung wurde vor allem angeführt, ${ }^{2}$ daß

- die Ad-hoc-Regelungen nicht auf Dauer akzeptiert werden könnten und noch kein Vorschlag einer umfassenden längerfristigen Lösung vorliege; zudem würden durch die Anwendung des Kriteriums "Nettobeitrag" die Grundsätze der Finanzautonomie der Gemeinschaft und das System der eigenen Einnahmen in Frage gestellt;

- die Sondermaßnahmen nur unzureichend in die bestehende Gemeinschaftspolitik integriert seien und sie teilweise lediglich

Zahlungen zur Entlastung des britischen Staatshaushalts darstellten;

- die bisherige Einstufung der Ausgleichszahlungen als obligatorische Ausgaben ${ }^{3}$ nicht den haushaltsrechtlichen Grundsätzen entspreche und sie stattdessen den nichtobligatorischen Ausgaben zuzuordnen seien.

Diesen Einwänden wurde zum einen mit einer veränderten haushaltsmäBigen Einstufung der Ausgaben und zum anderen mit der Verpflichtung der Kommission, baldmöglichst Vorschläge zur Haushalts- und Fi-

\footnotetext{
${ }^{1}$ Vgl. EG-Kommission der Bulletin der EG, 1982, Nr. 5, S. 74 f. und Nr. 10, S. 62 .

2 EG-Parlament, Sitzungsdokumente 1982 - 1983, Dok. 1-1004/82, S. $19 \mathrm{ff}$.

${ }^{3}$ Es sei daran erinnert, daß im Haushaltsverfahren der Rat für die obligatorischen, das Parlament für die nichtobligatorischen Ausgaben die Entscheidungskompetenz besitzt.
} 
nanzreform vorzulegen, teilweise Rechnung getragen, ${ }^{1}$ so daB die Ausgleichsmaßnahmen im ersten Berichtigungs- und Nachtragshaushalt 1983 im März des Jahres endgültiq festgestellt werden konnten. Darin sind 692 Mio. ECU für zusätzliche - obligatorische - Ausgaben zugunsten des Vereinigten Königreichs auf dem Gebiet der gemeinschaftlichen Regionalpolitik und - nichtobligatorische - Ausgaben in Höhe von 610 Mio. ECU für Maßnahmen im Energiebereich, davon wie vorgesehen 210 Mio. ECU für die Bundesrepublik, veranschlagt. ${ }^{2}$ Zur Finanzierung dieser Maßnahmen kann auf Haushaltsüberschüsse vergangener Jahre zurückgegriffen werden.

Die angekündigten Vorschläge der Kommission zur künftigen Finanzierung der Gemeinschaften hatten eine neue Dimension zu berücksichtigen. Es ging inzwischen nicht mehr allein um die Lösung des Problems der ungleichmäßigen Belastung der Mitgliedsstaaten, sondern auch darum, daB der Finanzierungsspielraum zunehmend geringer wurde. Konnten die Ausgaben bis dahin noch aus einer Erhöhung des Ausschöpfungssatzes der Mehrwertsteuereigenmittel (1981: 0,787 \&) finanziert werden, so war die im Eigenmittelbeschluß festgelegte 18 -Grenze mit dem zweiten Nachtragshaushaltsplan 1983, der hauptsächlich wegen gestiegener Agrargarantieausgaben erforderlich geworden war, praktisch erreicht. Angesichts der Abhängigkeit der Agrarausgaben von Ernteergebnissen und Weltmarktpreisschwankungen bestanden bereits ernsthafte Zweifel, ob die bisherige Politik auch $1984 \mathrm{zu}$ finanzieren sein würde, ${ }^{3}$ ganz zu schweigen von einer stets geforderten Intensivierung der Strukturpolitiken. Hinzu kommt die angestrebte Erweiterung der Gemeirschaft um Spanien und Portugal,

${ }^{1}$ Vgl. R. Arndt, Leitlinien einer Finanzierung der Gemeinschaft, in: wirtschaftsdienst, 63. Jg., 1983, S. 378 ff., hier: S. 379.

2 Diese Ausgleichsbeträge wurden im zweiten Nachtragshaushalt 1983 um insgesamt 463 Mio. ECU aufgestockt, da der aktualisierte Nettobeitrag GroBbritanniens (2.036 Mio. ECU) höher als die ursprünglich festgesetzte Richtzahl ausfiel.

${ }^{3}$ Vgl. G. Stahl, Budgetary Policy of the European Communities: Squarring the Circle, in: Intereconomics, Vol. 18, 1983, S. 213 ff., hier: S. 215 . 
deren Nettokosten von der Kommission auf 5 - 7 \& des Haushaltsvolumens geschätzt werden. 1

Der Vorschlag der Kommission ${ }^{2}$ bezüglich einer Erweiterung des Finanzierungsrahmens richtete sich auf eine Anhebung des Mehrwertsteuer-Eigenmittelsatzes auf zunächst 1,4 8 , mit der Möglichkeit, weitere Tranchen von jeweils 0,4 \& ohne erneute Anderung des Eigenmittelbeschlusses allein von Rat und Parlament beschlieBen zu können. In diesem Rahmen zielen die Vorschläge zur Lösung der Verteilungsprobleme auf eine vorübergehende Diversifizierung der Mehrwertsteuererhebung ab: Solange die Agrargarantieausgaben ein Drittel des Haushaltsvolumens übersteigen, soll die Differenz zwischen einem Drittel und den gesamten Agrarausgaben über variable Mehrwertsteuersätze der Mitgliedsstaaten finanziert werden. Diese Sätze sollen sich an dem nationalen Anteil an der Agrarproduktion, dem BIP pro Kopf und dem sog. Nettobetriebsüberschuß der Volkswirtschaft orientieren. Sie berücksichtigen damit zum einen die verschieden großen Vorteile aus der Agrarpolitik und zum anderen die unterschiedliche Leistungsfähigkeit der Mitgliedsstaaten sowie die Wirtschaftlichkeit ihrer Volkswirtschaften. Eine dauerhafte Lösung sieht die Kommission nach wie vor in einem Ausbau der Gemeinschaftspolitik in den nicht landwirtschaftlichen Bereichen zusammen mit einer Eindämmung der Agrarproduktion.

Mit diesem Fragenkomplex stand für das Stuttgarter Gipfeltreffen im Juni 1983 ein ganzes Paket zur Verhandlung an. Endgültige Entscheidungen konnten nicht getroffen werden; stattdessen wurden die vorliegenden Probleme als Leitlinien für künftige Verhandlungen vorgegeben, über deren Ergebnisse auf dem nächsten Gipfeltreffen im De-

${ }^{1}$ Vgl. C. Tugendhat, Die Kontroverse um die EG-Finanzierung, Der Finanzierungsvorschlag der Kommission, in: Wirtschaftsdienst, 63. Jg., 1983, S. $371 \mathrm{ff}$.

2 Kommission der EG, Die künftige Finanzierung der Gemeinschaft Vorschlag für einen Beschluß über die eigenen Mittel - (Mitteilung der Kommission an den Rat), KOM (83) 270 endg., Brüssel, 6.5.1983. 
zember in Athen - gemeinsam, d.h. gleichzeitig - entschieden werden sollte. ${ }^{1}$ Dies schließt auch die vorläufige Einigung über britische Ausgleichszahlungen für 1983 in Höhe von 750 Mio. ECU netto ein - gemeinsam mit Entlastungen für die Bundesrepublik von etwa 150 Mio. ECU. Die britische Ausgleichsleistung liegt erheblich unter den Entlastungen der vergangenen Jahre und ist auch weit entfernt von den ursprünglich geforderten 1,3 Mrd. ECU. "Damit mag der Rat in gewisser Weise den "Irrtum zugunsten Großbritanniens" korrigiert haben, der ihm bei der Regelung für die Jahre 1980 und 1981 unterlaufen war." 2

Die Ausgleichszahlungen wurden bereits in den Haushaltsentwurf für 1984 eingestellt. Das Europäische Parlament hatte daraufhin allerdings angekündigt, daß es ohne eine klare Konzeption für die künftige Finanzierung keiner weiteren Ad-hoc-Ubergangsregelung mehr zustimmen wollte. Diesen Forderungen verlieh das Parlament dadurch Nachdruck, daß es im Rahmen der Haushaltsberatungen die für die Ausgleichsmaßnahmen vorgesehenen Mittel - zusammen mit 5 \& der obligatorischen Agrarausgaben - durch Einstellung in ein Reservekapitel vorläufig gesperrt hat, und mit der Globalablehnung des Haushalts für den Fall drohte, daß der Athener Gipfel scheitern sollte.

Die Zeit bis zu diesem Gipfeltreffen war gekennzeichnet durch rege Aktivitäten der Kommission und der nationalen Regierungen in Ausführung der vorliegenden Prüfungs- und Verhandlungsaufträge. In diesem Rahmen legte die Kommission Vorschläge zu einer effizienteren und gleichzeitig intensiveren strukturpolitik ${ }^{3}$ und zu einer sparsa-

\footnotetext{
${ }^{1}$ Vgl. Erklärung des Europäischen Rates, in: Bulletin der EG, 1983, Nr. 6, S. $20 \mathrm{ff}$.

${ }^{2} v$. Götz, Das Finanzierungssystem der Europäischen Gemeinschaften in der Krise, in: K.-H. Böckshegel u.a. (Hrsq.), Finanzverfassung der Europäischen Gemeinschaften, Beilecung internationaler Rechtsstreitigkeiten: Heidelberger Kolloquium aus Anlaß des 70. Geburtstags von Günter Jaenicke, Studien zum internationalen Wirtschaftsrecht und Atomenergierecht, Bd. 70, Berlin, Bonn u.a. 1984, S. 3 ff., hier: S. 14 .

${ }^{3}$ EG-Kommission, Stärkuna der Effizienz der Strukturfonds der Gemeinschaft, Bulletin der EG, Beilage $3 / 83$.
} 
meren Agrarpolitik ${ }^{1}$ vor, die in ihrem Zusammenwirken auch zur Lösung der bestehenden Verteilungsprobleme beitragen würden. Auf der Verhandlungsebene der nationalen Regierungen um die Neuregelung der Finanzierungsbeteiligungen knüpften die britischen Vorschläge an ihre bisherige Forderung eines langfristigen Automatismus, der ihnen einen Ausgleich von zwei Drittel ihres Nettosaldos gewährleisten sollte, an. Die deutschen Vorstellungen liefen nach wie vor darauf hinaus, daß insbesondere wohlhabende Länder, die zudem von der Agrarpolitik im besonderen Maße profitieren, verstärkt zur Finanzlerung herangezogen werden sollten. Die meisten übrigen Mitgliedsländer befürworteten eine Korrektur der Ungleichgewichte über die Ausgabenseite im Rahmen einer Erweiterung des Finanzierungsspielraums durch eine Anhebung des Mehrwertsteuerhöchstsatzes. Damit wurden die bisher vertretenen Positionen prinzipiell unverändert aufrechterhalten und bestanden die "Gemeinsamkeiten" darin, daB sich die Vorschläge zuvorderst an einer Verringerung der eigenen Belastungen bzw. an dem Erhalt der einmal "erworbenen" Besitzstände orientierten.

Auf den zahlreichen Sonderratstagungen der Fachminister konnten weder diese Gegensätze ausgeräumt werden, noch erwiesen sich die übrigen Fragen des auf dem Stuttgarter Gipfeltreffen "geschnürten" Paketes mit ihren wechselseitigen Abhängigkeiten als lösbar. So konnte es nicht verwundern, daß das Gipfeltreffen der Regierungschefs in Athen ohne Entscheidung $z u$ Ende ging. Das Parlament reagierte darauf - angesichts der angespannten Haushaltslage - zwar nicht mit der angedrohten Globalablehnung des Etats für 1984. Es sperrte allerdings die vorgesehenen Ausgleichszahlungen für das Vereinigte Königreich und die Bundesrepublik bis mindestens zum März 1984 und setzt damit eine erneute Frist zur Lösung der anstehenden Probleme.

${ }^{1}$ EG-Kommission, Anpassung der gemeinsamen Agrarpolitik, Bulletin der EG, Beilage $4 / 83$. 
Das folgende Gipfeltreffen des Europäischen Rates im März 1984 in Brüssel blieb nochmals ohne konkrete Ergebnisse. Allerdings wurde hier bereits sichtbar, daß der ursprüngliche Kommissionsvorschlag, den Haushaltsorganen weitere autonome Erhöhungen des Mehrwertsteuersatzplafonds zu gestatten, ebensowenig Aussicht auf Erfolg hatte, wie das im Februar 1984 vorgelegte Konzept ${ }^{1}$ einer Heraufsetzung des Mehrwertsteuersatzes auf $2 \%$ der Bemessungsgrundlage. ${ }^{2}$ Auch hatte man sich auf eine Korrektur des britischen Beitrags bereits im Grundsatz verständigt; eine konkrete tbereinkunft zwischen dem Vereinigten Königreich und den übrigen Mitgliedsstaaten scheiterte offenbar an wenigen $100 \mathrm{Mio}$. ECU. ${ }^{3}$

Ein gewisser Durchbruch gelang erst auf der Tagung des Europäischen Rates in Fontainebleau im Juni 1984. Die Regierungschefs der Mitgliedsländer einigten sich darauf, den Höchstsatz für die Abführung der Mehrwertsteuer-Eigenmittel mit Beginn des Jahres 1986 auf 1,4 \& anzuheben. Diese Neuregelung machte eine Anderung des Eigenmittelbeschlusses vom 21. April 1970 erforderlich ${ }^{4}$ und bedurfte der Ratifikation durch die Parlamente der Mitgliedsländer. Gleichzeitig wurde festgelegt, daß der Höchstsatz auf einstimmigen Beschluß des Rates und nach Zustimmung der nationalen Parlamente $z u$ Beginn des Jahres 1988 auf 1,6 \& heraufgesetzt werden kann.

Der zweite Teil der Neuregelungen betraf den Ausgleich zugunsten des Vereinigten Königreichs. Hier wurde für das Jahr 1984 ein Pauschalbetrag von $1 \mathrm{Mrd}$. ECU festgelegt, der über den Haushalt 1985 abzuwickeln war. Für die folgenden Jahre wurde eine Korrektur des britischen Nettobeitrags wie folgt vereinbart: Aus dem prozentualen

${ }^{1}$ Vgl. Bulletin der EG, Nr. 2, 1984, S. 31.

2Vgl. V. Götz, s. 20.

${ }^{3} \mathrm{Vgl}$. Bulletin der EG, Nr. 3, 1984, S. 8 .

${ }^{4}$ Vgl. EG-Rat, Beschluß vom 7. Mai 1985 über das system der eigenen Mittel der Gemeinschaften. 
Mehrwertsteuer-Anteil des Vereinigten Königreichs und seinem prozentualen Anteil an den aufgeteilten Gesamtausgaben des EG-Haushalts wird der Differenzbetrag ermittelt, der dem Vereinigten Königreich zu zwei Drittel erstattet wird. Der Ausgleich erfolgt durch einen Abzug bei der Abführung der Mehrwertsteuer an den Gesamthaushalt im jeweiligen Folgejahr. Der dadurch entstehende Haushaltsfehlbetrag ist von den übrigen Mitgliedsstaaten im Verhältnis ihres regulären Mehrwertsteuer-Anteils zu tragen. Diese zusätzlichen Belastungen werden - in Anlehnung an die bisherige Praxis allerdings so angepaßt, daß sich der Anteil der Bundesrepublik daran nur auf zwei Drittel ihres Mehrwertsteuer-Anteils beläuft.

Zur Bemessung des britischen Nettobeitrags und der damit verbundenen Korrekturbeträge werden also - entgegen den ursprünglichen Forderungen des Vereinigten Königreichs - die Zölle und Agrarabschöpfungen nicht mit einbezogen, da die übrigen neun Mitgliedsstaaten diese Einnahmen als "Gemeinschaftseigentum" einstuften." Damit hatte es den Anschein, als würde die Zweiteilung der Eigenmittel, die sich im Rahmen des Dubliner Korrekturmechanismus bereits abzeichnete, konsequent fortgeführt. ${ }^{2}$ Inwieweit solche Grundsatzpositionen allerdings bei der getroffenen Regelung von Bedeutung waren, ist fraglich. Entscheidend könnte vielmehr der Tatbestand gewesen sein, daß eine Berechnung der britischen Finanzierungsbeiträge nicht nur auf der Grundlage des Mehrwertsteuer-Anteils, sondern einschließlich der übrigen abgeführten Eigenmittel einen höheren britischen Nettobeitrag und damit auch höhere Ausgleichszahlungen bewirkt hätte.

\footnotetext{
${ }^{1}$ Vgl. Bulletin der EG, Nr. 3, 1984, S. 8.

2vgl. v. Götz, S. 19.
} 
Mit den Neuregelungen wurde vor allem auch das bisher praktizierte Verfahren aufgegeben, die Entlastungsmaßnahmen über die Ausgabenseite des EG-Haushalts abzuwickeln und damit die budgetären Ungleichgewichte im Rahmen der Gemeinschaftspolitiken zu beseitigen. Dies mußte naturgemäß den Widerstand des Parlaments hervorrufen, das sich stets gegen bloße Rückzahlungen, die zudem keine langfristige Perspektive gemeinschaftlicher Politik aufweisen, ausgesprochen hatte.

Das Parlament weist auch darauf hin, das mit den getroffenen Ausgleichsregelungen das Vereinigte Königreich sich von den Belastungen weiterer Ausgabenerhöhungen zu einem großen Teil abkoppelt, denn "jede neue Maßnahme für eine gemeinschaftliche Politik, die nicht zu überproportionalen Ausgaben im Vereinigten Königreich führt sowie alle Kosten, die aufgrund des Beitritts von Spanien und Portugal zu den Gemeinschaften entstehen, (belasten) die übrigen (...) Mitgliedsstaaten neben ihrem 'normalen' Mehrwertsteueranteil zusätzlich mit der durch das Vereinigte Königreich verursachten Mindereinnahme" ${ }^{1}$. Weiterhin würden die Haushaltsbehörden (Parlament und Rat) ihre Kontrolle uber die tatsächliche Höhe der Eigenmittel verlieren, denn künftig setzt die Kommission die tatsächliche Höhe des an die Gemeinschaft abzufuhrenden Mehrwertsteueranteils für jeden einzelnen Mitgliedsstaat fest, da sie aufgrund ihrer Ausgleichsberechnungen entscheidet, in welcher Höhe das Vereinigte Königreich Mindereinnahmen der Gemeinschaft verursachen darf und mit welcher tatsächlichen zusätzlichen Belastung die übrigen Mitgliedsstaaten zur Deckung der Mindereinnahmen herangezogen werden. Da die Ausgleichsregelungen zumindest bis zur geplanten zweiten Anhebung

\footnotetext{
${ }^{1}$ EG-Parlament, Berlcht im Namen des Haushaltsausschusses über den geänderten Vorschlag der Kommission der Europäischen Gemeinschaften an den Rat (KOM (84) 384 endg. - Dok. 2-368/84) für einen BeschluB uber das System der eigenen Mittel der Gemeinschaften, Berichterstatter: Herr Gero Pfennig, Sitzungsdokumente 1984-1985, Dok. 2-799/84, 22.10.1984, S. 20 .
} 
des Mehrwertsteuersatz-Plafonds Gültigkeit besitzen, diese aber nur durch einstimmigen Beschluß aller Mitgliedsstaaten herbeigeführt werden kann, besteht zudem die Gefahr, daß eine solche Erhöhung einseitig von einem Mitgliedsland blockiert werden kann. 1

Die Neuregelung wirkt sich schlieslich auch in einer relativen Reduzierung des Haushaltsvolumens aus, denn gleichzeitig fallen die bisher über die Ausgabenselte abgewickelten Ausgleichszahlungen weg und werden die Einnahmen reduziert. Der neue MehrwertsteuerAnteil der Gemeinschaft beträgt durch die Entlastung des Vereinigten Königreichs fakt1sch nur noch etwa $1,3 \%{ }^{2}$

Das Haushaltsverfahren des Jahres 1984 wurde mit der Verabschiedung des Berichtigungs- und Nachtragshaushalts 1/1984 und der Freigabe der bislang noch gesperrten britischen und deutschen Entlastungsbetrăge für das Haushaltsjahr 1983 fortgefuhrt. Hierbel handelt es sich um brutto 991 Mio. ECU fur das Vereinigte Königreich zur Finanzlerung von Sondermaßnahmen Im Beschäftiqungs-, Energie- und Verkehrsinfrastrukturberelch sowle um 211 M10. ECU zugunsten der Bundesrepublik für Energle und VerkehrsinfrastrukturmaBnahmen. 3

Ein solcher Nachtragshaushalt war vor allem deshalb erforderlich geworden, well zusätzliche Ausgaben - vornehmlich im Bereich des Agrargarantiefonds - zu finanzieren waren, hierfur allerdings die zur Zeit verfugbaren elgenen Mittel nicht mehr ausreichten. Um diese Lucke zu schließen, haben sich die Reglerungen der Mitgliedsstaaten verpflichtet, der Gemeinschaft rulckzahlbare Vorschüsse zur Verfügung zu stellen. Die zwischenstaatliche Verteilung dieser Fi-

${ }^{1}$ Vgl. EG-Parlament, Sitzungsdokumente 1984 - 1985, Dok. 2-799/84, S. 20.

2 Vgl. Ebenda.

3 Vgl. Bulletin der EG, Nr. 6, 1984, s. 98 . 
nanzierungsmittel entspricht den nationalen Mehrwertsteuer-Anteilen. 1

Das gleiche Verfahren, allerdings mit dem Unterschied, daß es sich um nicht rückzahlbare zuschüsse handelt, wurde im Rahmen des Budgets für das Jahr 1985 praktiziert. Dabei gestaltete sich dieses Haushaltsverfahren noch problematischer. Der Rat hatte ursprünglich einen Entwurf vorgelegt, der nur die mit den derzeit zur Verfügung stehenden Eigenmitteln zu finanzierenden Ausgaben abdeckte und damit die Finanzierung nur fur zehn Monate gewahrleistete. Diese Vorgehensweise verstie $\beta$ nach Auffassung des Parlaments gegen die Budgetprinzipien der Haushaltswahrheit und -klarheit sowie gegen den Jährlichkeltsgrundsatz und führte zur massiven Ablehnung des Haushaltsplans. Erstmals seit 1980 mußte ein Haushalt wieder nach dem System der vorlaufigen Zwölftel flnanziert werden. ${ }^{2}$

Die parlamentarische Genehmigung des nunmehr fur 12 Monate erstel1ten neuen Entwurfs Im Jun1 1985 wurde möglich, nachdem in einem Reglerungsabkommen die zusatzliche Finanzierung durch nicht rückzahlbare Zuschlisse slchergestellt war. Konnte sich das Parlament mit diesen Forderungen auch durchsetzen, so blieben seine wïnsche bel der Frage der Ausglelchszahlungen zugunsten des Vereinigten Königreichs und der Bundesrepublik unerfüllt. Die Ausgleichsmaßnahmen werden - wie in den SchluBfolgerungen des Europäischen Rates von Fontainebleau vereinbart - nunmehr ubber die Einnahmenseite abgewickelt.

Eine welterrelchende Entwicklung zeichnet sich für den Haushalt 1986 $a b$. Obwohl in diesem Jahr erstmals der Mehrwertsteuer-EigenmittelSatz von 1,4 \& gllt, könnte diese Obergrenze bereits errelcht wer-

\footnotetext{
${ }^{1}$ Vgl. Bulletin der EG, Nr. 10, 1984, S. $7 \mathrm{f}$.

${ }^{2}$ Ein solches System erlaubt monatliche Zahlungen fur jedes Kapitel des Haushaltsplans in Höhe elnes Zwölftels der im vergangenen Haushaltsplan bereltgesteliten Mittel oder des in Vorbereitung befindlichen Haushaltsentwurfs, wobel jeweils der niedrigere Betrag maßgebend 1st. Vgl. G. Ott (1982), S. 367 .
} 
den. Ursachen dieser unerwarteten Entwicklung sind hauptsächlich währungsbedingte Zusatzausgaben bei den Agrarexporterstattungen und daneben die Erfüllung in den vorangegangenen Haushaltsjahren eingegangener Verpflichtungen. Die Frage einer weiteren Anhebunq des Mehrwertsteuersatz-Plafonds dürfte sich also schon bald stellen. Damit wäre nicht nur die vereinbarte Korrektur der britischen Entlastungsregelungen verbunden, sondern würde die gesamte Verteilung der Finanzierungslasten der Gemeinschaft erneut in den Mittelpunkt der Diskussion rücken.

\subsubsection{Ausgabensystem}

Anders als das Einnahmensystem des Gesamthaushalts ${ }^{1}$ unterlaqen die Ausgaben in ihrer Entwicklung keinem Umbruch, sondern ist ihre Struktur weitgehend konstant geblieben. Schon bald nach der Gründung der EWG wurde die finanzwirksame Gemeinschaftspolitik von den Ausqaben zur Finanzierung des qemeinsamen Aqrarmarktes dominiert. Daneben bestanden zunächst nur die Verwaltungsaufaaben und die Mittel des Sozialfonds sowie - noch in getrennten Haushalten - die Verwaltungsund Forschungs/Investitionsausgaben der EAG. Im Laufe der siebziaer Jahre traten neue Aufgaben- und Ausgabenberelche hinzu: vor allem der Regionalfonds, die Entwicklungshilfemaßnahmen und im Zusammenhanq mit dem Eigenmittelsystem die Erstattung der Erhebunoskosten an die Mitgliedsstaaten. Dieses äderte an der Dominanz der Agrarausgaben nur wenig, da die ubrigen Ausgaben insgesamt ein quantitativ zu geringes Gewicht besaßen.

Tabelle 24 zeigt die Entwlcklung der Ausqaben in Form der tatsächlich erfolgten Zahlungen in der letzten Dekade. Dabei lassen sich drei wesentliche Entwicklungslinien unterscheiden: Die Aqrarausqa-

${ }^{1}$ Siehe hierzu Abschnitt 4.1.1. 
Tabelle 24

Ausgaben (Zahlungen) des EG-Gesamthaushalts 1975 - 1984

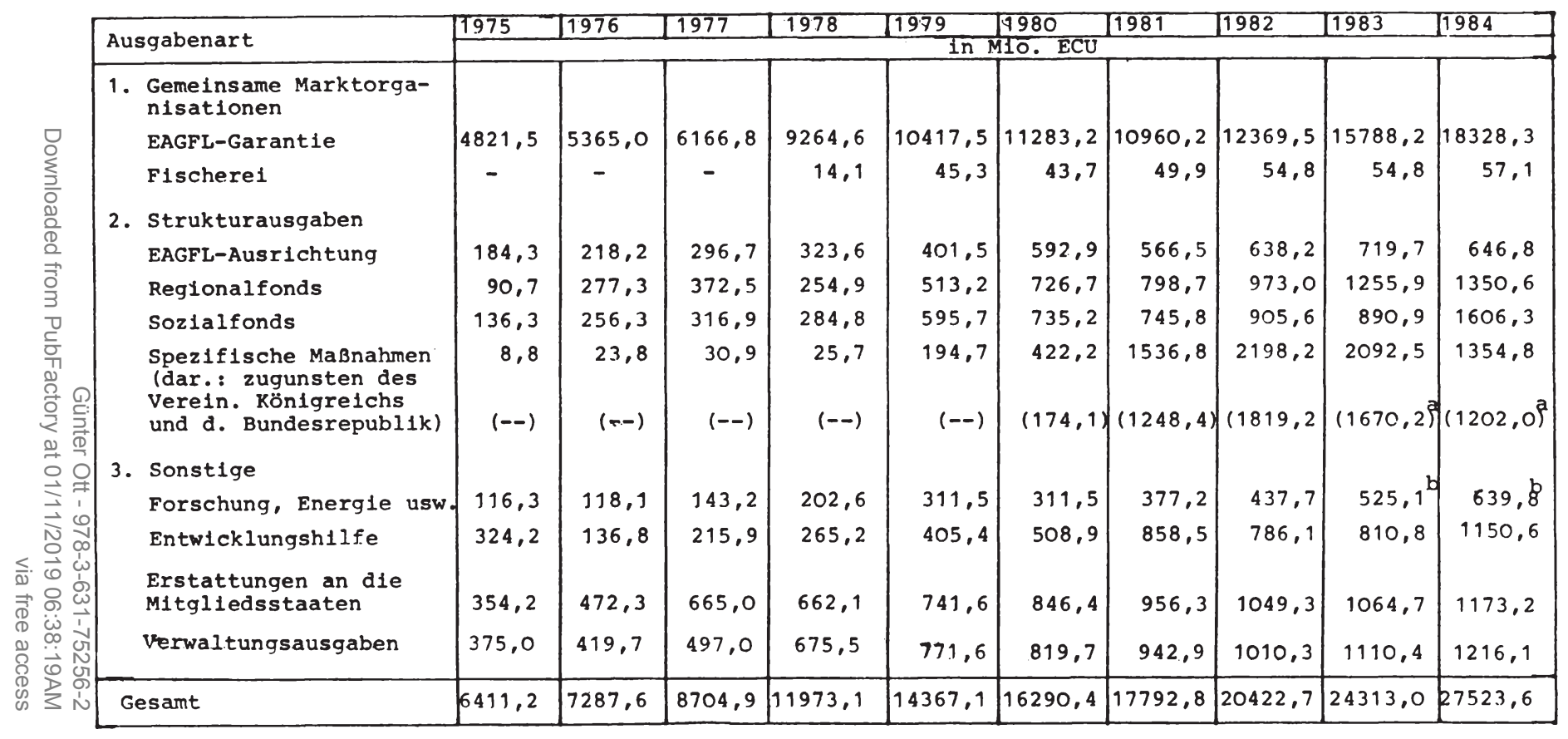


Tabelle 24 (Fortsetzung)

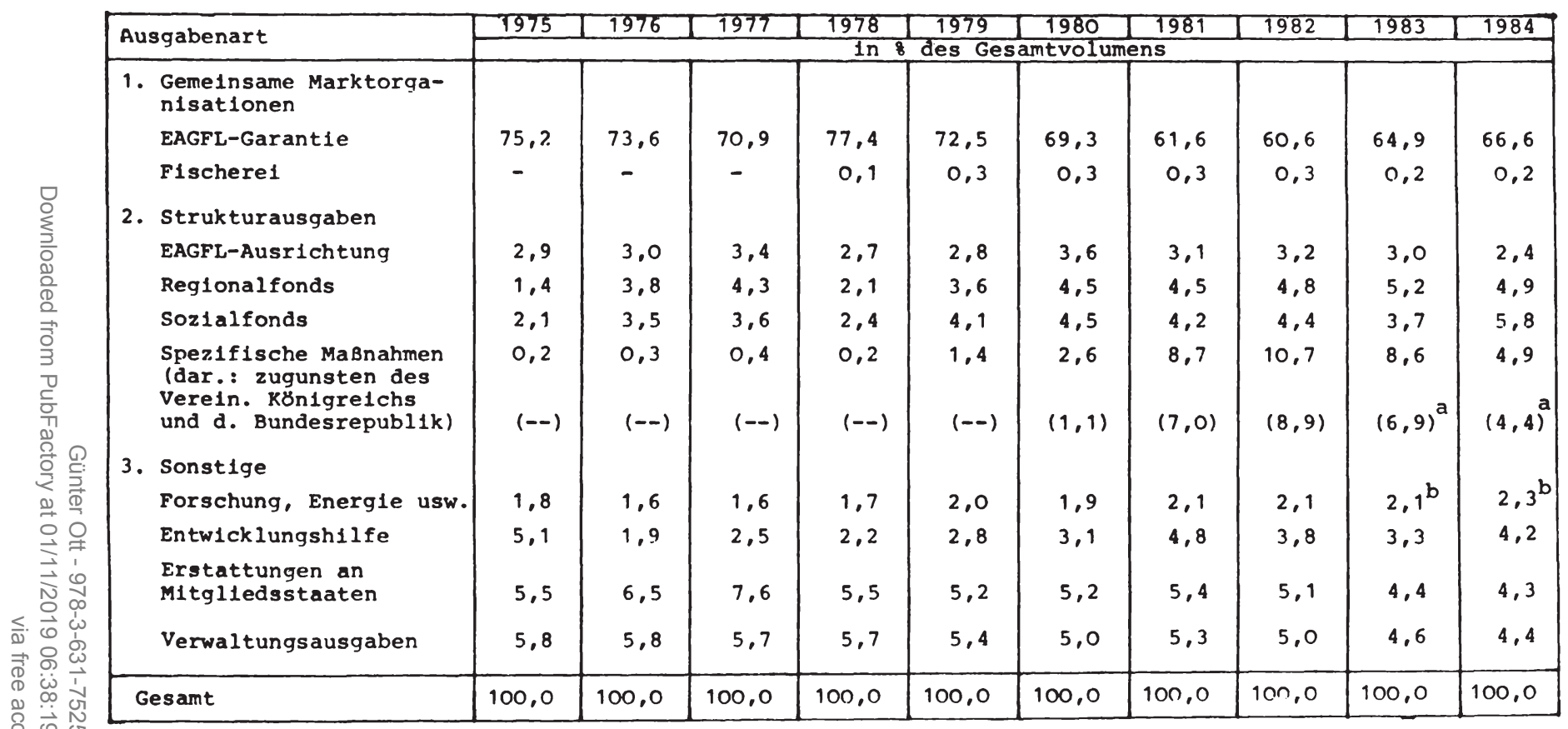

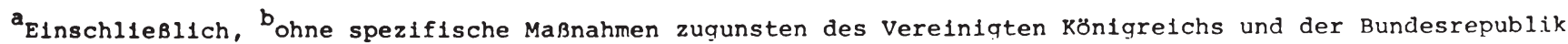
Deutschland auf dem Energle-, Verkehrs- und Beschäftiqungssektor.

Quelle: EG-Rechnungshof, Jahresberichte, versch. Jg. 
ben zur Finanzierung der gemeinsamen Marktorganisation machen zumeist etwa zwei Drittel bis drei Viertel der Gesamtausgaben aus. Die uber eine Reihe von Jahren sinkende Tendenz dieses Ausgabenteils hat sich ab 1983 wieder umgekehrt. ${ }^{1}$ Bei den strukturausgaben ist zeitweise zwar ein bemerkenswerter Anstieg des Ausgabenanteils zu beobachten, der sich etwa parallel zur Entwicklung der Agrarausgaben vollzogen hat. Ohne die britischen Entlastungsmaßnahmen liegen die Strukturausgaben mit 13,6 \& im Jahre 1984 allerdings nur mäßig über dem $1976 \mathrm{er}$-Wert $(10,6 \%)$, 30 daß sich insgesamt kein grundlegender Wandel in der Ausgabenstruktur vollzogen hat.

Die übrigen Ausgabenkategorien weisen zusamen elnen relativ konstanten Ausgabenantell von etwa 15 \% auf. Bemerkenswert sind dabei die teilweise starken Schwankungen der Entwicklungshilfeleistungen sowle die sinkende Tendenz des Verwaltungsausgabenanteils. 2

\subsubsection{Agrarfonds - Abteilung Garantie}

Die gemeinsame Agrarpolitik (GAP) ist mit der älteste, wohl wichtigste, zugleich aber auch am heftigsten kritislerte Bereich der Gemeinschaftspolitik. Der Europäische Ausrichtungs- und Garantiefonds für die Landwirtschaft (EAGFL) wurde 1962 durch die Verordnung Nr. $25 / 1962$ des Rates ${ }^{3}$ geschaffen. Er umfaßt die Abteilung Garantie, von der die Kosten der Durchführung von Marktordnungsausgaben getragen werden, und die Abtellung Ausrichtung zur Finanzierung der

\footnotetext{
${ }^{1}$ Nach dem Haushaltsplan des Jahres 1985 betrăgt der Anteil der Agrarausgaben 69,3 \%. Vgl. Bulletin der EG, Nr. 6, 1985, S. 110 f.

${ }^{2}$ Im folgenden werden die Ausgaben für Fischereierzeugnisse wegen ihrer sehr geringen Bedeutung vernachlässigt. Für diesen sektor gilt prinzipiell das gleiche Marktordnungssystem wie für die meisten groBen Agrarmärkte. Neben den Interventionsausgaben werden auch Mittel fur strukturelle Zwecke zur Verfügung gestellt.

3 Verordnung Nr. 25 uber die Finanzierung der gemeinsamen Agrarpolitik, in: Amtsblatt der FG vom 20.4.1962.
} 
gemeinsamen Agrarstrukturpolitik. In der Verordnung war vorgesehen, die Gesamtausgaben des EAGFL im Verhältnis 3:1 auf die Abteilungen Garantie und Ausrichtung aufzuteilen. Im Laufe der Entwicklung wurden die strukturausgaben allerdings auf bestimmte Höchstbeträge begrenzt; ${ }^{1}$ seitdem beanspruchen die Ausgaben der Abteilung Garantie etwa $95 \&$ der Gesamtmittel des EAGFL.

Die wichtigsten Ziele der Agrarpolitik werden in Art. 39 des EWGVertrages definiert. Für die Markt- und Preispolitik sind dies vor allem die Einkommensstützung für die Landwirtschaft, die Stabilisierung der Märkte sowie die Sicherstellung der Versorgung. Diese Zielvorstellungen sollen im Rahmen einer Agrarmarktordnung realisiert werden, die sich auf folgende drei Prinzipien gründet ${ }^{2}$ : den Grundsatz der Markteinheit mit einem ungehinderten Warenverkehr zwischen den Mitgliedsstaaten; einer Gemeinschaftspräferenz, die durch die Errichtung eines Zoll- und Abschöpfungssystems an den Grenzen die europäische Landwirtschaft von den Weltmärkten abschirmt sowie das Prinzip der finanziellen Solidarität der Mitgliedsstaaten, nach dem die erforderlichen Ausgaben gemeinschaftlich finanziert werden.

Der EAGFL ist kein Fonds im eigentlichen Sinne, da er weder über eine elgene Rechtsfähigkelt noch über Finanzautonomie verfügt.. Er ist bis 1967 Bestandtell des EWG-Haushalts gewesen und seither in den Gesamthaushalt der EG integriert. Seine Ausgaben - wie auch die übrigen Leistungen des Gesamthaushalts - werden grundsätzlich ${ }^{3}$ nach dem Nonaffektionsprinzip aus den gesamten dem Gemeinschaftshaushalt zur Verfügung stehenden Elnnahmen finanziert.

\footnotetext{
${ }^{1}$ Siehe hierzu Abschnitt 4.1.3.2.1.

2Vgl. EG-Kommission, Die Agrarpolitik der Europäischen Gemeinschaft, 3. Ausgabe, Europäische Dokumentation 6/1982, Luxemburg 1982, S. 14 f. ${ }^{3} \mathrm{zu}$ den Ausnahmen slehe welter unten.
} 
Die Mittel des Garantiefonds gehören zu den obligatorischen Ausgaben, ${ }^{1}$ für die im Haushaltsverfahren der Rat die letztendliche Entscheidungskompetenz besitzt. ${ }^{2}$ Diese Ausgaben sind die finanzielle Konsequenz aus den Regelungen der verschiedenen Marktorganisationen, die vom Rat auf Vorschlag der Kommission getroffen werden. ${ }^{3}$ Da die Kompetenz für die Markt- und Preispolitik auf die Gemeinschaft übertragen worden ist, ist die Agrarmarktpolitik "in ihren Entscheidungs- und Finanzmechanismen voll gemeinschaftlich orientiert." 4

\subsection{Organisation und Instrumentarium der gemeinsamen} Agrarpolitik

Heute unterliegen fast sämtliche landwirtschaftlichen Erzeugnisse der Mitgliedsländer dem gemeinsamen Marktordnungssystem, das über $90 \&$ des Wertes der landwirtschaftlichen Enderzeugung erfaßt. ${ }^{5}$ Wenn damit auch nahezu der gesamte Agrarbereich reglementiert wird, so erfolgt dies ausgabenseitig doch nur marginal, denn die Ausgaben für die gemeinsame Agrarpolitik (GAP) machen weniger als 108 der landwirtschaftlichen Enderzeugung aus. 6

\footnotetext{
${ }^{1}$ So werden in Art. 203 des EWG-Vertrages diejenigen Ausgaben des Gesamthaushalts definiert, die sich zwingend aus dem Gemeinschaftsvertrag oder aufgrund hieraus erlassener Rechtsakte ergeben.

${ }^{2}$ Vgl. hierzu etwa G. Ott, Das Haushaltsverfahren in den Europäischen Gemeinschaften, in: Wirtschaftswissenschaftliches Studium (WiSt), 11. Jg., 1982, S. $364 \mathrm{ff}$.

${ }^{3}$ Vgl. zu Einzelheiten P. Gilsdorf, Gemeinschaftssubventionen im Bereich der Landwirtschaft, in: B. Börner, M. Bullinger (Hrsg.), Subventionen im gemeinsamen Markt, Kölner Schriften zum Europarecht, Bd. 29, Köln, Berlin u.a. 1978, S. 215 ff., hier: S. 262 ff. ${ }^{4}$ D. Strasser, S. 172 .

${ }^{5}$ Vgl. EG-Kommission, Die Lage der Landwirtschaft in der Gemeinschaft, Bericht 1983, S. $190 \mathrm{f}$.

${ }^{6}$ Vgl. Ebenda sowie die Angaben der Tabelle 25.
} 
Grundlegendes Element der $\mathrm{GAP}^{1}$ ist die Festsetzung einheitlicher (Richt-) Preise für die einzelnen Marktordnungsprodukte durch die alljährlichen Preisbeschlüsse des Ministerrates. Daraus werden zum einen - als untere Grenze des angestrebten Erzeugerpreisniveaus die sog. Interventionspreise abgeleitet, zu denen die Interventionsstellen zum Ankauf der ihnen angebotenen Erzeugnisse verpflichtet sind, und die damit eine Mindestpreisgarantie darstellen. Zum anderen ist das Gemeinschaftspreisniveau Bestimmungsq̣öße für die Höhe der Exporterstattungen, die bei der Ausfuhr von Agrarprodukten die Differenz zu den Weltmarktpreisen ausgleichen und den Agrarexporteuren ebenfalls das Gemeinschaftspreisniveau garantieren sollen. Durch diese Markt- und Preispolitik haben also zum einen die Verbraucher höhere Preise zu tragen und wird zum anderen der Gemeinschaftshaushalt über die Interventionsmaßnahmen belastet, um das einkommenspolitische $\mathrm{ziel}$ für die Landwirtschaft $z$ u erreichen. ${ }^{2}$

Die Preispolitik konkretisiert sich in den Marktordnungen für die einzelnen Erzeugnisse, die je nach spezifischen Produkteigenschaften, Produktions- und Marktbedingungen unterschiedlich ausgestaltet sind. Vereinfachend lassen sie sich in folgende Grundtypen einteilen: Uber 70 \& der landwirtschaftlichen Erzeugung fällt unter Marktordnungen mit obligatorischen bzw. fakultativen Mindestpreisen und gleichzeitigem Außenhandelsschutz (Ausfuhrerstattungen, Einfuhrabschöpfungen). Dazu gehören die Produkte Getreide, Milch- und Milcherzeugnisse, Zucker und Rindfleisch. Für weitere 25 \& der Erzeugung bleibt die Marktordnung im wesentlichen auf den Außenschutz beschränkt. Daneben werden im Rahmen einiger Marktordnungen Ergänzungshilfen mit dem ziel tragbarer Verbraucherpreise bei gleichbleibendem Erzeugereinkommen und bei anderen Produkten Pauschalbeihilfen als Produktionsanreiz gewährt. ${ }^{3}$

\footnotetext{
${ }^{1}$ Ausführliche Darstellungen der GAP finden sich neben den bereits genannten Belträgen auch in R. Fennell, The Common Agricultural Policy of the European Community, London, Toronto u.a. 1979; J. S. Marsh, P. J. Swanney, Agriculture and the European Community, London 1980.

2Vgl. J. Ф. Mфller, S. 159; V. Petersen (1983), S. 39 f.

${ }^{3}$ Vgl. hierzu etwa EG-Kommission, Die Agrarpolitik der Europäischen Gemeinschaft, S. $18 \mathrm{ff}$.;
} 
Die Gemeinschaftsinterventionen im Rahmen der Agrarmarktpolitik sind in ihrer Vielfalt auch von Fachleuten kaum zu überblicken und unterliegen zudem einem ständigen wandel. ${ }^{1}$ Für die Zwecke dieser Arbeit reichte es allerdings aus, das Instrumentarium der GAP in seinen wesentlichen zügen zu erfassen und die einzelnen Maßnahmen nach ihrer wirtschaftlichen Art zu differenzieren. Die quantitative Bedeutung der einzelnen Instrumente geht aus der Tabelle 25 hervor.

Dabei hat sich das Schwergewicht der Ausgaben in den letzten Jahren auf die Binnenmarktinterventionen verlagert, die gegenwärtig etwa zwei Drittel des Fondsvolumens beanspruchen, während etwa ein Drittel auf die Ausfuhrerstattungen entfällt. Von den Maßnahmen zur Stützung des Binnenmarktes stellen die Preisausgleichsbeihilfen das mit Abstand wichtigste Marktordnungsinstrument dar. Es umfaßt einen breiten Fächer von Subventionen, die die Kommission in Erzeugerund Verarbeitungshilfe gliedert. Die Erzeugerbeihilfen werden an die produzenten direkt gezahlt und stellen für sie ein zusätzliches Einkommen im Sinne einer Transferzahlung dar, während die Beihilfen zur Verarbeitung der Grunderzeugnisse eine Absatzgarantie bewirken. ${ }^{2}$

Die Lagerhaltungskosten ergeben sich aus der Verpflichtung der. Interventionsstellen zum Ankauf der landwirtschaftlichen Erzeugnisse zu garantierten Mindestpreisen. Hierfür finanziert der EAGFL die technischen Kosten der Lagerhaltung, die Zinskosten der für den Ankauf erforderlichen Mittel sowie die Ausgaben zur Deckung des Unter-

${ }^{1}$ Vgl. P. Gilsdorf, S. 216 f.; P. Ipsen, Europäisches Gemeinschaftsrecht, München 1972, S. 896.

${ }^{2}$ Vgl. EG-Kommission, Elfter Finanzbericht über den Europäischen Ausrichtungs- und Garantiefonds für die Landwirtschaft 1981, Abteilung Garantie und finanzielle Durchführung der Nahrungsmittelhilfe, KOM (82) 439 endg., Brüssel, 23. Juli 1982, S. 15 f.

${ }^{3} \mathrm{Vgl}$. EG-Kommission, Die Finanzierung der Gemeinsamen Agrarmarktpolitik: Der EAGFL, Garantie, Grünes Europa, Nr. 182, Brüssel, Luxemburg 1982, S. 9. 
Tabelle 25

Ausgaben des EAGFL-Garantie nach wirtschaftlichen Arten und Erzeugnissen $1980-1984$

\begin{tabular}{|c|c|c|c|c|c|c|c|c|c|c|}
\hline \multirow{2}{*}{$\begin{array}{l}\text { Ausgabenart } \\
\text { Erzeugnisse }\end{array}$} & \multicolumn{5}{|c|}{ Mio. ECU } & \multicolumn{5}{|c|}{ in 8} \\
\hline & 1980 & \begin{tabular}{|l}
1981 \\
\end{tabular} & \begin{tabular}{|l}
1982 \\
\end{tabular} & 1983 & 1984 & 1980 & 1981 & 1982 & 1983 & 1984 \\
\hline $\begin{array}{l}\text { Ausfuhrer } \\
\text { stattungen }\end{array}$ & 5452,4 & 4938,5 & 4764,2 & 5220,5 & 6203,6 & 48,2 & 44,3 & 38,4 & 32,8 & 33,7 \\
\hline $\begin{array}{l}\text { Interventio } \\
\text { nen: }\end{array}$ & & & & & & & & & & \\
\hline $\begin{array}{l}\text { Lagerhal tungs - } \\
\text { kosten }\end{array}$ & 1616,9 & 1631,3 & 1818,0 & 2893,4 & 3540,4 & 14,3 & 14,7 & 14,7 & 18,2 & 19,3 \\
\hline $\begin{array}{l}\text { Preisaus- } \\
\text { gleichsbei- } \\
\text { hilfen }\end{array}$ & 3448,3 & 3663,6 & 4745,2 & 6409,8 & 6727,0 & 30,5 & 32,9 & 38,2 & 40,3 & 36,6 \\
\hline Sonstige & 499,1 & 669,4 & 765,1 & 907,4 & 1524,7 & 4,4 & 6,0 & 6,2 & 5,7 & 8,3 \\
\hline $\begin{array}{l}\text { hathrungsaus } \\
\text { glelchsbe- } \\
\text { träge }\end{array}$ & 298,5 & 238,4 & 313,1 & 488,6 & 376,2 & 2,6 & 2,1 & 2,5 & 3,0 & 2,1 \\
\hline Cesant & 11315,2 & $10980,2^{2}$ & 12405,6 & 15919,7 & 18371,9 & 100,0 & 100,0 & 100,0 & 100,0 & 100,0 \\
\hline Getrelde & 1669,3 & 1921,4 & 1824,5 & 2441,2 & 1650,0 & 15,2 & 17,6 & 15,1 & 15,8 & 9,2 \\
\hline $\begin{array}{l}\text { Milcherzeug- } \\
\text { nisse }\end{array}$ & 4752,0 & 3342,7 & 3327,7 & 4396,1 & 5441,7 & 43,1 & 30,7 & 27,5 & 28,5 & 30,2 \\
\hline Fette & 687,3 & 1025,4 & 1213,8 & 1620,9 & 1752,0 & 6,2 & 9,4 & 10,0 & 10,5 & 9,7 \\
\hline Zucker & 575,2 & 767,5 & 1241,9 & 1316,2 & 1631,5 & 5,2 & 7,0 & 10,3 & 8,5 & 9,1 \\
\hline Rindfletsch & 1363,3 & 1436,9 & 1158,6 & 1736,5 & 2546,8 & 12,4 & 13,2 & 9,6 & 11,3 & 14,2 \\
\hline $\begin{array}{l}\text { Obst und ce- } \\
\text { milse }\end{array}$ & 687,3 & 641,1 & 914,3 & 1196,1 & 1454,6 & 6,2 & 5,9 & 7,6 & 7,8 & 8,1 \\
\hline Sonstige & 1282,3 & 1767,8 & 2411,7 & 2723,5 & 3519,1 & 11,7 & 16,2 & 19,9 & 17,6 & 19,5 \\
\hline $\operatorname{cesamt}^{b}$ & 11016,7 & 10902,8 & 12092,5 & 15431,1 & 17995,7 & 100,0 & 100,0 & 100,0 & 100,0 & 100,0 \\
\hline
\end{tabular}

AEinschlleblich des Fehlbetrages aus den Rechnungsabschlussen 1974/75 von 161 M1O. ECU.

bohne Währungsausgleichsbetrăge.

Quelle: EG-Kormission, Finanzbericht llber den EAGFL-Garantie, versch. Jg.; elgene Berechnungen. 
schieds zwischen Ankaufspreis bei der Einlagerung und Verkaufspreis bei der Auslagerung. ${ }^{1} \mathrm{zu}$ den sonstigen Interventionen auf dem Binnenmarkt gehören die Kosten für die Rücknahme von Produkten aus dem Markt sowie bestimmte Prämien, die die Erzeugung und den Verbrauch in ihrer Ausrichtung beeinflussen sollen.

Die Währungsausgleichsbeträge resultieren aus den Differenzen zwischen den Wechselkursen auf den Devisenmärkten und den für den gemeinsamen Agrarmarkt vereinbarten "grünen Paritäten". Mit diesen gespaltenen Wechselkursen wird das Agrarpreissystem von der Entwicklung der Devisenmarktkurse abgekoppelt und so verhindert, daß sich Auf- und Abwertungen auf die nationalen Agrarpreise bzw. Erzeugereinkommen auswirken. Um dieses Wechselkurssystem vor Arbitragegeschäften zu schützen, wurden WAB eingeführt, deren jeweilige Höhe die Differenzen zwischen Devisenmarktkursen und grünen Paritäten abdeckt. ${ }^{2}$ Dieses system wird prinzipiell in der Form praktiziert, daß die - im Verhältnis zu den grünen Kursen - aufwertenden Mitgliedsstaaten Ausgleichszahlungen für Exporte leisten und Ausgleichsabgaben für Importe erheben, während die Abwertungsländer umgekehrt verfahren. Es fuhrt damit sowohl zu Einnahmen als auch zu Ausgaben, die jeweils uber den Gemeinschaftshaushalt abgewickelt, allerdings nur saldiert budgetiert werden.

Wie die Tabelle 25 ebenfalls ausweist, konzentrieren sich etwa 80 \& der EAGFL-Ausgaben auf nur sechs Marktordnungen, von denen die Sektoren Milch und Getrelde fast die Hälfte der Fondsmittel beanspruchen. Die auf diesen Märkten vorliegenden Bedingungen determinieren damit maßgeblich die Entwicklung der Agrarausgaben sowie ihre Verteilung auf die Mitgliedsstaaten. Bestimmungsgründe sind vor allem das administrierte Preisniveau, die Produktion, der Verbrauch und die Welt-

\footnotetext{
${ }^{1}$ Vgl. EG-Kommission, Die Finanzierung der Gemeinsamen Agrarmarktpolitik: Der EAGFL, Garantie, Grünes Europa, Nr. 182, Brüssel, Luxemburg 1982, S. 9 .

${ }^{2}$ Vgl. C. Thoroe, Die europäische Agrarpolitik als Finanzausgleichsproblem, in: D. Pohmer (Hrsg.), Probleme des Finanzausgleichs III. Finanzausglelch 1m Rahmen der Europäischen Gemeinschaften, Schriften des Verelns für Socialpolitik, N.F., Bd. 96/III, Berlin 1981, S. 85 ff.; hier: S. $99 \mathrm{f}$.
} 
marktlage. 1 Dabei ist neben einer stetigen Erhöhung der Agrarpreise die Produktion stets stärker als der Verbrauch gestiegen und hat bei den meisten der in Tabelle 25 angegebenen produkten zu einem gemeinschaftlichen selbstversorgungsgrad von über $100 \%$ geführt. ${ }^{2}$ Damit muB die UberschuBproduktion entweder auf dem Weltmarkt abgesetzt werden oder sind Interventionen auf dem Binnenmarkt erforderlich. 3 Im Rahmen des Weltmarktangebots spielen die Absatzbedingungen sowie die Weltmarktpreise und ihre Differenz zum Gemeinschaftspreisniveau eine entscheidende Rolle für die Höhe der Exporterstattungen. ${ }^{4}$

Nach dieser skizzenhaften Darstellung der Organisation und des Instrumentariums der GAP sind die Ausgaben des EAGFL dahingehend $z u$ differenzieren, inwieweit sie sachlich im Dienste der Marktordnungspolitik stehen. Dabei zeigt sich, daß ein Teil des Fondsvolumens nicht in diesem sinne verwendet wird, sondern als Folgekosten anderer Polltikberelche einzuordnen ist.

Dazu werden die über den Agrarfonds abgewickelten Ausgaben im Rahmen der gemeinschaftlichen Entwicklungspolitik gezählt. ${ }^{5}$ Dies sind zum einen die Exporterstattungen im Zusammenhang mit den Nahrungsmittellieferungen an Entwicklungsländer. ${ }^{6}$ Daneben entstehen auf indirektem Wege Kosten dadurch, daB die Gemeinschaft sich im Zuckerabkommen mit den AKP-Staaten und Indien zur Einfuhr bestimmter Zuckermengen veroflichtet hat, die bei der qeqenwärticen oemeinschaftlichen tiber-

${ }^{1}$ Vgl. V. Petersen (1983), s. 47.

${ }^{2} \mathrm{Vgl}$. EG-Komission, Die Lage der Landwirtschaft in der Gemeinschaft, Bericht 1983, S. $239 \mathrm{f}$.

3vgl. J. Ø. Mфller, S. 127.

${ }^{4}$ Im Hinblick auf die Gesamtausgaben des EAGFL ist auf die mögliche Interdependenz zwischen den Exporterstattungen und den Lagerkostenausgaben hinzuweisen, da etwa Einsparungen bei einer Ausgabenart Aufwendungen in der anderen Kategorie nach sich ziehen können.

${ }^{5}$ vgl. D. Strasser, S. $177 \mathrm{ff}$.

${ }^{6}$ Siehe hierzu Abschnitt 4.1.3.4. 
schuBproduktion reexportiert werden müssen, und die damit ebenfalls Ausgaben für Ausfuhrerstattungen bewirken. Weiterhin importiert die Gemeinschaft $z u$ herabgesetzten oder Null-Zollsätzen Agrarerzeugnisse, die unmittelbar mit den Gemeinschaftsprodukten in Wettbewerb treten, und die Ausgleichsbeihilfen zur Förderung ihr̈es Absatzes nach sich ziehen. Dies betrifft Buttereinfuhren aus Neuseeland sowie den Import von Rindfleisch und Getreide. ${ }^{1}$ Ebenso als nicht zum Marktordnungsbereich gehörend werden die Währungsausgleichsbeträge angesehen, deren Existenz auf die Weigerung der betroffenen Mitgliedsstaaten zurückgeführt wird, die für den Agrarmarkt geltenden Währungsrelationen den laufenden Devisenkursen anzupassen, ${ }^{2}$ und die - so Strasser - "die Unfähigkeit der Mitgliedsstaaten wiederspiegeln, eine Konvergenz ihrer Volkswirtschaften zu erreichen und eine Wirtschafts- und Währungsunion einzurichten." 3

Die uneingeschränkte Zuordnung dieser Ausgaben zum nichtmarktordnungspolitischen Bereich ist allerdings problematisch. So sind mit der handelspolitischen Zusammenarbeit, in deren Rahmen die begünstigten Importe fallen, auch positive Effekte für den Gemeinschaftshaushalt verbunden, ${ }^{4}$ und lassen sich die finanziellen Nettowirkungen wohl nur schwer abschätzen. Weiterhin ist in den Fällen, in denen die Nahrungsmittellieferungen lediglich Ersatz für einen Exportverkauf darstellen oder die Güter anderenfalls zu Garantiepreisen an die Interventionsstellen abgegeben werden, die dafür gewährte Exporterstattung durchaus als Marktordnungsmaßnahme anzusehen. 5 Schließlich stehen die mit den WAB verbundenen Berichtigungen der

${ }^{1}$ Die hlerflur veranschlagten Kosten beliefen sich im Haushaltsplan 1983 auf über $1 \mathrm{Mrd}$. ECU. Vgl. EG-Parlament, Endgültige Feststellung des Gesamthaushaltsplans der Europäischen Gemeinschaften für das Haushaltsjahr 1983, S. 407.

${ }^{2}$ vgl. J. $\varnothing$. Mфller, S. $119 \mathrm{f}$.

3 D. Strasser, S. 198 .

${ }^{4}$ Vgl. EG-Parlament, Endgültige Feststellung des Gesamthaushaltsplans der Europäischen Gemeinschaften für das Haushaltsjahr 1983, S. 407 .

${ }^{5}$ Vgl. hierzu die Diskussion im Rahmen der Entwicklungsausgaben in Abschnitt 4.1.3.4. 
Marktpreise beim innergemeinschaftlichen Handel bzw. mit Drittländern vor allem im Zusammenhang mit dem einkommenspolitischen $\mathrm{Ziel}$ der Agrarpolitik ${ }^{1}$ und stellen damit durchaus mit der Bewirtschaftung der Agrarmärkte zusammenhängende Ausgaben dar. ${ }^{2}$

Zur Bestimung des Nettovolumens der Agrarmarktordnungspolitik wird auch auf die damit verbundenen Einnahmen aus dem Außenschutz, die Agrarabschöpfungen sowie die zucker- und Isoglucoseabgaben zur Finanzierung des Zuckermarktes ${ }^{3}$ verwiesen. ${ }^{4}$ Diese Einnahmen machten in den letzten Jahren etwa 16 bis 18 \& des EAGFL-Volumens aus, so daß unter Berücksichtigung auch der nicht marktordnungsbedingten Ausgaben das Nettovolumen der Marktordnungspolitik durchaus auf nur etwa drei Viertel der EAGFL-Ausgaben geschätzt werden kann.

In diesem Zusamenhang ist allerdings auch von Interesse, daB innerhalb des Agrarfonds bereits einige Einnahmen mit den Ausgaben verrechnet werden. Dazu gehören vor allem die Mitverantwortungsabgabe für Milch, eine an produzierte Mengen anknüpfende und von den Erzeugern erhobene zweckgebundene Einnahme zur Finanzierung der Kosten des Milchmarktes, sowie die WAB, bei denen Erstattungen und Abfuhrungen gegenelnander aufgerechnet werden. Diese Saldierungen, die einen Versto $B$ gegen das Budgetprinzip der Bruttoetatisierung darstellen, wlirden bel einem getrennten Ausweis zu Erhöhungen des haushaltsmäBigen EAGFL-Volumens der Jahre 1980 bis 1983 um 5 bis 10 \& führen. 6

${ }^{1}$ Dies hat nicht zuletzt auch die jüngste Entwicklung beim Abbau der Währungsausgleichsbeträge und die gleichzeitig getätigten nationalen Entlastungsmaßnahmen für die Landwirtschaft in der Bundesrepublik gezeigt.

2Vgl. EG-Rechnungshof, Jahresbericht zum Haushaltsjahr 1982 , S. $39 \mathrm{f}$.

${ }^{3}$ Siehe hierzu Abschnitt 4.1.1.4.

${ }^{4}$ Vgl. D. Strasser, S. 198; J. Ø. Mфller, S. 120.

${ }^{5}$ Diese Einnahme entspricht in ihren wirtschaftlichen wirkungen den Zuckerabgaben.

${ }^{6}$ Vgl. EG-Rechnungshof, Jahresbericht zum Haushaltsjahr 1983, S. 49. 
4.1.3.1.2. Mitglledsstaatliche Ausgabenverteilung

Entsprechend der in dieser Arbeit verfolgten zielsetzung, die Distributionswirkungen des EG-Budgets $\mathrm{zu}$ quantifizieren und sie im Hinblick auf verteilungspolitische Erfordernisse zu beurteilen, steht auch bei den Agrarausgaben der Bezug zur wirtschaftlichen Stärke der Mitgliedsländer im Vordergrund. Es geht damit nicht primär um die Frage - so wichtig sie für sich genommen auch ist -, die zielgruppe der Landwirtschaft zu betrachten und $z u$ prüfen, inwieweit die agrarpolitischen zielsetzungen der Gemeinschaft adaquat und konsistent verwirklicht werden. Solche Betrachtungen anhand der globalen gemeinschaftlichen Agrarausgaben vorzunehmen, ist zudem problematisch, da diese Zahlungen nicht allein den Landwirten zufließen, sondern der größte Tell etwa an den Handel oder andere mit der Verarbeitung und Verteilung der landwirtschaftlichen Produkte beschaftigte Gruppen gezahlt werden. ${ }^{1}$ Dieser Tatbestand gibt vielmehr Rechtfertigung dafür, gerade auch die Agrarausgaben unter allgemeinen vertellungspolitischen Aspekten zu bewerten.

Die Verteilung der Ausgaben des EAGFL auf die Mitgliedsstaaten ist in der Tabelle 26 angegeben. Eine erste Interpretation dieser Werte kann anhand der mitgliedsstaatlichen BIP-Anteile am Gemeinschaftssozialprodukt und ihrer Finanzierungsbeteiligungen am Gesamthaushalt erfolgen. ${ }^{2}$ Bel einem Vergleich der Agrarausgaben mit den Finanzlerungsantellen werden gewissermaBen die Verteilungswirkungen allein der Agrarmarktpolitik betrachtet; dabei wird unterstellt, daB die Finanzierungsstruktur der Agrarausgaben mit der des Gesamthaushalts ubereinstimmt.

\footnotetext{
1Vgl. H. Relchenbach (1984), S. 455.

${ }^{2}$ siehe hierzu die Angaben in den Tabellen 1 und 19.
} 
Tabelle 26

Ausgaben des EAGFL-Garantie nach Mitgliesstaaten 1980 - 1984

\begin{tabular}{|c|c|c|c|c|c|}
\hline Mitgliedsland & 1980 & 1981 & 1982 & 1983 & 1984 \\
\hline & \multicolumn{5}{|c|}{ in Mio. ECU } \\
\hline Belgien & 571,1 & 489,1 & 535,1 & 611,9 & 686,4 \\
\hline Dänemark & 614,5 & 507,8 & 556,7 & 680,7 & 879,6 \\
\hline Deutschland & 2451,4 & 2031,5 & 2027,5 & 3075,8 & 3323,0 \\
\hline Frankreich & 2827,6 & 3014,2 & 2866,2 & 3566,6 & 3592,0 \\
\hline Griechenland & -- & 146,2 & 684,6 & 1007,4 & 961,2 \\
\hline Irland & 563,6 & 437,9 & 496,5 & 619,4 & 884,4 \\
\hline Italien & 1824,0 & 2092,1 & 2502,6 & 2820,5 & 3909,4 \\
\hline Luxemburg & 11,6 & 4,1 & 2,6 & 4,2 & 3,6 \\
\hline Niederlande & 1538,8 & 1157,2 & 1416,7 & 1707,8 & 1964,2 \\
\hline Verein. Königreich & 880,5 & 1080,1 & 1278,3 & 1691,0 & 2121,3 \\
\hline \multirow[t]{2}{*}{ EG } & $11283,2^{a}$ & 10960,2 & $12369,5^{a}$ & $15788,2^{a}$ & $18328,3^{a}$ \\
\hline & \multicolumn{5}{|c|}{ in 8} \\
\hline Belgien & 5,1 & 4,5 & 4,3 & 3,9 & 3,8 \\
\hline Dänemark & 5,4 & 4,6 & 4,5 & 4,3 & 4,8 \\
\hline Deutschland & 21,7 & 18,5 & 16,4 & 19,5 & 18,1 \\
\hline Frankreich & 25,1 & 27,5 & 23,2 & 22,6 & 19,6 \\
\hline Griechenland & -- & 1,3 & 5,5 & 6,4 & 5,3 \\
\hline Irland & 5,0 & 4,0 & 4,0 & 3,9 & 4,8 \\
\hline Italien & 16,2 & 19,1 & 20,2 & 17,9 & 21,3 \\
\hline Luxemburg & 0,1 & 0,0 & 0,0 & 0,0 & 0,0 \\
\hline Niederlande & 13,6 & 10,6 & 11,5 & 10,8 & 10,7 \\
\hline Verein. Königreich & 7,8 & 9,9 & 10,3 & 10,7 & 11,6 \\
\hline EG & 100,0 & 100,0 & 100,0 & 100,0 & 100,0 \\
\hline
\end{tabular}

a Nicht aufgeteilt: 1980: 0,1 Mio. ECU, 1982: 2,7 Mio. ECU, 1983: 2,9 Mio. ECU, 1984: 3,2 Mio. ECU.

Quelle: EG-Rechnungshof, Jahresberlchte, versch. Jg. 
Unter diesem Aspekt erhalten traditionell Irland und neuerdings auch Griechenland die mit Abstand höchsten Zuflüsse, nämlich etwa viermal soviel als es ihrem Finanzierungs-bzw. BIP-Anteil entspricht. Für Dänemark und die Niederlande machen die Agrarzuweisungen etwa das Doppelte ihrer Finanzierungs- und Sozialproduktanteile aus. Ebenfalls überproportionale Ausgaben - etwa das eineinhalbfache seines BIP-Anteils - fließen Italien $z u$, während in den Fällen Frankrelchs und Belgiens das Verhältnis annähernd ausgeglichen ist. Vergleichswelse geringere Ausgabenzuflüsse erhalten nur Luxemburg, die Bundesrepublik und Großbritannien, wobei die deutschen Zahlungen ungefahr zwel Drittel und die britischen zuweisungen nur etwa die Hălfte ihres BIP- bzw. Finanzierungsanteils ausmachen.

Damit läßt sich feststellen, daß die drei wirtschaftsschwächsten Lănder Griechenland, Irland und Italien, bei denen der Anteil des landwirtschaftlichen Wirtschaftszweiges am inländischen Sozialprodukt im ubrigen am höchsten in der Gemeinschaft ist, ${ }^{1}$ am stärksten durch den EAGFL begünstigt werden. Ein Bruch dieser Vertellungswirkungen tritt allerdings im Falle Großbritanniens ein, das als wirtschaftlich viertschwächstes Gemelnschaftsland die relativ geringsten Zuflüsse erhält. Hierin liegt auch die finanzielle Ursache für die hohe britische Nettozahlerposition. Ebenso werden auch die deutschen Nettotransfers maßgeblich durch die relativ geringen Betelligungen an den Agrarausgaben bestimmt. Andererseits erhalten mit Dänemark und die Niederlanden wohlhabende Länder überdurchschnittliche Zuweisungen aus dem Agrarfonds. Damit wird deutlich, daB die Ausgaben des EAGFL nur partiell einer an der Wirtschaftskraft der Mitgliedsländer orientierten Verteilung Rechnung tragen.

Fur eine erste Ursachenanalyse seien der Ausgabenverteilung die entsprechenden Werte der landwirtschaftlichen Enderzeugung gegenubergestellt (Tabelle 27). Dieser Vergleich welst in vielen Fällen

${ }^{1}$ Diese Anteile betragen im Jahre $1983 \quad 16,3$ \& für Griechenland, 11,5 \& fur Irland und 6,6 \& für Italien bei einem Durchschnittswert fur die EG von 3,7 \%. Vgl. EG-Kommission, Die Laqe der Landwirtschaft in der Gemeinschaft, Bericht 1984, S. 195. 
Tabelle 27

Landwirtschaftliche Enderzeugung ${ }^{\mathbf{a}}$ nach Mitgliedsstaaten und Im Verhältnis zu den Marktordnungsausgaben 1980 - 1984

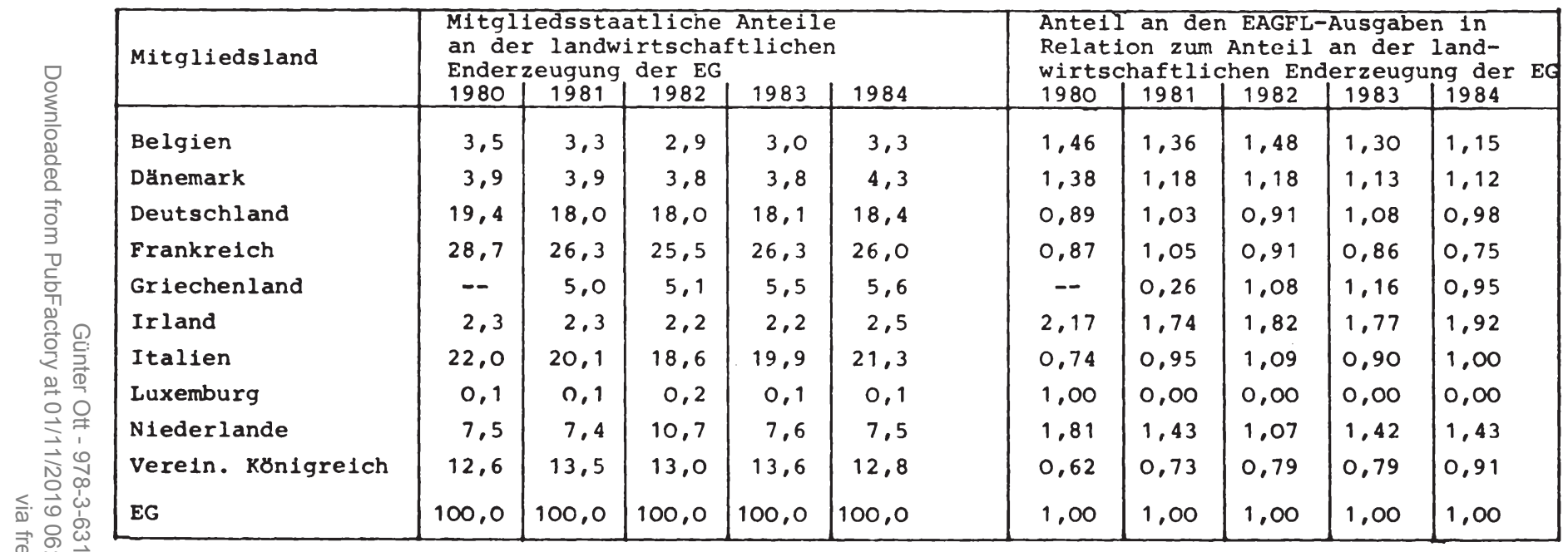

avur Erzeugnisse mit gemeinsamer Marktorganisation.

Quelle: EG-Kommission, Die Lage der Landwirtschaft in der Gemeinschaft, versch. Jg.; elgene Berechnungen. 
eine relativ gute Ubereinstimmung der Ausgaben mit den Produktionsanteilen auf. Weniger treten diese zusammenhänge allerdings für Belgien und die Niederlande auf, wofür teilweise Zurechnungsprobleme im Rahmen der Exporterstattungen verantwortlich sein werden. 1 Am ausgeprägtesten sind die Unterschiede im Falle Irlands, dessen vergleichsweise sehr hohe Ausgabenzuflüsse vor allem auf einen großen Anteil hochprotektionierter Erzeugnisse an der landwirtschaftlichen Erzeugung zurückzuführen sind. Bemerkenswert sind zudem die - zumindest lange Zeit - relativ geringen Agrarausgaben zugunsten Großbritanniens, die auf einen insgesamt geringen Protektionsgrad der britischen Agrarproduktion hinweisen.

Wenn auch das Volumen der landwirtschaftlichen Produktion der Mitgliedsstaaten als maßgeblicher Bestimmungsgrund der ihnen zuflieBenden Marktordnungsausgaben eingestuft werden kann, so ist davon selbstverständlich keine vollständige Erklärung $z$ u erwarten. Hierzu müßten vor allem der bereits angesprochene unterschiedliche Subventionsgrad der einzelnen Marktordnungserzeugnisse, die landwirtschaftliche Produktionsstruktur sowie der Selbstversorgungsgrad bzw. die Uberschußproduktion bei den einzelnen Produkten und für die Mitgliedsstaaten herangezogen werden. An diesen Faktoren hätte eine umfassendere Analyse der Garantieausgaben anzusetzen, die dann auch die Ausgabenentwicklung im Zeitablauf zuverlässig erklären könnte.

\subsection{Problematik des formalen Verteilungsansatzes}

Die bisherige Betrachtung der Verteilungswirkungen der Agrargarantleausgaben stellt auf die Zahlungsströme ab. Damit müssen nicht zugleich auch die finanziellen Vorteile für die Mitgliedsländer erfaßt werden; denn die mit den Exporterstattungen, Lagerhaltungskosten und WAB den Mitgliedsländern zugerechneten Haushaltsrückflüsse

\footnotetext{
${ }^{1}$ Siehe hierzu Darstellung im Abschnitt 4.1.3.1.3.1.
} 
erfolgen nicht immer zugunsten der heimischen wirtschaftssubjekte. Diese zusammenhänge sind im folgenden $z u$ analysieren. Daneben ist im Rahmen eines umfassenderen Ansatzes zu fragen, inwieweit eine zurechnung der - wenn auch um die eben genannten Verzerrungen korrigierten - Haushaltsausgaben nach Maßgabe der empfangenen Leistungen überhaupt ein geeigneter Ansatz zur Bemessung der Vorteile aus der gemeinsamen Agrarpolitik ist.

\subsection{Exporterstattungen}

Der hier vorliegende Sachverhalt stellt gewissermaßen die Kehrseite des mit den zöllen und Agrarabschöpfungen verbundenen Rotterdam-Antwerpen-Effekts dar, ${ }^{1}$ Dort wurde danach gefragt, wie groß die bei Drittlandsimporten mit Bestimmung fur ein anderes Mitgliedsland erhobenen und an den Gemelnschaftshaushalt abgefuhrten zölle und Abschöpfungen sind. Hier geht es um den Tatbestand, daB Exporterstattungen im allgemeinen im Ausfuhrland gezahlt und diesem Land auch als Haushaltsausgaben zugerechnet werden. Insofern nun die ausgeführten Agrargliter ursprünglich aus einem anderen Mitgliedsland stammen und nur aus transporttechnischen Gründen auf diese Weise abgewickelt werden, werden dem Ausfuhrland Leistungen aus dem EG-Haushalt zugerechnet, die nicht seinen Landwirten, sondern denen des Ursprungsland zugute kommen, d.h. den Absatz ihrer Produkte ermöglichen. ${ }^{2}$ Analog zu den zöllen und Agrarabschöpfungen kann davon ausgegangen werden, daß hiervon vor allem Belgien und die Niederlande als Ausfuhrländer und die Bundesrepublik als Ursprungsland betroffen sind.

Im folgenden soll nun versucht werden, diese Effekte abzuschätzen und auf dieser Grundlage die ausgewiesenen Zahlungsströme zu korrigieren. Da hierfür Schätzungen der zuständigen statistischen Ämter

${ }^{1}$ Siehe hierzu die Abschnitte 4.1.1.2.3. und 4.1.1.3.3. 2Vgl. hierzu A. Edwards, S. 752; J. Ø. Møller, Der Haushalt der EG, in: Europa-Archiv, 37. Jg., 1982, S. 231 ff.; hier: S. 234 f.; E. Seebohm, S. 179 . 
nicht zur Verfügung stehen, soll die empirische tberprüfung anhand der Produktions- und Selbstversorgungsstruktur der betroffenen Länder erfolgen. Die Arbeitsgrundlage lautet konkret: Je höher der auf einzelne Agrarprodukte bezogene - Selbstversorgungsgrad eines Landes ist, desto höher wird im allgemeinen auch sein (Drittlands-) Exportvolumen sein und um sohöher sind auch die Exporterstattungen In 8 der Produktion. Signifikante Abweichungen von diesen Zusammenhängen in der Weise, daß ein Mitglledsland mit geringem Selbstversorgungsniveau weit überproportionale Exporterstattungen erhält, können dann als Existenz des 'umgekehrten' Rotterdam-AntwerpenEffekts interpretiert werden.

Die empirische Analyse erfolgt fir vier Marktordnungsprodukte, die zwischen 80 und 90 \& der Exporterstattungen für sich beanspruchen. Ihre Ergebnisse sind in der Tabelle 28 zusammengestellt.

Betrachtet man zunächst die Situation für Getreide, dann fällt hier sofort auf, daB die relative Höhe der Exporterstattungen zugunsten Belgiens und der Niederlande welt aus dem üblichen Zusammenhang fällt, denn diese Länder erhalten trotz der mit Abstand niedrigsten Selbstversorgungsniveaus die bel weltem höchsten Exporterstattungen in bezug auf ihre produktion. Diese hohen werte können auch nicht durch vergleichswetse geringe Elnlagerungen erklärt werden (siehe Tabelle 29), die als mögltche alternative Verwendung mit der Höhe der Exporte $1 \mathrm{~m}$ Zusarmenhang stehen können. Die auf dem Getreidesektor zu beobachtenden Ergebnisse deuten also auf die Existenz des Rotterdam-Antwerpen-Effekts bel den Exporterstattungen hin.

Geht man noch einen Schritt weiter und wagt auf der Grundlage dieser Ergebnisse etne erste Quantlfizlerung dieses Effekts, dann gelangt man $z u$ folgenden Größenordnungen: Legt man vorsichtigerweise trotz des geringen belgischen und niederländischen selbstversorgungsniveaus das gemeinschaftsdurchschnittliche Verhältnis von Exporterstattungen und Produktion von 1 \& zugrunde und wertet die daruber hinausgehenden Erstattungen als nicht zur landeseigenen Produktion gehörend, dann erhalt man als Ergebnis, daß in den Jahren des Un- 
Tabelle 28

Exporterstattungen, Produktion und Selbstversorgungsgrad ausgewählter Agrarerzeugnisse nach Mitgliedsländern 1980 - 1983

\begin{tabular}{|c|c|c|c|c|c|c|c|c|c|c|c|}
\hline \multirow{12}{*}{ 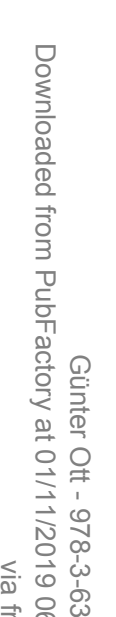 } & \multicolumn{11}{|c|}{ Getreide } \\
\hline & \multirow{2}{*}{$\begin{array}{l}\text { Mitgliedsland } \\
\text { Belgien/Luxemburg }\end{array}$} & \multicolumn{4}{|c|}{ Exporterstattungen in MiO. ECU } & \multicolumn{6}{|c|}{$\begin{array}{l}\text { Exporterstattungen in o der Produktiona und } \\
\text { Selbstversorqunasarad (in Klammern) } \\
1980^{\mathrm{b}} \\
\end{array}$} \\
\hline & & 107,2 & 97,4 & 79,6 & 131,7 & 5,0 & (57) & 4,8 & $(48)$ & 4,0 & $(49)$ \\
\hline & Dänemark & 51,8 & 23,3 & 17,7 & 21,6 & 0,7 & $(112)$ & 0,3 & $(100)$ & 0,2 & $(105)$ \\
\hline & Deutschland & 158,6 & 115,3 & 129,1 & 135,8 & 0,7 & (87) & 0,5 & (91) & 0,6 & $(89)$ \\
\hline & Frankreich & 632,1 & 707,3 & 510,2 & 745,5 & 1,4 & $(168)$ & 1,5 & $(179)$ & 1,1 & $(170)$ \\
\hline & Griechenland & -- & 18,6 & 55,3 & 64,8 & & & 0,4 & $(124)$ & 1,1 & (95) \\
\hline & Irland & 1,2 & 2,3 & 2,1 & 4,4 & 0,1 & $(82)$ & 0,1 & (79) & 0,1 & (83) \\
\hline & Italien & 64,5 & 69,3 & 67,3 & 142,5 & 0,4 & (69) & 0,4 & (73) & 0,4 & (78) \\
\hline & Niederlande & 54,0 & 45,6 & 58,7 & 67,6 & 4,2 & $(26)$ & 3,6 & $(27)$ & 4,6 & (28) \\
\hline & Verein. Königreich & 105,5 & 127,2 & 144,9 & 211,1 & 0,6 & $(80)$ & 0,7 & (92) & 0,7 & $(105)$ \\
\hline & EG & 1174,7 & 1206,3 & 1064,9 & 1525,0 & 1,0 & $(98)$ & 1,0 & $(106)$ & 0,9 & $(105)$ \\
\hline
\end{tabular}

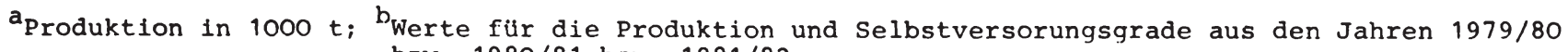
bzw. 1980/81 bzw. 1981/82. 
Tabelle 28 (Fortsetzung)

\begin{tabular}{|c|c|c|c|c|c|c|c|c|c|c|c|}
\hline \multirow{12}{*}{ 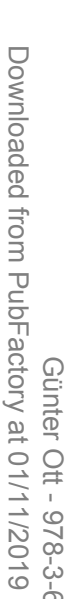 } & \multicolumn{11}{|c|}{ M1lch- und Milcherzeugnisse } \\
\hline & \multirow{2}{*}{ Mitgliedsland } & \multicolumn{4}{|c|}{ Exporterstattungen in Mio. ECU } & \multicolumn{6}{|c|}{$\begin{array}{l}\text { Exporterstattungen in } 8 \text { der Produktion a und } \\
\text { Selbstversorgungsgrad }{ }^{\mathrm{b}} \text { (in klammern) } \\
1980\end{array}$} \\
\hline & & 253,8 & 101,2 & 107,3 & 52,7 & 35,1 & $(112 / 260)$ & 13,0 & $(115 / 470)$ & 25,7 & $(110 / 208$ \\
\hline & Dänemark & 254,3 & 171,6 & 196,3 & 189,0 & 22,9 & $(209 / 97)$ & 14,3 & $(214 / 103)$ & 28,2 & $(233 / 173)$ \\
\hline & Deutschland & 429,5 & 311,4 & 208,9 & 188,6 & 7,9 & $(132 / 238)$ & 5,5 & $(124 / 227)$ & 6,4 & $(128)$ \\
\hline & Frankre1ch & 555,8 & 462,9 & 274,3 & 207,0 & 10,1 & $(122 / 121)$ & 8,5 & $(120 / 122)$ & 8,6 & $(124 / 139)$ \\
\hline & Griechenland & -- & 0,1 & 1,6 & 1,7 & & -- & 0,0 & $(70 /--)$ & 0,5 & $(43 /--)$ \\
\hline & Irla & 212,6 & 119,3 & 85,2 & 75,2 & 26,8 & $(295 /-)$ & 13,5 & $(266 /--)$ & 16,1 & $(298 /--)$ \\
\hline & Ita & 19,1 & 19,0 & 17,9 & 21,5 & 0,7 & $(68 /-)$ & 0.7 & $(63 /--)$ & 1,1 & $(64 /--)$ \\
\hline & Niede & 878,3 & 579,9 & 548,4 & 500,3 & 34,0 & $(255 / 67)$ & 22,4 & $(270 / 83)$ & 32,5 & $(467 / 79)$ \\
\hline & Verein. Königreich & 142,5 & 120,9 & 81,2 & 90,6 & 4,3 & $(51 / 329)$ & 3,1 & $(53 / 181)$ & 3,7 & $(67 / 315)$ \\
\hline & EG & 2745,9 & 1886,3 & 1521,3 & 1326,8 & 12,4 & $(120 / 132)$ & 7,7 & $(118 / 142)$ & 10,9 & $(128 / 153)$ \\
\hline
\end{tabular}

$a_{\text {in Mio. ECU; }}{ }^{b}$ (Butter/Magermilchpulver) 
Tabelle 28 (Fortsetzung)

\begin{tabular}{|c|c|c|c|c|c|c|c|c|c|c|c|}
\hline \multirow{12}{*}{ 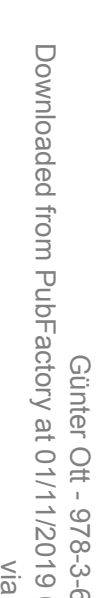 } & \multicolumn{11}{|c|}{ Zucker } \\
\hline & Mitgliedsland & \multicolumn{4}{|c|}{ Exporterstattungen in Mio. ECU } & \multicolumn{6}{|c|}{$\begin{array}{l}\text { Exporterstattungen in o der Produktiona und } \\
\text { Selbstversorqungsorad (in Klammern) } \\
1980^{\mathrm{b}} \\
\end{array}$} \\
\hline & Belgien/Luxemburg & 45,8 & 66,0 & 135,8 & 113,0 & 0,8 & (277) & 1,2 & $(222)$ & 2,0 & $(282)$ \\
\hline & Dänemark & 6,0 & 17,6 & 30,9 & 21,8 & 0,2 & (192) & 0,6 & (187) & 1,0 & (208) \\
\hline & Deutschland & 59,7 & 76,4 & 125,5 & 132,1 & 0,3 & $(124)$ & 0,4 & $(123)$ & 0,5 & (152) \\
\hline & Frankreich & 148,8 & 203,3 & 336,1 & 386,2 & 0,6 & $(208)$ & 0,8 & $(221)$ & 0,9 & $(263)$ \\
\hline & Griechenland & -- & -- & -- & 0,0 & & & -- & (74) & -- & (115) \\
\hline & Irland & 3,2 & -- & -- & 5,0 & 0,2 & $(117)$ & -- & $(101)$ & -- & $(115)$ \\
\hline & Italien & -- & 13,7 & 20,7 & 0,2 & & & 0,1 & $(102)$ & 0,1 & $(132)$ \\
\hline & Nieder lande & 19,5 & 25,7 & 51,8 & 50,1 & 0,4 & $(142)$ & 0,4 & $(161)$ & 0,7 & (179) \\
\hline & Verein. Königreich & 3,2 & 6,4 & 43,1 & 49,8 & 0,0 & $(48)$ & 0,1 & (49) & 0,6 & $(47)$ \\
\hline & EG & 286,2 & 409,2 & 744,0 & 758,1 & 0,4 & $(125)$ & 0,5 & $(127)$ & 0,7 & $(154)$ \\
\hline
\end{tabular}

${ }^{a}$ Produktion zuckerrüben in $1000 \mathrm{t}$; $b_{\text {Werte }}$ für die Produktion und Selbstversorgungsgrade aus den Jahren $1979 / 80$ bzw. 1980/81 bzw. $1981 / 82$. 
Tabelle 28 (Fortsetzung)

Rindfleisch

\begin{tabular}{|c|c|c|c|c|c|c|c|c|c|c|}
\hline \multicolumn{11}{|c|}{ Rindfleisch } \\
\hline Mitgliedsland & $\begin{array}{l}\text { Export } \\
1980 \\
\end{array}$ & $\begin{array}{l}\text { stattun } \\
1981 \\
\end{array}$ & $\begin{array}{l}\text { en in } \\
1982 \\
\end{array}$ & $\begin{array}{l}0 . \mathrm{ECU} \\
1983 \\
\end{array}$ & $\begin{array}{r}\text { Expo } \\
\text { Selb }\end{array}$ & $\begin{array}{l}\text { erstatt } \\
\text { versor } \\
30\end{array}$ & $\begin{array}{l}\text { ngen } \\
\text { ngsgr }\end{array}$ & $\begin{array}{l}8 \mathrm{der} \\
81^{\text {(in }} \\
\end{array}$ & $\begin{array}{l}\text { rodukt } \\
\text { mmern }\end{array}$ & $\begin{array}{l}\mathrm{n}^{\mathrm{a}} \text { und } \\
32\end{array}$ \\
\hline Belgien/Luxemburg & 13,8 & 15,9 & 7,1 & 10,2 & 4,5 & $(108)$ & 5,0 & $(116)$ & 2,5 & $(106)$ \\
\hline Dänemark & 44,1 & 40,7 & 33,6 & 44,6 & 18,1 & $(351)$ & 17,2 & $(372)$ & 14,6 & $(413)$ \\
\hline Deutschland & 211,9 & 215,0 & 109,7 & 165,1 & 13,5 & $(106)$ & 14,0 & $(111)$ & 7,5 & $(111)$ \\
\hline Frankreich & 134,6 & 165,2 & 169,1 & 167,0 & 7,3 & $(111)$ & 9,0 & $(116)$ & 9,7 & $(112)$ \\
\hline Griechenland & -- & -- & -- & -- & -- & $(46)$ & -- & (57) & -- & $(42)$ \\
\hline Irland & 121,8 & 166,8 & 190,3 & 238,4 & 27,4 & $(655)$ & 53,0 & $(482)$ & 55,3 & $(483)$ \\
\hline Italien & 46,9 & 60,0 & 41,7 & 48,1 & 4,1 & $(63)$ & 5,4 & $(60)$ & 3,8 & (59) \\
\hline Niederlande & 104,0 & 85,5 & 29,2 & 49,1 & 24,8 & $(141)$ & 19,6 & $(156)$ & 6,7 & $(154)$ \\
\hline Verein. Königreich & 38,5 & 76,2 & 62,8 & 105,7 & 3,5 & $(84)$ & 7,2 & $(83)$ & 6,5 & (83) \\
\hline EG & 715,5 & 825,3 & 643,5 & 828,2 & 10,1 & $(103)$ & 11,9 & $(104)$ & 9,7 & $(102)$ \\
\hline
\end{tabular}

aproduktion in $1000 t$

Quelle: Eurostat, Agrarstatistisches Jahrbuch, versch. Jg.; EG-Kommission, Finanzbericht über den EAGFL-Garantie; versch. Jg.; dies., Die Lage der Landwirtschaft in der Gemeinschaft, Bericht, versch. Jg.; eigene Berechnungen. 
tersuchungszeitraums etwa drei Viertel der jeweils für Belgien und die Niederlande ausgewiesenen Exporterstattungen auf die Ausfuhr von Erzeugnissen anderer Mitgliedsländer, vor allem wohl der Bundesrepublik, zurückzuführen sind.

Weniger eindeutig ist dagegen die Situation bei den übrigen Produkten. Im Milchsektor ${ }^{1}$ sind zwar ebenfalls Tendenzen einer Uberbewertung der ursprünglich zu Belgien und den Niederlanden gehörigen Erstattungen erkennbar, die angesichts des relativ hohen Erstattungsvolumens nicht unbedeutend sein könnten. Andererseits mögen aber auch zusammenhänge mit den relativ niedrigen Einlagerungen (Tabelle 28) bestehen und eine gewisse Kompensation bewirken. Insgesamt lassen sich auf der Grundlage der vorliegenden Daten allerdings kaum Schätzungen über elne effektive zugehörigkeit der Exporterstattungen angeben. Für den Zucker- und Rindfleischbereich sind keine gravlerenden Unregelmäßigkelten erkennbar; gewisse Abweichungen vom elngangs beschrlebenen Grundzusammenhang sollten nicht uberinterpretiert werden.

Die hier vorgestellten Ergebnisse bllden den ersten Versuch einer Quantifizlerung der mit der herkömmlichen Zurechnung der Exporterstattungen verbundenen Verzerrungen. Sle können selbstverständlich keinen Anspruch auf große Zuverlässlgkeit erheben. Denn es konnte bel ihrer Ermittlung nicht an die unmittelbaren, die Erstattungen auslösenden Transaktionen angeknlipft werden, sondern es wurde auf die zugrundellegenden Produktions- und Versorgungsverhältnisse abgestellt; etwalge Besonderheiten auf einzelnen Märkten bleiben dabe1 unberückslchtigt. Die Resultate dürften - angesichts der vorsichtigen Interpretation und Auswertung der zugrundeliegenden Daten - dennoch zumindest einen ersten ungefähren Eindruck von den Größenverhältnissen der Verzerrungen des 'umgekehrten' RotterdamAntwerpen-Effekts vermitteln und ermoglichen es, die Nettopositionen in Richtung auf eine verbesserte Aussagefähigkeit auch quantitativ zu korrigieren.

${ }^{1}$ Die Angaben zu den Selbstversorgungsgraden beziehen sich auf Butter und Magermilchpulver, die zusammen etwa die Hälfte der Exporterstattungen für Milch und Milcherzeugnisse beanspruchen, wovon die Butter wiederum mit etwa drei Viertel den größten Anteil innehat. 


\subsection{Lagerhaltungskosten}

Die Ausgaben für Lagerhaltung fallen bei den Interventionsstellen an und werden den betreffenden Gemeinschaftsländern als Rückflüsse aus dem EG-Haushalt zugerechnet. Diese Rückflüsse stellen aber im Rahmen des bisher verfolgten Verteilungsansatzes dann keine Vorteile für die Landwirtschaft des betreffenden Mitgliedsstaates dar, wenn die eingelagerten Erzeugnisse in einem anderen Land produziert worden sind, das dann als eigentlicher Nutznießer dieser Haushaltsausgaben anzusehen ist. Es ist nämlich den Agrarproduzenten in dem Gemeinsamen Markt freigestellt, in welchem Mitgliedsland sie ihre Uberschußproduktion zur Intervention bringen. Diese Möglichkeit, die Uberschußproduktion nicht an die Interventionsstellen des He1matlandes zu geben, wird durchaus genutzt, wobei solche Transaktionen vor allem in der Weise zu beobachten sind, daß deutsche Interventionsstellen in signifikantem Umfang Erzeugnisse aus anderen Mitgliedsstaaten einlagern.

Die Beweggründe für diese Richtung der Transaktionen liegen zum einen darin, daß die deutschen Interventionsstellen offenbar eine schnellere Abwicklung der Zahlungen vornehmen ${ }^{1}$ und damit $\mathrm{Zinsvor-}$ teile bieten. Sie sind aber vor allem währungspolitischer Natur und resultieren aus der gegenläufigen Entwicklung der Währungen der beteiligten Gemeinschaftsländer. Davon sind die Bundesrepublik als zumeist aufwertendes Mitgliedsland einerseits und die relativ währungsschwachen Länder wie etwa Frankreich andererseits betroffen. Als Nebenbedingung treten die Transportkosten und -fähigkeit der Produkte hinzu, so daß dadurch der Kreis der ausländischen Einlagerer begrenzt wird. Die währungspolitischen Bedingungen, die die Einlagerung von Produkten bei Interventionsstellen eines währungsstärkeren Mitgliedslandes lukrativ erscheinen lassen, bestehen nun darin, daß die Differenz zwischen den Devisenmarktkursen und

${ }^{1}$ So auch A. Nottelmann, S. 56. 
den grünen Paritäten im Rahmen der im EWS vorhandenen Bandbreite für die floatenden währungen nicht voll über die währungsausgleichsbeträge ausgeglichen werden kann. Diese ungedeckte Marge bletet die Möglichkeit, im Zusammenhang mit den Interventionen währungsgewinne zu erzielen.

Zur empirischen Uberprüfung dieser Sachverhalte wird analog den Exporterstattungen von der Produktions- und Versorgungsstruktur in den Mitgliedsländern ausgegangen. Die Arbeitsqrundlaqe lautet wiederum: je höher der produktspezifische Selbstversorgungsgrad eines Mitgliedslandes ist, desto gröBer ist auch das potentiell für eine Lagerung in Frage kommende Volumen und umso höher sind die Lagerhaltungskosten. Bestehen gravierende Abweichungen von diesen Zusammenhängen, indem etwa ein Mitgliedsland im Verhältnis zu seinem Selbstversorgungsgrad weit überdurchschnittliche Lagerhaltungskosten aufweist, dann können diese als die eingangs beschriebenen Verzerrungen interpretiert werden.

Die Analyse erfolgt im Vergleich zu den Exporterstattungen nur fur die drel Marktordnungsprodukte Getreide, Zucker und Rindfleisch, auf die in den Jahren 1980 bis 1983 etwa 60 \& der Lagerhaltungskosten entfallen. Im Rahmen der Milcherzeugnisse ist ein Ankauf ausländischer Butter durch deutsche Interventionsstellen nicht gestattet, und die Lagerkosten für Magermilchpulver fallen erst 1983 quantitativ ins Gewicht. 1 Die Ergebnisse der Berechnungen sind in der Tabelle 29 dargestellt.

Im Bereich Getreide ist unmittelbar ersichtlich, daß die Bundesrepublik weit aus dem Rahmen der übrigen Werte herausfallt: trotz eines unter dem Gemeinschaftsdurchschnitt liegenden Selbstversorgungsgrades entfallen auf sie die weitaus höchsten Lagerhaltungskosten in \& ihrer Produktion. Dies stützt die Vermutung, daB bel deutschen Interventionsstellen maßgebliche Mengen von Getreide

${ }^{1}$ Die übrigen Produkte der Milcherzeugnisse beanspruchen nur einen unwesentichen Teil der Lagerhaltungskosten. 
Tabelle 29

Lagerhaltungskosten, Produktion und Selbstversorungsgrad ausgewählter Agrarerzeugnisse nach Mitgliedsländern 1980 - 1983

\begin{tabular}{|c|c|c|c|c|c|c|c|c|c|c|c|}
\hline \multirow[b]{2}{*}{$\underset{O}{O}$} & \multicolumn{11}{|c|}{ Getreide } \\
\hline & Mitgliedsland & \multicolumn{4}{|c|}{ Lagerhaltungskosten in Mio. ECU } & \multirow{2}{*}{\multicolumn{6}{|c|}{$\begin{array}{l}\text { Lagerhaltungskosten in o der Produktion a und } \\
\text { Selbstversorqungsarad (in Klammern) } \\
1980^{\mathrm{b}}\end{array}$}} \\
\hline$\sum_{3}^{0}$ & & 1980 & 1981 & 1982 & 1983 & & & & & & \\
\hline 气 & Belgien/Luxemburg & 4,6 & 4,6 & 2,7 & 1,4 & 0,22 & $(57)$ & 0,22 & $(48)$ & 0,14 & (49) \\
\hline$\stackrel{1}{\Rightarrow}$ & Dänemark & 1,6 & 6,4 & 19,0 & 41,6 & 0,02 & $(112)$ & 0,09 & $(100)$ & 0,26 & $(105)$ \\
\hline$\frac{\overrightarrow{0}}{3}$ & Deutschland & 143,2 & 179,0 & 145,1 & 258,8 & 0,62 & $(87)$ & 0,78 & (91) & 0,64 & (89) \\
\hline$\underset{C}{Z}$ & Frankreich & 65,3 & 132,6 & 132,0 & 221,9 & 0,15 & $(168)$ & 0,28 & (179) & 0,29 & $(170)$ \\
\hline$\overline{\bar{T}}$ & Griechenland & -- & -- & 67,5 & 4,9 & & & - & & 0,14 & (95) \\
\hline$\frac{0}{0}$ & Irland & 0,3 & 0,7 & 3,2 & $-7,3$ & 0,02 & $(82)$ & 0,04 & (79) & 0,16 & (83) \\
\hline غ & Italien & $-15,2$ & 12,2 & 1,8 & $-33,4$ & $-0,09$ & $(69)$ & 0,07 & (73) & 0,01 & (78) \\
\hline & Niederlande & 2,0 & 1,7 & 9,1 & 11,3 & 0,16 & (26) & 0,13 & (27) & 0,71 & $(28)$ \\
\hline $\bar{z}$ & Verein. Königreich & 11,0 & 70,7 & 72,8 & 66,4 & 0,06 & $(80)$ & 0,36 & (92) & 0,38 & $(105)$ \\
\hline & EG & 212,8 & 407,9 & 453,4 & 565,6 & 0,19 & $(98)$ & 0,33 & (105) & 0,37 & $(105)$ \\
\hline
\end{tabular}

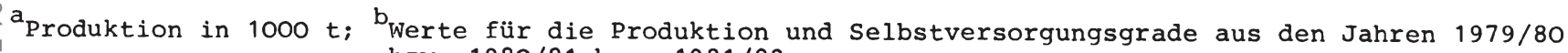
bzw. 1980/81 bzw. 1981/82 
Tabelle 29 (Fortsetzung)

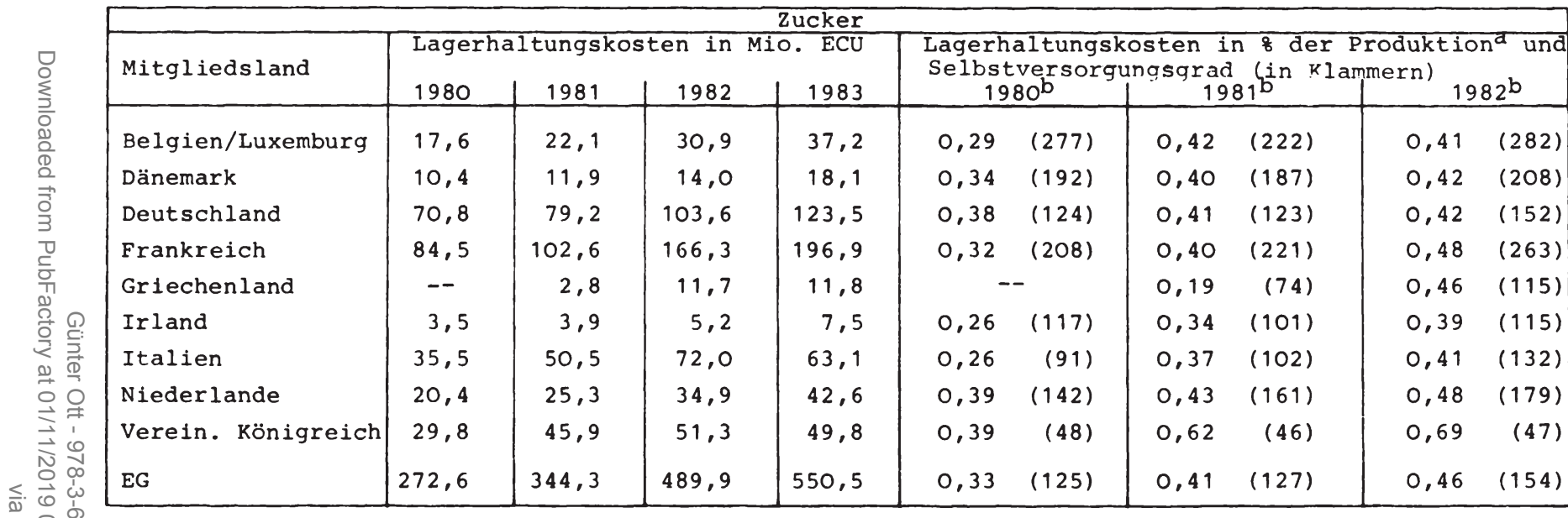

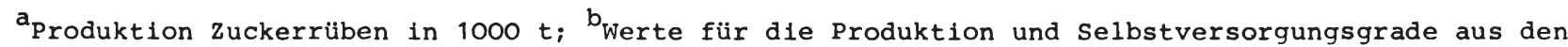
Jahren $1979 / 80$ bzw. 1980/81 bzw. 1981/82 
Tabelle 29 (Fortsetzung)

\begin{tabular}{|c|c|c|c|c|c|c|c|c|c|c|}
\hline & & & & fleisch & & & & & & \\
\hline Mitgliedsland & Lager & teungs & ten in & & $\begin{array}{l}\text { Lager } \\
\text { Selbs }\end{array}$ & $\begin{array}{l}\text { IItung } \\
\text { ersor }\end{array}$ & $\begin{array}{l}\text { Osten } \\
\text { nasar: }\end{array}$ & (in $\mathrm{K})$ & $\begin{array}{l}\text { roduk } \\
\text { nern) }\end{array}$ & on $^{a}$ und \\
\hline & 1980 & 1981 & 1982 & 1983 & & & & & & \\
\hline Belgien/Luxemburg & 2,8 & 1,8 & 1,3 & 4,9 & 0,9 & $(108)$ & 0,6 & $(116)$ & 0,5 & (106) \\
\hline Dänemark & 15,1 & 13,8 & 14,7 & 38,3 & 6,2 & (351) & 5,8 & (372) & 6,4 & $(413)$ \\
\hline Deutschland & 107,6 & 72,7 & 62,9 & 87,5 & 6,9 & (106) & 4,7 & (111) & 4,3 & (111) \\
\hline Frankreich & 133,3 & 187,7 & 70,5 & 192,0 & 7,3 & (111) & 10,2 & (116) & 4,0 & $(112)$ \\
\hline Griechenland & -- & -- & -- & 0,5 & & & & & & - \\
\hline Irland & 110,8 & 46,1 & 59,4 & 76,8 & 25,0 & (655) & 14,6 & (482) & 17,3 & $(483)$ \\
\hline Italien & 78,1 & 38,6 & 114,5 & 191,8 & 6,8 & (63) & 3,4 & $(60)$ & 10,3 & (59) \\
\hline Niederlande & 23,9 & 14,0 & 8,2 & 19,4 & 5,7 & (141) & 3,2 & (156) & 1,9 & $(154)$ \\
\hline Verein. Königreich & 32,4 & 18,4 & 9,9 & 21,2 & 2,9 & $(84)$ & 1,7 & $(83)$ & 1,0 & (83) \\
\hline EG & 504,1 & 393,1 & 341,5 & 632,4 & 7,1 & (103) & 5,7 & (104) & 5,1 & $(102)$ \\
\hline
\end{tabular}

aproduktion in $1000 \mathrm{t}$.

Quelle: Eurostat, Agrarstatistisches Jahrbuch, versch. Jg.; Kommission der EG, Finanzbericht über den EAGFL, versch. Jg.; eigene Berechnungen. 
eingelagert sind, deren Produzenten anderen Gemeinschaftsländern angehören. Versucht man auf dieser Grundlage eine Quantifizierung und legt als Maßstab für die tatsächlich aus Deutschland stammenden Interventionsprodukte vorsichtigerweise die gemeinschaftsdurchschnittlichen Lagerhaltungskosten/Produktions-Verhältnisse zuqrunde und wertet die daruber hinausgehenden Lagerhaltungskosten als ursprünglich zu anderen Gemeinschaftsländern gehörend, dann erhält man als Ergebnis, daß von den der Bundesrepublik in den Jahren des Untersuchungszeitraums zugerechneten Lagerhaltungskosten uber die Hälte letztlich den Produzenten und Händern anderer Gemeinschaftsländer zugute kommt. Als solches Land käme nach den ausgewiesenen Relationen vor allem Frankreich in Frage.

Für die Produkte Zucker und Rindfleisch lassen sich die bisher beschriebenen Zusammenhänge nicht feststellen, so daß davon ausgegangen wird, daB die Lagerhaltungskosten weitgehend an die heimischen Wirtschaftssubjekte geflossen sind.

Ebenso wle bel den nach der gleichen Methode geschätzten Ergebnissen der Exporterstattungen sind auch die hier erzielten Resultate nur mit Vorbehalten aufzunehmen. Zwar konnte die Beobachtung von Interventionsverkăufen ausländischer produzenten an deutsche Stellen empirisch gestutzt werden. In welchem Maße allerdings die geschätzten $\mathrm{Zahlen}$ der Realität nahekommen, muß zunächst dahingestellt bleiben. Dennoch durften die erstmals ermittelten Größenordnungen zumindest als Ausgangspunkt dafür herangezogen werden können, das Problem der Verzerrungen in der mitgliedsstaatlichen zurechnung der Lagerhaltungskosten auch quantitativ zu relativieren. 
4.1.3.1.3.3. Wăhrungsausgleichsbeträge

Ein lange Zeit gravierender Streitpunkt zwischen den Mitgliedsstaaten war die Zurechnung der Währungsausgleichsbeträge. Diese in Einnahmen und Ausgaben saldierten Größen betragen in den letzten Jahren zwar nur 2 bis 3 der EAGFL-Ausgaben. In der zweiten Hälfte der siebziger Jahre machten sie allerdings zwischen 7 und 14 o der Agrarausgaben aus ${ }^{1}$, und die Art ihrer zurechnung auf die einzelnen Mitgliedsländer hatte signifikante Auswirkungen auf die Höhe ihrer Nettopositionen. Wenn diese problematik gegenwärtig auch nicht mehr in diesem AusmaB besteht, so sind die daraus resultierenden Verzerrungen gleichwohl nicht zu vernachlässigen.

Um störungsfrei funktionieren zu können, setzt das system gemeinsamer Agrarpreise stabile Wechselkurse zwischen den beteiligten nationalen Wăhrungen voraus. Diese Bedingungen waren bis 1969 weltgehend erfullt. Danach gerieten die Wechselkurse jedoch in Bewegung. Dies betraf zunächst die deutsche und französische währung und insbesondere nach Aufhebung des Bretton-Woods-Systems auch die Wechselkurse der ubrigen Mitgliedsstaaten. Ohne Intervention wären damit die in elner (fiktiven) Rechnungseinheit festgesetzten gemeinsamen Agrarpreise bel ihrer Umrechnung in die nationalen Währungen Schwankungen unterworfen gewesen. Im Falle einer Aufwertung hätte dies für die Landwirte des betroffenen Mitgliedslandes Einkommensverluste zur Folge gehabt, glelchzeltig wären allerdings geringere Nahrungsmittelpreise zu erwarten gewesen. Umgekehrt hätten sich die landwirtschaftlichen Elnkommen eines abwertenden Mitgliedsstaates erhöht und wären damit grundsätzlich Preiserhöhungen der Agrarprodukte elnhergegangen. 2

Da nun die Regierungen der Gemeinschaftsstaaten nicht bereit waren, je nach wăhrungssituation Einkommensverluste ihrer Bauern bzw. verstärkte Inflationäre Tendenzen zu akzeptieren, ${ }^{3}$ wurden vorübergehend

\footnotetext{
${ }^{1}$ Vgl. EG-Kommission, Finanzberichte des EAGFL, versch. Jg.

2vgl. A. Nottelmann, S. 57; D. Strasser, S. $178 \mathrm{ff}$.

${ }^{3} \mathrm{Vgl}$. A. Nottelmann, S. 59 .
} 
Einzelmaßnahmen getroffen und 1971 das System der währungsausgleichsbeträge durch die Grundverordnung $974 / 71^{1}$ generalisiert. Dieses System besteht zunächst darin, daß für den Agrarsektor stabile Währungsbeziehungen geschaffen wurden - die sog. grünen oder repräsentativen Paritäten. Mit diesen Wechselkursen wird das Agrarpreissystem von der Entwicklung der Kurse auf den Devisenmärkten abgekoppelt und so verhindert, daß sich Auf- und Abwertungen unmittelbar auf die nationalen Agrarpreise bzw. Erzeugereinkommen auswirken. Allein die Schaffung grüner Kurse reichte allerdings nicht aus, denn aufgrund der Kursspaltung wäre es möglich, Warenarbetragegeschäfte von den Abwertungsländern in die Aufwertungsländer vorzunehmen, die zu einer Verzerrung der innergemeinschaftlichen Handelsströme geführt und für den EAGFL zusätzliche finanzielle Belastungen bedeutet hätten. ${ }^{2}$ Um diese Effekte zu verhindern, wurden WAB einqeführt, deren jeweilige Höhe die Abweichungen zwischen Devisenmarktkursen und grünen Paritäten ausgleicht.

Da die WAB für die jeweiligen Marktordnungsprodukte sich aus der Abweichung zwischen dem aktuellen Devisenmarktkurs und den grünen Kursen ergeben, wird ihre Höhe - neben dem Volumen der gehandelten Güter - maßgeblich von dieser Differenz bestimmt. Die in den Jahren des Untersuchungszeitraums angewendeten WAB-Sätze sind in der Tabelle 30 angegeben. Sie zeigt, daß 1983 wieder sämtliche Mitgliedsstaaten WAB angewendet haben, wobei ein positiver währungsausgleich vor allem in den währungsstarken Ländern ${ }^{3}$ Deutschland und Niederlande und ein negativer Ausgleich insbesondere in den währungsschwachen Ländern Italien, Frankreich und Griechenland durchgeführt wurde.

\footnotetext{
${ }^{1}$ Verordnung (EWG) Nr. 974/71 des Rates vom 12. Mai 1971 úber bestimmte konjunkturpolitische Maßnahmen, die in der Landwirtschaft im AnschluB an die vorubergehende Erweiterung der Bandbreiten der Währungen elniger Mltgliedsstaaten zu treffen sind, in: Amtsblatt der EG, Nr. L 106 vom 12.5.1971, S. 1 ff.

${ }^{2} \mathrm{Vgl}$. EG-Kommission, Die Agrarpolitik der Europäischen Gemeinschaft, S. $24 \mathrm{ff}$.

${ }^{3}$ siehe hierzu Tabelle 9.
} 
Tabelle 30

Im Handelsverkehr angewendete WAB-Sätze ${ }^{\mathrm{a}} 1980-1984$

\begin{tabular}{|l|c|c|c|c|c|c|c|c|c|c|}
\hline Währung des & \multicolumn{2}{|c|}{1980} & \multicolumn{2}{|c|}{1981} & \multicolumn{2}{|c|}{1982} & \multicolumn{2}{|c|}{1983} & \\
Mitgliedslandes & $\mathrm{n}$ & $\mathrm{h}$ & $\mathrm{n}$ & $\mathrm{h}$ & $\mathrm{n}$ & $\mathrm{h}$ & $\mathrm{n}$ & $\mathrm{h}$ & $\mathrm{n}$ & $\mathrm{h}$ \\
\hline Belgien/Luxemburg & & & & & & & & & & \\
(BFR/LFR) & $+1,7$ & $+2,4$ & 0 & $+2,2$ & $-8,1$ & 0 & $-3,1$ & $+1,0$ & 0 & 0 \\
Dänemark (DKR) & 0 & 0 & 0 & 0 & $-1,8$ & 0 & 0 & $+1,3$ & 0 & $+1,0$ \\
Deutschland (DM) & $+8,8$ & $+10,8$ & $+3,2$ & $+11,4$ & $+5,1$ & $+11,2$ & $+8,4$ & $+13,0$ & $+6,8$ & $+10,8$ \\
Frankreich (FF) & $-3,7$ & 0 & 0 & 0 & $-5,3$ & 0 & $-9,5$ & $-2,2$ & $-4,4$ & $-1,0$ \\
Griechenland (DRA) & - & - & 0 & 0 & $-2,3$ & 0 & $-23,3$ & $+4,5$ & $-17,7$ & 0 \\
Irland (IRL) & 0 & 0 & 0 & 0 & 0 & 0 & $-2,3$ & 0 & 0 & 0 \\
Italien (LTT) & $-11,1$ & 0 & 0 & $-4,4$ & $-6,6$ & -1 & $-5,0$ & 0 & $-1,8$ & 0 \\
Niederlande (HFL) & $+1,7$ & $+2,4$ & 0 & $+4,3$ & $+2,0$ & $+7,3$ & $+5,4$ & $+8,4$ & $+2,6$ & $+6,6$ \\
Verein. König- & & & & & & & & & & \\
reich (UKL) & $-6,6$ & $+12,1$ & $+1,9$ & $+18,2$ & $+1,4$ & $+10,7$ & $-1,1$ & $+7,9$ & $-1,9$ & $+7,6$ \\
\hline
\end{tabular}

$a_{n}=$ niedrigster, $\mathrm{h}=$ höchster Satz

Quelle: EG-Kommission, Finanzberichte des EAGFL, versch. Jg.

Solche Abweichungen zwischen grünen und effektiven Kursen waren in der zweiten Hälfte der siebziger Jahre weitaus gravierender, als vor allem das Vereinigte Königreich aufgrund der starken Abwertungen des Pfund Sterling negative WAB-Sätze von bis $z u \quad 40 \%$ anwenden mußte und auch Italien sowie zeltweise Irland und Frankreich negative Sätze von etwa 20 \& aufwiesen. 1 In den Jahren 1979 bis 1981 fand speziell eine massive Aufwertung des pfund sterling mit dem Ergebnis positiver britischer WAB sowie allgemein eine rückläufige Entwicklung der Ausgleichssätze statt. Nach den Währungsanpassungen vom Oktober 1981, Juni 1982 und März 1983 haben die Ausgleichssätze allerdings erneut zugenommen.

${ }^{1}$ Vgl. J. $\emptyset$. Mфller, S. $220 \mathrm{f}$. 
Die WAB werden nun von den Mitgliedsländern bel folgenden Gegebenheiten erhoben bzw. dezahlt (die hieraus resultierenden Einnahmen und Ausgaben sind beispielhaft für das Jahr 1983 in der Tabelle 31 dargestellt): Im innergemeinschaftlichen Handel

- gewähren Mitgliedsländer mit einer im Verhältnis zu den grünen Kursen aufgewerteten Währung WAB bei der Ausfuhr und erheben WAB bei der Einfuhr (positiver Währungsausgleich);

- erheben Mitgliedsländer mit einer im Verhältnis zu den grünen Kursen abgewerteten Währung WAB bei der Ausfuhr und gewăhren WAB bei der Einfuhr (negativer Währungsausgleich)

Von diesen Regelungen der Grundverordnung wird aus verwaltungstechnischen Gründen ${ }^{1}$ im Falle des Vereinigten Königreichs und Italiens seit 1976 abgewichen: ${ }^{2}$ Die in diesen Abwertungsländern ${ }^{3}$ eigentlich für EG-Importe zu gewährenden WAB werden in den Ausfuhrländern gezahlt und stellen für diese auch ein Mittelzufluß des EG-Haushalts dar. Zur Ermittlung der auf die einzelnen Mitgliedsstaaten entfallenden Ausgaben aus dem Währungsausgleich und der Nettopositionen werden diese Korrekturbeträge allerdings im nachhinein wiederum berichtigt, da die WAB dem Land zugerechnet werden sollen, dessen währungsentwicklung die betreffenden Ausgaben verursacht hat. ${ }^{4}$ Durch diese belden slch aufhebenden - im Ergebnis technischen Korrekturen sind faktisch die Bedingungen der Grundverordnung wiederhergestellt.

Das System der Wăhrungsausglelchsbetrăge erstreckt sich nicht nur auf den innergemeinschaftlichen Handel, sondern gilt auch für den Handel mit Drittländern. Die in Tabelle 31 ausgewiesenen Beträge resultieren aus Drittlandsexporten verschiedener Mitgliedsstaaten

\footnotetext{
1Vgl. hlerzu G. Denton, S. 156; D. Strasser, S. 182

2 Auf der Grundlage von Art. 2a der Verordnung 974/71.

${ }^{3}$ Fur das Vereinigte Königreich sind 1980 positive WAB eingefihrt worden, so daB das System flir dieses Land faktisch nicht mehr angewendet wird.

${ }^{4}$ Grundlage fur diese Berichtigungen ist Art. 3 Verordnung (EWG) $1172 / 76$ zur Schaffung elnes Finanzmechanismus und die in diesem Zusammenhang $z u$ ermittelnden Nettopositionen als Kriterium der Rủckzahlungsbegrenzung (siehe hierzu Abschnitt 4.1.2.1.). Diese Verordnung lief Ende 1982 aus.
} 
Tabelle 31

wahrungsausgleichsbeträge ${ }^{a}$ der Mitgliedsstaaten 1983

\begin{tabular}{|c|c|c|c|c|c|c|c|c|c|}
\hline \multirow[t]{2}{*}{ Mitgliedsland } & \multicolumn{2}{|c|}{$\begin{array}{l}\text { Von Elnfuhr- } \\
\text { ländern } \\
\begin{array}{c}\text { gezahlte } \\
\text { WAB }\end{array} \\
\end{array}$} & \multicolumn{2}{|c|}{$\begin{array}{l}\text { Von Ausfuhr- } \\
\text { ländern } \\
\frac{\text { gezahlted } / \text { erhobene }}{\text { WAB }}\end{array}$} & $\begin{array}{l}\text { Von Ausfuhrl. } \\
\text { für Rechnung f } \\
\text { d. Elnfuhrl. } \\
\text { gezahlte WAB }\end{array}$ & \multirow[t]{2}{*}{$\begin{array}{l}\text { Berichti- } \\
\text { gungen }\end{array}$} & $\begin{array}{l}\text { (Berichtig- } \\
\text { te) Innerge- } \\
\text { meinschaftl. } \\
\text { WAB }\end{array}$ & \multirow[t]{2}{*}{$\begin{array}{l}\text { WAB im } \\
\text { Handel } \\
\text { mit } \\
\text { Drittl. }\end{array}$} & \multirow[t]{2}{*}{$\begin{array}{l}\text { WAB } \\
\text { gesamt }\end{array}$} \\
\hline & & & & & 10. ECU & & & & \\
\hline Belgien & 35,9 & $-\quad 0,9$ & 1,0 & $-\quad 12,9$ & $+3,9$ & $-\quad 3,9$ & 23,0 & 0,5 & $+23,5$ \\
\hline Dănemark & -- & 1,4 & $+14,0$ & -- & 6,4 & 6,4 & 12,6 & 8,4 & 20,9 \\
\hline Deutschland & -- & $-391,5$ & $+358,1$ & -- & $+21,0$ & $-21,0$ & $-\quad 33,4$ & $+159,9$ & $+126,5$ \\
\hline Frankre1ch & $+111,5$ & $-\quad 0,0$ & -- & $-194,2$ & $+26,8$ & $-26,8$ & 82,6 & -- & $-82,6$ \\
\hline Griechenland & 25,6 & 2,2 & 1,9 & 0,0 & -- & $+0,1$ & 25,4 & -- & 25,4 \\
\hline Irland & 0,0 & -- & -- & 2,4 & $+2,1$ & $-1,6$ & 1,8 & -- & 1,8 \\
\hline Italien & 1,3 & -- & -- & 2,5 & -- & $+68,7$ & 67,5 & 0,0 & 67,5 \\
\hline Luxemburg & $+0,4$ & $-\quad 0,0$ & -- & 0,4 & $+0,0$ & -- & $+0,0$ & -- & $+0,0$ \\
\hline Niederlande & -- & $-139,0$ & $+296,5$ & -- & $+10,8$ & $-10,8$ & $+157,5$ & $+123,4$ & $+280,9$ \\
\hline $\begin{array}{l}\text { Verein. } \\
\text { Königrelch }\end{array}$ & $+0,0$ & $-100,3$ & $+79,5$ & $-1,4$ & $+1,3$ & $+1,7$ & $-\quad 19,2$ & $+\quad 47,1$ & $+27,9$ \\
\hline EG & $+174,8$ & $-635,3$ & $+750,9$ & $-213,8$ & $+72,4$ & 0 & $+149,1$ & +339.3 & $+488,3$ \\
\hline
\end{tabular}

$a_{+}$: Haushaltsausgaben; -: Haushaltseinnahmen

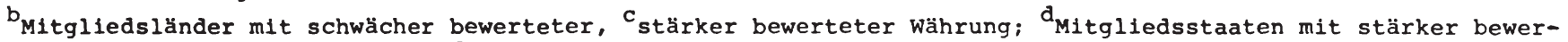

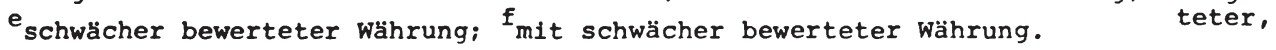

Quelle: EG-Kommission, Finanzbericht des EAGFL 1983, KOM (84) 485 endg., S. 31 und 83 f.; eigene Berechnungen 
mit positivem Währungsausgleich. Prinzipiell müßten hier auch die bei Drittlandsimporten gewährten WAB ausgewiesen werden. Diese Beträge werden jedoch mit den Agrarabschöpfungen verrechnet, so daß nur der die Abschöpfungen überschreitende Teil unter den WAB erfaßt wird. 1

Die WAB führen also sowohl zu Ausgaben als auch zu Einnahmen des Gesamthaushalts, je nach Kombination aus Import-/Exportverhältnissen und Währungssituation der Mitgliedsstaaten. So ergaben sich im innergerneinschaftlichen Handel des Jahres 1983 im Saldo Einnahmen durch die Bundesrepublik, Frankreich, GroBbritannien und - faktisch unbedeutend - Irland, während im Zusammenhang mit den ubrigen Ländern Nettoausgaben anfielen. Weitaus höher waren, wie auch in den vergangenen Jahren, die WAB im Rahmen der Drittlandsexporte, die fast ausschließlich durch die währungsstarken Länder Deutschland, Niederlande und Vereinigtes Königreich induziert worden sind.

Nach diesen zur Charakterisierung des Sachverhalts notwendigen Vorbemerkungen ist nunmehr der im Hinblick auf das Untersuchungsziel eigentlich interessierenden Frage nachzugehen, ob und inwieweit die praktizierte Zurechnung der WAB auf die einzelnen Mitgliedsländer den ihnen zufließenden finanziellen Vorteilen bzw. Belastungen entspricht.

In diesem zusammenhang ist vor allem die Frage umstritten, ob die in einem Agrarimportland mit abgewerteter währung gezahlten Ausgleichsbeträge - dazu gehören auch die berichtigten Zahlungen der Ausfuhrländer flir Rechnuna der Einfuhrländer - diesem Land auch "gerechterweise" als Zuflüsse des EG-Haushalts angerechnet werden können. Quantitativ bedeutsam waren diese Ausgaben vor allem in den Jahren 1977 und 1978 für das seinerzeit sehr währungsschwache

${ }^{1}$ Dieser Betrag war im Jahre 1983 faktisch gleich Null. 
Agrarimportland Vereinigtes Königreich; im abgeschwächten Maße galt dieser Sachverhalt für Italien. Die jährlich gezahlten britischen WAB lagen bei 700 MiO. ERE bzw. 300 bis 400 MiO. ERE im Falle Italiens. 1

Während mit dieser zurechnungsweise für Italien kaum weitere Konsequenzen verbunden waren, war sie für das Vereinigte Königreich im Zusammenhang mit seiner wachsenden Nettozahlerposition und den Verhandlungen über Korrekturmechanismen zu ihrer. Begrenzung von Bedeutung; ${ }^{2}$ denn durch die praktizierte Zurechnung der WAB wurde nach britischer Ansicht der Nettobeitrag als zu gering ausgewiesen. Mit dieser Art der Zurechnung würden nämlich die WAB als Subventionen der Verbraucher des Importlandes gewertet. Gegen diese offizielle Sichtweise ${ }^{3}$ wehrte sich das Vereinigte Königreich, da es diese Ausgleichsbeträge vielmehr als Subvention dex Exporteure in den Ausfuhrländern ansah; denn aufgrund des überhöhten Gemeinschaftspreisniveaus wären solche Subventionen erforderlich, um den Exporteuren überhaupt Absatzchancen zu eröffnen. Daraus ergäbe sich die Konsequenz, die WAB als Haushaltsausgaben den Ausfuhrländern zuzurechnen. 4

Während diese Argumentation von den Verhältnissen auf dem Weltmarkt als Normalsituation ausgeht und das Preisniveau auf dem gemeinsamen Agrarmarkt als anormal ansieht, geht die offizielle sichtweise von dem jährlich beschlossenen Gemeinschaftspreisniveau als Basis aus. Danach haben die abwertenden Mitgliedsstaaten, deren Verbraucher die importierten Agrarprodukte zu geringeren als den Gemeinschaftspreisen erwerben können, die hierfür gezahlten WAB als Subvention anzuerkennen. Zieht man also das Prinzip der gemeinsamen Agrarpreise als Maßstab heran, ist die praktizierte Zurechnung der Grenzausgleichsbeträge gerechtfertigt. ${ }^{5}$ Dennoch fällt es unter Berücksichtigung der zuvor genannten Argumentation schwer, die den

\footnotetext{
${ }^{1}$ Vgl. EG-Rechnungshof, Jahresbericht 1979, S. 234.

${ }^{2}$ Siehe hierzu Abschnitt 4.1.2.

$3 \mathrm{vgl}$. A. Nottelmann, s. 60 .

${ }^{4} \mathrm{Vgl}$. J. Ø. Mфller, S. 148 .

${ }^{5} \mathrm{Vgl}$. ebenda, S. $148 \mathrm{ff}$.
} 
Importländern zugerechneten Haushaltsausgaben uneingeschränkt als finanzielle Vorteile anzusehen. Dieser Kontroverse kommt in den letzten Jahren allerdings keine überragende Bedeutung mehr zu. Denn nach der massiven Aufwertung der britischen währung gelten für das Vereinigte Königreich seit 1980 fast nur positive Ausgleichssätze. So betrifft während des Untersuchungszeitraums dieser Sachverhait über die berichtigten, zunächst von Ausfuhrländern gezahlten WAG noch Italien mit abnehmender Tendenz in einem jährlichen Volumen von etwa 100 bis 25 Mio. ECU, 1983 in nicht unbedeutendem Umfang aber auch Frankreich und in geringfügigem Maße Belgien und Griechenland.

Von den übrigen währungs- und handelsspezifischen Konstellationen, die zur Gewährung oder Erhebung von Grenzausgleichsbeträgen führen, ist nunmehr zu hinterfragen, ob im Falle der von Aufwertungsländern bei Import erhobenen und an den Gesamthaushalt abgeführten WAB auch von einem finanzwirtschaftlichen Verzicht ausgegangen werden kann. Wollte man diese Frage bejahen, so müBte man dies damit begründen, daß der Verzicht darin besteht, daß die heimischen Verbraucher die importierten Waren nicht $z u$ den niedrigeren Gemeinschaftspreisen erwerben können. Andererseits wird in der durch die WAB bedingten Verteuerung der Produkte von den Agrarexportländern eine Einschränkung des Wettbewerbs gesehen, und dem Grenzausgleich kommt eine Schutzfunktion für den Markt des Aufwertungslandes zu. Mit dieser Sichtweise ließe sich allerdings schwerlich vereinbaren, die resultierenden Ausgleichsbeträge auch noch als finanziellen Verzicht des Importlandes - budgetmäßig gleichzusetzen etwa mit den zöllen oder Mehrwertsteuerabfuhrungen - anzuerkennen. Diese Fälle betreffen vor allem die Bundesrepublik in einem jährlichen Umfang von etwa 200 Mic. bis 460 Mio. ECU; daneben aber auch das Vereinigte Königreich und die Niederlande. ${ }^{1}$

\footnotetext{
${ }^{1}$ Vgl. EG-Kommission, Finanzberichte des EAGFL 1980 - 1984 .
} 
Insbesondere diese Wettbewerbsverzerrungen haben seit langem $\mathrm{zu}$ Forderungen nach einem Abbau des Währungsausgleichs geführt. Zwar wurde bei der Einführung des EWS im März 1979 vereinbart, die WAB im Rahrnen der jährlichen Agrarpreisbeschlüsse schrittweise abzubauen; erneute Leitkursänderungen führten jedoch dazu, daß der Grenzausgleich nicht entscheidend reduziert werden konnte. Ratsbeschlüsse in Richtung auf einen nachhaltigen Abbau der WAB wurden erst 1984 gefaBt. Beginnend mit dem Wirtschaftsjahr 1984/85 sehen sie vor allem die Umwandlung positiver in neqative WAB sowie die verringerung der deutschen WAB um 5 Prozentpunkte vor. ${ }^{1}$

Die übrigen Fälle der Erhebung und Zahlung von Grenzausgleichsbeträgen und ihre haushaltsmäßige zurechnung dürften den damit verbundenen finanziellen Vortellen bzw. Belastungen eher entsprechen: Die von Aufwertungsländern für Ausfuhren gewährten WAB, bedeutsam auch im Handel mit Drittländern, garantieren den Exporteuren den heimischen Preis und können deshalb als Subvention für die Exportländer akzeptiert werden. Die von den Abwertungsländern - 1983 faktisch nur von Frankreich - belm Export erhobenen und an den EGHaushalt abgefuhrten WAB stellen in erster Linie einen Verzicht auf günstigere Absatzchancen dar, so daß ihre Zurechnungsweise ebenfalls den ökonomischen Wirkungen weitgehend entsprechen dürfte.

Insgesamt hat sich gezelgt, daß es tellweise problematisch ist, die mit den WAB verbundenen Haushaltseinnahmen und -ausgaben einfach entsprechenden Belastungen bzw. Vorteilen der betroffenen Mitgliedsländer gleichzusetzen. Wenn es hier auch nicht möglich ist, eine den finanziellen wirkungen adăquate Aufteilung anzugeben, bleibt gleichwohl etwa fur das Haushaltsjahr 1983 festzuhalten, daß die in der Tabelle 31 dargestellten mitgliedsstaatlichen Zurechnungen vor allem in nachstehenden Fällen und mit folgender Tendenz zu korrigieren sind: Die von den währungsstarken Einfuhrländern Deutschland,

${ }^{1}$ Vgl. EG-Kommission, Bulletin der F, , Heft $3 / 1984$, S. 13 und 55 ff. 
Niederlande und Verefniates Königreich erhobenen und an den EG-Haushalt abgeführten WAB in Höhe von 391,5 Mio. ECU, 139,0 Mio. ECU und 100,3 Mio. ECU sind nur zu einem Teil als Verzichte zugunsten der Gemeinschaft anzusehen. Die Nettozahlerpositionen Deutschlands und des Vereinigten Königreichs werden um die entsprechenden Beträge zu hoch ausgewiesen und die Nettoempfängerposition der Niederlande wird sich in entsprechendem Umfang erhöhen. Daneken sind die von den währungsschwachen Elnfuhrländern gezahlten bzw. die berichtigten WAB diesen Ländern zumindest nicht uneingeschränkt als Haushaltsausgaben der EG anzuerkennen. Dies betrifft im Jahr 1983 vor allem Frankreich $(111,5 \mathrm{Mio}$. ECU) bzw. Italien $(68,7 \mathrm{Mio}$. ECU) . In entsprechendem Ausmaß wird sich die Nettozahlerposition Frankreichs erhöhen bzw. die itallenische Nettoempfängerposition vermindern.

Konkretere Aussagen als die der vorstehenden Art sind derzeit kaum möglich. Denn es 1st nichtklar anzugeben, welcher Effekt der WAB den produzenten und welche Wirkungen den Konsumenten in den jeweils beteiligten Ländern zuzurechnen 1st. Eine solche gemeinsame Betrachtung der insgesamt entstehenden Wohlfahrtsverluste bzw. -gewinne müßte aber stets angestellt werden. W1ll man dennoch nicht darauf verzichten, auch die Vertetlung der WAB angemessener als bisher auf die tatsachlich von thnen ausgehenden finanziellen wirkungen abzustimmen, so könnte man viellelcht als vorläufigen, gewissermaßen "mittleren" Ansatz wählen, nur die Hälfte der oben angegebenen Beträge den dort genannten Ländern zuzurechnen. Die jeweiliqe Gegenbuchung fur die partnerländer mubte dann allerdings mangels statistischerInformattonen unberucksichtigt bleiben. Eine solche Verfahrensweise ist selbstverständlich mit großen Vorbehalten aufzunehmen. Sie eröffnet aber die Möglichkeit, zumindest der Tendenz nach auch die WAB realistischer als bisher in thren finanziellen Auswirkungen zu erfassen und ste in einem solchen Gesamtkonzept zu relativieren. 
Eine weiterfuhrende Frage betrifft die Auswirkungen des Systems der WAB auf die Entwicklung der landwirtschaftlichen Produktion und des innergemeinschaftlichen Handels sowie langfristig die daraus möglicherweise resultierenden Belastungen des EG-Budgets. ${ }^{1}$ Diese Fragestellungen gehen allerdings über den in dieser Arbeit verfolgten Ansatz der formalen Inzidenz hinaus und sind der Analyse der effektiven Verteilungswirkungen zuzuordnen, so daß sie hier nicht weiter verfolgt werden sollen.

4.1.3.1.3.4. Zurechnung nach Maßgabe der landwirtschaftlichen Produktion

Trotz der Verbesserungen der an die reinen Zahlungsströme anknüpfenden zurechnung der Agrarausgaben in Richtung auf eine die tatsächlichen finanziellen Nutzen berücksichtigende Verteilung ist es fraglich, ob damit die Bedeutung der GAP für die Mitgliedsstaaten zutreffend erfaßt wird. Vergegenwärtigt man sich etwa, daß die Garantieausgaben weniger als 10 \& des Wertes der landwirtschaftlichen Enderzeugung ausmachen, dann wird unmittelbar deutlich, daß der weitaus größte Teil der Agrarprodukte über den Markt abqesetzt und entgolten wird. Nun sind die finanzwirksamen Gemeinschaftsinterventionen aber Voraussetzung für die Aufrechterhaltung des Preisniveaus sowohl der ủber die Gemeinschaft abgesetzten Produkte als auch der über den Markt verkauften Erzeugnisse ${ }^{2}$, so daß faktisch sämtliche Erzeuger in den Genuß der Abnahme- und Preisgarantie gelangen, unabhängig von der Bestimmung ihrer Produkte. Insoweit die

\footnotetext{
1 tuber diesen Problemkreis liegt eine umfangreiche Diskussion in der agrarwissenschaftlichen Literatur vor. Zur jüngsten umfassenden Stellungnahme der Kommission hierzu vgl. EG-Kommission, Mitteilung der Kommission an den Rat über die wirtschaftlichen Auswirkungen der Währungsregelung für die Landwirtschaft (1984 überarbeiteter Text), KOM (84) 95 endg., Brüssel 26.4.1984.

${ }^{2}$ Dies gilt uneingeschränkt für die Exporterstattungen und Lagerhaltungsausgaben; bei den Preisausgleichsbeihilfen, die tellweise nach sozio-ökonomischen Kriterien gewährt werden, ist der Zusammenhang nicht eindeutig.
} 
Kosten der Interventionsmaßnahmen als vom Produktionsvolumen abhängig betrachtet werden, kann jedes Mitgliedsland nach Maßgabe seines Anteils an der Gesamterzeugung als Kostenverursacher angesehen bzw. können ihm in dieser Höhe Nutzen der GAP zugerechnet werden. ${ }^{1}$

Die Konsequenz dieses Ansatzes besteht also darin, die Verteilung der Garantieausgaben proportional nach den landwirtschaftlichen Produktionsanteilen auf die Mitgliedsstaaten vorzunehmen. Eine solche alternative Aufteilung hat auch die Kommission vorgeschlagen, wobei allerdings die den Drittländern zugute kommenden Ausgaben (Nahrungsmittelhilfe, zuckerabkommen u.a.) von vornherein auszuklammern sind. 2

In der Tabelle 32 sind die Ergebnisse dieses Konzepts dargestellt. Die den Mitgliedsstaaten zugewiesenen Beträgen nach Maßgabe ihrer landwirtschaftlichen Produktionsanteile sind die Sume der für die einzelnen Marktordnungsprodukte durchgeführten Berechnungen. Eine solche zusammengefaBte Einzelberechnung - im Gegensatz zu einer globalen Aufteilung der Gesamtausgaben nach dem Gesamtwert der Agrarproduktion - ist erforderlich, um dem unterschiedlichen subventionierungsgrad der einzelnen Marktordnungserzeugnisse Rechnung $z u$ tragen. In die Verteilung nicht einbezogen wurden die WAB, deren Aufteilung auf die einzelnen Produktgruppen in den zugrundeliegenden Statistiken nicht vorhanden ist, sowie ein sehr geringer Teil der Marktordnungsausgaben (kleiner als 2 \& der Gesamtausgaben), für den die mitgliedsstaatlichen Produktionsanteile nicht ausgewiesen sind. Dagegen sind - im Gegensatz zur Auffassung der Kommission auch die sachlich mit der Entwicklungshilfepolitik verbundenen Exporterstattungen im Rahmen der Nahrungsmittelhilfe sowie die Folgeausgaben aus den Zuckerabkommen nicht aus den Berechnungen ausgeklammert worden. Dies geschah zum einen, um die Vergleichsbasis zu den Zahlungsstromergebnissen ${ }^{3}$, für die eine mitgliedsstaatliche Auf-

1vgl. J. $\varnothing$. Mфller, s. $152 \mathrm{f}$.

${ }^{2}$ Vgl. Kommission der EG, Korrektur der Haushaltsungleichgewichte (Vermerk der Kommission an den Besonderen Ministerrat), SEK (83) 1759 endg., Brüssel 7.11.1983, S. 2 . 3 Tabelle 26 . 


\section{Tabelle 32}

Vertellung der EAGFL-Garantieausgaben nach der landwirtschaftlichen Enderzeugung und Differenzen zur Vertellung der Zahlungsströme 1980 - 1984

\begin{tabular}{|c|c|c|c|c|c|c|c|c|c|c|}
\hline Mitgliedsland & $\begin{array}{l}\text { Verte } \\
1980\end{array}$ & $\begin{array}{l}\text { lung nach } \\
\perp \quad 1981\end{array}$ & $\begin{array}{r}\text { landw. } \\
1982\end{array}$ & $\begin{array}{r}\text { derzeugu } \\
1983^{a}\end{array}$ & 1984 & $\begin{array}{l}\text { Differer } \\
\text { zeugng } \\
1980\end{array}$ & $\begin{array}{l}\text { nz: Vertel } \\
\text { Verteilu } \\
\text { | } 1981\end{array}$ & $\begin{array}{l}\text { Iung nach } \\
\text { ng der } \mathrm{Za} \\
1982\end{array}$ & $\begin{array}{l}\text { landw. } \\
\text { hlungsst } \\
1983\end{array}$ & $\begin{array}{l}\text { Ender- } \\
\text { röme } \\
1984\end{array}$ \\
\hline & & & & & Mio & ECU & & & & \\
\hline Belgien & 312,8 & 283,1 & 293,6 & 372,8 & 475,9 & $-231,0$ & $-195,3$ & $-244,3$ & $-211,7$ & $-221,0$ \\
\hline Dänemark & 416,5 & 381,4 & 408,6 & 536,1 & 616,8 & $-177,5$ & -121.7 & $-140,2$ & $-117,3$ & $-234,4$ \\
\hline Deutschland & 2092,8 & 1791,1 & 2004,1 & 2580,4 & 3093,9 & $-213,9$ & $-\quad 98,2$ & $+\quad 77.7$ & $-347,9$ & $-96,3$ \\
\hline Frankreich & 3111,3 & 2925,3 & 3123,4 & 4007,1 & 4680,9 & $+302,5$ & $-74,4$ & $+264,5$ & $+384,7$ & $+990,0$ \\
\hline Griechenland & -- & 794,0 & 1114,5 & 326,9 & 1313,0 & -- & +647.8 & $+430,2$ & $+345,1$ & $+372,9$ \\
\hline Irland & 281,9 & 244,3 & 256,1 & 339,9 & 476,2 & $-261,7$ & $-193,3$ & $-239,4$ & $-279,7$ & $-414,4$ \\
\hline Italien & 2494,8 & 2263,8 & 2428,1 & 3034,4 & 3702,1 & $+656,7$ & $+165,2$ & $-\quad 84,0$ & $+212,7$ & $-290,9$ \\
\hline Luxemburg & 12,9 & 10,7 & 10,8 & 14,4 & 18,6 & $+1,1$ & $+6,8$ & $+\quad 7,7$ & $+10,3$ & $+\quad 14,9$ \\
\hline Niederlande & 803,7 & 666,6 & 721,3 & 934,6 & 1165,6 & $-637,1$ & $-419,0$ & $-480,8$ & $-481,5$ & $-572,7$ \\
\hline $\begin{array}{l}\text { Verein. Könlg- } \\
\text { relch }\end{array}$ & 1399,5 & 1384,1 & 1480,4 & 1941,1 & 2069,7 & $+501,8$ & $+304,4$ & $+196,8$ & $+276,3$ & $-\quad 6,8$ \\
\hline EG & 10962,2 & 10744,4 & 11840,9 & 15087,7 & 17612,7 & $-\quad 59,1$ & $+22,3$ & $-211,8$ & $-211,9$ & $-361,6$ \\
\hline
\end{tabular}

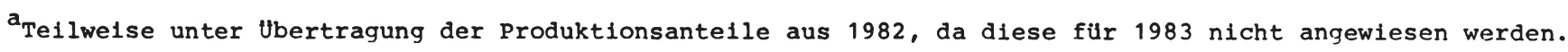

Quelle: EG-Kommission, Die Lage der Landwirtschaft in der Gemeinschaft, Bericht, versch. Jg.; Finanzbericht uber den EAGFL, versch. Jg.; eigene Berechnungen. 
teilung dieser Ausgaben nicht $\mathrm{zu}$ isolieren ist, nicht $\mathrm{zu}$ schmälern; zum anderen aber auch deshalb, weil die sachliche Bestimung der Nahrungsmittelhilfeausgaben nicht eindeutig ist. ${ }^{1}$ Angesichts der GröBenordnungen dieser Ausgaben ${ }^{2}$ dürfte allerdings ihre Berücksichtigung bzw. Nichtberücksichtigung die Aussagefähigkelt der Ergebnisse nur marginal beeinflussen.

Neben der Verteilung der Garantieausgaben nach Maßgabe der landwirtschaftlichen Enderzeugung sind in der Tabelle 32 die Unterschiede zur Verteilung nach den Zahlungsströmen ${ }^{3}$ angegeben. Die ausgewiesenen Differenzen zeigen nicht nur, wie sich die mitgliedsstaatlichen Vorteile aus der Agrarmarktpolitik im Vergleich zur traditionellen Berechnungsweise erhöhen (+) bzw. vermindern (-). Sie geben gleichzeitig auch die Verbesserung bzw. Verschlechterung der nationalen Nettopositionen an, die aus der Anwendung eines solchen alternativen Zurechnungsverfahrens resultieren würden.

Einer Interpretation und Bewertung dieser Ergebnisse bietet sich ein vielschichtiges Bild. Ohne auf sämtliche Einzelresultate einzugehen und ihre zeltliche Entwicklung möglicherweise uberzuinterpretieren, lassen sich folgende generelle Aussagen treffen:

Bei der Gruppe der vier großen Gemeinschaftsländer sind die Differenzen zwischen den belden Vertellungsansätzen relativ - bezogen auf die Zahlungsstromgrößen - zumeist gering. Dies gilt insbesondere für Deutschland und, mit Ausnahme 1984, für Frankreich, neuerdings aber auch fur Italien und das Vereinigte Könlgreich. Damit wurde ein Ubergang $z u$ einem Verteilungskonzept entsprechend den landwirtschaftlichen Produktionsantellen für diese Länder auch keine gravierenden Änderungen der bestehenden Nettopositionen bewirken. ${ }^{4}$

\footnotetext{
${ }^{1}$ Siehe hierzu Abschnitt 4.1.3.1.1. 2 siehe Tabelle 40 .

${ }^{3}$ Die Ergebnisse der Tabelle 27 wurden um die (nichtberichtigten) WAB korrigiert, um eine jeweils einheitliche Verteilungsbasis herzustelien.

${ }^{4}$ Siehe hierzu Tabelle 2.
} 
Für eine zweite Gruppe der Mitgliedsländer ergeben sich dagegen relativ gröBere Verănderungen. Im Falle Belgiens, Dänemarks, der Niederlande und Irlands würde die veränderte Zurechnung dazu führen, daß die bisher an den Zahlungsströmen gemessenen Begünstigungen aus der GAP sich um etwa ein Drittel bis die Hälfte verminderten. In bezug auf die Nettopositionen dieser Länder bedeutete dies, daß sich die Empfängerposition Irlands um ca. ein Drittel und die Dänemarks um etwa die Hälfte reduzieren, diejenige Belgiens sich fast ganz aufheben und die der Niederlande sich sogar in eine Nettozahlerposition verwandeln würde.

Von den übrigen Gemeinschaftsländern würden Griechenland nach dem veränderten Zurechnungsverfahren wesentlich größere Vorteile als bisher aus der Agrarpolitik zufließen und auch elne entsprechend höhere Nettoempfängerposition aufweisen. Dagegen besitzen die Agrarausgaben flir Luxemburg und mithin auch ihre Aufteilung traditionell kaum Bedeutung.

Insgesamt lassen sich bel einer mitgliedsstaatlichen Zurechnung der Garantleausgaben nach den landwirtschaftlichen Produktionsanteilen im Vergleich zum bisherigen Konzept der Zahlungsströme in etwa glelchvielen Fällen relativ geringfügige wie größere Veränderungen feststellen. Dlese Unterschlede würden die Relationen der Zahlungsstromrechnungen allerdings nicht fundamental verändern und sich damit auch nicht entscheldend auf die Nettopositionen auswirken. Insbesondere behielten die deutschen und britischen Nettozahlerposit1onen weitgehend thre bisherigen Größenordnungen, während wohl nur Im Falle der Niederlande elne bedeutsame Verschlechterung der bisherigen Nettoposition einträte und Frankreich im Jahre 1984 vom Nettozahler zum Nettoempfänger werden würde. 
4.1.3.1.4. Exkurs: AuBerbudgetäre Verteilungswirkungen des innergemeinschaftlichen Agrarhandels

VerläBt man das dieser Analyse zugrundeliegende Konzept der formalen Zahlungsinzidenz, dann ergeben sich Verteilungswirkungen der gemeinsamen Agrarpolitik nicht nur aus der Finanzierung und den Ausgaben des Agrarfonds, sondern auch über den innergemeinschaftlichen Handel mit Agrarerzeugnissen.

Diese Vorteils- und Belastungswirkungen sind darauf zurückzuführen, $\mathrm{daB}$ der innergemeinschaftliche Agrarhandel in den gemeinsamen Markt einbezogen wurde und zwischen den Gemeinschaftsländern keine $\mathrm{Ab}-$ schöpfungen erhoben und keine Ausfuhrerstattungen geleistet werden müssen. In diesem System haben Mitgliedsländer mit einem Netto-Einfuhrsaldo gegenüber anderen Gemeinschaftsländern dadurch einen Nachteil, daß sie die Importe nicht zu den Weltmarktpreisen, sondern $z u$ den höheren Gemeinschaftspreisen vornehmen müssen, während Länder mit einem Netto-Ausfuhrsaldo im innergemeinschaftlichen Handel Vorteile besitzen, da sie statt der Weltmarktpreise die innergemeinschaftlichen Preise erhalten. 1

Zur Quantifizierung dieser Verteilungswirkungen kann von einem Referenzsystem ausgegangen werden, "dem das System der Marktordnungen der gemeinsamen Agrarpolitik unverändert zugrunde liegt, bei dem die Durchführung und Finanzierung der Marktordnungen aber nicht bei der EG, sondern bei den nationalen Regierungen liegt." 2 Damit wird eine Situation 'ohne EG' simuliert, in der das Prinzip der finanziellen Solidarität aufgegeben wird und die Bevorzugung eines abgabenfreien innergemeinschaftlichen Handels entfällt. Dagegen soll sich für die Agrarproduzenten und die Konsumenten nichts ändern, und auch das Agrarhandelsvolumen und die -struktur werden als kon-

\footnotetext{
${ }^{1}$ Vgl. H. Ahrens, W. von Urff, Neuere Tendenzen der EG - internen Einkommenstransfers über den innergemeinschaftlichen Agrarhandel, in: Agrarwirtschaft, 33. Jg., 1984, S. 1 ff., hier: S. 1.

${ }^{2}$ C. Thoroe, Die Agrarpolitik in der EG im Konflikt mit Finanzausgleichszielen, in: Agrarwirtschaft, 29. Jg., 1980, S. 78 ff., hier: S. 81.
} 
stant unterstellt. ${ }^{1}$ In diesem Referenzsystem müssen - ebenso wie im Rahmen des Drittlandshandels - auch im innergemeinschaftlichen Agrarhandel die Produktpreise vom exportierenden Gemeinschaftsland durch Ausfuhrerstattungen auf das Weltmarktpreisniveau gesenkt werden, während die importierenden Partnerländer die Einfuhren durch Agrarabschöpfungen auf das Inlandspreisniveau anheben. Im Vergleich zu einem solchen Marktordnungssystem in nationaler Regie verzichten die Gemeinschaftsländer durch die praktizierte gemeinsame EG-Agrarpolitik also auf Abschöpfungseinnahmen bzw. sparen Ausfuhrerstattungen. Die hieraus resultierenden Salden für die einzelnen Mitgliedsstaaten stellen die außerbudgetären Transfers des innergemeinschaftlichen Agrarhandels dar.

Zur Ermittlung dieser Handelstransfers können die innergemeinschaftIichen Nettoimporte bzw. Nettoexporte der Mitgliedsstaaten bei den einzelnen Agrarerzeugnissen mit der jeweiligen Differenz aus EGPreis und Weltmarktpreis bewertet und daraus die Abschöpfungsbeträge bzw. Exporterstattungen abgeleitet werden. Solche Berechnungen sind erstmals ${ }^{2}$ von Koester ${ }^{3}$ für den Zeitraum von 1971 bis 1975 vorgenommen worden; sie wurden in der Folgezeit von anderen Autoren - mit teilweise unterschiedlichen methodischen Ansätzen - fortgeführt. 4

${ }^{1}$ Vgl. W. Scheper, Gesamtwirtschaftliche Umverteilungsaspekte der Agrarpolitik, in: C. C. von Weizsäcker (Hrsg.), staat und Wirtschaft, Schriften des Vereins für Socialpolitik, N.F., Bd. 102, Berlin 1979, S. 559 ff.

${ }^{2}$ Vgl. H. Ahrens, W. von Urff, s. 1.

${ }^{3} \mathrm{U}$. Koester, The redistributional effects of the common agricultural financial system, in: European Review of Agricultural Economics, Vol. 4-4, 1977, s. 321 ff.

${ }^{4} \mathrm{Vgl}$. hierzu: University of Cambridge, Department of Applied Economics, Cambridge Economic Policy Review, Nr. 5, April 1979, S. $23 \mathrm{ff.;}$ C. Thoroe (1981), S. $103 \mathrm{ff}$; A. E. Buckwell, D. R. Harvey, K. J. Thomson, K. A. Parton, The Costs of the Common Agricultural Policy, Beckenham 1982, S. 47 ff.; H. Ahrens, W. von Urff, S. 1 ff.; V. Petersen, Die Finanzierung der gemeinsamen Agrarpolitik - Eine theoretische und empirische Analyse ihrer Grundlagen, Wirkungen und Alternativen, Agrarwirtschaft, Sonderheft 102, Frankfurt/M., 1984, S. 95 ff. 
In der folgenden Abbildung 2 sind die bis 1980 reichenden neuesten Ergebnisse der Handelstransfers dargestellt. ${ }^{1}$ Danach ergibt sich folgendes Bild: Hohe Belastungen während des gesamten Beobachtungszeitraums haben die Nettoimportländer Italien und Vereinigtes Königreich - hier allerdings mit stark abnehmender Tendenz - hinzunehmen, während die Nettoexportländer Dänemark und Irland relativ kontinuierliche Vorteile aus dem abgabenfreien innergemeinschaftlichen Agrarhandel ziehen. Uneinheitlich ist die Situation vor allem für Frankreich, das traditionell als Nettoexportland galt, 1980 allerdings ebenso wie die Bundesrepublik und mit Abstrichen auch die Beneluxstaten eine weitgehend neutrale Position einnahm. Insgesamt läßt sich in diesem Zeitraum eine allgemeine Verminderung der Handelstransfers feststellen, die in den Fällen Frankreichs und des Vereinigten Königreichs am stärksten ausgeprägt ist. Hier zeigen sich auch am deutlichsten die Zusammenhänge zwischen den Entwicklungen der einzelstaatlichen Handelstransfers, denn die britisch/französische Entwicklung ist maßgeblich durch britische Produktionssteigerungen bestimmter Produkte und gleichzeitige Verdrängung Frankreichs vom britischen Markt bedingt. 2

Der hier verwendete Ansatz zur Quantifizierung der innergemeinschaftlichen Handelstransfers ist allerdings nicht unproblematisch. Dies betrifft zum einen die Annahme, daß im Referenzsystem sämtliche Mi.tgliedsstaaten das gleiche Protektionsniveau wie im Falle des bestehenden EG-Agrarsystems realisieren. ${ }^{3}$ Eine solche Hypothese ist zwar aus operationellen Gründen geboten, zur Charakterisierung einer realen Alternativsituation muB sie jedoch zweifelhaft bleiben. Zum anderen richten sich Einwände darauf, daß die Abschöpfungen und Erstattungen anhand der geltenden Weltmarktpreise ermittelt werden. Denn diese stellen lediglich preise für marginale gehandelte Produktmengen dar, ${ }^{4}$ während der größte Teil des Handelsvolumens

\footnotetext{
1 Die hier ausgewählten Agrarprodukte machen etwa $2 / 3$ der landwirtschaftlichen Enderzeugung der EG-Marktordnungsprodukte aus.

${ }^{2}$ Vgl. H. Ahrens, W. von Urff, S. $3 \mathrm{ff}$.

${ }^{3} \mathrm{Vgl}$. C. N. Morris, The Common Agricultural Policy, in: Fiscal

Studies, Vol. 1, 1980, Nr. 2, S. 26.

${ }^{4} \mathrm{Vgl}$. C. Thoroe (1981), S. 104 .
} 
Abbildung 2

Transfers im innergemeinschaftlichen Handel

mit ausgewählten Agrarprodukten 1976/77 - 1979/80

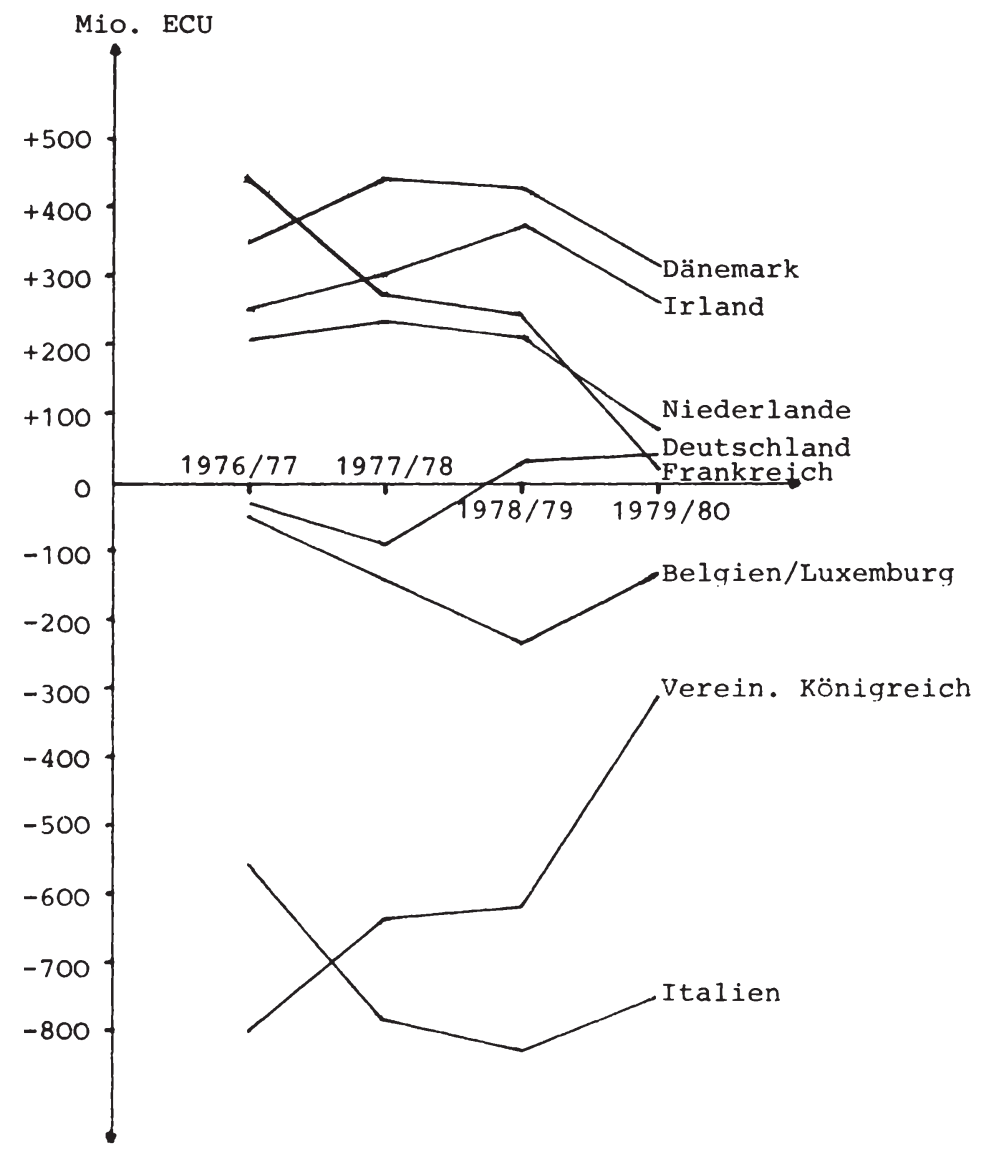

Quelle: V. Petersen (1984), S. $98 \mathrm{ff}$. 
über Kontrakte abgewickelt wird. ${ }^{1}$ Im Rahmen einer stringenten Anwendung des zugrunde liegenden Referenzsystems wären diese Einwände zwar gegenstandslos ${ }^{2}$, in der Realität wird man allerdings nicht von einem konstanten Handelsvolumen und einer unveränderten Handelsstruktur ausgehen dürfen und werden sich Auswirkungen auf das EG- bzw. Weltmarktpreisniveau ergeben.

Bezieht man die ermittelten Handelstransfers trotz dieser Vorbehalte in die Vorteils- und Belastungswirkungen der finanzwirksamen Gemeinschaftsaktivitäten mit ein, dann verändern sie - bei zugrundelegung der neuesten verfügbaren Ergebnisse für 1979/80 - die einzelstaatlichen finanziellen Nettopositionen ${ }^{3}$ wie folgt: Für die Bundesrepublik, Frankreich und die Benelux-Länder ergeben sich zumeist absolut bzw. relativ geringe Auswirkungen. Dagegen verbessern sich die Nettoempfängerpositionen Dänemarks und Irlands in signifikanter Weise, während sich die britische Nettozahlerposition erhöht und vor allem aber Italien gravierende Abstriche an seiner bisherigen Nettoempfängerposition hinzunehmen hat.

Eine solche Einbeziehung der Handelstransfers liegt allerdings nicht im Rahmen der in der vorliegenden Arbeit angestrebten $\mathrm{ziel-}$ setzung, die finanzwirtschaftlichen Verteilungswirkungen der Gemeinschaftstätigkeit zu bestimmen, sondern läuft auf eine umfassendere wohlfahrtsökonomische Analyse hinaus. ${ }^{4}$ Bei einer solchen Betrachtungsweise müßten dann allerdings nicht nur die wohlfahrtsökonomischen Effekte der GAP, sondern auch der übrigen Gemeinschaftspolitiken - wie etwa die aus der Handelsliberalisierung resultierenden Wirkungen - berücksichtigt werden, so daß eine einseitige Einbeziehung der Handelstransfers insofern $z$ verzerrten Ergebnissen führt.

1Vgl. V. Petersen (1983), S. 103.

${ }^{2}$ Vgl. J. M. C. Rollo, K. S. Warwick, The CAP and Ressource Flows among EEC Member States, Ministry of Agriculture, Fisheries and Food, London 1979, S. 6., zitiert nach V. Petersen (1983), S. 103. ${ }^{3}$ Siehe hierzu Tabelle 2 .

${ }^{4} \mathrm{Vgl}$. J. $\varnothing$. Mфller, S. $158 \mathrm{f}$. 
Gleichwohl sind die Größenordnungen der Handelstransfers für die Relativierung der budgetären Verteilungswirkungen hilfreich. Auch wenn die konkreten Ergebnisse mit Vorbehalten zu interpretieren sind und sich die GröBenordnungen aufgrund von Handels-, Ernteund Preisschwankungen schnell ändern können, bietet sich dadurch die Möglichkeit, den Stellenwert der Nettopositionen im Rahmen eines umfassenderen Verteilungskonzepts beurteilen zu können: Danach werden zwar die Nettopositionen teilweise signifikant verändert, die grundlegende mitgliedsstaatliche Verteilungsstruktur und insbesondere die alleinigen und hohen Nettozahlerpositionen Deutschlands und des Vereinigten Königreichs werden allerdings nicht entscheidend berührt. 
4.1.3.2. Interventionen im Bereich der Strukturpolitik

Die finanzwirksame Strukturpolitik der Gemeinschaft vollzieht sich auf verschiedenen Ebenen. Kernstücke sind die Abteilung Ausrichtung des Agrarfonds, der Europäische Sozialfonds und der Europäische Fonds für regionale Entwicklung, die den weitaus größten Teil dieser Mittelansätze beanspruchen. Ebenso wie der Agrargarantiefonds sind auch diese Fonds keine autonomen Institutionen, sondern Bestandteile des Gesamthaushalts. Uber den Haushalt werden vor allem in letzter zeit einige weitere Maßnahmen mit struktureller Zweckbestimmung durchgeführt. Außerhalb des Gesamthaushalts erfolgen strukturpolitisch motivierte Aktivitäten durch den Funktionshaushalt der EGKS sowie im Rahmen der gemeinschaftlichen Darlehensvergabe. 1

Gemeinsam verfolgen diese Finanzierungsinstrumente die zielsetzung, einen direkten Einfluß auf eine konvergente wirtschaftsentwicklung in den Mitgliedsstaaten zu nehmen. 2 Inwieweit solche gemeinschaftlichen Bestrebungen zu realisieren sind, oder ob die Maßnahmen lediglich finanzielle Umverteilungen bewerkstelligen können, ist eine der im folgenden $\mathrm{zu}$ untersuchenden Fragestellungen.

\subsection{Agrarfonds - Abteilung Ausrichtung}

Die Abteilung Ausrichtung des EAGFL ist Bestandteil sowohl der gemeinsamen Agrarpolitik als auch Instrument der strukturpolitik. Anders als die Abteilung Garantie sieht der Ausrichtungsfonds keine Vollfinanzierung der geförderten Maßnahmen, sondern Beteiligungen an den mitgliedsstaatlichen Aufwendungen vor. Beim Beginn der Agrarfinanzierung ging man davon aus, daß die für die Agrarstrukturpolitik bereitgestellten Mittel ein Drittel der Ausgaben des Garantiefonds betragen sollten. ${ }^{3}$ Infolge des Anstiegs der Garantie-

${ }^{1}$ Diese Zusammenhänge werden in den Abschnitten 4.2.2. und 4.2.3. behandelt.

${ }^{2}$ Vgl. D. Strasser, S. $205 \mathrm{ff}$.

${ }^{3}$ Verordnung Nr. 25 über die Finanzierung der gemeinsamen Agrarpolitik. 
leistungen wurden die Ausgaben für die Abteilung Ausrichtung allerdings schon 1967 auf einen jährlichen Plafonds begrenzt, der nach der Erweiterung der Gemeinschaft 1973 heraufgesetzt wurde. ${ }^{1} \mathrm{Ab}$ 1980 wird auf eine jährliche Plafondierung verzichtet. Statt dessen sind die Mittel für den Fünfjahreszeitraum von 1980 bis 1984 auf insgesamt 3,6 Mrd. ECU festgesetzt und nach dem Beitritt Griechenlands auf 3,755 Mrd. ECU erhöht worden. ${ }^{2}$ Die jährlich zu veranschlagenden Mittel werden entsprechend dem zu finanzierenden Ausgabenbetrag im jeweiligen Haushaltsverfahren festgelegt. Damit unterliegen die Mittel des EAGFL-Ausrichtung einer vorab festgelegten Höchstgrenze, und wird die globale Haushaltsplanung bereits weitgehend durch Ratsverordnungen determiniert.

Die Mittel des Ausrichtungsfonds sind gegenwärtig etwa je zur Hälfte für die Finanzierung sog. horizontaler Aktionen und regionaler Maßnahmen bestimmt. ${ }^{3}$ Die horizontalen Maßnahmen sind in sämtlichen Gebieten bzw. allen landwirtschaftlichen Betrieben anwendbar, die die Förderungsbedingungen erfüllen. Sie dienen zur Verbesserung der Agrarinfrastruktur und der Verarbeitung und Vermarktung landwirtschaftlicher Produkte, sozio-ökonomischen Vorhaben wie der Betriebs-

${ }^{1}$ Vgl. D. Strasser, S. $221 \mathrm{f}$.

2 Damit ist das ursprünglich angestrebte Beteiligungsverhältnis zu keiner zeit und zunehmend weniger erreicht worden. Für die Jahre 1965 bis 1974 beliefen sich die für die Abteilung Ausrichtung bereitgestellten Mittel auf etwa 10 \& der Gesamtverpflichtungen des EAGFL (vgl. EG-Kommission, Bestandsaufnahme der gemeinsamen Agrarpolitik, Bulletin der EG, Beilage 2/75, S. 24). In den folgenden Jahren reauzierte sich dieser Anteil auf etwa $5 \%$ (vgl. EG-Rechnungshof, Jahresberichte, versch. Jg.).

${ }^{3}$ Für den Zeitraum von 1964 bis 1982 betragen die horizontalen Maßnahmen noch 71 \% der Gesamtfinanzierung. Daraus wird eine verstärkte regionalpolitische Orientierung in den letzten Jahren deutlich. Vgl. EG-Parlament, Bericht im Namen des Landwirtschaftsausschusses über neue Leitlinien für die Agrarstrukturpolitik der Gemeinschaft, Teil B: Begründung, Berichterstatter: Herr Bernhard Thareau, Europäisches Parlament, Sitzungsdokumente 1983 - 1984, Dok. 1-923/83 B, vom 14.11.1983, S. 24; C. Thoroe, Die Interdependenzen von Agrarund Regionalpolitik im Hinblick auf die wirtschaftliche und soziale Integration der EG, in: Agrarwirtschaft, 30. Ja., 1981, S. 204 ff., hier: S. 206. 
modernisierung und Förderung der beruflichen Qualifikationen der Landwirte sowie zur Bewältigung spezifischer Probleme in Verbindung mit den gemeinsamen Marktorganisationen. Die regional begrenzten Aktionen betreffen vor allem die Landwirtschaft in Berggebieten und daneben spezifische Maßnahmen, die von forstwirtschaftlichen Vorhaben über Produktionsumstellungsmaßnahmen bis $\mathrm{zu}$ integrierten Entwicklungsprogrammen, bei denen verschiedene Maßnahmen auf bestimmte Regionen konzentriert werden, reichen. Der Beteiligungssatz des Ausrichtungsfonds beträgt je nach Maßnahmeart und betroffener Region zwischen $25 \%$ und 65 \& der Gesamtkosten. 1

Die Grundsatzentscheidungen über Art und Umfang der Maßnahmen werden vom Rat auf Vorschlag der Kommission in Form von Richtlinien oder Verordnungen ${ }^{2}$ beschlossen. ${ }^{3}$ Um eine Abstimmung mit den zur Verfügung stehenden Fondsmitteln zu gewährleisten, "werden die Maßnahmen nur nach einer eingehenden Finanzplanung erlassen, und die voraussichtlichen Gesamtkosten werden bei Erlaß festgelegt." 4

Im Hinblick auf die Entscheidungsfindung über die Strukturausgaben, die zumeist gemeinsam mit den jährlichen Preisbeschlüssen der Abteilung Garantie erfolgt, läßt sich insofern von einem gewissen Interessenausgleich sprechen ${ }^{5}$, als die preisstützenden Beschlüsse vor allem den (Uberschuß-) Erzeugerstaaten Vorteile verschaffen und die strukturellen Beihilfen in erster Linie den Mitgliedsstaaten mit zurückgebliebenen strukturen zugute kommen sollen. Insoweit dabei allerdings die eigentlichen strukturpolitischen ziele des Fonds in den Hintergrund treten, und er - so J. $\varnothing$. M $\phi 11$ er ${ }^{6}$ - lediglich

\footnotetext{
${ }^{1}$ Vgl. hierzu EG-Kommission, Die Agrarpolitik der Europäischen Gemeinschaften, S. 34 ff. sowie dies., Stärkung der Effizienz der Strukturfonds der Gemeinschaft, Bulletin der EG, Beilage 3/83, $19 \mathrm{f}$.

$2 \mathrm{zu}$ den Unterschieden val. B. Beutler, R. Bieber, J. Ripkorn, J. Streil, Die Europäische Gemeinschaft - Rechtsordnung und Politik, Baden-Baden 1979, S. $164 \mathrm{ff}$.

${ }^{3}$ Vgl. P. Gilsdorf, S. $262 \mathrm{ff}$.

4 Ebenda, S. 231.

5 ËEenda, S. $265 \mathrm{f}$.

${ }^{6} \mathrm{Vgl}$. J. Ø. Mфller, S. 102.
} 
die Funktion einer "Extra-Kasse" zur Kompensation unzureichender Begünstigungen aus den Preisbeschlüssen einnimmt, wird er zu einem Mechanismus rein finanzieller Umverteilung zugunsten bestimmter Mitgliedsstaaten. $\mathrm{Zu}$ einer ähnlichen Wertung, allerdings nur in bezug auf die horizontalen Maßnahmen, kommt auch das Europäische Parlament, das diese strukturbeihilfen mit einem "SelbstbedienungsBuffet" vergleicht, von dem einige Länder "mehr als ihren Anteil" erhalten und die Bedürftigen "vor einem prall gefüllten Tisch hungrig" bleiben. 1

Ohne daß an dieser Stelle eine eingehende Analyse der Verteilungswirkungen der Agrarstrukturausgaben erfolgen soll, läßt sich aus der Aufgliederung der Ausgaben nach Mitgliedsstaaten in Tabelle 33 doch feststellen, daß Umvertellungen zugunsten der struktur- und wirtschaftsschwachen Länder - mit Ausnahme Irlands und neuerdings auch Griechenlands - nicht bewirkt werden konnten. ${ }^{2}$ Die Abteilung Ausrichtung des Agrarfonds wird damit kaum als Instrument zwischenstaatlicher Umvertellung genutzt.

Neben den tatsächlichen Zahlungen sind in der Tabelle 33 auch die uber den Untersuchungszeitraum kumulierten Differenzen zu den Verpflichtungsermächtigungen angegeben. Damit werden noch bestehende Ansprliche aus den bisherigen Entscheidungen angegeben und wird der Trend der Zahlungen in künftigen Perioden sichtbar. Vor allem im Falle Italiens vermitteln die kumulierten Verpflichtungsermächtigungen ein zutreffenderes Blld von der Fondsbeteiligung, sie weisen aber gleichzeltig auch auf administrative probleme bei der Inanspruchnahme der Mittel hin.

Anders als die Ausgaben des Garantlefonds sind die strukturbeihilfen nicht mit dem Problem elner den tatsächlichen finanziellen Vorteilen nicht entsprechenden Zurechnung auf die Mitgliedsstaaten behaftet. Denn die Betelligungen der Gemelnschaft kommen unmittelbar

${ }^{1}$ Vgl. EG-Parlament, Sitzungsdokumente 1983 - 1984, Dok. 1-923/83 B, S. 26 .

2Als Maßstab siehe die Finanzierungsanteile in Tabelle 20 und die BIP-Relationen in Tabelle 1 . 
Tabelle 33

Ausgaben des EAGFL-Ausrichtung nach Mitgliedsstaaten $1980-1984$

\begin{tabular}{|c|c|c|c|c|c|c|}
\hline \multirow[t]{2}{*}{ Mitgliedsland } & 1980 & 1981 & lungen & 1983 & 1984 & $\begin{array}{c}\text { Verpflichtungen ab- } \\
\text { züglich Zahlungen } \\
1980-1984 \\
\end{array}$ \\
\hline & \multicolumn{6}{|c|}{ in Mio. ECU } \\
\hline Belgien & 25,2 & 21,7 & 17,7 & 18,1 & 12,9 & $-\quad 25,4$ \\
\hline Dänemark & 24,5 & 20,7 & 20,8 & 20,5 & 13,9 & 6,9 \\
\hline Deutschland & 142,1 & 134,1 & 107,1 & 107,7 & 89,3 & $-170,8$ \\
\hline Frankreich & 133,0 & 120,8 & 167,4 & 182,0 & 143,9 & $+87,1$ \\
\hline Griechenland & -- & -- & 14,5 & 21,9 & 52,8 & $+134,9$ \\
\hline Irland & 39,8 & 57,6 & 84,3 & 84,1 & 64,2 & $+40,4$ \\
\hline Italien & 97,0 & 78,5 & 125,0 & 103,0 & 127,8 & $+464,7$ \\
\hline Luxemburg & 1,0 & 2,3 & 1,6 & 0,6 & 3,9 & $+0,6$ \\
\hline Niederlande & 26,4 & 21,9 & 32,2 & 32,2 & 24,8 & $-18,4$ \\
\hline Verein. Königreich & 103,9 & 108,9 & 67,6 & 149,6 & 113,3 & $+\quad 5,8$ \\
\hline \multirow[t]{2}{*}{ EG } & 592,9 & 566,5 & 638,2 & 719,7 & 646,8 & $+512,0$ \\
\hline & \multicolumn{6}{|c|}{ in 8} \\
\hline Belgien & 4,2 & 3,8 & 2,8 & 2,5 & 2,0 & \\
\hline Dänemark & 4,1 & 3,6 & 3,2 & 2,8 & 2,2 & \\
\hline Deutschland & 24,0 & 23,7 & 16,8 & 15,0 & 13,8 & \\
\hline Frankreich & 22,4 & 21,3 & 26,2 & 25,3 & 22,2 & \\
\hline Griechenland & -- & -- & 2,3 & 3,0 & 8,2 & \\
\hline Irland & 6,7 & 10,2 & 13,2 & 11,7 & 9,9 & \\
\hline Italien & 16,4 & 13,9 & 19,6 & 14,3 & 19,8 & \\
\hline Luxemburg & 0,2 & 0,4 & 0,3 & 0,1 & 0,6 & \\
\hline Niederlande & 4,5 & 3,9 & 5,0 & 4,5 & 3,8 & \\
\hline Verein. Königreich & 17,5 & 19,2 & 10,6 & 20,8 & 17,5 & \\
\hline EG & 100,0 & 100,0 & 100,0 & 100,0 & 100,0 & \\
\hline
\end{tabular}

Quelle: EG-Rechnungshof, Jahresberichte, versch. Jg.; eigene Berechnungen. 
und in voller Höhe den jeweiligen Mitgliedsstaaten bzw. den dort ansässigen Landwirten zugute. Die Mittel des Agrarstrukturfonds gehören damit unter dem Aspekt der Zurechenbarkeit zu den "gesicherten" Ausgaben.

\subsection{Europäischer Fonds für regionale Entwicklung}

Im Unterschied zur Agrar- und Sozialpolitik wird die Regionalpoli$t i k^{1}$ in den europäischen Verträgen nicht zusammenhängend als $\mathrm{Be}-$ fugnis $z u$ einer gemeinschaftlichen Politik geregelt.

Es wird zwar in der Präambel des EWG-Vertrages das Ziel formuliert, "den Abstand zwischen einzelnen Gebieten und den Rückstand weniger begünstigter Gebiete zu verringern", es ist gemäß Art. 2 des EWGVertrages Aufgabe der Gemeinschaft, "eine harmonische Entwicklung des Wirtschaftslebens innerhalb der Gemeinschaft zu fördern", und es werden auch darüber hinaus noch einzelne regionalpolitische Probleme vertraglich erfaßt. Diese reichten aber als Grundlage für eine gemeinsame Regionalpolitik nicht aus. ${ }^{2}$ Die relative Bedeutungslosigkeit regionaler Aspekte beim Abschluß der Gemeinschaftsverträge wird auf die damalige Vorstellung zurückgeführt, daß die Entwicklung des Gemeinsamen Marktes - im Sinne einer Philosophie des laissez-faire - auch die bestehenden reciionalen Ungleichgewichte beseitigen wïrde. ${ }^{3}$

Allerdings zeigte sich, daB der wirtschaftliche Aufschwung innerhalb des gemeinsamen Marktes nicht die erwarteten positiven Auswirkungen hinsichtlich einer besseren Verteilung der wirtschaftli-

\footnotetext{
${ }^{1} \mathrm{Zu}$ einem Uberblick val. etwa D. Pinder, Regional Economic Development and Policy, Theory and Practice in the European Community, London 1983; P. Wäldchen, Einzelbericht zur Subventionspraxis des Europäischen Fonds für regionale Entwicklung, in: B. Börner, $M$. Bullinger (Hrsg.) subventionen im gemeinsamen Markt, Kölner Schriften zum Europarecht, Bd. 29, Köln, Berlin u.a. 1978, S. $345 \mathrm{ff}$.

2Vgl. B. Beutler, R. Bieber, J. Pipkorn, J. Streil, S. 405.

${ }^{3}$ Vgl. R. Jochimsen, P. Treuner, Grundprobleme der europäischen Regionalpolitik, in: W. von Urff (Hrsg.), Der Agrarsektor im Integrationsprozess, Baden-Baden 1975, S. 289 ff., hier: S. 289.
} 
chen Tätigkeit hatte, sondern daß sich die Ungleichgewichte zwischen den Regionen noch vergrößerten. ${ }^{1}$ Die Kommission unternahm deshalb bereits Ende der sechziger Jahre verstärkte Bemühungen, um eine gemeinschaftliche Regionalpolitik zu verwirklichen. ${ }^{2}$ Die Errichtung des Fonds verzögerte sich allerdings bis zum Jahre 1974, da zwischen den Mitgliedsstaten lange Zeit Uneinigkeit über seine Dotierung und Finanzierung sowie uber die Kriterien der Mittelvergabe bestand. 3

Neben der Zielsetzung, nunmehr elne wirksame Regionalpolitik betreiben zu können, wurde der neu geschaffene Fonds auch unmittelbar als ein Instrument dafür angesehen, die sich abzeichnende ungünstige Haushaltssituation des 1973 neu aufgenommenen Mitglieds Vereinigtes Königreich durch eine bevorzugte Mittelvergabe zu kompensieren. Zuaiem erhoffte sich das Parlament einen verstärkten EinfluB auf die Gemeinschaftspolitik, da es die Haushaltsmittel des Regionalfonds als nicht-obligatorische Ausgaben ansah, über deren haushaltsmäBige Festlegung es weltgehend autonom entscheiden konnte, ${ }^{4}$ und die etn Gegengewicht zu den vom Rat zu bestimmenden Agrarausgaben bilden sollten. 5

Der europäische Regionalfonds hat selne Tätigkeit im Jahre 1975 aufgenommen. 6 Setne Ausgestaltung wurde in den Jahren bis 1984 nur in elnigen Punkten modifizlert. ${ }^{7}$ Die Mittel des Fonds, die jährlich

${ }^{1}$ Vgl. EG-Kommisston, Europalscher Fonds fur regionale Entwicklung, Erster Jahresbertcht (1975), Bulletin der EG, Beilage 7/76,

S. 6 ff.; dies., Orlentierungsrahmen fur die Regionalpolitik der Gemeinschaft, Bulletin der EG, Bellage 2/77, S. 6 f.

2 Vgl. R. B. Talbot, The European Community's Regional Fund, in:

D. Diamond, J. B. Mcloughlin (Hrsg.), Progress in Planning, Vol. 8, S. 183 ff., hier: S. 192 ff.

${ }^{3}$ Vgl. etwa B. Beutler, R. Bleber, J. Pipkorn, J. Stre11, S. 407; E. Reister, S. 89.

${ }^{4} \mathrm{Zu}$ den $\mathrm{h}$ teraus entstandenen Auseinandersetzungen $\mathrm{zw}$ ischen den Gemelnschaftsorganen vgl, etwa D. Strasser, S. $233 \mathrm{f}$.

${ }^{5} \mathrm{vgl}$. J. $\emptyset$. Mфller, S. $109 \mathrm{f}$.

${ }^{6}$ Er wurde errichtet mit der Verordnung (EWG) Nr. 724/75 des Rates vom 18. März 1975 uber die Errichtung eines Europäischen Fonds für regtonale Entwicklung, in: Amtsblatt der EG, Nr. L 73 vom 21.3.1975. 7 Vgl. hterzu etwa D. Strasser, s. $228 \mathrm{f}$. 
im Gesamthaushaltsplan festgesetzt werden, sind für folgende Maßnahmen bestimmt: ${ }^{1}$

- Bis zu 95 \& seiner Ausstattung für Unterstützungsaktionen der von den Mitgliedsstaaten getroffenen regionalpolitischen Maßnahmen, wobei die Mittel nach einem festgelegten Verteilungsschlüssel auf die einzelnen Mitgliedsstaaten aufgestellt werden (Tabelle 34).

- Mit den restlichen 5 \& für nicht quotengebundene spezifische Gemeinschaftsmaßnahmen zur regionalen Entwicklung.

Tabelle 34

Länderquoten im Regionalfonds

\begin{tabular}{|l|c|c|c|r|r|}
\hline Mitgliedsland & $\begin{array}{l}\text { Ursprlingliche } \\
\text { Quoten }\end{array}$ & $\begin{array}{r}\text { 1. Revision } \\
\text { ab } 1979\end{array}$ & $\begin{array}{c}\text { 2. Revision } \\
\text { (Einbezie- } \\
\text { hung Grie- } \\
\text { chenlands) } \\
\text { ab } 1981\end{array}$ & $\begin{array}{r}\text { 3. Revision } \\
\text { ab 1985 } \\
\text { Unter- } \\
\text { grenze }\end{array}$ & $\begin{array}{r}\text { Ober- } \\
\text { grenze }\end{array}$ \\
\hline Belgien & 1,5 & 1,39 & 1,11 & 0,90 & 1,20 \\
Dänemark & 1,3 & 1,20 & 1,06 & 0,51 & 0,67 \\
Deutschland & 6,4 & $6, \infty 0$ & 4,65 & 3,76 & 4,81 \\
Frankreich & 15,0 & 16,86 & 13,64 & 11,05 & 14,74 \\
Griechenland & - & - & $13, \infty$ & 12,35 & 15,74 \\
Irland & 6,0 & 6,46 & 5,94 & 5,64 & 6,83 \\
Italien & 40,0 & 39,39 & 35,49 & 31,94 & 42,59 \\
Luxemburg & 0,1 & 0,09 & 0,07 & 0,06 & 0,08 \\
Niederlande & 1,7 & 1,58 & 1,24 & 1,00 & 1,34 \\
Verein. Königreich & 28,0 & 27,03 & 23,80 & 21,42 & 28,56 \\
\hline
\end{tabular}

Quelle: Bundesminister der Finanzen, Der Haushalt der Europäischen Gemeinschaften 1981, Dokumentation 3/81 vom 10.3.1981, S. 19; EG-Rat, Verordnung (EWG) Nr. 1787/84 des Rates vom 19. Juni 1984 betreffend den Europäischen Fonds für regionale Entwicklung, in: Amtsblatt der EG, Nr. L 169 vom 28.6 .1984$, S. 3 .

${ }^{1}$ Diese Regelungen gelten erst seit 1979. Vgl. Verordnung (EWG) Nr. 214/79 des Rates vom 6. Februar 1979 zur Änderung der Verordnung (EWG) Nr. $724 / 25$ uber die Errichtung eines Europäischen Fonds für regionale Entwicklung, in: Amtsblatt der EG, Nr. L 35 vom 9.2.1979. Seit dem 1. Januar 1985 tritt eine erneute Revision des Fonds in Kraft. 
Im Rahmen der quotengebundenen Abteilung beteiligt sich der ERFE an der Finanzierung von Investitionen vor allem im Infrastrukturbereich sowie im Industrie-, Handels- und Dienstleistungssektor. Dabei müssen die Vorhaben in Gebieten liegen, die von den nationalen Regierungen unter Anwendung der von der EG aufgestellten Beihilfegrundsätze als Förderregionen ausqewiesen sind und sich die Investitionen in ein regionales Entwicklungsprogramm einfügen. Die gemeinschaftlichen Beteiligungen betragen bis zu 30 \& der Investitionskosten bzw. 50 der von der öffentlichen Hand gewährten Beihilfen. 1

Im Rahmen des nicht quotengebundenen Teils des Fonds beteiligt sich die Gemeinschaft an regionalpolitischen Vorhaben, die sich sowohl in bezug auf die Förderungsprojekte als auch hinsichtlich der Förderungsregionen von den quotengebundenen Maßnahmen unterscheiden können. Damit soll den spezifisch regionalpolitischen Prioritäten der Gemeinschaft Rechnung getragen werden. Angesichts der beschränkten Mittel kann allerdings auch nach Auffassung der Kommission "von einer wirklichen Änderung der Aktionsbedingungen des ERFE" ${ }^{2}$ nicht gesprochen werden.

Mit der vertraglichen Fixierung der länderspezifischen Relationen in der quotengebundenen Abteilung sind die Verteilungswirkunqen des Regionalfonds praktisch festgelegt. Danach sind über drei Viertel der Fondsmittel für die vier wirtschaftsschwächeren Gemeinschaftsländer Griechenland, Irland, Italien und Großbritannien vorgesehen. Stellt man in diesem zusammenhang sinnvollerweise auf die Zuflüsse pro kopf der jeweiligen Bevölkerung ab, dann erhält Irland fast doppelt soviel zuwendungen wie Griechenland und mehr als fünf- bzw. sechsmal soviel wie Italien bzw. Großbritannien. Daneben ist bemerkenswert, daß von den wohlhabenderen Gemeinschaftsländern Frankreich und Dänemark im Vergleich zu ihren BIP-Anteilen zwar unter-

${ }^{1}$ Vgl. Art. 2 ff. der Fondsverordnung.

${ }^{2}$ EG-Kommission, Stärkung der Effizienz der strukturfonds der Gemeinschaft, S. 22. 
durchschnittlich, aber immer noch erheblich an dem Fonds partizipieren, während die Betelligung der übrigen Mitgliedsstaaten recht gering ist. Ein ähnliches Bild ergibt sich, wenn man die Mittelverteilung des quotenfreien Fonds betrachtet. 1 Insgesamt bewirkt der Regionalfonds damit - im Rahmen seines Volumens - erhebliche Umverteilungen zugunsten der wirtschaftsschwächeren Gemeinschaftsländer.

Die quotale Aufteilung der Fondsmittel entspricht im wesentlichen den jährlich uber den Haushalt geleisteten Zahlungen, wie sie in Tabelle 35 dargestellt sind. Die bestehenden Unterschiede resultieren daraus, daß die ursprünglichen Mittelbindungen noch nicht und nicht gleichmäBig ausgenutzt werden, und daß die im Haushalt angewiesenen Zahlungsermächtigungen nicht immer vollständig in Anspruch genommen werden. Diese Differenzen werden auch durch den Bestand an noch nicht ausgezahlten Mittelbindungen verdeutlicht. Darin zeigt sich etwa die starke Ausnutzung der Mittel vor allem durch Irland und - wie bei den Agrarstrukturausgaben - die recht schwache Inanspruchnahme im Falle Italiens.

Für die weitere verteilungspolitische Würdigung der über den Regionalfonds geleisteten Ausgaben ist zunächst die Feststellung wichtig, daß die länderspezifische Zurechnung der Mittel insofern unproblematisch ist, als sie den Wirtschaftseinheiten der Mitgliedsstaaten unmittelbar zufließen und keinerlei Abgrenzungsprobleme auftreten. Die Rückflüsse aus dem Regionalfonds können daher ebenso wie diejenigen des landwirtschaftlichen Ausrichtungsfonds unter dem Gesichtspunkt der verteilungsmäßigen Zurechenbarkeit als "gesicherte" Ausgaben angesehen werden.

In diesem zusammenhang ist weiterhin die Frage nach dem Verhältnis von gemeinschaftlicher und mitgliedsstaatlicher Regionalpolitik bedeutsam, d.h. ob die Mittel wie beabsichtigt eine Ergänzung zu den einzelstaatlichen Hilfen sind, oder ob sie nicht nur an deren stel-

\footnotetext{
${ }^{1}$ Vgl. EG-Kommission, Europäischer Fonds für regionale Entwicklung (Achter Bericht der Kommission an den Rat), KOM (83) 566 endg., Brüssel, 3.10.1983, S. 46.
} 
Tabelle 35

Ausgaben des Regionalfonds nach Mitgliedsstaaten $1980-1984$

\begin{tabular}{|c|c|c|c|c|c|c|}
\hline Mitgliedsland & 1980 & 1981 & 1982 & 1983 & 1984 & $\begin{array}{l}\text { noch nicht gezahlte Mit- } \\
\text { telbindungen zum } \\
31.12 .1984\end{array}$ \\
\hline \multicolumn{7}{|c|}{ in MiO. ECU } \\
\hline Belgien & 6,6 & 9,2 & 10,8 & 7,0 & 6,0 & 48,5 \\
\hline Dänemark & 9,4 & 10,7 & 14,6 & 16,7 & 28,1 & 25,0 \\
\hline Deutschland & 50,4 & 36,2 & 61,6 & 45,0 & 43,9 & 156,2 \\
\hline Frankreich & 99,7 & 66,8 & 130,0 & 219,3 & 201,7 & 320,5 \\
\hline Griechenland & -- & 122,0 & 152,3 & 214,6 & 216,7 & 616,1 \\
\hline Irland & 69,6 & 80,3 & 93,5 & 94,5 & 104,1 & 139,7 \\
\hline Italien & 249,1 & 211,7 & 281,8 & 344,5 & 435,1 & 2129,8 \\
\hline Luxemburg & 1,0 & 0,9 & 0,1 & 0,0 & 2,5 & 4,9 \\
\hline Niederlande & 7,7 & 5,7 & 3,2 & 18,1 & 14,8 & 77.8 \\
\hline Verein. Königreich & 233,2 & 255,2 & 225,1 & 296,2 & 297,7 & 1023,3 \\
\hline EG & 726,7 & 798,7 & 973,0 & 1255,9 & 1350,6 & 4541,7 \\
\hline \multicolumn{7}{|c|}{ in 8} \\
\hline Belgien & 0,9 & 1,2 & 1,1 & 0,6 & 0,4 & 1,1 \\
\hline Dänemark & 1,3 & 1,3 & 1,5 & 1,3 & 2,1 & 0,6 \\
\hline Deutschland & 6,9 & 4,5 & 6,3 & 3,6 & 3,3 & 3,4 \\
\hline Frankreich & 13,7 & 8,4 & 13,4 & 17,5 & 14,9 & 7,1 \\
\hline Griechenland & - & 15,3 & 15,7 & 17,1 & 16,1 & 13,6 \\
\hline Irland & 9,6 & 10,1 & 9,6 & 7,5 & 7,7 & 3,1 \\
\hline Italien & 34,3 & 26,5 & 29,0 & 27,4 & 32,2 & 46,3 \\
\hline Luxemburg & 0,1 & 0,1 & 0,0 & 0,0 & 0,2 & 0,1 \\
\hline Niederlande & 1,1 & 0,7 & 0,3 & 1,4 & 1,1 & 1,7 \\
\hline Verein. Königreich & 32,1 & 31,9 & 23,1 & 23,6 & 22,0 & 22,5 \\
\hline EG & 100,0 & 100,0 & 100,0 & 100,0 & 100,0 & 100,0 \\
\hline
\end{tabular}

Quelle: EG-Rechnungshof, Jahresbericht, versch. Jg.; EG-Kommission, Neunter Bericht uber den EFRE. 
le treten, und der Regionalfonds damit ein reines Umverteilungsinstrument darstellt. Zwar wird in der Fondsverordnung ${ }^{1}$ und auch von der Kommission ${ }^{2}$ der Aspekt der generellen Zusätzlichkeit als Kriterium fur die Vergabe der Gemeinschaftsmittel betont; ${ }^{3}$ ein fundiertes Urteil uber die praktische Umsetzung dieses Grundsatzes ist allerdings außerordentlich schwierig. ${ }^{4}$ Es kann sicherlich davon ausgegangen werden, daB die Mitgliedsstaaten zumindest teilweise die Fondsbeihilfen als Ersatz für ihre nationalen Subventionen verwenden $^{5}$. J. $\emptyset$. Mфller kommt sogar zu dem Ergebnis, daß "the EEC Regional Fund does not therefore have much, if anything, to do with regional policy. It is instead a mechanism for the transfer of appropriations to member states using specific scales of distribution. Roughly speaking, the purpose is to transfer amounts to the UK, Italy and Ireland ${ }^{6}$ from the other member states." 7

Diese Auffassung wird durch die Ergebnisse eines Sondergutachtens des EG-Rechnungshofes weitgehend bestätigt. ${ }^{8}$ Darin wird festgestellt, daß die von den Mitgliedsstaaten vorgelegten Entwicklungsprogramme für die Beurteilung der Vorhaben durch die Kommission nur von geringem Nutzen sind, die Auswahl nicht den vorrangig zu fördernden Bereichen entspricht, und daß die Mitgliedsstaaten kaum mehr Beihilfeanträge vorlegen, als es ihrer Quote entspricht und

1Vgl. Art. 4 der Verordnung.

${ }^{2}$ Vg1. Kommission der EG, Europäischer Fonds für regionale Entwicklung, Erster Jahresbericht, 1975, S. $17 \mathrm{ff}$.

${ }^{3}$ Vgl. B. Beutler, R. Bieber, J. Pipkorn, J. Streil, S. 409.

${ }^{4} \mathrm{Vgl}$. Kommission der EG, Europäischer Fonds für regionale Entwicklung (Achter Bericht der Kommission an den Rat), S. $71 \mathrm{ff}$.

${ }^{5}$ So die EG-Kommission, Entschließung zum Vierten Jahresbericht (1978) der Kommission der EG über den Europäischen Fonds für regionale Entwicklung (ERFE), in: Amtsblatt der EG, Nr. C 117 vom 12.5 .1980$, S. 22 .

${ }^{6} \mathrm{Ab} 1981$ gehört zu diesen wirtschaftsschwächeren Gemeinschaftsländern auch Griechenland.

7. $\varnothing$. Mфller, S. 110.

${ }^{8}$ EG-Rechnungshof, Bericht des Rechnungshofes der Europäischen Gemeinschaften aufgrund der Erklärung des Europäischen Rates vom 18. Juni 1983, in: Amtsblatt der EG, Nr. C 287 vom 24.10.1983, S. $12 \mathrm{ff}$. 
damit der Auswahlvorgang von vornherein begrenzt wird. Zudem lie $B$ sich ein ergänzender Charakter des EFRE nicht feststellen, so daB die gemeinsame Regionalpolitik offenbar kaum mehr bewirkt, als einen Teil der Kosten für Maßnahmen zu übernehmen, die ausschließlich im nationalen Interesse liegen.

Die in dem Regionalfonds liegenden Umverteilungsmöglichkeiten werden allerdings durch das relativ geringe Volumen begrenzt. Die Regionalausgaben sind zwar seit der Gründung des ERFE weit stärker als das Haushaltsvolumen angestiegen, hatten sich aber $\mathrm{zu}$ Beginn der achtziger Jahre bei 4,5 \& des Budgetumfangs eingependelt, sind 1984 auf über 58 angestiegen ${ }^{1}$ und liegen nach den Haushaltsplänen für 1985 und 1986 bei 5,7 bzw. $7,4 \% .^{2}$

Mit dem Jahre 1985 tritt eine erneute Revision des Regionalfonds in Kraft. ${ }^{3}$ Die wesentlichen Neuerungen im Vergleich zur bisherigen Regelung bestehen darin, da $\beta$

- das System starrer Länderquoten von einem Margensystem abgelöst wird, innerhalb dessen die Anteile der Mitgliedsstaten variieren können (Tabelle 34 ).

- die nicht-quotengebundene Abteilung aufgehoben wird; dafür sollen insgesamt die gemeinschaftlichen Interessen stärker berücksichtigt und die gemeinschaftliche Einflußnahme verstärkt werden.

- der Beteiligungssatz des Regionalfonds für Infrastrukturprojekte merklich erhöht wird.

Bei einer ersten Beurteilung der Neuregelung ist festzustellen, da $\beta$ sich daraus kaum Veränderungen im Hinblick auf die länderspezifische Verteilung der Fondsmittel ergeben, denn die Mittelwerte der neuen Ländermargen entsprechen im wesentlichen den derzeit garan-

\footnotetext{
${ }^{1}$ Siehe Tabelle 25.

${ }^{2}$ EG-Kommission, Bulletin der EG, Nr. 6/1985, S. $110 \mathrm{f}$.

${ }^{3}$ Niedergelegt in der Ratsverordnung $\mathrm{Nr}$. 1787/84.
} 
tierten Länderquoten. Dem ursprünglichen Vorschlag der Kommission, die Ausgaben ausschlieBlich auf die ärmsten Regionen zu konzentrieren, wurde damit nicht gefolgt. In welchem Maße der Gestaltungsspielraum der Kommission bei der Auswahl der künftigen Projekte tatsächlich ausgeweitet wird, und eine Abkehr von der bisherigen Erstattungspraxis hin $z u$ einer gemeinschaftlichen Mitbestimmung vollzogen werden kann, bleibt abzuwarten.

\subsection{Europäischer Sozialfonds}

Für eine gemeinschaftliche Sozialpolitik ${ }^{1}$ war neben weiteren, allgemein gehaltenen sozialpolitischen Zielvorstellungen im Gründungsvertrag die Errichtung eines Europäischen Sozialfonds (ESF) vorgesehen. In Relation zu den Bestimmungen über die Zollunion und die gemeinsame Agrarpolitik waren diese Vorschriften allerdings nur von begrenzter Tragweite. Ursache hierfür war ähnlich wie im Falle der Regionalpolitik die ursprüngliche Vorstellung, daß die Entwicklung des gemeinsamen Marktes schon für eine optimale Allokation der Ressourcen und ein ökonomisches Wachstum Sorge tragen werde und der Sozialfonds lediglich Fehlentwicklungen zu korrigieren hätte und Ubergangsschwierigkeiten mildern sollte. ${ }^{2}$ Im Laufe der Zeit und unter dem Eindruck der wirtschaftlichen, strukturellen und arbeitsmarktpolitischen Entwicklung wurde die Notwendigkeit einer eigenständigen Sozialpolitik allerdings immer deutlicher und führte auch zu einem verstärkten sozialpolitischen Engagement der Gemeinschaft.

\footnotetext{
${ }^{1} \mathrm{zu}$ einem - auch die nichtfinanzielle Politik berücksichtigenden Uberblick vgl. M. Shanks, European Social Policy: Today and Tomorrow, Oxford 1977; R. Rummel, Soziale Politik für Europa. Ein integrationspolitisches Konzept, Bonn 1975; W. Stabenow, Subventionen im gemeinsamen Markt: Subventionen aus Gemeinschaftsmitteln - Der Europäische Sozialfonds -, in: B. Börner, M. Bullinger (Hrsg.), Subventionen im gemeinsamen Markt, Kölner Schriften zum Europarecht, Bd. 29, Köln, Berlin u.a. 1978, s. $313 \mathrm{ff}$.

${ }^{2}$ Vgl. D. Masberg, P. Pintz, Die Sozialpolitik der Europäischen Gemeinschaft, in: M. v. Hauff, B. Pfister-Gaspary, Internationale Sozialpolitik, Stuttgart, New York 1982, S. 51 ff., hier: S. 52 ff.
} 
Das in Art. 123 des EWG-Vertrages formulierte ziel des Sozialfonds, "die Beschäftigungsmöglichkeiten der Arbeitskräfte im gemeinsamen Markt zu verbessern und damit zur Hebung der Lebenshaltung beizutragen" weist den Fonds vornehmlich als arbeitsmarktpolitisches Instrument aus, das den strukturwandel in der Gemeinschaft sozial absichern helfen soll. Der erste Sozialfonds ${ }^{1}$ hatte eine Laufzeit von 1960 bis 1976. Er wurde 1972 von einem zweiten Fonds ${ }^{2}$ abgelöst, der durch eine Ausdehnung des Aktionsbereichs, eine nunmehr ${ }^{3}$ an Gemeinschaftskriterien orientierte Mittelvergabe und eine umfangreichere finanzielle Ausstattung gekennzeichnet war und damit ein flexibleres und wirksameres Instrument der Sozial- und Beschäftigungspolitik darstellte. ${ }^{4}$ Im Gegensatz zu seinem Vorgänger, der durch spezielle Beiträge der Mitgliedsstaaten finanziert wurde, war der "neue" Fonds voll in den Gesamthaushalt integriert. Seine Mittel werden den nicht-obligatorischen Ausgaben zugerechnet und liegen damit in der Entscheidungskompetenz des Parlaments. Dieses hat die quantitative Entwicklung des Sozialfonds insofern positiv beeinflußt, als es seine haushaltspolitische Manövriermasse zumeist voll ausnutzte. 5

Eine Revision des Sozialfonds erfolgt Ende der siebziger Jahre, dessen wichtigste Ergebnisse eine allgemeine Ausdehnung des personalen Anwendungsbereichs, eine stärkere regionalpolitische Ausrichtung sowie weitergehende Maßnahmen zugunsten der beschäftigungslosen Jugendichen waren. ${ }^{6}$ Nach einer erneuten Modifikation der

${ }^{1}$ Verordnung Nr. 9 über den Europäischen Sozialfonds, in: Amtsblatt der EG 1960, S. 1189 ff.

2 Beschluß des Rates vom 1. Februar 1971 über die Reform des Europäischen Sozialfonds, in: Amtsblatt der EG Nr. L 28 vom 4.2.1971.

3 zuvor wurden die Anträge - im Rahmen der verfügbaren Mittel quasi automatisch gebilligt und der Fonds hatte eher den Charakter einer "Reservekasse der nationalen Arbeitsmarktinstanzen". Vgl. P. Gloystein, Finanzierung des industriellen Strukturwandels durch die EG, Hamburg 1978, S. 221.

${ }^{4}$ Vgl. EG-Kommission, Die Sozialpolitik der Europäischen Gemeinschaften, Europäische Dokumentation 5/1983, S. 19.

${ }^{5}$ Vgl. D. Strasser, S. 216.

${ }^{6}$ Sämtliche diesbezügliche Rechtsakte sind im Amtsblatt der EG, Nr. L 337 vom 27.12.1977 veröffentlicht. 
Fondsvorschriften sollen ab 1984 mindestens $75 \%$ der insgesamt verfügbaren Mittel zur Förderung der Beschäftigung von Jugendlichen eingesetzt werden. 1

Die Interventionen der Gemeinschaft bestehen in Beteiligungen, die normalerweise 50 \& der einzelstaatlichen Ausgaben betragen. Dabei sind zwei Maßnahmentypen zu unterscheiden. Bei Vorhaben nach Art. 4 der Fondsverordnung ist für die Intervention ein besonderer $\mathrm{Be}-$ schluß des Rates erforderlich, der bisher in sechs Fällen zur Unterstützung verschiedener Personenkreise erfolgt ist. ${ }^{2}$ Die Maßnahmen nach Art. 5, die jährlich mindestens $50 \%$ der gesamten Fondsmittel ausmachen müssen, sind für schwerwiegende sektorale und regionale Beschäftigungsprobleme vorgesehen und haben sich in bestimmte nationale Arbeitsmarktprogramme einzufügen. In allen Fällen werden die von den Mitgliedsstaaten eingereichten Anträge von einem Fondsausschuß geprüft und von der Kommission im Rahmen der vorhandenen Mittel bewilligt. Ihre Entscheidungspraxis orientiert sich an den jährlich festzulegenden Leitlinien, die auf die wirtschaftliche und soziale Lage in der Gemeinschaft abstellen, vor allem aber von der Situation der Arbeitsmärkte bestimmt werden und auch die Erfordernisse regionaler und sektoraler Wirtschaftspolitik berücksichtigen. 3

Entsprechend der Aufgabenstellung fließt der größte Teil - im Durchschnitt der letzten Jahre etwa zwei Drittel - der Fondsmittel den drei bzw. vier wirtschaftsschwächsten Mitgliedsstaaten zu (Tabelle 36), die gleichzeitig auch mit die gröBten Beschäftigungsprobleme aufweisen. ${ }^{4}$ Stellt man auch hier wieder auf die Pro-Kopf-Relationen

${ }^{1}$ Vgl. EG-Kommission, Der Europäische Sozialfonds, Stichwort Europa 2/84, Januar 1984, S. 4 .

${ }^{2}$ Vgl. EG-Kommission, Die Sozialpolitik der Europäischen Gemeinschaft, S. 20.

${ }^{3}$ Vgl. W. Stabenow, S. 337. Zum Vergabeverfahren im einzelnen vgl. EG-Kommission, Elfter Tätigkeitsbericht des Europäischen Sozialfonds, Haushaltsjahr 1982, KOM (83) 434 endg., Brüssel, 30.6.1983, S. $11 \mathrm{ff}$.

${ }^{4} \mathrm{Vgl}$. Eurostat, Employment and Unemployment, Statistical Bulletin, 2 - 1983 . 
Tabelle 36

Ausgaben des Sozialfonds nach Mitgliedsstaaten $1980-1984$

\begin{tabular}{|c|c|c|c|c|c|c|}
\hline Mitgliedsland & 1980 & 1981 & 1982 & 1983 & 1984 & $\begin{array}{l}\text { noch nicht gezahl- } \\
\text { te Mittelbindungen } \\
\text { zum } 31.12 .1984\end{array}$ \\
\hline & \multicolumn{6}{|c|}{ in Mio. ECU } \\
\hline Belgien & 12,1 & 15,3 & 16,8 & 20,6 & 52,1 & 90,6 \\
\hline Dänemark & 14,7 & 18,5 & 17,6 & 14,7 & 68,7 & 79,7 \\
\hline peutschland & 80,5 & 72,3 & 89,9 & 81,5 & 63,8 & 167,8 \\
\hline Frankreich & 195,8 & 155,3 & 119,3 & 140,5 & 225,7 & $42.5,1$ \\
\hline Griechenland & -- & 6,6 & 23,5 & 20,4 & 71,3 & 164,0 \\
\hline Irland & 72.5 & 60,4 & 115,0 & 134,2 & 131,4 & 216,5 \\
\hline Italien & 194,4 & 207,1 & 235,1 & 221,2 & 368,5 & 953,5 \\
\hline Luxemburg & 0,4 & 0,6 & 1,1 & 0,3 & 0,5 & 1,8 \\
\hline Niederlande & 5,1 & 14,3 & 9,0 & 12,6 & 14,1 & 72,4 \\
\hline Verein. Königreich & 159,7 & 195,4 & 278,3 & 244,9 & 610,2 & $6 \cap 3,6$ \\
\hline \multirow[t]{2}{*}{ EG } & 735,2 & 745,8 & 905,6 & 890,9 & 1606,3 & 2775,0 \\
\hline & \multicolumn{6}{|c|}{ in 8} \\
\hline Belgien & 1,6 & 2,1 & 1,9 & 2,3 & 3,2 & 3,3 \\
\hline Dänemark & 2,0 & 2,5 & 1,9 & 1,7 & 4,3 & $2, a$ \\
\hline Deutschland & 11,0 & 9,7 & 9,9 & 9,1 & 4,0 & 5,0 \\
\hline Frankreich & 26,6 & 20,8 & 13,2 & 15,8 & 14,1 & 15,3 \\
\hline Griechenland & -- & 0,9 & 2,6 & 2,3 & 4,4 & 5,9 \\
\hline Irland & 9,9 & 8,1 & 12,7 & 15,1 & 8,2 & 7,8 \\
\hline Italien & 26,4 & 27,7 & 26,0 & 24,8 & 22,9 & 34,4 \\
\hline Luxemburg & 0,1 & 0,1 & 0,1 & 0,0 & 0,0 & 0,1 \\
\hline Niederlande & 0,7 & 1,9 & 1,0 & 1,4 & 0,9 & 2,6 \\
\hline Verein. Königreich & 21,7 & 26,2 & 30,7 & 27,5 & 38,0 & 21,8 \\
\hline EG & 100,0 & 100,0 & 100,0 & 100,0 & 100,0 & 100,0 \\
\hline
\end{tabular}

Quelle: EG-Rechnunashof, Jahresbericht, versch. Jg.; F.G-Kommission, Zwölfter und Nreizehnter Tätigkeitshericht des FSF. 
ab, dann erhält - wie beim Agrarstruktur- und Regionalfonds - Irland mit Abstand die höchsten Zuweisungen, etwa sechsmal soviel wie Italien und Großbritannien. Daneben ist aber - analog zum Regionalfonds - auch auf die relativ hohen Beteiligungen der wirtschaftsstarken Länder Frankreich und Dänemark hinzuweisen. 1 Die Berücksichtigung dex noch zu zahlenden Beträge aus den bisher vorgenommenen Mittelbindungen ergibt zwar ein ähnliches Bild. Bemerkenswert ist allerdings auch hier wiederum die stark unterschiedliche Ausnutzung Italiens und die überproportionale Inanspruchnahme durch Irland und Großbritannien.

Obwohl die gemeinschaftlichen Möglichkeiten für eine aktive Beschäftigungs- und Sozialpolitik seit der Institutionalisierung des zweiten Sozialfonds stark ausgeweitet worden sind, betragen die gegenwärtig zur Verfügung stehenden Mittel nur etwa 6 \& des Gesamthaushaltsvolumens (Tabelle 25). Die Gemeinschaftsausgaben machen auch nur einen Bruchteil der im nationalen Bereich getätigten Sozialausgaben aus, so daß die Sozialpolitik nach wie vor als einzelstaatliche Domäne bestehen bleibt. ${ }^{2}$ Dem Sozialfonds wird deshalb auch in erster Linie eine Anreizfunktion für die Maßnahmen der Mitgliedsstaaten zuerkannt werden können. ${ }^{3}$ Allerdings handelt es sich auch hier häufig nicht um Maßnahmen, die auf Initiative der Gemeinschaft durchgeführt werden, sondern bewirken unzureichende zieldefinitionen und Kriterien für die Auswahl der Förderungsprogramme, daß es sich bei den Ausgaben lediglich um Rückerstattungen für Maßnahmen handelt, die die Mitgliedsstaaten ohnehin finanziert hätten. ${ }^{4}$

Im Hinblick auf eine korrekte Zuweisung der Ausgaben auf die Mitgliedsstaaten ergeben sich keine Probleme, so daß auch die Rückflüsse aus dem Sozialfonds unter dem Aspekt der verteilungspolitischen Zurechenbarkeit als "gesicherte" Ausgaben einzuordnen sind.

${ }^{1}$ Siehe auch hierzu als Maßstab die Finanzierungsanteile in Tabelle 20 und die BIP-Relationen in Tabelle 1.

${ }^{2} \mathrm{Vgl}$. D. Masberg, P. Pintz, S. 51.

${ }^{3}$ Vgl. E. Reister, S. 72; R. A. B. Leaper, Social Policy, in: D. Lasok, P. Soldatos (Hrsg.), Les communautéés européeunes en fonctiomnement, Brüssel 1981, S. $273 \mathrm{ff}$., hier: S. $28 \overline{3}$.

${ }^{4} \mathrm{Vgl}$. EG-Rechnungshof, Bericht aufgrund der Erkläruna des Europäischen Rates vom 18. Juni 1983, S. $14 \mathrm{f}$. 
4.1.3.2.4. Spezifische Maßnahmen

Außerhalb der Strukturfonds werden vor allem in den letzten Jahren eine Reihe weiterer Aktionen mit regional- und sozialpolitischem Charakter durchgeführt.

Im weiteren Sinne zur Regionalpolitik gehören die Zinssubventionen für Darlehen, die im Zusammenhang mit dem Europäischen Währungssystem gewährt werden. ${ }^{1}$ Dabei handelt es sich um flankierende Maßnahmen, mit deren Hilfe die Wirtschaft der weniger wohlhabenden Mitgliedsstaaten des EWS gestärkt werden soll, um die wirtschaftlichen Unterschiede $z u$ den übrigen Staaten $z u$ vermindern. Die Darlehen, die vorwiegend zur Förderung von Infrastrukturinvestitionen zu verwenden sind, werden von der Europäischen Investitionsbank und von der EWG im Rahmen des Neuen Gemeinschaftsinstruments aufgebracht $^{2}$ und für den Zeitraum von 1979 bis 1983 in Jahresraten von 1 Mrd. ECU den Ländern Irland und Italien zur Verfügung gestellt. 3 Sie sind mit einer über den Gesamthaushalt zu finanzierenden $\mathrm{Z}$ insverbilligung von 3 \& ausgestattet, für die - abgezinst - jährlich 200 Mio. ECU $z u$ veranschlagen sind. Weiterhin werden $z$ inszuschüsse für Darlehen zugunsten von Wiederaufbaumaßnahmen in erdbebengeschädigten Gebieten Italiens und Griechenland gewährt.

${ }^{1}$ Niedergelegt in der Verordnung (EWG) Nr. $1736 / 79$ des Rates vom 3. August 1979 über Zinszuschüsse für bestimmte im Rahmen des Europäischen Währungssystems gewährte Darlehen, in: Amtsblatt der EG, Nr. L 200 vom 8.8.1979, S. $1 \mathrm{ff}$. 2

hierzu Abschnitt 4.2.3.

${ }^{3}$ Da das Entwicklungsgefälle zwischen den Mitgliedsstaaten nach wie vor besteht, hat die Kommission dem Rat einen Verordnungsvorschlag übermittelt, mit dem das zinszuschußsystem für bestimmte Darlehen um zwei weitere Jahre verlängert werden soll. Kommission der EG, Vorschlag für eine Verordnung (EWG) des Rates über Zinszuschüsse für bestimmte im Rahmen des Europäischen Währungssystems gewährten Darlehen, in: Amtsblatt der EG Nr. C 163 vom 22.6.1983. Dieser Vorschlag ist bis zum Jahre 1985 vom Rat noch nicht angenommen worden. Vgl. EG-Kommission, 18. Gesamtbericht 1984 , S. 68. Dieser Vorschlaq ist his zum Jahre 1985 vom Rat noch nicht. anqenommen worden. Val. EG-Kommission, 18. Cesamtbericht 1984, S. 68. 
Tabelle 37

Zinszuschüsse des Gesamthaushalts 1980 - 1984

\begin{tabular}{|c|c|c|c|c|c|}
\hline Empfängerland & 1980 & 1981 & $19 \overline{82}$ & 1983 & 1984 \\
\hline & \multicolumn{4}{|c|}{ MiO. ECU } & \\
\hline Irland & 67,2 & 66,6 & 72,7 & 50,8 & -- \\
\hline Italien & 129,8 & 122,3 & 145,6 & 190,0 & 21,7 \\
\hline Griechenland & -- & -- & -- & 1,2 & 2,7 \\
\hline Gesamt & 197,0 & 188,9 & 218,3 & 242,0 & 24,4 \\
\hline
\end{tabular}

Quelle: EG-Kommission, Stärkung der Effizienz der Strukturfonds der Gemeinschaft, S. 27; EGRechnungshof, Jahresberichte; versch. Jg.

Als spezifische Maßnahmen mit regionaler Zweckbestimmung gelten nach der Haushaltssystematik auch diejenigen Ausgaben, die im Rahmen der Entlastungsmaßnahmen zugunsten des Vereinigten Königreichs ausgeführt werden. Denn dabei handelt es sich um Ausgaben, die vereinbarungsgemä $B$ für regionale Vorhaben $z u$ verwenden sind. Seit 1983 werden die Entlastungen auch in Form von Ausgaben für den Energie- und Verkehrsbereich durchgeführt, so daß auch sie im Interesse einer sachlich zusammengehörigen Darstellung an dieser Stelle berücksichtigt werden sollen. Die Finanzausgleichsregelungen und Entlastungsmaßnahmen sind im einzelnen im Kapitel 4.1.2. dargestellt und analysiert worden, so daß im folgenden nur noch auf zwei Gesichtspunkte einzugehen ist.

Der erste Aspekt betrifft die zeitliche Ausführung der Entlastungsmaßnahmen und ihre Berücksichtigung bei der Ermittlung der korrigierten Nettopositionen. Dabei gehen sowohl die Ausgaben als auch die damit verbundenen Finanzierungsvorgänge jeweils in dem Jahr in die Berechnung der Nettopositionen ein, für das sie vorgesehen und beschlossen wurden, während sie erst im darauffolgenden Jahr im 
Haushalt veranschlagt und ausgeführt werden (Tabelle 38). Insofern liegt hier also ein Unterschied zur Behandlung der übrigen Ausgaben und Einnahmen vor.

\section{Tabelle 38}

Ausgaben im Zusammenhang mit den britischen

Entlastungsmaßnahmen - Ausführung und Auswirkungen auf die Nettopositionen $1980-1984$

\begin{tabular}{|c|c|c|c|c|c|}
\hline \multirow[t]{2}{*}{ Jahr } & $\begin{array}{l}\text { Ausfül } \\
\text { Verein. } \\
\text { König- } \\
\text { reich }\end{array}$ & $\begin{array}{l}\text { Denga } \\
\text { Deutsch- } \\
\text { land }\end{array}$ & \multicolumn{3}{|c|}{$\begin{array}{l}\text { Berücksichtigung bei der Ermittlung } \\
\text { der Nettopositionen } \\
\begin{array}{r|r|r}\text { Verein. } & \text { Königreich } & \text { Deutschland } \\
\text { Bruttoent- } & \text { Nettoent- } & \text { Bruttoentla- } \\
\text { lastung } & \text { lastung } & \text { stung } \\
\end{array}\end{array}$} \\
\hline & \multicolumn{5}{|c|}{ Mio. ECU } \\
\hline 1980 & 174,2 & -- & 1437,6 & 1175 & -- \\
\hline 1981 & 1248,4 & -- & 1804,2 & 1410 & -- \\
\hline 1982 & 1819,2 & -- & $1400^{b}$ & 1048 & $272,5^{\mathrm{C}}$ \\
\hline 1983 & 1400 & 272,5 & $991^{d}$ & 750 & $211^{e}$ \\
\hline 1984 & 991 & 211,0 & & & \\
\hline
\end{tabular}

$a_{b i s}$ einschl. 1982 Ist-Ergebnisse, danach Haushaltsansätze

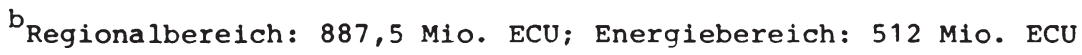

$c_{\text {Energiebereich }}$

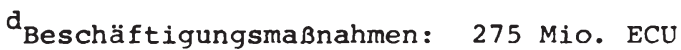

Energiebereich: 255 Mio. ECU

Verkehrsbereich: 461 Mio. ECU

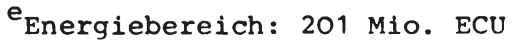

Verkehrsbereich: $10 \mathrm{MIO}$. ECU

Quelle: EG-Rechnungshof, Jahresbericht 1981, S. 116; Kommission der EG, Bulletin 12/1983, S. 95; 6/1984, S. 98 .

Der zweite Aspekt bezieht sich auf die Frage, inwieweit die Entlastungen überhaupt als Bestandteil einer gemeinschaftlichen Politik angesehen werden können oder in welchem Maße es sich lediglich um allgemeine finanzielle Rückzahlungen handelt. Bezüglich der für die 
Haushaltsjahre 1980 und 1981 durchgeführten Maßnahmen ist der gemeinschaftliche Einfluß bestenfalls als gering $\mathrm{zu}$ veranschlagen. So konnte der Rechnungshof nicht bestätigen, daß die Finanzierungsmittel zielgerichtet für die vorgesehenenmehrjährigen Sonderprogramme verwendet wurden, ${ }^{1}$ und spricht das Parlament davon, daß die Zuweisungen in Wirklichkeit nichts anderes waren, "als Erstattungen an die britische Staatskasse zur Deckung von schon erfolgten Ausgaben für schon durchgeführte Vorhaben und Maßnahmen." 2

Diese Situation änderte sich insofern, als eine Verlängerung der bisherigen Maßnahmen am Widerstand des Parlaments scheiterte und die Kommission neue Vorschläge zur Ausführung der Entlastungsregelungen vorlegen mußte. Darin waren nun zu einem großen Teil auch Sondermaßnahmen im Rahmen der Energiestrategle vorgesehen, die dem Gemeinschaftsinteresse besser entsprechen sollten. ${ }^{3}$ Im Rahmen der Entlastungsregelungen fur das Haushaltsjahr 1983 fand eine weitere Diversifizierung der Maßnahmen statt, die nun auch Beschäftigungsprogramme und Investitionen im Verkehrsinfrastrukturbereich umfaßten, und die ebenfalls elne Intensivierung bestehender Gemeinschaftsstrategien darstellten.

Entlastungen des Vereinigten Königreichs über besondere Ausgabenprogramme wurden letztmalig für das Jahr 1983 beschlossen und in der darauffolgenden Haushaltsperiode ausgefuhrt. 1984 wird dem Vereinigten Königreich von seinem normalen Mehrwertsteuer-Anteil ein Pauschbetrag von 1 Mrd. ECU abgezogen, der von den übrigen Mitgliedsländern - von der Bundesrepublik in vermindertem Ausmaß finanziert wird. ${ }^{4}$ Für dieses Jahr wird der Betrag auch bel der Ermittlung der Nettopositionen berijcksichtigt; die haushaltsmäBige Durchführung erfolgt in der Budgetperiode 1985.

\footnotetext{
${ }^{1}$ Vgl. EG-Rechnungshof, Jahresbericht zum Haushaltsjahr 1982, S. 69. 2 EG-Parlament, Sitzungsdokumente 1982 - 1983, Dok. 1-1004/82, Anlage I, Stellungnahme des Ausschusses für Regionalpolitik und Raumordnung.

${ }^{3}$ Vgl. EG-Kommisston, Bulletin Nr. 1/1983, s. 28. ${ }^{4}$ Siehe hierzu Abschnitt 4.1.2.3.
} 
Als wichtigste Ausgaben für spezifische Aktionen im Sozialbereich sind der Beitrag zugunsten der EGKS für Sozialmaßnahmen im zusammenhang mit der Umstrukturierung der Eisen- und Stahlindustrie sowie die Hilfen für die von Katastrophen heimgesuchte Bevölkerung der Gemeinschaft zu nennen (Tabelle 39). Zu den sonstigen Ausgaben gehören die Beihilfen zur Förderung der Berufsausbildung, Aktionen im Umweltbereich sowie verschledene Maßnahmen im Bereich des Sozialwesens.

Tabelle 39

Ausgaben für spezifische Maßnahmen im Sozialbereicha 1980 - 1984

\begin{tabular}{|l|r|r|r|r|r|}
\hline Maßnahmen & 1980 & 1981 & 1982 & 1983 & 1984 \\
\hline & \multicolumn{5}{|c|}{ M10. ECU } \\
Beiträge zur & & & & & \\
EGKS & - & 62,0 & 50,0 & 50,0 & 122,5 \\
Katastrophen- & & & & & \\
hilfen & 45,1 & 18,8 & 29,8 & 48,8 & 31,8 \\
Sonstige & 26,6 & 26,0 & 33,5 & 46,4 & 55,0 \\
Gesamt & 71,7 & 106,8 & 113,3 & 145,2 & 209,3 \\
\hline
\end{tabular}

$a_{\text {Haushaltsansätze }}$

Quelle: EG-Parlament, Endgültige Feststellung der Gesamthaushaltspläne, versch. Jg.

Während die Vertellungswirkungen der EGKS-Beiträge im Zusammenhang mit den zugehörigen Ausgaben des EGKS-Funktionshaushalts analysiert werden, ${ }^{1}$ stehen uber die Aufteilung der übrigen Ausgaben auf die Mitgliedsstaaten keine Angaben zur Verfügung. Angesichts der Größenordnungen und der tellweise breiten streuung ist ihr EinfluB auf die Vertellungspositionen der Mitgliedsstaaten ohnehin gering.

'Siehe hierzu Kapitel 4.2.2. 
Kennzeichnend für die Gemeinschaftspolitik auf den Gebieten Forschung, Energie, Wirtschaft und Verkehr sind langjährige Bemiihungen um einheitliche, den gewachsenen Anforderungen entsprechende Regelungen, doch - mit Ausnahme der vor allem auf die EAG zurückgehenden Forschungspolitik - nur sehr begrenzte Erfolge. 1 Dem entspricht auch das finanzielle Engagement. Der Anteil dieser Ausgaben am Gesamthaushaltsvolumen betrug in den letzten Jahren etwa $2 \%$. Außerhalb des Gesamthaushalts erfolgen Transaktionen über den Funktionshaushalt der EGKS sowie im Rahmen der Darlehensvergabe der EWG, EAG und EIB.

Die über den Gesamthaushalt finanzierten Maßnahmen werden dominiert von den Forschungsausgaben, die etwa drei Viertel dieses Ausgabenvolumens ausmachen. Die Forschungstätigkeit der EG wird durch die Vorschriften des EAG-Vertrages (Kernforschung) und durch die nach Art. 235 des EWG-Vertrages erlassenen Vorschriften (sonstige Forschung) geregelt. Dabei handelt es sich zum einen um sog. direkte Aktionen, d.h. Forschungsvorhaben, die ausschließlich in der Gemeinsamen Forschungsstelle (GFS) vom Personal der Gemeinschaft durchgeführt werden. Die GFS setzt sich aus der Generaldirektion in Brüssel sowie den vier Forschungsanstalten in Italien (der weitaus größten), Belgien, den Niederlanden und der Bundesrepublik ${ }^{2}$ zu- $^{-}$ sammen. Die indirekten Maßnahmen bestehen aus finanziellen Beteiligungen der Gemeinschaft zu Forschungsarbeiten, die gemeinsam mit vertraglich verbundenen Dritten durchgeführt werden. Dazu gehört vor allem das Versuchsprojekt JET (Joint European Torus) mit sitz

\footnotetext{
1Vgl. hierzu etwa EG-Kommission, Die Europäische Gemeinschaft und die Energiefrage, Europäische Dokumentation 1/1983; dies., Die Industriestrategie der Europäischen Gemeinschaft, Europäische Dokumentation 5/1982, EG-Parlament, Wirtschafts- und Sozialausschuß, Die Verkehrspolitik der 80er Jahre, Stellungnahme, Brüssel, März 1983.

${ }^{2}$ In diesem Zusammenhang ist auf den Betrieb eines Reaktors in den Niederlanden hinzuweisen, der im Rahmen eines Ergänzungsprogramms durch gesonderte Beiträge der Niederlande und der Bundesrepublik finanziert wird.
} 
in Culham, dessen Kosten die Gemeinschaft $\mathrm{zu} 80$ \& ubernimmt. An diesem Projekt sind im Rahmen von Kooperationsabkommen auch die Drittländer Schweiz und Schweden beteiligt. 1

Die finanziellen Maßnahmen auf den übrigen Gebieten Energie, Wirtschaft, Industrie und Verkehr sind quantitativ von untergeordneter Bedeutung. Dabei handelt es sich zum größten Teil um Zuschüsse zu Vorhaben, die von Unternehmen der Mitgliedsstaaten durchgeführt werden.

Die Ausgaben für Forschung, Energie etc. basieren im allgemeinen auf den von der Kommission vorgeschlagenen und vom Rat verabschiedeten Programmen. Da diese Mittel zu den nicht-obligatorischen Ausgaben gehören, entscheidet das Parlament in letzter Instanz über ihre Veranschlagung im Haushaltsplan. Auf diesen Umstand ist es zurückzuführen, daß etwa in den Bereichen Energie und Industrie überhaupt bzw. zusätzliche Mittel zur Verfügung gestellt wurden, da die Versammlung - und die Kommission - im Gegensatz zum Rat stets bemüht waren, auch die finanzwirksamen Maßnahmen dieser Politiken auszuweiten. 2

Die Tabelle 40 enthält Angaben über die Verteilung der Ausgaben auf die Mitgliedsstaaten. Dafür steht allerdings nur der Verteilungsschlüssel für das Jahr 1980 zur Verfügung, ${ }^{3}$ da solche Ausgaben in der Folgezeit nicht mehr von der Kommission veröffentlicht wurden. Allerdings dürfte sich die Ausgabenstruktur nicht gravierend geändert haben, da - wie anhand der Haushaltspläne nachzuvollziehen ist - die bestehenden Projekte im wesentlichen fortgeführt wurden. Daneben sind die spezifischen MaBnahmen im Rahmen der britischen Entlastungsregelungen gesondert ausgewlesen.

\footnotetext{
${ }^{1}$ Vgl. hierzu etwa D. Strasser, S. $267 \mathrm{ff}$.

2 Vgl. ebenda, S. 260 und 264.

3 Dabel handelt es slch um die von der Kommission anläßl1ch der Vorbereitung des Europäischen Rates 1979 in Dublin veröffentlichten Daten (EG-Kommission, KOM (79) 462 endg.), die auf den Stand von März 1980 fortgeschrieben wurden; vgl. D. Strasser, S. 275 f.
} 
Tabelle 40

Ausgaben für Forschung, Energie, Wirtschaft und Verkehr nach Mitgliedsstaaten 1980 - 1984

\begin{tabular}{|c|c|c|c|c|}
\hline \multirow[t]{2}{*}{ Mitgliedsland } & gesamt & $\begin{array}{l}\text { darunter: } \\
\text { Forschung| }\end{array}$ & Energie & $\begin{array}{l}\text { Wirtschaft/ } \\
\text { Verkehr }\end{array}$ \\
\hline & Verte & ilungsschli & ssel 1980 & $0^{2}$ in 8 \\
\hline Belgien & 8,5 & 10,0 & 3,3 & 13,5 \\
\hline Dänemark & 0,6 & 0,6 & 0,7 & 1,3 \\
\hline Delutschland & 18,3 & 19,1 & 16,0 & 13,5 \\
\hline Frankreich & 14,8 & 7,6 & 36,6 & 27,0 \\
\hline Griechenland & -- & -- & -- & -- \\
\hline Irland & 0,4 & 0,2 & 1,4 & 0,0 \\
\hline Italien & 27,0 & 31,4 & 12,5 & 31,1 \\
\hline Luxemburg & 0,0 & 0,0 & 0,0 & 0,0 \\
\hline Niederlande & 8,3 & 9,6 & 4,7 & 4,1 \\
\hline Verein, Königreich & 20,2 & 21,5 & 16,8 & 8,1 \\
\hline Sonstiges & 1,9 & -- & 8,0 & 1,4 \\
\hline Gesamt & 100,0 & 100,0 & 100,0 & 100,0 \\
\hline Jahr & \multicolumn{4}{|c|}{ Mio. ECU } \\
\hline 1980 & 311,5 & 249,8 & 40,3 & 12,0 \\
\hline 1981 & 377,2 & 295,6 & 63,1 & 8,3 \\
\hline 1982 & 437,7 & 367,6 & 44,0 & 16.3 \\
\hline $\begin{aligned} & \text { 1983: } \text { herkömmliche Aus- } \\
& \text { gaben } \\
& \text { Sondermaßnahmen }\end{aligned}$ & $\begin{array}{l}525,1 \\
782,6\end{array}$ & $\begin{array}{c}423,4 \\
--\end{array}$ & $\begin{array}{r}45,3 \\
782,6\end{array}$ & $\begin{array}{c}44.7 \\
--\end{array}$ \\
\hline $\begin{aligned} \text { 1984: } & \text { herkömmliche Aus- } \\
& \text { gaben } \\
& \text { Sondermaßnahmen }\end{aligned}$ & $\begin{array}{l}639,8 \\
835,9\end{array}$ & $\begin{array}{c}441,0 \\
--\end{array}$ & $\begin{array}{l}133,7 \\
412,0\end{array}$ & $\begin{array}{r}50,8 \\
423,9\end{array}$ \\
\hline
\end{tabular}

${ }^{a}$ Soll-Zahlen; ${ }^{b}$ im Rahmen der britischen Entlastungsregelungen.

Quelle: D. Strasser, S. 276; EG-Rechnungshof, Jahresbericht, versch, Jg. 
In der Verteilung der traditionellen Ausgaben kommt die Dominanz der Forschungsausgaben und hier speziell die Standortwahl der Forschungsanlagen zum Ausdruck. 1 Dabei zeigt sich, daß mit diesen Politikbereichen kaum eine Umverteilung im Sinne einer Begünstigung der wirtschaftsschwächeren Gemeinschaftsländer erreicht werden kann und soll, sondern vor allem andere Bestimmungsgründe wie etwa die wissenschaftlich-technologischen Kapazitäten und deren Entwicklung für die Mittelverteilung maßgeblich sind.

Abschließend ist $\mathrm{zu}$ hinterfragen, inwieweit mit den $\mathrm{Zahlungen}$ auch entsprechende Vorteile für diese Länder verbunden sind. Dabei ist zwar allgemein davon auszugehen, daß mit der Ansiedlung der Forschungseinrichtungen gewisse nachfrage- und arbeitsmarktwirksame Impulse einhergehen. ${ }^{2}$ Auf der anderen seite handelt es sich hier um gemeinsame und koordinierte Forschungstätigkeit, deren Durchführung von gemeinschaftlichem Interesse ist, und deren Ergebnisse sämtlichen Mitgliedsstaaten - und darüber hinaus den kooperierenden Nichtmitgliedsländern - zur Verfügung stehen, wenngleich sie im Hinblick auf eine spätere wirtschaftliche Verwendung vor allem denjenigen staaten mit den entsprechenden technologischen Voraussetzungen von Nutzen sein dürften. Die in den übrigen Bereichen Energie, Wirtschaft und Verkehr gewährten Zuschüsse bedeuten für die begünstigten Unternehmen zwar eine unmittelbare Entlastung; allerdings ist bei den Beihilfen für energiepolitische Entwicklungsvorhaben zu berücksichtigen, daß diese im Falle einer späteren wirtschaftlichen Rentabilität zurückgezahlt werden müssen.

Insgesamt ist offensichtlich, daß mit den Forschungs-, Energieetc.-Ausgaben vielfältige Verteilungswirkungen verbunden sind, die sich ohne eine eingehende Analyse - sie würde den Rahmen dieser Arbeit übersteigen - nur schwer abschätzen lassen. An dieser Stelle

1Vgl. J. Ø. Mфller, S. 100.

${ }^{2}$ Siehe hierzu auch die Ausführungen im Zusammenhanq mit den Verwaltungsausgaben im Abschnitt 4.1.3.6. 
kann deshalb nur festgestellt werden, daß die in diesem Abschnitt betrachteten Ausgaben nicht im gleichen Maße wie diejenigen im Rahmen der Strukturfonds $z u$ den vertellungstechnisch "gesicherten" Ausgaben zu rechnen sind. Damit ist die Aussagefähigkeit der Nettopositionen mit einem weiteren Unsicherheitsfaktor behaftet, dem angesichts der GröBenordnungen und den insgesamt wohl nur begrenzten Abweichungen zwischen Zahlung und tatsächlicher Beglinstigung allerdings kein entscheidendes Gewicht zukommen düfte.

\subsubsection{Entwicklungshilfe}

Die in diesem Abschnitt zu analysierenden Ausgaben für die zusammenarbeit mit Entwicklungsländern und Drittländern, wie sle über den Titel 9 des Gesamthaushaltsplans abgewickelt werden, sind nur ein Bestandteil aller Gemeinschaftsausgaben im Bereich der Entwicklungshilfepolitik. Um einen Gesamtüberblick zu erhalten, sind hierzu auch die zu Lasten des Agrargarantlefonds verbuchten Ausgaben für Ausfuhrerstattungen im Rahmen der Nahrungsmittelhilfe und zugunsten der Zuckerabkommen ${ }^{1}$, die uber den EEF abgewlckelten Zahlungen ${ }^{2}$ sowie die Einnahmenausfulle durch die Gewahrung von Zollpräferenzen hinzuzurechnen. ${ }^{3}$ Eine zusammenstellung dieser Maßnahmen ist in Tabelle 41 enthalten. ${ }^{4}$ Der finanzielle Stellenwert der Gemeinschaftshilfe läBt sich etwa im Verglelch mit den nationalen Entwicklungshilfeleistungen der Mitgliedsstaaten elnordnen. In der Abgrenzung der Tabelle 41 machen die Gemeinschaftsleistungen in den letzten Jahren etwa $10 \%$ der von den Mitgliedsstaaten gewährten öffentlichen Entwicklungshilfe aus. 5

\footnotetext{
${ }^{1}$ Siehe hierzu Kapitel 4.1.3.1.1.

${ }^{2}$ Siehe hierzu Kapitel 4.2.1.

${ }^{3}$ Daneben ist auch die Darlehensvergabe an Entwicklungsländer zu berücksichtigen. Slehe hierzu Kapitel 4.2.3.

${ }^{4}$ In Anlehnung an D. Strasser, S. 308.

${ }^{5} \mathrm{Zu}$ den Ausgaben fur die einzelstaatliche offentliche Entwicklungshilfe, vgl. EG-Kommission, Antwort auf die schriftliche Anfrage $\mathrm{Nr} .751 / 83$ von Herrn Bonde, Mitteilungen der Sprechergruppe vom 12.10.1983. Vgl. hierzu auch EG-Kommission, Memorandum zur Entwicklungspolitik der Europalschen Gemelnschaft, Bulletin der EG, Beilage 5/1982, S. 16 .
} 
Der Anteil der über den Titel 9 des Gesamthaushalts abgewickelten Ausgaben für Entwicklung und Zusammenarbeit betrug in den letzten Jahren zwischen 3 \& und 5 \& des Gesamthaushaltsvolumens.

Tabelle 41

Leistungen der EG für die Zusammenarbeit mit Entwicklungsländern und Drittländern 1980 - 1984

\begin{tabular}{|c|c|c|c|c|c|}
\hline Instrument & 1980 & 1981 & 1982 & 1983 & 1984 \\
\hline & \multicolumn{5}{|c|}{ Mio. ECU } \\
\hline \multicolumn{6}{|l|}{ Gesamthaushalt } \\
\hline - Titel 9 & 508,9 & 858,5 & 786,1 & 810,7 & 1150,6 \\
\hline $\begin{aligned} \text { dar.: } & \text { Nahrungsmittel- } \\
& \text { hilfe }\end{aligned}$ & $(318,8)$ & $(601,6)$ & $(540,2)$ & $(388,5)$ & $(709,2)$ \\
\hline - EAGFL - Garantie & & & & & \\
\hline - Ausfuhrerstattungen & 193,4 & 181,9 & 166,4 & 200 & -- \\
\hline - zuckerabkommen ${ }^{\mathrm{a}}$ & 200 & 140 & 330 & 390 & 455 \\
\hline $\begin{array}{l}\text { Europäischer Entwick- } \\
\text { lungsfonds }\end{array}$ & 481,9 & 663,7 & 647,2 & 718,8 & 703,0 \\
\hline Gesamt & 1384,1 & 1844,1 & 1929,7 & 2119,5 & -- \\
\hline $\begin{array}{l}\text { Einnahmeausfälle } \\
\text { durch das Allgemeine } \\
\text { Zollpräferenzsystem }\end{array}$ & -- & -- & $\begin{array}{l}596 \\
\text { bis } \\
673\end{array}$ & -- & -- \\
\hline
\end{tabular}

$a_{\text {Haushaltsansätze }}$

Quelle: EG-Rechnungshof, Jahresbericht, versch. Jg.; EG-Parlament, Endgültige Feststellung des Gesamthaushaltsplans, versch. Jg.

Diese Ausgaben fließen sowohl den im Rahmen des EEF verbundenen Entwicklungsländern des afrikanischen, karibischen und pazifischen Raums (AKP-Staaten) als auch den nichtassoziierten Entwicklungsiändern sowie den durch verschiedene Abkommen verbundenen Staaten des 
Mittelmeerraumes zu. Mit dieser breiten Streuung verfolgt die Gemeinschaft das Ziel, einen gewissen Ausgleich zur einseitig auf die AKP-Länder ausgerichteten Entwicklungspolitik des EEF zu bewirken. 1 Neben gezielten Sondermaßnahmen wie etwa für Katastrophenhilfen sowie finanzieller und technischer Unterstïtzung zur Förderung der wirtschaftlichen Entwicklung ${ }^{2}$ spielt die Nahrungsmittelhilfe mit zumeist etwa zwei Dritteln der Ausqaben die quantitativ herausragende Rolle. Dabei handelt es sich fast ausschließlich um die kostenlose Abgabe von Getreide, Milchpulver und Butteröl Produkte, bei denen die Gemeinschaft erhebliche Uberschußmengen aufweist. 3

Die Nahrungsmittelhilfelieferungen werden über Ausschreibungsverfahren von den Unternehmen der Mitgliedsstaaten durchgeführt und die dafür bereitgestellten Mittel über die Interventionsstel.len an die zuschlagsempfänger ausgezahlt. Dabei wird derjenige werthestandteil der Erzeugnisse, der dem Weltmarktpreis entspricht - das sog. Geschenk-Element - von Titel 9 gedeckt, während die Differenz zwischen dem Weltmarkt- und dem Gemeinschaftspreis als Erstattuna über den Agrargarantiefonds abgerechnet wird. Die Transportkosten sowie die administrativen Leistungen der Verteilung müssen zumeist von den Empfängerländern übernommmen werden, da die Gemeinschaft in der Regel nur die fob-Kosten ${ }^{4}$ übernimmt. 5

${ }^{1}$ Vgl. J. $\varnothing$. M $\varnothing$ ller, S. $98 \mathrm{f} .$, sowie EG-Kommission, Entwicklungshilfe, Skizze der Gemeinschaftsaktion von morgen, Bulletin der EG, Beilage $8 / 74$.

${ }^{2}$ Vgl. hierzu ausführlicher etwa D. Strasser, S. 293 ff.

${ }^{3}$ Vgl. EG-Kommission, Die Lage der Landwirtschaft in der Gemeinschaft, Bericht 1983, S. 124 und $133 \mathrm{f}$.

4 "fob" stellt eine Abkürzung für 'free on board' dar; fob-Kosten umfassen nur die Leistungen bis zur Beladung im Hafen des Exportlandes, vgl. etwa J. E. Meade, The Theory of International Economic Policy, Vol. I, The Balance of Payments, Oxford 1962, S. $20 \mathrm{f}$.

${ }^{5}$ Vgl. zu einer àetaillierten Darstellung dieser Zusammenhänge H. H. Schumacher, Die Nahrungsmittelhilfe der Europäischen Gemeinschaft. Entstehung, System und Rechtsgrundlagen, Baden-Baden 1981, insbes. $\mathrm{S} .74 \mathrm{ff}$. 
Die Entwicklungshilfeausgaben des Titel 9 werden von der EG-Kommission bei der Berechnung der Nettopositionen ausgeklammert - zusammen mit den zugehörigen Finanzierungsmitteln entsprechend den mitgliedsstaatlichen Beteiligungen an der Finanzierung des Gesamthaushalts. ${ }^{1}$ Damit ist allerdings die Frage nach den Verteilungswirkungen der gemeinschaftlichen Entwicklungshilfepolitik noch nicht beantwortet.

Geht man von dem Ansatz aus, daß mit den Entwicklungshilfeausgaben keinerlei finanzielle Vorteile zugunsten der Mitgliedsstaaten verbunden sind, dann verbleibt als primare Verteilungswirkung, da $B$ sich die Nettopositionen der Mitgliedsstaaten nach Maßgabe ihrer Finanzierungsbetelligungen verschlechtern.

Betrachtet man alleraings die konkrete situation, in der die Hilfe abgewickelt wird, so ist zu prufen, ob die Finanzierungsleistungen der Mitgliedsstaaten bel einer Nichtgewăhrung der Hilfe konsequenterweise auch entfallen. Analysiert man zunăchst die Nahrungsmittelhilfe, dann ist zu unterschelden, ob die Hilfelieferungen aus kurzfristigen Lagerüberschüssen, die nur vorübergehende Marktschwankungen ausgleichen sollen, oder aus strukturellen Lagerbeständen, die aufgrund ständig produzierter Uberschüsse aufgebaut werden müssen, ${ }^{2}$ erfolgen. Im letztgenannten Fall ergibt sich bel einem Wegfall der Nahrungsmittelhilfe kelne volle Verminderung der Finanzierungslast, da davon auszugehen ist, daß die Agrarproduzenten thre Erzeugnisse dann an die Interventionsstelien $z u$ den Garantiepreisen abgeben und die eingelagerten Produkte langfristig anderweitig verwertet werden müBten. Durch die Nahrungsmittellieferungen würden zudem Lagerkosten vermieden, so daß sie in diesem Fall die Funkton eines tberschußventils ${ }^{3}$ mit kostensenkender Wirkung ausüben.

1'siehe hierzu Abschnitt 3.

${ }^{2}$ Vgl. H. Bartling, Landwirtschaft, in: P. Oberender (Hrsg.), Marktstruktur und Wettbewerb in der Bundesrepublik Deutschland, Branchenstudian zur deutschen Volkswirtschaft, Munchen 1984, s. 1 ff., hier: S. 33 .

${ }^{3}$ Vgl. etwa H. P. Wiesebach, Entwicklungspolitik II: Entwicklungshilfe, in: Handworterbuch der Wirtschaftswissenschaft, Bd. 2 , stuttgart, New York u.a. 1980, S. 407 ff., hier: S. 409. 
Da die im Rahmen der Entwicklungshilfe kostenlos abgegebenen Nahrungserzeugnisse eher den strukturellen Uberschüssen zuzuordnen sind, spricht einiges dafür, das wertmäßige Äquivalent jeweils denjenigen Mitgliedsstaaten als empfangene Vorteile "gutzuschreiben", die dje Hilfeleistungen durchgeführt haben. Damit müßten die Beteiligungen an den Nahrungsmittelhilfelieferungen den Finanzierungsre-lationen gegenübergestellt und aus den Differenzen die Auswirkungen auf die Nettopositionen abgeschätzt werden. ${ }^{1}$ Dabei würden - nicht nur für 1984, sondern ähnlich auch in den Vorjahren - vor allem Frankreich nennenswerte Vorteile zufallen, während eine derartige Einbeziehung der Nahrungsmittelhilfeausgaben in die Nettopositionen insbesondere das Vereinigte königreich schlechtstellen würde.

Die übrigen Ausgaben im Rahmen des Titel 9 bestehen überwiegend aus finanziellen und technischen Hilfen. Ihnen stehen Finanzierungsbeteiligungen der Mitgliedsstaaten gegenüber, die bei einer Nichtgewährung der Hilfen entfielen und die damit als einseitige Leistungen gewertet werden können. Wirtschaftliche Rückwirkungen aus die-

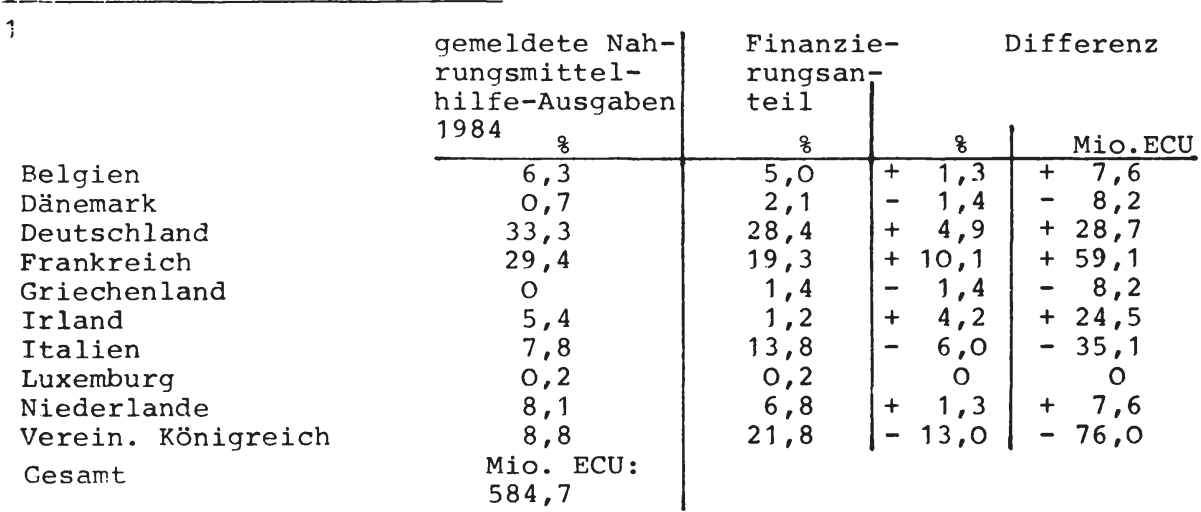

Vgl. Vierzehnter Gesamtbericht über den Europäischen Ausrichtungsund Garantiefonds für die Landwirtschaft - Jahr 1984 - Abteilung Garantie und finanzielle Durchführung der Nahrungsmittelhilfe, KOM (85) 492 endg., Brüssel, 17.9.1985, S. 101; Tabelle 19; eigene Berechnungen. 
sen Entwicklungshilfeausgaben auf die Gemeinschaft sind etwa bei Katastrophen- oder Soforthilfen zur Linderung unmittelbarer Not nicht zu erwarten. Insoweit die übrigen finanziellen und technischen Hilfen zur Industrialisierung der Entwicklungsländer beitragen, dürften sich prinzipiell die Möglichkeiten für die Ausweitung des Handels vergrößern und die Rohstoffmärkte stabilisiert werden, und würde daraus langfristig auch ein gewisser Nutzen für die Gemeinschaft erwachsen.' Eine Quantifizierung solcher Vorteile ist jedoch nicht mögllch; die Ergebnisse würden angesichts der für diese Maßnahmen zur Verfügung stehenden geringen Beträge auch nur von untergeordneter Bedeutung sein.

\subsubsection{Erstattungen an die Mitgliedsstaaten}

Zu dieser Ausgabenkategorie gehören drei Typen von Mittelzuweisungen an die Mitgliedsstaaten: die Pauschalerstattungen der bei der Erhebung der Eigenmittel entstandenen Kosten, Finanzausgleichszahlungen im Zusammenhang mit gewährten $Z$ insvergünstigungen für Darlehen im Rahmen des Europäischen Währungssystems sowie Rückerstattungen an Griechenland innerhalb der Beitrittsübergangsregelung (Tabelle 42$)^{2}$

Die Zahlungen der Eigenmittel-Erhebungskosten haben ihre rechtliche Grundlage im Eigenmittelbeschluß, der bestimmt, daß den Mitqliedsstaaten 10 \& der an Zöllen und Agrarabschöpfungen abgeführten Beträge der Erhebungskosten erstattet werden. ${ }^{3}$ Diese Ausgaben sind sachlich den Verwaltungsausgaben gleichzusetzen, nur daß sie unmittelbar auf die Mitgliedsstaaten entfallen, deren Zoll- und Finanz-

1Vgl. E. Grabitz, Die Entwicklungspolitik der Europäischen Gemeinschaften, Ziele und Kompetenzen, in: Europarecht, 12. Jq. 1977, S. 217 ff., hier: S. 222 .

2 Bei den EWS-Finanzausqleichsbeträqen und Rückerstattungen an Griechenland handelt es sich um veranschlagte Mittel der Haushaltspläne, so daß die Gesamitsummen der Erstattunqen nicht qenau mit denjenigen in Tabelle 25 übereinstimmen.

${ }^{3}$ Art. 3, Abs. 1, 4. Unterabsatz des Eiqenmittelbeschlusses. 
Tabelle 42

Erstattungen an die Mitgliedsstaaten $1980-1984$

\begin{tabular}{|c|c|c|c|c|c|c|c|c|c|c|c|c|c|c|c|c|}
\hline \multirow{13}{*}{ 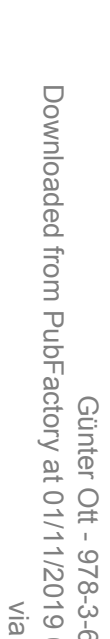 } & Mitgliedsland & \multirow{2}{*}{\multicolumn{5}{|c|}{$\begin{array}{c}\text { Erhebungskosten der } \\
\text { E1genmittel } \\
1980|1981| 1992|1983| \\
\end{array}$}} & \multirow{2}{*}{\multicolumn{5}{|c|}{$\begin{array}{l}\text { Finanzausgle1ch für ewS- } \\
\text { Zinsvergünst1gungen } \\
1980|1981| 1982|1983| 1984 \\
\end{array}$}} & \multicolumn{5}{|c|}{$\begin{array}{l}\text { Erstattungen an Griechen- } \\
\text { land } \\
1980|1981| 1982 \not 1983 \mid 1984\end{array}$} \\
\hline & \multicolumn{6}{|c|}{ Mio. ECU } & & & & & & & & & & \\
\hline & Belgien & 61,5 & 61,8 & 68,3 & 77,7 & 82,0 & & & & & & & & & & \\
\hline & Dănemark & 15,7 & 16,2 & 17,6 & 20,3 & 26,0 & & & & & & & & & & \\
\hline & Deutschland & 212.7 & 225,4 & 237,0 & 241,8 & 302,5 & & & & & & & & & & \\
\hline & Frankre1ch & 113,8 & 123,1 & 134.7 & 142,5 & 169,7 & & & & & & & & & & \\
\hline & Griechenland & -- & 8,9 & 18,3 & 16,6 & 14,8 & -- & 1,2 & 2,2 & 2,8 & -- & -- & 105,7 & 98,0 & 65,1 & 46,2 \\
\hline & Irland & 7,6 & 8,8 & 10,2 & 11,6 & 16,0 & & & & & & & & & & \\
\hline & Itallen & 106,7 & 94,9 & 102,6 & 105,9 & 117,5 & & & & & & & & & & \\
\hline & Luxemburg & 0.5 & 0,4 & 0,4 & 0,5 & 0.7 & & & & & & & & & & \\
\hline & Niederlande & 82,9 & 77,3 & 83,9 & 83,4 & 101,1 & & & & & & & & & & \\
\hline & $\begin{array}{l}\text { Vere1n. Konlg- } \\
\text { reich }\end{array}$ & 189,8 & 190,2 & 236,3 & 221,6 & 279,6 & 45,4 & 39,6 & 60,4 & 54,2 & - & & & & & \\
\hline & Ges ant & 791,2 & 807,0 & 909,3 & 921,9 & 1109,9 & 40,8 & 62,6 & 62,6 & 57,0 & -- & - & 105,7 & 98,0 & 65,1 & 46,2 \\
\hline
\end{tabular}

Quelle: EG-Rechnungshof, Jahresbericht, versch. Jg., EG-Parlament, Endgültige Feststellung des Gesamthaushaltsplans, versch. Jg. 
verwaltungen die Eigenmittel im Wege einer Befugnisübertragung erheben und dann an die Gemeinschaft abführen. ${ }^{1}$ Da das Aufkommen an Zölien und Agrarabschöpfungen langsamer als das Budgetvolumen gewachsen ist, hat auch die prozentuale Haushaltsbelastung durch diese Erstattungen abgenommen.

Die den Mitgliedsstaaten Vereinigtes Königreich und Griechenland erstatteten Finanzausgleichsbeträge gehen auf die Zinsvergünstigungen für Darlehen, die den am EWs teilnehmenden, weniger wohlhabenden Mitgliedsstaaten für den Zeitraum von 1979 bis 1983 gewährt wurden, ${ }^{2}$ zurück. Diese $\mathrm{zinssubventionen} \mathrm{wurden} \mathrm{über} \mathrm{den} \mathrm{Haushalt}$ gezahlt und damit zunächst von sämtlichen Mitgliedsstaaten getragen. Da allerdings vereinbart worden war, daß die Mitgliedsstaaten, die sich nicht an dem währungssystem beteiligen, auch nicht zu einer Finanzierung heranzuzlehen sind, erhielten das Vereinigte Königreich und Griechenland Ausgleichszahlungen nach Maßgabe ihres marginalen, d.h. an den Mehrwertsteuerabführungen orientierten Finanzierungsanteils.

Schließlich erfolgen Rlickerstattungen an Griechenland im Rahmen der Beitrittsübergangsregelungen. Gemäß Art. 127 der Beitrittsakte wird für die Zett von 1981 bis 1985 ein degressiv gestaffelter Teil der nach dem BSP berechneten Finanzbelträge erstattet. Dieses sind für dle Jahre 1981: 70 \&, 1982: 50 \%, 1983: 30 \% , 1984: 20 \% und 1985: 10 \& der abgefuhrten Finanzmittel. Diese Erstattungen sollen während der Anpassungszelt entlastende Wirkungen für den neuen Mitgliedsstaat ausuben. Hinslchtlich der Degression und der nicht an irgendwelche Bedingungen geknüpfte Rückzahlungen sind Parallelen zum Finanzmechanismus der siebziger Jahre ${ }^{3}$ erkennbar. ${ }^{4}$

${ }^{1}$ Vgl. D. Strasser, S. 315 .

${ }^{2}$ Siehe Abschnitt 4.1.3.2.4.

${ }^{3}$ Siehe hierzu Abschnitt 4.1.2.1.

${ }^{4} \mathrm{vgl}$. D. Strasser, S. 131. 


\subsubsection{Verwaltungsausgaben}

Die Ausgaben für die Verwaltungstätigkeit der Gemeinschaftsorgane werden in einem gesonderten Kapitel des Haushaltsplans zusammengefaßt. Ihr Anteil am Gesamthaushaltsvolumen ist in der letzten Dekade von $5,8 \%$ auf $4,4 \%$ gesunken.

Die Verwaltungsausgaben lassen sich untergliedern in Personal- und Sachausgaben (Tabelle 43). Die Personalausgaben, die über zwei Drittel der gesamten Verwaltungsausgaben beanspruchen, bestehen vor allem aus den Dienstbezügen des Verwaltungspersonals $(89 \%)^{1}$, den Vergütungen für die Mitglieder der Gemeinschaftsorgane ${ }^{2}$ (5 \%) sowie Nebenkosten etwa fur Dienstreisen, berufliche Fortbildung und soziale Zwecke. Zu den Sachmitteln gehören Ausgaben für unterschiedliche Bereiche: Grundstuckskäufe und Gebäudemieten (32 \%), Käufe beweglicher Sachen und laufende Sachausgaben (24 \%), Ausgaben für Sitzungen, Sachverständige und Studien (13\%) sowie Zuschüsse an bestimmte mit der Gemeinschaft eng verbundenen Einrichtungen (13 \%).

Tabelle 43

Verwaltungsausgaben $1980-1984$

\begin{tabular}{|l|r|r|r|r|r|}
\hline Ausgabenart & 1980 & 1981 & 1982 & 1983 & 1984 \\
\hline & \multicolumn{5}{|c|}{ Mio. ECU } \\
\hline Personalausgaben & 561,3 & 635,2 & 695,5 & 751,3 & 837,0 \\
Sachausgaben & 214,1 & 251,2 & 314,7 & 353,7 & 379,2 \\
Gesamt & 775,4 & 886,4 & 1010,2 & 1105,0 & 1216,2 \\
\hline
\end{tabular}

Quelle: EG-Rechnungshof, Jahresberichte, versch. Jg.

${ }^{1}$ Die hier und im folgenden angegebenen Prozentzahlen beziehen sich auf das Haushaltsjahr 1982. Vgl. EG-Rechnungshof, Jahresbericht 1982 , S. 102. Die Werte der übrigen Jahre des Untersuchungszeitraums weisen ähnliche Größenordnungen auf.

2 Dieses betrifft nicht die Bezi̊ge der Abgeordneten des Parlaments, die nach nationalem Recht gewährt werden, sowie die Mitqlieder des Rates, die von der Gemeinschaft weder Dienstvergütungen noch Entschädigungen erhalten. Vgl. D. Strasser, S. 314. 
Die Beantwortung der Frage, welche Anteile der mit den Verwaltungsausgaben verbundenen Vorteile den einzelnen Mitgliedsländern zuzurechnen sind, ist problematisch. Im Rahmen der von der Kommission durchgeführten Berechnungen der Nettopositionen werden die Verwaltungsausgaben denjenigen Mitgliedsländern als empfangene Leistungen zugewiesen, in denen die Zahlungen anfallen. ${ }^{1}$ Diese Methode führt dazu, daß etwa 95 \& der Verwaltungsausgaben auf Belgien und Luxemburg entfallen (Tabelle 44), da die organe der Gemeinschaft fast ausschlieblich in diesen Ländern thren Sitz haben.

Gegen die Verfahrensweise, die von der Kommission selbst bereits früheitig in Frage gestellt wurde, ${ }^{2}$ lassen sich eine Reihe von Einwänden vorbringen:

- Anders als etwa bel den gemeinschaftlichen Beteiligungen an nationalen Maßnahmen bel den Strukturausgaben oder den Ausgaben zur Absatzsicherung und Einkommensniveaustabilisierung im Rahmen des Agrargarantiefonds handelt es sich bei den Verwaltungsmitteln um Ausgaben, denen zumelst Gegenlelstungen in Form von Güterlieferungen oder Dienstleistungen gegenuberstehen. Die Verwaltungsausgaben uneingeschrănkt als empfangene zuflusse zu bewerten, dürfte kein zutreffendes Blld von den tatsăchlichen Vortellen ergeben.

- Gleichwohl 1st nicht zu bestreiten, das mit der Ansiedlung von Verwaltungen vielfältige ökonomische Vortelle verbunden sind, die in ihrer Gesamtwlrkung die ursprunglichen Ausgaben durchaus ubersteigen können. Daß dlese Zusammenhänge in ähnlicher Weise auch von den Mitgliedsstaaten beurtellt werden, wird durch das starke Interesse der Mitgliedslander an der Attrahierung bzw. dem Erhalt des Sitzes von Gemeinschaftsorganen und -verwaltungen bestatigt. Dieses wird etwa auch dadurch unterstrichen, daB Belgien - im Sinne eines Koppelungsgeschafts - mit der Inst1tutionalisierung

${ }^{1}$ Siehe hierzu Kapitel 3.

${ }^{2} \mathrm{Vgl}$. Kommission der EG, Gemeinsame Antwort auf die schriftlichen Anfragen, Nrn. 1022/77, 604/78 und 607/78, S. 2; dies., Antwort auf die schriftliche Anfrage Nr. 50/79 von Lord Bessborough, S. 9 . 
der gemeinsamen Agrarpolitik offenbar nur deshalb eine Nettozahlerposition in Kauf genommen hat, weil als Sitz der Kommission und des Rates Brüssel gewählt wurde. 1

Die Vorteile für die Länder mit einem Verwaltungssitz können zum einen darin bestehen, daß hierdurch die Ansiedlung für andere Unternehmen attraktiver wird. ${ }^{2}$ Vor allem aber dürften sich angesichts der gegenwartigen gesamtwirtschaftlichen Lage nicht unerhebliche Vorteile aus der uber die Personal- und Sachausgaben entfalteten Nachfrage ergeben. Die hiermit verbundenen positiven Beschäftigungswirkungen werden durch die Entlastung des Arbeitsmarktes über die direkt in den Verwaltungsstellen $\mathrm{zu}$ besetzenden Arbeitsplätze verstärkt. Die Bedeutung des letztgenannten direkten Beschäftigungseffekts geht daraus hervor, daß knapp 40 \% der etwa 14.000 Bediensteten der Kommission ${ }^{3}$ Angehörige der BeneluxStaaten sind und daß hier der Anteil der in mittleren und unteren Laufbahngruppen Besoldeten uberproportional hoch 1st. ${ }^{4}$

- Allerdings dürfte der beschäftigungspolitische Nutzen der Verwaltungsausgaben nicht auf die Länder mit Verwaltungssitzen beschränkt bleiben, sondern werden in einem bestimmten Umfang auch die übrigen Mitgliedsländer daran partizipieren. Denn nach dem Beamtenstatut der Gemeinschaft sind die Bediensteten auf breiter geographischer Basis auszuwählen, ${ }^{5}$ und ist dieses auch zu einem großen Teil in die Praxis umgesetzt worden. ${ }^{6}$ Auf diese Weise kön-

1Vgl. G. Cordts, Finanzierung des EWG-Agrarmarktes, in: Institut für das Recht der Europäischen Gemeinschaften der Universität Köln (Hrsg.), Agrarrecht der EWG, Kölner Schriften zum Europarecht, Bd. 10, Köln, Berlin u.a., S. 189 ff., hier: S. 201.

2Vgl. Edwards, S. 752 .

${ }^{3}$ Die Kommission stellt den Hauptteil der insgesamt etwa $18.000 \mathrm{Be}-$ schäftigten der EG.

${ }^{4} \mathrm{Vgl}$. D. Strasser, S. 326.

${ }^{5}$ Vgl. Art. 27 des Statuts der Beamten der Europäischen Gemeinschaften, in: Amtsblatt der EG, Nr. C 100 vom 28.9.1972, S. 12.

${ }^{6}$ Vgl. D. Strasser, S. $325 \mathrm{f}$. 
nen auch für die übrigen Mitgliedsstaaten gewisse arbeitsmarktentlastende Wirkungen ausgehen. Daneben mögen äie Beziehungen der Beschäftigten zum Heimatland sich auch darin niederschlagen, daB sie einen Teil ihres Einkommens im Heimatland verausgaben. ${ }^{1}$

Insgesamt sind mit den Verwaltungsausgaben sehr vielschichtige Vertellungswirkungen verbunden, die einer einfachen Lösung nicht zugänglich sind. Es kann zunächst lediglich konstatiert werden, daß das bisherige, an Zahlungsvorgänge anknüpfende Zurechnungsverfahren keine befriedigenden Ergebnisse liefert. Nach den vorangegangenen Uberlegungen wird man allerdings davon ausgehen können, daß den tatsächlichen Verhältnissen generell besser Rechnung getragen wird, wenn den beiden Ländern Belgien und Luxemburg ein geringerer und den übrigen Mitgliedsstaaten ein gröBerer Anteil der Verwaltungsausgaben als nach dem bisherigen Verfahren zugerechnet wird.

So schlägt die Kommission vor, elnen bestimmten Prozentsatz der gesamten Verwaltungsausgaben pauschal den Hauptsitzen der Organe, also Brissel und Luxemburg, zuzuweisen und die restlichen Ausgaben - im Sinne einer Gesamtlast - nicht mehr in die Verteilungsrechnung einzubeziehen. ${ }^{2}$ Ein solcher Ansatz bedeutet im Vergleich zum gegenwärtig praktizierten Verfahren, daß auf der Finanzierungsseite auch geringere Mittelabfuhrungen der Mitgliedsstaaten zu berücksichtigen sind. Unter der Prämisse, daß die Finanzierungsstruktur der Verwaltungsausgaben derjenigen der Gesamtausgaben entspricht, ${ }^{3}$ werden die Mitgliedsstaaten nach Maßgabe ihres Finanzierungsantei is am Gesamthaushalt entlastet. Ein solches Verfahren läßt sich auch dahingehend interpretieren, daß der nicht mehr in der Verteilungsrechnung berücksichtigte Tell der Ausgaben den Mitgliedsstaaten in Höhe

${ }^{1}$ Vgl. A. Edwards, S. 752.

2Vgl. Kommisston der EG, SEK (83), S. 2 .

${ }^{3}$ Diese Annahme wird etwa auch bei der Ausklammerung der Entwicklungshilfeausgaben aus der Berechnung der Nettopositionen getroffen. 
Tabelle 44

Auswirkungen eines veränderten zurechnungsverfahrens der Verwaltungsausgaben auf die Höhe der Nettopositionen $1980-1984$

\begin{tabular}{|c|c|c|c|c|c|c|}
\hline \multirow[t]{2}{*}{ Mitgliedsland } & \multirow[t]{2}{*}{\begin{tabular}{|} 
Verteilung der \\
Verwaltungsaus- \\
gaben \\
$1980^{a}$ \\
in 8
\end{tabular}} & \multicolumn{5}{|c|}{$\begin{array}{l}\text { Anderumg der Nettopositionen nach veränder- } \\
\text { tem Verteilungsschlüssel }\end{array}$} \\
\hline & & \multicolumn{5}{|c|}{ Mio. ECU } \\
\hline Belgien & 62,2 & $-215,6$ & $-249,7$ & $-285,2$ & $-314,1$ & $-347,2$ \\
\hline Dänemark & 0,1 & $+8,2$ & $+8,5$ & $+9,2$ & $+11,3$ & $+12,3$ \\
\hline Deutschland & 1,8 & $+109,0$ & $+116,1$ & $+126,0$ & $+145,5$ & $+161,3$ \\
\hline Frankreich & 1,0 & $+72,1$ & $+82,3$ & $+97,1$ & $+104,4$ & $+112,3$ \\
\hline Griechenland & - & - & $+2,2$ & 4,5 & 3,8 & 2,9 \\
\hline Irland & 0,5 & $-\quad 0,2$ & $-\quad 0,2$ & $-\quad 0,3$ & 1,5 & 1,6 \\
\hline Italien & 1,0 & $+43,7$ & $+56,9$ & $+53,2$ & $+65,5$ & $+76,8$ \\
\hline Luxemburg & 31,7 & $-122,5$ & $-140,0$ & $-159,5$ & $-174,8$ & $-191,5$ \\
\hline Niederlande & 0,7 & $+28,7$ & $+27,2$ & $+29,9$ & $+32,3$ & $+35,4$ \\
\hline Verein. Königreich & 0,5 & $+80,4$ & $+96,7$ & $+124,6$ & $+124,6$ & $+134,6$ \\
\hline
\end{tabular}

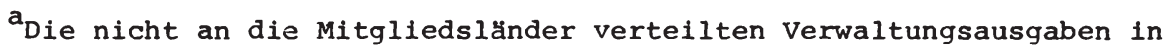
Höhe von 0,5 \& wurden aus Vereinfachungsgrïnden ab 1981 Griechenland zugerechnet.

$\mathrm{b}_{50}$ \& der nach dem bisher angewandten Verteilungsschlüssel auf Belgien und Luxemburg entfallenden Verwaltungsausgaben wurden diesen Ländern pauschal als empfangene Leistungen zugerechnet. Die restlichen Verwaltungsausgaben und der auf sie entfallende Finanzierungsantell der Mitgliedsländer wurden aus den Berechnungen der Nettopositionen ausgeklammert.

Quelle: Eigene Berechnungen unter Zuhilfenahme von Daten aus: EG-Rechnungshof, Jahresbericht 1983, D. Strasser, S. 335.

ihrer Finanzierungsbeteiligungen als Verwaltungsleistungen zufliest. Wählt man als die den Hauptsitzen Brüssel und Luxemburg pauschal zuzurechnenden Betrăge jeweils 50 \& der bisher auf Belgien bzw. Luxem- 
burg entfallenden Verwaltungsausgaben, ${ }^{1}$ dann zeigt Tabelle 44 die sich im Vergleich $z u$ bisherigen Verfahren ergebenden Auswirkungen auf die Höhe der Nettopositionen. ${ }^{2}$

Wie in den Annahmen impliziert, verschlechtern sich die Nettopositionen Belgiens und Luxemburgs etwa um die Hälfte der ihnen bisher aus den Verwaltungsausgaben zugewiesenen Vorteile, während sich die positionen der übrigen Länder im Verhältnis ihrer Finanzierungsanteile am Gesamthaushalt verbessern. Im Hinblick auf die Nettopositionen aus der gesamten Gemeinschaftstatigkeit (Tabelle 2) führt allein die hier vorgenommene veränderte Zurechnung der Verwaltungsausgaben dazu, daß sich die ursprüngliche Nettoempfängerposition Belgiens etwa auf Null reduziert und die Luxemburgs sich etwa halbiert.

${ }^{1}$ Ein solcher Antell der bisher in diesen Ländern gezahlten Verwaltungsausgaben ließe slch vor allem angesichts der Dominanz der Personalausgaben und der damit unmittelbar bereltgestellten Arbeitsplätze durchaus rechtfertigen.

2

Da eine länderspezifische Aufgliederung der gezahlten Verwaltungsausgaben nur bis zum Jahre 1980 vorllegt, mußten diese Relationen auch flir die folgenden Jahre zugrundegelegt werden. Die daraus resultierenden Verzerrungen werden angesichts der konstanten Verteilungsstruktur dieser Ausgaben vernachlässlgbar gering sein. 


\subsection{AuBerbudgetäre Aktivitäten}

Die Einnahmen und Ausgaben des Gesamthaushalts stellen nur einen Teilbereich der finanzwirksamen Aktivitäten der Gemeinschaften dar. Dazu gehören auch die außerhalb des Gesamthaushalts durchgeführten Tatigkeiten des EEF, der Funktionshaushalt der EGKS sowie die Anleihe- und Darlehensoperationen sämtlicher Gemeinschaftsinstitutionen. Damit sind insofern verschiedenartige Distributionseffekte verbunden, als im EGKS-Funktionshaushalt - analog zum Gesamthaushalt - den mitgliedsstaatlichen Finanzierungsleistungen auch Ausgaben an die Mitgliedsstaaten gegenuberstehen, während mit der Durchführung des EEF ausschlleßlich bzw. überwiegend Belastungen für die Mitgliedsstaaten verbunden sind und auch Im Rahmen der Anleihe- und Darlehensströme nicht von elnem Nullsummensplel ausgegangen werden kann, In dem sich finanzielle Vor- und Nachteile der Mitgliedsstaaten in der Summe ausgleichen.

Die Rechtfertigung der gemeinsamen Betrachtung von Gesamthaushalt und auBerbudgetären flnanzlellen Gemeinschaftsaktivităten resultiert nicht nur aus dem hier gewählten vertellungspolitischen Ansatz. Sie wird unterstutzt durch die mehr oder wentger ausgeprăgten Forderungen von Parlament und Kommission, die gesamten finanzwirksamen Aktivitäten - mit Ausnahme der EIB - in den Gemeinschaftshaushalt und damit in die umittelbare Verantwortung der gemeinschaftlichen Haushaltsorgane elnzubeziehen. 


\subsubsection{Europäischer Entwicklungsfonds}

Neben der weltweit gestreuten Entwicklungshilfe im Rahmen des Gesamthaushalts besteht als zusätzliches Instrument der Europäische Entwicklungsfonds (EEF), durch den die Gemeinschaft denjenigen Entwicklungsländern Hilfe leistet, die mit ihr durch ein umfassendes Kooperationsabkommen verbunden sind. Dieses war - auf der Grundlage der Art. 131 bis 136 des EWG-Vertrages ursprünglich für diejenigen uberseeischen Länder und Hoheltsgebiete konzlplert, die sich bei der Gründung der EWG noch in Abhănglgkelt elnzelnzer Mitgliedsstaaten, nämlich Belgien, Frankrelch, Italien, Niederlande, befanden, zu denen mit dem Beltritt Großbritanniens noch einige CommonwealthLänder hinzukamen. Mit der Unabhănglgkelt der melsten dieser Entwlcklungslander anderte sich zwar deren Vertragsstatus, doch blieben sie zumelst weiterhin mit der Gemelnschaft assozilert. zudem wurden die Abkommen auch fur elnige andere Entwicklungsländer geoffnet. 1

Der 5. EEF ${ }^{2}$ regelt von 1981 bis 1985 die Bezlehungen zu 63 staaten tm afrikantschen, tm kartbischen und pazifischen Raum (sog. AKPStaaten). Daneben 1st etn besonderer Tell der Fondsmittel fur die Entwlcklung der derzett noch zu Frankrelch, den Niederlanden und dem Vereinlgten Köntgretch gehorenden uberseelschen Lănder und Gebiete (ULG) bestimnt.

Neben handelspolttıschen Vortellen, denen zufolge die AKP-Staaten den wettaus gröBten Tell threr Produkte zollfrel in die Gemelnschaft ausfuhren konnen ohne den EG-Erzeugntssen threrselts Zollfreihelt etnraumen zu mussen, besteht die ausgabenwirksame Gemeinschaftshilfe $t m$ wesentlichen in der Finanzierung von Projekten zum Aufbau der wirtschaftltchen Infrastruktur, zur Modernisierung der Landwirtschaft sowte fur sozlalpolitische MaBnahmen; daneben in der Finan-

${ }^{1}$ Vgl. D. Strasser, S. 282 ff.

2 Er knupft an die ebenfalls auf 5 Jahre befristeten yler vorhergehenden Fonds an, die erstmals Im Jahre 1959 in Kraft getreten sind. 
zierung eines Systems zur Stabilisierung der Ausfuhrerlöse (Stabex) sowie eines Systems zur Aufrechterhaltung der Produktions- und Exportkapazitäten bei bestimmten Bergbauerzeugnissen. 1 Die Gesamtausstattung des 5. EEF beläuft sich auf 4.802,3 Mio. ECU, von denen ca. 80 \& als nichtrückzahlbare Zuschüsse vergeben werden. Hinzu kommen 700 Mio. ECU, die die Europäische Investitionsbank im Rahmen threr Darlehenspolitik für Investitionsvorhaben auBerhalb der Gemeinschaftsländer zur Verfügung stellt.

Die Finanzierung der Ausgaben des EEF durch die Mitgliedsstaaten erfolgt nach einem besonderen Aufbringungsschlüssel, der für jeden Fonds jeweils in einem internen Abkommen festgelegt wird. Der für den 5. EEF geltende Beitragsschlüssel ist in Tabelle 45 angegeben. Dabei wird deutlich, ${ }^{2}$ daß zu seiner Festlegung - wie auch bei den vorhergehenden Fonds - in erster Linie die Wirtschaftskraft der Mitgliedsländer als Bestimmungsfaktor herangezogen wurde, daneben - wie im Falle Belgiens, Frankreichs und der Niederlande erkennbar - aber auch die historischen Verbindungen mit den unterstützten Entwicklungsländern berücksichtigt werden. ${ }^{3}$

Die Einbeziehung des EEF in den Gesamthaushalt wird vom Parlament und von der Kommission seit langem gefordert. Die hierfür vorgebrachten Argumente ${ }^{4}$ sind im wesentlichen, daß auf diese Weise

- die gemeinschaftliche Entwicklungspolitik im Haushalt zusammengefast und damit auch dem Budgetgrundsatz der Einheit Rechnung getragen wird;

${ }^{1} \mathrm{Vg}$ 1. etwa: EG-Kommission, Die Gemeinschaft und die Entwicklungsländer, Stichwort Europa, 3/83, Februar 1983, S. 4

${ }^{2}$ siehe hierzu die in Tabelle 1 dargestellten BIP-Anteile der Mitgliedsländer.

${ }^{3}$ Vgl. E. Reister, s. 164 .

${ }^{4}$ Vgl. hierzu etwa EG-Parlament, Bericht im Namen des Haushaltsausschusses uber die parlamentarische Kontrolle der Finanztätigkeit des Europäischen Entwicklungsfonds, Berichterstatter: M. Bangemann, Europäisches Parlament, Sitzungsdokumente 1978 - 1979, Dok. 203/78, insbes. S. 17 f.; EG-Kommission, Einbeziehung des Europäischen Entwicklungsfonds in den Haushaltsplan (Mitteilung der Kommission an den Rat), KOM (78) 732 endg., Brüssel, 22.12.1978. 
- die parlamentarische Kontrolle gewährleistet ist;

- eine stärkere Diversifizierung der von den Agrarausgaben dominierten Ausgabenstruktur möglich ist;

- sich die oft langwierigen Diskussionen über die Aufbringungsschlüssel erübrigen und das Problem der tbergangsregelungen zwischen zwei Assozilerungsabkommen entfällt.

Diesen Vorschlägen ist der Rat bisher allerdings nicht gefolgt.

Die verteilungspolitischen Auswirkungen einer Einbeziehung des EEF in den Gesamthaushalt ergeben sich aus einer veränderten Aufbringung der Finanzierungsmittel. An die stelle des besonderen Beitragsschlüssels tritt dann eine Finanzierung aus den Eigenmitteln und in diesem Rahmen konkret über erhöhte Mehrwertsteuerzahlungen der Mitgliedsländer. Tabelle 45 zeigt, welche finanziellen Effekte für das Haushaltsjahr $1983^{1}$ resultieren würden. Danach hätten insbesondere Belgien, Frankreich und die Niederlande geringere, Italien und das Vereinigte Königreich dagegen höhere Zahlungen im Vergleich zur bestehenden Regelung zu leisten und würden damit vor allem diejenigen Gemeinschaftsländer entlastet, die mit assoziierten Staaten durch besondere Beziehungen verbunden sind. Allerdings wird auch deutlich, daß sich die Veränderungen in der Mittelaufbringung zumeist in einem sehr bescheidenen Rahmen bewegen. Dies gilt auch dann, wenn man berücksichtigt, daß die 1983 zu leistenden Beiträge zum EEF nur etwa die Hälfte der jahresdurchschnitt]ich fälligen Mittel ${ }^{2}$ ausmachen. Von einer Einbeziehung des EEF würden bei dem gegenwärtigen Volumen also nur relativ geringe Verteilungswirkungen ausgehen.

Neben der Verteilung der Finanzierungslasten ist nach den Rückwirkungen auf die EG-Mitgliedsstaten zu fragen, die sich aus der Durchführung der Entwicklungshilfeprogramme ergeben. Denn hiermit

${ }^{1}$ Das Problem der Erschöpfung der Mehrwertsteuer-Eiqenmittel sei hier unberücksichtigt gelassen.

${ }^{2}$ Diese betragen bei einem Volumen des EEF von $4.802,3$ Mio. F.CU bei einer fünfjährigen Laufzeit etwa 1 Mrd. ECU. 
Tabelle 45

Finanzierung des 5. EEF nach Beitragsschlüssel und nach Maßgabe der Mehrwertsteuer-Anteile 1983

\begin{tabular}{|c|c|c|c|c|c|}
\hline Mitgliedsland & $\begin{array}{l}\text { Beitrags- } \\
\text { schlüssel } \\
5 . \text { EEF } \\
8 \\
\end{array}$ & $\begin{array}{l}\text { Beiträge } \\
\text { Mio. ECU } \\
\end{array}$ & \begin{tabular}{|c|} 
Mehrwert- \\
steuer- \\
Anteile \\
8
\end{tabular} & $\begin{array}{l}\text { Differenz } \\
\text { steuer-Bei } \\
\text { nanzierung } \\
\text { Anteile }\end{array}$ & $\begin{array}{l}\text { Mehrwert- } \\
\text { itragsfi- } \\
\text { Mio. ECU }\end{array}$ \\
\hline Belgien & 5,82 & 29,2 & 3,14 & $-2,68$ & $-13,4$ \\
\hline Dänemark & 2.47 & 12,4 & 2,00 & $-0,47$ & $-2,4$ \\
\hline Deutschland & 27,92 & 140,0 & 29,42 & $+1,50$ & $+7,5$ \\
\hline Frankreich & 25,26 & 126,7 & 22,45 & $-2,81$ & $-14,1$ \\
\hline Griechenland & 1,34 & 6,7 & 1,57 & $+0,23$ & $+1,2$ \\
\hline Irland & 0,59 & 3,0 & 1,10 & $+0,51$ & $+2,5$ \\
\hline Italien & 11,34 & 56,9 & 14,00 & $+2,66$ & $+13,3$ \\
\hline Luxemburg & 0,20 & 1,0 & 0,29 & $+0,09$ & $+0,5$ \\
\hline Niederlande & 7,30 & 36,6 & 5,20 & $-2,10$ & $-10,5$ \\
\hline $\begin{array}{l}\text { Verein. König- } \\
\text { reich }\end{array}$ & 17,76 & 89,1 & 20,83 & $+3,07$ & $+15,4$ \\
\hline EG & 100,00 & 501,5 & 100,00 & 0 & 0 \\
\hline
\end{tabular}

Quelle: EG-Rechnungshof, Jahresbericht zum Haushaltsjahr 1983, S. 185; EG-Kommission, Vermögensübersichten und Haushaltsrechnungen des Europäischen Entwicklungsfonds für das Haushaltsjahr 1982, KOM (82) 479 endg., Brüssel, 25.7.1983, S. 8; Tabelle 5; elgene Berechnungen.

sind zu einem großen Tell Bau- und Lieferaufträge sowie Dienstleistungsverträge über technische zusammenarbeit verbunden, deren Vergabe bei Bauleistungen und Lieferungen im Wege internationaler Ausschreibungen erfolgt und die vor allem auf die EG-Mitgliedsländer sowie auf die AKP-Staaten abstellen. ${ }^{1}$ Insoweit Gemeinschaftsstaaten die Zuschläge erhalten, darf wohl prinzipiell mit entsprechenden positiven wirtschaftlichen Wirkungen gerechnet werden. Diese werden

\footnotetext{
${ }^{1}$ Vgl. Kommission der EG, Teilnahme an den vom Europäischen Entwicklungsfonds finanzierten Auftrăgen, Sammlung Aktuelle Fragen, Reihe Entwicklung, Nr. 3, Brüssel 1981, s. $11 \mathrm{ff}$.
} 
sich zunächst aus der unmittelbaren Auftragserfüllung und darüber hinaus eventuell aus einer Intensivierung der wirtschaftlichen Beziehungen ergeben.

Tabelle 46 zeigt die Aufschlüsselung der im Rahmen des 5. EEF bis zum 31. Dez. 1982 finanzierten Aufträge nach Empfängerländern. ${ }^{1}$ Dabei wird deutlich, daß die ökonomischen Vorteile der Auftragserfüllung den Finanzierungsbeteiligungen der Mitgliedsstaaten in vielen Fällen nicht entsprechen. So führen Frankreich und Irland vergleichsweise weit mehr Aufträge aus und sind vor aliem das vereinigte Königreich und Dänemark in dieser Hinsicht stark unterrepräsentiert. Auch wenn sich die hieraus resultierenden ökonomischen und finanziellen Wirkungen nicht quantifizieren lassen, ist angesichts der Größenordnungen ${ }^{2}$ doch festzustellen, daß diese Effekte insgesamt recht gering sein werden.

\subsubsection{Funktionshaushalt der Europäischen Gemeinschaft für Kohle und Stahl}

Neben dem Gesamthaushalt der EG werden die operationellen Ausgaben der EGKS und die zu ihrer Deckung vorgesehenen Einnahmen über einen gesonderten Haushalt, den sog. Funktionshaushalt abgewickelt. Die Finanz- und Ausgabenautonomie der EGKS wurde durch die Fusion einzelner Haushalte der Gemeinschaften zum Gesamthaushalt ${ }^{3}$ nur insoweit beruhrt, als der Verwaltungshaushalt in den Gesamthaushalt integriert worden ist. Dafür leistet die EGKS - zur Entlastung ihres Funktionshaushalts bewußt zu niedrig angesetzte - Pauschalzahlungen ${ }^{4}$

${ }^{1}$ Die hier ausgewiesenen 304,7 Mio. ECU machen 58 der bis dahin erfolgten Zahlungen des 5. EEF von 526 Mio. ECU (vgl. EG-Rechnungshof, Jahresbericht zum Haushaltsjahr 1982, S. 191) aus.

2 Auch hier ailt allerdings, daß mit wachsender tatsächlicher Inanspruchnahme des EEF sich auch diese Beträge erhöhen werden.

${ }^{3}$ Siehe hierzu Abschnitt 4.1 .

${ }^{4}$ Diese Zahlungen waren in Art. 20 des Fusionsvertrages ursprünglich mit 18 Mio. Rechnungseinheiten festgesetzt, wurden aber 1978 auf 5 Mio. ECU reduziert. 
Tabelle 46

Aufschlüsselung der vom 5. EEF finanzierten Aufträge nach Nationalitäten. Stand: 31. Dez. 1982

\begin{tabular}{|l|r|r|}
\hline $\begin{array}{l}\text { Staatsangehörigkeit } \\
\text { der Unternehmen }\end{array}$ & $\begin{array}{r}\text { Bau- und Lieferaufträge und Verträge } \\
\text { uiber technische Zusammenarbeit }\end{array}$ \\
\hline Belgien & Mio. ECU & 8 \\
Dänemark & 11,0 & 5,70 \\
Deutschland & 1,4 & 0,72 \\
Frankreich & 56,7 & 29,34 \\
Griechenland & 65,1 & 33,66 \\
Irland & - & -- \\
Italien & 5,6 & 2,91 \\
Luxemburg & 24,0 & 12,42 \\
Niederlande & 0,4 & 0,21 \\
Verein. Königreich & 10,9 & 5,65 \\
EG-Staaten & 18,1 & 9,39 \\
\hline AKP-tuLG & 193,3 & 100,00 \\
Dritte Länder & 93,8 & \\
Gesamt & 17,6 & \\
\hline
\end{tabular}

Quelle: Supplement zum Amtsblatt der Europäischen Gemeinschaften S. 91 vom 16.5 .1983 , S. 4 .

an den Gesamthaushalt.

Der Umfang des Funktionshaushalts ist mit 1 bis 1,5 \% des Gesamthaushaltsvolumens relativ gering. Daneben führt die EGKS allerdings umfangreiche Anleihe- und Darlehensaktivitäten durch. Diese erscheinen jedoch nicht im Funktionshaushaltsplan, sondern in der jährlichen Bilanz, in der die gesamte Finanztätiqkeit der EGKS dargestellt wird. Eine Verbindung der Anleihe- und Darlehenstätigkeit 
mit dem Funktionshaushalt erfolgt dadurch, daß die $\mathrm{Z}$ insen der Darlehensnehmer teilweise über den Funktionshaushalt subventioniert werden. ${ }^{1}$ Während die hieraus resultierenden Verteilungswirkungen mit Gegenstand dieses Abschnittes sind, werden die mit der Anleiheaufnahme und Darlehensvergabe verbundenen Effekte zusammen mit den übrigen Anleihe- und Darlehensaktivitäten der EG in Abschnitt 4.2.3. behandelt.

Als generelle Zielsetzung der EGKS bestimmt Art. 2 des EGKS-Vertrages, das wirtschaftliche Wachstum und die Beschäftiquna im Integrationsraum zu steigern und abzusichern sowie die Voraussetzungen für eine rationellere Verteilung der Erzeugnisse zu schaffen. Dazu ist gemäß Art. 3 der technische Fortschritt in der Montanindustrie zu fördern, wobei gleichzeitig auf eine Verbesserung der Lebensund Arbeitsbedingungen der Arbeiter hinzuwirken ist. 2 Die operationellen Aufgaben der EGKS in ihrer gegenwärtigen quantitativen Bedeutung - sie sind maßgeblich durch die Krise der Stahlindustrie bestimmt - kommen in den einzelnen Positionen auf der Ausgabenseite des Funktionshaushalts (Tabelle 47) ${ }^{3}$ zum Ausdruck.

Auf der Einnahmenseite des Funktionshaushalts stellt das Umlageaufkommen die wichtigste Finanzierungsquelle dar. Während sie allerdings bis zum Ende der siebziger Jahre die übrigen Einnahmen weit überstieg, ${ }^{4}$ ist ihre relative Bedeutung insbesondere auch aufgrund der außerordentlichen Einnahmen in den letzten Jahren gesunken. Die Umlage wird auf die Produktion von Kohle und Stahl von den Unternehmen des Montanbereichs erhoben. Der Umlagesatz wird jährlich von der Kommission (Hohen Behörde) autonom festgelegt und be-

1 Daneben dienen das Umlageaufkommen sowie die aus überschüssigen Einnahmen gebildeten Rücklagen zur Besicherung der Anleihen.

2vgl. auch U. Nittka, S. 18 .

${ }^{3}$ Da für die im folgenden durchzuführende Distributionsanalyse wichtige Aufgliederungen einzelner Ausgabenarten erst ab 1981 in den Finanzberichten der EGKS verfügbar sind, wird - abweichend von der bisherigen Vorgehensweise - auch die Gesamtbetrachtung erst mit dem Jahre 1981 begonnen.

${ }^{4}$ Vgl. U. Nittka, S. 462 f. 
Tabelle 47

Ausfuhrung der Funkt1onshaushaltspläne der EGKS 1981 - 1984

\begin{tabular}{|c|c|c|c|c|c|c|c|c|c|}
\hline Finanzbedarf & 1981 & 1982 & 1983 & 1984 & Deckung & T981 & 1982 & 1983 & 1984 \\
\hline & \multicolumn{4}{|c|}{ M10. ECU } & & \multicolumn{4}{|c|}{ MiO. ECU } \\
\hline Verwaltungsausgaben & 5 & 5 & 5 & 5 & Umlageaufkommen & 126,6 & 117,3 & 136,8 & 136,4 \\
\hline Anpassungsbeihilfen & 124 & 115 & 125 & 140 & Zlnsen aus Geldanlagen & 40 & 75 & 80 & 70 \\
\hline $\begin{array}{l}\text { SozialmaBnahmen zur Um- } \\
\text { strukturierung der Elsen-, } \\
\text { Stahl- u. Kohlenindu- } \\
\text { strie }\end{array}$ & 48,6 & 113,4 & 50 & 122,5 & $\begin{array}{l}\text { Aufhebung von Mittel- } \\
\text { bindungen }\end{array}$ & 27,7 & 2,4 & 7,3 & 31,3 \\
\hline Forschungsbethilfen & 44 & 50 & 53 & 50,9 & $\begin{array}{l}\text { N1chtverwendete Ein- } \\
\text { nahmen des vorjahres }\end{array}$ & 11,4 & 63,4 & -- & 13,2 \\
\hline $\begin{array}{l}\text { Zinsverbilligungen } \\
\text { - Investitionen }\end{array}$ & 7 & 7 & 13,6 & 20,9 & $\begin{array}{l}\text { Außerordentliche E1n- } \\
\text { nahmen }\end{array}$ & 48,6 & 50 & 50 & 122,5 \\
\hline - Umstellungen & 26,2 & 38,9 & 44 & 63 & RUckariff auf RUck- & & & & \\
\hline Beihilfe fur Kokskohle & & & & 6 & $\begin{array}{l}\text { stellungen für Unvor- } \\
\text { hergesehenes }\end{array}$ & 7,6 & 29 & 25 & 26,1 \\
\hline Sonstiges & 1,7 & 1,8 & 13,6 & -- & Sonstiges & 1.9 & -- & & 8,8 \\
\hline Haushaltsplan insgesamt & 263,8 & 337.1 & 310,2 & 408,3 & Haushaltsplan insgesamt & $263 ; 8$ & 337,1 & 310,2 & 408,3 \\
\hline $\begin{array}{l}\text { Aus Darlehen aus Nicht- } \\
\text { anleihemitteln finan- } \\
\text { zlerte MaBnahmen Im So- } \\
\text { zlalwohnungsbau }\end{array}$ & 11 & 15 & 15 & 10 & $\begin{array}{l}\text { Spezialreserve und ehe- } \\
\text { mallqe Penstonsfonds }\end{array}$ & 11 & 15 & 15 & 10 \\
\hline
\end{tabular}

Quelle: EG-Kommission, Finanzbericht der EGKS, versch. Jg. 
trägt seit $1980 \quad 0,31 \% .{ }^{1}$ Die aufgrund eines hoheitlichen Aktes mögliche Festsetzung der Umlage sowie die unmittelbare, d.h. ohne die Mitwirkung nationaler Steuerbehörden erfolgende zahlung an die EGKS lassen es gerechtfertigt erscheinen, die Umlage als erste europäische steuer zu charakterisieren. 2

Im Zusammenhang mit der Umlage wird deutlich, daß das Haushaltsrecht der EGKS maßgeblich von dem Grundsatz der Zweckbindung bestimmt wird. Denn das Umlageaufkommen darf nur zur Deckunq der Verwaltungsausgaben, der Anpassungsbeihilfen, der Ausgaben zur Förderung der technischen und wirtschaftlichen Forschuna sowie im Rahmen der Anleihe- und Darlehenstätigkeit der EGKS zur Deckung von Kreditausfällen und Gewährleistungsverpflichtungen aus Bürgschaften verwendet werden. ${ }^{3}$

In der Beteiligung der Mitgliedsstaaten am Umlageaufkommen (Tabelle 48) spiegelt sich aufgrund der am Produktionswert der Montanerzeugnisse anknüpfenden Bemessungsgrundlage die relative Bedeutung der nationalen Montanindustrie wider, und aus einem Vergleich mit den zugehörigen BIP-Anteilen wird deren Stellung im Rahmen der Gesamtwirtschaft deutlich. So weisen Belgien, Deutschland und Luxemburg einen bedeutenden Anteil der Montanindustrie an ihrer Wirtschaft auf und leisten die Unternehmen dieser Länder entsprechend hohe Umlagezahlungen. Großbritannien und Italien weisen einen etwa durchschnittlichen, Frankreich und die Niederlande nur einen schwachen Anteil auf, während die Kohle- und Stahlindustrie in Dänemark und Irland kaum Bedeutung hat. ${ }^{4}$

Eine quantitativ bedeutende Einnahmequelle stellen auch die $\mathrm{Z}$ insen aus Anlagemitteln dar. Sie resultieren im wesentlichen aus der $\mathbf{z i n s -}$ tragenden Anlage der gebildeten Rücklaqen. Diese bestehen zum einen

1 Der Umlagesatz darf gemäß Art. 50 \& 1 EGKS-Vertrag 1 \& nur übersteigen, wenn der Rat mit Zweidrittelmehrheit zustimmt.

${ }^{2}$ Vgl. hierzu etwa N. Andel (1983), S. 344 f.; W. Bickel, Der Finanzausgleich, in: W. Gerloff, F. Neumark (Hrsg.), Handbuch der Finanzwissenschaft, 2. Bd., 2. Auf1., Tübingen 1956, S. 759; E. Reister, S. 155.

${ }^{3}$ Vgl. Art. 50, § 1 EGKS-Vertrag.

${ }^{4} \mathrm{Vgl}$. hierzu auch D. Strasser, S. 117. 
Tabelle 48

Aufkommen aus der EGKS-Umlage nach Mitgliedsstaaten 1981 - 1984

\begin{tabular}{|l|r|r|r|r|r|r|r|r|r|}
\hline Mitgliedsland & \multicolumn{5}{|c|}{ MiO. ECU } & \multicolumn{3}{c|}{ 年achrichtl.: } \\
& 1981 & 1982 & 1983 & 1984 & 1981 & 1982 & 1983 & 1984 & $\begin{array}{c}\text { BIP-Anteile } \\
1984\end{array}$ \\
\hline Belgien & 9,7 & 7,9 & 9,7 & 10,9 & 7,7 & 6,3 & 7,2 & 8,0 & 3,5 \\
Dänemark & 0,5 & 0,4 & 0,4 & 0,5 & 0,4 & 0,3 & 0,3 & 0,4 & 2,5 \\
Deutschland & 44,3 & 41,6 & 47,3 & 51,8 & 35,0 & 35,5 & 35,1 & 38,1 & 28,2 \\
Frankreich & 18,9 & 16,4 & 18,8 & 20,6 & 14,9 & 14,0 & 14,0 & 15,1 & 22,6 \\
Griechenland & 0,8 & 0,7 & 0,9 & 0,8 & 0,6 & 0,6 & 0,7 & 0,6 & 1,5 \\
Irland & 0,0 & 0,0 & 0,1 & 0,1 & 0,0 & 0,0 & 0,1 & 0,1 & 0,8 \\
Italien & 18,7 & 16,9 & 19,4 & 21,5 & 14,8 & 14,4 & 14,4 & 15,8 & 15,9 \\
Luxemburg & 2,8 & 2,5 & 2,9 & 3,6 & 2,2 & 2,1 & 2,1 & 2,6 & 0,1 \\
Niederlande & 3,9 & 3,0 & 3,8 & 4,8 & 3,1 & 2,6 & 2,8 & 3,5 & 5,6 \\
Verein. Königreich & 27,0 & 27,8 & 31,4 & 21,4 & 21,3 & 23,7 & 23,4 & 15,7 & 19,3 \\
EG & 126,6 & 117,3 & 134,7 & 136,0 & 100,0 & 100,0 & 100,0 & 100,0 & 100,0 \\
\hline
\end{tabular}

Quelle: EG-Kommission, Finanzberichte der EGKS, versch. Jg.; Tabelle 1 .

aus einem Garantiefonds zur Sicherung des Schuldendienstes aus den aufgenommenen Anleihen, der in den Anfangsjahren der Gemeinschaft aus Umlagemitteln angelegt und mit dem Beitritt der neuen Mitgliedsstaaten 1973 aufgestockt wurde. Daneben besteht eine Spezialreserve aus anderen als Umlagemitteln, die gegenwärtig zur Vergabe von Darlehen für den Bau von Sozialwohnungen dient. 1 Diesen Fonds werden die jährlichen Uberschüsse der Erträge über die Aufwendungen zugewiesen. Die Rücklagen hatten Ende 1984 eine Höhe von 543 Mio. ECU.

\footnotetext{
1 Zusammen mit Mitteln aus dem ehemaligen Pensionsfonds der EGKS. ${ }^{2}$ Vgl. EG-Kommission, Finanzbericht der EGKS 1982, KOM (85) 430 endg., Brüssel, 25.7.1985, S. 41.
} 
Eine exakte Zurechnung der Zinseinnahmen auf die Mitgliedsstaaten ist infolge der vielfältigen und auf einen langen zeitraum zurückreichenden Bestimmungsgründe nicht möglich. Da die Rücklagen jedoch aus den Eigenmitteln der EGKS aufgebaut worden sind und die thnen zugewiesenen überschüssigen laufenden Mittel ebenfalls zum großen Teil hieraus resultieren, wird man näherungsweise davon ausgehen dürfen, daß die über den Zeitablauf durchschnittlichen nationalen Anteile am Umlageaufkommen als maßgeblicher Einflußfaktor für die länderspezifische Verteilung der Zinsen aus Anlagemitteln anzusehen sind.

Die Einnahmen aus der Aufhebung von Mittelbindungen - dabei handelt es sich um nicht verwendete Mittel aus vorhergehenden Haushaltsjahren - ${ }^{1}$ und die nichtverwendeten Einnahmen des Vorjahres ${ }^{2}$ sowie die Rluckgriffe auf Rückstellungen für unvorhergesehene Ereignisse sind Finanzierungsquellen, die ursprünglich aus den Eiqenmitteln der EGKS gespeist worden sind. Ihre Verteilungswirkungen auf die Mitgliedsstaaten düften deshalb ebenfalls maßgeblich auf die nationalen Beteiligungen am Umlageaufkommen zurückzuführen sein.

Eine in den letzten Haushaltsjahren bedeutsame Rolle spielen die auBerordentlichen Einnahmen, die der EGKS zur Finanzierung eines Soforthilfeprogramms zur Umstrukturierung der Fisen- und Stahlund Kohlenindustrie zur Verfügung stehen. Diese Mittel entstammen In Höhe der ersten Tranche von 50 Mio. ECU für das Jahr $1981^{3}$ aus unmittelbaren Beiträgen der Mitgliedsstaaten, die nach einem besonderen Aufbringungsschlüssel finanziert wurden ${ }^{4}$, in Höhe von 62 Mio.

${ }^{1}$ Vgl. D. Strasser, S. $120 \mathrm{f}$.

${ }^{2}$ Die hierfür in Tabelle 47 ausgewiesenen 63,4 Mio. ECU stehen sachlich im Zusammenhang mit den außerordentlichen Einnahmen. ${ }^{3}$ Hiervon wurden nur 48,6 Mio. ECU vergeben.

${ }^{4} \mathrm{Vgl}$. EG-Kommission, Bulletin der EG, Nr. 6-1981, s. 21. 
ECU zu Lasten des Gesamthaushaltsplans 1981', in Höhe von 100 Mio. ECU zu gleichen Teilen aus den Gesamthaushaltsplänen 1982 und $1983^{2}$ und in Höhe von insgesamt 122,5 Mio. aus dem EG-Haushalt des Jahres 1984. An der Aufbringung dieser Mittel sind die Mitgliedsstaaten im Rahmen der ersten Tranche entsprechend dem besonderen Aufbringungsschlüssel beteiligt. Die anschließend aus dem Gesamthaushalt übertragenen Mittel können den Mitgliedsstaaten nach ihren Beteiligungen an der Finanzierung des Gesamthaushalts zugerechnet werden.

Die Ausgabenseite des Funktionshaushalts wird dominiert von den Sozialmaßnahmen zugunsten der vom Beschäftigungsrückgang in der Eisen- und Stahlindustrie betroffenen Arbeitnehmer. In diesem Rahmen stellen die nicht rückzahlbaren Anpassungsbeihilfen Entschädigungszahlungen, Beihilfen zur Erlangung eines neuen Arbeitsplatzes oder Finanzierungsunterstützungen für Umschulungsmaßnahmen dar. Dabei handelt es sich um Beteiligungen an den vom betreffenden Mitgliedsstaat getragenen Gesamtkosten. ${ }^{3}$ Die Aufteilung der Anpassungsbeihilfen auf die Mitgliedsstaaten in Tabelle 49 zelgt, daß zumeist etwa drei Viertel der Mitఓel an Großbritannien geflossen sind, das damit etwa drei- bis viermal soviel zuweisungen erhielt, als es dem relativen Anteil seiner Montanindustrie in der Gemeinschaft ${ }^{4}$ entspricht.

Die Sozialmaßnahmen durch die traditionellen Anpassungsbeihilfen werden in den letzten Jahren durch die oben berelts angesprochenen zusätzlichen - von den Mitgliedsstaaten ad hoc bzw. über den Gesamthaushalt finanzlerten - Programme ergänzt. Dabei handelt es sich um Zuschüsse zu den Beihilfen, die die Mitgliedsstaaten im

\footnotetext{
${ }^{1}$ EG-Rat, Beschluß vom 23. Februar 1982 über einen Beitrag zu Lasten des Gesamthaushaltsplans der Europäischen Gemeinschaften an die Europäische Gemelnschaft für Kohle und Stahl, in: Amtsblatt der EG $\mathrm{Nr}$. L 74 vom 18.3.1982, S. $27 \mathrm{f}$.

2EG-Rat, Beschluß vom 21. September 1982 über einen Beitrag zu Lasten des Gesamthaushaltsplans der Gemeinschaften an die Europäische Gemeinschaft für Kohle und Stahl, in: Amtsblatt der EG Nr. L 277 vom 29.9.1982, S. $13 \mathrm{f}$.

${ }^{3} \mathrm{Vgl}$. D. Strasser, S. 214 .

${ }^{4}$ Siehe Tabelle 48 .
} 
Tabelle 49

Anpassungsbeihilfen der EGKS nach Mitgliedsstaaten $1981-1984$

\begin{tabular}{|c|c|c|c|c|c|c|c|c|}
\hline \multirow[t]{2}{*}{ Mitgliedsland } & \multicolumn{4}{|c|}{ MiO. $\overline{\mathrm{BCU}}$} & \multicolumn{4}{|c|}{8} \\
\hline & 1981 & 1982 & 1983 & 1984 & 1981 & 1982 & 1983 & 1984 \\
\hline Belgien & 2,4 & 2,0 & 7,5 & 2,6 & 2,0 & 1,7 & 6,0 & 1,9 \\
\hline Dänemark & - & - & - & 1,9 & - & - & - & 1,4 \\
\hline Deutschland & 16,5 & 16,4 & 26,0 & 35,7 & 13,3 & 14,2 & 20,8 & 25,5 \\
\hline Frankreich & 4,5 & 2,0 & 0,4 & 21,1 & 3,9 & 1,7 & 0,3 & 15,1 \\
\hline Griechenland & - & - & - & - & - & - & - & - \\
\hline Irland & - & - & 0,1 & - & - & - & 0,1 & - \\
\hline Italien & 0,9 & - & - & 0,1 & 0,7 & - & - & 0,0 \\
\hline Luxentburg & 0,3 & - & - & 9,0 & 0,2 & - & - & 6,4 \\
\hline Niederlande & 1,8 & - & - & 2,2 & 1,4 & - & 一 & 1,6 \\
\hline Verein. Königreich & 97,2 & 94,7 & 91,0 & 67,4 & 78,4 & 82,3 & 72,8 & 48,1 \\
\hline EG & 124,0 & 115,0 & 125,0 & 140,0 & 100,0 & 100,0 & 100,0 & 100,0 \\
\hline
\end{tabular}

Quelle: EG-Kommission, Finanzberichte der EGKS, versch. Jg.; eigene Berechnungen.

Rahmen der Umstrukturierungsprogramme im Montansektor für Maßnahmen bel vorzeitigem Ausscheiden aus dem Erwerbsleben sowie bei Kurzarbeit gewăhren.' 1 Die Verteilungswirkungen dieser Sozialmaßnahmen auf die M1tgliedsstaaten in Tabelle 50 zeigen bei stark schwankenden zuflüssen, daB vor allem Frankreich im Vergleich zum Antell seiner Montanindustrie ${ }^{2}$ uberproportional groBe Vorteile erhalten hat.

1Vgl. EG-Kommission, Bulletin der EG, Nr. 6-1981, s. 21. 2 siehe Tabelle 48 . 
Tabelle 50

Sozialmaßnahmen zur Umstrukturierung der Eisen- und Stahl- und Kohlenindustrie nach Mitgliedsstaaten 1981 - 1984

\begin{tabular}{|l|c|c|c|c|c|c|c|c|}
\hline Mitgliedsland & 1981 & 1982 & 1983 & 1984 & 1981 & 1982 & 1983 & 1984 \\
\hline Belgien & 4,7 & 14,6 & 3,5 & 5,2 & 9,7 & 12,9 & 7,0 & 4,2 \\
Dänemark & - & - & 1,6 & 0,3 & -- & - & 3,2 & 0,2 \\
Deutschland & 0,8 & 8,2 & 21,0 & 39,6 & 1,6 & 7,2 & 42,0 & 32,3 \\
Frankreich & 21,4 & 36,8 & 10,3 & 28,6 & 44,0 & 32,5 & 20,6 & 23,3 \\
Griechenland & - & - & - & - & -- & - & -- & -- \\
Irland & - & - & - & - & -- & - & - & - \\
Italien & 2,1 & 13,8 & 2,0 & 15,9 & 4,3 & 12,2 & 4,0 & 13,0 \\
Luxemburg & 0,3 & 0,9 & 0,2 & 1,9 & 0,6 & 0,8 & 0,4 & 1,6 \\
Niederlande & - & 1,2 & 1,2 & 0,6 & - & 1,1 & 2,4 & 4,9 \\
Verein. Königreich & 19,3 & 37,9 & 10,2 & 30,5 & 39,7 & 33,4 & 20,4 & 24,9 \\
EG & 48,6 & 113,4 & 50,0 & 122,5 & 100,0 & 100,0 & 100,0 & 100,0 \\
\hline
\end{tabular}

Quelle: EG-Kommission, Sechzehnter, Siebzehnter und Achtzehnter Gesamtbericht 1982,1983 und 1984 über die Tätigkeit der Europäischen Gemeinschaften.

Mit den Forschungsbeihilfen kommt die Hohe Behörde ihrer Verpflichtung aus Art. 55 EGKS-Vertrag nach, die technische und wirtschaftliche Forschung für die Erzeugung und die Steigerung des Verbrauchs von Kohle und Stahl sowie die Betriebssicherheit in diesen Industrien zu fördern. Diese Beihilfen stellen ebenfalls nicht rückzahlbare Beteiligungen an den Gesamtkosten der Forschungsprojekte dar. 1 Eine Auftellung dieser Mittel nach Mitgliedsstaaten steht nicht zur Verfügung.

\footnotetext{
${ }^{1}$ Vgl. D. Strasser, S. $266 \mathrm{f}$.
} 
Die Zinszuschüsse im Rahmen der von der EGKS gewährten Darlehen werden über den Funktionshaushalt finanziert. ${ }^{1}$ solche subventionen gewährt die EGKS für Darlehen, die Umstrukturierungs- und Umstellungszielsetzungen dienen sollen. Dabei ist zu unterscheiden in zinsverbilligte Darlehen für Investitionsvorhaben zur strukturellen Verbesserung und Sanierung der Montanindustrie sowie in Umstellungsdarlehen zur Schaffung neuer Arbeitsplätze in Regionen sinkender Beschäftigung der Kohle- und Stahlunternehmen. 2 Die Zinszuschüsse werden zumeist in der Form gewährt, daß der Zinssatz während eines Zeitraums von 5 Jahren 3 Prozentpunkte unter dem Normalsatz liegt. ${ }^{3}$ In der Tabelle 51 sind die auf die Mitgliedsstaten entfallenden $\mathrm{Zinszuschüsse} \mathrm{angegeben,}{ }^{4}$ die ebenfalls starke Schwankungen im Zeltablauf aufweisen.

Zur Bestimmung von Umverteilungswirkungen im Rahmen der EGKS-Funktionshaushalte sind die den Mitgliedsstaaten zugeflossenen Ausgaben den von ihnen geleisteten Finanzierunqsbeiträgen gegenüberzustellen. Ein solcher Verglelch ist allerdings nur für diejenigen Ausgaben- und Einnahmenkategorien möglich, die den Mitgliedsstaaten zurechenbar sind. Dazu gehören auf der Ausgabenseite die Anpassungsmaßnahmen, die zusätzlichen Sozialmaßnahmen sowie die zinszuschüsse, während auf der Einnahmenseite die länderspezifische Verteilung des Umlageaufkommens vorliegt und die Zurechnung der außerordentlichen Einnahmen auf die Mitgliedsstaaten nach Maßgabe ihrer

${ }^{1}$ Vgl. EG-Parlament, Bericht im Namen des Ausschusses für Haushaltskontrolle über die Finanztätigkeiten der EGKS, Berichterstatter: Herr Volkmar Gabert, Europäisches Parlament, Sitzungsdokumente 1983 - 1984, Dok. 1-464/83, S. 12.

${ }^{2} \mathrm{Vgl}$. D. Strasser, S. $231 \mathrm{f}$.

${ }^{3}$ Val. EG-Kommission, Finanzbericht der EGKS 1982, s. 38.

${ }^{4}$ Im Vergleich $z u$ den Anqaben der Tabelle 47 erqeben sich in der Gesamtsume tellweise nicht unerhebliche Abweichungen. 
Tabelle 51

EGKS-Zinszuschüsse nach Mitgliedsstaaten

$1981-1984$

\begin{tabular}{|c|c|c|c|c|c|c|c|c|}
\hline Mitgliedsland & 1981 & $\begin{array}{r}\text { Mio } \\
1982 \\
\end{array}$ & $\begin{array}{r}\text { ECU } \\
1983 \\
\end{array}$ & 1984 & 1981 & 1982 & 1983 & 1984 \\
\hline Belgien & 3,5 & 2,4 & 12,1 & 0,7 & 11,1 & 8,1 & 35,0 & 1.7 \\
\hline Dănemark & -- & 0.3 & 0,8 & 0,8 & -- & 1,0 & 2,3 & 2,0 \\
\hline Deutschland & 6,0 & 11,1 & 4,1 & 25,8 & 19,1 & 37,2 & 11,8 & 64,0 \\
\hline Frankreich & 3,0 & 0,5 & 4,7 & 0,1 & 9,6 & 1,7 & 13,6 & 0,2 \\
\hline Griechenland & -- & -- & -- & -- & -- & -- & -- & \\
\hline Irland & -- & 0,4 & -- & -- & -- & 1,3 & -- & -- \\
\hline Italien & 1,2 & -- & 6,5 & 2,2 & 3,8 & -- & 18,8 & 5,5 \\
\hline Luxemburg & -- & 0,7 & -- & -- & -- & 2,4 & -- & \\
\hline Niederlande & -- & 1,2 & -- & -- & -- & 4,0 & -- & -- \\
\hline Verein. Königreich & 17,7 & 13,2 & 6,4 & 10,6 & 56,4 & 44,3 & 18,5 & 26,3 \\
\hline EG & 31.4 & 29,8 & 34,6 & 40,3 & 100,0 & 100,0 & 100,0 & 100,0 \\
\hline
\end{tabular}

Quelle: EG-Kommission, Bericht Uber die Anleihe- und Darlehenstätigkeit der Gemeinschaft, versch. Jg.

Finanzierungsantelle am Gesamthaushalt ${ }^{1}$ möglich ist. Diese vom Gesamthaushalt ubertragenen Mittel werden bel der Berechnung der Nettopositionen durch die EG-Kommission - ebenso wie die Entwicklungshilfeausgaben - ausgeklammert, so dab sie mit ihrer Berücksichtigung im Rahmen des EGKS-Funktionshaushalts wieder in die Betrachtung einbezogen werden. Mit den hier genannten Budgetpositionen werden auf der Ausqaben- und Einnahmenseite knapp zwei Drittel des Funktionshaushaltsvolumens in die Verteilungsrechnung einbezogen.

${ }^{1}$ siehe Tabelle 19. 
Wie Tabelle 52 zeigt, fallen die Nettovorteile aus dem hier zugrundeliegenden Ausgaben-Finanzierungs-System hauptsächlich dem Vereinigten Königreich zu. Dieses Ergebnis wird mit der Einbeziehung der ubrigen Ausgaben und Einnahmen zwar modifiziert, dürfte sich aber nicht grundlegend verändern.

Tabelle 52

Umverteilungswirkungen ausgewăhlter Einnahmen und Ausgaben des EGKS-Funktionshaushalts 1983

\begin{tabular}{|l|c|c|c|}
\hline Mitgliedsland & Ausgaben & $\begin{array}{c}\text { Elnnahmen } \\
\text { Mio. ECU }\end{array}$ & Differenz \\
\hline Belgien & 23,1 & 12,4 & $+10,7$ \\
Dänemark & 2,4 & 1,5 & $+0,9$ \\
Deutschland & 51,1 & 61,4 & $-10,3$ \\
Frankreich & 15,4 & 28,6 & $-13,2$ \\
Griechenland & -- & 1,7 & $-1,7$ \\
Irland & 0,1 & 0,7 & $-0,6$ \\
Italien & 8,5 & 25,9 & $-17,4$ \\
Luxemburg & 0,2 & 3,0 & $-2,8$ \\
Niederlande & 1,2 & 7,2 & $-6,0$ \\
Verein. Königreich & 107,6 & 42,5 & $+65,1$ \\
EG & 209,6 & 184,9 & $+24,7$ \\
\hline
\end{tabular}

Quelle: Tabellen 20, 50 bis 53; elgene Berechnungen.

Die vorangegangene Analyse hat gezeigt, daß die ausgabenwirksamen Aktivitäten im Rahmen des Funktionshaushalts zum weit uberwiegenden Tetl soztal- bzw. regionalpolitisch motiviert sind. Diese MaBnahmen welsen Parallelen $z u$ den Ausgabenprogrammen des EG-Gesamthaushalts auf. So sind die traditionellen und zusatzlichen Belhilfeprogramme 
der EGKS etwa mit den Maßnahmen des Sozialfonds vergleichbar ${ }^{1}$, trägt der Regionalfonds ebenso wie die über den Funktionshaushalt finanzierten $\mathrm{Z}$ insverbilligungen zur Schaffuna von Arbeitsplätzen in den von der Stahlkrise betroffenen Gebieten bei ${ }^{2}$ und bestehen auch bei den Forschungsbeihilfen Paralellen zu den über den Gesamthaushalt abgewickelten Projekten. ${ }^{3}$

Die Einnahmensituation des Funktionshaushalts ist gekennzeichnet durch eine relativ geringe Flexibilität in der Ausschöofung der Eigenmittelquellen. Denn es besteht gegenwärtig allgemeine Ubereinstimmung darin, daß eine Heraufsetzung des Umlagensatzes angesichts der Krisensituation der Eisen- und Stahlindustrie nicht vertretbar ist, 4 so daß die Hauptfinanzierungsquelle nicht in der Lage ist, ein dem gestiegenen Finanzbedarf angemessenes Aufkommen zu liefern. zur Lösung dieses Problems hatte die Kommission bereits 1978 vorgeschlagen, die im warenverkehr mit den Nichtmitgliedsstaaten auf EGKS-Erzeugnisse entfallenden zölle der EGKS zuzuweisen. Diese Einnahmen gehören nämlich nicht zu den eigenen Mittlen der Gemeinschaft, sondern fallen immer noch in den Verfügungsbereich der Mitgliedsstaaten. ${ }^{5}$ Allerdings kam hierüber nie ein Beschluß des Rates zustande. Stattdessen beschränkte er sich darauf, in den Jahren 1978 bis 1980 die Finanzierungsdefizite des Funktionshaushalts tellweise über Ad hoc Beiträge der Mitgliedsstaaten, die nach einem besonderen Aufbringungsschlüssel aufgebracht wurden, zu dek-

\footnotetext{
${ }^{1}$ Vgl. EG-Rechnungshof: Studie über die Finanzsysteme der Europäischen Gemeinschaften (1981), in: Amtsblatt der EG, Nr. C 342 vom 31.12 .1981 , s. 39 .

2Vgl. EG-Parlament, Erläuterungen der Kommission zur Festsetzung des Umlagesatzes der EGKS und der Aufstellung des EGKS-Funktionshaushaltsplans für 1983, Europäisches Parlament, Sitzungsdokumente 1982 - 1983, Dok. 1-865/82, S. 34 .

${ }^{3}$ Vgl. EG-Parlament, Bericht im Namen des Haushaltsausschusses über die Festsetzung des Umlagesatzes der EGKS und über den EGKS-Funktionshaushaltsplan für 1983 (Dok. 1-865/82, KOM (82) 706 endg.), Berichterstatter: Herr Konrad Schön, Europäisches Parlament, Sitzungsdokumente 1982 - 1983, Dok. 1-959/82, S. 21.

${ }^{4} \mathrm{Vgl}$. etwa Europäisches Parlament, Bericht im Namen des Haushaltsausschusses uber die Festsetzung des Umlagesatzes der EGKS und uber den EGKS-Funktionshaushaltsplan 1983, S. 12.

${ }^{5}$ Vgl. D. Strasser, s. $118 \mathrm{f}$.
} 
ken. 1 Diese Beiträge wurden dann ab 1981 durch die Ubertragungen aus dem Gesamthaushalt zugunsten spezieller Sozialprogramme abge1öst. Damit entstand auch über die Finanzierungsseite eine enge Verbindung mit dem Gesamthaushalt.

Diese Zusammenhänge in der Aufgabenerfüllung und im Finanzierungsbereich zwischen dem Gesamthaushalt und dem Funktionshaushalt stützen maßgeblich die Forderungen des Parlaments nach einer Abstimmung sämtlicher Gemeinschaftsinstrumente im EWG- und EGKSBereich und als Konsequenz daraus nach einer weitgehenden Koordinierung des Funktionshaushalts der EGKS mit dem Gesamthaushalt der $E G$, wobei langfristig eine Verschmelzung der beiden Haushalte anzustreben sei. ${ }^{2}$ An dieser Stelle soll eine Diskussion über eine Integration des Funktionshaushalts in den Gesamthaushalt und die damit verbundenen Auswirkungen auf den Sonderstatus der EGkS nicht erfolgen. Im Hinblick auf die verteilungspolitische Analyse im Rahmen der EG kam es hier lediglich darauf an, die Einbeziehung auch dess Funktionshaushalts in das Gesamtkonzept der Distributionswirkungen zusätzlich rechtfertigen zu können.

${ }^{1}$ Vgl. Eg-Parlament, Sitzungsdokumente 1982 - 1983, Dok. 1-865/82, S. 12 .

${ }^{2}$ Vgl. EG-Parlament, Entschließung zur Festsetzung des Umlagesatzes und zum EGKS-Funktionshaushaltsplan für 1983, in: Amtsblatt der EG, Nr. C 13 vom 17.1 .1983 , S. 36 ff. 


\subsubsection{Anleihe- und Darlehenstransaktionen}

Die Entwicklung der Anleihe- und Darlehenstätigkeit gehört zu den bedeutendsten Ereignissen der Gemeinschaftsfinanzen in den letzten Jahren. 1 Dies kommt auch in der Tabelle 53 zum Ausdruck, die die Anleiheaufnahme und Darlehensvergabe der verschiedenen Gemeinschaftsinstitutionen seit 1980 wiedergibt. Gegenwärtig betragen die aufgebrachten und bereitgestellten Mittel etwa ein Viertel, im Jahre 1983 aufgrund einer neuen EWG-Zahlungsbilanztransaktion sogar etwa die Hälfte des Gesamthaushaltsvolumens. Dabei besteht die Tendenz, auch weiterhin in zunehmendem Maße solche Aktivitäten außerhalb des Haushalts durchzuführen, und dürften diese Bestrebungen un sostärker an Bedeutung gewinnen, je mehr das system der eigenen Mittel an seine Grenzen stößt.

Die Anleihe- und Darlehenstätigkeit wird über fünf Institutionen mit unterschicdlicher quantitativer Intensität durchaeführt. Darunter ist die EGKS als älteste der Gemeinschaften durch Art. 49 bis 51 des EGKS-Vertrages seit 1952 zur Aufnahme von Anleihen und zur Gewährung von Krediten für bestimmte Zwecke der Montanindustrie ermächtigt. Die EGKS weitete zunächst ihre Aktivitäten kontinuierlich aus; die Krise in der Eisen- und Stahlindustrie bewirkte in den letzten Jahren jedoch eine Verminderung des Niveaus.

Für die EAG sieht deren Gründungsvertrag (Art. 172, Abs. 4) die Aufnahme von Anleihen zur Finanzierung von Forschungsaufgaben und Investitionen vor. Zwar wurde bereits 1959 ein begrenztes Kreditabkommen mit der amerikanischen Eximbank zur Finanzierung von Kernkraftwerken in der Gemeinschaft geschlossen. Die eigentliche Anleihe- und Darlehenstätigkeit begann allerdings erst im Jahre 1977 mit der Genehmigung eines Kreditplafonds von 500 Mio. ECU, der nach

\footnotetext{
${ }^{1}$ Vgl. D. Strasser, S. 153 .
} 
Tabelle 53

Anleihe- und Darlehenstätigkeit der EG 1980 - 1984

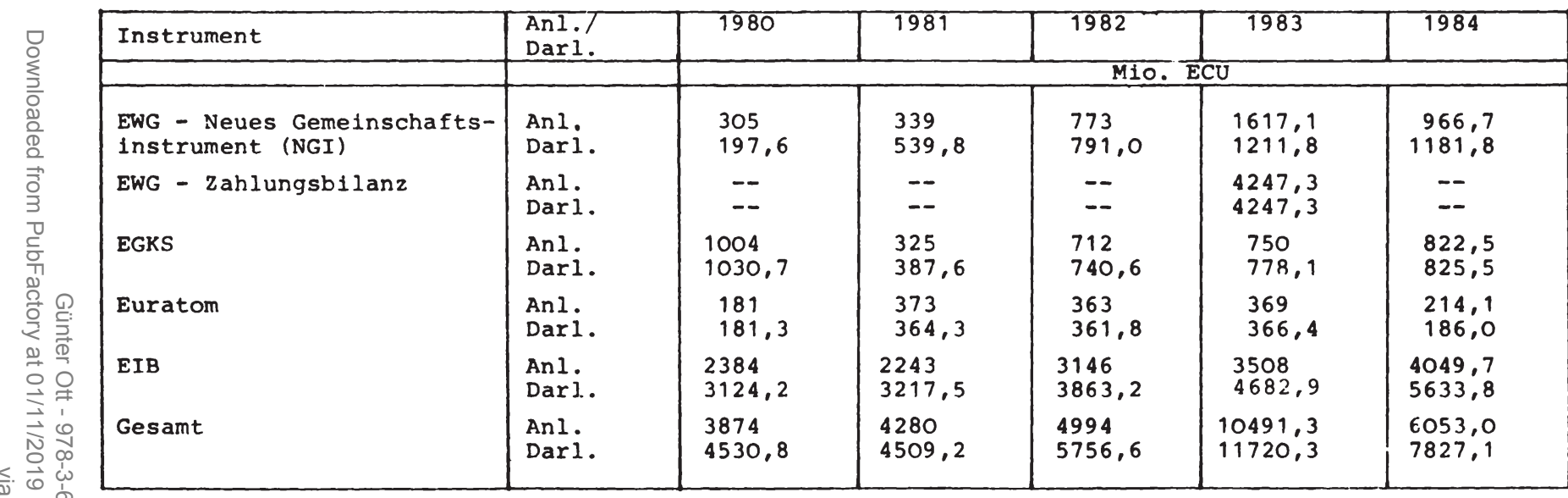

Quelle: EG-Kommission, Bericht uber die Anleihe- und Darlehenstätigkelt, versch. Jg. 
dem jüngsten Ratsbeschluß von $1982^{1}$ auf 2 Mrd. ECU erhöht wurde.

Anders als der EGKS-Vertrag und der EAG-Vertrag sieht der EWG-Vertrạ eine Anleihebefugnis der Gemeinschaft nicht ausdrücklich vor, so daß mit der Anleihe- und Darlehenstätiqkeit erst relativ spät begonnen wurde. ${ }^{2}$ zunächst wurden als Reaktion auf die erste $61 k r i-$ se 1975 Gemeinschaftsanleihen zur stützung der Zahlungsbilanzen der in Schwieriqkeiten geratenen Gemeinschaftsländer geschaffen, deren Obergrenze auf 3 Mrd. US-Dollar festgesetzt war. Hiervon sind 1,8 Mrd. $\mathrm{ECU}^{3}$ in Anspruch genommen und in den Jahren 1976 und 1977 an Italien und Irland als Antraqsteller vergeben worden. Angesichts der wiederholten Verteuerungen der Erdölerzeugnisse ist der Höchstbetrag der im Rahmen dieses Systems genehmigten Anleihen auf 6 Mrd. ECU heraufgesetzt worden, ${ }^{4}$ obwohl zunächst keine Darlehensanträge vorlagen. Ein solcher Antrag wurde 1983 von Frankreich in Höhe von 4 Mrd. ECU zur Durchführung seines wirtschafts- und währungspolitischen Programms gestellt und vom Rat genehmigt. ${ }^{5}$ Die abgewickelten Maßnahmen beliefen sich auf 4247,3 Mio. ECU ${ }^{6}$ entsprechend den Anleihetransaktionen über 150 Mio. ECU und 3390 Mio. US-Dollar. ${ }^{7}$

1EG-Rat, Beschluß vom 15 März 1982 zur Änderunq des Beschlusses 77/ 271 /Euratom hinsichtlich des Höchstbetrages der Euratom-Anleihen, welche die Kommission im Hinblick auf einen Beitraq für die Finanzierung von Kernkraftanlagen aufnehmen kann, in: Amtsblatt der EG, Nr. L 78 vom 24.3.1982, S. 21

${ }^{2}$ Grundlage bildete der Art. 235 des EWG-Vertrages nach dem der Rat auf Vorschlag der Kommission die qeeigneten Vorschriften erlassen kann, sofern ein Tätigwerden der Gemeinschaft erforderlich erscheint, um im Rahmen des gemeinsamen Marktes eines ihrer ziele zu verwirklichen und im Vertrag die hierfür notwendigen Mittel nicht vorgesehen sind. Vgl. H. Pfisterer, Zur Anleihe- und Darlehenspolitik der EG, in: Sparkasse, $98 \mathrm{Jg} ., 1981, \mathrm{~S} .415 \mathrm{ff} .$, hier: S. 417; M. Seidel, Ausgestaltung und rechtliche Begrenzungen der Anleihebefugnis der Furopäischen Wirtschaftsgemeinschaft, in: Recht der Internationalen Wirtschaft, $23 \mathrm{Jg} ., 1977$, s. 665 ff., hier: S. 665 .

3 Einer ECU entsprachen im Jahresdurchschnitt 1976 1,12 us-Dollar.

${ }^{4}$ EG-Rat, Verordnung (EWG) Nr. 682/81 vom 16. März 1981 für die Anpassung des Systems der Gemeinschaftsanleihen zur Stützung der Zahlungsbilanzen der Mitgliedsstaaten, in: Amtsblatt der EG, Nr. L 72 vom 19.3.1981, S. 1 f.

${ }^{5} \mathrm{Vgl}$. Bulletin der EG, Nr. 5, 1983, Ziff. 2.1.3.

${ }^{6}$ Umrechnungskurse von Dezember 1983.

${ }^{7} \mathrm{Vgl}$. EG-Kommission, Siebzehnter Gesamtbericht, Ziff. 107 und 146. 
Neben den zahlungsbilanzpolitisch motivierten Anleihen wurde der EG im Jahre 1978 durch einen Grundsatzbeschluß des Rates - ebenfalls gestutzt auf Art. 235 des EWG-Vertrages - eine allgemeine Kompetenz der Anleiheaufnahme zur Finanzierung von Energie-, Industrie- und Infrastrukturmaßnahmen unter Berücksichtiqung regionaler und beschäftigungspolitischer Auswirkunqen zugewiesen. Der Höchstbetrag dieses "Neuen Gemeinschaftsinstruments (NGI) " war zunächst auf 1 Mrd. ECU begrenzt, wurde aber durch eine erneute Ermächtigung des Rates im Jahre $1983^{1}$ um weitere 3 Mrd. ECU erhöht.

Die Europäische Investitionsbank wurde 1958 auf der Grundlaqe von Art. 129 des EWG-Vertrages mit dem Auftraq gegründet, "zu einer ausgewogenen und reibungslosen Entwicklung des gemeinsamen Marktes im Interesse der Gemeinschaft beizutragen." 2 sie wickelt vor allem seit Ende der siebziger Jahre den weitaus arößten Teil der Anleiheund Darlehenstätigkeit innerhalb der Gemeinschaftsinstitutionen ab und hat während dieser Zeit eine rasche Zunahme ihres Geschäftsvolumens zu verzeichnen.

Die EIB besitzt eine besondere autonome Stellung. Sie verfüat iber eine eigene Rechtspersönlichkeit und ist finanziell und orqanisatorisch selbständiq. Uber die Fntscheidunas- und Kontrollaremien, die aus den Vertretern der Mitgliedsstaten bestehen bzw. von ihnen bestellt werden, ist allerdings der Einfluß der Gemeinschaft gewährleistet. Die Mitaliedsstaaten statten die Bank mit Eiaenkapital aus, das zusammen mit den aebildeten Rücklaqen die finanzielle Basis für ihre Anleihe- und Darlehenstätiqkeit bildet. ${ }^{3}$ Die Aufteilung des Kapitalsauf die Mitgliedsstaaten entspricht ungefähr ihrer Bevölkerungsgröße, ihrer wirtschaftlichen Leistungsfähigkeit sowie

1EG-Rat, Beschluß vom 19. April 1983 zur Ermächtiguna der Kommission, im Rahmen des Neuen Gemeinschaftsinstruments Anleihen zur Investitionsförderung in der Gemeinschaft aufzunehmen, in: Amtsblatt der EG, Nr. L 112 vom 24.4.1983, s. 26 f.

${ }^{2}$ Art. 130 EWG-Vertrag.

${ }^{3} \mathrm{zu}$ einem therblick vol. etwa J. Harrop, The European Investment Bank, in: National Westminster Bank Quarterly Review, Mai 1978, S. $18 \mathrm{ff} . ; K_{.}-$G. Schmidt, Die Europäische Investitionsbank, in: R. Regul, H. Wolf (Hrsg.), Das Bankwesen im größeren Euroda, Baden-Baden 1974 , S. 43 ff. 
ihrer politischen Bedeutung, ${ }^{1,2}$ ist aber auch Resultat des politischen Kräftespiels und Kompromisses. ${ }^{3}$

Innerhalb der Gemeinschaft finanziert die EIB vor allem Infrastruktur-, Modernisierungs- und Umstellungsinvestitionen, die zur Konvergenz und Integration der Mitgliedsstaaten beitragen und der Schaffung von Arbeitsplätzen dienen sollen. ${ }^{4}$ Der räumliche Tätiqkeitsbereich der Bank wurde in den vergangenen Jahren auf assozilerte Staaten und Drittländer ausgedehnt; der hierauf entfallende Anteil der Darlehenstätigkeit beträgt aber nur etwa $10 \%$. Neben der Darlehensgewährung aus eigenen Mitteln schließt die EIB auch Sonderkredite auf Rechnung und Gefahr der EWG ab und übernimmt Aufgaben der Prüfung und Abwicklung bei der Darlehensvergabe von EAG und EWG.

Während die Rolle der EIB als autonome öffentlich-rechtliche Institution innerhalb der Gemeinschaft prinzipiell akzentiert und lediqlich die Transparenz ihres Finanzgebarens und ihrer Mitwirkung bei den übrigen Darlehenstransaktionen gefordert wird, ist vor allem seit 1978 auf die fehlende systematische politische Kontrolle der NGI-, Zahlungsbilanz und EAG-Aktivitäten hingewiesen und deren Einbeziehung in den Gesamthaushaltsplan verlangt worden. In diesen

\footnotetext{
${ }^{1}$ Vgl. D. Killmer, Die Europäische Investitionsbank. Eine rechtliche Untersuchung ihrer Aufgaben, ihres Aufbaus und ihrer Geschäftsgrundsätze, Diss., Frankfurt 1963, S. 41; J. Müller-Borle, Die Europäische Investitionsbank, Sonderdruck aus dem Kommentar zum EWG-Vertrag, Baden-Baden 1983.

${ }^{2}$ Die ursprünglichen Quoten der sechs Gründunasstaaten entsprachen den nach ähnlichen Kriterien fest.gesetzten Anteilen der damaliaen Verwaltungshaushalte von EWG und EAG, dem Sozialfonds und dem Forschungs- und Investitionshaushalt der EAG. Siehe Abschnitt 4.1.1.

${ }^{3}$ Vgl. B. Meyer, Die Europäische Investitionshank zwischen Markt und Lenkung, Untersuchunçen zur Wirtschaftspolitik des Instituts für Wirtschaftspolitik an der Universität zu Köln, Bd. 60, Köln 1984 , S. 294 f.

${ }^{4} \mathrm{Vgl}$. Art. 129 und 130 EWG-Vertraq. Die EIB finanziert im allgemeinen nicht mehr als 50 \& der Projektkosten.
} 
Auffassungen stimmen Parlament ${ }^{1}$, Kommission ${ }^{2}$ und Rechnunashof ${ }^{3}$ ubberein, die in ihren Vorschlägen zusätzlich zum Gesamthaushalt die Einführung eines Kapitalbudgets vorsehen, in das die Anleiheund Darlehenstransaktionen eingesetzt werden. Diese Forderungen haben jedoch bislang nicht die Zustimmung des Rates gefunden.

\subsubsection{Mitgliedsstaatliche Verteilung der Kapitaltransaktionen}

Als Kapitaltransaktionen der gemeinschaftlichen Anleihe- und Darlehenstätigkeit, aus denen empirisch erfaßbare finanzielle Verteilungswirkungen resultieren, lassen sich vor allem die Anleihenaufnahme, die Darlehensvergabe sowie die Kapitalbeteiligungen der Mitgliedsstaaten an der EIB ausmachen. Ihre Verteilung auf die Mitgliedsstaaten ist zunächst zu analysieren, bevor auf dieser Basis versucht wird, die finanziellen Beqünstigungen und Belastungen $z u$ quantifizieren.

Die Gemeinschaftsanleihen werden nicht nur in den währungen der Mitgliedsstaaten, sondern fast zur Hälfte in Drittlandsvaluta aufgenommen (Tabelle 54). Unter den Gemeinschaftswährungen werden vor allem der deutsche und daneben der niederländische Kapitalmarkt beansprucht, während bei den Drittlandswährungen vor allem auf den US-Dollar - insbesondere im Rahmen der 1983er Zahlungsbilanz-Anleihe - zurückgegriffen wird. Die Anteile der einzelnen Währunqen an der Mittelaufnahme schwanken von Jahr zu Jahr. Sie hängen ange-

${ }^{1}$ Vgl. EG-Parlament, Bericht im Namen des Ausschusses für Haushaltskontrolle üher die Intensivierung der politischen Kontrolle über die Anleihe- und Darlehenstätiqkeiten der Gemeinschaften, Berichterstatter: Herr Pierre-Bernard Couste, Europäisches Parlament, Sitzungsdokumente 1981 - 1982, Dok. 1-175/81 vom 8.5.1981.

${ }^{2}$ Vgl. EG-Kommission, Vorschlag einer Verordnung (Euratom, EGKS, EWG) des Rates zur Änderung der Haushaltsordnung vom 21. Dezember 1977 für den Gesamthaushaltsplan der Europäischen Gemeinschaften, in: Amtsblatt der EG, Nr. C 160 vom 6.7.1978, S. $11 \mathrm{ff}$.

${ }^{3} \mathrm{Vgl}$. EG-Rechnungshof, Stellungnahme des Rechnungshofes zu dem Entwurf einer Verordnung (EWG) des Rates zur Abänderuna der Haushaltsordnung vom 21. Dezember 1977 für den Gesamthaushalt der Europäischen Gemeinschaften, in: Amtsblatt der EG, Nr. C 139 vom 5.6.1979, S. $25 \mathrm{ff}$. 
Tabelle 54

Gemelnschaftsanle1hen nach Anleihewährungen $1980-1984$

\begin{tabular}{|l|r|r|r|r|r|r|r|r|r|r|}
\hline $\begin{array}{l}\text { Wăhrungen der Mitglieds- } \\
\text { staaten } \\
\text { Drittländerwährungen }\end{array}$ & 1980 & 1981 & 1982 & 1983 & 1984 & 1980 & 1981 & 1982 & 1983 & 1984 \\
\hline & & &
\end{tabular}

a einschl. 150 Mio. ECU bzw. baufgenommene USD-Anlethen 1m werte von 4097,3 M10. ECU im Rahmen der EWG-Zahlungsbilanzanle1hen.

Quelle: EG-Kommission, Bericht uber die Anleihe- und Darlehenstätigke1t, versch. Jg. 
botsseitig vor allem von den $\mathrm{z}$ insrelationen und den Wechselkurserwartungen in Verbindung mit der Geld- und Kreditpolitik und der wirtschaftlichen Lage der einzelnen Länder ab. Auf der Nachfrageseite sind die Präferenzen der Darlehensnehmerländer für die verschiedenen währungen mitbestimmend. 1

Die Mittelbeschaffung erfolgt in der Form öffentlicher Anleihen und Privatplazierungen vornehmlich bei Banken und bedeutenden institutionellen Anlegern. Die Anleihenaufnahme auf den Kapitalmärkten der Mitgliedsstaaten unterliegt besonderen kapitalmarktrechtlichen Begrenzungen. Bei der Aufnahme von Finanzierungsmitteln zugunsten der Darlehensvergabe an ein anderes Gemeinschaftsland ist die Zustimmung des mittelaufbringenden Landes erforderlich; ${ }^{2}$ bei der Anleiheaufnahme der EIB $^{3}$ und ähnlich der EAG ${ }^{4}$ kann ein Mitgliedsstaat der Auflage einer Anleihe widersprechen, wenn infolge der Emission Störungen seines Kapitalmarktes zu befürchten sind. 5 Diese Beschränkungen haben bei der bisherigen Anleihepraxis allerdings keine entscheidende Rolle gespielt.

Die mitgliedsstaatliche Verteilung der gewährten Darlehen ist in der Tabelle 55 dargestellt. Aus den Ergebnissen ist erkennbar, daß zumeist etwa zwei Drittel der Darlehen an die vier wirtschaftsschwächsten Gemeinschaftsländer vergeben worden sind, wobei Italien den weitaus arößten Teil auf sich vereinigen konnte. Ein solches Verteilungsmuster gilt jedoch nicht durchgängig für sämtliche der darlehensgewährenden Gemeinschaftsinstitutionen. So stehen im Falle der EAG und der EGKS eneraiepolitische und standortbedingte Motive im Vordergrund, die überwiegend die wirtschaftsstärkeren Länder begünstigen, und wurde das Zahlungsbilanzdarlehen 1983 von einem der wohlhabenderen Mitgliedsstaaten in Anspruch genommen. Da-

\footnotetext{
${ }^{1}$ Vgl. EG-Kommission, KCM (83) 527 endg., S. 15; EIB, 25 Jahre, 1958 - 1983, Luxemburg 1983, S. 96.

${ }^{2}$ Vgl. Art. 68 Abs. 3 EwG-Vertrag.

${ }^{3}$ Vgl. Art. 22 der satzung der EIB.

${ }^{4} \mathrm{Vgl}$. Art. 172 Abs. 4 EAG-Vertrag.

${ }^{5} \mathrm{vgl}$. M. Seidel, S. $668 \mathrm{f}$.
} 
Tabelle 55

Gemeinschaftsdarlehen nach Empfängerländern 1980 - 1984

\begin{tabular}{|c|c|c|c|c|c|c|c|c|c|c|}
\hline $\begin{array}{l}\text { Mitgliedsländer } \\
\text { Außerhalb der EG }\end{array}$ & 1980 & 1981 & 1982 & 1983 & 1984 & 1980 & 1981 & 1982 & 1983 & 1984 \\
\hline & \multicolumn{5}{|c|}{ M10. ECU } & \multicolumn{5}{|c|}{ in 8} \\
\hline Belgien & 264,6 & 408,2 & 151,3 & 36,4 & 133,0 & 5,8 & 9,1 & 2,6 & 0,3 & 1, \\
\hline Dänemark & 103,5 & 142,9 & 307,1 & 365,1 & 327,8 & 2,3 & 3,2 & 5,3 & 3,2 & 4,2 \\
\hline Deutschland & 143,3 & 384,3 & 205,5 & 301,6 & 411,2 & 3,2 & 8,5 & 3,6 & 2,7 & 5. \\
\hline Frankreich & 653,6 & 477,2 & 789,8 & $5617,9^{a}$ & 1472,7 & 14,4 & 10,6 & 13,7 & 49,8 & 18,8 \\
\hline Griechenland & -- & 159,2 & 469,8 & 450,0 & 344,9 & -- & 3,5 & 8,2 & 4,0 & 4, \\
\hline Irland & 377,1 & 353,6 & 408,6 & 308,4 & 174,1 & 8,3 & 7,9 & 7,1 & 2,7 & 2,2 \\
\hline Italien & 1430,0 & 1834,7 & 2133,4 & 2732,2 & 3101,5 & 31,5 & 40,7 & 37,1 & 24,2 & 39,6 \\
\hline Luxemburg & 68,6 & 0,1 & 5,5 & 0,0 & 86,0 & 1,5 & 0,0 & 0,1 & 0,0 & 1,1 \\
\hline Niederlande & 4,9 & 0,5 & 44,1 & 39,5 & 0,7 & 0,1 & 0,0 & 0,8 & 0,4 & 0,0 \\
\hline Verein. Königreich & 1056,0 & 323,0 & 759,7 & 1008,2 & 1015,6 & 23,3 & 7,2 & 13,2 & 8,9 & $13, c$ \\
\hline EG & 4105,5 & 4083,7 & 5274,8 & 10859,3 & 7067,5 & 90,5 & 90,7 & 91,6 & 96,2 & 90,3 \\
\hline Außerhalb der EG & 428,5 & 418,8 & 481,8 & 427,0 & 759,6 & 9,5 & 9,3 & 8,4 & 3,8 & 9,7 \\
\hline Gesamt & 4534,0 & 4502,5 & 5756,6 & 11286,3 & 7827,1 & 100,0 & 100,0 & 100,0 & 100,0 & 100,0 \\
\hline
\end{tabular}

aeinschließlich der EWG-Zahlungsbilanzanleihe in Höhe von 4.247,3 Mio. ECU.

Quelle: EG-Kommission, Bericht über die Anleihe- und Darlehenstätigkeit, versch. Jg. 
gegen kommt in der Darlehensvergabe des NGI und der EIB die zielsetzung der Förderung einer ausgewogenen, die wirtschaftsschwächeren Gemeinschaftsstaaten bevorzugenden ökonomischen Entwicklung voll zum Ausdruck. Da die EIB im allgemeinen den weitaus gröBten Tell der gemeinschaftlichen Darlehenstätigkeit bestreitet, wird das Gesamtbild der Vertellungswirkungen hierdurch auch maßgeblich bestimmt.

Während die Basıs der Anleihe- und Darlehenstätigkeit von EWG, EAG und EGKS in der Garantie durch den Gesamthaushalt bzw. den EGKSFunktionshaushalt besteht, ist diese Grundlage bel der EIB das von den Mitgliedsstaaten bereitgestellte Elgenkapital. Da es sich hierbel um Kapitaltransfers von den M1tgliedsländern zugunsten gemeinschaftlicher Aktivitäten handelt, sind sie ebenfalls bel einer Vertellungsanalyse $z u$ berücksichtigen.

Dabel ist zu unterschelden in das gezeichnete Kapital, das eingezahlte Kapital und die tatsuchlich geleisteten Zahlungen (Tabelle 56). Der weltaus grobte Tell des gezelchneten Kapitals besteht aus dem nicht elngezahlten Kapttal, das bel Bedarf ganz oder teilweise eingefordert werden kann, um den Verpflichtungen der Bank gegenüber ihren Anleiheglaubigern nachzukomen. ' Eln solcher Fall dürte allerdings rein hypothetischer Natur ${ }^{2}$ sein, so daß dieser Kapitalanteil praktisch eine satzungsmäBlg verankerte zusätzliche sicherheit darstellt. ${ }^{3}$ Das eingezahlte Kapital macht stets einen bestimmten Prozentsatz des gezeichneten Kapitals - 1983 10,2 \& - aus. Diese Mittel waren am Ende dieses Jahres allerdings erst zu knapp zwei Drittel tatsachlich elngezahlt, da die infolge der letzten Kapitalerhöhungen erforderlichen Nachschusse von den Mitgliedsstaaten ratenweise zu leisten sind. Neben diesen Kapitalbeständen schreibt

\footnotetext{
${ }^{1}$ Art. 5 Abs. 2 der Satzung der EIB. ${ }^{2}$ Vgl. D. Strasser, S. 157; J. Muller-Borle, S. $2140 \mathrm{f}$. 3 vgl. EIB, 25 Jahre 1958 - 1983, S. 93.
} 
die Satzung der Bank (Art. 24) die Bildung eines Reservefonds bis zum Höchstbetrag von 10 \% des gezeichneten Kapitals vor, der 1983 erreicht wurde. Die eigenen Mittel der Bank aus eingezahltem Kapital, Reserven und sonstigen Rücklagen betrugen Ende 1983 gut 2 Mrd. ECU. 1

Tabelle 56

Zusammensetzung des EIB-Kapitals 1983

\begin{tabular}{|l|c|c|}
\hline Mitgliedsland & $\begin{array}{c}\text { Gezeichnetes Kapital } \\
1981-1985\end{array}$ & $\begin{array}{c}\text { Geleistete Zahlungen } \\
\text { am 31.12.1983 }\end{array}$ \\
\hline Deutschland & 3150 & 202,5 \\
Frankreich & 3150 & 202,5 \\
Verein. Königreich & 3150 & 202,5 \\
Italien & 2520 & 162 \\
Belgien & 829,5 & 53,3 \\
Niederlande & 829,5 & 53,3 \\
Dänemark & 420 & 27 \\
Griechenland & 225 & 14,5 \\
Irland & 105 & 6,8 \\
Luxemburg & 21 & 1,4 \\
Gesamt & 14400 & 925,7 \\
\hline
\end{tabular}

Quelle: Europäische Investitionsbank, Jahresberichte 1980 bis 1983 .

Mit Wirkung vom 1.1.1986 wurde eine Anhebung des gezeichneten Kapitals der Bank von 14.400 Mio. ECU auf 26508,15 Mio. ECU beschlossen. Gleichzeitig erhöhte sich dieses Kapital mit dem Beitritt Spaniens und Portugals zur Gemeinschaft um weitere 2291,85 Mio. ECU auf insgesamt $28.800 \mathrm{MiO}$. ECU.

1Vgl. EIB-Jahresbericht 1983, s. 91. 
4.2.3.2. Mitgliedsstaatliche Vorteils- und Belastungswirkungen

Auf der Grundlage der vorangegangenen Verteilungsanalyse der Kapitaltransaktionen ist nunmehr zu prüfen, welche finanziellen Vorteile und Belastungen daraus für die Mitgliedsstaaten abzuleiten sind. Dabei kann hier - anders als im Rahmen des Gesamthaushalts - nicht davon ausgegangen werden, daß sich Vor- und Nachteile der Mitgliedsstaaten in der Summe ausgleichen. Zum einen sind nämlich sowohl bei der Mittelaufbringung als auch bei der Darlehensgewährung Staaten außerhalb der Gemelnschaft betroffen. Vor allem aber ist es erklärtes Ziel der gemeinschaftlichen Anleihe- und Darlehenstätigkeit, daß ihre Abwicklung uber Gemeinschaftsinstitutionen insgesamt zu kostengünstigeren Ergebnissen im Vergleich zu einzelwirtschaftlichen und -staatlichen Aktivitäten fuhrt.

Im Zusammenhang mit den Vorteilen sind zunächst die Zinssubventionen zu nennen, mit denen einlge Darlehen der EWG, EIB und EGKS ausgestattet sind. ${ }^{1}$ Diese Zuschüsse werden allerdings vom Gesamthaushalt bzw. EGKS-Funkttonshaushalt getragen und wurden in der bisherigen Vertellungsanalyse bereits berucksichtigt. ${ }^{2}$

Als wichtigste Quelle finanzieller Vorteile der gemeinschaftlichen Anleihe- und Darlehenstätigkelt werden im allgemeinen die indirekten $\mathrm{Z}$ inseffekte angesehen. Diese entstehen grundsätzlich dadurch, daß die Darlehen der Gemetnschaftsinstitutionen zu günstigeren Konditionen gewahrt werden können, als dies bel alternativen Finanzierungsarten möglich 1st. Um diese Unterschiede herauszuarbeiten, ist es erforderlich, auf die Modalitaten der Anleiheaufnahme und Darlehensvergabe der EG naher elnzugehen.

\footnotetext{
1 Im Rahmen des EWS, belm Wlederaufbau erdbebengeschädigter Gebiete sowie bet Umstrukturierungsmaßnahmen in der Montanindustrie. Vgl. hierzu die Abschnitte 4.1.3.3.2. und 4.2.2.

2 Eine Zusammenstellung dieser Zinszuschüsse findet sich in: EGKommission, Europä1s che Nirtschaft Nr. 21, S. 60.
} 
Hinsichtlich der Mittelaufnahme an den Kapitalmärkten gehören sämtliche Gemeinschaftsinstitutionen $\mathrm{zu}$ den sog. ersten Adressen und wird Ihrem Kreditstanding die beste Bewertung, das "AAA-Ratinq" ertellt. ${ }^{1}$ Die Gründe hierfur liegen vor allem in den Haushaltsgarantien fur EWG-, Euratom, NGI- und EGKS-Anle1hen bzw. in der Kapital- und Haftungsgrundlage und den zusätzlichen mitqliedsstaatlichen Besicherungen im Falle der EIB. ${ }^{2}$ Diese Bedingungen führen da$z u, d a ß$ die Gemeinschaftsinstitutionen eine bestmögliche Plazierung ihrer Anleihen auf allen Kapitalmärkten vornehmen können und daB vor allem vom Risiko her die gunstigsten zinssätze zu erzielen sind.

Die Vergabe der Gemeinschaftsdarlehen erfolgt entweder an die Gemeinschaftslander direkt - wle bel den Zahlungsbilanz-Krediten oder zumelst an offentliche und private Unternehmen wie im Rahmen der Euratom-, NGI-, EGKS- und EIB-Darlehen. Sie lassen vor allem durch folgende Merkmale charakterisieren:

- Die Darlehen werden den Kreditnehmern nicht nur in der wahrung ihres Landes, sondern auch in anderen whrurungen zur Verfügung gestellt. In welchem Umfang dies jewells geschleht, hängt vom Wunsch der Darlehensnehmer sowle den Bedingungen des Kapitalmarktes $\mathrm{ab}$.

- Die Ruckzahlbarkett der Darlehen erfolgt in allen Fällen in der Währung, in der sie zur Verfugung gestellt wurden. Das damit verbundene Wechselkursrislko wird also von den Darlehensnehmern getragen.

\footnotetext{
${ }^{1}$ Vgl. etwa ETB, 25 Jahre, S. 97; D. Strasser, S. 143 und 158. Dieser Bonitatsstandard wird allerdings ebenso einzelnen Gemeinschaftslandern zugetellt.

2 Die Bank macht satzungsmäBlg die Darlehensvergabe von einer Bürgschaft des Staates bzw. seiner offentlich-rechtlichen Instituionen abhanglg, in dem das Vorhaben verwirklicht wird. Die außerhalb der Gemelnschaft gewährten Darlehen werden durch elne Globalbürgschaft der EG in Hohe von 75 \& der eröffneten Kredite abgesichert. Vgl. EIB, 25 Jahre, S. 99.
} 
- Die Darlehen werden zumeist langfristig gewährt. Ihre Laufzeit liegt in der Regel zwischen 5 und 15 Jahren.

- Die Darlehenszinssätze varileren nicht mit den unterschiedlichen geförderten Projekten in den verschiedenen Ländern, sondern sind allein von den Bedingungen der Anleiheaufnahme abhängig. Während der Darlehenslaufzelt bleiben die Zinssätze unverändert. 1

Aus diesen Modalitaten der gemelnschaftlichen Anleiheaufnahme und Darlehensgewährung ergeben sich nun Vortelle fur die Darlehensnehmer daraus, wenn sie die Kapitalmärkte nicht selbst oder nicht zu vergleichbaren Bedingungen in Anspruch nehmen können. Dies betrifft zum einen die Darlehenskonditionen, zum anderen aber auch den wunsch der Mitgliedsländer, uber den Umweg der EG Devisen zu erlangen, da nationale Anleiheauflagen an den Kapitalmärkten nicht immer in der gewünschten Hohe durchzufuhren sind.

In diesen Zusamenhang fallen auch die Vorzlige aus dem Gewinnverzicht der darlehensgewahrenden Inst1tutionen: Die EIB verfolgt satzungsgemaß kelnen Erwerbszweck und die Abwlcklung der Anlethen und Darlehen erfolgt bei den ubrigen Gemeinschaftsinstitutionen im Rahmen ihrer Verwaltungstätigke1t. Die Darlehenszinssätze entsprechen also den Zinssutzen der Anletheaufnahme bzw. liegen in der Nuhe der zinssutze auf den Beschaffungsmärkten. 2

Als Ausdruck fur dte Vortelle der Darlehensnehmer aus den indirekten $\mathrm{Z}$ inseffekten kann nun die Differenz aus den Gemeinschaftszinssätzen und den zinssatzen bel alternativer Finanzlerungsform her-

1 Im Rahmen der Zahlungsbllanz-Anleihen und -Darlehen wurden allerdings variable zinssätze angewendet.

2 Die EIB berechnet thre Darlehenszinssätze, Indem sie den Rosten der Anleihemittel etwa 0,15 \& fur Verwaltungsausgaben zuschlägt. Vgl. EIB, 25 Jahre, S. 100. 
angezogen werden. Eine solche Aussage läßt sich zwar leicht formulieren, sie ist in praxi allerdings kaum exakt quantifizierbar. Denn die Zinssätze alternativer Finanzierungsmöglichkeiten lassen sich nicht ohne weiteres mit den Gemeinschaftszinssätzen vergleichen, da diese von den aufgenommenen und ausgezahlten währungen abhängen. Dabei ist vor allem das vom Darlehensnehmer zu tragende Wechselkursrisiko $\mathrm{zu}$ berücksichtigen, das etwaige $\mathrm{zinsdifferenzen}$ tellweise oder ganz ausgleichen oder im Extremfall sogar überkompensieren kann.

Eine Berücksichtigung des Wechselkursrisikos wäre für die gewährten und berelts zurückgezahlten Darlehen zwar möglich und ließen sich im Verglelch mit konkreten Finanzierungsalternativen die indirekten 2 inseffekte der Vergangenheit prinzipiell quantifizieren. ${ }^{2}$ Für die gegenwărtig laufenden Darlehen ist dies aufgrund der unsicheren Wechselkursentwicklungen jedoch nicht möglich.

Verspricht also der Versuch, die indirekten Zinseffekte auf der Basis der Im einzelnen gewährten Gemeinschaftsdarlehen abschätzen zu wollen, wenig Aussicht auf Erfolg, so ist doch zumindest allgemein danach zu fragen, wie sich die Vortelle für die Darlehensnehmer konkretisieren lassen.

Berücksichtigt man, daß mit der gemeinschaftlichen Anleihe- und Darlehenstätigkeit kein Erwerbszweck verfolgt wird, keine Verzinsung des eingesetzten und haftenden Kapitals verbunden ist und das Kreditstanding der Gemeinschaftsinstitutionen $z u$ den günstigsten an den Kapitalmärkten zu erzlelenden $\mathrm{z}$ inssätzen führt, dann dürfte

\footnotetext{
${ }^{1}$ Um diese Risiken zu mildern, sind im Rahmen von EIB-Darlehen von einigen nationalen Regierungen Wechselkursgarantien gegeben worden. Vgl. J. Harrop, An Evoluation of the European Investment Bank: Prospect and Retrospect, Société Universitaire Europeene de Recherches Financières, Series 23A 1978, S. 4.

${ }^{2}$ Dazu mußten allerdings die Konditionen der einzelnen gewährten Kredite bekannt seln, die nlcht veröffentlicht werden.
} 
hiermit generell ein geringeres zinsniveau im Vergleich $z u$ alternativen Finanzierungsformen verbunden sein. Da weiterhin die gemeinschaftlichen Darlehenszinssätze nicht nach den finanzierten Projekten und den Darlehensnehmerländern differenziert werden, wird der Zinsvorteil der Darlehensempfänger umso größer sein, je risikoreicher die Projekte sind und je geringer die Bonität ist. 1 Dagegen ist die Frage, ob die zumeist langfristig gewährten EG-Darlehen mit festen Zinssätzen $z u$ Vorteilen fü die Kreditnehmer führen, nicht eindeutig beantwortbar. Langfristige Ausleihungen vermindern zwar das Risiko einer Anschlußfinanzierung zu steigenden $\mathrm{zinsen,} \mathrm{ver-}$ hindern aber auch die Möglichkeit, bei sinkenden $\mathrm{Z}$ insen die Kreditkosten zu reduzieren. ${ }^{2}$

Eine Quantifizlerung der indirekten Zinsvortelle kann daher nur über eine grobe Abschätzung erfolgen. In diesem Zusammenhang spricht Strasser ${ }^{3}$ von einem um 1 bis 1,5 \& geringeren $\mathrm{z}$ inssatz der Gemeinschaftsdarlehen im Vergleich $z u$ den üblichen Marktkonditionen und rechnen andere Autoren nauschal mit einem solchen Subventionselement von $1 \%{ }^{4}$ Solche zinssatzdifferenzen sind nun auf den jährlichen Bestand der laufenden Darlehen anzuwenden, um die länderspezifischen indirekten zinsvortelle abzuschätzen.

Legt man dabel die von Strasser angegebenen Zinsvortelle von 1 bis 1,5 \& zugrunde und differenzlert in diesem Rahmen danach, daß die Alternativkosten der risikoärmeren Länder an der unteren und die

1Vgl. P.K. Woolley, The European Investment Bank, in: The Three Banks Review, No. 105, 1975, S. 53 ff., hler: S, 57 ff.

2vgl. B. Meyer, S. 149.

${ }^{3}$ Für EIB und EGKS-Darlehen. Vgl. D. Strasser, S. 143 und 158.

${ }^{4}$ Vgl. F. Franzmeyer, S. Schultz, D. Schumacher, B. Seldel, Einflüsse der EG auf die Reglonalpolitik in der Bundesrepublik Deutschland, Gottingen 1975, S. 79; P. Gloysteln, S. 257. 
risikoreicheren Länder ${ }^{1}$ an der oberen Grenze liegen, dann lassen sich auf der Grundlage der Tabelle 57 die absoluten indirekten zinsvorteile wie folgt angeben:

Danach fallen solche Vorteile vor allem Italien $z u$, an das die mit Abstand ${ }^{2}$ meisten Darlehen vergeben wurden und dessen $\mathrm{Zinsvorteile}$ sich für 1983 durchaus mit etwa 150 Mio. ECU beziffern lassen. Nach diesen Maßstäben dürften weitere nennenswerte $\mathrm{Z}$ insvorteile dem Vereinigten Königreich, Frankreich und Irland zufallen. Die Darlehen an Länder außerhalb der Gemeinschaft werden aufgrund der geringen Bonität mit einem erheblich höheren subventionselement verbunden sein, so daß der übrigen gemeinschaftlichen Entwicklungshilfe über die günstige Kreditvergabe durchaus noch einmal 50 bis 100 Mio. ECU hinzugerechnet werden dürften.

Im Rahmen der finanziellen Vorteilswirkungen der gemeinschaftlichen Anleihe- und Darlehensaktivitäten ist - analog zu den Verwaltungsausgaben des Gesamthaushalts - konsequenterweise auch auf die hier-

${ }^{1}$ Als Grundlage zur Beurteilung der Bonität der Mitgliedsländer mag die Länderliste der internationalen Kreditwürdigkeit herangezogen werden, die vom Banken-Magazin 'Institutional Investor' erstelit wird. Danach ergab sich für september 1983 folgende Rangliste der Gemeinschaftsländer:

\begin{tabular}{clc} 
Rang & Mitgliedsland & Bonitätsindex \\
\hline 4 & Deutschland & 92,7 \\
5 & Verein. Königreich & 88,6 \\
8 & Niederlande & 85,6 \\
11 & Frankreich & 78,8 \\
15 & Belgien & 72,3 \\
19 & Italien & 71,1 \\
21 & Dänemark & 69,6 \\
27 & Irland & 61,3 \\
34 & Griechenland & 52,3
\end{tabular}

Quelle: Handelsblatt vom 18.4.1984, S. 8. K.C. Engelen, Länderrisiken. Die Philippinen und Brasilien sacken stark ab, in: Handelsblatt vom 18.4.1984.

${ }^{2}$ Sieht man einmal von der Ausnahmesituation des Zahlungsbilanz-Darlehens an Frankreich ab. 
Tabelle 57

Ausstehende EG-Darlehen nach Mitgliedsstaaten $1980-1983^{a}$

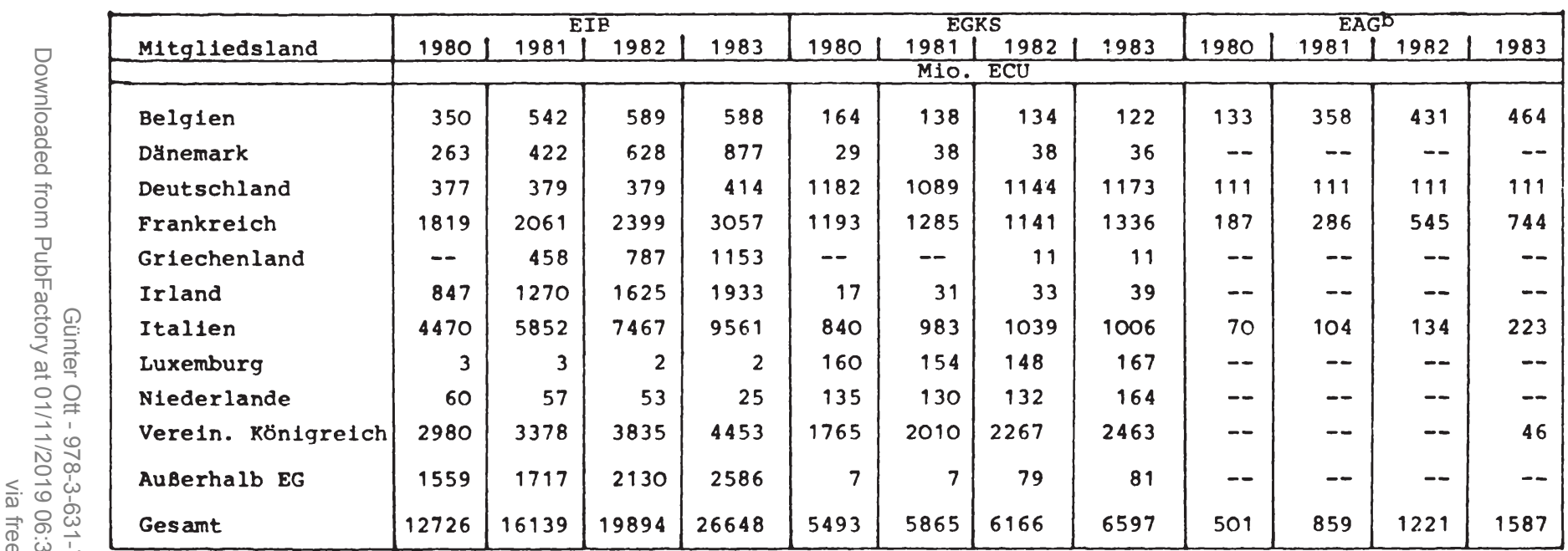

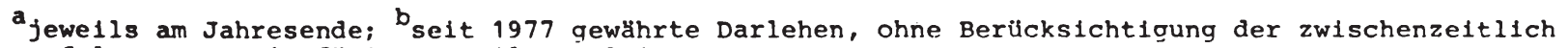
erfolgten - geringfugigen - Tilgungsle1stungen. 
Tabelle 57 (Fortsetzung)

\begin{tabular}{|l|c|c|c|c|c|c|c|c|c|r|r|r|r|}
\hline Mitgliedsland & \multicolumn{9}{|c|}{ EWG-Zahlungsbilanz } \\
\hline
\end{tabular}

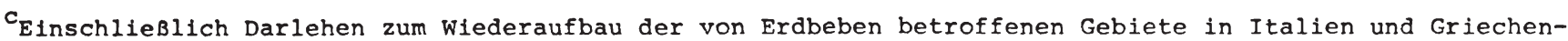
land.

Quelle: EIB, Jahres berichte 1980 - 1983; EG-Kommission, Finanzberichte des EGKS 1980 - 1983; dies., Europäische Wirtschaft Nr. 21, S. 52 und 54 , Gesamthaushaltspläne $1982-1984$. 
mit verbundene Verwaltungstätigkeit einzugehen. Da die Ausgaben zur Durchführung der EWG-, Euratom- und EGKS-Anleihen und -Darlehen bereits über den Gesamthaushalt finanziert werden, bleiben an dieser Stelle nur noch die Verwaltungsausgaben der EIB zu untersuchen. Diese betrugen in den Jahren 1980 bis 1983 zwischen 30 und 43 Mio. ECU und bestanden zum weit überwiegenden Teil aus Personalkosten. 1 Sie fallen fast ausschließlich am Sitz der EIB in Luxemburg an und dürten damit vornehmlich diesem Land Vorteile erbringen. Diese bestehen - wie bei den übrigen Verwaltungsausgaben auch ${ }^{2}$ - allgemein in positiven Effekten der Ansiedlung öffentlicher Unternehmen und unmittelbar wohl hauptsächlich in arbeitsmarktentlastenden und nachfragestimulierenden Wirkungen aus der Beschäftigung der Bediensteten und den damit verbundenen Einkommenszahlungen. ${ }^{3}$ Angesichts der Größenordnungen der EIB-Verwaltungsausgaben sind die daraus resultierenden finanziellen Effekte jedoch als vernachlässigbar gering einzustufen.

Nachdem die Vorteilswirkungen der Anleihe- und Darlehenstransaktionen analysiert worden sind, ist nunmehr nach möglichen Belastungen für die Mitgliedsstaten $z$ fragen. Sie ergeben sich durch die Bereitstellung des EIB-Eigenkapitals, die Risikoübernahme im Rahmen der Darlehenssicherung sowie durch mögliche Belastungen der Kapitalmärkte bei der Anleihebegebung. Diese Wirkungen sind allerdings in unterschiedlichem Maße empirisch identifizierbar.

Einigermaßen zuverlässig quantifizierbar sind die Belastungswirkungen aus der Bereitstellung vor EIB-Kapital, insoweit dies die tatsächlich geleisteten Zahlungen betrifft. Da diese Einlagen nicht verzinst werden, entsteht für die Anteilseigner ein Zins-bzw. Dividendenverzicht im Vergleich $z u$ einer alternativen Kapitalanlage. 4

${ }^{1}$ Vgl. EIB-Jahresberichte 1980 - 1983 .

${ }^{2}$ Siehe hierzu Abschnitt 4.1.3.5.

${ }^{3}$ Wenngleich auch hier zu berücksichtigen ist, daB ein Teil der Beschäftigten Staatsbürger anderer Mitgliedsstaaten sind, und damit auch diese Länder an den Ausgaben partizipieren.

${ }^{4}$ Vgl. B. Meyer, S. 281. 
Der finanzielle Belastungseffekt kann demnach aus der Anwendung alternativer zinssätze auf die Höhe der geleisteten Zahlungen ermittelt werden. Läßt man unberücksichtigt, daß die in den einzelnen Jahren getätigten Einlagen korrekterweise mit den jeweils geltenden $\mathrm{z}$ inssätzen bewertet werden müßten und legt vereinfachend eine durchschnittliche Verzinsung von $108^{1}$ zugrunde, dann lassen sich die jährlichen finanziellen Verzichte der Mitgliedsstaaten unschwer aus Tabelle 57 ablesen: also etwa im Jahre 1983 für die Bundesrepublik, Frankreich und das Vereinigte Königreich jeweils 20,25 Mio. ECU, für Belgien und die Niederlande jeweils 5,3 Mio. ECU etc.. Auch wenn man andere Anlagebedingungen zugrundelegen würde, handelt es sich insgesamt nur um relativ geringe Beträge.

Kaum quantifizierbar sind dagegen die im zusammenhang mit der Rückzahlung der gewährten Darlehen erwachsenden finanziellen Risiken der Mitgliedsstaaten. Diese resultieren bei der EIB aus dem nicht eingezahlten Teil des gezeichneten Kapitals, der bei Bedarf von den Mitgliedsstaaten nachgefordert werden kann. Die EWG-, Euratomund EGKS-Darlehen werden durch Garantien der Haushalte gesichert, in die diese Eventualverbindlichkeiten in Form von z. E.-Vermerken aufgenommen werden. Im Eventualfall ergibt sich also eine Absicherung der notleidenden Kredite durch Haushaltsmittel, die die Mitgliedsstaaten in Form höherer Finanzierungsbeteiligungen aufzubringen hätten.

Eine Grundlage zur quantitativen Ermittlung solcher Risiken gibt es derzeit nicht. Bisher sind weder Fälle aufgetreten, in denen die Eventualverbindlichkeiten des Haushalts wirksam geworden sind, noch hat die EIB Nachschüsse anfordern müssen, so daß eine Basis für wahrscheinlichkeitstheoretische Risikobestimmungen nicht besteht. Allgemein wird man solche Wahrscheinlichkeiten auch als sehr gering anzusetzen haben. Denn die meisten Darlehen sind entweder direkt durch die unmittelbare Haftung des kreditnehmenden Mitgliedsstaates

\footnotetext{
${ }^{1}$ Dieser wert kommt dem durchschnittlichen zinssatz für Gemeinschaftsanleihen in ECU in den Jahren 1980 bis 1983 nahe.
} 
oder indirekt durch zusätzliche Bürgschaften der betreffenden Staaten besichert, und stehen bei der EIB das eingezahlte Grundkapital, die Rückstellungen und der Reservefonds als Kreditsicherung zur Verfügung.

Dennoch wäre es konsequent, den Zinsvorteilen gemeinschaftlicher Anleiheaufnahme, die aus dem Kreditstanding der Gemeinschaftsinstitutionen resultieren, die sicher unterschiedlichen Beiträge der einzelnen Mitgliedsländer hierzu gegenüberzustellẹ, wobei als Kriterien die Kreditwürdigkeit und die Wirtschaftskraft dieser Länder heranzuziehen wären. Da es jedoch schon nicht möglich ist, die auf der günstigen Risikokomponente basierenden $\mathrm{Z}$ inseffekte isoliert abzuschätzen, wäre es rein spekulativ, die Beiträge der einzelnen Mitgliedsstaaten hierzu bestimmen zu wollen. Damit müssen diese Uberlegungen unberücksichtigt bleiben, wodurch implizit unterstellt wird, daß den positiven indirekten $\mathrm{Z}$ inseffekten keine Risikobeiträge gegenüberstehen.

Schließlich ist die Frage, ob und inwieweit eine Belastung der nationalen Kapitalmärkte durch die gemeinschaftliche Anleihenbegebung vorliegt, ebenfalls nicht eindeutig beantwortbar und faktisch nicht $z u$ quantifizieren. Solche Belastungen werden allgemein dann konstatiert werden können, wenn die nationale Kapitalmarktpolitik gestört wird und sich dabei aufgrund der zusätzlichen Nachfrage vor allem das $\mathrm{z}$ insniveau entgegen den nationalen $\mathrm{Z}$ ielsetzungen erhöht.

Im Rahmen der gesamten Anleihetätigkeit können die Gemeinschaftsländer von solchen Wirkungen allerdings nur zu einem Teil betroffen werden, da etwa 40 \& der Mittelbeschaffung in währungen außergemeinschaftlicher Länder erfolgt (Tabelle 55). Von den Gemeinschaftsstaaten sind - in Relation zum Sozialprodukt - im wesentlichen nur die Bundesrepublj.k, die Niederlande, Belgien und Luxembura betroffen, wobei allein etwa ein Viertel bis ein Drittel der Anleiheaufnahme in deutscher Währung erfolgt. Dabei ist allerdings zu berücksichtigen, daß diese Anleihen nicht nur beim nationalen Publikum untergebracht, sondern zu einem Teil auch von ausländischen 
Anleihezeichnern ubernommen worden sind (ebenso wie die in fremder Währunq aufgelegten Anleihen teilweise von dem heimischen Publikum gezeichnet werden), so daß die Herkunft der Mittel nicht eindeutia $\mathrm{zu}$ bestimmen ist. 1

Die Vorbehalte der Mitgliedsländer gegen eine uneingeschränkte Beanspruchung ihrer Kapitalmärkte durch die gemeinschaftlichen Anleihen haben sich in den vertraglichen bzw. satzungsmäßigen Bestimmungen niedergeschlagen, nach denen ein Mitgliedsland der Anleihenbegebung widersprechen kann, wenn dadurch Störungen seines Kapitalmarktes $\mathrm{zu}$ befürchten sind, Solche Störungen, die bei der EIB-Refinanzierung sogar ernstlicher Natur sein müssen, ${ }^{2}$ sind allerdings ökonomisch kaum zweifelfrei $\mathrm{zu}$ bestimmen; ${ }^{3}$ formelle widersprüche wurden bisher auch nicht erhoben. Dennoch sind etwa von der Bundesrepublik bereits Einwände gegen eine relativ hohe Inanspruchnahme des heimischen Kapitalmarktes vorgebracht worden, ${ }^{4}$ und wurde speziell bei der Operation zugunsten der EWG-Zahlungsbilanzanleihe für Frankreich darauf hingewiesen, daß die Kapitalmärkte der EG nicht überfordert werden dürften, und daß deshalb - wie auch geschehen - auf währungen außerhalb der Gemeinschaft zurückgegriffen werden müssen. 5

Diese Hinweise erlauben allerdings nicht, von signifikaten störungen der einzelstaatlichen Kapitalmärkte zu sprechen. Auch angesichts der Größenordnungen der Anleihen, die etwa im Falle der Bundesrepublik weniger als 5 o der öffentlichen Nettokreditaufnahme betragen, und deren Kapitalmarktwirkungen um die erhaltenen Gemeinschaftsdarlehen und die Rückzahlungen früherer Kredite zu korrigieren sind, dürften eventuelle Belastungswirkungen vernachlässigbar gering sein.

1Vgl. hierzu P. Gloystein, S. $174 \mathrm{ff}$ und S. $210 \mathrm{ff}$.

2vgl. Art. 22 der EIB-Satzung.

${ }^{3}$ vgl. B. Meyer, S. 59.

${ }^{4} \mathrm{Vgl}$. Bundesministerium der Finanzen, Finanznachrichten Nr. 27/ 1980 vom 18.6.1980, S. 3 .

${ }^{5}$ Vgl. O.V., Der Frankreich-Kredit soll die Geldmärkte schonen, in: Handelsblatt $\mathrm{Nr} .94$ vom 17.8.1983. 
Zusammenfassend für die gesamte Anleihen- und Darlehenstätiqkeit der EG läßt sich feststellen, daß gegenüber alternativen einzelstaatlichen bzw. einzelwirtschaftlichen Finanzierungsformen von Nettovorteilen auszugehen ist, die maßgeblich durch die indirekten $\mathrm{Z}$ inseffekte bestimmt werden. Als diesen Vorteilen qeqenüberzustellende Nachteile konnten lediglich die (relativ geringen) Verzichte auf eine EIB-Kapitalverzinsung quantitativ erfaßt werden. Mögliche weitere Belastungen aus Darlehenshaftung und Kapitalmarktinanspruchnahme konnten nicht größenmäßig bestimmt werden; sie dürften allerdinqs auch nicht als sehr bedeutsam einzustufen sein. Die mitgliedsstaatlichen Verteilungswirkungen der gemeinschaftlichen Anleihe- und Darlehenstätigkeit ergeben sich also im wesentlichen aus den Salden aus indirekten $\mathrm{Z}$ insvorteilen und den $\mathrm{Z}$ insverzichten auf die EIB-Kapitaleinlagen.

Die dabei erzielten Ergebnisse sind nochmals zu relativieren, da vor allem das Wechselkursrisiko nicht berücksichtigt werden konnte und die verwendete prozentuale Größe für die indirekten $\mathrm{z}$ inseffekte nur eine grobe und globale SchätzgröBe darstellt. Allerdings kommt es im Rahmen der vorliegenden Untersuchung auch nicht auf exakte Angaben an, sondern geht es vornehmlich darum, die ungefähren GröBenordnungen zu ermitteln, um damit die bisher ausgewiesenen Nettopositionen relativieren zu können.

Schließlich ist darauf hinzuweisen, daß gemäß dem hier verwendeten Konzept der formalen Inzidenz nur die unmittelbaren finanzwirksamen Verteilungswirkungen betrachtet wurden. Unberücksichtigt bleiben dabei - wie auch bei den übrigen finanziellen Gemeinschaftsaktivitäten - die Zahlungsbilanzeffekte und die daraus resultierenden ökonomischen Wirkuncen. Abgesehen davon, daß diese kaum emoirisch erfaßbar sind, ginge eine solche Analyse in Richtung auf ein Konzept effektiver Inzidenz und würde damit den Ansatz der vorliegenden Arbeit überschreiten. 
5. Erweiterter Maßstab der finanzwirtschaftlichen Verteilungswirkungen

Ausgehend von den vielfältigen Bestimmungsgriłnden im finanzwirtschaftlichen, ökonomischen und politischen Bereich, die ein umfassender Indikator für die Vor- und Nachtelle einer EG-Mitgliedschaft zu berücksichtiqen hätte, bestand eine Aufgabe dieser Arbeit darin, die auf der Ebene der finanzwirksamen Transaktionen liegenden Einflußfaktoren zu analysieren. Dazu war zunächst eine Abgrenzung zur herkömmlichen Erfassung der gemeinschaftlichen Verteilungswirkungen erforderlich. Während die Berechnungen dieser traditionellen Nettopositionen auf die Zahlungsströme des EG-Gesamthaushalts abstellen, liegt der vorllegenden Untersuchung ein sowohl in aualitativer als auch in quantitativer Hinsicht erweiterter Ansatz zugrunde. Dies bedeutet zum einen, daß zwar auch hier nur das Konzept der formalen Inzidenz Basis der empirischen Untersuchung sein kann, dieses slch aber nicht auf die Erfassung der Zahlungsströme beschrănkt, sondern auf die damit verbundenen finanziellen vortetle bzw. Belastungen abstellt. Daneben umfaßt die Untersuchung nicht nur die uber den Gesamthaushalt abgewickelten Transaktionen, sondern berücksicht1gt auch die außerbudgetären Gemeinschaftsaktivitaten tm. Furopäischen Entwicklungsfonds (FEF), in der Furopäischen Gemeinschaft für Kohle und Stahl (EGKS) sowie im Rahmen der quantitativ 1mmer bedeutsamer werdenden Anleihe- und Darlehensaktivitäten der Gemeinschaft.

Aus diesen Ansätzen ergab sich folgende Vorgehensweise der empirischen Untersuchung: In detallilerter Form waren zunächst die mitgliedsstaatliche Vertellung der Einnahmen und Ausgaben des Gesamthaushalts sowle die der außerbudgetären Aktivitäten zu analysieren und die Ergebnisse $1 \mathrm{~m}$ Hinblick auf die vertellungspolitischen $\mathrm{ziel-}$ setzungen der Gemeinschaft $z u$ beurteilen. Auf dieser Grundlage konnte dann die Aussagefahhigkeit des traditionellen Konzepts der Nettopositionen uberprüft und die Basis für die Berechnung eines erwetterten Indikators geschaffen werden. Ein solcher Versuch ist nunmehr $z u$ unternehmen. 
Die Qualität der Aussage über mitgliedsstaatliche Verteilungswirkungen ist grundsätzlich abhängig davon, inwleweit die finanzwirksamen Gemeinschaftsaktivitäten den Mitgliedsstaaten zuverlässiq als f1nanzielle Belastungen bzw. Vortelle zugerechnet werden können. Hinsichtlich der Finanzierungsmittel sind mit den abzufuhrenden Mehrwertsteuern - und damit bei über der Hälfte der Gesamthaushaltseinnahmen - kaum Probleme verbunden. Dies ist für die zölle und Agrarabschöpfungen aufgrund der Verzerrungen durch den sog. RotterdamAntwerpen-Effekt zwar nicht im gleichen Maße der Fall. Ein Ergebnis der Analyse 1st jedoch, daß diese Verzerrungen die mitgliedsstaatliche Vertellung der Zahlungsströme nicht gravierend beeinträchtigen und damit auch die darauf basierenden Ergebnisse den tatsächlich zu tragenden finanziellen Belastungen weitgehend entsprechen durften.

Dagegen sind die Ausgaben des Gesamthaushalts zum größten Teil nicht eindeutia den Mitgliedsländern als empfangene Leistungen im Sinne unmittelbarer finanzieller Vorteile zuzurechnen. Zweifelsfrel zurechenbar sind mit den Interventionsausgaben im Bereich der strukturpolitik sowle den Erstattungen an die Mitgliedsstaaten nur etra ein Viertel des Ausgabenvolumens. Problematisch bleibt dageqen die mitgliedsstaatliche Aufteilung der Vorteile aus den Agrarausgaben, den Verwaltungsausgaben und der Entwicklungshilfe. Die Verteilungswirkungen der außerbudgetären Gemeinschaftsaktivitäten konnten für den größten Tell der Elnnahmen und Ausgaben der EGKS recht zuverlăssig ermittelt werden. Dagegen sind die Ergebnisse für die Anleihe- und Darlehenstätigkeit mit relativ großen Schätzrisiken verbunden.

Ausgehend von den traditionellen Nettopositionen sind im einzelnen folgende Änderungen $z u$ berilcksichtigen, die beispielhaft fiur das Jahr 1983 in der Tabelle 58 angegeben sind:

- Zur Schätzung der durch den Rotterdam-Antwerpen-Effekt bewirkten Verzerrungen bel den zöllen und Agrarabschöpfungen werden die Ergebnisse des Statistischen Bundesamtes und der Deutschen Bundes- 
Tabelle 58

Korrekturen der traditionellen Nettopositionen $\mathbf{a}_{1983}$

\begin{tabular}{|c|c|c|c|c|c|c|c|c|c|c|c|}
\hline \multirow{2}{*}{ Mitgliedsland } & \multirow{2}{*}{ Zölle } & \multirow[b]{2}{*}{$\begin{array}{l}\text { Agrar- } \\
\text { abschöpfọ. }\end{array}$} & \multirow[b]{2}{*}{$\begin{array}{l}\text { Export- } \\
\text { erst. }\end{array}$} & \multicolumn{2}{|c|}{$\begin{array}{l}\text { Korrekturen } \\
\text { Lager-| WAB }\end{array}$} & \multirow{2}{*}{$\mid \begin{array}{l}\text { Entwick- } \\
\text { lungs- } \\
\text { hilfe }\end{array}$} & \multirow{2}{*}{$\begin{array}{l}\text { U } \\
\text { Verw. }- \\
\text { Ausg. }\end{array}$} & \multirow{2}{*}{ BGKS } & \multirow[b]{2}{*}{$\begin{array}{l}\text { Anl. u. } \\
\text { Darlehen }\end{array}$} & \multicolumn{2}{|c|}{ Surme } \\
\hline & & & & $\begin{array}{l}\text { Lager- } \\
\text { kosten }\end{array}$ & & & & & & a) & b) \\
\hline Belgien & +52 & +66 & -99 & & & +5 & -314 & +11 & + & -270 & -501 \\
\hline Dänemark & & & & & & -5 & +11 & +1 & +11 & +18 & -99 \\
\hline Deutschland & -131 & -97 & +150 & -129 & +196 & +19 & +145 & -10 & -3 & +140 & -197 \\
\hline Frankreich & & & & +129 & -56 & +39 & +104 & -13 & +86 & +289 & +601 \\
\hline Griechenland & & & & & & -5 & +4 & -2 & +19 & +16 & +361 \\
\hline Irland & & & & & & +16 & +2 & -1 & +31 & +48 & -232 \\
\hline Italien & & & & & -34 & -26 & +66 & -17 & +141 & +130 & +377 \\
\hline Luxemburg & & & & & & 0 & -175 & -3 & +1 & -177 & -167 \\
\hline Niederlande & +79 & +31 & $\therefore 51$ & & +70 & +5 & +32 & -6 & -3 & +157 & -454 \\
\hline $\begin{array}{l}\text { Verein. König- } \\
\text { reich }\end{array}$ & & & & & +50 & -51 & +125 & +65 & +52 & +241 & +467 \\
\hline EG & 0 & 0 & 0 & o & +226 & 0 & 0 & +25 & +344 & +595 & +156 \\
\hline
\end{tabular}

a + Vorteile; -: Belastungen

a) als Ergebnis der vorstehenden Spalten.

b) unter Einbeziehung der Verteilung der EAGFL-Ausgaben nach Maßgabe der landwirtschaftlichen Enderzeugung.

Quelle: Eigene Berechnungen. 
bank über die sog. ausgefallenen Zoll- und Abschöpfungserträqe der Bundesrepublik Deutschland herangezogen ${ }^{1}$ und manaels zusätzlicher Informationen vereinfachend unterstellt, daß diese vollständig und entsprechend den anteiligen Zoll-, bzw. Abschöpfungseinnahmen aus dem Handelsverkehr mit Belgien und den Niederlanden resultieren.

- Problematisch ist die Schätzung der den tatsächlichen Vorteilen entsprechenden Exporterstattungen und Laqerhaltungskosten, denn dabel mußte auf die zugrundelieqenden globalen Produktions- und Versorgungsverhältnisse abgestellt werden. Die in Tabelle 58 ausgewlesenen Ergebnisse geben auch nur die Schätzungen an, die aus dem zugrundeliegenden Datenmaterial hinreichend gesichert erscheinen. Dabel handelt es sich um drei Viertel der niederländischen und belgischen Exporterstattungen für Getreideausfuhren, die der Bundesrepublik als empfanqene Gemeinschaftsausgaben zuqerechnet werden mußten, ${ }^{2}$ und die Hälfte der bundesdeutschen Laqerhaltungskosten für Getreide, die vermutlich den französischen Landwirten zugute kommen. 3

- Zumindest ebenso problematisch 1st der Versuch, auch die währungsausgleichsbetrăge (WAB) in die Korrekturen einzubeziehen. Dabei sind auch nur die offensichtlich verzerrt zugerechneten und guantitativ bedeutsamen Haushaltseinnahmen bzw. -ausgaben berücksichtigt und mit der Halfte ihres Betrages berichtigt worden. Die Gegenbuchung für die Partnerländer mußte mangels statistischer Information unberücksichtigt bleiben. ${ }^{4}$

- Von den Entwicklungshilfeausgaben sind die Nahrungsmittellieferungen, die 1983 knapp die Hälfte dieser Ausqaben ausmachten, entsprechend der 1984er Verteilung auf die Mitgliedsstaaten und mit den zugehörigen Finanzierungsanteilen in die Berechnung einbezogen worden. Damlt wird dem Ansatz qefolat, daß die Nahrungsmittellieferungen auch lanqfistiq in der Gemeinschaft nicht absetzbare

\footnotetext{
1Vgl. Tabelle 13, S. 101 und Tabelle 17, S. 112.

2vgl. S. $166 \mathrm{ff}$.

${ }^{3} \mathrm{Vgl}$. S. $173 \mathrm{ff}$.

${ }^{4} \mathrm{Vgl}$. S. $186 \mathrm{f}$.
} 
Uberschüsse darstellen, deren Lagerungs-, Wertminderungs- und Verwertungskosten die haushaltsmäßig ausgewiesenen Beträge gewissermaBen auf Null reduzieren wirden. ${ }^{1}$ Eine solche Bewertung der Nahrungsmittelhilfeausgaben ist zwar extremer Art; angesichts mangelnder zuverlassiger Alternativen und der in Frage kommenden relativ geringen GröBenordnungen dürfte sie im hier vorllegenden Gesamtzusammenhang allerdings vertretbar sein.

- Die Korrekturen der Verwaltungsausqaben resultieren aus einem Ansatz, nach dem die Hälfte der in Belgien und Luxemburg gezahlten Ausgaben diesen Ländern pauschal als empfangene Leistungen zugerechnet und die restlichen Verwaltungsausqaben und die auf sie entfallenden Finanzierungsantelle der Mitgliedsländer aus der Berechnung ausgeklammert werden. Eine solche Aufteilung ist zwar in gewisser Weise wlllkürlich, dürfte den tatsächlich auf die Mitgliedslander entfallenden Vorteilen aber wesentlich eher entsprechen als die bisher praktizierte Zurechnung nach dem $\mathrm{Zah}-$ lungsort. ${ }^{2}$

- Die Angaben für den EGKS-Funktionshaushalt stellen zuverlässig ermittelte Umverteilungswirkungen von etwa zwei Drittel dieses Haushaltsvolumens dar. ${ }^{3}$

- Dagegen beruhen die Ergebnisse für die Anleihe- und Darlehenstätigkelt auf relativ groben Schätzungen liber die indirekten $\mathrm{zins-}$ effekte und die entgangenen $\mathrm{Z}$ inseinnahmen aus den Kapitaleinlagen. 4

Mit diesen Korrekturen tst die Grenze der derzeit quantifiziexbaren Vertetlungswirkungen wohl erreicht. Unberücksichtigt bleiben lediaİch die Ausgaben und Flnanzierungsbeltrăge des Europäischen Entwicklungsfonds, deren Einbeziehung die Ergebnisse faktisch jedoch ntcht verändern würde.

\footnotetext{
${ }^{1}$ Vgl. S. $226 \mathrm{ff}$.

2Vgl. S. 238 .

3 vgl. s. 257 .

${ }^{4}$ Vgl. S. 275 ff.
} 
Tabelle 58 zeigt, das sich die Änderungen in Vorteilen und Belastungen tellweise saldieren, so daß die summen der nationalen Korrekturbeträge zumeist relativ gering bleiben. Eine Aussage dieser globalen Art war im Rahmen der vorangegangenen Untersuchung auch lediglich möglich. Damit werden gleichzeitig die zweifellos vorhandenen Probleme und Unzulänglichkeiten im Ansatz wie im Ergebnis einzelner Schätzungen relativiert. Von ihnen waren von vornherein keine exakten Ergebnisse zu erwarten, sondern sie sollten allein die Grössenordnungen mögl1cher Korrekturen aufzeigen.

In der Tabelle 58 sind welterhin die Korrekturbeträge angegeben, die sich aus dem alternativen Ansatz einer Berücksichtiguna der Auftellung der Agrargarantleausgaben nach Maßqabe der nationalen landwirtschaftilchen Produktionsanteile an Stelle der herkömmlichen Vertellung der zahlungsströme ergeben. ${ }^{1}$ Dabel fällt auf, daß diese Gesamtbetrăge allgemein größer als die vorstehenden Summen aus der korriglerten Zahlungsstromanalyse sind, so daß sich auch stärkere Auswlrkungen auf die Hobe der Nettopositionen ergeben.

Dte tradtttonellen und korrigierten Nettopositionen sind in der Tabelle 59 dargestellt. Bel den von der EG-Kommission ermittelten herkömmlichen Größen (Tabelle 2) handelt es sich um die Werte unter Ełnschluß der Entlastungsregelungen zugunsten des Vereinigten Königrelchs. Dieser Ansatz wird gewählt, da trotz aller Vorbehalte bezuglich des gemeinschaftsoriginăren Charakters der Entlastungsmaßnahmen hterin doch die letztlich gemeinsam beschlossene Politik zum Ausdruck kommt, die in thnlicher weise auch fur die kommenden Jahre festgeschrteben 1st.

${ }^{1}$ Dte in der letzten Spalte der Tabelle 58 ausgewiesenen Beträge resultteren daraus, daß die entsprechenden Differenzen $z w i s c h e n$ der Vertellung nach der landwlrtschaftlichen Enderzeugung und der Vertetlung nach den Zahlungsströmen aus Tabelle $32 \mathrm{mit}$ den Korrekturbetraggen der Entwicklungshilfeausgaben, Verwaltungsausgaben, des EGKS-Tetlhaushaltsvolumens und der Anleihen- und Darlehenseffekte aus Tabelle 58 zusamengefaßt worden sind. 
Tabelle 59

Traditionelle und korrigierte Nettopositionen 1983

\begin{tabular}{|c|c|c|c|c|c|c|}
\hline Mitgliedsland & \multicolumn{3}{|c|}{$\begin{array}{l}\text { Nettopositionen in MIO. ECU } \\
\text { traditionel19 }\end{array}$} & \multicolumn{3}{|c|}{$\begin{array}{c}\text { Nettopositionen pro kopf in ECU } \\
\text { traditionell| }\end{array}$} \\
\hline Belgien & +166 & -104 & $-\quad 335$ & +17 & -11 & -34 \\
\hline Dänemark & +307 & +325 & +208 & +60 & +63 & +41 \\
\hline Deutschland & -2435 & -2295 & -2632 & -40 & $-\quad 37$ & -43 \\
\hline Frankreich & -269 & 20 & +332 & - & + & + \\
\hline Griechenland & +923 & 939 & +1284 & +94 & +95 & +130 \\
\hline Irland & +757 & +805 & +525 & +216 & +230 & +150 \\
\hline Italien & +1140 & +1270 & +1517 & +20 & +22 & +27 \\
\hline Luxemburg & +266 & 89 & +99 & +727 & +243 & +270 \\
\hline Niederlande & +308 & 465 & -146 & +21 & + & \\
\hline Verein. Königreich & -1163 & 922 & -696 & 21 & 16 & \\
\hline
\end{tabular}

a) Korriaierte Betryae aus der Zahlunasstromanalyse.

b) unter Finbeziehung der Vertetlung der EAGFL-Ausqaben nach Maßaabe der landwirtschaftlichen Enderzeuoung.

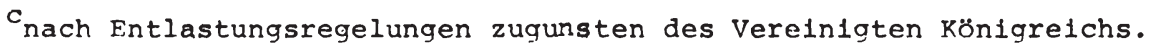

Quelle: Tabelle 2 und elgene Berechnungen. 
Die korrigierten Nettopositionen sind analog zur Tabelle 58 in zwei Versionen angegeben: Neben den aus den korriglerten Zahlunqsströmen resultierenden Werten (a)) sind die Nettopositionen ausgewiesen, die sich unter Berücksichtigung einer Verteilung der Agrargarantieausgaben nach der landwirtschaftlichen Enderzeugung ergeben (b)). Diese Alternative wird herangezogen, um das Spektrum der gerade auch hinsichtlich der Agrarausgaben problematischen Verteilungswirkungen der Zahlungsstromanalyse zu erweitern.

Betrachtet man zunächst die aus der korrigierten Zahlungsstromanalyse resultierenden Nettopositionen, dann ergibt sich im Vergleich $z u$ den traditionellen Größen folgendes Bild:

- Die Bundesrepublik, gefolgt von dem Vereinigten Königreich, bleibt mit Abstand gröBter Nettozahler der Gemeinschaft.

- Die Nettopositionen Belgiens und Luxemburgs werden sich allein aufgrund der veränderten zurechnung der Verwaltungsausqaben erheblich vermindern; dabei wird Belaien sogar zum Nettozahler.

- Dänemark und die Niederlande als relativ wohlhabende Gemeinschaftslunder bleiben Nettoempfänger bzw. bauen ihre Position noch aus.

- Frankrelch verbessert seine Position ebenfalls und wird, wenn auch tn unbedeutendem Umfana, zum Nettoempfäncer. Hier ist allerdings auf die veränderten Nettopositionen des Jahres $1984 \mathrm{zu}$ verwetsen $^{1}$, die dieses Land vermutlich wieder als Nettozahler ausweisen werden.

- Die Nettoempfängernositionen der drei wirtschaftsschwächsten Länder Italien, Irland und Griechenland werden sich nur unwesentItch verändern.

${ }^{1}$ Vgl. Tabelle 2 . 
Unter diesem Gesichtspunkt hat die vorliegende Arbeit also keine völlig neuen Ergebnisse hervorgebracht. Sie hat zwar die Korrekturbedürftigkeit der traditionellen Nettopositionen aufgezeigt und hierfür in den meisten Fällen auch Schätzergebnisse vorgestellt. Daraus wird aber auch deutlich, daß mit den bisher ermittelten Nettotransfers die Verteilungswirkungen der finanzwirtschaftlichen Ebene der EG in ihrer Grundstruktur und in ihren Größenordnungen im wesentlichen zutreffend erfaßt werden.

Einen etwas anderen Eindruck erhält man, wenn an die Stelle der zahlungsstromorlentierten Erfassung der EAGFL-Ausgaben die Vertellung nach den landwirtschaftlichen Produktionsanteilen der Mitgliedsländer tritt:

- Danach bleibt die Bundesrepublik, sogar noch leicht verstärkt, zwar großter Nettozahler der Gemeinschaft. Zieht man die ProKopf Werte heran, dann tritt an die zweite Stelle bereits Belaien. Es folgen mit einigem Abstand das Vereinigte Königreich und nunmehr sogar die Nlederlande, die etwa gleich hohe Nettozahlerpositionen pro Kopf der Bevölkerung aufwelsen.

- Dänemark bletbt, wenn auch etwas abgeschwächt, in einer Nettoempfängerposttton, die nunmehr auch von Frankreich eindeutig eingenommen wird.

- Die dret wirtschaftsschwächsten Länder bleiben nach wie vor Nettoempfänger und rücken in ihren Pro-Kopf Werten näher zusammen.

Diese Ergebnisse bestätigen also teilweise die vorangegangenen Resultate der korrtglerten Zahlungsstromanalyse, teilweise - vor allem für die Niederlande - werden sie modifiziert. 


\section{Normative Ansätze zur Beurteilung der Verteilungswirkungen}

Auf der Grundlage der im vorhergehenden Abschnitt quantifizierten Nettopositionen der EG-Mitgliedsländer ist nunmehr danach zu fragen, wie diese Ergebnisse unter verteilungspolitischen Aspekten zu beurteilen sind. Dabei wird auf die im Abschnitt 2 dieser Arbeit diskutierten Zielsetzungen der EG zurückqeariffen, nach denen der Ressourcen-Transfer von den wirtschaftsstärkeren zu den wirtschaftsschwächeren Gemeinschaftsländern grundsätzlich als Vertellungsmaxime anzusehen ist.

\subsection{Vergleich der Nettopositionen mit der nationalen} Wirtschaftskraft

In einem erstèn, elnfachen Ansatz zur verteilungspolitischen würdigung des gemeinschaftlichen Finanzierungs- und Ausqabensystems werden in Tabelle 60 die mitgliedsstaatlichen Nettopositionen den zugehörigen Sozlalproduktgrößen in einer Pro-Kopf-Betrachtuna gegenübergestellt. Die Höhe des Pro-Kopf BIP wird dabei als Maßstab für die Leistungs- und Umvertellungskraft des jeweiligen Mitgliedslandes herangezogen, und es 1st zu prüfen, in welchem Umfang die mitgliedsstaatlichen Pro-Koof Nettopositionen diesen Relationen entsprechen.

Die Ergebnisse ${ }^{1}$ bestätigen noch einmal die größtenteils bereits in den vorangegangenen Abschnitten getroffenen Aussagen, daß nämlich konsistente Vertellungsw1riunqun im Sinne des Ressourcentransfers nur tellweise vollzogen werden. Sieht man einmal von dem Sonderfall Luxemburg mit selner geringen absoluten quantitativen Relevanz ab, dann wird in bezug auf den hier gewählten Maßstab vor allem Däne-

${ }^{1}$ Im folgenden werden die Nettopositionen, die slch unter Berücksichtigung der Vertellunq der Agrargarantleausgaben nach den Anteilen der landwirtschaftlichen Produktion ergeben, nicht mehr herangezogen. Sle fuhren arößtentells zu ähnlichen Resultaten. 
Tabelle 60

Mitgliedsstaatliche Bruttoinlandsprodukte und Nettopositionen pro Kopf 1983

\begin{tabular}{|c|c|c|c|c|}
\hline \multirow[b]{2}{*}{ Mitgliedsland } & \multicolumn{3}{|c|}{ BIP pro Kopf } & \multirow{2}{*}{$\begin{array}{l}\text { Nettopositionen } \\
\text { pro Kopf in ECU }\end{array}$} \\
\hline & & ECU & $\begin{array}{l}\text { Abweichungen } \\
\text { vom EG-Durch- } \\
\text { schnitt in } \%\end{array}$ & \\
\hline Dänemark & 12 & 458 & $+31,3$ & +63 \\
\hline Deutschland & 11 & 947 & $+25,9$ & $-\quad 37$ \\
\hline Frankreich & 10 & 672 & $+12,4$ & + \\
\hline Niederlande & 10 & 327 & $+8,8$ & +32 \\
\hline Luxemburg & & 754 & $+2,8$ & +243 \\
\hline EG (Durchschnitt) & 9 & 491 & 0 & - \\
\hline Belgien & 9 & 141 & $-\quad 3,7$ & -11 \\
\hline Verein. Königreich & & 013 & $-5,0$ & -16 \\
\hline Italien & 6 & 969 & $-26,6$ & 22 \\
\hline Irland & 5 & 503 & $-42,0$ & +230 \\
\hline Griechenland & 3 & 924 & $-58,7$ & +95 \\
\hline
\end{tabular}

Quelle: Tabellen 1 und 59.

mark, in abgeschwächtem Maße aber auch die Niederlande und Frankreich nicht in genügendem Maße zur Umverteilung herangezogen. Auf der anderen Seite bleibt auch nach den Entlastungsmaßnahmen das Vereinigte königreich noch zu wenig begünstigt, allerdings - im Vergleich etwa zu Belgien - nicht unbedingt mehr in einem gravierendem Ausmaß.

Die dre1 wirtschaftsschwächsten Mitgliedsländer erhalten ganz im Sinne der Vertellungsmaxime durchweg positive Nettotransfers. In diesem Rahmen schelnt Irland uberproportional stark begünstigt, doch ist auf die seit selnem Beitritt stets gestiegenen Nettozah- 
lerpositionen Griechenlands hinzuweisen ${ }^{1}$, die in den vergangenen Jahren bereits eine gewisse Annäherung gebracht haben. Im Vergleich zu diesen beiden Ländern scheint Italien nur unterproportional begünstigt $z u$ werden, während die italienischen Pro-Kopf Vorteile in Relation $z u$ den nächst wirtschaftsstärkeren Mitoliedsländern eher vertretbar sein dürften.

$\mathrm{Ob}$ und inwieweit die deutsche Nettozahlerposition innerhalb der Relationen der Pro-Kopf Sozialprodukte zu rechtfertiaen ist, läßt sich nur im Vergleich mit den übrigen wirtschaftsstarken Mitgliedsländern beantworten. Bei den derzeitigen Verhältnissen ist die Bundesrepublik sicher uberproportional beansprucht. Wïrden auch andere leistungsfuhlge Mitgliedsländer stärker zur Umverteilung herangezogen, könnte die deutsche Nettoposition vermindert und/oder die Mittel etwa zu einem welteren Ausgleich der britischen Nettozahlerposition verwendet werden.

\subsection{Verminderung der Differenzen zwischen den mitgliedsstaat- lichen Pro-Kopf Sozialprodukten als Masstab normativer Umvertellungskraft}

Der bisher durchgeführte Vergleich der Nettopositionen mit den Relationen der mitgliedsstaatlichen Sozlalprodukte gestattet zwar eine erste Wertung der gemeinschaftlichen Verteilungswirkungen an der relativen Wirtschaftskraft der Mitqliedsländer. Dabei bleibt allerdings die Frage nach einem konkreten Maßstab unbeantwortet, d.h. es kann nlcht festgelegt werden, welche Höhe die nationalen Nettopositionen unter normativen Gesichtspunkten aufweisen sollten.

${ }^{1}$ Siehe Tabelle 2. 
Als ein solcher Ansatz kann entsprechend dem Prinzip des Ressourcen-Transfers eine Verteilung der Nettopositionen herangezogen werden, unter deren Einschluß sich die Differenzen zwischen den mitgliedsstaatlichen Pro-Kopf Sozialprodukten um einen bestimmten Prozentsatz reduzieren. Das dazu erforderliche analytische Konzept ist im Mac Dougall-Bericht von Reichenbach entwickelt und von ihm in einem späteren Beitrag als möglicher Ansatz einer normativen Verteilung der gemeinschaftlichen Budgetwirkungen dargestellt worden.

Diese normativen Nettotransfers $\left(N_{i}\right)$ für die einzelnen Mitgliedsstaaten ( $i=1 \ldots$ 10) lassen sich ermitteln, indem man auf das BIP der Gemeinschaft $(Y)$ den gewählten prozentualen Umverteilungsgrad ( $r$ ) anlegt und den so errechneten Wert mit der Differenz aus dem jeweiligen mitgliedsstaatlichen Anteil an der Bevölkerung der Gemeinschaft $\left(D_{i}\right)$ und an dem Gemeinschafts-BIP $\left(y_{i}\right)$ multipliziert ${ }^{2}$ :

$$
N_{1}=r Y\left(p_{i}-y_{i}\right)
$$

Reichenbach hat die entsprechenden Nettopositionen ermittelt, die sich bei elner einprozentigen Verminderung der Differenzen in den nationalen Pro-Kopf BIP ergeben. ${ }^{3}$ Das daraus resultierende Umver-

\footnotetext{
${ }^{1} \mathrm{Vgl}$. Mac Dougall Bericht, Bd. II: Einzelbeiträge und Arbeitsunterlagen, S. $143 \mathrm{ff}$; H. Reichenbach, 1984, S. $458 \mathrm{ff}$.

2 zur Ableitung dieser Beziehung vgl. ebenda.

${ }^{3} \mathrm{Vgl}$. H. Reichenbach, 1984, S. 458. Bezogen auf das in der vorliegenden Arbeit betrachtete Untersuchungsjahr 1983 ergeben sich folgende Werte (in Mio. ECU):
}

\begin{tabular}{l|r} 
Mitgliedsland & $\begin{array}{c}\text { normative Verteilung } \\
\text { der Nettopositionen }\end{array}$ \\
\hline Belgien & +34 \\
Dänemark & -152 \\
Deutschland & -1507 \\
Frankreich & -642 \\
Griechenland & +550 \\
Irland & +139 \\
Italien & +1432 \\
Luxemburg & -121 \\
Niederlande & -121 \\
Verein. Königreich & +270 \\
Umverteilungsvolumen: &
\end{tabular}


teilungsvolumen von 2423 Mio. ECU ist allerdings geringer, als das in diesem Jahr tatsächlich realisierte, das bel den korriqierten Nettopositionen mit 3321 Mio. ECU einer 1,37 sigen Verminderung und bei den traditionellen Nettopositionen mit $3867 \mathrm{Mio}$. ECU ${ }^{1}$ einer 1,60 sigen Reduzierung der nationalen Pro-Kopf BIP Differenzen entspräche.

Legt man nun die aktuellen Umverteilungsvolumina zuarunde und berechnet auf der Basis der entsprechenden höheren Umverteilunqsgrade die zugehörigen normativen Nettopositionen, dann erhält man die in Tabelle 61 ausgewiesenen Werte. Sie stellen für das Jahr 1983 die Verteilung der normativen Nettotransfers unter der Voraussetzung eines unveränderten Umverteilungsvolumens dar. Die Differenzen $z u$ den realen korrigierten bzw. traditionellen Nettopositionen ermöglichen eine Beurteilung dieser Größen an einem konkreten Maßstab.

Die Ergebnisse bestätigen insgesamt die in den vorangegangenen Abschnitten getroffenen Aussagen. Teilweise gehen die Verteilungswirkungen des gemeinschaftlichen Finanzierungs- und Ausqabensystems zumindest der Tendenz nach in die dem Prinzip des Ressourcen-Transfers entsprechende Richtung. Daneben sind allerdings wie zuvor folgende Fehlentwlcklungen festzustellen:

- Die Bundesrepublik als größter Nettozahler der Gemeinschaft stößt zumindest an die Grenzen seiner Belastung bzw. müßte leicht entlastet werden.

- Die lange zeit und intensiv geforderten Fntlastunasregelunaen zugunsten des Verelnigten Königreichs erwelsen sich an diesem Maßstab normativer Verteilungswirkungen nicht nur als gerechtfertigt, sonder: müßten sogar noch verstärkt werden.

${ }^{1}$ Siehe Tabelle 59. 
Tabelle 61

Normative und reale Nettopositionen 1983 (in Mio. ECU)

\begin{tabular}{|c|c|c|c|c|}
\hline Mitgliedsland & $\begin{array}{l}\text { Nomativ } \\
\text { tionen }^{\mathrm{a}} \\
\text { verteilur } \\
1,378\end{array}$ & $\begin{array}{l}\text { ettoposi- } \\
\text { einem Um- } \\
\text { grad von } \\
1,60 \%\end{array}$ & $\begin{array}{c}\text { Differ } \\
\text { korrigierten } \\
\text { Nettopositionen }\end{array}$ & $\begin{array}{l}\text { nzen zu den } \\
\text { traditionellen } \\
\text { Nettopositionen }\end{array}$ \\
\hline Belgien & $+\quad 46$ & +54 & +150 & $-\quad 112$ \\
\hline Dänemark & -209 & $-\quad 244$ & $-\quad 534$ & -551 \\
\hline Deutschland & -2064 & -2411 & +231 & 24 \\
\hline Frankreich & -880 & -1028 & -900 & -759 \\
\hline Griechenland & +753 & +879 & $-\quad 186$ & 44 \\
\hline Irland & +191 & +223 & -614 & 534 \\
\hline Italien & +1962 & +2291 & $+\quad 692$ & +1151 \\
\hline Luxemburg & - & - & 91 & -268 \\
\hline Niederlande & -166 & -194 & - 631 & $-\quad 502$ \\
\hline $\begin{array}{l}\text { Verein. König- } \\
\text { reich }\end{array}$ & $\begin{array}{l}+\quad 371 \\
+\end{array}$ & $+\quad 433$ & +1293 & +1596 \\
\hline
\end{tabular}

a Nettotransfers, die zu einer Verminderung der Differenzen zwischen den mitgliedsstaatlichen Pro-Kopf-BIP um den angegebenen Prozentsatz führen. Dabei entspricht der Umverteilungsgrad von 1,37 8/ 1,60 \& dem Umverteilungsvolumen bei den korrigierten/traditionellen Nettopositionen von 3321 Mio. ECU/3 867 Mio. ECU im Jahre 1983.

Quelle: Eigene Berechnungen auf der Grundlage von H. Reichenbach, 1984 , S. 458 .

- Für einen solchen Ausgleich wären vor allem die wirtschaftsstärkeren Mitgliedsländer Dänemark, Niederlande und Frankreich heranzuziehen, wobei im Falle Frankreichs allerdings auf die im Zeitablauf offenbar stark schwankenden Nettopositionen hinzuweisen ist. 
- Von den drei wirtschaftsschwächsten Gemeinschaftsstaaten wird Irland weit überproportional, Italien dagegen verqleichsweise gering begünstigt.

Der hier gewählte Ansatz zur Bestimmung einer normativen Verteilungsstruktur der gemeinschaftlichen Verteilungswirkungen erlaubt es auch, einen Ausblick auf die Situation nach der Erweiterung der EG um Portugal und Spanien zu werfen. ${ }^{1}$ Unterstellt man dabei einen Umvertellungsqrad von 1 \&, dann führt dies auf der Grundlaqe der Daten des Jahres $1983 \mathrm{zu}$ einem Umverteilunqsvolumen von 3733 Mio. ECU, das etwa der Größenordnung der in der Gemeinschaft der zehn derzeit tatsächlich realisierten Redistributionswirkungen entspricht. Unter dlesen Voraussetzungen müßten den neu aufgenommenen Mitgliedsländern Nettouberweisungen in Höhe von insqesamt 2170 Mio. ECU zufließen. Das sind 6 o des Haushaltsvolumens des Jahres $1986^{2}$, und dieser Wert entspricht den ursprünglich geschätzten Nettokosten für die neuen Gemeinschaftsstaaten. ${ }^{3}$

Verglelcht man nun die Werte der normativen Nettopositionen der erweiterten Gemeinschaft in Tabelle 62 mit den tatsächlich realisierten Größen der zehn Mitgliedsstaaten in Tabelle 59, dann wird deut$11 \mathrm{ch}$, daß selbst bel elner über das normative Maß hinausgehenden Belastung der Bundesrepublik und vor allem des Vereinigten Königretchs die ubrigen Mitgliedsstaaten größtenteils eine gravierende Verschlechterung threr Nettopositionen hinzunehmen hätten, wenn innerhalb des hier angenommenen Umverteilungsrahmens die neuen Mitglledsstaaten entsprechend berücksichtigt werden sollen.

${ }^{1}$ Wie bel Reichenbach, 1984.

2 Nach dem ersten Vorentwurf des Haushaltsplans 1986 beträgt das Budaetvolumen $36051 \mathrm{MlO}$. ECU. Vgl. EG-Kommission, Bulletin 61985 , S. $110 \mathrm{f}$.

$3 \mathrm{vgl}$. S. $138 \mathrm{f}$. 
Normative Nettopositionen der erweiterten Gemeinschaft auf der Basis des Jahres 1983 (Mio. ECU)

\begin{tabular}{|l|c|}
\hline Mitgliedsland & $\begin{array}{c}\text { Normative Nettopositionen bei } \\
\text { einem Umverteilungsgrad von } 1 \text { a }\end{array}$ \\
\hline Belgien & -44 \\
Dănemark & -192 \\
Deutschland & -2000 \\
Frankreich & -1076 \\
Griechenland & +470 \\
Irland & +114 \\
Italien & +979 \\
Luxemburg & -4 \\
Niederlande & -236 \\
Verein. Königreich & -181 \\
Portugal & +629 \\
Spanien & +1541 \\
\hline
\end{tabular}

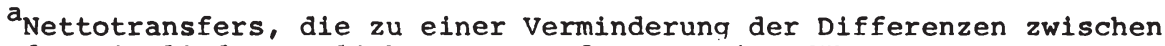
den mitgliedsstaatlichen Pro-Kopf BIP um 1 o führen.

Quelle: Eigene Berechnungen auf der Grundlage von H. Reichenbach, 1984 , S. 458 .

Eine solche Betrachtung macht die künftigen Probleme beim praktischen Vollzug der erweiterten Gemeinschaft deutlich. Sie weist gleichzeitig darauf hin, daß es zunehmend dringlicher wird, die gemeinschaftlichen Vertellungswirkungen möglichst genau und umfassend $z u$ ermitteln und eine den gemeinschaftlichen zielsetzungen entsprechende Vertellungsstruktur zu realisieren. 


\section{Schlußbemerkungen}

Die zielsetzung der vorliegenden Arbeit besteht darin, die Verteilungswirkungen des Finanzierungs- und Ausgabensystems der EG auf breiterer Basis und zuverlässiger als bisher zu quantifizieren und die Resultate anhand der aus gemeinschaftichen Zielsetzungen ableltbaren Maßstäbe zu beurteilen. Dies führt zum elnen zu der Erkenntnis, daß die quantitativ wie qualitativ verbesserten Erqebnisse die bisherigen Berechnungen zwar modifizieren, sie die traditionell ermittelte Verteilungsstruktur aber nur in wenigen Fällen maßgeblich verändern. Zum anderen bestätigt ein Verqleich der tatsächlichen Verteilungswirkungen mit normativen Maßstäben, daß im gemelnschaftlichen Finanzausgleichssystem teilweise durchaus zieladăquate Vertellungswirkungen realisiert werden. Diese Zusammenhänge werden allerdings vor allem dadurch gestört, daß die Nettobelastungen der Bundesrepublik und vor allem des Vereinigten Königreichs uber die vertretbaren Grenzen hinausgehen, und auf der anderen Selte wirtschaftsstarke Mitgliedsländer nicht genügend zur Umvertellung herangezogen werden.

Die Frage, wie durchgehend konsistente Umverteilungen erreicht werden können, llegt außerhalb der Themenstellung dieser Arbeit, so daB darauf abschliebend nur im tberblick eingeganaen werden soll. Insowe1t diese Änderungen uber eine Korrektur der oriqinären Verteilungswirkungen, also vor allem im Rahmen der Einnahmen und Ausgaben des Gesamthaushalts und daneben uber die außerbudgetären Aktivitäten erfolgen soll, 1st auf verschiedene Ansätze zu verweisen.

Als Maßnahme auf der Einnahmenseite des EG-Haushalts ist von der Kommission in threm Grünbuch zur künftigen Finanzierung der Gemeinschaft die Möglichkeit gepruft worden, die Mehrwertsteuerbete1ligung mit progressiven Sätzen entsprechend der relativen wirtschaftskraft der Mitgliedsländer auszugestalten bzw. alternativ eine progressive Sozlalproduktsteuer zusätzlich zur verbrauchsabhängigen Mehrwertsteuer einzufüren. ${ }^{1}$ Abgesehen von den belastungstechnischen

${ }^{1} \mathrm{Vgl}$. EG-Kommission, KOM (83), 10 endg., s. $11 \mathrm{ff}$. 
und vor allem politischen Schwierigkeiten solcher Vorhaben, die im Falle einer Sozialproduktsteuer auch als Rückschritt zum früheren System der Finanzbeiträge angesehen werden, weisen diese Modelle vor allem einen generellen Nachteil auf: Sie stellen ausschließlich auf die Finanzierungsseite ab und beriicksichtiqen nicht, daß die verteilungspolitischen Probleme auch durch die Ausgabenstruktur des Gesamthaushalts bestimmt werden. Ein zielgerichteter Ausgleich müßte konsequenterweise an den globalen Nettopositionen ansetzen.

So werden dann auch in zahlreichen Ausgleichsmodellen die die Ausgabenseite dominierenden Agrarausgaben einbezogen. Dazu gehört der im oben erwähnten Grünbuch vorgetragene Vorschlaq zur Diversifizierung der Mehrwertsteuerregelung 1 ebenso wie andere Verfahren, die die mttgliedsstaatlichen Finanzausgleichsbeträge aus der Differenz zwischen den mit den nationalen Haushaltsfinanzierunas- bzw. Sozialproduktquoten gewichteten EAGFL-Ausgaben und den tatsächlich empfangenen $\mathrm{Zahlungen} \mathrm{bzw.} \mathrm{mit} \mathrm{den} \mathrm{durch} \mathrm{die} \mathrm{nationalen} \mathrm{Anteile}$ an der EG-Agrarproduktion qewichteten ?:ahlunaen ies Arraraarantiefonds ermttteln. ${ }^{2}$ Diese vor allem auf die Korrektur des britischen Ungleichgewichts ausgerichteten Ansätze führen allerdings nicht zu befrłedıgenden Lösungen, da die Ergebnisse des Ausgleichs insqesamt zu unausgewogen sind und vor allem die weniger wohlhabenden Mitgliedsländer stärker als bisher belastet werden.

Als dauerhafte Maßnahme zur Steuerung der gemeinschaftlichen Vertetlungswtrkungen wird von der EG-Kommission und vom Europäischen Parlament seit langem gefordert, den Anteil der Strukturausgaben am EG-Budget bei gleichzeitiger Eindämmung der Agrarausqaben zu erhöhen. Auf diese Weise soll über gemeinschaftlich initiierte

\footnotetext{
${ }^{1}$ Siehe S. 139.

${ }^{2}$ Vgl. v. Petersen, 1983, s. 198 ff.
} 
regional-, sozial- und sonstige strukturpolitische Programe der Ressourcentransfer im Sinne der gemeinschaftlichen Zielsetzunqen gefördert werden. Die wirtschaftsschwächeren Mitgliedsländer werden durch solche Maßnahmen bisher schon überproportional beaünstiat, und darauf sind nicht zuletzt ihre derzeitiaen Nettoempfängerpositionen zurückzuführen. In diesen Rahmen fielen bis 1983 auch die Entlastungsreqelungen zugunsten des Vereinigten Köniareichs und der Bundesrepublik, die damit gleichzeitig als Ausdruck gemeinschaftlicher Politik gewertet werden konnten.

Wie die Entwicklung in den vergangenen Jahren allerdings gezeigt hat, ist der Anteil der Strukturausgaben nicht in dem für die beschriebene $\mathrm{zlelsetzung} \mathrm{erforderlichen} \mathrm{Ausmaß} \mathrm{gestiegen,} \mathrm{da} \mathrm{zum}$ einen die Agrarausgaben nicht nachhaltig reduziert werden konnten und zum anderen das gesamte Ausgabenvolumen schnell den Finanzierungsrahmen ausschöpfte. Eine begrenzte zukünftige Erhöhung des Strukturausgabenanteils kann zudem nur partiell zu einer qünstiaeren Vertellungsstruktur beitragen. Denn einige wirtschaftsstarke Mitgliedsländer wie Dänemark und die Niederlande befinden sich derzett vor allem deshalb in einer Nettoempfängerposition, well dte Zuflusse aus der Agrarpolitik ihre Finanzierungsbeteiliqungen bei wettem ubersteigen.

Angestchts der vielschichtigen Ursachen der ungleichgewichtiqen Verteilungsstruktur sind befriedigende Lösungen vor allem von Finanzausgleichsmodellen $z u$ erwarten, die auf den gesamten Finanztransfer ausgerichtet sind und die sich an gesamtwirtschaftlichen Daten ortentieren. Als Beispiel dafür könnten etwa Ausgleichsbeträge angesehen werden, unter deren Einschluß eine bestimmte Verminderung der Differenzen zwischen den nationalen Pro-Kopf Sozialprodukten erreicht wird, der oben verwendete normative Ansatz also zur effektiv realisierbaren Vorgabe erhoben wird. Daneben sind andere, ähnlich konstruierte Modelle vorstellbar. Ein solcher nach- 
träglicher Finanzausglelch würde die über die Haushaltseinnahmen und -ausgaben bewirkten Ergebnisse der Gemeinschaftspolitik korrigieren und setzt zweifellos einen hohen Integrationswillen insbesondere der gegenüber dem derzeitigen System benachteiligten Mitgliedsländer voraus.

Das bedeutet vor allem, daß die Mitgliedsstaaten die Notwendigkeit eines solchen redistributionspolitisch motivierten Finanzausqleichs auch angesichts der nur qualitativ und sicher unterschiedlich beurtelibaren relativen Vorteils- und Belastungswirkungen auf dem handels-, wirtschafts- und allgemeinpolitischen Gebiet gemeinschaftlicher Politik akzeptieren. Die finanziell belasteten Gemeinschaftsstaaten müssen diesen "Preis" für die Vorteile aus der europäischen Integration zu zahlen bereit sein, und insgesamt alle Länder ein Ausgleichssystem fur die weitere Entwicklung der Gemeinschaft für unbedingt erforderlich halten.

Die Basis solcher Finanzausgleichsmodelle ist in jedem Fall eine klare Vorstellung darüber, welche GröBenordnungen die derzeitiaen Vertetlungswirkungen des gemeinschaftlichen Finanzierungs- und Ausgabensystems uberhaupt aufweisen. Hierzu beizutragen ist das vornehmliche ziel der vorliegenden Arbeit. 


\section{Literaturverzeichnis}

Ahrens, H., von Urff, W., Neuere Tendenzen der EG-internen Einkommenstransfers über den innergemeinschaftlichen Agrarhandel, in: Agrarwirtschaft, 33. Jg., 1984, S. $1 \mathrm{ff}$.

Albers, W., Steuer- und Finanzprobleme in einem gemeinsamen Markt, in: W. Gerloff, F. Neumark (Hrsg.), Handbuch der Finanzwissenschaft, 4. Bd., 2. Aufl., Tübingen 1965, S. 349 ff.

Andel, N., Zur Harmonisierung öffentlicher Ausqaben in einem qemeinsamen Markt, in: Finanzarchiv, N.F. Bd. 24, 1965, S. 1 ff.

Ders., Finanzwissenschaftliche Aspekte regionaler wirtschaftlicher Zusammenschlüsse, a Europäische Gemeinschaften, in: F. Neumark (Hrsg.), Handbuch der Finanzwissenschaft, 3. Aufl., Bd. 4, Tübingen 1983 , S. 329 ff.

Arndt, R., Leitlinien einer Finanzierung der Gemeinschaft, in: Wirtschaftsdienst, $63 \mathrm{Jg} ., 1983, \mathrm{~S} .378 \mathrm{ff}$.

Balassa, B., Trade creation and diversion in the European Common Market: an appraisal of the evidence, in: Balassa, B. (Hrsq.), European Economic Integration, Amsterdam, Oxford u.a. 1975, S. $79 \mathrm{ff}$.

Bartling, H., Landwirtschaft, in: P. Oberender (Hrsg.), Marktstruktur und Wettbewerb in der Bundesrepublik Deutschland, Branchenstudien zur deutschen Volkswirtschaft, München 1984, S. 1 ff.

Bea, F. X., Umsatzsteuern I: Ziele und Ausgestaltung, in: Handwrrterbuch der wirtschaftswissenschaft, Bd. 8, stuttqart u.a.o. 1980 , S. 27 ff.

Beutler, B., Bleber, R., Pipkorn, J., Streil, J., Die Euronäische Gemeinschaft - Rechtsordnung und Politik -, Baden-Baden 1979.

Bickel, W., Der Finanzausgleich, in: Gerloff, W., Neumark, F. (Hrsa.), Handbuch der Finanzwissenschaft, 2. Bd., 2. Aufl., Tilbingen 1956.

Btehl, D., Zur Rolle der öffentlichen Finanzen in der Europäischen Integration, in: Integration, 2. Jq., 1978, S. 35 ff.

Ders., Finanzausgleich IV: Internationaler Finanzausgleich, in: Hanäwörterbuch der Wirtschaftswissenschaft (HdWW), Bd. 2, Stuttgart, Tübingen u.a. 1980, S. 608 ff.

Böhnke, R., Der Intrahandel in der FWG, in: Wirtschaftsdienst, 55. Jg., 1975, S. 208 ff.

Börner, B., Das Interventionssustem der landwirtschaftlichen Marktordnungen der FWG, in: Aqrarrecht der F.WG, Kölner Schriften zum Furonarecht, Köln, Berlin u.a., 1969. 
Borrmann, A., Borrmann, C., Stegger, M., Das Allgemeine Zollpräferenzsystem der EG und seine Auswirkungen auf die Einfuhren aus Entwicklungsländern - Zusammenfassung - HWWA - Institut für Wirtschaftsforschung Hamburg, Sept. 1979.

Britisches Schatzamt, The European Community budget: net contribulutions and receipts, in: Economic Progress Report, Supplement Oktober 1982 .

Buchanan, J. M., Fiscal Theory and Political Economy. Selected Essays, 1960 .

Buchholz, H. E., Agrarmarkt: EWG-Marktordnungen, in: Handwörterbuch der Wirtschaftswissenschaft (HDWW), Bd. 1, stuttgart, New York, Tübingen u.a. 1977, S. $87 \mathrm{ff}$.

Buckwell, A. E., Harvey, D. R., Thomson, K. J., Parton, K. A., The Costs of the Common Agricultural Policy, Beckenham 1982.

Bundesministerium der Finanzen, Finanznachrichten Nr. 27/1980 vom 18.6.1980 und $\mathrm{Nr}$. 30/1983 vom 10.11.1983.

Dass., Finanzbericht 1984, Bonn 1983.

Dass., Der Haushalt der Europäischen Gemeinschaften, Dokumentation, Bonn, versch. Jahre.

Christiansen, R., Die Europäische Zollunion, Erreichtes und Erstrebtes, in: Zeitschrift für Zölle und Verbrauchsteuern, 54. Jg. (1978), S. $354 \mathrm{ff}$.

Cnossen, S., The Netherlands, in: H. J. Aaron (Hrsg.), The ValueAdded Tax, Experiences of some European Countries, Antwerpen, Boston u.a. 1982 .

Conrad, H., Der Gleitzoll, Hamburg, Berlin, Bonn 1955.

Controller General's Report to the Congress of the United States, The Value-Added Tax in the European Economic Community, Washington, 5. Dez. 1980.

Cordts, G., Finanzierung des EWG-Agrarmarktes, in: Institut für das Recht der Europäischen Gemeinschaften der Universität Köln (Hrsg.), Agrarrecht der EWG, Kölner Schriften zum Europarecht, Bd. 10, Köln, Berlin u.a., S. $189 \mathrm{ff.}$

Denton, G., The Economics of Renegotiation, London 1975.

Ders., Der Beitrag Großbritanniens zum EG-Haushalt - eine Untersuchung aus britischer Sicht, in: Integration, 2. Ja., 1979, S. $149 \mathrm{ff}$. 
Ders., Finanzföderalismus und der Haushalt der Europäischen Gemeinschaft, in: Integration, 2. Jg., 1979, S. $1 \mathrm{ff}$.

Deutsche Bundesbank, Monatsberichte, 37. Jg., 1986, Nr. 8 .

Dodsworth, J. R., European Community Financing: An Analysis of the Dublin Amendement, in: Journal of Common Market studies, Vol. 14, 1976, S. $129 \mathrm{ff}$.

Druker, I. E., Financing the European Communities, Leyden 1975.

Edwards, A., Der Haushalt der Europäischen Gemelnschaft: das Konzept der Nettopositionen, 1n: Europa-Archiv, 37. Jq., 1982, S. 747 ff.

Ehle, D., Meier, G., EWG-Warenverkehr. Außenhandel-Zölle-Subventionen, Köln 1971.

Ehlermann, C. C., The Financing of the Community: The Distinctions between Financial Contributions and own Resources, in: Common Market Law Review, Vol. 19, 1982, S. 571 ff.

Emmerson, M. R., Scott, T. W., The Financlal Mechanism in the Budget of the European Community: The Hard core of the British "Renegotiations" of 1974 - 1975, 1n: Common Market Law Review, Vol. 14, 1977, S. 209 ff.

Engelen, K. C., Lănderrislken. Dle Philipplnen und Brasilien sacken stark ab, 1n: Handelsblatt vom 18.4.1984.

Europäische Gemeinschaften, Compte de gestion et bilan financier, afferents aux operations du budget de l'exercise, Vol. I, Analyse de la gestion financière, 1980 - 1984.

Dies., Amt für amtliche Veröffentlichungen. Eurostat revue 1974 1983, Luxemburg 1985.

Dies., Kommission, Antwort auf die schriftliche Anfrage von Herrn L'Estrange vom 31. Januar 1978, in: Amtsblatt der EG, Nr. C 56 vom 6.3.1978, S. $28 \mathrm{f}$.

Dies., Kommission, Gemeinsame Antwort der auf die schriftliche Anfragen Nr. 1020/77 von Lord Bessborough, Nr, 604/78 von Herrn Cointat und $\mathrm{Nr} .607 / 78$ von Lord Bessborough vom 22. Dezember 1978, in: Amtsblatt der EG, Nr. C 28 vom 31.1.1979, S. 2 f.

Dies., Kommission, Antwort auf die schriftliche Anfraqe Nr. 50/79 von Lord Bessborough vom 8. Juni 1979, in: Amtsblatt der EG, Nr. C 104 vom 2.6.1979, S. 9 ff. 
Dies., Kommission, Antwort auf die schriftliche Anfrage Nr. 1260/ 81 der Herren Seeler und Rogalla, in: Amtsblatt der EG, Nr. C 47 vom 22.2.1982, S. 16 .

Dies., Kommission, Antwort auf die schriftliche Anfrage Nr. 289/ 83 von Herrn Kellett-Bowman, Mitteilungen der Sprechergruppe vom 9.8.1983.

Dies., Kommission, Antwort von Herrn Pisani im Namen der Kommission auf die schriftliche Anfrage $\mathrm{Nr}$. 751/83 von Herrn Bonde, Mitteilungen der Sprechergruppe vom 12.10.1983.

Dies., Kommission, Antwort von Herrn Tugendhat im Namen der Kommission auf die schriftlichen Anfragen Nrn. 1680/83, 1681/83 und 1682/83 von Herrn Paisley, Mitteilungen der Sprechergruppe vom 30.5 .1984 .

Dies., Kommission, Entwicklungshilfe, Skizze der Gemeinschaftsaktion von morgen, Bulletin der EG, Beilage $8 / 74$.

Dies., Kommission, Bestandsaufnahme der Wirtschafts- und Finanzlage in der Gemeinschaft seit der Erweiterung und Uberblick über die künftige Entwicklung, KOM (74) 1800 endg., Brüssel, 25.10.1974.

Dies., Kommission, Bestandsaufnahme der gemeinsamen Agrarpolitik, Bulletin der EG, Beilage 2/75.

Dies., Kommission, Unannehmbare Situation und Korrekturmechanismus, KOM (75) 40, Brüssel, 30.1.1975.

Dies., Kommission, Europäischer Fonds für regionale Entwicklung, Erster Jahresbericht, 1975, Bulletin der EG, Beilage 7/76.

Dies., Kommission, Orientierungsrahmen für die Regionalpolitik der Gemeinschaft, Bulletin der EG, Beilage 2/77.

Dies., Kommission (Hrsg.), Bericht der Sachverständigengruppe zur Untersuchung der Rolle der öffentlichen Finanzen bei der Europäischen Integration, Brüssel, April 1977, Bd. I und II, ('Mac Dongall-Bericht').

Dies., Kommission, Vorschlag einer Verordnung (Euratom, EGKS, EWG) des Rates zur Änderung der Haushaltsordnung vom 21. Dezember 1977 für den Gesamthaushaltsplan der Europäischen Gemeinschaften, in: Amtsblatt der EG, Nr. C 160 vom 6.7.1978.

Dies., Kommission, Einbeziehung des Europäischen Entwicklungsfonds in den Haushaltsplan (Mitteilung der Kommission an den Rat), KOM (78) 732 endq., Brüssel, 22.12.1978. 
Dies., Kommission, Arbeitsdokument über Haushaltsfragen, KOM (79) 462 endg. , Brüssel, 12.9.1979.

Dies., Kommission, Konvergenz- und Haushaltsfragen, KOM (79) 620 endg., Brüssel, 31.10.1979.

Dies., Kommission, Konvergenz- und Haushaltsfragen (Mitteilung der Kommission an den Europäischen Rat - Dublin, 29. und 30. November 1979), KOM (79) 680 endg., Brüssel, 21.11.1979.

Dies., Kommission, Entschließung zum Vierten Jahresbericht (1978) der Kommission der EG über den Europäischen Fonds für regionale Entwicklung (ERFE), in: Amtsblatt der EG, Nr. C 117 vom 12.5 .1980 .

Dies., Kommission, Bericht der Kommission der Europäischen Gemeinschaften an den Rat in Durchführung des Mandats vom 30. Mai 1980, Bulletin der EG, Beilage $1 / 81$.

Dies., Kommission, Bericht zu dem Mandat vom 30. Mai 1980, KOM (81) 300 endg. , Brüssel, 24.6.1981.

Dies., Kommission, Mandat vom 30. Mai 1980. Bericht über die Anwendung des Finanzmechanismus, KOM (81) 704 endg., Brüssel, 13.11 .1981 .

Dies., Kommission, Teilnahme an den vom Europäischen Entwicklungsfonds finanzierten Aufträgen, Sammlung Aktuelle Fragen, Reihe Entwicklung, $\mathrm{Nr} .3$, Brüssel 1981.

Dies., Kommission, Die Landwirtschafts- und Agrarhandelspolitik der Gemeinschaft, Grünes Europa, Nr. 187, Brüssel 1981.

Dies., Kommission, Die Industriestrategie der Europäischen Gemeinschaft, Europäische Dokumentation 5/1982.

Dies., Rommission, Memorandum zur Entwicklungspolitik der Europäischen Gemeinschaft, Bulletin der EG, Beilage 5/1982.

Dies., Kommission, Die Agrarpolitik der Europäischen Gemeinschaften, Europäische Dokumentation 6/1982, Luxemburg 1982.

Dies., Kommission (Hrsg.), Gemeinsame Agrarpolitik und Agrarhandel mit den Entwicklungsländern, Grünes Europa, Nr. 192, Brüssel, Nov. 1982 .

Dies., Kommission, Die Finanzierung der Gemeinsamen Agrarmarktpolitik: Der EAGFL, Garantie, Grünes Europa, Nr. 182, Brüssel, Luxemburg 1982 .

Dies., Kommission, Vermögensübersichten und Haushaltsrechnungen der Europäischen Entwicklungsfonds für das Haushaltsjahr 1982, KOM (82) 479 endg., Brüssel, 25.7.1983. 
Dies., Kommission, Die Europaische Gemeinschaft und die Energiefrage, Europäische Dokumentation, 1/1983.

Dies., Kommission, Die Gemeinschaft und die Entwicklungsländer, Stichwort Europa, 3/83, Februar 1983.

Dies., Kommission, Vierter Bericht der Kommission an Rat und Parlament zur Anwendung der Verordnung (EWG) des Rates Nr. 2744/ 80 vom 27. Oktober 1980 betreffend zusätzliche Maßnahmen zugunsten des Vereinigten Königrelchs, KOM (83) 100 endq̣., Brüssel, 1.3.1983.

Dies., Kommission, Stărkung der Effizienz der Strukturfonds der Gemeinschaft, Bulletin der EG, Beilaqe $3 / 83$.

Dies., Kommission, Anpassung der gemeinsamen Agrarpolitik, Bulletin der EG, Bellage $4 / 83$.

Dies., Kommission, Die Sozialpolitik der Europä1schen Gemeinschaften, Europä1sche Dokumentation 5/1983.

Dies., Kommission, Die künftige Finanzierung der Gemeinschaft Vorschlag für elnen Beschluß über dle elgenen Mittel - (Mitteilung der Kommission an den Rat), KOM (83) 270 endg., Brüssel, 6.5 .1983 .

Dies., Kommission, Die Zollunion, Europä1sche Dokumentation 6/1983, Luxemburg 1983.

Dies., Kommission, Vorschlag für elne Verordnung (EWG) des Rates über Zinszuschüsse für bestimmte $1 \mathrm{~m}$ Rahmen des Europä1schen Währungssystems gewăhrte Darlehen, 1n: Amtsblatt der EG, Nr. C 163 vom 22.6.1983.

Dies., Kommission, Korrektur der Haushaltsungleichgewichte (Vermerk der Kommission an den Besonderen Ministerrat), SEK (83) 1759 endg., Brlissel, 7.11.1983.

Dies., Kommission, Europäische Wirtschaft, Nr. 22, November 1984 .

Dies., Kommission, Der Europäische Sozialfonds, Stichwort Europa 2/84, Januar 1984 .

Dies., Kommission, Mitteilung der Kommission an den Rat über die wirtschaftlichen Auswirkungen der Währungsregelung für die Landwirtschaft (1984 liberarbeiteter Text), KOM (84) 95 enda., Brüssel, 26.4.1984.

Dies., Kommisston, Bericht über die Anwendung der Verordnungen (EWG, Euratom, EGKS) Nr. 2891/77 und 2892/77 des Rates vom 19. Dezember 1977 zur Durchführung des Beschlusses vom 21. Apr11 1970 über die Ersetzung der Finanzbeiträge der Mitgliedsstaaten durch eigene Mittel der Gemeinschaften; Vorschlag für eine Verordnung (EGKS, EWG, Euratom) des Rates zur Verlängerung der Verordnung (EWG, Euratom, EGKS) Nr. 2892/77 über die Anwendunq des Beschlusses vom April 1970 über die Ersetzung der Finanzbeiträqe der Mitqliedsstaaten durch eigene Mittel der Gemeinschaften auf die Mehrwertsteuerelgenmtttel, KOM (85) 170 endq., Brüssel, 19.4.1985. 
Dies., Kommission, Die ECU, Europäische Dokumentation 6/1984.

Dies., Kommission, Bericht über die Anleihe- und Darlehenstätigkeit der Gemeinschaft, versch. Jg.

Dies., Kommission, Bulletin der Europäischen Gemeinschaften, versch. Jg.

Dies., Kommission, Europäischer Fonds für reqionale Entwicklung, Bericht, versch. Jg.

Dies., Kommission, Finanzbericht der Europäischen Gemeinschaft für Kohle und Stahl, versch. Jq.

Dies., Kommission, Finanzberichte über den Europäischen Ausrichtungs- und Garantiefonds für die Landwirtschaft, Abteilung Garantie und finanzielle Durchführung der Nahrungsmittelhilfe, Brüssel, versch. Jg.

Dies., Kommission, Finanzbericht des Europäischen Ausrichtungsund Garantiefonds für die Landwirtschaft - Abteilung Ausrichtung, versch. Jg.

Dies., Kommission, Gesamtbericht über die Tätigkeit der Europäischen Gemeinschaften, Brüssel, Luxemburg, versch. Jg.

Dies., Kommission, Die Lage der Landwirtschaft in der Gemeinschaft. Bericht, Brüssel, Luxemburg, versch. Jg.

Dies., Kommission, Tätigkeitsbericht des Europäischen Sozialfonds, versch. Jq.

Dies., Parlament, Bericht im Namen des Finanz- und Haushaltsausschusses uber die Vorschläge der Kommission der Europäischen Gemeinschaften an den Rat (Dok. 99/69 und 147/69) für die Schaffung von Eigenmitteln der Gemeinschaften und die Erweiterung der Haushaltsbefugnisse des Europäischen Parlaments, Berichterstatter: Herr Spénale, Furopäisches Parlament, Sitzungsdokumente 1969 - 1970, Dok. 174 vom 8.12.1969, S. 41.

Dies., Parlament, Bericht im Namen des Haushaltsausschusses über die parlamentarische Kontrolle der Finanztätigkeit des Europäischen Entwlcklungsfonds, Berichterstatter: M. Bangemann, Sitzungsdokumente 1978 - 1979, Dok. 203/78.

Dies., Parlament, Bericht im Namen des Haushaltsausschusses über die Elgenmittel der Gemeinschaft, Berichterstatter: Herr Altiero Spinell1, Europäisches Parlament, Sitzungsdokumente 1980 - 1981, Dok. 1-772/80, 5.1.1981.

Dies., Parlament, Bericht im Namen des Ausschusses für Haushaltskontrolle uber die Intensivierung der politischen Kontrolle uber die Anle1he- und Darlehenstätigkeiten der Gemeinschaften, Berichterstatter: Herr Pierre-Bernard Couste, Europäisches Parlament, Sitzungsdokumente 1981-1982, Dok. 1-175/81 vom 8.5.1981. 
Dies., Parlament, Bericht im Namen des Haushaltsausschusses über den neuen Entwurf des Gesamthaushaltsplans der Europäischen Gemeinschaften für das Haushaltsjahr 1985, Berichterstatter: Herr Ove Fich, Dok. A 2-30/85, 7.5.1985.

Dies., Parlament, Bericht im Namen des Haushaltsausschusses über den Vorschlag der Kommission der Europäischen Gemeinschaften an den Rat (Dok. C2-33/85-KOM (85) 170 endg.) für eine Verordnung zur Verlängerung der Verordnung (EWG, Euratom, EGKS) Nr. 2892/77 uber die Anwendung des Beschlusses vom 21. April 1970 über die Ersetzung der Finanzbelträge der Mitgliedsstaaten durch eigene Mittel der Gemeinschaften auf die MehrwertsteuerEigenmittel und uber den Bericht der Kommission über die Anwendung der Verordnungen (EWG, Euratom, EGKS) $\mathrm{Nr} .2891 / 77$ und $2892 / 77$ des Rates vom 19. Dezember 1977 zur Durchfuhrung des Beschlusses vom 21. April 1970 über die Ersetzung der Finanzbeiträge der Mitgliedsstaaten durch Elgenmittel der Gemeinschaften, Berichterstatter: Herr Petrus A. M. Cornelissen, Dok. A2$126 / 85,21.10 .1985$.

Dies., Rat, BeschluB vom 21. Apr11 1970 über die Ersetzung der Finanzbeltrăge der Mitgliedsstaaten durch eigene Mittel der Gemeinschaften, in: Amtsblatt der EG, Nr. L 94 vom 28.4.1970, S. 19 ff.

Dies., Rat, Beschluß vom 1. Februar 1971 über die Reform des Europäischen Sozialfonds, in: Amtsblatt der EG, Nr. L 28 vom 4.2 .1971 .

Dies., Rat, BeschluB vom 23. Februar 1982 über elnen Beitrag zu Lasten des Gesamthaushaltsplans der Europäischen Gemelnschaften an die Europä1sche Gemelnschaft für Kohle und Stahl, in: Nr. L 74 vom 18.3 .1982 , S. $27 \mathrm{ff}$.

Dies., Rat, Beschluß vom 15. März 1982 zur Änderung des Beschlusses 77/271/Euratom hinsichtlich des Höchstbetrages der EuratomAnlethen, welche die Kommission im Hinblick auf einen Beitraq für die Fłnanzierung von Kernkraftanlagen aufnehmen kann, in: Amtsblatt der EG, Nr. L 78 vom 24.3.1982.

Dies., Rat, BeschluB vom 21. September 1982 über elnen Beitrag zu Lasten des Gesamthaushaltsplans der Gemeinschaften an die Europälsche Gemeinschaft für Kohle und Stahl, in: Nr. L 277 vom 29.9.1982, S. $13 \mathrm{ff}$.

Dies., Rat, Beschluß vom 19. Apr1l 1983 zur Ermächtigung der Kommisston, Im Rahmen des Neuen Gemeinschaftsinstruments Anleihen zur Investitionsforderung in der Gemeinschaft aufzunehmen, in: Amtsblatt der EG, Nr. L 112 vom 24.4.1983.

Dies., Rat, BeschluB vom 7. Mai 1985 über das System der eigenen Mittel der Gemeinschaften, in: Amtsblatt der EG, Nr. L 128 vom 14.5 .1985 . 
Dies., Parlament, Erläuterungen der Kommission zur Festsetzung des Umlagesatzes der EGKS und der Aufstellung des EGKS-Funktionshaushaltsplans für 1983, Sitzungsdokumente 1982 - 1983, Dok. $1-865 / 82$.

Dies., Parlament, Bericht im Namen des Haushaltsausschusses über die Festsetzung des Umlagesatzes der EGKS und über den EGKSFunktionshaushaltsplan für 1983 (Dok. 1-865/82, KOM (82) 706 endg.), Berichterstatter: Herr Konrad Schön, Sitzunqsdokumente 1982 - 1983, Dok. 1-959/82.

Dies., Parlament, Bericht im Namen des Haushaltsausschusses über den Vorschlag der Europäischen Gemeinschaften an den Rat (Dok. 1-936/82 KOM (82) 728 endg.) für eine Verordnung zur Ainderung der Verordnung (EWG) $\mathrm{Nr} .2744 / 80$ betreffend zusätzliche MaBnahmen zugunsten des Vereinigten Königreichs, Berichterstatterin: Frau Carla Barbarella, in: Europäisches Parlament, Sitzungsdokumente 1982 - 1983, Dok. 1-1004/82, 13.12.1982.

Dies., Parlament, Entschließung zur Festsetzung des Umlagesatzes und zum EGKS-Funktionshaushaltsplan für 1983, in: Amtsblatt der EG, Nr. C 13 vom 17.1.1983.

Dies., Parlament, Bericht im Namen des Ausschusses für Haushaltskontrolle uber die Finanztätigkeiten der EGKS, Berichterstatter: Herr Volkmar Gabert, In: Europäisches Parlament, Sitzunasdokumente 1983 - 1984, Dok. 1-464/83.

Dies., Parlament, Bericht im Namen des Landwirtschaftsausschusses uber neue Leitlinien fur die Agrarstrukturpolitik der Gemeinschaft, Teil B: Begrundung, Berichterstatter: Herr Bernhard Thareau, Europäisches Parlament, Sitzungsdokumente 1983 - 1984 , Dok. 1-923/83 B vom 14.11.1983.

Dies., Parlament, Endgültige Feststellung des Gesamthaushaltsplans der Europäischen Gemeinschaften für das Haushaltsjahr .... in: Amtsblatt der EG, versch. Jg.

Dies., Parlament, Wirtschafts- und SozialausschuB, Die Verkehrspolitik der 80er Jahre, Stellungnahme, Brüssel, März 1983.

Dies., Parlament, Bericht Im Namen des Haushaltsausschusses über den geänderten Vorschlag der Kommission der Europäischen Gemeinschaften an den Rat (KOM (84) 384 endg. - Dok. 2-368/84) für einen BeschluB liber das System der eigenen Mittel der Gemeinschaften, Berlchterstatter: Herr Gero Pfennig, Sitzungsdokumente 1984 - 1985, Dok. 2-799/84, 22.10.1984.

Dies., Parlament, Berlcht im Namen des Haushaltsausschusses über die Ablehnung des Entwurfs des Haushaltsplans der Gemeinschaften fur das Haushaltsjahr 1985, Berichterstatter: Herr Ove Fich, S1tzungsdokumente 1984 - 1985, Dok. 2-1185/84, 11.12.1984. 
Dies., Rat, Schlußfolgerungen von der Tagung vom 30. Mai 1980 betreffend den britischen Beitrag zur Finanzieruna des Haushaltsplans der Gemeinschaft, in: Amtsblatt der EG, $\mathrm{Nr}$. C 158 vom 27.6 .1980 , S. 1 .

Dies., Rat, Statut der Beamten der Europäischen Gemeinschaften, in: Amtsblatt der EG, Nr. C 100 vom 28.9.1972.

Dies., Rat, Verordnung Nr. 9 über den Europäischen Sozialfonds, in: 1960 , S. 1189 ff.

Dies., Rat, Verordnung Nr. 25 über die Finanzierung der gemeinsamen Agrarpolitik, in: Amtsblatt der EG vom 20.4.1962.

Dies., Rat, Verordnung (EWG) 950/68 vom 28. Juni 1968 über den Gemeinsamen Zolltarif, in: Amtsblatt der EG, Nr. L 172 vom 22.7 .1968 .

Dies., Rat, Verordnung (EWG) $\mathrm{Nr}$. 974/71 vom 12. Mai 1971 über bestimmte konjunkturpolitische Maßnahmen, die in der Landwirtschaft im Anschluß an die vorübergehende Erweiterung der Bandbrelten der währungen einiger Mitgliedsstaaten zu treffen sind, in: Amtsblatt der EG, Nr. L 106 vom 12.5.1971, S. 1 ff.

Dies., Rat, Verordnung (EWG) Nr. 724/75 vom 18. März 1975 über die Errichtung eines Europäischen Fonds für regionale Entwicklung, in: Nr. L 73 vom 21.3.1975.

Dies., Rat, Verordnung (EWG) Nr. 1172/76 vom 17. Mai 1976 zur Schaffung elnes Finanzmechanismus, in: Amtsblatt der EG Nr. L 131 vom 20.5.1976, S. 7 ff.

Dies., Rat, Verordnung (EWG, Euratom, EGKS) Nr. 2892/77 vom 19. Dezember 1977 uber die Anwendung des Beschlusses vom 21. Apr 11970 uber die Ersetzung der Finanzbeiträge der Mitgliedsstaaten durch elgene Mittel der Gemeinschaften auf die Mehrwertsteuer-Eigenmittel, in: Nr. L 336 vom 27.12.1977, S. $8 \mathrm{ff}$.

Dies., Rat, Verordnung (EWG, Euratom, EGKS) Nr, 2891/77 vom 19. Dezember 1977 zur Durchfuhrung des Beschlusses vom 21. Aprıl 1970 uber die Ersetzung der Finanzbeiträge der Mitgliedsstaaten durch elgene Mittel der Gemeinschaften, in: Nr. I 336 vom 27.12 .1977 , S. 1 ff.

Dies., Rat, Haushaltsordnung vom 21. Dezember 1977 für den Gesamthaushaltsplan der Europäischen Gemeinschaften, in: Amtsblatt der EG, Nr. L 356 vom 31.12.1977, S. 1 ff.

Dies., Rat, Verordnung (EWG) Nr. 214/79 des Rates vom 6. Februar 1979 zur Anderung der Verordnung (EWG) Nr. 724/25 über die Errichtung eines Europäischen Fonds für regionale Entwickluna, in: Amtsblatt der F,G, Nr. L 35 vom 9.2.1979. 
Dies., Rat, Verordnung (EWG) Nr. $1736 / 79$ vom 3. Auqust 1979 über Zinszuschüsse für bestimmte im Rahmen des Europäischen Wăhrungssystems gewährte Darlehen, in: Amtsblatt der EG, Nr. L 200 vom 8.8 .1979 , S. 1 ff.

Dies., Rat, Verordnung (EWG) Nr. 2743/80 vom 27. Oktober 1980 zur Änderung der Verordnung (EWG) Nr. 1172/76 zur Schaffung eines Finanzmechanismus, in: Amtsblatt der EG, Nr. L 284 vom 29.10 .1980 , S. 1 ff.

Dies., Rat, Verordnung (EWG) Nr. 2744/80 vom 27. Oktober 1980 betreffend zusätzliche Maßnahmen zugunsten des Vereinigten Königreichs, in: Amtsblatt der EG, Nr. L 284 vom 29.10.1980, S. 4 ff.

Dies., Rat, Verordnung (EWG) Nr. 682/81 vom 16. März 1981 für die Anpassung des Systems der Gemeinschaftsanleihen zur Stützung der Zahlungsbllanzen der Mitgliedsstaaten, in: Amtsblatt der EG, $\mathrm{Nr}$. L 72 vom 19.3.1981.

Dies., Rat, Verordnung (EWG) Nr. 1785/81 vom 30. Juni 1981 über die gemeinsame Marktorganisation für Zucker, in: Amtsblatt der EG, Nr. L 177 vom 1.7.1981, S. 4 ff.

Dies., Rat, Verordnung (EWG) Nr. 1787/84 vom 19. Juni 1984 betreffend den Europälschen Fonds für regionale Entwicklung, in: Amtsblatt der EG, Nr. I, 169 vom 28.6.1984.

Dies., Rat, Verordnung (EWG) Nr. 2626/84 vom 15. September 1984 zur Anderung von Artikel 1 der Verordnung (EWG) Nr. 3180/78 zur Änderung des Wertes der von Europäischen Fonds für wăhrungspolitische Zusammenarbeit verwendeten Rechnungseinheit, in: Amtsblatt der EG, Nr. L 247 vom 16.9.1984, S. 1 f.

Dies., Rechnungshof, Stellungnahme des Rechnungshofes zu dem Entwurf einer Verordnung (EWG) des Rates zur Abänderung der Haushaltsordnung vom 21. Dezember 1977 für den Gesamthaushalt der Europä1schen Gemeinschaften, in: Amtsblatt der EG, Nr. C 139 vorn 5.6.1979.

Dies., Rechnungshof, Studie über die Finanzsysteme der Europäischen Gemeinschaften (1981), in: Amtsblatt der EG, Nr. C 342 vom 31.12 .1981 .

Dies., Rechnungshof, Bericht des Rechnungshofes der Europäischen Gemelnschaften aufgrund der Erklärung des Europäischen Rates vom 18. Juni 1983, in: Amtsblatt der EG, Nr. C 287 vom 24.10.1983.

Dies., Rechnungshof, Jahresbericht zum Haushaltsjahr... zusammen mit den Antworten der Organe, in: Amtsblatt der EG, versch. Jg.

Dies., Rechnungshof, Statistisches Amt, Konten und Statistiken des Staates 1971 - 1982, Luxemburg 1984. 
Europäische Investitionsbank, Jahresberichte, versch. Jq.

Dies., 25 Jahre, 1958 - 1983, Luxemburg 1983.

Eurostat, Agrarstatistisches Jahrbuch, versch. Jg.

Dass., Employment and Unemployment, Statistical Bulletin 2-1983.

Dass., Monatsbulletin der Außenhandelsstatistik, versch. Jg.

Fennell, R., The Common Agricultural Policy of the Furopean Community, London, Toronto u.a. 1979.

Franke, S. F., Entwicklung und Begründung der Einkommensbesteuerung, Darmstadt 1981 .

Franzmeyer, F., Reglonapolitische Erfordernisse für die weitere Entwicklung der Europäischen Gemeinschaft, in: Konjunkturpolitik, 18. Jg., 1972, S. 28 ff.

Franzmeyer, F., Schultz, S., Schumacher, D., Seidel, B., Einflüsse der EG auf die Regionalpolitik in der Bundesrepublik Deutschland, Göttingen 1975.

Gildsdorf, P., Gemeinschaftssubventionen im Bereich der Landwirtschaft, in: B. Börner, M. Bullinger (Hrsg.), Subventionen im gemeinsamen Markt, Kölner Schriften zum Europarecht, Bd. 29, Köin, Berlin u.a. 1978, S. 215 ff.

Gloystein, P., Finanzierung des industriellen Strukturwandels durch die EG, Hamburg 1978.

Götz, V., Das Finanzierungssystem der Europäischen Gemeinschaften in der Krise, in: K. H. Böckstiegel u.a. (Hrsg.), Finanzverfassung der Europäischen Gemeinschaften, Beilegung internationaler Rechtsstreitigkeiten: Heidelberger Kolloquium aus Anlaß des 70 . Geburtstags von Günter Jaenicke, Studien zum internationalen Wirtschaftsrecht und Atomenergierecht, Bd. 70, Berlin, Bonn u.a. 1984, S. 3 ff.

Gottsmann, 0., Der gemeinsame Agrarmarkt. Texte mit Kommentar. Loseblattsammlung, Baden-Baden.

Grabitz, E., Die Entwicklungspolitik der Europäischen Gemeinschaften, Ziele und Komnetenzen, in: Europarecht, 12. Jg., 1977,

S. $217 \mathrm{ff}$.

Haller, H., Finanzpolitik, 3. Aufl., Tübingen, Zürich 1965.

Ders., Die Steuern, 3. Auf1,. Tübingen 1981.

Harrop, J., The European Investment Bank, in: National Westminster Bank Quarterly Review, Mai 1978, S. 18 ff. 
Ders., An Evaluation of the European Investment Bank: Prospect and Retrospect, Société Universitaire Europeene de Recherches Financières, Series 23A, 1978.

Heck, W., Die neue Europäische Rechnungseinheit, in: Wirtschaftsdienst, $58 \mathrm{Jg} ., 1978$, S. $87 \mathrm{ff}$.

Heiduk, G., Integrationseffekte - Theorie und Praxis, in: Wirtschaftsdienst, 55. Jg., 1975, S. $303 \mathrm{ff}$.

Heming, R. and Kay, J. A., Unitedt Kingdom, in: Aaron, H. J. (Hrsg.), The Value-Added Tax, Experiences of some European Contries, Antwerpen, Boston u.a. 1982.

Henke, K.-D., Die Finanzierung der Europäischen Gemeinschaften. Zur integrationsfördernden Fortentwicklung des europäischen Einnahmensystems, in: D. Pohmer (Hrsg.), Probleme des Finanzausgleichs III. Finanzausgleich im Rahmen der Europäischen Gemeinschaften. Schriften des Vereins für Socialpolitik, N.F., Bd. 36/ III, Berlin 1981, S. 11 ff.

Hrubesch, P., Regelungen des Außen- und Binnenhandels der Europäischen Gemeinschaft mit Agrarprodukten, in: Deutsches Institut für Wirtschaftsforschung, Vierteljahresheft 1/1975, S. 33 ff.

Ipsen, H. P., Europäisches Gemeinschaftsrecht, München 1972 .

Jenkins, M., Britain and the Community Budget: The End of a Chapter, in: Common Market Law Review, Vol. 17, 1980, S. 493 ff.

Jochimsen, R., Treuner, P., Grundprobleme der europäischen Regionalpolitik, in: W. von Urff (Hrsg.), Der Agrarsektor im Integrationsprozeß, Baden-Baden 1975, S. 289 ff.

Killmer, D., Die Europäische Investitionsbank. Eine rechtliche Untersuchung threr Aufgaben, thres Aufbaus und ihrer Geschäftsgrundsätze, Diss., Frankfurt 1963.

Kitterer, w., Belastungswirkungen der Umsatzsteuer: Gutachten im Auftrage des Bundesministers für Wirtschaft. Unter Mitarbeit von J. Fronia, Tübingen: Institut für Angewandte Wirtschaftsforschung, 1981.

Koester, U., The redistributional effects of the common agricultural financial system, in: European Review of Agricultural Economics, Vol. 44, 1977, S. $321 \mathrm{ff}$.

Ders., EG-Agrarpolitik in der Sackgasse, Baden-Baden 1977.

Kolms, H., Finanzwissenschaft IV, 2. Aufl., Berlin, New York 1976.

Krämer, H. R., Instrumente und Gestaltung der Gemeinsamen Agrarpolitik, Publikation des Instituts für Weltwirtschaft an der Universität Kiel, Kiel 1981. 
Leaper, R. A. B., Social Policy, in: D. Lasok, P. Soldatos (Hrsg.), Les communautées européennes en foncitonnement, Brüssel 1981, S. $273 \mathrm{ff}$.

Lipsey, R. G., The Theory of Customs Unions: Trade Diversion and Welfare, in: Economica, 5/1957, S. $40 \mathrm{ff}$.

Littmann, K., Ein Valet dem Leistungsfähigkeitsprinzip, in: H. Ha1ler u.a. (Hrsg.), Theorie und Praxis des finanzpolitischen Interventionismus. Fritz Neumark zum 70. Geburtstag, Tübingen 1970, S. 113 ff.

Lux, M., Allgemeines Zolltarifrecht, in: Regul, R. (Hrsg.), Gemeinschaftszollrecht, Baden-Baden 1982, S. 59 ff.

Marsh, J. S., Swanney, P. J., Agriculture and the European Community, London 1980 .

Masberg, D., Pintz, P., Die Sozialpolitik der Europäischen Gemeinschaft, in: $M$, $v$. Hauff, B. Pfister-Gaspary, Internationale Sozialpolitik, Stuttgart, New York 1982, S. $51 \mathrm{ff}$.

May, B., Kosten und Nutzen der deutschen EG-Mitgliedschaft, Bonn 1982 .

Meade, J. E., The Theory of Customs Unions, Amsterdam 1955.

Ders., The Theory of International Economic Policy, Vol. I, The Balance of Payments, Oxford 1962.

Meyer, B., Die Europäische Investitionsbank zwischen Markt und Lenkung. Untersuchungen zur Wirtschaftspolitik des Instituts für Wirtschaftspolitik an der Universität Köln, Bd. 60, Köln 1984 .

Möller, H., Europäische Gemeinschaften, in: Handwörterbuch der Wirtschaftswissenschaft (HdWW), 2. Bd., Stuttgart, New York u.a. 1980 , S. 472 ff.

Mфller, J. $\varnothing .$, Member States and the Community Budget, Kopenhagen 1982 .

Ders., Der Haushalt der EG, in: Europa-Archiv, 37. Jg., 1982, S. $231 \mathrm{ff}$.

Mohr, E., Die Finanzprobleme der Gemeinschaft - Spiegelbild des Integrationsstandes und -willens der EG -, in: Ifo-Schnelldienst, 33. Jg., 1980, Heft 8, S. 5 ff.

Morris, C. N., The Common Agricultural Policy, in: Fiscal Studies, Vol. 1, 1980, Nr. 2

Musgrave, R. A., Musgrave, P. B., Kullmer, L., die öffentlichen Finanzen in Theorie und Praxis, Bd. 3, Tübingen 1977. 
Narjes, K. H., Die EG ist mehr als ein Haushalt, in: Wirtschaftsdienst, 62. Jg., 1982, S. $111 \mathrm{ff}$.

Neumark, F., Steuern I: Grundlagen, in: Handwörterbuch der Wirtschaftswissenschaft, Bd. 7, S. $295 \mathrm{ff}$.

Nittka, U., Das Finanzierungssystem der Europäischen Gemeinschaften, Bochum 1979.

Nottelmann, A., Der Haushalt der Europäischen Gemeinschaften Probleme und Reformvorstellungen -, Hamburg 1982.

Ott, G., Das Haushaltsverfahren in den Europäischen Gemeinschaften, in: Wirtschaftswissenschaftliches Studium (Wist), 11. Jg., 1982, S. $364 \mathrm{ff}$.

Ders., Das brit1sche Haushaltsproblem im Finanzausqleich der Europäischen Gemeinschäten, Diskussionsbeitrag aus dem Institut für Finanzwissenschaft der Universität Kiel, Nr. 10, Sept. 1983.

Ders., Zur Diskussion um "Zahlmeister" und "Nutznießer" der Europäischen Gemelnschafter, in: W. A. S. Koch, H. -G. Petersen (Hrsg.), Staat, Steuern und Finanzausgleich. Probleme nationaler und internationaler Finanzwirtschaften im zeitlichen Wandel. Festschrift für Heinz Kolms zum 70. Geburtstag, Berlin 1984, S. $333 \mathrm{ff}$.

o. V., Der Frankrelch-Kredit soll die Geldmärkte schonen, in: Handelsblatt Nr. 94 vom 17.8.1983.

o. V., EC: The Evolution of VAT Rates Applicable in the Member States of the Community, in: Intertax, 1984, S. $252 \mathrm{ff}$.

Pedone, A., Italiy, in: H. J. Aaron (Hrsg.), The Value-Added Tax, Experiences of some European Countries, Antwerpen, Boston u.a. 1982 , S. 203 ff.

Peffekoven, R., Das Bestimmungs- und Ursprungslandprinzip bei Steuern im grenzüberschreitenden Verkehr, in: Albers, W. (Hrsg.), Besteuerung und $\mathrm{Zahlungsbilanz.} \mathrm{Schriften} \mathrm{des} \mathrm{Vereins} \mathrm{für} \mathrm{Social-}$ politik, N.F. Bd. 68, Berlin 1972, S. $33 \mathrm{ff}$.

Ders., Einführung in die Grundbegriffe der Finanzwissenschaft, 2. Aufl., Darmstadt 1986.

Ders., Finanzausgleich I: Wirtschaftstheoretische Grundlagen, in: Handwörterbuch der Wirtschaftswissenschaft (HdWW), Bd. 2, stuttgart, Tlubingen u.a. 1980, S. $608 \mathrm{ff}$.

Ders., Verteilung internationaler Finanzierungslasten, in: List Forum, Bd. 11, 1981/82, S. $14 \mathrm{ff}$.

Ders., Das Finanzierungssystem der Europäischen Gemeinschaften, in: Wirtschaftswissenschaftliches Studium (Wist), 11. Jg., 1982, S. 413 . 
Ders., Probleme der internationalen Finanzordnung, in: F. Neumark (Hrsg.), Handbuch der Finanzwissenschaft, 3. Aufl., Bd. IV, Tilbingen 1983, S. 219 ff.

Petersen, V., Agrarpolitik und EG-Haushalt. Gemeinsame Agrarpolitik und der Haushalt der Europäischen Gemeinschaft. Eine Untersuchung des Finanztransfers innerhalb der EG sowie Analyse alternativer Finanzmodelle. Schriftenreihe des Bundesministers für Ernährung, Landwirtschaft und Forsten, Reihe A: Angewandte Wissenschaft Heft 278, Münster-Hiltrup 1983.

Ders., Die Finanzierung der gemeinsamen Agrarpolitik - Eine theoretische und empirische Analyse ihrer Grundlagen, Wirkungen und Alternativen, Agrarwirtschaft, Sonderheft 102, Frankfurt/M. 1984 .

Pfisterer, H., Zur Anlelhe- und Darlehenspolitik der EG, in: Sparkasse, 98. Jg., 1981, S. 415 ff.

Pinder, D., Regional Economic Development and Policy, Theory and Practice in the European Community, London 1983.

Pohmer, D., Allgemeine Umsatzsteuern, in: Handbuch der Finanzwissenschaft, 3. Aufl., Bd. II, Tübingen 1980, S. 647 ff.

Ders., Zur Systematik und Praxis der Mehrwertsteuern unter besonderer Berücksichtigung der Entwicklung in Europa, in: Fischer, I. (Hrsg.), Unternehmung und Steuer, Festschrift zur Vollendung des 80. Lebensjahres von Peter Scherpf, Wiesbaden 1983, S. 375 ff.

Presse- und Informationsamt der Bundesregierung, Bulletin Nr. 68 vom 10.6 .1980 .

Reding, K., Zur Problematik eines Finanzausgleichs in der Europäischen Gemeinschaft, in: $H$. von der Groeben, H. Möller, Möglichkeiten und Grenzen einer Europäischen Union, Bd. 2, Verteilung der wirtschaftlichen Kräfte im Raum, Baden-Baden 1976, S. 199 ff.

Reichenbach, H., Les déséquilibres des flux budgétaires, in: Revue francaise de Finances publiques, 1983, S. $35 \mathrm{ff}$.

Ders., EC Budgetary Imbalances: A Conceptual Framework, in: Finanzarchiv, N.F., Bd. 43, 1984 .

Reister, E., Haushalt und Finanzen der Europäischen Gemeinschaften, Baden-Baden 1975.

Ries, A., Das ABC der Europäischen Agrarpolitik, Baden-Baden 1979.

Rohr, H.-J. Cordts, G., EWG Abschöpfungen und Ausfuhrerstattungen, Kommentar und Texte, Loseblattausgabe, Frankfurt/M., o. J. 
Rollo, J. M. C., Warwick, K. S., The CAP and Resource Flows among EEC Member States, Ministry of Agriculture, Fisheries and Food, London 1979.

Rose, K., Theorie der AuBenwirtschaft, 3. Aufl., Berlin 1970.

Rose, M., Finanzwissenschaftliche Verteilungslehre, München 1977.

Rummel, R., Soziale Politik für Europa. Ein integrationspolitisches Konzept, Bonn 1975.

Scheper, W., Gesamtwirtschaftliche Umverteilungsaspekte der Agrarpolitik, in: C. C. von Weizsäcker (Hrsg.), Staat und Wirtschaft, Schriften des Vereins für Socialpolitik, N.F., Bd. 102, Berlin 1979, S. 559 ff.

Schmidt, K.-G., Die Europäische Investitionsbank, in: R. Regul, H. Wolf ( $\mathrm{Hrsg.)}$, Das Bankwesen im größeren Europa, Baden-Baden 1974 , S. 43 ff.

Schriftliche Anfrage $\mathrm{Nr} .888 / 77$ von Herrn L'Estrange vom 16. Dezember 1977, in: Amtsblatt der EG, Nr. C 56 vom 6.3.1978, S. 28.

Schriftliche Anfrage Nr. 1260/81 der Herren Seeler und Rogalla vom 5. November 1981, in: Amtsblatt der EG, Nr. C 47 vom 22.2.1982, S. 15.

Schriftliche Anfrage Nr. 2258/82 von Herrn E. Kellett-Bowman, EGKommission, Mitteilungen der Sprechergruppe vom 6.5.1983.

Schriftliche Anfrage Nr. $289 / 83$ von Herrn E. Kellett-Bowman, EGKommission, Mitteilungen der Sprechergruppe vom 9.8.1983.

Schriftliche Anfrage Nr. 1682/83 von Herrn I. Paisley, EG-Kommission, Mitteilungen der Sprechergruppe vom 30.5.1984.

Schumacher, H. H., Die Nahrungsmittelhilfe der Europäischen Gemeinschaft. Entstehung, System und Rechtsgrundlagen, Baden-Baden 1981 .

Seidel, M., Ausgestaltung und rechtliche Begrenzungen der Anleihebefugnis der Europäischen Wirtschaftsgemeinschaft, in: Recht der Internationalen Wirtschaft, 23. Jg., 1977, S. $665 \mathrm{ff}$.

Sellekaerts, W., How Maningful Are Empirical Studies on Trade Creation and Diversion?, in: Weltwirtschaftliches Archiv, B. 109, 1973 , S. 519 ff.

Shanks, M., European Social Policy: Today and Tomorrow, Oxford 1977.

Siebels, F., Lenke, H., Kapitalistische Vergesellschaftung in der Landwirtschaft und "Gemeinsamer Agrarmarkt" in der EWG, in: F. Deppe (Hirsg.), Europäische Wirtschaftsgemeinschaft (EWG). Zur politischen రkonomie der westeuropäischen Integration, Reinbek 1975 , S. 338 ff. 
Siebert, H., Zölle IV: Zollunion und Präferenzzonen, In: Handwörterbuch der Wirtschaftswissenschaft (HdWW), Bd. 9, stuttgart/ New York u.a. 1982, S. 666 ff.

Spanakakis, G., Mehrwertsteuer-Eigenmittel für die EG - Verteilung der Lasten auf die Mitgliedsstaaten nicht unproblematisch -, in: Ifo-Schnelldienst, 32. Jg., 1979, Heft 11/12, S. 9 ff.

Stabenow, W., Subventionen im gemeinsamen Markt: Subventionen aus Gemeinschaftsmitteln - Der Europäische Sozialfonds -, in: B. Börner, M. Bullinger (Hrsg.), Subventionen im gemeinsamen Markt, Kölner Schriften zum Europarecht, Bd. 29, Köln, Berlin u.a. 1978 , S. 313 ff.

Stadlmarn, H., Was man in Bonn vor lauter Sparen an Europa nicht vergessen sollte, in: Kommission der EG (Hrsg.), EG-Magazin 10/ 1981 , S. 3 ff.

Stahl, G., Die Lösung läßt noch auf sich warten, in: Wirtschaftsdienst, 61. Jg., 1981, S. $326 \mathrm{ff}$.

Ders., Budgetary Policy of the European Communities: Squarring the Circle, in: Intereconomics, Vol. 18, 1983, S. $213 \mathrm{ff}$.

Steinberg, E. B., Yager, J. A., New Means of Financing International Needs, Washington 1978.

Stobbe, A., Volkswirtschaftslehre I. Volkswirtschaftliches Rechnungswesen, 5. Aufl., Berlin, Heidelberg u.a. 1980.

Strasser, D., Die Finanzen Europas, Luxemburg 1982.

Talbot, R. B., The European Community's Regional Fund, in: D. Diamond, J. B. McLoughlin (Hrsg.), Progress in Planning, Vol. 8, S. $183 \mathrm{ff}$.

Thoroe, C., Die Agrarpolitik in der EG im Konflikt mit Finanzausgleichszielen, in: Agrarwirtschaft, 29. Jg., 1980, S. 78 ff.

Ders., Die Interdependenzen von Agrar- und Regionalpolitik im Hinblick auf die wirtschaftliche und soziale Integration der EG, in: Agrarwirtschaft, 30. Jg., 1981, S. $204 \mathrm{ff}$.

Ders., Die europäische Agrarpolitik als Finanzausgleichsproblem, in: Pohmer, D. (Hrsg.), Probleme des Finanzausgleichs III. Finanzausgleich im Rahmen der Europäischen Gemeinschaften, Schriften des Vereins für Socialpolitik, N.F., Bd. 96/III, Berlin 1981 , S. 85 ff.

Tugendhat, C., Die Kontroverse um die EG-Finanzierung, Der Finanzierungsvorschlag der Kommission, in: Wirtschaftsdienst, 63. Jg. 1983 , S. 371 ff. 
University of Cambridge, Department of Applied Economics, Cambridge Economic Policy Review, Nr. 5, April 1979, S. 23 ff.

Vanden Abeele, M., The Mandate of 30 May 1980, Budget Financing and the Revitalization of the Community: An Unfinished Journey, in: Common Market Law Review, Vol. 19, 1982, S. $501 \mathrm{ff}$.

Vaulant, N., Die Zollunion der Europäischen Wirtschaftsgemeinschaft, Luxemburg 1981.

Viner, J., The Customs Union Issue, New York 1950.

Wäldchen, P., Einzelbericht zur Subventionspraxis des Europäischen Fonds für regionale Entwicklung, in: B. Börner, M. Bullinger (Hrsg.), S. $345 \mathrm{ff}$.

Wagner, O., Die preisdifferenzierenden Wirkungen von Außenhandelsinstrumenten, Abschöpfungen, Zölle und Kontingente im Außenhandel der EWG, Hamburg 1968.

Wahl, J., Die "weitsichtigen" Enkel der Gründungsväter, in: Kommission der EG (Hrsg.), EG-Magazin 7-8/1982, S. 3 f.

Wallace, H., Budgetary Politics: The Finances of the European Communities, London 1980.

Watrin, C., Integrationstheorie. Was ist die EG wert?, in: Der Volkswirt, $\mathrm{Nr} .31 / 1970, \mathrm{~S} .35 \mathrm{ff}$.

Weber, J. H., The Financing of the Common Agricultural Policy, in: Common Market Law Review, Vol. 4, 1966, S. 263 ff.

Weltbank, Weltentwicklungsbericht 1984.

Wiesebach, H. P., Entwicklungspolitik II: Entwicklungshilfe, in: Handwörterbuch der Wirtschaftswissenschaft, Bd. 2, Stuttgart, New York u.a. 1980, S. 407 ff.

Woolley, P. K., The European Investment Bank, in: The Three Banks Review, No. 105, 1975, S. 53 ff.

Zimmermann, H., Henke, K.-D., Einführung in die Finanzwissenschaft, 3. Aufl., München 1982 . 
Günter Ott - 978-3-631-75256-2

Downloaded from PubFactory at 01/11/2019 06:38:19AM

via free access 


\section{FINANZWISSENSCHAFTLICHE SCHRIFTEN}

Band 1 Werner Steden: Finanzpolitik und Einkommensverteilung. Ein Wachstums- und Konjunkturmodell der Bundesrepublik Deutschland. 1979.

Band 2 Rainer Hagemann: Kommunale Finanzplanung im föderativen Staat. 1976.

Band 3 Klaus Scherer: Maßstäbe zur Beurteilung von konjunkturellen Wirkungen des öffentlichen Haushalts. 1977.

Band 4 Brita Steinbach: „Formula Flexibility” - Kritische Analyse und Vergleich mit diskretionärer Konjunkturpolitik. 1977.

Band 5 Hans-Georg Petersen: Personelle Einkommensbesteuerung und Inflation. Eine theoretisch-empirische Analyse der Lohn- und veranlagten Einkommensteuer in der Bundesrepublik Deutschland. 1977.

Band 6 Friedemann Tetsch: Raumwirkungen des Finanzsystems der Bundesrepublik Deutschland. Eine Untersuchung der Auswirkungen der Finanzreform von 1969 auf die Einnahmenposition der untergeordneten Gebietskörperschaften und ihrer regionalpolitischen Zieladäquanz. 1978.

Band 7 Wilhelm Pfähler: Normative Theorie der fiskalischen Besteuerung. Ein methodologischer und theoretischer Beitrag zur Integration der normativen Besteuerungstheorie in der Wohlfahrtstheorie. 1978.

Band 8 Wolfgang Wiegard: Optimale Schattenpreise und Produktionsprogramme für öffentliche Unternehmen. Second-Best-Modelle im finanzwirtschaftlichen Staatsbereich. 1978.

Band 9 Hans P. Fischer: Die Finanzierung des Umweltschutzes im Rahmen einer rationalen Umweltpolitik. 1978.

Band 10 Rainer Paulenz: Der Einsatz finanzpolitischer Instrumente in der Forschungs- und Entwick lungspolitik. 1978.

Band 11 Hans-Joachim Hauser: Verteilungswirkungen der Staatsverschuldung. Eine kreislauftheoretische Inzidenzbetrachtung. 1979.

Band 12 Gunnar Schwarting: Kommunale Investitionen. Theoretische und empirische Untersuchungen der Bestimmungsgründe kommunaler Investitionstätigkeit in Nordrhein-Westfalen 1965-1972. 1979.

Band 13 Hans-Joachim Conrad: Stadt-Umland-Wanderung und Finanzwirtschaft der Kernstädte. Amerikanische Erfahrungen, grundsätzliche Zusammenhänge und eine Fallstudie für das Ballungsgebiet Frankfurt am Main. 1980.

Band 14 Cay Folkers: Vermögensverteilung und staatliche Aktivität. Zur Theorie distributiver Prozesse im Interventionsstaat. 1981.

Band 15 Helmut Fischer: US-amerikanische Exportförderung durch die DISC-Gesetzgebung. 1981.

Band 16 Günter Ott: Einkommensumverteilungen in der gesetzlichen Krankenversicherung. Eine quantitative Analyse. 1981.

Band 17 Johann Hermann von Oehsen: Optimale Besteuerung. (Optimal Taxation). 1982.

Band 18 Richard Kössler: Sozialversicherungsprinzip und Staatszuschüsse in der gesetzlichen Rentenversicherung. 1982.

Band 19 Hinrich Steffen: Zum Handlungs- und Entscheidungsspielraum der kommunalen Investitionspolitik in der Bundesrepublik Doutschland. 1983.

Band 20 Manfred Scheuer: Wirkungen einer Auslandsverschuldung des Staates bei flexiblen Wechselkursen. 1983.

Band 21 Christian Schiller: Staatsausgaben und crowding-out-Effekte. Zur Effizienz einer Finanzpolitik keynesianischer Provenienz. 1983. 
Band 22 Hannelore Weck: Schattenwirtschaft: Eine Möglichkeit zur Einschränkung der öffentlichen Verwaltung? Eine ökonomische Analyse. 1983.

Band 23 Wolfgang Schmitt: Steuern als Mittel der Einkommenspolitik. Eine Ergänzung der Stabilitätspolitik? 1984.

Band 24 Wolfgang Laux: Erhöhung staatswirtschaftlicher Effizienz durch budgetäre Selbstbeschränkung? Zur Idee einer verfassungmäßig verankerten Ausgabengrenze. 1984.

Band 25 Brita Steinbach-van der Veen: Steuerinzidenz. Methodologische Grundlagen und empirisch-statistische Probleme von Länderstudien. 1985.

Band 26 Albert Peters: Ökonomische Kriterien für eine Aufgabenverteilung in der Marktwirtschaft. Eine deskriptive und normative Betrachtung für den Allokationsbereich. 1985.

Band 27 Achim Zeidler: Möglichkeiten zur Fortsetzung der Gemeindefinanzreform. Eine theoretische und empirische Analyse. 1985.

Band 28 Peter Bartsch: Zur Theorie der längerfristigen Wirkungen 'expansiver' Fiskalpolitik. Eine dynamische Analyse unter besonderer Berücksichtigung der staatlichen Budgetbeschränkung und ausgewählter Möglichkeiten der öffentlichen Defizitfinanzierung. 1986.

Band 29 Konrad Beiwinkel: Wehrgerechtigkeit als finanzpolitisches Verteilungsproblem. Möglichkeiten einer Kompensation von Wehrungerechtigkeit durch monetäre Transfers. 1986.

Band 30 Wolfgang Kitterer: Effizienz- und Verteilungswirkungen des Steuersystems. 1986.

Band 31 Heinz Dieter Hessler: Theorie und Politik der Personalsteuern. Eine Kritik ihrer Einkommens- und Vermögensbegriffe. 1987.

Band 32 Wolfgang Scherf: Die beschäftigungspolitische und fiskalische Problematik der Arbeitgeberbeiträge zur Rentenversicherung. Eine Auseinandersetzung mit der Kritik an der lohnbezogenen Beitragsbemessung. 1987.

Band 33 Andreas Mästle: Die Steuerunion. Probleme der Harmonisierung spezifischer Gütersteuern. 1987.

Band 34 Günter Ott: Internationale Verteilungswirkungen im Finanzausgleich der Europäischen Gemeinschaften. 1987. 